\title{
WHOI-83-41 1983
}

Copg 1

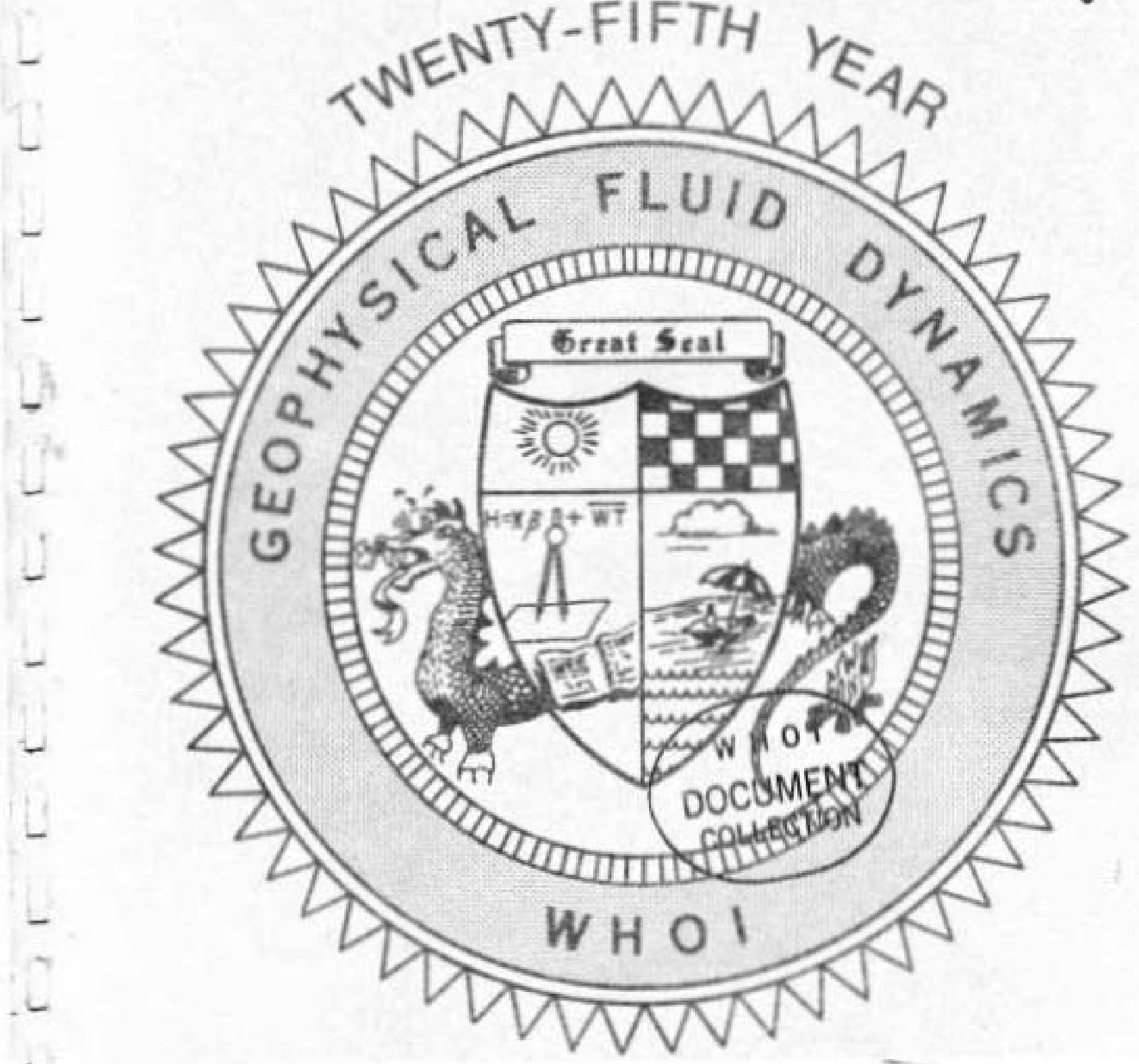

COURSE LECTURES ABSTRAOTSOF SEMINARS LECTUAES OF TI!EFELLOWS 


\title{
Summer Study Program
}

in

\section{Geophysical Fluid Dynamics}

The Woods Hole Oceanographic Institution

\section{Baroclinic Instability and Ocean Fronts}

\section{by}

\author{
Melvin E. Stern, Director \\ and
}

Florence K. Mellor, Editor

Woods Hole Oceanographic Institution

Woods Hole, Massachusetts 02543

November 1983

\section{Technical Report}

Prepared for the Office of Naval Research under Contract N00014-82-G-0079 and the National Science Foundation under Grant MCS-82-00450. Partial support acknowledged from the Office of Naval Research Contract N00014-76-C-0197; NR 083-400.

Reproduction in whole or in part is permitted for any purpose of the United States Government. This report should be cited as: Woods Hole Oceanog. Inst. Tech. Rept. WHOI-83-41.

Approved for public release; distribution unlimited.

Approved for Distribution:

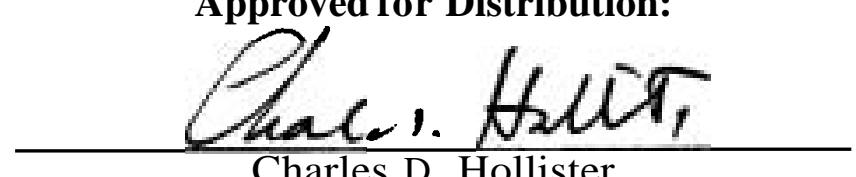

Charles D. Hollister

Dean of Graduate Studies 


\section{SUMMER - 1983}

$\underline{\text { STAFF }}$

Flierl, Glenn

Howard, Louis

Malkus, Willem

Killworth, Peter

Spiegel, Edward

Stern, Melvin

Whitehead, John

Veronis, George
Massachusetts Institute of Technology Florida State University Massachusetts Institute of Technology Cambridge University, England Columbia University University of Rhode Island Woods Hole Oceanographic Institution Yale University

\section{VISITORS}

Aref, Hassan

Armi, Larry

Branscome, Lee

Bretherton, Francis

Brown, Otis

Cushman-Roisin, Benoit

Drazin, Phillip

Garrett, Christopher

Gordon, Arnold

Griffiths, Ross

Hughes, Roger

Ingersoll, Andrew

Martin, Seely

Maxworthy, Tony

Olson, Donald

Osborne, Thomas

Rooth, Claes

Rossby, Thomas

Ruddick, Barry

Watts, Randy

Young, William
Brown University

Scripps Institution of Oceanography

University of Miami

NCAR

Rosenstiel School of Marine Science

Florida State University

Bristol University

Dalhousie University

Lamont-Doherty Geological Observatory

Australian National University

Yale University

California Institute of Technology

University of Washington

University of Southern California

Rosenstiel School of Marine Science

Naval Post Graduate School

Rosenstiel School of Marine Science

University of Rhode Island

Dalhousie University

University of Rhode Island

Scripps Institution of Oceanography 
WOODS HOLE OCEANOGRAPHIC PARTICIPANTS

Meacham, Stephen

Joyce, Terrence

Pedlosky, Joseph

\section{FELLOWS}

Corfield, Charles

Flament, Pierre

Kunze, Eric

Lele, Sanjiva

Mory, Mathieu

Niino, Hiroshi

Paldor, Nathan

Samelson, Roger

Tryggvason, Gretar

Voropaev, Sergey

Zatsepin, Andrey
Columbia University

University of California

University of Washington

Corne11 University

Institut de Mechanique de Grenoble Japan Meteorological Agency

Weizmann Institute of Science'

Oregon State University

Brown University

Moscow University

Moscow University 


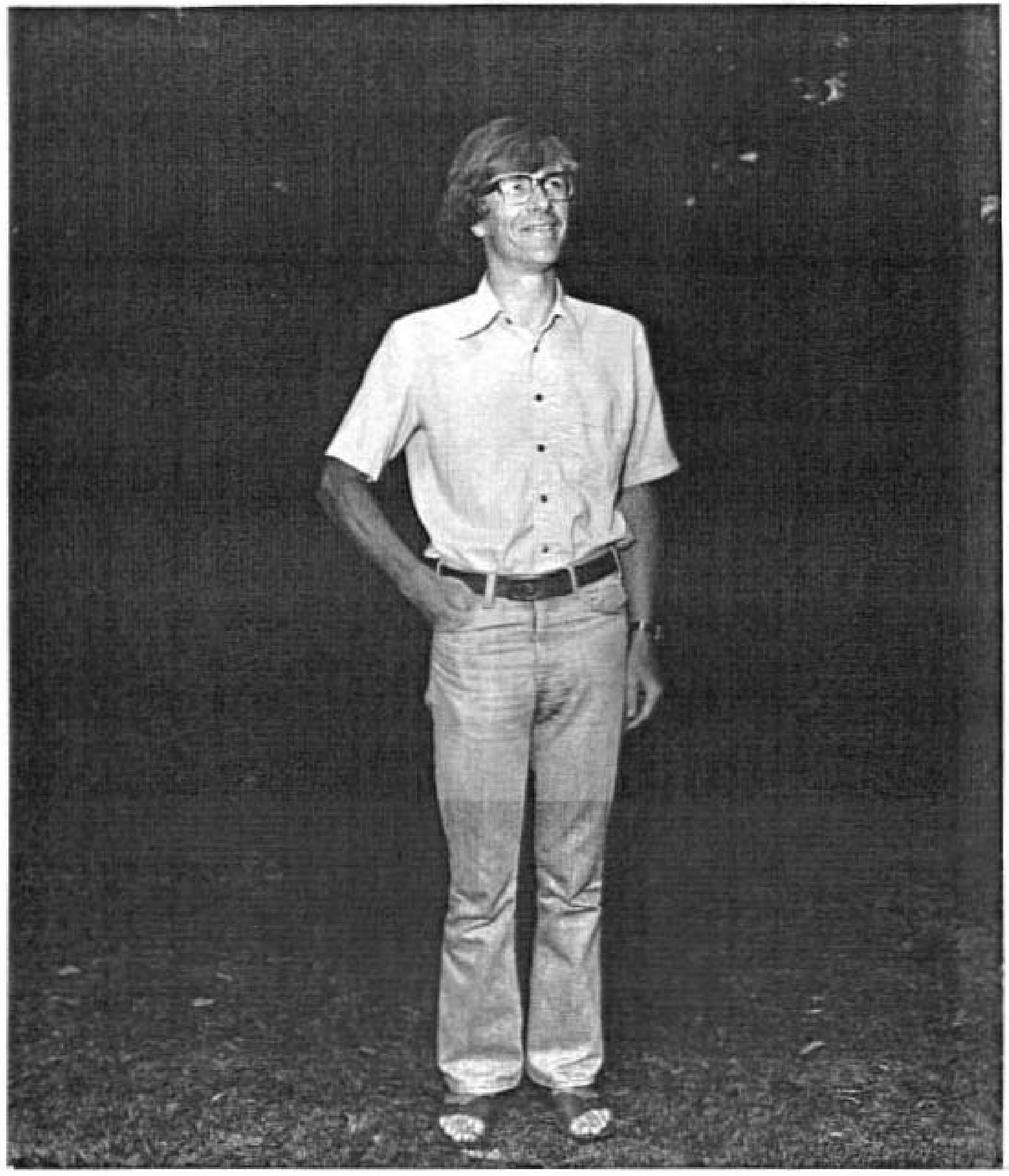

PRINCIPAL LECTURER

Peter Xillworth 


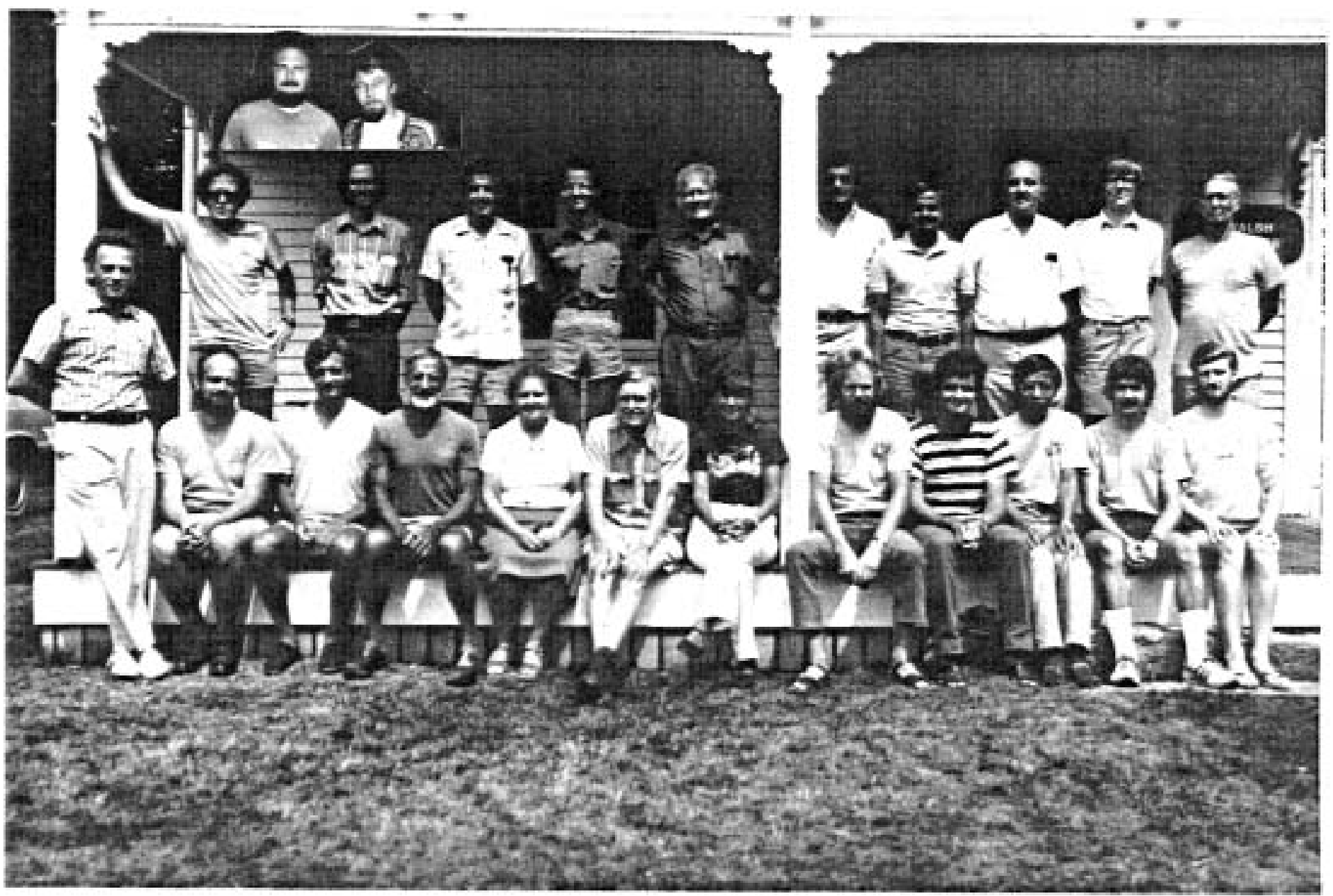

GH FELOWS AND STAFF

Back Row:

Front Row:

Inset:

Absentees:
Paldor, Flier1, Salmon, Kunze, Rooth, Cushman-Roisin, Lele, Stern, Killworth, Malkus

Splegel, Aref, Samelson, Veronis, Mellor, Howard, Jones, Tryggvason, Mory, Niino, Flament, Corfield

Voropaev, Zatsepin

Armi, Branscome, Bretherton, Brown, Drazin, Garrett, Gordon, Griffiths, Hughes, Ingersoll, Joyce, Martin, Maxworthy, Meacham, Olson, Osborne, Pedlosky, Rossby, Ruddick, Watts, Whitehead, Young 


\section{Editor's Preface}

"Baroclinic instability as the largest scale of motion participating in the cross frontal oceanic transport process" was the theme of the 25 th summer program at G.F.D. Killworth (Cambridge University) reviewed classical baroclinic instability theory, leading up to recent studies in which the distinctive structure of an ocean front is included. Finite amplitude baroclinic instability in the classical model was discussed by Pedlosky (WHOI) (and the corresponding effect in the frontal model was studied by one of the G.F.D. Fellows).

Laboratory experiments on baroclinic frontal theory was surveyed by Griffiths (Australian National University) (and one of our Fellows extended that work in Whitehead's (WHOI) lab this summer).

The different kinds of oceanic fronts were surveyed by Joyce (WHOI), and additional observations were supplied by several of the invited staff. The smallest scales of motion relevant to the cross-frontal transfer problem were discussed from the oceanic standpoint by Osborn (Naval Postgraduate School) and from the point of view of laboratory experiments by Ruddick (Dalhousie University), among others. (This subject was also one which was followed up by a G.F.D. Fellow.)

The main theme was supplemented, as in a 11 previous years, by a diversity of geophysical fluid dynamical subjects, the nature of which can be seen in the abstracts included in this report.

The smooth running of the program is due in no small measure to the secretarial expertise of Florence Mellor and Joanne Jones and to the administrative expertise of A.L. Peirson. Finally we thank the Office of Naval Research and the National Science Foundation for their financial support.

Melvin E. Stern 
"Monin-Obukhov" and Stability Theory Applied

to Circular Couette Flow Willem V.R. Malkus . . . . . . . . . . . . . 80

Turbulence Measurement from DOLPHIN

Thomas Osborn . . . . . . . . . . . . . . . . .

JFD (Jovian Fluid Dynamics)

1. Non-Hydrostatic, Quasi-Geostrophic Flow

2. Inertial Oscillations, Barotropic and Baroclinic

Instability in a Rotating Fluid Planet

Andrew P. Ingersoll . . . . . . . . . . . . .

Double-Diffusively Driven Mixing Across Thermohaline

Fronts in the Laboratory

Barry Ruddick

A Three-Layer Baroclinic Instability Analysis and

its Application to Subtropical Fronts in the Ocean

Donald B. Olson ..................

Fronts by Confluence -- The Brazil and Falkland Currents Meet

Arnold L. Gordon . . . . . . . . . . . . . 92

Satellite Derived Kinematics of Warm Core Rings - An Overview

0 t is B. Brown .......... . . . . . . . . . 93

Meandering of the Gulf Stream and Long Wave Instability

Glenn F11er1

Baroclinic Amplitude Vacillation

Philip Drazin ........................

The Gulf Stream: Barrier or Blender or Isopycnal

Signatures of Advection and Mixing

Thomas Rossby . . . . . . . . . . . . . . . . .

Mixing, Waves,. and Gravity Currents in the Laboratory and in Nature

Tony-Maworthy . . . . . . . . . . . . . . . 98

Boundary Forced Nonlinear Planetary Radiation ' ..

Paola Malanotte Rizzoli .. . . . . . . . . . 101

What Maintains the Subtropical Front and its Associated Countercurrent..

Benoit Cushman-Roisin . . ...... . 3 . . . . 103

Turbulently Generated Eastern Boundary Currents

Roger L. Hughes . . . . . . . . . . . . 106

A Two-Layer Model of the Subpolar Gyre and the Subtropica1 Gyre

Rui Xin Huang . . . . . . . . . . . . . . . 106 
Visco-Double-Diffusive Density Intrusions

William R. Young. . . . . . . . . . . . . . . . 107

On the Interaction between Wind and Thermohaline

Forcing in Subtropical Gyres

Claes G.H. Rooth . . . . . . . . . . . . . . . . . . 110

Restraining Baroclinic Instability: Some Effects

of Horizontal Shear

Stephan P. Meacham . . . . . . . . . . . . . . , 112

The Interaction of the Winter Bering Sea Ice Edge with

an Oceanic Front

Seelye Martin , . . . . . . . . . . . . , . , 113

LECTURES OF FELLOWS

Theory and Experiment of Isolated Baroclinic Vortices

Mathieu Mory . . . . . . . . . . . . . . . . . . . . . . . . 114

Langmuir Circulations in a Strong Shear Flow

Sanjiva Lee . . . . . . . . . . . . . . . 133

Free Jet and Frontogenesis Experiments in Shear Flow

Sergey I. Voropaev . . . . . . . . . . . . . . . . . . 147

Waves in a Polytropic Atmosphere

Charles Corfield . . . . . . . . . . . . . . . 160

The Influence of Wave-Wave Interactions on Near-Inertial Waves

Eric Kunze . . . . . . . . . . . . . . . . . . . . . 199

Numerical Experiment on Slumping Gravity Current

Gretar Tryggvason . . . . . . . . . . . . . 207

A Linear Stability Theory of Double Diffusive Horizontal

Intrusioins in a Temperature-Salinity Front

Hiroshi Niino. . . . . . . . . . . . . . . . . . 229

Weakly Nonlinear Frontal Waves

Nathan Paldor . . . . . . . . . . . . . . . 245

A Multi-Timing Analysis of the Heat-Up Problem

Roger Samelson . . . . . . . . . . . . . . . 257

Some Experiments on Rotating Baroclinic Vortices

Andrey Zatsepin . . . . . . . . . . . . . . . . . 272

Some Three-Dimensional Aspects of Coastal Upwelling

Pierre J. Flament . . . . . . . . . . . . . 286 
In addition, we have (empirical) equations of state for $\rho$ and $\eta$ as functions of the state variables $\mathrm{S}, \mathrm{T}$, $\mathrm{p}$ :

$$
\begin{aligned}
& \rho=\rho(s, T, p) \\
& 1=1(s, T, p)
\end{aligned}
$$

Equations (2) to (6) are a set of 8 equations in 8 unknown fields, first order in time, which, with top, bottom, and lateral boundary conditions, completely describes the motion. A discussion of the boundary conditions is found in Gill (1982).

Conservation of energy per unit mass can be derived by taking $\underline{\mathrm{v}}$ - (3), while conservation of vorticity is obtained by taking $\mathrm{V} \mathrm{x}$ (3).

This system of primitive equations describes a wide class of possible motions; in the next pages, it will be simplified to filter out processes that have little effect on large-scale oceanic motions.

\section{Isentropic Approximation}

A rigorous treatment of the molecular transport terms is found in Kamenkovich (1977). It is not reproduced here because dissipation enters most oceanographic models often through crude parameterization of smaller scale processes rather than directly through molecular diffusion.

Wave motion and large-scale flows are adequately described by setting $\mathrm{D}_{\boldsymbol{\eta}}=0, \mathrm{D}_{\mathbf{s}}=0 \mathrm{in}$ (4) and (5) (this-approximation filters out differential diffusive processes). However, in many cases the stability of inviscid flows is quite different from that of a viscous fluid, and $D_{\mathbf{n}}$ should be kept in (3). This is thermodynamically inconsistent, but the viscous dissipation of a 11 the kinetic energy of $1 \mathrm{~kg}$ of water with fluid velocities of $0.1 \mathbf{m s}^{-1}$ would only raise the temperature by a neglectible $10^{-6} \mathrm{~K}$.

The disagreeable entropy is then readily eliminated from the equations (after some nontrivial manipulation of Maxwell relations). Plug (6b) into (5), and get:

$D_{t} T=\frac{\alpha T}{c_{p}} D_{t} p$

where $\alpha(\mathrm{s}, \mathrm{T}, \mathrm{p})=-\frac{1}{\rho}\left(\frac{\partial \rho}{\partial T}\right)_{P, S}{ }^{\mathrm{C}}$ is the coefficient of thermal expansion and $c_{p}(s, T, p)=T\left(\frac{\partial M}{\partial T}\right)$ P,S the specific heat. (The coefficient in the RHS is the adiabatic gradient of temperature).

Plugging (6) into (2), we can also derive

$\mathrm{D}_{\mathrm{t} \rho}=\frac{1}{\mathrm{c}^{2}} \mathrm{D}_{\mathrm{t}} \mathrm{P}$
where $\mathrm{c}(\mathrm{S}, \mathrm{T}, \mathrm{P})=\left(\frac{\partial \rho}{\partial \rho}\right)_{\eta, s^{\prime}}$ is the sound velocity. (The coefficient in the RHS is the adiabatic gradient of density). 


\section{Incompressibility Approximation}

The period of acoustic waves is generally less than $1 \mathrm{~s}$, much less than the periods of other oceanic waves; acoustic waves have a group velocity of $c_{2} \sim 1.510^{3} \mathrm{~ms}^{-1}$, while the fastest surface gravity wave has

$\sim 210^{2} \mathbf{m s}^{-1}$, and most other waves - internal, planetary - have $\mathbf{c g}^{\prime} \mathbf{s}$ less than $1 \mathbf{~ m s}^{-1}$; a fortiori, $c$ is much larger than any particle velocity, and we can expect sound waves to play no role in the dynamics of the ocean.

Plugging eq. (8) in eq. (2), we get:

$$
\underline{\nabla} \cdot \underline{v}=\frac{-1}{c^{2} p} D_{t} p
$$

which, for $\mathrm{c} \rightarrow \infty$, is:

$$
\underline{\nabla} \cdot \underline{\mathrm{V}} \sim 0
$$

This approximation makes the fluid kinematically incompressible, while retaining the effects of compressibility in (7) and (8) sound waves are filtered out. Equation (9) means that, in (2), $\partial v_{i} / \partial x_{i} \gg D_{t} \rho$ and that the main balance is between the components of the divergence.

\section{Boussinesq Approximation}

Assume that the fields can be decomposed in an adiabatic, hydrostatic equilibrium state $\left({ }^{\circ}\right)$, and a perturbation component ('):

$$
\begin{aligned}
& \rho=\rho^{\circ}(\underline{r})+\rho^{\prime} \\
& v=v^{\prime} \\
& \bar{S}=s^{\circ}(\underline{r})+s^{\prime} \\
& T=T^{\circ}(\underline{r})+T^{\prime} \\
& P=p^{\circ}(\underline{r})+p^{\prime}
\end{aligned}
$$

To be a solution of the equations, the equilibrium state must satisfy

$$
\begin{aligned}
\rho^{\circ} & =\rho^{\circ}\left(\mathrm{s}^{\circ}, \mathrm{T}^{\circ}, \mathrm{p}^{\circ}\right) \\
\underline{\nabla} \mathrm{p}^{\circ} & =\rho^{\circ} \underline{\nabla} \phi
\end{aligned}
$$

Plug (10) in (3), (7) and (8), use (1la) and (11b), replace remaining

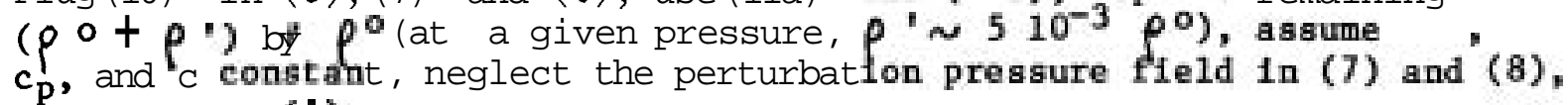
and drop the (").

The final system of equations for an isentropic, incompressible, Boussinesq fluid is:

$$
\begin{aligned}
& \mathrm{D}_{\mathrm{t}} \rho=-\underline{\mathrm{v}} \cdot\left(\underline{\nabla} \rho^{0}+\frac{\rho_{0}}{\mathrm{c}^{2}} \underline{\nabla} \phi\right) \\
& \underline{\nabla} \cdot \underline{\mathrm{v}}=0 \\
& \mathrm{D}_{\mathrm{t}} \mathrm{v}=-\frac{1}{\rho_{0}} \underline{\nabla} \mathrm{p}-\frac{\rho}{\rho_{0}} \underline{\nabla} \phi-2 \underline{\Omega} \underline{\underline{v}}+\underline{D}_{\mathrm{v}}
\end{aligned}
$$




$$
\begin{aligned}
& \mathrm{D}_{\mathrm{t}} \mathrm{S}=\underline{-\underline{v}} \cdot \underline{\nabla} \mathrm{s}^{\circ} \\
& \mathrm{D}_{\mathrm{t}} \mathrm{T}=\underline{-\underline{\mathrm{v}}} \cdot\left(\underline{\nabla} \mathrm{T}{ }^{\circ}-\frac{\alpha_{\mathrm{T}}^{0}}{\mathrm{c}_{\mathrm{p}}} \underline{\nabla} \phi\right)
\end{aligned}
$$

These equations are a system of 7 equations in the 7 unknown perturbation fields $P, \underline{v}, s, T, p$.

Note that $S$ and $T$ have lost their dynamical role, and are simply advected by the velocity field $\stackrel{\nu}{ }$. consequently we will drop them in the subsequent discussion.

\section{Tangent Plane Approximation}

Equipotential surfaces of the earth's gravity and centrifugal field are oblate ellipsoids, with small axis along the axis of rotation, and eccentricity e $\sim 11300$; the equilibrium sea level is one of these surfaces.

$\underline{z}=-\nabla \phi$, the gradient of geopotential, defines the local vertical. The relative variation of $\mathrm{g}$ is $10^{-3}$ from equator to pole and through the depth of the ocean: it has no dynamical effects and can be ignored in (12) and (14).

The natural coordinate system would be the oblate ellipsoidal coordinates; Veronis (1973) explicitly writes (12) to (14) in that system.

The principal effect of sphericity is the variation of the angle $(\Omega, g$ ) with latitude -- and hence of the horizontal components of the Coriolis acceleration, but the exact oblate ellipsoidal metric contains several other geometric effects of minor dynamical importance: poleward convergence of equipotentials and meridians, downward convergence of verticals, which may be neglected.

Because of the mathematical complication of the full ellipsoidal metric, it is customary to introduce an approximate cartesian metric in the neighborhood of a reference point Po, adequate to model meso-scale oceanic motions. The full derivation is found in Mysak and LeBlond (1979). Assume the scales: H, depth of the ocean; L, lateral scale of the motion; R, radius of the earth; $U$ and $W \sim U H / L$, horizontal and vertical velocities. Provided that

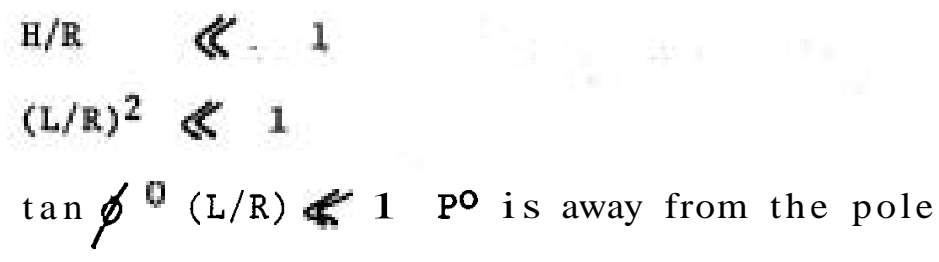

a simplified form of (12) to (14) can be written in a local cartesian system centered at $\mathrm{p}^{\circ}\left(\lambda^{\circ}, \varnothing^{\circ}, \mathrm{R}\right)$, with $\mathrm{x}$ eastward, y northward and $z$ upward:

$$
D_{t} P=w \frac{\rho}{g} \mathrm{~N}^{2}
$$




$$
\begin{aligned}
& \partial_{x^{u}}+\partial_{y^{v}}+\partial_{z^{w}}=0 \\
& D_{t^{u}}=\frac{-1}{\rho^{0}} \partial_{x} p+\left(f^{\circ}+\beta y\right) v+\tilde{f}_{w}+D_{x} \\
& D_{t^{v}}=\frac{-1}{\rho^{0}} \partial_{y} p-\left(f^{\circ}+\beta y\right) u+D_{y} \\
& D_{t^{w}}-\frac{u^{2}+v^{2}}{R}=\frac{1}{\rho^{0}} \partial_{z} p-\frac{\rho}{\rho^{0}} 8-\tilde{f} u+D_{z}
\end{aligned}
$$

where

$$
\begin{aligned}
& \mathrm{D}_{\mathrm{t}}=\partial_{\mathrm{t}}+\mathrm{u} \partial_{\mathrm{x}}+\mathrm{v} \partial_{\mathrm{y}}+\partial_{\mathrm{z}} \\
& \mathrm{f} 0=2 \Omega \sin \phi^{\circ} \sim 10^{-4} \mathrm{~s}^{-1} \\
& \tilde{\mathrm{f}}=2 \Omega \cos \phi \circ \\
& \beta=\tilde{\mathrm{f}} / \mathrm{R} \sim 210^{-11} \mathrm{~m}^{-1} \mathrm{~s}^{-1}
\end{aligned}
$$

and

$$
\left.N^{2}(z)=-\frac{g}{\rho_{0}} \partial_{z} \rho 0+\frac{g^{2}}{c^{2}}\right)
$$

is the buoyancy frequency of the adiabatic equilibrium state.

Except for geometric distortions intolerable only at the planetary scale, damage to the physics has been minimal so far. Most oceanic motions, surface waves, internal waves, and planetary waves are still fully described by this system of equations.

The coming simplifications are the most drastic - the hydrostatic approximation will low pass surface gravity waves and internal gravity waves; the quasigeostrophic approximation, developed in lecture 2, will remove gravity waves altogether.

\section{Hydrostatic Approximation.}

Provided that

$$
\begin{aligned}
& \mathrm{H} / \mathrm{L} \ll 1, \quad \text { the aspect ratio is small } \\
& \cot \phi^{\circ}(\mathrm{H} / \mathrm{L}) \ll 1, \quad \text { Po }^{\mathrm{i}} \text { is away from the equator }
\end{aligned}
$$

the main balance in (19) is hydrostatic:

$$
0=-\frac{1}{\rho^{0}} \partial_{z^{P}}-\frac{\rho}{\rho^{\circ}} g
$$




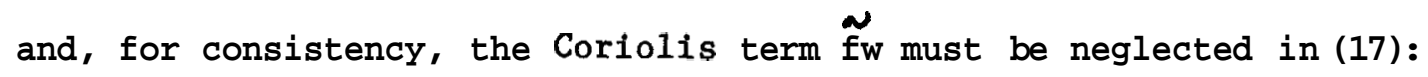

$$
\mathrm{D}_{\mathrm{t}} \mathrm{u}=-\frac{1}{\rho \circ} \partial_{\mathrm{x}} \mathrm{p}+(\mathrm{fo}+\beta \mathrm{y}) \mathrm{v}+\mathrm{D}_{\mathrm{x}}
$$

ag can be verified by a worst-case order-of-magnitude estimation; if

P/P० $\sim 10^{-3}$, U $\sim 10^{-1} \mathrm{~ms}^{-1}, \mathrm{~W} \sim \mathrm{UH} / \mathrm{L}_{\mathrm{p}} \mathrm{H} \sim 10^{3} \mathrm{~m}, \mathrm{~L} \sim 10^{3} \mathrm{~m}$,

the magnitude of the terms in (19) are:

$$
\begin{aligned}
& \mathrm{D}_{\mathrm{t}} \sim 10^{-5} \\
& \mathrm{U}^{2} / \mathrm{R} \sim 210^{-4} \\
& \mathrm{~g} \rho / \rho \circ \sim 10^{-2} \\
& \mathrm{fu} \sim 10^{-5}
\end{aligned}
$$


Initial comments

The quasigeostrophic approximation assumes large-scale flow structures, and geostrophy in the fundamental balance. It has the advantage that it describes the dynamic's in terms of a single dynamic variable, the potential vorticity,

$$
\nabla^{\alpha} \psi+\frac{\partial}{\partial z}\left[\frac{f^{\alpha}}{N^{*}} \frac{\partial \psi}{\partial z}\right]+\beta y .
$$

Some disadvantages of the method are i) it is still nonlinear; ii) by assuming small Rossby number Ro $=U / f L$, one is restricted to low frequency $(\omega<<f)$, large-scale flows which may not include important physical phenomena such as fronts. Conservation of potential vorticity can be derived without first invoking quasigeostrophy (Charney, 1973; Pedlosky, 1979); and iii) only small perturbations of the stratification are allowed. Note that the potential vorticity is difficult to measure in the ocean since it involves determining horizontal gradients of the geostrophic flow, 1.e., from arrays of instruments. These gradients can be contaminated by noise data and underestimated by inadequate sampling.

Equations of Motion: from Lecture 1

Conservation of mass: (really conservation of volume or continuity)

$$
\frac{\partial u}{\partial x}+\frac{\partial v}{\partial y}+\frac{\partial w}{\partial z}=0
$$

Conservation of momentum:

$$
\begin{aligned}
\frac{\partial u}{a t}+(\vec{u} \cdot \vec{\nabla}) u-f v & =-\frac{\partial P}{\rho_{*}} \frac{\partial P}{\partial x}+F_{x} \\
\frac{\partial v}{\partial t}+(\vec{u} \cdot \vec{\nabla}) v+f u & =-\frac{1}{\rho_{*}} \frac{\partial P}{\partial y}+F_{y} \\
\frac{\partial P}{\partial z} & =-\rho g
\end{aligned}
$$

Hydrostatic balance:

Conservation of buoyancy:

$$
\frac{\partial \rho}{\partial t}+(\vec{u} \cdot \vec{\nabla}) \rho=Q
$$

Informal Derivation

Neglecting forcing terms Fx, Fy, and $Q$ and assuming a geostrophic/ hydrostatic balance, the equations of motion to lowest order reduce to:

$$
\begin{aligned}
-f_{0} v_{0} & =-\frac{1}{\rho} \frac{\partial p_{0}}{\partial x} \\
f_{0} u_{0} & =-\frac{1}{\rho} \frac{\partial p_{0}}{\partial y} \\
\frac{\partial p_{0}}{\partial z} & =-\rho .9
\end{aligned}
$$


These equations are degenerate. To obtain prediction equations, one must go to the next order

$$
\begin{aligned}
& \frac{D_{0} u_{0}}{D t}-f_{0} v_{1}-\beta y v_{0}=-\frac{1}{\rho_{0}} \frac{\partial P_{1}}{\partial x} \\
& \frac{D_{0} v_{0}}{D t}+f_{0}+\beta y u_{0}=-\frac{1}{\rho_{0}} \frac{\partial P_{1}}{\partial y} \\
& \frac{\partial u_{1}}{\partial x}+\frac{\partial v_{1}}{\partial y}+\frac{\partial w_{1}}{\partial z}=0 \\
& \frac{\partial P_{1}}{\partial z}=-\rho_{1} g ; \quad \frac{D \cdot \rho_{0}}{D t}+\frac{\partial \bar{\rho}}{\partial z} \omega_{1}=0 \\
& \text { where } \frac{D_{0}}{D t}=\frac{\partial}{\partial t}+\left(\vec{u}_{0} \cdot \vec{\nabla}_{H}\right)
\end{aligned}
$$

and $\partial \bar{\rho} / \partial z$ is the unperturbed density gradient which is large compared to the perturbed stratification $\partial \boldsymbol{\rho} / \partial \boldsymbol{Z}$.

From the two momentum equations, eliminating $\mathbf{P}_{\mathbf{1}}$,

$$
\frac{D_{0}}{D t}\left[\frac{\partial v_{0}}{\partial x}-\frac{\partial u_{0}}{\partial y}\right]+f\left(\frac{\partial u_{0}}{\partial x}+\frac{\partial v_{0}}{\partial y}\right)+\beta v_{0}=0
$$

Substituting for Uo and Vo from (1) and using continuity (16)

$$
\frac{D_{0}}{D t}\left[\frac{\nabla_{H}^{2} P_{0}}{\rho_{0} f}\right]+\frac{\beta}{f_{0} \rho_{0}} \frac{\partial P_{0}}{\partial x}=f_{0} \frac{\partial w_{1}}{\partial z}
$$

Then from the buoyancy equation and hydrostatic balance

$$
w_{1}=\frac{-\frac{1}{\partial \bar{\rho}}}{\left(\frac{D_{0} \rho_{0}}{\partial z}\right)}=\frac{\rho_{0}}{\left(\frac{\partial \bar{\rho}}{\partial t}\right)} \frac{D_{0}}{D t}\left[\frac{1}{\rho_{0} g} \frac{\partial P_{0}}{\partial z}\right]=-\frac{1}{N^{2}} \frac{D_{0}}{D t}\left[\frac{1}{\rho} \frac{\partial P_{0}}{\partial z}\right]
$$

where $\mathbb{k}^{2}=-\frac{3}{\rho} \frac{\partial \bar{\rho}}{\partial z}$

Therefore,

$$
\frac{D}{D t}\left[\nabla_{*}^{2} P_{0}+\frac{\partial}{\partial z}\left[\frac{f_{0}^{2}}{N^{2}} \frac{\partial p_{0}}{\partial z}\right]\right]+\beta \frac{\partial P_{0}}{a x}=0
$$


Formal Derivation

Definition: The Rossby number, $\quad$ Ro $=\frac{\mathrm{U}}{\mathrm{fL}}$

describes the magnitude of the nonlinear vs. Coriolis terms. For the large ocean gyres, $\mathrm{U}=0.1 \mathrm{~m} / \mathrm{s}, \mathrm{f}=10^{-4}, \mathrm{~L}=10^{6} \mathrm{~m}=1000 \mathrm{~km}$. and then Ro $\approx 0.001$ (small). For fronts $U=0.5 \mathrm{~m} / \mathrm{s}, \mathrm{L}=10^{4}=10 \mathrm{~km}$, and $R O \simeq 0.5 * 0(1)$ and the quasigeostrophic formalism is not strictly valid.

Nondimensionalization: $(\mathbf{x}, \mathbf{y})=\mathbf{L}\left(\mathbf{x}^{\prime}, \mathbf{y}^{\prime}\right)$ where $\mathrm{L}$ is the smaller of the two dimensions

$$
\begin{aligned}
& \mathbf{Z}=\mathbf{H} \mathbf{Z}^{\prime} \\
& t=\frac{\mathrm{L} \mathbf{t}^{\prime}}{\mathrm{U}} \\
& (\mathbf{u}, \mathbf{v})=\mathbf{U}\left(\mathbf{u}^{\prime}, \mathbf{v}^{\prime}\right) \begin{array}{l}
\text { assuming horizontal isotropy (may } \\
\text { not be valid) }
\end{array} \\
& \mathrm{w}=\frac{\mathrm{HU} \mathbf{w}^{\prime}}{\mathrm{L}} \quad-\text {-this will prove to be too large }
\end{aligned}
$$

Now, scale P AND $\boldsymbol{\rho}$ assuming geostrophy; this is a strong restriction and will not be valid near fronts where isopycnals intersect the surface)

$$
\begin{aligned}
& P=\bar{P}_{(\mathrm{z})}+\rho_{\mathrm{x}} f_{\mathrm{*}} U L \cdot P^{\prime} \\
& \rho=\bar{\rho}(z)+\frac{\rho_{*} f_{0} U L}{g+t^{*}} \cdot \rho^{r}
\end{aligned}
$$

Then $\quad \frac{\Delta \rho}{\rho_{0}}=\frac{\rho_{*}}{\rho_{0}} \cdot \frac{f_{0}}{g H}=R_{0} f_{g}^{2} L^{2}=10^{-2} R_{0}<<1$

where the Mach number $\frac{f_{0}^{2} L^{2}}{g H}$ being small is a condition for incompres-
sibility.

The nondimensional equations of motion then become

$$
\begin{aligned}
& \frac{\partial u^{\prime}}{\partial x^{\prime}}+\frac{\partial v^{\prime}}{\partial y^{\prime}}+\frac{\partial w^{\prime}}{\partial z^{\prime}}=0 \\
& R_{0} \frac{D_{0} u^{\prime}}{D^{\prime}}-\left(1+\frac{\beta L}{f_{0}} y^{\prime}\right) v^{\prime}+\frac{\partial p^{\prime}}{\partial x^{\prime}}=0 \\
& R_{0} \frac{D_{0} v^{\prime}}{D t^{\prime}}+\left(1+\frac{\beta L}{f_{0}} y^{\prime}\right) u^{\prime}+\frac{\partial p^{\prime}}{\partial y^{\prime}}=0 \\
& \frac{\partial p^{\prime}}{\partial z^{\prime}}=-\rho^{\prime}
\end{aligned}
$$




$$
R \cdot \frac{D_{0} \rho^{\prime}}{D t}-\frac{N^{2} H^{2}}{f_{0}^{2} L^{2}} \omega^{\prime \prime}=0
$$

Let $\beta^{r}=\beta L^{*} \quad 8.800$

so the $\beta$-effect cannot be neglected except for small $y$-scales;

and $\mathrm{k}$ 的 $\sim 0.01-1.0$.

$\overline{\mathrm{fL}}$

Two extreme cases of $\beta^{\prime}$ :

i) for $\beta^{\prime}$ large,

$$
\left\{\begin{array}{l}
v=\frac{1}{\rho f} \frac{\partial P}{\partial x} \\
u=-\frac{1}{\rho \cdot f} \frac{\partial p}{\partial y} \quad \Rightarrow \frac{\partial w^{\prime}}{\partial z}=-\frac{\partial u}{\partial x}-\frac{\partial v}{\partial y}=\frac{\beta}{f} v
\end{array}\right.
$$

and long Rossby waves exist.

Under the $\boldsymbol{\beta}^{\boldsymbol{\gamma}}$ large approximation, complications can arise due to nonlinearity in the buoyancy equation leading to fronts and shocks and the assumptions are violated (Anderson and Killworth, 1979).

$$
\frac{\partial \rho}{\partial t}+A(\rho) \frac{\partial \rho}{\partial x}=0 \quad \Rightarrow c=c(\rho)
$$

so, given a density contrast, the differential phase speed of Rossby waves can lead to convergence and discontinuities

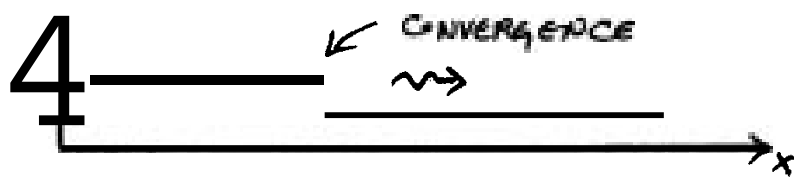

ii) For $\beta^{*} \sim O(1)$, can get a consistent quasigeostrophie regime.

Expand variables in Ro: $U=U_{0}+\operatorname{RoU}_{1}+\ldots$

Then the nondimensional equations of motion to lowest order become to $0(1)$ :

$$
\begin{aligned}
& \frac{\partial u_{0}^{\prime}}{\partial x^{\prime}}+\frac{\partial v_{0}^{\prime}}{\partial y^{\prime}}+\frac{\partial w_{0}^{\prime}}{\partial z^{\prime}}=0 \\
& v_{0}^{\prime}=\frac{\partial P_{0}^{\prime}}{\partial x^{\prime}} \\
& u_{0}^{*}=-\frac{\partial P_{0}^{\prime}}{\partial y^{\prime}}
\end{aligned}
$$




$$
\begin{aligned}
& \frac{\partial p_{0}^{\prime}}{\partial z^{\prime}}=-\rho^{\prime} \\
& \left(\frac{N H}{f . L}\right)^{2} W_{0}^{\prime}=0
\end{aligned}
$$

From (33) and (34), Po $=\psi_{\text {is a streamfunction of the flow. }}$

$$
V_{0}^{\prime}=\frac{\partial \psi}{\partial x^{r}} ; u_{0}^{\prime}=-\frac{\partial \psi}{\partial y^{\prime}} \quad \text { (horizontally nondivergent) }
$$

From (36), Wo' $^{\prime}=0$

and $\rho_{0}^{\prime}=-\frac{\partial \psi}{\partial z^{\prime}}$

Note: To lowest order, there is no evolution in time in the equations.

To next order $0(\mathrm{Ro}): \quad \frac{\partial u_{1}^{\prime}}{\partial x^{\prime}}+\frac{\partial v_{1}^{\prime}}{\partial y^{\prime}}+\frac{\partial \omega_{1}^{\prime}}{\partial L^{\prime}}=0$

$\frac{D_{0} u_{0}^{\prime}}{D t^{\prime}}-v_{1}^{\prime}-\beta^{\prime} y^{\prime} v_{0}^{\prime}+\frac{\partial P_{1}^{\prime}}{\partial x^{\prime}}=0$

$$
\frac{D v_{0}^{\prime}}{D t^{\prime}}+u_{1}^{\prime}+\beta^{\prime} y^{\prime} u_{0}^{\prime} t \frac{\partial P_{1}^{\prime}}{\partial y^{\prime}}=0
$$

$$
\frac{D_{0} \rho_{0}^{\prime}}{D E}-\left(\frac{N H}{f_{0} L}\right)^{2} \omega_{1}^{\prime}=0
$$

where $\frac{D_{a}}{D t^{\prime}}=\frac{\partial}{\partial t^{\prime}}-\frac{\partial^{N}}{\partial y^{\prime}} \frac{\partial}{a x^{*}}+\frac{\partial^{\psi}}{\partial x^{\prime}} \frac{\partial}{\partial y^{\prime}}$

Eliminating $P_{1}{ }^{\prime}$ between (41) and (42)

$$
\frac{D_{0}}{D t^{\prime}} \nabla_{H}^{\nu^{2}} \psi+\left(\frac{\partial u_{1}^{\prime}}{\partial x^{\prime}}+\frac{\partial v_{1}^{\prime}}{\partial y^{\prime}}\right)+\beta^{\prime} \frac{\partial \psi}{\partial x^{\prime}}=0
$$


northward and particle (3) towards south, as a result of which particles (2) and (3) develop a negative and positive vorticity respectively. The induced flow resulting from these acts to bring particle (1) back to its original position and the net result is a westward propagation of phase.
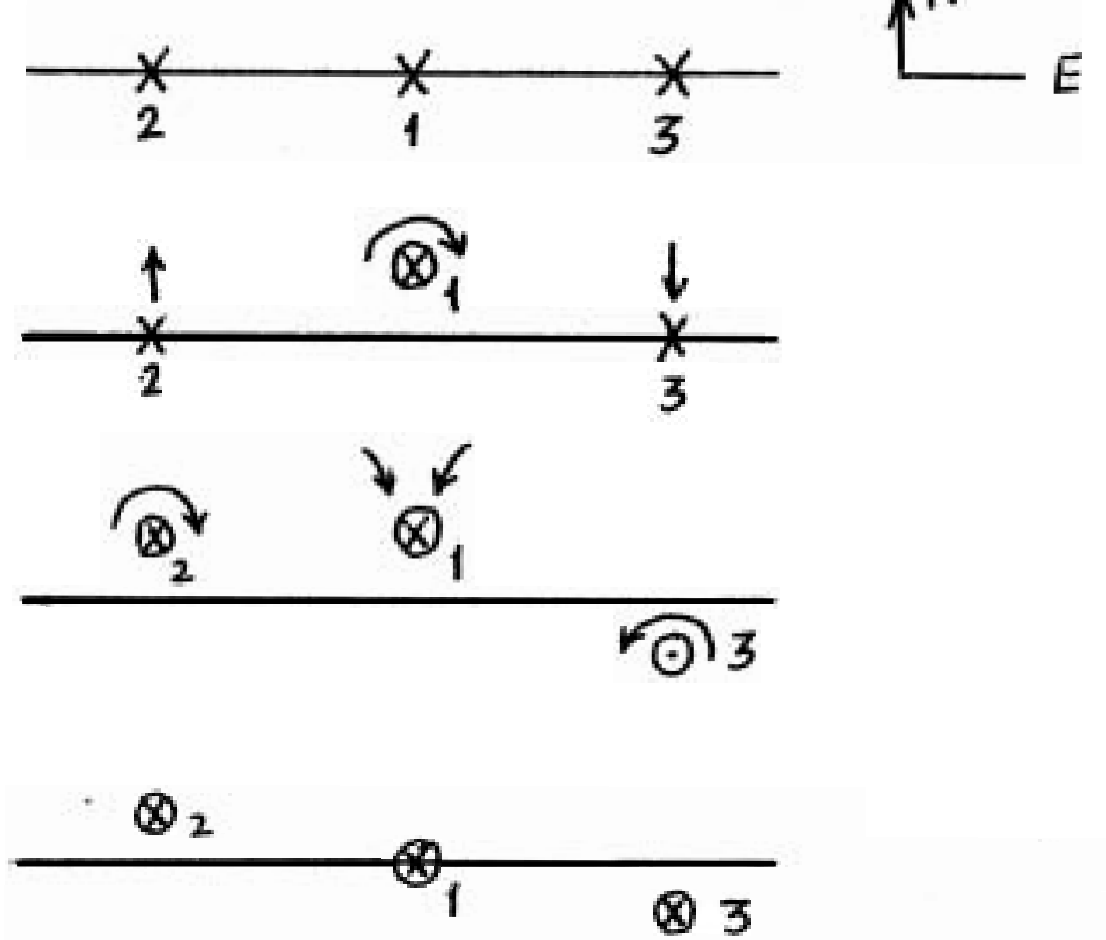

The presence of $\beta$ is absolutely crucial to the above example; if $\beta$ is zero then the system cannot support any waves.

But there is another way in which we can produce wave propagation, and that is by modifying our boundary. Let $N$ be a constant and $\beta=0$. The potential vorticity equation then says

$$
\text { at }=0 \text { where } \quad q=\nabla^{2} \psi+\left(\frac{f^{2}}{N^{2}} \psi z\right)_{z}
$$

$q=0$ is then a perfectly good solution.

$$
\therefore \nabla^{2} \psi+\frac{f^{2}}{N^{2}} \psi_{z z}=0
$$

Now imagine that we have a sloping bottom as sketched:

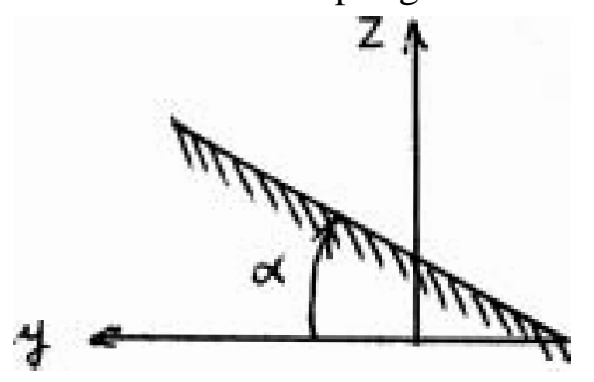

The boundary condition on the bottom is the usual no normal flow condition which gives: $\omega=\alpha v^{*}$ Now since we want $\omega^{*} \sim Q\left(R_{0}\right) v \Rightarrow$ $\alpha \leqslant \frac{H}{L} R o$ 
1.e. we have to remain contented with a small slope, but, aha! we can also conveniently linearize the boundary condition and apply it at $\mathbf{z}=0$.

$$
\begin{aligned}
& \text { Solving for eq. (6) gives } \\
& \psi=\exp i\{k x+l y-\omega t\} \exp \left[-\frac{N}{f}\left(k^{2}+l^{2}\right)^{1 / 2} 3\right]
\end{aligned}
$$

where the -ve sign has been chosen so that all disturbances decay as $\mathbf{z} \rightarrow \infty$.

The linearized boundary condition is

$$
-\frac{f}{N^{2}} \psi_{z t}=\alpha \psi_{x} \text { at } z=0
$$

On substitution we get

$$
\begin{aligned}
& -\frac{f}{N^{2}}\left\{-\frac{N}{f}\left(k^{2}+l^{2}\right)^{1 / 2}\right\}(-i \omega)=i k \alpha \\
& \omega=\frac{-\alpha N k}{\left(k^{2}+l^{2}\right)^{1 / 2}}
\end{aligned}
$$

Note that if $\alpha>0, \omega / k<0$ which implies that the phase of these waves travels left of upslope.

Interestingly the dispersion relation of these waves does not involve $f$, nonetheless existence of $f$ is crucial to be able to support wavepropagation. Let's now compare the mechanisms which bring about vorticity change in the two types of wave we have considered.

1) Rossby waves require the existence of a potential vorticity gradient which was provided by the planetary vorticity in our example.

2) In topographically generated waves vorticity changes because of the stretching.

We can now make our second example look like the first one by considering the following mathematical problem

$$
\begin{aligned}
& \frac{\partial q}{\mathrm{a} t}+f \alpha \delta(z) \psi_{x}=0 \quad \text { where } \delta(z) \text { is the Dirac } \delta \text {-function } \\
& \text { with } \psi_{z}=0 \text { at } z=0- \\
& \text { and a s } z \rightarrow \infty
\end{aligned}
$$

It can be shown that the above mathematical problem has the same solution as we obtained for our second example. The result is that we can think of the boundary as providing a $\delta$-function in potential vorticity and hence making wave propagation possible.

Note: A question we raised about why any internal wave modes did not appear in the examples illustrated and whether they would appear at higher order. After some discussion it was agreed upon that internal waves had been filtered out of present analysis (by Hydrostatic Approx.). 
$\underline{\text { Baroclinic Instability }}$

Let's choose a basic state which is not at rest and here we chose one which requires no forcing and the basic question to be asked is when do we 'wave' and when we do not.

Choose an East-West mean flow.

$$
\mathrm{U}(\mathrm{y}, \mathrm{z}), \quad \mathrm{V}=\mathrm{W}=\mathrm{O}
$$

Note that any function $U(y, z)$ will satisfy the dynamical equations. Let the streamfunction be $\Psi(y, z)$, 1.e. $\Psi_{y}=-U$.

Now the Mean Potential vorticity is given by

$$
Q(y, z)=\Psi_{y y}+\left(\frac{f^{2}}{N^{2}} \Psi_{z}\right)_{z}+\beta y
$$

It is useful to keep in mind the typical size of $U$

Atmosphere

Ocean away from boundaries

(in mid latitudes)

In boundary currents
$\mathrm{U}=70 \mathrm{~m} / \mathrm{s}$

$\mathrm{U}=5 \mathrm{~cm} / \mathrm{s}$

$\mathrm{U}=1-2 \mathrm{~m} / \mathrm{s}$

Now consider small perturbations to these

$$
q(x, y, z, t)=\nabla^{2} \psi+\left(\frac{f_{0}^{2}}{N^{2}} \psi z\right) z
$$

The perturbation vorticity $q$ is governed by

$$
\left\{\frac{\partial}{\partial t}+U \frac{\partial}{\partial x}\right\} q+\psi_{x} Q_{y}=0
$$

where the first term represents the advection by the mean and the second term is the northward advection of the mean by the perturbation velocity field. $Q_{y}$ is the potential vorticity gradient and is given by

$$
Q_{y}=\beta-U_{y y}-\left(\frac{f^{2}}{N^{2}} U_{z}\right)_{z}
$$

Here we note that we have forced an anisotropy in the problem since total $\mathrm{U} \gg$ total $t^{2}$. Furthermore, we now have a mean flow and so we anticipate the answers to be qualitatively different from what we have seen so far.

Before we get entangled into the mathematics, let's look at some basic physics. Since we have a nonzero $U_{z}$, the thermal wind reminds us that

$P_{y} \propto U_{z}$, hence the isopycnals are tilted in the $(y, z)$ plane. Let's sketch them for definiteness.

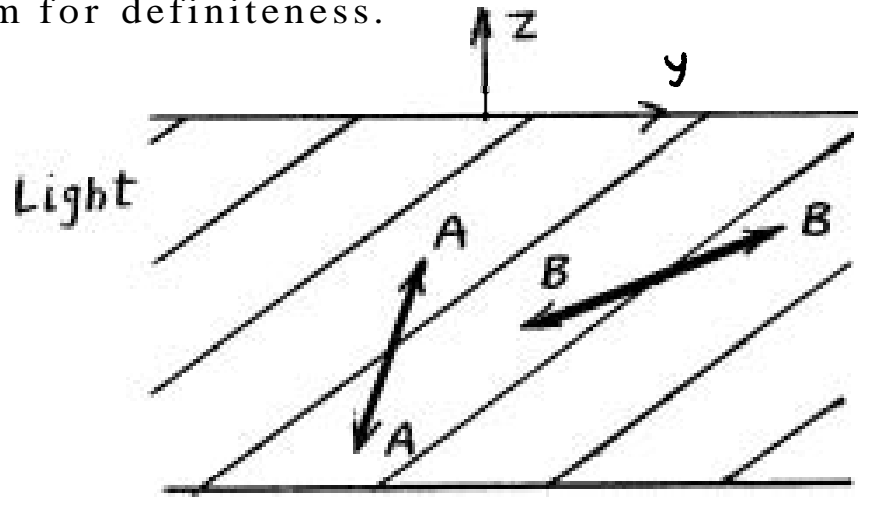


Now we can go through the usual argument of particle exchange and arrive at the following conclusions. i) If the vector joining the exchanged particles makes an angle $>$, the tilt of the isopycnals (as sketched in AA), then the buoyancy force tends to restore them to their original position. 1i) However, if this vector makes an angle that lies within the tilt of the isopycnals and horizontal (as sketched in BB), a component of the buoyancy force acts to displace the particles further, hence this range of angles can be potentially unstable.

The argument just presented is incomplete since i) it neglected the Coriolis force; i i) it did not consider the geometry, 1.e. 'did the perturbations have enough room to grow ${ }^{\text {; }}$ i i i) it ignored a 11 dynamics.

Let's now consider a simple example. Here we choose $\beta=0$ and $\mathrm{N}=$ constant. We also choose a linear profile for the mean flow, i.e. $U=U z Z+U_{1}$ which gives

$$
Q y=0
$$

our past experience tells us that

$$
q=0
$$

is a good solution.

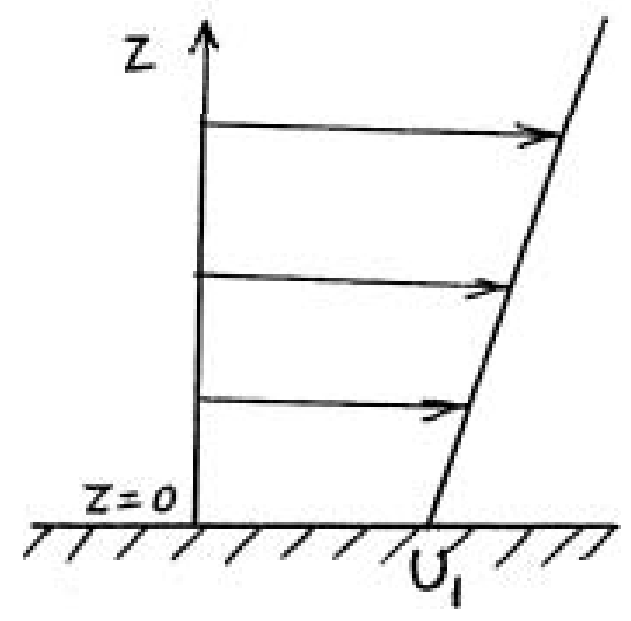

We also recall that this leads to

$$
V=\exp i(k x+l y-\omega t) \exp \left(-\frac{N}{f}\left(k^{2}+l^{2}\right)^{1 / 2} z\right)
$$

The boundary condition at $Z=0$ is $\boldsymbol{\omega}=0$. This leads to

$$
\left(\frac{\partial}{\partial t}+U \frac{\partial}{\partial x}\right) \psi_{z}+\psi_{x} \Psi_{z y}=0 \quad \text { at } z=0
$$

Since $\Psi_{z y}=U_{z}$ the above simplifies to

$$
(U-\omega / k) \psi_{z}=U_{z} \psi
$$

which on substituting for $\psi$ gives

$$
\omega=k U_{1}+\frac{f U_{z} k}{N\left(k^{2}+l^{2}\right)^{1 / 2}}
$$


We can identify the first term on the RHS as a doppler shift and the second term reminds us of the sloping bottom example we did previously. If we make the following identification $f U_{z} / N=-\not N$, then we obtain exactly the same expression as in the sloping bottom case (without the doppler shift). We can also interpret the phase speed (ignoring the doppler shift) as the mean speed at scale height $f /\left(N \sqrt{R^{2}+l^{2}}\right)$. The logical question to ask now is why do the answers resemble each other. The reason is as follows:

1) $Q y=0$

i i) Both problems actually had a $\delta$-function $Q_{y}$ Here $Q_{y}=-\frac{f^{2}}{N^{2}} U_{z} \delta(z) \quad \begin{aligned} & \text { which identifies } \alpha \text { to be precisely what } \\ & \text { we just did. }\end{aligned}$

We did not find an instabiity and did we release potential energy? Compute the net mass flow in the horizontal direction

$$
\iint d z d x p v=\int d x \rho_{\text {mean }}^{v} \propto \iint d z d x \psi_{x}^{\psi_{x}} \underbrace{\psi_{z}} \propto \iint d z d x\left(\psi^{2}\right)_{x}=0
$$

So we did not release any potential energy!

It is then an obvious question to ask as to what conditions must hold for instability. There are a fair number of theorems that exist most of which are discussed in Pedlosky (1979). These theorems are not very illuminating and at best provide bounds on growth rate, etc. which are correct to an order of magnitude (i.e. they are not very tight bounds). We shall nevertheless consider one necessary condition for instability.

$$
\begin{aligned}
& \text { Let's go back to the equation governing the perturbations } \\
& \left(\frac{\partial}{\partial t}+U \frac{\partial}{\partial x}\right)\left(\nabla^{2} \psi+\left(f^{2} / N^{2} \psi_{z}\right)_{z}\right)+Q_{y} \psi_{x}=0
\end{aligned}
$$

where $U=U(y, z)$. We note that the above PDE is nonseparable in $y$ and $z$ but is separable in $\mathrm{x}$ and $\mathrm{t}$.

We consider $\psi$ to be proportional to $\exp i k(x-c t)$ which leads to

$$
(U-c)\left\{\psi_{y y}-k^{2} \psi+\left(\frac{f^{2}}{N^{2}} \psi_{z}\right)_{z}\right\}+Q_{y} \psi=0
$$

Notice that if $\mathrm{c}$ is real, the solution is stable and if $\mathrm{c}$ is complex with Im $C>0$ it is unstable.

The boundary conditions we consider follow:

In $x$ : Long box or cyclic or infinite.

In $y$ : no normal flow on $y=0$ and $y=L$ or 1.e. $\psi=0$

or

$$
|\psi| \rightarrow 0 \text { as }|y| \rightarrow \infty
$$

In $z$ : $\quad=0$ on $z=0$ and $z=-H$ which implies

$$
(\mathrm{U}-\mathrm{c}) \psi_{\mathrm{z}}-\mathrm{U}_{\mathrm{z}} \psi=0 \text { at } \mathrm{z}=0 \text { and }-\mathrm{H}
$$


$*$
Multiply eq. (20) by $\frac{\psi}{U-C}$ and integrate over y and $z$ giving
$\iint d y d z\left\{\psi_{y y} \psi^{*}-k^{2}|\psi|^{2}+\psi^{*}\left(\frac{f^{2}}{N^{2}} \psi_{z}\right)_{z}+\frac{|\psi|^{2}}{U-c} Q_{y}\right\}=0$ Let's manipulate the individual terms

$$
\begin{aligned}
\iint d y d z \psi_{y y} \psi^{*}= & -\iint d y d z\left|\psi_{y}\right|^{2} \\
\iint d z \psi^{*}\left(\frac{\psi_{z} f^{2}}{N^{2}}\right)_{z} & =\left[\frac{\psi^{*} \psi_{z} f^{2}}{N^{2}}\right]_{-H}^{0}-\int \frac{\left|\psi_{z}\right|^{2} f^{2}}{N^{2}} d z \\
& =\left[\frac{\mid \psi^{2} f^{2} U_{z}}{N^{2}(U-C)}\right]_{-H}^{0}-\int \frac{\left|\psi_{z}\right|^{2} f^{2}}{N^{2}} d z
\end{aligned}
$$

With these eq. (23) can be written as

$\iint d y d z\left\{-\left|\psi_{y}\right|^{2}-k^{2}|\psi|^{2}-\frac{f^{2}}{N^{2}}\left|\psi_{z}\right|^{2}\right\}+\int d y\left[\frac{f^{2} V_{z}|\psi|^{2}}{N^{2}(U-c)}\right]_{-H}^{0}+\iint \frac{d y d z|\psi|^{2} Q_{y}}{U-c}=0$

Note that $\operatorname{Im}\left(\frac{1}{U-c}\right)=I_{m} \frac{U-c^{*}}{|U-c|^{2}}=\frac{C_{i}}{|U-c| 2}$

Hence by taking the imaginary part of eq. (24) we get

$$
C_{i}\left\{\int d y\left[\frac{f^{2} U_{z}|f|^{2}}{N^{2}|U-c|^{2}}\right]_{-H}^{0}+\iint d y d z \frac{|\psi|^{2} Q_{y}}{|U-C|^{2}}\right\}=0
$$

which leads to the result due to Charney and Stern (1962), namely,

$c_{i} \neq\left. 0 \Rightarrow U_{z}\right|_{z=0}, Q_{y},-\left.U_{z}\right|_{z=-H}$ All cannot have the same sign,

or, generalized Qy must change sign somewhere for instability.

Notes Submitted By

Sanjiva Lele 
There is a big number of models involving baroclinic or barotropic instability. The conditions for the mean flow are often very ideal conditions. We are more interested in predicting the different kind of instabilities, which will occur for different situations. We will therefore not present all the classical models in detail. We will only present the ideal case of the Eady Model (for baroclinic instability) and a case of barotropic instability close to Rayleigh's problem.

\section{BAROCLINIC INSTABIITY: EADY MODEL}

This model is a purely baroclinic one since the mean flow is taken uniform in a horizontal plane

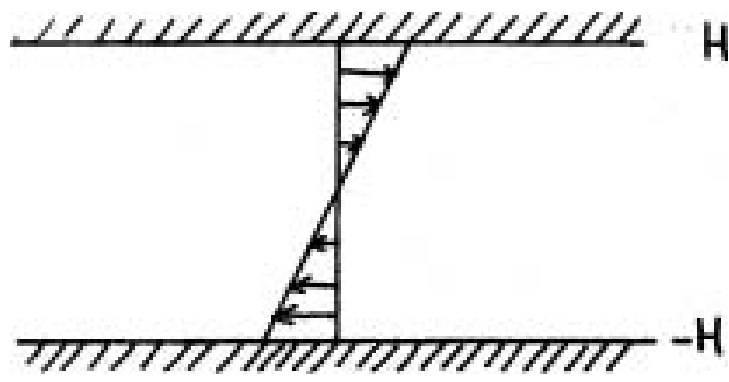

$$
\mathrm{v}_{\mathrm{y}} \equiv 0
$$

The flow is supposed to be contained between two rigid planes, with no $\beta$ effect

$$
\beta=0
$$

The stratification is taken as a constant, and we assume a linear vertical shear

$$
\mathrm{N}=\text { const } \quad \mathrm{U}=2 \mathrm{~J}_{z}
$$

We look at a solution for (1) which has the form

$$
\psi \propto e^{i\left(R_{y}+k x\right)}
$$

For the present case (1) reduces to

$$
\psi_{33}=\frac{N^{2} K^{2}}{R^{2}} \Psi
$$

with $\mathbb{K}^{2}=k^{2}+1^{2}$, a general solution is

$$
\Psi=A \sinh \left(\frac{N K}{f} z\right)+B \cosh \left(\frac{N K}{f} z\right)
$$

the condition of 0 vertical velocity on boundaries (Eq. 3) leads to

$$
\begin{aligned}
& \left(U_{3} H-c\right) \Psi_{3}(H)=U_{3} \Psi(H) \\
& \left(-U_{3} H-c\right) \Psi_{3}(-H)=U_{3} \Psi(-H)
\end{aligned}
$$

A nontrivial solution for $\Psi$ is found when

$$
c^{2}=\frac{U_{z}^{2} f^{2}}{N^{2} K^{2}}\left\{\frac{N K H}{f} \tanh \left(\frac{N K H}{f}\right)-1\right\}\left\{\frac{N K H}{f} \operatorname{coth}\left(\frac{N K H}{f}\right)-1\right\}
$$


Negative values for $\mathrm{C}^{2}$ are found when NKH/f $<1.1997$. Calling $\mathrm{L}=1 / \mathrm{K}$ the scale of the disturbance the perturbation appears to be unstable when its scale is large enough so that

\section{$L>\frac{N H}{f(1.1997)}$}

the imaginary part $\boldsymbol{C}_{\boldsymbol{i}}$ of $\mathrm{C}$ and the growth rate $\mathbf{K C}_{\boldsymbol{i}}$ are plotted on this figure.

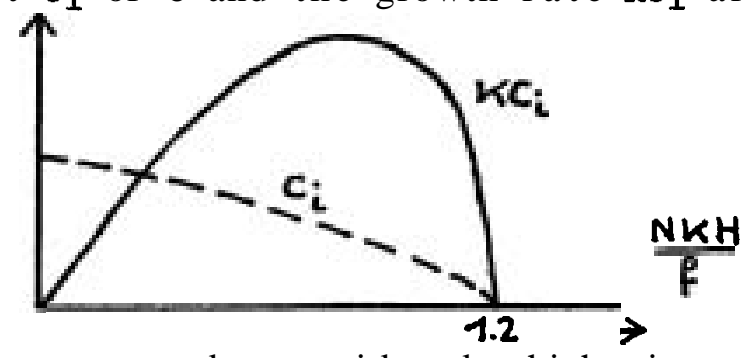

Two li mit cases may be considered which give a picture of the instability

$$
\text { - Lone: waves: } \quad \frac{\mathrm{NKH}}{\mathrm{f}} \ll 1
$$

It comes from (15)

$$
c \approx \pm i \frac{U_{3} H}{\sqrt{3}}
$$

Long waves are unstable. Using the boundary condition (14) $\Psi$ may be calculated from Eqs. (11) and (13). It may be shown (Pedlosky, 1979, that a $z$-dependent phase appears. $\Psi$ is written.

$$
\psi \alpha \cos \left(e_{y}+k x+\alpha(z)\right) e^{c_{i} t}
$$

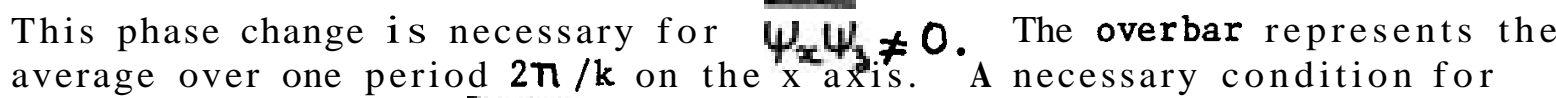
instability is then $2 \alpha / \partial z>0$, which means physically that the surface of constant $\Psi$ must tilt upward and westward.

- short waves:

We now get from (15)

\section{$\frac{\text { NKH }}{\mathrm{f}} \gg 1$}

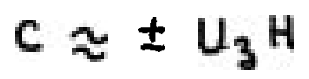

The solution consists then in two stable waves

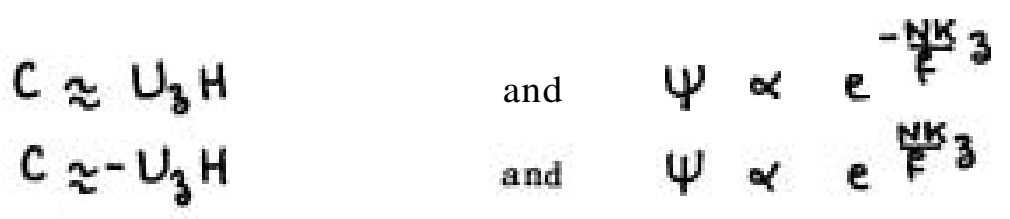

The wave travelling in positive direction is trapped near the lower boundary, and the wave travelling in negative direction is trapped near the upper boundary. These waves are trapped so close to the boundary that they may be considered as waves travelling in a domain where one of the boundaries is at infinity. Such waves are known to be stable, and this explains physically the stabiity of short waves in Eady's Model. 
Although Eady's Model gives a good idea about baroclinic instability, the fact that instability results from the boundary conditions remains one important defect of this model.

\section{BAROTROPIC INSTABILITY: RAYLEIGH'S PROBLEM}

This problem is the horizontal equivalent of Eady's problem. All baroclinic terms are no longer considered

$$
\mathrm{v}_{\mathrm{z}} \equiv 0 \quad \Psi_{\mathrm{z}} \equiv 0
$$

As in Eady's model, we do not either consider $\boldsymbol{\beta}$ effect: $\boldsymbol{\beta} \equiv 0$

A horizontal mean flow is considered as follows:

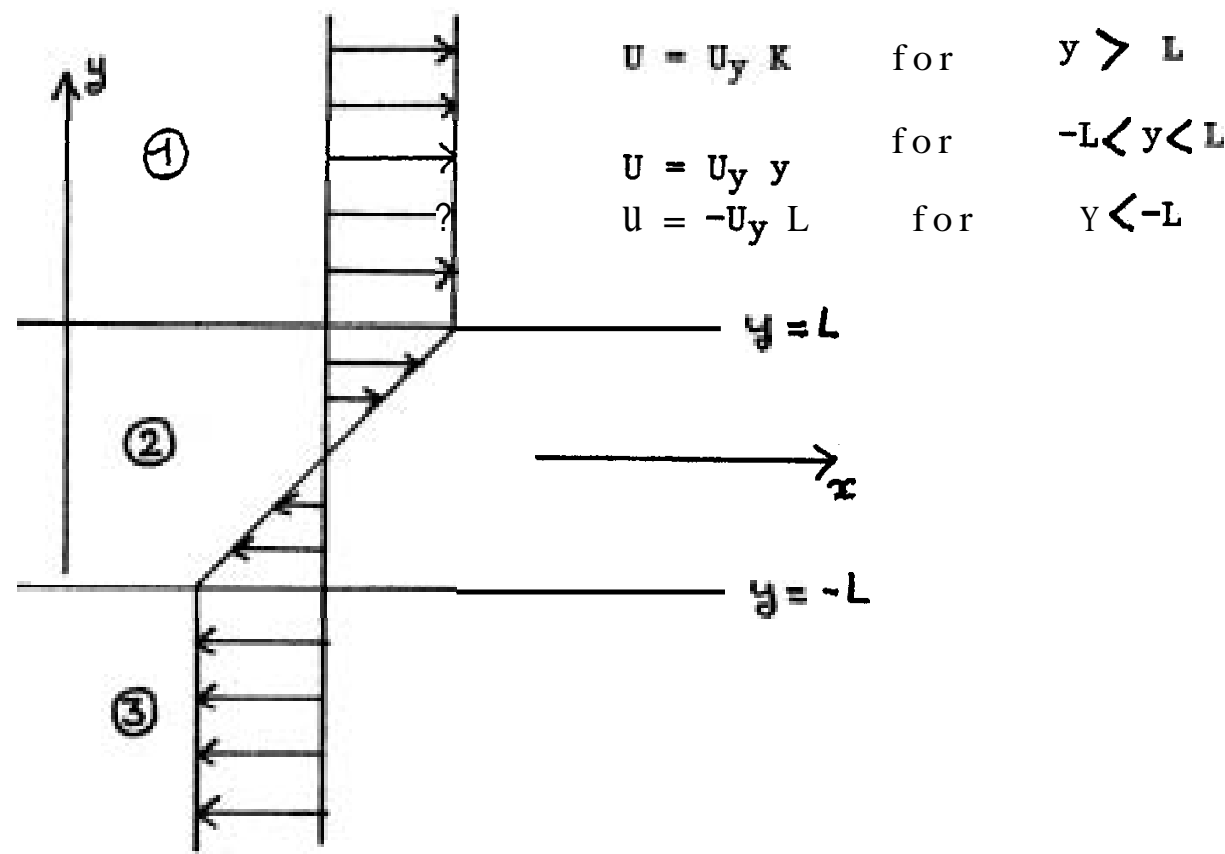

We get $Q_{y}=U_{y}\{S(y-L)-\delta(y+L)\}$. Therefore $Q_{y}=0$ except

for $y= \pm L$. Instab4ity will depend on the jump conditions at $y= \pm L$ $\left(\Psi\right.$ cont1 nuous, $\left.(U-c)\left[Q_{y}\right]+\Psi[Q]=0\right)$.

Eq. (1) is integrated in each region of the domain (1), (2), (3):
Region (1)
$\Psi_{1}=\left(A e^{k L}+B e^{-k L}\right)$
$e^{-k(y-L)}$
$y>L$
Region (2)

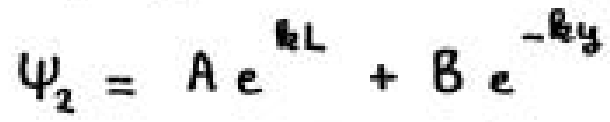$$
-L<y<L
$$
Region (3)

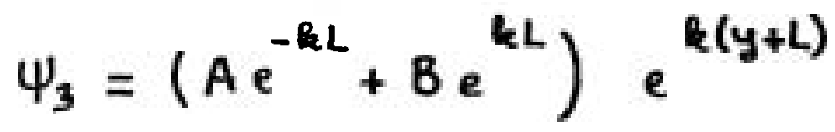

so that $\Psi$ remains continuous at $y= \pm L$. 
Integration of Eq. (1) at the points $y= \pm$ L leads to a linear system where $\mathbf{A}$ and $B$ are unknown. Nontrivial solutions for $A$ and $B$ are found only if

$$
k^{2} c^{2}=U_{y}^{2}\left\{k^{2} L^{2}-k L+\frac{1}{4}-\frac{1}{4} e^{-4 k L}\right\}
$$

The stability diagram shows that sufficiently long waves are unstable, and that short waves remain stable.

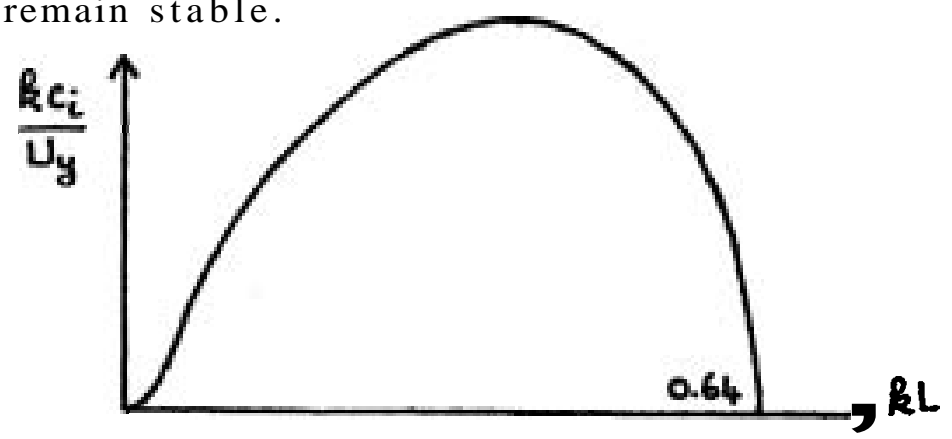

This fact may be easily understood if one recalls the fact that $\Psi$ is as sensitive to the jump conditions at $y= \pm L$ as $k$ is small. Then for small $k$ (long wave), the wave "sees the flow" as a sharp discontinuity, which is a well known case of instability.

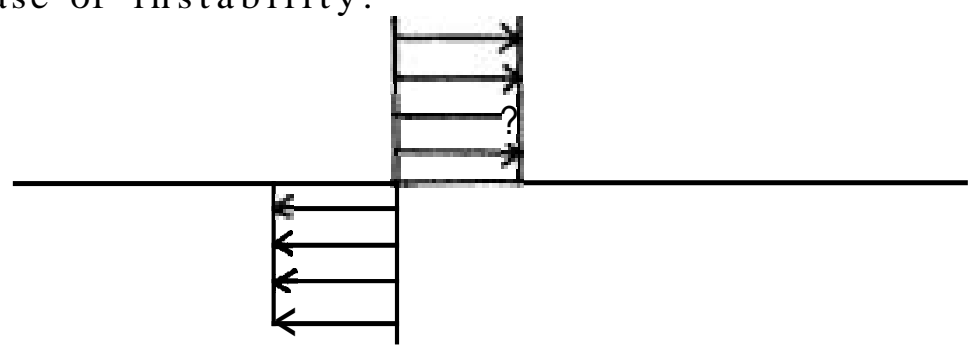

On the other hand, for large values of $k$ (short waves), the perturbation decreases very rapidly as $|z|$ increases, and the wave ignores the jump conditions at $y= \pm I$. The waves behave like a wave in a domain of constant shear.

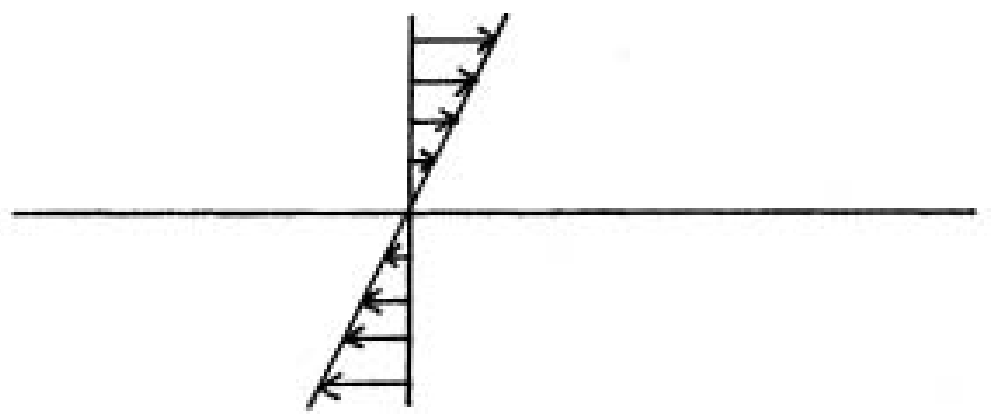

Many other problems of instability have been treated in the literature, like Charney's or Phillip's models. These models present specific cases involving either barotropic or baroclinic instability. We will not consider them since we are more interested in predicting the different kind of instabilities which will occur for different situations. In fact, in physical situations the mean flow possesses horizontal shear as well as vertical. Therefore either barotropic and baroclinic instabilities could occur. The problem is, in fact, to know which one will arise or whether the instability will be a mixed one. 
We will present a theory which will distinguish different possible situations of instability. The reader will find the details in Killworth (1980) and Holland and Haidvogel (1980).

First this theory tends to take into account the fact that the Brunt Väisälä does not remain constant through the whole fluid.

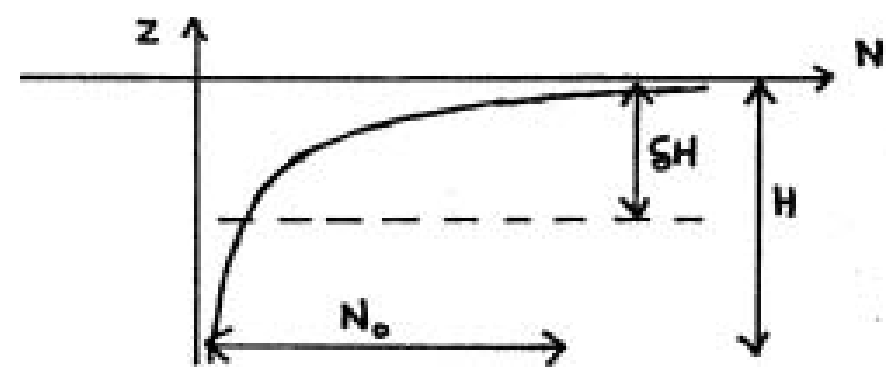

For a fluid of depth $\mathrm{H}$, N varies only over a layer of depth $\boldsymbol{\sigma} \mathrm{H}$ in the upper part of the fluid: $\boldsymbol{\delta} \lesssim 0(1)$. A suitable definition for the deformation radius is therefore

$$
a=\frac{\text { No } S \text { H }}{f}
$$

We are interested in treating the general problem, i.e. without throwing out either the vertical or horizontal shear.

The equation for potential vorticity remains

$$
(U-c)\left\{\Psi_{y y}-k^{2} \Psi+\left(\frac{f^{2}}{N^{2}} \Psi_{z}\right)_{a}\right\}+\left(\beta-U_{y y}-\left(\frac{f^{2}}{N^{2}} U_{z}\right)_{z}\right) \Psi=0
$$

For boundary conditions, we assume rigid boundaries at the top and a $t$ the bottom of the fluid, i.e.

$$
(U-c) \Psi_{z}=U_{3} \Psi \quad z=0,-H
$$

and for lateral conditions, the perturbation is assumed to vanish a

$$
\Psi \rightarrow 0 \quad \text { as } \quad|y| \rightarrow \infty
$$

which imposes no further horizontal scales on the problem. In order to get (21) i n a nondimensional form we use the following scaling:

$$
\begin{array}{ll}
(x, y) \sim L & (z) \sim H \\
(c, u) \sim U & \sim(k) \sim L^{-1} \\
(\beta) \sim U / L^{2} & (N) \sim N_{0}
\end{array}
$$

The length scale L, as well as the velocity scale U, denote typical scales of 'the MEAN HOW. Then (22) becomes

$$
(U-c)\left(\Psi_{y y}-k^{2} \Psi+\lambda^{2} \delta^{2}\left(\frac{\Psi_{z}}{N^{2}}\right)_{z}\right)+\left(\beta-\lambda^{2} \delta^{2}\left(\frac{U_{z}}{N^{2}}\right)_{z}-U_{y y}\right) \Psi=0
$$


where $\lambda$ is taken as $\lambda=L / a$ and $\delta$ is the fractional variation of $N$. The two parameters $\lambda$ and $\boldsymbol{\delta}$ are the two essential parameters which will govern a 11 the asymptotic expansion we want to provide in order to determine the type of instability if any will occur. It is useful at this stage to keep in mind the different orders of the physical quantities and get an idea of typical values of $\lambda$ and $\delta$.

The following table summarizes different situations:

$\begin{array}{lllllll} & \mathrm{L}(\mathrm{km}) & \mathbf{a}(\mathbf{k m}) & \mathrm{U}\left(\mathbf{m} \mathbf{~ s}^{-1}\right) & \boldsymbol{\delta} & \boldsymbol{\lambda} & \boldsymbol{\beta} \\ \text { Ocean } & 1000 & 30 & 1.5 \times 10^{-2} & 0.1-0.2 & 30 & 40-2000 \\ \text { Gulf-Stream } & 60-100 & 30 & \mathbf{1} & 0.1-0.2 & 2-3 & 0.2 \\ \text { North-Atlant Eddy } & 100 & 30 & 10^{-1} & 0.1-0.2 & 3 & 2 \\ \text { Atmosphere } & 5000 & 1000 & 20 & \mathbf{1} & 5 & 25 \\ \text { Anticyclone } & 1000 & 1000 & 20 & \mathbf{1} & \mathbf{1} & \mathbf{1} \\ \text { Laboratory } & 10^{-4} & 10^{-4} & 1.5 \times 10^{-2} & \mathbf{1} & 0.5-3 & ?\end{array}$

We may evaluate from Eq (7) the energy transfer term which feeds energy to the disturbance or take some from it. It follows that

$$
\frac{\partial}{\partial t} \int E d V=\frac{1}{2} \operatorname{Re}\left\{i k \iint d y d z U_{y} \Psi \Psi_{y}^{*}+i k \iint d y d z U_{z} \Psi \Psi_{z}^{*} \frac{\lambda^{2} \delta^{2}}{N^{2}}\right\}
$$

T.K.E.

T.A.P.E.

It comes out that $x^{2} \delta^{2} / N^{2}$ characterize the ratio of potential energy to kinetic energy which is transferred to (or from) the perturbation. We would therefore expect to get a mixed baroclinic-barotropy instability when $\lambda^{2} \delta^{2} / N^{2} \approx 1$. On the other hand the instability would be rather baroclinic if $\lambda^{2} \delta^{2} / N^{2} \gg 1$ and rather barotropic if $\lambda^{2} \delta^{2} / N^{2} \ll-1$.

We will now examine more carefully a 11 these different possibilities, considering limit cases.

1. $\underline{\lambda \text { large }}$

In this case we expect an instability, when it occurs, to be mainly baroclinic. We do not want to examine a 1D problem along the vertical axis, but we will consider two cases, namely, when the y scale is of the order of unity, and when it is small

\section{(i) y scale is of order unity}

$\delta$ is assumed to be $O(1)$ and $\beta=\beta_{0} \lambda^{2}+\beta_{1}$. This allows to consider either important $\boldsymbol{\beta}$ effects $\left(\beta_{0} \neq 0\right)$ or weak $\left(\boldsymbol{\beta}_{0}=0\right)$.

It comes from the energy integral that for $k=O(\lambda)$ the solution is stable if $\mathrm{k}$ is sufficiently large. We will therefore assume $\mathrm{k}=0(1)$.

$\Psi$ and $c$ are expanded at the different orders of the parameter $1 / \lambda^{2}$

$$
\begin{aligned}
& \psi=\psi_{0}+1 / \lambda^{2} \psi_{1}+\ldots \\
& c=c_{0}+1 / \lambda^{2} c_{1}+\ldots .
\end{aligned}
$$


To the first order (23) becomes

$$
\left(U-c_{0}\right)\left(\frac{\Psi_{03}}{N^{2}}\right)_{z}=\left(\frac{B_{0}}{S^{2}}+\left(\frac{U_{3}}{N^{2}}\right)_{z}\right) \Psi_{0}
$$

Unless $\beta_{a}=0$, there is no solution to first order. Assuming then $\beta_{0}=0$, a trivial solution to (26) is

$$
\Psi_{0}=A(y)\left(u-c_{0}\right)
$$

Substituting $\Psi_{a}$ in the second order equation and integrating along the $z$ axis, we obtain

$$
\left(\Phi A_{y}\right)_{y}-R^{2} \Phi A+B_{1} A Q=0
$$

where $\Phi$ and $Q$ are defined as

$$
\begin{aligned}
& \Phi\left(y, c_{0}\right)=\int_{0}^{H}\left(U-c_{0}\right)^{2} d z \\
& Q\left(y, c_{0}\right)=\int_{0}^{H}\left(U-c_{0}\right) d z
\end{aligned}
$$

The second order equation (28) determines A, taking into account the $y$ structure of U. Solving (28) by computer shows unstable solutions whose growth rate is of the order of unity. The instability is in fact found to be baroclinic in the sense that the ratio T.K.E/(T.K.E + T.A.P.E) calculated as defined from Eq. (24) is close to 0.

Notes Submitted By Mathieu Mory 
LECTURE 5

$\mathrm{y}-$ scale $\ll 0(1)$

One can think of two methods which may be applicable to the present case; one is the WKBJ-method and the other is the local expansion technique.

If one poses a WKBJ-type solution of the form $\psi=\varnothing(y, z) \exp (\lambda D(y))$ and provided that $C_{o}$ is known, $D_{y}^{2}$ can be determined, to the leading order, as an eigenvalue of the Iocal y-independent vertical problem. Since it is generally difficult to know how the eigenvalue varies with $\mathrm{y}, \mathrm{c}_{\mathrm{o}}$, or $\mathrm{k}$, however, one cannot proceed analytically (except for special cases such as a two-layer case).

Here we apply the local expansion technique near a specified point $\mathrm{y}=\mathrm{y}_{\mathrm{o}}$. Define a local stretched coordinate $\eta$ by

$$
\eta=\lambda^{1 / 2}\left(\mathbf{y}-y_{0}\right)
$$

a s suggested by Simmons (1974). The $\mathrm{x}$-scale of the solution is assumed to be

$$
O(a): k=\lambda k_{0}\left(k_{0} \text { 1s } O(1)\right)
$$

and $\beta=\beta_{0} \lambda^{2}$ will suffice for the present purpose. Expanding the variables in terms of $\lambda^{-1 / 2}$ about $y_{0}$, we have

$$
\begin{aligned}
& u=u_{0}(z)+\lambda^{-\frac{1}{2}} \eta u_{1}(z)+\lambda^{-1} \frac{\eta^{2}}{2} u_{2}(z)+\cdots \cdots \\
& \psi=\psi_{0}(\eta, z)+\lambda^{-\frac{1}{2}} \psi_{1}(\eta, z)+\lambda^{-1} \psi_{2}(\eta, z)+\cdots \\
& \text { and }=c_{0}+c_{1} \pi^{-\frac{1}{2}}+c_{2} \lambda^{-1}+\cdots \cdots
\end{aligned}
$$

Substituting eqs. (3) n(5) into eq. ( ), we obtain

$\left[u_{0}-c_{0}+\left(u_{1} \eta-c_{1}\right) \lambda^{-\frac{1}{2}}+\left(\frac{u_{2} \eta^{2}}{2}-c_{2}\right) \lambda^{-1}+\cdots \cdot\right]\left\{\lambda^{2}\left[\delta^{2}\left(\frac{f^{2}}{N^{2}} \psi_{0,8}\right)-k_{0}^{2} \psi_{0}\right]\right.$

$\left.+\lambda^{\frac{3}{2}}\left[\delta^{2}\left(\frac{f^{2}}{N^{2}} \psi_{1 z}\right)_{z}-k_{i}^{2} \psi_{i}\right]+\lambda\left[\delta^{2}\left(\frac{f^{2}}{N^{2}} \psi_{2 z}\right) z-k_{0}^{2} \psi_{2}+\psi_{0, z}\right]+\cdots\right\}$ $+\left\{\lambda^{2}\left[\beta_{0}-\delta^{2}\left(\frac{u_{c z}}{N^{2}}\right)_{z}\right]-\lambda^{\frac{3}{2}} \delta^{-2}\left(\frac{u_{i z}}{N^{2}}\right)_{z}-\lambda \delta^{2}\left(\frac{u_{2 z}}{N^{2}}\right)_{z}\right\}\left(\psi_{0}+\lambda^{-\frac{1}{2}} \psi_{i}+\pi^{-1} \psi_{2}\right)$
$=0$,

while from the boundary conditions at $\mathbf{z}=0,-\mathbf{1}$,

$$
\begin{aligned}
& {\left[\left(u_{0}-c_{0}\right)+\left(u_{i} \eta-c_{1}\right) \pi^{-\frac{1}{2}}+\left(\frac{u_{2} \eta^{2}}{2}-c_{2}\right) \pi^{-1}+\cdots \cdot\left(\psi_{0 z}+\psi_{i z} \pi^{-\frac{1}{2}}+\psi_{2 z} \pi^{-1}+\cdots\right)\right.} \\
& =\left(u_{0 z}+u_{i z} \eta \pi^{-\frac{1}{2}}+u_{2 z} \frac{\eta^{2}}{2} \pi^{-1}+\cdots\right)\left(\psi_{0}+\psi_{1} \pi^{-\frac{1}{2}}+\psi_{2} \pi^{-1}+\cdots\right) .
\end{aligned}
$$

To the leading order, that is $0\left(\chi^{2}\right)$, we have

$O\left(\pi^{2}\right): h_{0}\left(\psi_{s}\right) \equiv\left[u_{c}(z)-C_{c}\right]\left[\delta^{2}\left(\frac{\psi_{c o}}{N^{2}}\right)_{z}-k_{0}^{2} \psi_{0}\right]-\left[\beta_{0}-\delta^{2}\left(\frac{u_{c t}}{N^{2}}\right)_{z}\right] \psi_{0}=0,(7)$ 


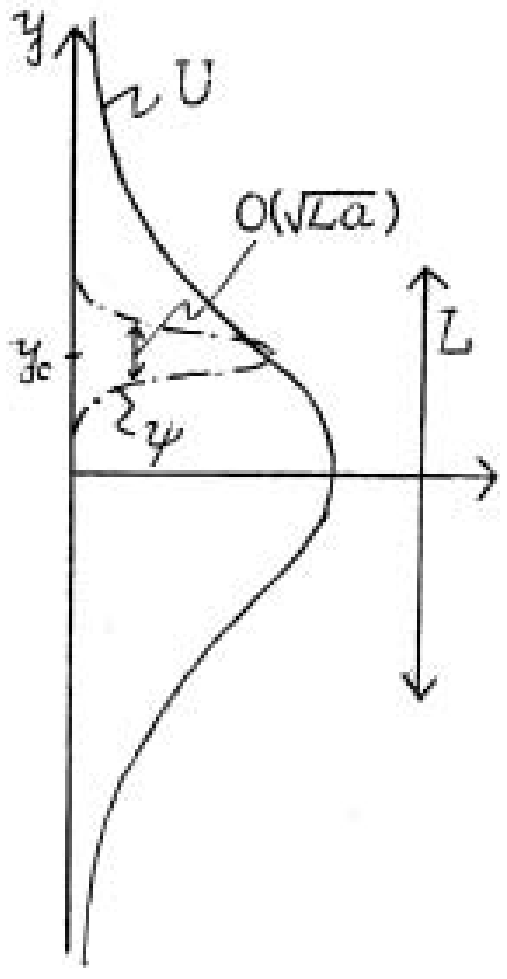

Figure 1

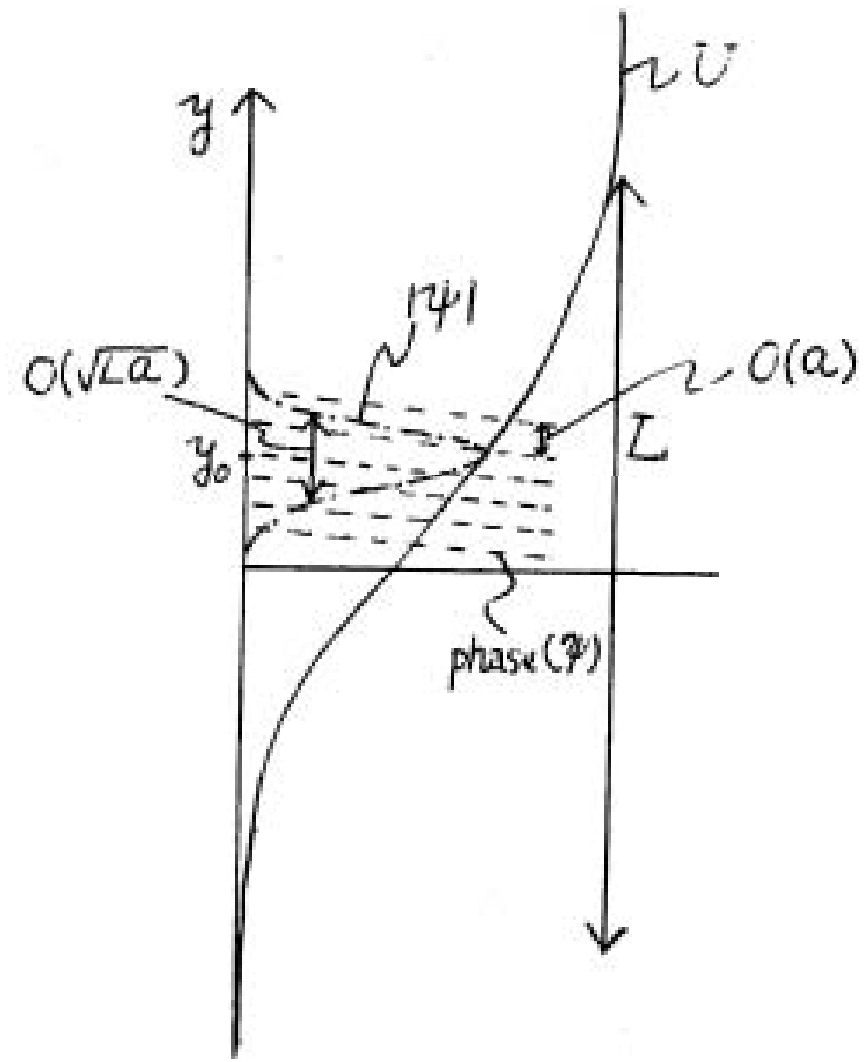

Figure 2

Notes Submitted By

Hiroshi Niino 


\section{LECTURE 6}

In the last lecture we investigated the large $\boldsymbol{\lambda}$ asymptotic behavior of the solution of the equation:

$$
(u-c)\left(\psi_{y y}-\hbar^{2} \psi+\lambda^{2} \delta^{2}\left(\frac{\psi_{z}}{N^{2}}\right)_{z}\right)+\left(\beta-u_{y y}-\lambda^{2} \delta^{2}\left(\frac{u_{z}}{N^{2}}\right)_{z}\right) \psi=0
$$

where:

$$
\begin{aligned}
& =\mathrm{L} / \mathrm{a} \\
& =\mathrm{depth} \text { scale of the } \mathrm{N} \text { variations } \\
\beta & =\boldsymbol{\beta}_{\boldsymbol{\alpha}} \leq \mathrm{L}^{2} / \mathrm{V}
\end{aligned}
$$

In this lecture we will focus on the small $\boldsymbol{\lambda}$ asymptotic as well as on the $\delta \ll 1$ limit.

The limiting case when $\lambda \ll \mathbf{1}$

We first anticipate that since $L \ll$ a the flow is very narrow compared with the radius of deformation, a, and therefore the stratification does not. significantly affect the flow. This might be an indication that the flow is barotropic. We get another indication in the same direction by considering the ratio of total kinetic energy to total available potential energy,

TKE/TAPE; this can be shown to be of the order of $\lambda^{-2}$. Hence for small $\lambda$ the kinetic energy seems to be the source from which the instabilities can draw their energy.

Indeed for a two-layer problem it can be shown that this is the case (Killworth, 1980), but for the continuous case things are not as simple.

$$
\begin{aligned}
\text { If we naively expand } C \text { and } \Psi \text { in power series of } \\
\qquad=C_{0}+\lambda^{2} C_{1}+\ldots \\
\Psi=\Psi_{0}+\lambda^{2} \Psi_{1}+\ldots
\end{aligned}
$$

then the leading order equation is

$$
\left(u-c_{0}\right)\left(\psi_{0 y y}-k^{2} \Psi_{c}\right)+\left(\beta-u_{y y}\right) \Psi_{0}=0
$$

This equation is identical in form to the Rayleigh equation. However, $U$ in (3) is a function of $Z$ also, so that for each value of $Z$ there exists an eigenvalue, Co, of (3). For different values of $Z$, different eigenvalues $C$ will be encountered, and therefore in general (3) has no solutions. An exception to this unfortuitous situation is if U varies slowly with $\mathrm{Z}$ (i.e. $u_{z} \leqslant 0(\lambda)$ ).

Thus, the neglected $\mathrm{Z}$ variations of $\boldsymbol{\Psi}$ must be important somewhere, and we distinguish between various possibilities; a boundary layer at the surface or a the bottom, or a region at an intermediate depth in which $\Psi$ is nonzero, vanishing above and below it.

For the last case, where $\boldsymbol{\Psi}$ vanishes outside a region around Zo we can transform $Z$ to a stretched coordinate about $\mathrm{Zo}, \quad \zeta=\lambda^{-1 / 2}(\mathrm{Z}-\mathrm{ZO})$; (we could also formally proceed by a WKBJ expansion). In the local coordinate 3 
the leading order equation which we get from (1) is equation (3) with $U(y, z)$ replaced by $\mathrm{U}(\mathrm{y}, \mathrm{zo})$. Therefore, the leading order equation yields Co as a function of Zo. The next order equation, $\left(0\left(\lambda^{1 / 2}\right)\right)$ then provides the solubility condition $\left.\partial C D Z\right|_{\mathbf{Z}, Z_{*}}=0$, and this determines Zo.

If there exists zo such that $U_{z}(y, z o)=0, \forall y$, then Co is indeed the eigenvalue of the local Rayleigh problem near Zo. In general, however, we have to consider the case when $\mathrm{Zo}$ is complex. .

The next order equation $(O(\lambda))$ then determines the variation of $\boldsymbol{\Psi}$ with depth, and it turns out to be a parabolic cylinder function of the form

$$
\psi(z) \sim \operatorname{Ex\rho }\left(-\alpha \frac{\left(z-z_{0}\right)^{2}}{\lambda}\right)
$$

for some complex number $\alpha$ with $\operatorname{Re}(\alpha)>0$. Thus, the solution is confined in the vertical to range $0\left(\lambda^{1 / 2}\right.$ ) with rapid oscillations (in phase) on depth scale $0(\lambda)$ (since $Z_{0}$ is complex). We see again a behavior similar to the large $\lambda$ solutions (but with $Z$ replacing $y$ and $\lambda^{-1}$ replacing $\lambda$ ). The schematic of the solution is:

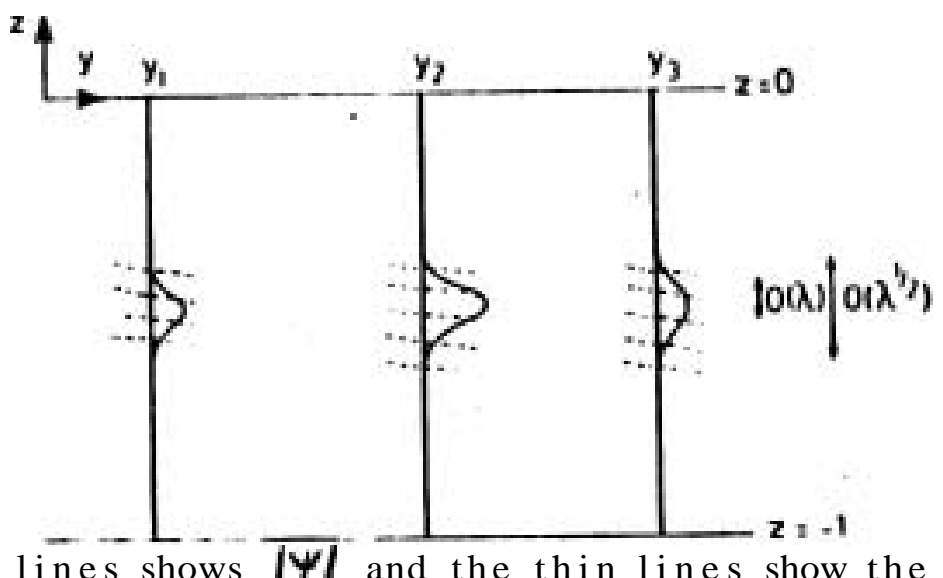

where the thick lines shows $|\boldsymbol{\Psi}|$ and the thin lines show the phase of $\psi$. If $\mathrm{U}_{\mathrm{z}}(\mathrm{y}, \mathrm{zo})=0, \mathbf{v y}$ then the phase of $\Psi$ varies on $\lambda^{1 / 2}$ scale, too.

The instabilities in this case are indeed barotropic as we have anticipated.

In the other cases mentioned where the boundary layer is (say) at the top, it can be shown (Killworth, 1980) that no solutions exist unless U-C is suitably small somewhere at the surface. In the y-independent case of this problem (i.e. with U-C suitably small at the boundary layer) the instabilities are baroclinic, and this is contrary to our naive expectation.

Other parameter ranges which are of interest:

$\underline{1} \ll 1$

In this case the stratification is confined to a very thin top layer and $N_{z}$ changes on a $\delta \ll 1$ scale, while $\lambda$ is $0(1)$ and so is $\beta$. The velocity field will be assumed to vary on vertical scales of order 1 so that $\left(\frac{\mathrm{Uz}}{\mathrm{N}^{2}}\right)_{\mathrm{z}}$ is large below depths of the order of $\delta$. We consider two cases: 


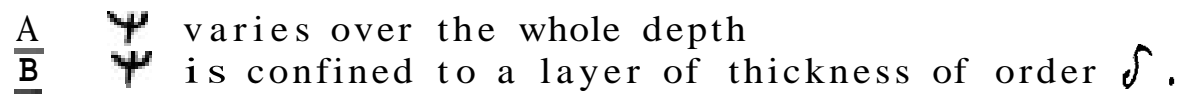

In case $A$, below $Z=\oint$, the main terms in (1) are

$$
(u-c)\left(\frac{\Psi_{2}}{N^{2}}\right)_{z} \approx\left(\frac{u_{z}}{N^{2}}\right)_{z} \Psi
$$

and this has a solution

$$
\psi(y, z)=A(y)(u-c)=\psi(y, 0)(u-c) /(\bar{u}-c)
$$

where $\overline{\mathrm{U}}=U(\mathrm{y}, 0)$.

Since near the surface $(|z| \leqslant \delta)$ the vertical derivatives are small when (6) is substituted in (1), we get the near-surface equation

$$
(\bar{u}-c)\left(\psi(y, 0)_{y y}-k^{2} \psi(y, 0)\right)+\left(\beta-\bar{u}_{y y}\right) \psi(y, 0)=0
$$

Therefore, (6) is a solution if the surface velocity is unstable in the sense of the Rayleigh problem (7). The instability is baroclinic in this case.

In case B, $\Psi$ is confined to the surface, and we transform to the stretched coordinates $\xi=z \delta^{-1}$. In that case the leading order of (1) yields

$$
(\bar{u}-c)\left(\psi_{y y}-h^{2} \psi+\lambda^{2}\left(\frac{\psi_{z}}{N^{2}}\right)_{z}\right)+\left(\beta-\bar{u}_{y y}\right) \psi=0
$$

where $\widetilde{u}(y)=u(y, 0)$ a s in the preceding case. The boundary conditions are:

$$
\begin{aligned}
& \psi_{\xi}=0 \quad \xi=0 . \\
& \psi \rightarrow 0, \quad \zeta \rightarrow-\infty
\end{aligned}
$$

Equation (8) has a separable solution in $y$ and 3 of the form $\boldsymbol{\Psi}(y, \zeta)=a(y) g(\zeta)$. Substituting this form in (8) we get the O.D.E. for $g$

$$
\left(\frac{g 3}{N^{2}}\right)_{z}=-\alpha^{2} g
$$

where g has to satisfy the boundary conditions (9) and where $\propto$ is the separation constant. Equation (10) has a countable infinity of solutions and eigenvalues $\propto$ since $\mathrm{N}^{3} 0, \zeta \rightarrow \cdot \infty$. For each of these eigenvalues $\propto$ we get the following Rayleigh equation for $A(y)$

$$
(\bar{u}-c)\left(a_{y y}+\left(\alpha^{2} \lambda^{2}+k^{2}\right) a\right)+\left(\beta-\bar{u}_{y y}\right) a=0
$$

Again the surface velocity has to be unstable in the Rayleigh (barotropic) sense, and the resulting instability is barotropic. 
There are other limiting cases discussed in Killworth (1980) as well as two-layer versions of all these limits, but we will stop here and summarize the finding for the continuous case in the following $(\lambda, \delta)$ diagram showing which instabiities are found in the various parameter regions.

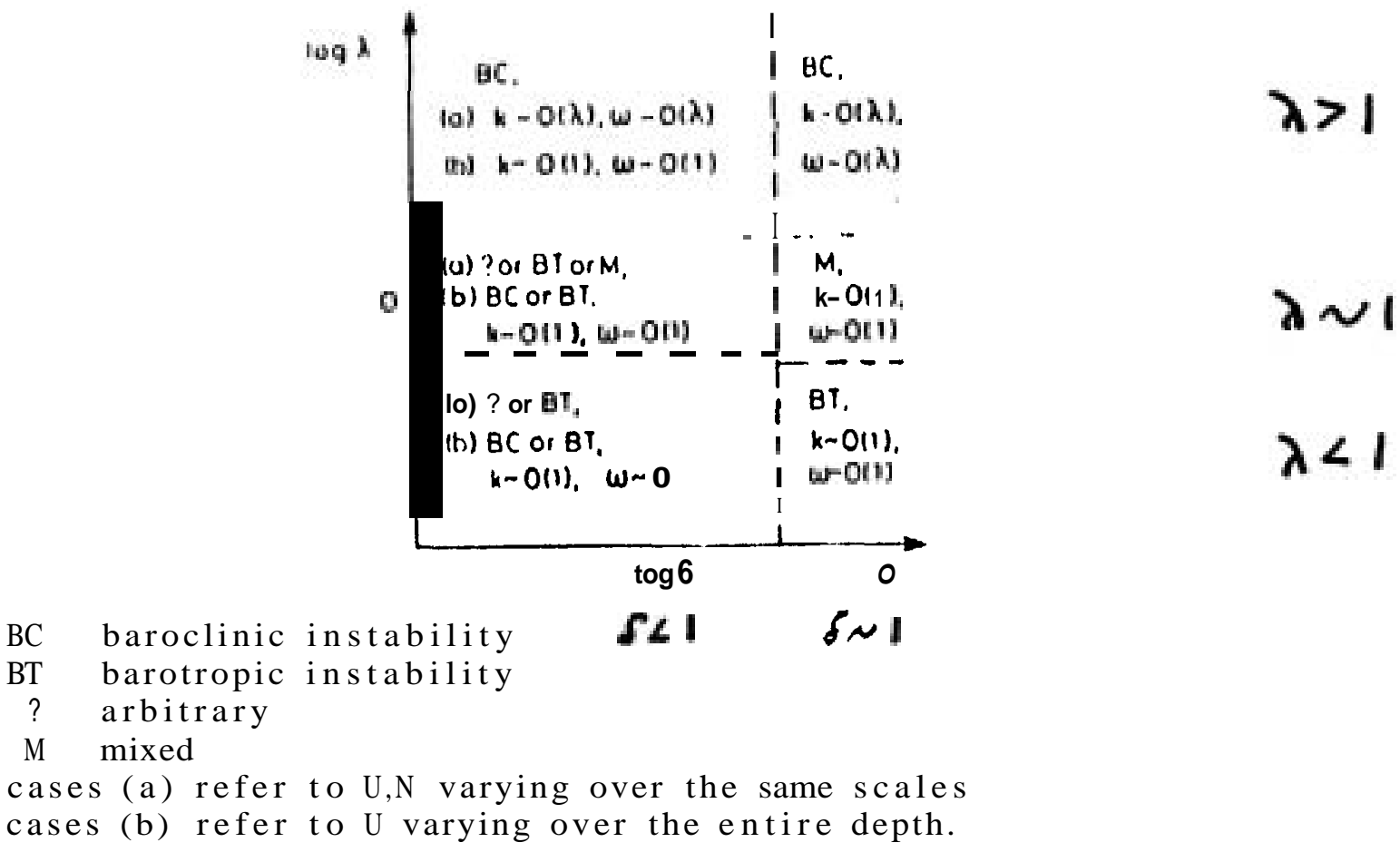

Until now we used the normal mode approach to study the stability of various quasi-geostrophic systems. We will focus our attention now on a time-dependent baroclinic instability and study as a particular case of the time evolution of the instability in Eady's problem (for a more complete treatment, see Simmons and Hoskins, 1979).

We start with a simple velocity field

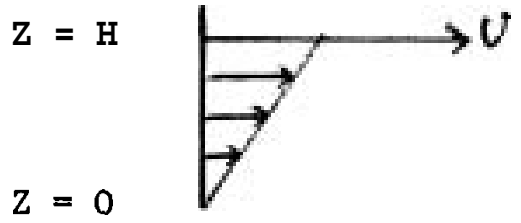

$$
\mathbf{U}=\mathbf{A Z}
$$

and look at a meridionally homogeneous stream function

$$
\operatorname{Re} \Psi(z, t) E X
$$

Scaling $\mathrm{Z}$ by $\mathrm{H}, \mathrm{t}$ by $\mathrm{Af} / \mathrm{N}$ and $\mathrm{k}$ by $\mathrm{NH} / \mathrm{f}$ ( so that $\mathrm{U}=\mathrm{Z}$ ), we get the following equation for $\Psi$ from the quasi-geostrophic potential vorticity equation

$$
\left(\frac{S t}{\partial t}+i k z\right)\left(\psi_{z z}-k^{2} \psi\right)=0
$$

and the boundary conditions are no normal flow at $\mathrm{Z}=0,1$ i.e.

$$
\psi_{z t}+i \hbar \psi_{z}-i h \psi=0
$$




$$
\psi_{z t}-i k \psi=0
$$

$$
z=0
$$

The integration of (12) with respect to t yields

$$
\left(\psi_{z z}-k^{2} \Psi\right)=\left.\left(\psi_{z z}-k^{2} \Psi\right)\right|_{t=0} \operatorname{Ex\rho }(-i \beta z t)
$$

The general solution of (15) is

$$
\Psi(z, t)=\frac{E X P(-i k z t)}{1+t^{2}}+A(t) \cos H(\alpha z)+B(t) \operatorname{srnH}(\alpha z)
$$

where $\mathbf{A}(t), \mathbf{B}(t)$ are determined by the boundary conditions (13), (14).

We now introduce the complex growth rates of the normal mode problem, i.e., $A(t), B(t) \sim \exp \left(c_{1} t\right), \exp \left(c_{2} t\right)$ where

$$
l_{1,2}=-\frac{i k}{2} \pm\left[\left(\frac{k}{2}-\operatorname{TANH}\left(\frac{k}{2}\right)\right)\left(\operatorname{Cot} H\left(\frac{k}{2}\right)-\frac{k}{2}\right)\right]^{1 / 2}
$$

We apply an initial disturbance which is independent of depth

$$
\Psi(x, z, 0)=\cos ^{2}\left(\frac{\pi x}{L}\right) \cdot E x p\left(\frac{-x^{2}}{d^{2}}\right) \quad-L / 2<x<L / 3
$$

with periodic boundary condition at $x= \pm L / 2$. What the numerical integration of the equation showed is that the disturbance was advected downward at about the phase speed of the fastest growing normal mode but its growth rate is significantly larger. The vertical variation of the disturbance approached the normal mode form very slowly but the downstream development of the disturbance at the bottom was much faster than the normal mode calculation suggests.

These numerical findings demonstrate the need for a more accurate temporal analysis. Thus we look at a localized initial perturbation of the form

$$
\psi(x, z)=\int_{-\infty}^{\infty} A(k) \phi(z, h) \operatorname{Exp}(i k x) d h
$$

The symptotic theory for expressions of the form (18) is well established and we look for the solution at a point $X$ which moves downstream with velocity $\mathcal{U}$. After a 11 transients have decayed the perturbation can be written as

$$
\psi(x, z, t)=\int_{-\infty}^{2} A(k) \phi(z, k) E x P(-i \Omega(h) t), t \rightarrow \infty \text { (19) }
$$

where

$$
\Omega(k)=k(c(k)-U)
$$


The theory of Bers (1975) suggests that if there exists a complex $k_{s}$ such that $2 \Omega / \partial k_{\xi}=O$ with $\operatorname{Im} \Omega\left(\mathrm{k}_{\mathrm{g}}\right)>0$

then (19) can be approximated by

$$
a\left(h_{s}\right) \phi\left(z, h_{s}\right) t^{-1 / 2} \operatorname{ExP}\left(-i \Omega\left(k_{s}\right) t\right)
$$

This gives a local wavelength $2 \boldsymbol{r} / \operatorname{Re}\left(\mathbf{k}_{\mathbf{g}}\right)$, a local growth rate of $\mathrm{Im} \boldsymbol{\Omega}\left(\mathbf{k}_{\mathbf{g}}\right)$ and a local phase speed of $\operatorname{Re}\left(\Omega\left(\mathbf{k}_{\mathrm{g}}\right)\right) / \operatorname{Re}\left(\mathbf{k}_{\mathrm{g}}\right)+\mathrm{U}$.

If we apply this to the parameters of Eady's problem discussed previously $\Omega$ becomes

$$
\Omega(k)=k\left(\frac{1}{2}-U\right) \pm\left[\left(\frac{k}{2}-\operatorname{COTH}\left(\frac{k}{2}\right)\right)\left(\frac{k}{2}-\operatorname{TANH}\left(\frac{k}{2}\right)\right)\right]^{1 / 2}
$$

and $\mathbf{k}_{\mathbf{s}}$ is determined by

where

$$
\left.\frac{\partial \Omega}{\partial k}\right|_{k=B_{s}}=\frac{1}{2}-U \pm \frac{1}{4}\left(X+\frac{1}{x}\right)
$$

$$
X=T A N H^{2}\left(\frac{k}{2}\right)\left[\left(\frac{k}{2}-\operatorname{COTH}\left(\frac{k}{2}\right)\right)\left(\left(\frac{k}{2}-\operatorname{TANH}\left(\frac{h}{2}\right)\right)\right]^{1 / 2}\right.
$$

Taking the positive root of (22) we find that $\frac{\partial \Omega}{\partial h}=0$ at

$$
x=-(1-2 v) \pm 2\left(v^{2}-v\right)^{1 / 2}
$$
$k_{s}=\infty$.

A solution $X$ of (24) exists only if $U \leq 1$. When $U=1, X=1,1 . e$. ,

Analytical solutions for the case $U=1-\mathcal{E}, \mathcal{E} \ll \mathbf{1}$ can be obtained and they are:

$$
x=1 \pm 2 i \varepsilon^{1 / 2}+o(\varepsilon)
$$

and this gives

$$
k_{5}=\ln \left(\varepsilon^{1 / 2}\right)-\ln \left(\frac{1}{2}\right) \pm i r / 2+o\left(\varepsilon^{1 / 2}\right)
$$

Therefore this positive root of (26) gives a saddle point (where $2 \Omega / 3 k=0$ ) with Im $\Omega \geqslant 0$ and this demonstrates the existence of a local instability with a short zonal wavelength of order $-2 \% / \ln \varepsilon^{1 / 2}$, a local growth rate of order $\varepsilon_{K / 2}$ and a phase speed of order $1+1 / \ln \varepsilon^{1 / 2}$.

Although these estimates are valid for $t \rightarrow \infty$ they are in good agreement with the numerical simulation after $\mathbf{1}$ day. 


\section{LECTURE 7}

\section{$\underline{\text { Instabilities of Fronts }}$}

A few informal remarks are in order before we proceed with the theory. A front may be characterized as a narrow region with a steeply inclined density surface (isopycnal).

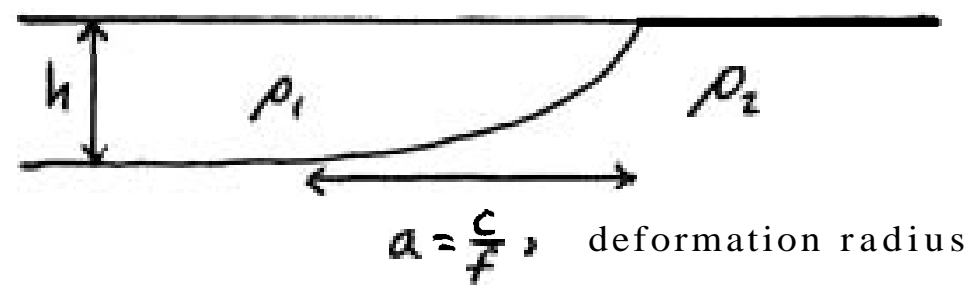

Fronts form on different scales, but in general the cross-front scale of the motion will be less than the deformation radius $a=c / f$, where $c$ is the internal wave speed (for two layers, $c \sim \sqrt{g \frac{\rho_{2}-\rho_{1}}{\nu_{x}}} h$ ) and $f$ the Coriolis parameter. Thus, the significant quasigeostrophic scale a, informally the width to which quasigeostrophy holds, will not be small enough to resolve the front. Typical speeds on fronts will be of the order of the internal wave speed c, so the vorticity will be typically of order c/a =f, and a typical Rossby number will be of order 1 . The density $\rho$ will not be a small

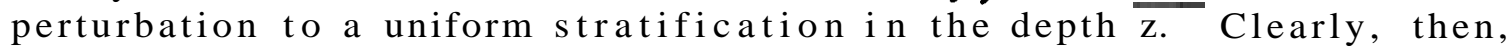
quasigeostrophy will not be a good theory for discussing frontal dynamics (formation, meanders, etc.).

For the most part we will ignore convergence fronts and any fronts produced by the strain rate and restrict ourselves to the fronts explored in the experimental work of Griffiths and Linden (1981). The basic state will be geostrophic, so kinetic and potential energy will be available, with the Coriolis force opposing the instability. Consider the simplest possible physics, two homogeneous layers of constant but differing densities.

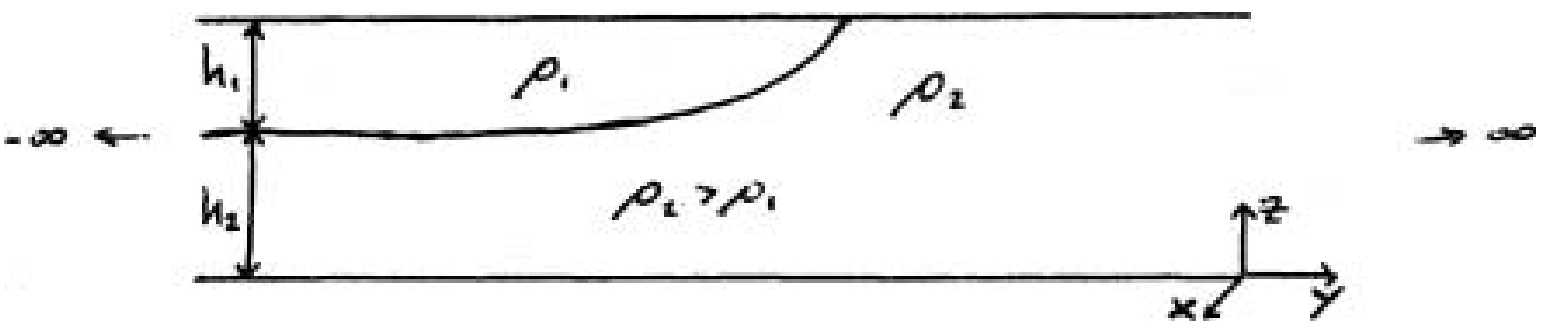

Assume that the horizontal scale of the motion is much greater than the vertical scale, so the hydrostatic balance holds (this will not be true for a horizontal gravity current). Then $\nabla_{H} P_{\mathbf{E}}=0$, so horizontal velocities will remain independent of $z$ if they are so fritially, which we take them to be. This represents a gross simplification of the vertical problem.

The horizontal momentum equations and the depth-integrated conservations of mass equations are

$$
\rho_{i}\left(\frac{D_{i}}{D t} u_{i}-f v_{i}\right)=-P_{i x} \quad, i=1,2 \quad\left(\frac{D_{i}}{D_{t}}=\frac{\partial}{\partial t}+u_{t}, \nabla\right)
$$




$$
\begin{array}{ll}
p_{i}\left(\frac{D_{i}}{D t} v_{i}+f u_{i}\right)=-p_{i y}, i=1,2 \\
h_{i t}+\nabla \cdot\left(u_{i} h_{i}\right)=0 \quad, i=1,2
\end{array}
$$

These are still very nonlinear and numerous. We further simplify by taking $\mathrm{h}_{2} \gg \mathrm{h}_{1}$. Then the flow in the lower layer is small, since a mass flux condition gives $\mathbf{u}_{1} \mathbf{h}_{1} \sim \mathbf{u}_{2} \mathbf{h}_{2}$, and we take it to be zero:

$$
u_{2}=v_{2}=p_{2 x}=p_{2 y}=0
$$

Here we have thrown away a layer that we know is important to baroclinic instability, so the theory we come up with will have gaps. (We are also avoiding the exact physics of the corner where the interface surfaces, where dissipation and other effects may be important.)

The hydrostatic equations can be integrated to determine $\mathrm{p}_{1}$, taking $\mathrm{p}=\mathrm{O}$ (or any constant) at the surface and $\mathbf{z}=0$ at the bottom:

Layer 1:

$$
\begin{aligned}
& P_{1 z}=-g \rho_{1} \\
& p_{1}=-g \rho_{1}\left(z-h_{1}-h_{2}\right)
\end{aligned}
$$

Interface:

$$
\begin{aligned}
& p_{1}\left(h_{2}\right)=g p_{1} h_{1} \\
& p_{2}\left(h_{2}\right)=p_{1}\left(h_{2}\right)=g p_{1} h_{1}
\end{aligned}
$$

Layer 2:

$$
\begin{aligned}
& p_{2 z}=-g \rho_{2} \\
& p_{2}=g \rho_{1} h_{1}-g \rho_{2}\left(z-h_{2}\right)
\end{aligned}
$$

Then eq. (4) implies

$$
\rho_{1} \nabla h_{1}+\rho_{2} \nabla h_{2}=0
$$

The horizontal gradient of $\mathrm{p}_{1}$ is

$$
\nabla_{H} P_{1}=g \rho_{1} \nabla\left(h_{1}+h_{2}\right)=\rho_{1} g^{\prime} \nabla h_{1}
$$

where

$$
g^{\prime}=g \frac{\left(\rho_{2}-\rho_{1}\right)}{\rho_{2}}
$$


is the reduced gravity. Dropping the subscript 1's, the upper layer equations become

$$
\begin{aligned}
& u_{t}+u u_{x}+v u_{y}-f v=-g ' h_{x} \\
& v_{t}+u v_{x}+v v_{y}+f u=-g^{\prime} h_{y} \\
& h_{t}+\nabla \cdot(\underline{u h})=0
\end{aligned}
$$

These are the nonlinear reduced gravity shallow water equations.

Let's briefly review the properties of these equations. Linearizing about a basic state $h=h_{0}, u=v=0$ gives

$$
\begin{aligned}
& u_{t}-f v=-g \cdot h_{x} \\
& v_{t}+f u=-g ' h_{y} \\
& h_{t}+h_{0}\left(u_{x}+v_{y}\right)=0
\end{aligned}
$$

A vorticity equation can be formed from the first two,

$$
\text { (B) } x-(A)_{y} 3\left(v_{x}-u_{y}\right)_{t}+f\left(u_{x}+v_{y}\right)=0
$$

(taking $f$ to be constant here) and, with the third, a potential vorticity equation,

$$
\left(v_{x}-u_{y}-\frac{f h}{h_{0}}\right)_{t}=0
$$

The terms multiplying $f$ are the stretched planetary vorticity terms; the velocity curl is the relative vorticity. Rotational gravity waves (Poincare? waves) are solutions, as are planetary waves if $f$ is not constant. A nonlinear potential vorticity equation can also be derived:

$$
\left(\frac{\partial}{\partial t}+u, \nabla\right)\left(\frac{v_{x}-u_{y}+f}{h}\right)=0
$$

so that potential vorticity is conserved following the motion. This allows a useful simplification: one nonlinear equation can be replaced by eq. (14) with a simple value for the potential vorticity, for instance, zero or any constant. However, conclusions drawn from such special cases may not be valid for the general case.

Now, let's get on with instability. Consider a front, which we define as somewhere where the interface hits the surface and the upper layer depth vanishes. To get a simple solution, consider a steady mean flow with no variation in the $\mathrm{x}$-direction,

$$
\overline{\mathrm{u}}=\overline{\mathrm{u}}(\mathrm{y}), \overline{\mathrm{v}}=0, \overline{\mathrm{h}}=\overline{\mathrm{h}}(\mathrm{y})
$$

The flow must satisfy eqs. (11-13), namely, the geostrophic balance must hold:

$$
f \bar{u}=-g^{\prime} \bar{h}_{y}
$$


It is useful to nondimensionalize the equations before proceeding with the analysis. Choose

$$
\begin{aligned}
& \text { depthscale } \mathrm{H} \text {, maximumdepthof upper layer } \\
& y \text {-scale } \quad a=\sqrt{g^{\prime}} H^{\prime} / f, \text { deformation radius } \\
& \text { u-scale } \sqrt{y^{\prime}} \mathrm{H} / f \text {, internal wave speed } \\
& x \text {-scale } \quad \boldsymbol{a} \boldsymbol{\epsilon}^{-1}=\sqrt{\boldsymbol{g}^{\prime} H^{\top}} / \boldsymbol{f} \boldsymbol{\epsilon}, \epsilon \text { nondimensional and arbitrary } \\
& \text { v-scale } \in \sqrt{g^{\prime} H} \\
& \text { t-scale } \epsilon^{-1} f^{-1}
\end{aligned}
$$

The $x$-scaling anticipates the long-wave approximation in the $\mathrm{x}$-direction, but at this point it is merely a nondimensionalization. The scales for $\mathrm{v}$ and $\mathrm{t}$ are obtained by requiring all terms in the continuity equation to be of the same scaled order. Thus, eqs. (11-13) become

$$
\begin{aligned}
& u_{t}+u u_{x}+v u_{y}-v=-h_{x} \\
& \epsilon^{2}\left(v_{t}+u v_{x}+v v_{y}\right)+u=-h_{y} \\
& h_{t}+(u h)_{x}+(v h)_{y}=0
\end{aligned}
$$

The scaled potential vorticity equation is

$$
D_{Z}\left(\frac{\epsilon^{2} v_{x}-u_{y}+1}{h}\right)=0 \text {. }
$$

For $\epsilon \ll 1$, we expect the geostrophic balance downstream $\left(u=-h_{y}\right)$, though we may not get it.

Consider perturbations to the undisturbed mean flow

$$
\overline{\mathrm{u}}(\mathrm{y})=-\overline{\mathrm{h}}_{\mathrm{y}}(y)
$$

of normal mode form

$$
\left(u^{\prime}, v^{\prime}, h^{\prime}\right)=(\hat{u}, \hat{v}, \hat{G}) e^{1}(x-c t)
$$

Note that has now taken on the role of a wave number. Substituting these into eqs. (17-19) and dropping the hats, we obtain

$$
\begin{aligned}
& (\bar{u}-c) u+\left(1-\bar{u}_{y}\right) i v+h=0 \\
& u+\epsilon^{2}(\bar{u}-c) i v+h_{y}=0 \\
& \bar{h} u-\frac{d}{d y}(1 v \bar{h})+(\bar{u}-c) h=0
\end{aligned}
$$

and defining $V=i v$ will get rid of the $i$ 's. (This suggests that $v$ is $\frac{\pi}{2}$ out of phase with $\mathbf{u}, \mathbf{h}$. However, $\mathrm{c}$ may also be complex - in fact it must be for instability!). Eqs. (22-24) are three equations for $\mathbf{u , v}, \mathbf{h}$ and the eigenvalue c. Boundary conditions are also needed. We consider three possibilities:

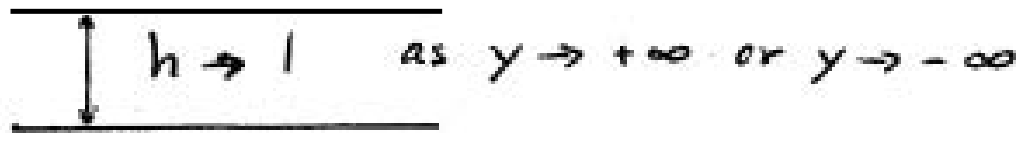


$-45-$

Requiring the disturbance to have no outgoing or incoming energy flux in this case gives $u, v, h \rightarrow 0$ as $y \rightarrow+\infty$ or $y \rightarrow-\infty$;

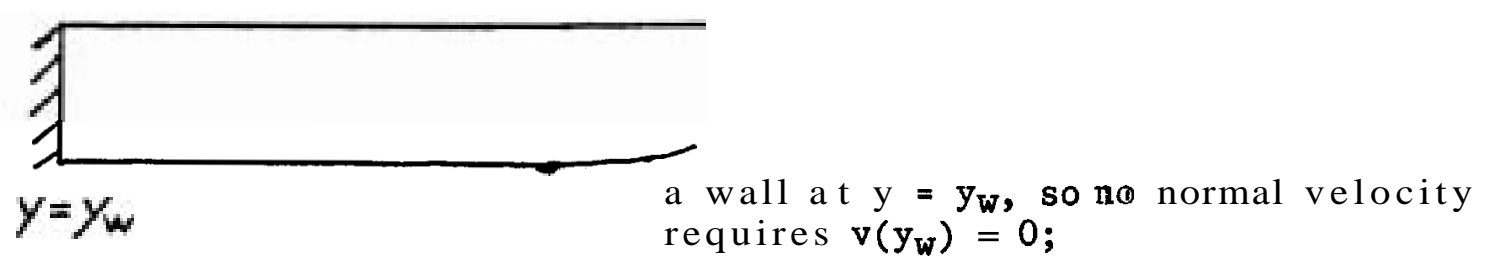

(111)

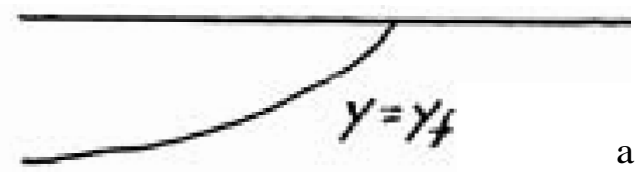

a front at $y=y_{f}$, where $\bar{h}\left(y_{f}\right)=0$.

Mathematically, eq. (24) is singular at $y=y_{f}$ in case (iii), and we require a regular solution. Formally, we must drop the linearization for the moment to get the condition at the front. The front is defined by

$$
\mathrm{h}=0 \text { on } \mathrm{y}=\mathrm{y}_{\mathrm{f}}+\mu \hat{\mathrm{y}}(\mathrm{x}, \mathrm{t})
$$

where $\mu \ll 1$ assures the position will be well defined (i.e. not multivalued), and we write

$$
\begin{aligned}
& \mathrm{u}=\overline{\mathrm{u}}+\mu \mathrm{u}^{\prime} \\
& \mathrm{v}=\mu \mathrm{v}^{\prime} \\
& \mathrm{h}=\overline{\mathrm{h}}+\mu \mathrm{h}^{\prime}
\end{aligned}
$$

The front is a string of material particles, so

$$
\frac{D}{D t}\left(y-y_{f}-\mu \hat{y}\right)=0 \text { on } y=y_{f}+\mu \hat{y}
$$

Then

$$
\mu v_{y=y_{f}}^{\prime}=\left.\mu\left(\hat{y}_{t}+\bar{u} \hat{y}_{x}\right)\right|_{y=y_{f}}+o\left(\mu^{2}\right)
$$

The condition that $h$ vanish at the front becomes

so

$$
\begin{aligned}
& \sum_{y=y_{f}+\mu \hat{y}}+\mu h_{y=y_{f}} \mid=0 \\
& \mu \hat{y} \bar{h}_{y=y f}+\left.\mu h^{\prime}\right|_{y^{\prime} y_{f}}=0
\end{aligned}
$$

Solving for $y$ and replacing it in the expression above,

or

$$
\begin{aligned}
& v_{y=y_{f}}=\left(\frac{\partial}{\partial t}+\bar{u} \frac{\partial}{\partial x}\right)\left(\frac{-h^{\prime}\left(x, y_{f}, t\right)}{\bar{h}_{y}\left(y_{f}\right)}\right) \\
& \bar{u} v=i(\bar{u}-c) h \quad \text { on } y=y f
\end{aligned}
$$


This is exactly eq. (24) expanded and evaluated at $\mathbf{y n}=\mathbf{7 y} \mathbf{f}$, where $\mathrm{h}=0$, so the condition says that eq. (24) is well-behaved at the front.

In general, the problem posed by eqs. (22-24) with boundary conditions is analytically intractable. We can get solutions on machines using numerical integrators, zero-finders, etc. But solving one problem gives no information on another. In particular, there are no known necessary conditions for instability such as there are for quasigeostrophic instability theory (i.e. the gradient of potential vorticity must vanish somewhere). What I'd like to develop here are some analytic statements which can be made about the instability of a one-layer frontal system. Specifically, we can deduce:

(1)

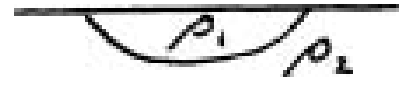

(11)

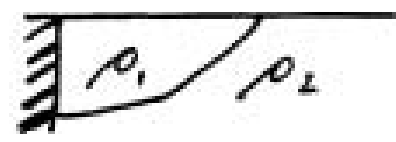

(iii)

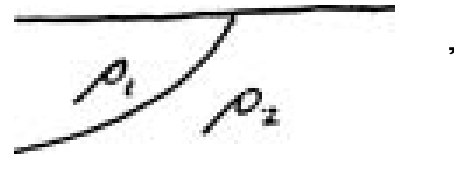

the two-front case is always unstable to long waves (Griffiths, Killworth, and Stern, 1981)

the coastal current is unstable to long waves if the potential vorticity increases towards the wall (Killworth and Stern, 1982)

the isolated front is unstable to long waves if $\lim _{y \rightarrow-\infty} \frac{u}{a_{y}}<1$, i.e. if the

front is sufficiently "narrow" (Killworth, 1983).

The short wave modes around the front are not understood in any of these cases. The full two-layer problem is still an area of active research.

Consider the two-front case for zero potential vorticity. Since the mean potential vorticity vanishes,

$$
1-\bar{u}_{y}=1+\bar{h}_{y y}=0
$$

and

$$
\overrightarrow{\mathrm{u}}=\mathrm{y}, \overrightarrow{\mathrm{u}}=1-\mathrm{y}^{2} / 2
$$

so $\bar{h}$ vanishes at $y= \pm \sqrt{2}(\bar{h}$ has been scaled to equal 1 at maximum). The depth is symmetric, the flow antisymmetric.

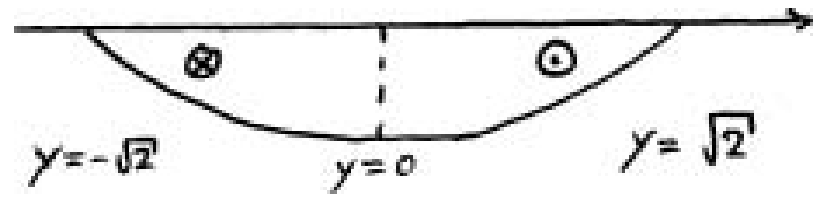

The perturbation potential vorticity must satisfy

$$
u_{y}-\epsilon^{2} i v \equiv u_{y}-\epsilon^{2} V=0
$$

Using eq. (26) instead of eq. (23) and noting that $1-\overline{\mathbf{u}}_{\mathbf{y}}=0$ in Eq. 22, we get

$$
\begin{aligned}
& (\bar{u}-c) u+h=0 \\
& \overline{h u}-(v \bar{h})_{y}+(\bar{u}-c) h=0 \\
& u_{y}=\epsilon^{2} v
\end{aligned}
$$


One equation for u can be obtained by eliminating $\mathrm{h}$ and $\mathrm{V}$,

$$
\left(\bar{h} u_{y}\right)_{y}-\epsilon^{2}\left(\bar{h}-(\bar{u}-c)^{2}\right) u=0
$$

with the boundary condition that $u$ be regular at $y= \pm \sqrt{2}$.

Eq. (30) can be integrated from $y=-\sqrt{2}$ to $y=\sqrt{2}$ to obtain

$$
-\sqrt{z}\left[\bar{h}-(\bar{u}-c)^{2}\right] \text { udy }=0
$$

from which we will be able to get a quick answer using a power series.

Notes Submitted By

Roger Samelson 


\section{$\underline{\text { LECTURE } 8}$}

Equation (31) derived in yesterday's lecture does not have any known algebraic solution, so far as $I$ know. For small $\epsilon$ we may try to expand the solution in powers of $\epsilon$. Doing that we write:

$$
\begin{aligned}
& c=c_{0}+\epsilon c_{1}+\epsilon^{2} c_{2}+\ddot{H}^{*} \\
& u=u_{0}(y)+\epsilon u_{1}(y)+\ldots
\end{aligned}
$$

We also normalize the amplitudes in such a way that $\mathrm{u}_{\mathrm{o}}(0)=\mathbf{1}$ and $\mathrm{u}_{i}(0)=0$ for $1>0$. Substituting the above expansion into Equation (30) we get:

$$
\begin{aligned}
{\left[\bar{h}\left(u_{o y}+\epsilon u_{1 y}+\epsilon^{2} u_{2 y}+\cdots \cdot\right]_{y}-\epsilon^{2}\left[\bar{h}-\left(\bar{u}-c_{0}\right)^{2}-2 \epsilon c_{1}\left(\bar{u}-c_{0}\right)+\right.\right.} \\
\epsilon^{2}\left(c_{1}-2\left(\bar{u}-c_{0}\right) c_{2}+\cdots\right]\left(u_{0}+\epsilon u_{1}+\epsilon^{2} u_{2}+\cdots\right) .
\end{aligned}
$$

To the leading order we have:

$$
\left(\bar{h} u_{o y}\right)_{y}=0 \Rightarrow \bar{h} u_{o y}=\operatorname{constant}
$$

at $\mathrm{y}= \pm \sqrt{2} \mathrm{~h}=0$, so if uoy is bounded, then the constant is zero, and

$$
u_{0}=1
$$

To find $c_{o}$ we substitute this into Equation (31) which gives to leading order:

$$
\int_{-\sqrt{2}}^{\sqrt{2}}\left[\bar{h}-\left(\bar{u}-c_{0}\right)^{2}\right] 1 d y=0
$$

Using the expression for $\bar{h}$ and $\bar{u}$ we get:

$$
\int_{-\sqrt{2}}^{\sqrt{2}}\left[\left(1-\frac{1}{2} y^{2}-\left(y-c_{0}\right)^{2}\right] d y=0 \Rightarrow c_{0}=0\right.
$$

To order $E$ we get

$$
\left(\bar{h} u_{2 y}\right)_{y}=0 \Rightarrow u_{1}=\text { Constant }=0
$$

since $u_{2}$ is bounded at $y= \pm \sqrt{2}$. There is no new information at this order. Be still have only geostrophic effects, and the boundaries are stable. To get ageostrophic effects we have to carry the expansion to order $\epsilon^{2}$ :

$$
\left(\bar{h} u_{2 y}\right)_{y}=\bar{h}-\bar{u}^{2}=1-\frac{3}{2} y^{2}
$$

Integrating we get

$$
u_{2}=1 / 2 y^{2}
$$


To find $c_{1}$ we substitute (39) into (31) and keep terms to order $\epsilon^{2}$.

$$
\epsilon^{2} \int_{-\sqrt{2}}^{\sqrt{2}} u_{0} c_{1}^{2} d y=\epsilon^{2} \int_{-\sqrt{2}}^{\sqrt{2}} u_{2}\left(\bar{h}-u^{2}\right) d y-\epsilon 2 \bar{u} u_{0} c_{1}
$$

substituting for $\bar{u}, \bar{h}, u_{0}$ and integrating we obtain

$$
2 \sqrt{2} c_{1}^{2}=-8 \sqrt{2} / 15 \Rightarrow c_{1}= \pm 2 i / \sqrt{15}
$$

so that the front is unstable, even though $Q_{y}=0$. The higher order terms are easily calculated, and in Figure 1 the growth rate $\epsilon\left|c_{1}\right|$ is plotted versus $\epsilon$. The solid line is the exact numerical solution of Eq. (31). The line denoted by -- - is a Pade approximation to $o\left(\epsilon^{12}\right)$ and the line denoted by - - is the above found approximation (to $\left.0\left(\epsilon^{2}\right)\right)$.

The eigenfunctions for this special case are proportional to $e^{i x}$, so if we take the real part we have:

$$
\begin{aligned}
& O(1) \quad u_{0} \sim \cos x, v_{0} \sim y \sin x, h_{0} \sim-y \cos x \\
& O(\epsilon) \quad u_{1}=0, \quad v_{1} \sim \frac{-4}{\sqrt{15}} \cos x, h_{1} \sim \frac{-2}{\sqrt{15}} \sin x
\end{aligned}
$$

Those two modes are plotted separately in Figure $2(a, b)$ and together in Figure $2 c$.

In the above case we assumed that there was no potential vorticity, and this made the problem relatively easy to handle. In general the problem is quite complicated and in order to see what happens we write Equations $(22)-(24)$ as a single equation for $h$.

$$
\left(\frac{\bar{h} h}{Q}\right)_{y}+\bar{h}\left\{1-\frac{\epsilon^{2} \bar{h}}{Q}-\left(\frac{\bar{h}}{Q}\right)_{y} \frac{1}{(\bar{u}-c)}\right\}=0
$$

where

$$
Q=\epsilon^{2}(\hat{\mathrm{u}}-\mathrm{c})^{2}+\mathrm{u}_{\mathrm{y}}-1
$$

$\mathrm{W}$ e may expect some problems when $\overline{\mathbf{h}}=0$, and $\overline{\mathbf{u}}-\mathrm{c}=0$.

Take now some general depth profile $\bar{h}(y)$, satisfying $\bar{h}( \pm L)=0$. The velocity is given by the geostrophic relation

$$
\overline{\mathrm{u}}=-\overline{\mathrm{h}}_{\mathrm{y}}
$$

Define an integrated depth perturbation $\phi(y)$ :

$$
\phi(y)=\int_{-L}^{y} h d y=\phi_{0}+\epsilon \phi_{1}+\epsilon^{2} \phi_{2}+\epsilon^{3} \log \epsilon \phi_{3}+\epsilon^{3} \phi_{4}+\cdots
$$

Similar expansion holds for $\mathrm{c}, \mathrm{u}$ and $\mathrm{v}$, and

$$
h_{n}=\phi_{\text {ny }},(n=0,1,2, \ldots)
$$




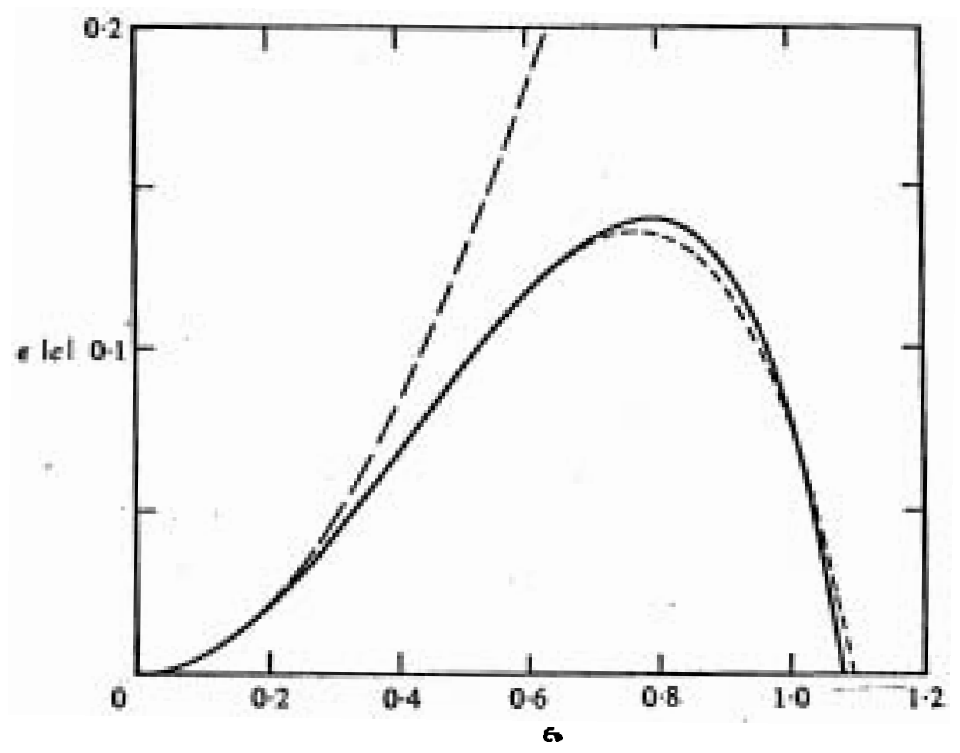

FIGURE 1 Growth rate of normal modes with wevenumber $\epsilon$ on a current with zero potential vorticity : - - exact numerical results; - - , first-order growth rate $\epsilon^{2} c_{1}$ given by the wavenumber expansion; - - - ., Pad6 approximation based on terms up to $O\left(\epsilon^{12}\right)$ in the expansion.

(a)

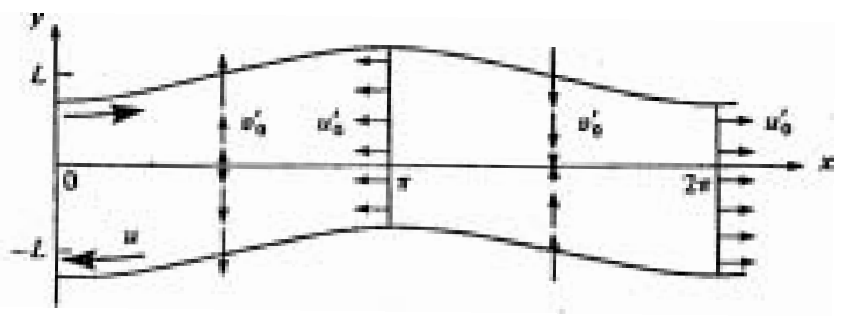

(b)

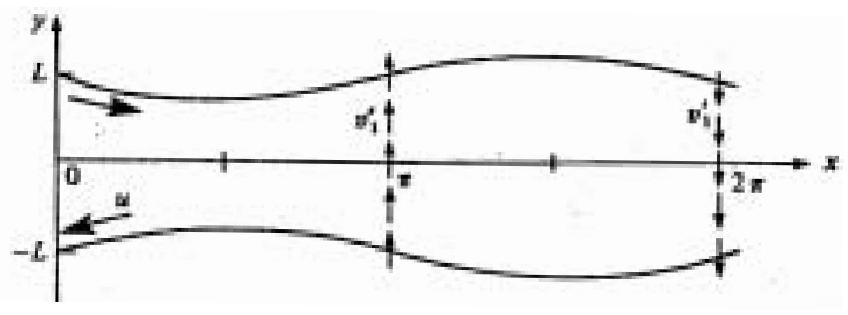

(c)

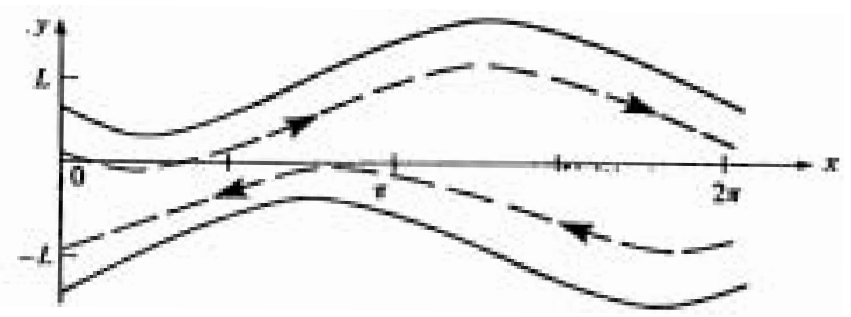

Figurf 2 The structure of the zeroth-order eigenfunctions: (a) the first-order eigenfunctions; (b) (from (3.16)) and the combination of these two modes; (c) for $\epsilon$ of order one and a flow with zero potential vorticity. The undisturbed flow $\tilde{u}$ is linear with $\mathrm{y}$. . 
From (40) we see that

$$
\phi(-\mathrm{L})=0
$$

For convenience we scale

$$
\begin{aligned}
& \phi_{\text {oy }}(-\mathrm{L})=-\mathrm{u}(-\mathrm{L}) \\
& \phi_{\text {ny }}(-\mathrm{L})=0, \mathrm{n}>0
\end{aligned}
$$

Applying the continuity equation at $y= \pm$ L and using that $V$ is well behaved gives

$$
\overrightarrow{u v}+(\dot{u}-c) \phi_{y}=0,(y= \pm L)
$$

Here $\mathrm{V}=\mathrm{iv}$ is as defined in Lecture 7. Where $\bar{u}=0$, we anticipate a critical layer of thickness $\epsilon$. A boundary layer expansion is necessary there, followed by asymptotic matching across these layers. Following is an outline of what occurs:

Write $c=c_{o}+\epsilon c_{1}+6^{2} c_{2}+\ldots$ and expand Equations (22-24) in powers of $\epsilon$. Also use (40) and (41) to obtain:

$$
\begin{aligned}
& \left(\overrightarrow{\mathrm{u}}-\mathrm{c}_{\mathrm{o}}\right) \mathrm{u}_{\mathrm{n}}+\left(1-\overline{\mathrm{u}}_{\mathrm{y}}\right) \mathrm{v}_{\mathrm{n}}+\phi_{\mathrm{ny}}+\mathrm{A}_{\mathrm{n}}=0 \\
& \mathrm{u}_{\mathrm{n}}+\mathrm{B}_{\mathrm{n}}=\phi_{\mathrm{nyy}} \\
& \overline{\mathrm{h}}_{\mathrm{n}}-\left(\overline{\mathrm{h}} \mathrm{v}_{\mathrm{n}}\right)_{\mathrm{y}}+\left(\overline{\mathrm{u}}-\mathrm{c}_{\mathrm{o}}\right) \mathrm{h}_{\mathrm{n}}+\mathrm{c}_{\mathrm{n}}=0
\end{aligned}
$$

for $n=0,1,2, \ldots$, where $A_{n}, B_{n}, C_{n}$ contains terms of order up to $\mathbf{n}-1$, so $A_{0}=B_{0}=C_{o}=0$. From (45) we get:

$$
V_{n}=\frac{\left(\vec{u}-c_{0}\right) \phi_{n y-1}-\phi_{n y}+B_{n}\left(\bar{u}-c_{0}\right)-A_{n}}{1-\bar{u}_{g}}
$$

where (46) has been used. Substitution of (48) into (47), integration from -L to y, and multiplication by $\left(1-\vec{u}_{y}\right) / \bar{h}$ gives

$$
\begin{aligned}
\left(\bar{u}-c_{0}\right) \phi_{n y g}-\dot{u}_{y} \phi_{n y} & +\frac{c_{0}\left(1-\bar{u}_{y}\right)}{\bar{h}} \phi_{n}= \\
& -\frac{1-u_{1}}{\bar{h}} \int_{-L}^{y}\left(\bar{h} B_{n}-C_{n}\right) d y-B_{n}\left(\bar{u}-c_{0}\right)+A_{n}
\end{aligned}
$$

Here we have used $h(-L)=0$. We now proceed to solve (49). To leading order we have, since $A_{O}=B_{O}=C_{O}=0$ :

$$
\left(\dot{u}-c_{0}\right) \phi_{0 y y}-\vec{u}_{y} \phi_{0 y}+\frac{c_{0}\left(1-u_{y}\right) \phi_{0}}{\bar{h}}=0
$$




$$
V_{0}=\frac{\left(\bar{u}-c_{0}\right) \phi_{0 y y}-\phi_{0 y}}{1-\bar{u}_{y}}
$$

The set (50), (43), and (44) is an eigenvalue problem for $c_{o}$, and may have many solutions. We chose one specific solution with $c_{0}$ real, namely:

$$
c_{0}=0, \phi_{0}=\bar{h}, v_{0}=\bar{u}, u_{0}=\bar{u}_{y}, h_{o}=-\bar{u}
$$

Here $v_{0}$ is real, so $v_{0}$ is $\pi / 2$ behind $u_{0}$ and $h_{0}$ as in the special case considered previously. To order Ewe have

$$
A_{1}=-c_{1} u_{0}, B_{0}=0, c_{1}=-c_{1} \phi_{\text {oy }}
$$

So (49) becomes

$$
\overline{\mathrm{u}} \phi_{1 \mathrm{yy}}-\tilde{\mathrm{u}}_{\mathrm{y}} \phi_{1 \mathrm{y}}+\mathrm{c}_{1}=0
$$

with the boundary conditions

$$
\phi_{1}=\phi_{1 y}=0 \text { at } y=-L
$$

and from (44) (terms of order $\epsilon$ )

$$
\dot{u} v_{1}+\dot{u} \phi_{1 y}-c_{1} \phi_{\text {oy }}=0 \text { at } y=L
$$

This appears to be a well-posed problem for $\mathbf{c}_{1}$, but in fact it is not so. Recall that from (48)

$$
\begin{aligned}
V_{1} & =\frac{\bar{u} \phi_{1 y y}-\phi_{19}+c_{1} \bar{u}_{y}}{1-\bar{u}_{y}} \\
& =\frac{\bar{u}_{i} \phi_{1 y}-c_{1}-\phi_{1 y}+c_{i} \bar{u}_{y}}{1-\bar{u}_{y}} \\
& =-\phi_{1 y}-c_{1}
\end{aligned}
$$

Using this expression for $\nabla_{1}$ we see that eq. (56) becomes $0=0$, so we have lost a boundary condition. Now Equation (54) has two independent solutions. One is

$$
\phi_{1 y} \times \bar{u} \quad \text { (i.e. zeroth order) }
$$

and the other is:

$$
\phi_{1 y}=-c_{i} \bar{u} \int^{y} \frac{d y}{\bar{u}^{2}}
$$

So-there is a singularity at $\overline{\mathrm{u}}=0$.

The solution Equation (58) is well behaved as u approaches zero only if $\bar{u}_{y y c}$ (where suffix c denotes values at the critical layer) is zero. 
Otherwise the solution contains logarithmically growing terms. A matching across a critical layer is necessary, and we write

$$
\begin{aligned}
& \phi_{1}=-c_{1} \int_{L}^{y} \bar{u}(\eta) d \eta \int_{-L}^{\eta} \frac{d \xi}{[u(\xi)]^{2}} ; y<y_{c} \\
& \phi_{1}^{+}=\beta-\alpha \bar{h}-c_{1} \int_{L}^{y} \bar{u}(\eta) d \eta \int_{L}^{\eta} \frac{d \xi}{[u(\xi)]^{2}} ; y>y_{c}
\end{aligned}
$$

where

$$
\overline{\mathrm{u}}\left(\mathrm{y}_{\mathrm{c}}\right)=0
$$

and we have used Equation (55) a t $-\mathrm{L}$ i n Equation (59). $\alpha$ and $\beta$ are unkown constants. To match across the critical layer we need the behavior of near $y=y_{c}$. We write $\boldsymbol{Z}=y_{-}-y_{c}$, where $\boldsymbol{Z}$ is small, then:

$$
\begin{aligned}
& \phi_{1}^{ \pm} \sim \phi_{1 c}^{ \pm}+\frac{c_{1}}{\bar{u}_{y c}} \eta+\left\{\begin{array}{c}
\frac{1}{2} \alpha u_{y c} \eta^{2} \\
0
\end{array}\right\}+\frac{c_{1} \bar{u}_{y g c}}{2 \bar{u}_{y c}^{2}} \eta^{2}\left(\log \eta-\frac{1}{2}\right) \\
& -\frac{1}{2} c_{i} \bar{u}_{y c} \eta^{2}\left\{\int_{ \pm L}^{g_{c} \pm \delta} \frac{d y}{\bar{u}^{2}} \pm \frac{1}{\delta \bar{u}_{g c}^{2}}+\frac{\bar{u}_{y y c}}{\bar{u}_{y c}^{2}} \log ( \pm \delta)\right\}+\frac{c_{i} \bar{u}_{y y c} \eta^{2}}{\bar{u}_{y c}^{2}}
\end{aligned}
$$

where $\delta 70$ is some small number, and $\bar{u}_{\mathrm{yc}}=\overline{\mathrm{u}}_{\mathrm{y}}\left(\mathrm{y}_{\mathrm{c}}\right)$, etc. Now $\log (+\delta)$ is well defined, but $\log (-\delta)$ requires a closer look. Near $\mathrm{y}=\mathrm{y}_{\mathrm{c}}$ Equation (54) really is

$$
\left(\bar{u}-\epsilon c_{1}\right) \phi_{1 y y}-\bar{u}_{y} \phi_{1 y}+c_{1}=0
$$

So when $\boldsymbol{Z}$ is of order 6 , the $\overline{\mathbf{u}}$ term, instead of giving terms like log $\bar{u}$, gives

$$
\log \left(\bar{u}-\epsilon c_{1}\right) \sim \log \left(\epsilon \bar{u}_{y} \eta-\epsilon c_{1}\right) \sim \log \epsilon+\log \left(\bar{u}_{\mathrm{y}} \eta-c_{1}\right)
$$

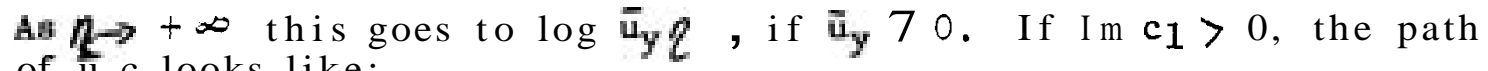
of $\overline{\mathrm{u}}-\mathrm{c}$ looks like:

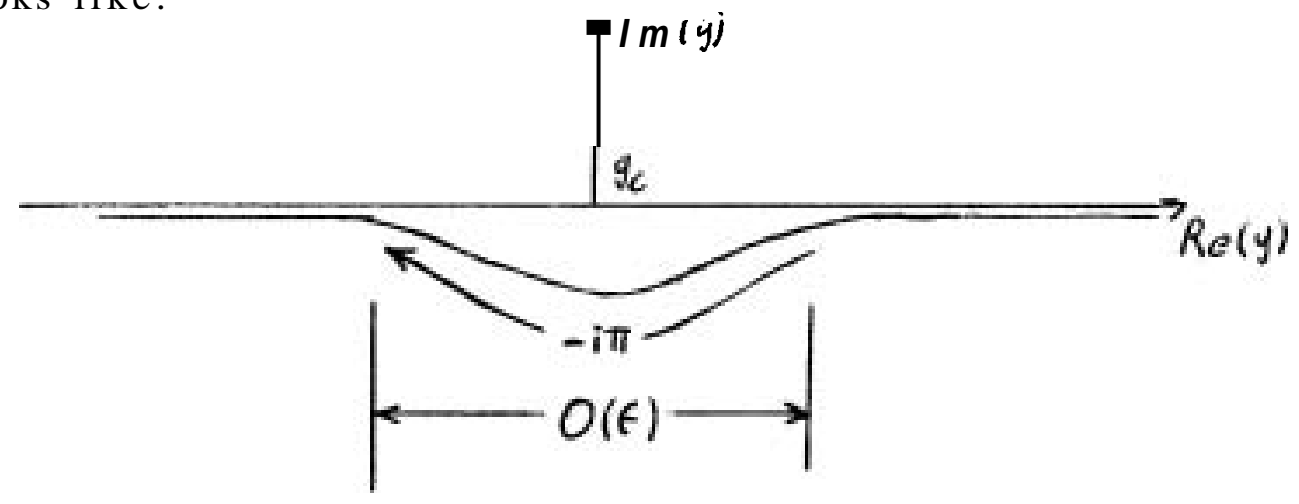


So as $\eta \rightarrow+\infty$, we get $\log \left(-\bar{u}_{y y}-c_{1}\right) \sim \log \left(\bar{u}_{y}|\ell|\right)-i \pi \operatorname{sgn}\left(\bar{u}_{y c}\right)$

$$
\Rightarrow \log (-\delta)=\log \partial-\mathbf{i} \pi \operatorname{sgn}\left(\bar{u}_{y c}\right)
$$

$c_{1}$ is still unknown, so we do the order $\boldsymbol{\epsilon}^{2}$ terms. We have

$$
A_{2}=c_{1} u_{1}-c_{2} u_{o}, B_{2}=\vec{u}^{2}, c_{2}=-c_{1} \phi_{1 y}-c_{2} \phi_{\text {oy }} .
$$

So the flow becomes ageostrophic at this order. $\phi_{2}$ satisfies

$$
\bar{u} \phi_{2 y y}-\vec{u}_{y} \phi_{2 y}+\frac{1-\bar{u}_{y}}{\bar{h}} \int_{-L}^{y} \bar{h} \bar{u}^{2} d y+\vec{u}^{3}-c_{1} \phi_{1 y y}+c_{2}+c_{1} \frac{i-\bar{u}_{y}}{\bar{h}} \phi_{1}=0
$$

and

$$
\phi_{2}=\phi_{2 y}=0 \text { at } y=-L
$$

At $y=I$ we want $\phi_{2}$ to be well behaved so it must satisfy:

or

$$
\begin{gathered}
\left(1-\vec{u}_{y}\right) \int_{-L}^{L} \bar{h} \bar{u}^{2} d y+c_{i}\left(1-\bar{u}_{y}\right) \phi_{1}=0 \quad \text { at } \mathrm{y}=\mathrm{L} \\
c_{i} \phi_{i}(L)=-\int_{-L}^{L} \bar{h} \bar{u}^{2} d y
\end{gathered}
$$

This is the missing condition for the $O(E)$ problem and the only information we need from the order $\epsilon^{2}$ terms. Equations (59) and (60) give $\phi^{ \pm}(y)$, the behavior near $\mathrm{y}=\mathrm{y}_{\mathrm{c}}$ is known so we can match using (63) and then close the problem by (66). From (62) we now see that $\phi_{1}$ and $\phi_{1 y}$ must be continuous at $y_{c}$, the $\log \ell$ was taken care of above, and the well-behaved part of $\dot{\phi}_{1 y y}$ being continuous. We now match the $O\left(\epsilon^{2}\right)$ terms of (62) for $\phi_{1}$ to find $\alpha$. On the - side we have

$$
-\frac{1}{2} c_{i} \bar{u}_{g c}\left[\int_{-L}^{y_{c}-d} \frac{d y}{u^{2}}-\frac{1}{\delta \bar{u}_{y c}^{2}}+\frac{\bar{u}_{g y c}}{\bar{u}_{g c}^{2}}\left\{\log \delta-i \pi \operatorname{sgn} \bar{u}_{y c}\right\}\right]
$$

This must be equal to the expression on the + side which is

So we get:

$$
\frac{1}{2} \alpha \bar{u}_{y c}-\frac{1}{2} c_{i} \bar{u}_{g c}\left[\int_{L}^{y_{c}+\delta} \frac{d y}{\bar{u}^{2}}+\frac{1}{\delta \bar{u}_{y c}^{2}}+\frac{\bar{u}_{y j c}}{\bar{u}_{y c}^{2}} \log \delta\right]
$$

$$
\alpha=\frac{i \pi \bar{u}_{y j k}}{\left[\bar{u}_{y c}\right]^{3}} c_{1}-c_{i}\left\{\left(\int_{-L}^{g_{i}-\delta}+\int_{y_{c}+\delta}^{L}\right) \frac{d y}{\bar{u}^{2}}-\frac{2}{\delta \bar{u}_{y c}^{2}}\right\}
$$

The term inside the brackets \{\} is the Hadmard 'finite part' of the divergent integral, written $F_{P} \int_{-L}^{L} d y / u^{2} \quad$ (evaluated by merely substituting in the end-point values and-ignoring the singularity). In order to calculate $c_{1}$ from Eq. (66) we need $\phi_{1}(\mathrm{~L})$ which is given by

$$
\phi_{1}(L)=\left(\int_{-L}^{y_{c}-\delta}+\int_{y_{L}-\delta}^{y_{c}+\delta}+\int_{y_{c+\delta}}^{L}\right) \phi_{1 y} d y
$$


Since $\phi_{1}(y)$ is continuous at $y=y_{c}$, the integrand in the middle is zero. Using (62) for $\phi_{1}$ we obtain, after some algebra:

$$
\phi_{1}(L)=-c_{1} F_{\rho} \int_{-L}^{L} \frac{\bar{h}}{\bar{u}^{2}} d y+\frac{i \pi \bar{u}_{y j c} \bar{h}_{c} c_{n}}{\left|\vec{u}_{j c}\right|^{3}}
$$

Substituting this in (66) we then get for $c_{1}$

We now note that:

$$
c_{i}^{2}=\frac{\int_{-L}^{L} \bar{h} \bar{u}^{2} d y}{F_{P} \int_{-L}^{L} \frac{\bar{h}}{\vec{u}^{2}} d y-\frac{i \pi \bar{u}_{y g_{c}} \bar{h}_{c}}{\left|\bar{u}_{y c}\right|^{3}}}
$$

i) If $\overrightarrow{\mathbf{u}}_{\mathrm{yyc}}=0$ (as in the special case done previously) then it can be shown that $F_{P} \int_{-L}^{L} \vec{h} / \tilde{u}^{2} d y<0$, so $c ?<0$ and we therefore have imaginary $c_{1}$. That gives an unstable mode.

i i ) If $\vec{u}_{\text {yyc }} \neq 0$ then $c^{2}$ is complex and there exists an unstable mode (with the imaginary part of $c_{1}>0$ ).

We therefore conclude that there is always a growing long wave mode.

We now look at the energetics, or where this mode gets its energy

from. Define a pseudo-energy

$$
E=1 / 2\left\langle\mathrm{~h}\left(\mathrm{u}^{2}+\epsilon^{2} \mathrm{v}^{2}\right)+\mathrm{h}^{2}\right\rangle
$$

where \langle\rangle denotes an $x$-average (i.e. $\left.\langle a b)=1 / 2 \operatorname{Re}\left\langle a b^{*}\right\rangle\right)$. From Equations (17-19) we get

$$
\frac{\partial E}{\partial t}=\bar{h} \bar{u}_{y}\langle u v\rangle+\{\bar{h}\langle h v\rangle\}_{y}=0
$$

Integrate this to obtain

$$
\frac{d}{d t} \int_{-L}^{L} E d y=-\int_{-L}^{L} \bar{h} \bar{u}_{y}\langle u v\rangle d y
$$

So the perturbations draw energy from the kinetic energy of mean flow through the mean shear $\bar{u}_{y}$ * Now we have

$$
\begin{aligned}
\langle u v\rangle & =\frac{1}{2} \operatorname{Re}\left(u_{0} v_{0}^{*}\right)+\frac{1}{2} \epsilon \operatorname{Re}\left(u_{0} v_{1}^{*}+u_{i} v_{0}^{*}\right)+\cdots \\
& =-\frac{1}{2} \epsilon c_{1 i}\left(1+\bar{u}_{y}\right)+O\left(\epsilon^{2}\right)
\end{aligned}
$$

so

$$
\begin{aligned}
\frac{d}{d t} \int_{-L}^{L} E d y & =\frac{1}{2} \in C_{t i} \int_{-L}^{L} \vec{u}_{y} \tilde{h}^{L}\left(1+\dot{u}_{y}\right) d y \\
& =\frac{1}{2} \in C_{l i} \int_{-L}^{L}\left(\bar{h}_{y}^{2}+\vec{u}^{2}\right) d y>0
\end{aligned}
$$


So the perturbation energy is growing. The mass transport in the y direction (across stream) is given by

$$
\langle v h\rangle=\frac{1}{2} \epsilon c_{t i} \bar{u}
$$

That is, the blob gets wider and thinner, so potential energy must be released. Indeed we have from (13)

$$
\frac{\partial}{\partial t}\left\{\frac{1}{2}\langle\bar{h}\rangle^{2}\right\}+\bar{h}\langle v \bar{h}\rangle_{y}=0
$$

for the rate of change of mean potential energy. Thus,

$$
\frac{d}{d t} \int_{-L}^{L} \frac{1}{2}\langle\bar{h}\rangle^{2} d y=-\frac{1}{2} \in C_{t i} \int_{-L}^{L} \bar{u}^{2} d y<0
$$

So release of mean potential energy is also necessary during the growth of an unstable mode. However, it can be shown that the total potential energy remains constant, so the mean potential energy is moved to the perturbation potential energy. Comparison with experiment shows good agreement for a narrow current, but poor for wider ones. This might be due to the effect of the bottom layer.

Other cases can be done in a similar way, but the algebra is more lengthy. We will briefly mention the main result below.

i) Boundary current.

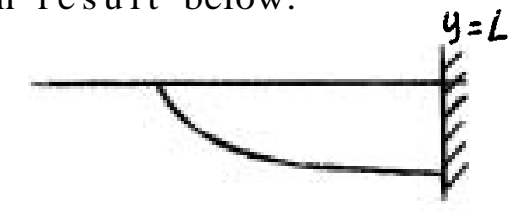

In the case considered above, the lowest order instability involved interactions between both fronts. If we put a wall along the $x$-axis at $y=L$, we may expect that to have stabilizing effects. This is indeed the case.

Recall that it is necessary for c to be complex for unstable modes. In the expansion $c=c_{0}+c_{1} \in+\ldots$ for the two front problem we found that $C_{1}$ was complex, but for the case of a boundary current it is the coefficient of $\epsilon^{4}$ that is the first one to be complex in the expansion. It can be shown that $\mathrm{c}$ has the Eorm

$$
c \sim \frac{-\epsilon^{2}}{1+\bar{u}_{y}(L)} \int_{0}^{L} \bar{h} \bar{u}^{2} d y+\epsilon^{4}\left(\text { real number }-\frac{i c_{1}^{2} \bar{u}_{y y}(L) \pi}{\bar{u}_{g}(L)\left(1+\bar{u}_{y}(L)\right)}\right)
$$

For instability we must therefore have $\overrightarrow{\mathbf{u}}_{\mathrm{yy}}<0$. That $\mathrm{is}$, at the wall, the potential vorticity must be increasing toward the wall.

ii) Isolated front.

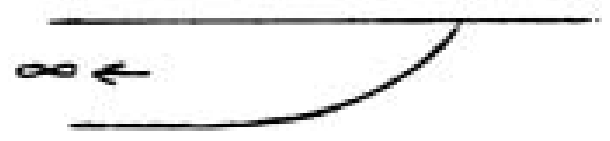

Here it can be shown that there exists instability if

$$
a^{-1} \equiv \operatorname{lit}_{y \rightarrow \infty} \frac{\bar{u}_{y}}{u}>1
$$


and $c_{i}$ has the form

$$
c_{i} \sim \epsilon^{4}\left(\int_{-\infty}^{0} \bar{h} \bar{u}^{2} d y\right)^{2} \pi a\left(1-a^{2}\right)
$$

The growth rate is therefore very weak. As a conclusion we note that the assumption of infinitely deep bottom layer may seriously affect our result, and work is in progress to incorporate finite depth. Nonlinear effects may also be important.

\section{Notes Submitted By}

Gretar Tryggvason 


\section{REFERENCES}

Anderson, and P. Killworth, 1979. Nonlinear propagation of long Rossby waves. Deep-Sea Res., 26A, 1033-1050.

Bers, A., 1975. Linear waves and instabilities. Physique de Plasmas, C. de Witt and J. Peyraud, eds., Gordon and Breach, 157-183.

Charney, 1973. Planetary fluid dynamics. Dynamic Meteorology, P. Morel, ed., 98-351.

Charney, J.G., and ME. Stern, 1962. On the stability of internal baroclinic jets in the rotating atmosphere. J. Atmos. Sci., 19, 159-177.

Gi11, A. E., 1982. Atmosphere-Ocean Dynamics. Academic Press.

Griffiths, R.W., and P.F. Linden, 1981. The stability of buoyancy-driven coastal currents. Dyn. Atmos. Sci., 5, 281-306.

Griffiths, R.W., P.D. Killworth, and M.E. Stern, 1981. Ageostrophkc

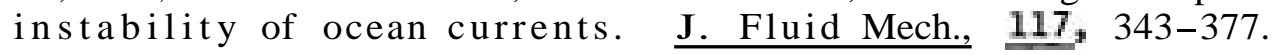

Holland, W.R., and D.B. Haidvoge1, 1980. A parameter study of the mixed instability of idealized ocean currents. Dyn. Atm. Oceans, 4 , $185-215$.

Kamenkovich, V.M., 1977. Fundamentals of ocean dynamics. Elsevier Oceanography Series, 16.

Killworth, 1983. Long wave instability of an isolated front. Geophys. and Astrophys. Fluid Dyn., in press.

Killworth, P.D., 1980. Barotropic and baroclinic instability in rotating stratified fluids. Dyn. Atm. Oceans, $\underline{4}, 143-184$.

Killworth, P.D., and M.E. Stern, 1982. Instabilities on density-driven boundary currents and fronts. Geophys. and Astrophys. Fluid Dyn., 22, $1-28$.

Mysak, L.A., and P.H. LeBlond, 1979. Waves in the Ocean. E1sevier Pub1. Co.

Pedlosky, J., 1979. Geophysical Fluid Dynamics, 624 pp.

Simmons, A.J., 1974. The meridional scale of baroclinic waves. J. Atmos. Sci., $31,1515-1525$.

Simmons, A.J. and B.J. Hoskins,, 1979. The downstream and upstream development of unstable baroclinic waves. J. Atmos. Sci., 36, 1239-1254.

Veronis, G., 1973. Large scale ocean circulation. Advances in Applied Mechanics, Vol. 113. Academic Press. 


\section{ABSTRACTS OF SEMINARS}

\section{VARIETIES OF OCEAN FRONIS}

Terrence M. Joyce

Fronts are regions of larger-than-average horizontal gradients of water properties such as temperature, salinity, density, turbidity, or color. Some characteristics of ocean fronts are large vertical and horizontal shears of currents, high rates of dissipation of mechanical energy as well as temperature and salinity variance, and high biological productivity or large gradients of productivity. Fronts are diverse in characteristics and do not possess all of the above characteristics. They vary in spatial dimensions from river plume fronts with horizontal changes on the scale of a few meters to open ocean, mid-latitude fronts having significant property changes on scales of $100 \mathrm{~km}$.

A number of "types" of fronts were presented which are more clearly separable by dynamical origin rather than physical scales of variability. While not exhaustive, the following list contains most of the fronts found in the ocean:

\section{Type of Front}

River Plume

Shallow Sea

Shelf Break

Coastal Upwelling

Mesoscale

Rings

Western Boundary Fronts

Zonal Fronts

Sub-surface Water Mass

Benthic

Other

\section{Forcing Mechanism}

discharge of fresh water

wind or tidal mixing in shallow water wintertime convection over shelf wind-driven Ekman flux eddy-induced convergence or confluence instability in major currents gyre-scale wind and thermohaline forcing Ekman convergence in subtropics tracer flux \& sharpening by mean currents variable bottom mixing

?

Some fronts do not clearly fall into the above breakdown. The polar front and sub-Antarctic front are associated with the zonally flowing Antarctic Circumpolar Current, which is forced by large-scale winds and thermohaline processes, yet contains fronts or geostrophic jets within its domain. Similar characteristics are found in the zonally flowing Kuroshio Extension.

Because fronts are regions of enhanced gradients, stirring and mixing phenomena are readily apparent and easily observed in some cases by satellites. Since the warm, tropical regions gain heat on average from the atmosphere and the cold, polar regions lose heat, the ocean must be the dissipative. Fronts are regions where most of this thermal dissipation occurs. 


\title{
LABORATORY EXPERIMENTS ON INSTABILITY AND TURBULENCE AT OCEAN FRONIS
}

\author{
Ross W. Griffiths
}

Two relationships between baroclinic instability and density fronts are illustrated by early qualitative experiments of Fultz (1952) and Faller (1956). Fultz created a long density front in a rotating two-layer fluid and observed the growth of frontal waves which tended to break up the front, while Faller showed that a smooth horizontal temperature gradient in rotation, with an associated geostrophic shear, could be unstable to baroclinic waves and that these waves lead to the formation of relatively sharp property gradients (fronts) on the scale of one wavelength. The latter approach has been refined in the differentially-heated rotating annulus experiments which have been used to investigate many properties of baroclinic waves (eg. Hide, 1958; Douglas, Hide, and Mason, 1972; Hide and Mason, 1975). An analogous two-layer system i n which a vertical shear is driven by a differentially rotating lid (Hart, 1972, 1979, 1980) has also been used to study stability conditions and the behavior of finite amplitude baroclinic waves in a flow that satisfies the quasi-geostrophic approximations.

Continuing from Fultz's experiment with a sharp density front, Saunders (1973) released a cylinder of heavy fluid on the bottom of a rotating layer of less dense fluid. The resulting vortex was unstable and the azimuthal wavenumber of the growing waves depended only on the parameter $\theta=g^{\prime} H / f^{2} R^{2}$, where $g^{\prime}=g \Delta \rho / \rho, H$ is the fluid depth, f is the Coriolis parameter, and $R$ is the radius of the cylinder. Griffiths and Linden (1981a) extended these measurements, placing the vortex on the free surface to reduce frictional effects and adding another variable - the ratio of layer depths $\gamma=h_{1} / h_{2}$. Instability depends primarily on release of potential energy when the depth ratio is small (and $\theta$ is small) but kinetic energy from the horizontal shear dominates when $\gamma<0.1$. At large disturbance amplitudes no amplitude vacillation or equilibration occurs (as does in the quasigeostrophic annulus models). The waves grow monotonically and give rise to dipolar eddy structures, or "modons", each modon consisting of an anticyclone containing upper layer fluid from the original vortex and a cyclone extending throughout the depth of the fluid (see also Flierl, 1979; Stern, 1975). These are stable, long-lived structures which eventually spin down due to the bottom friction or interact with neighboring modons. Further experiments with an "isolated" front around a very large buoyant blob and with a long front at the edge of a "coastal" current exhibit the same behavior, with large amplitude waves "breaking" backward and giving rise to vortex pairs (Griffiths and Linden, 1982). The wavelength in all cases scales either with the deformation radius (when the horizontal length scale of the flow is much greater than the deformation radius) or with the (smaller) current width (when the flow is narrow). Ekman dissipation can also influence the conditions for instability and the wavelength observed (Griffiths and Linden, 1981b; Linden and Van Heijst, 1983).

Similar laboratory arrangements can be used to obtain estimates of the horizonal spreading or cross-frontal mixing due to mesoscale baroclinic turbulence (Griffiths and Hoffinger, 1983b). The initial frontal instability creates a zone of turbulent eddy motions near the front, and this turbulence tends to spread in both directions. The transport of buoyant fluid in the 
direction normal to the original front may be parameterized in terms of a diffusion coefficient $\sigma$ which scales with the turbulence intensity $\mathbf{u}^{\prime} 1$, where $\mathbf{u}^{\prime}$ is the r.m.s. turbulence velocity and $\mathbf{1}$ is the dominant energy containing length scale. The experiments indicate that 1 scales with the deformation radius $\sqrt{g^{\prime} \mathrm{H}} / \mathrm{f}, \mathrm{u}^{\prime}$ scales with $\sqrt{\mathrm{g}^{\prime} \mathrm{H}}$ and $\sigma \approx 4 \mathrm{~g}^{\prime} \mathrm{H} / \mathrm{f}$. Another observation is that all of the stratified fluid (to one side of the front) eventually contains eddy motions, and these willact to keep the fluid away from the front relatively well mixed in the horizontal.

Another rotationally-dominated instability can occur when a current (perhaps on an abyssal slope) is bounded on both sides by a density front (Griffiths, Killworth, and Stern, 1982). So long as the current width is not too much greater than the deformation radius, the current tends to break up into a series of large anticyclonic eddies that are advected with the mean flow. This instability would occur even if only the one layer of fluid is present, and the theoretical analysis represents a first attempt at understanding the stability of fronts without making the quasi-geostrophic approximation (see also Killworth and Stern, 1982).

Other mechanisms for instability and mixing, some of which do not rely upon the presence of rotation, are also likely to occur at fronts.

Kelvin-Helmholtz billows form if the local Richardson number is sufficiently small and will act to produce marginally stable density and velocity gradients. These billows will occur, for example, whenever a fluid of one density intrudes into another of different density as a high Reynolds number

gravity current. In a rotating system the rotation constrains such a current to intrude along a side wall (Stern, 1980; Stern, Whitehead, and Hua, 1982; Griffiths and Hopfinger, 1983a). Shear instability can also occur at very much larger Richardson numbers if the density gradient also supports an angular momentum gradient (McIntyre, 1970). This is the case beneath a Gulf Stream ring or any mesoscale eddy. Instability then relies on the different rates of diffusion of density and momentum (Prandtl number not equal to one), and experiments indicate that a series of layers and sharp interfaces will form (Baker, 1971; Calman, 1977). This quasi-horizontal layering is somewhat similar to that which results from thermohaline double-diffusive convection, another mechanism for mixing whenever there are superposed horizontal gradients of temperature and salinity at ocean fronts (Ruddick and Turner, 1980).

\section{REFERENCES}

Baker, D.J., 1971. Density gradients in a rotating stratified fluid: Experimental evidence for a new instability. Science, 172, 1029-1031.

Calman, J., 1977. Experiments on high Richardson number instability of a rotating stratified shear flow. Dyn. Atmos. Oceans, 1, 277-297.

Douglas, H.A., R. Hide, and P.J. Mason, 1972. An investigation of the structure of baroclinic waves using three-level streak photography. Quart. Jour. Roy. Met. Soc., 98, 247-263.

Faller, A.J., 1956. A demonstration of fronts and frontal waves in atmospheric models. Jour. Metrol., 13, 13, 1-4. 
Flier1, G., 1979. Baroclinic solitary waves with radial symmetry. Dyn. Atmos. Oceans, 3 , 15-38.

Fultz, D., 1952. On the possibility of experimental models of the polar front wave. Jour. Metrol., 9, 379-384.

Griffiths, R.W., and E.J. Hopfinger, 1983a. Gravity currents moving along a lateral boundary in a rotating fluid. Jour. Fluid Mech., to appear.

Griffiths, R.W., and E.J. Hopfinger, 1983b. The structure of mesoscale turbulence and horizontal spreading at ocean fronts. Deep-Sea Res., submitted.

Griffiths, R.W., P.D. Killworth, and ME Stern, 1982. Ageostrophic instability of ocean currents. Jour. Fluid Mech., 117 343-377.

Griffiths, R.W., and P.F. Linden, 1981a. The stability of vortices in rotating, stratified fluid. Jour. Fluid Mech., 105, 283-316.

Griffiths, R.W., and P.F. Linden, 1981b. The stability of buoyancy driven coastal currents. Dyn. Atmos. Oceans, 5, 281-306.

Griffiths, R.W., and P.F. Linden, 1982. Laboratory experiments on fronts. Part I: Density driven boundary currents. Geophys. Astrophys. Fluid Dyn., 19, 159-187.

Hart, J.E., 1972. A laboratory study of baroclinic instability. Geophys. Fluid Dyn.. 3. 181-209.

Hart, J.E., 1979. Review. Finite amplitude baroclinic instability. Ann. Rev. Fluid Mech., 11, 147-172.

Hart, J.E., 1980. An experimental study of nonlinear baroclinic instability and mode selection in a large basin. Dyn. Atmos. Oceans, 4, 115-136.

Hide, R., 1958. An experimental study of thermal convection in a rotating liquid. Phil. Trans. Roy. Soc., A250, 441-478.

Hide, R., and P.J. Mason, 1975. Sloping convection in a rotating fluid. Adv. Phys., 24, 57-100.

Killworth, P.D., and ME. Stern, 1982. Instabilities on density-driven boundary currents and fronts. Geophys. Astrophys. Fluid Dyn., 22, 1-28.

Linden, P.F., and G.J.F. Van Heijst, 1983. Two-layer spin-up and frontogenesis. Jour. Fluid Mech., to appear.

McIntyre, M.E., 1970. Diffusive destabilization of the baroclinic circular vortex. Geophys. Fluid Dyn., 1, 19-57.

Ruddick, B.R., and J.S. Turner, 1980. The vertical length scale of doublediffusive intrusions. Deep-sea Res., 264, 903-913.

Saunders, P.M., 1973. The instability of a baroclinic vortex. Jour. Phys. Oceanogr., 3, 61-65. 


$$
-63-
$$

Stern, M.E., 1975. Minimal properties of planetary eddies. Jour. Mar. Res, 33, 1-13.

Stern, M.E., 1980. Geostrophic fronts, bores, breaking and blocking waves. Jour. Fluid Mech., 99, 687-704.

Stern, M.E., J.A. Whitehead, and Bach Lien Hua, 1982. The intrusion of a density current along the coast of a rotating fluid. Jour. Fluid Mech., 123, 237-266. 
The decay is by horizontal mixing of momentum $\left(v_{t} \geqslant A_{k} v_{x}\right)$ for $S \gg 1$ and lateral collapse $\left(f u=A_{y} v_{z z}\right)$ if $S \ll 1$.

On the other hand, if $\mathbf{A}_{\mathbf{h}}=\mathbf{K}_{\mathbf{h}}=0$, the front still follows trajectory (1) for $S \ll 1$, but trajectory (2) (S increasing) if $S \gg 1$.

Our understanding of frontal decay may be extended into the region Ro $\ll 1$ and $S \ll 1$, but no longer Ro $\ll S$, for the case of $A_{h}=K_{h}=0$ (Garrett, 1982a). The term ub can no longer be neglected in eq. (3), so that it becomes

$$
b_{t}=-f^{-2}\left(A_{v} b_{x}\right)_{t} b_{x}+f^{-2}\left(A_{v} b_{*}\right)_{x} b_{*}+\left(K_{v} b_{*}\right)_{*}
$$

(and remember $b_{z}=\mathbb{N}^{2}$ ). Changing to density coordinates

$$
\xi=x, b=b(x, z, t), \tau=t
$$

leads to

$$
z_{c}=\left[\left(N^{2} / f^{2}\right) A_{V} z_{\xi}\right]_{3}-\left[K_{V} N^{4} z_{b}\right]_{b}
$$

so that isopycnals diffuse laterally, with a diffusivity $\left(\mathbb{N}^{2} / \mathrm{f}^{2}\right) \mathrm{A}_{\mathrm{y}}$, until $S$ is about $\mathrm{Pr}^{-1}$.

A rough comparison may be made between the lateralfluxes due to the viscously induced mean flow and eddies arising from baroclinic instability. For the two-layer case with quadratic friction the flux $\mathbf{F}_{\operatorname{mf}}$ of the upper layer due to a quadratic interfacial stress is $C_{P} f^{-1}\left[g^{\prime} f^{-1}(\partial h / \partial x)^{2}\right]$ with $C_{D}=4 \times 10^{-4}$ (Csanady, 1978), whereas the flux Fbe associated with baroclinic eddies may be given by

$$
F_{b e}=\alpha\left(s^{\prime} H\right)^{1 / 2} H, \quad \alpha=5 \times 10^{-3}
$$

according to Pingree's (1979) extension of Green's (1970) work. The formula seems to be in reasonable agreement with eddy counts at some shallow sea fronts (eg. Pingree, 1979; Garrett and Loder, 1981). The ratio $\mathbf{F}_{m} / \mathbf{F}_{\text {be }}$ is of order 1 at these fronts; this is probably a coincidence. On the other hand, it is possible that initially stable isolated lenses start to spread viscously, then lose the spread fluid by baroclinic instability so that $F_{n f}=F_{b e}$ Baroclinic instability calculations need to be done for viscously spread interface profiles of axisymmetric blobs.

For diffuse fronts the lateral spread due to viscous forces may be parameterized by a lateral diffusivity $\left(\mathbb{N}^{2} / \mathrm{f}^{2}\right) \mathrm{A}_{\boldsymbol{\psi}}$, which is typically negligible in the deep ocean compared with the parameterization by Stone (1974) and Bryden (1979) of the effect of baroclinic instability. They take a diffusivity $\mathrm{K}_{\mathrm{hbe}} \approx \mathrm{CH}^{2} \mathrm{Nf}^{-1} \overline{\mathrm{V}}_{\mathbf{z}}$ (a constant $\mathrm{C}$ times a mixing length $\mathrm{Nf}^{-1} \mathrm{H}$ times a typical speed $\mathrm{BV}_{z}$ ) with $\bar{V}_{z}$ of course related to the lateral density gradient through the thermal wind equation. Stone (1974) proposes $\mathrm{C}=0.14$, Bryden (1979) finds $\mathrm{C}=0.28$ from Drake Passage data. 
One serious implication of this parameterization of baroclinic eddies is that it implies a diapycnal mixing coefficient of $K_{h b e} x$ (the isopycnal slope) $)^{2}$, with perhaps an extra factor of $1 / 2$ to allow for the trajectory of the water in the wedge of instability. The number is large for Bryden's (1979) data, several times $10^{-3} \mathrm{~m}^{2} \mathrm{~s}^{-1}$. Clearly we must take seriously the diapycnal mixing due to baroclinic eddies.

A rather different problem connecting horizontal stirring and diapycnal mixing arises from consideration of the thermohaline fronts that should develop as temperature and salinity anomalies are stirred by mesoscale eddies on isopycnal surfaces. By combining the formulae of Ruddick and Turner (1979) and Toole and Georgi (1981) on the thickness and growth rate of the double diffusive intrusions that develop at such fronts, and making various other arguable assumptions, Garrett (1982b) derived a formula

$$
K_{s}=10^{-3} D^{2} N\left(g \beta \bar{S}_{x} / N^{2}\right)^{3}
$$

for the net vertical eddy diffusivity of salt, in terms of the large-scale gradient $\bar{S}_{\mathbf{x}}$ of salinity along isopycnals. D is the typical diameter of a mesoscale eddy. The eddy diffusivity for heat is typically negative, but with observable consequences being unlikely due to the dominance of down-gradient diffusion along isopycnals.

Values for $\mathbf{K}_{\mathbf{S}}$ are small except in a few regions of large $\mathbf{S}_{\mathbf{X}}$ and weak stability $\left(\right.$ small $\mathbf{N}^{2}$ ). The theory is testable in the sense that it predicts typical intrusion thicknesses, and the frequency with which they should be found in CID casts.

\section{REFERENCES}

Bryden, H.L., 1979. Poleward heat flux and conversion of available potential energy i n Drake Passage. Jour. Mar. Res., 37, 1-22.

Csanady, G.T., 1978. Turbulent interface layers. Jour. Geophys. Res., 83, 2329-2342.

Garrett, C.J.R., and J.w. Loder, 1981. Dynamical aspects of shallow sea fronts. Phil. Trans. Roy. Soc.

Garrett, C., 1982a. On spindown in the ocean interior. Jour. Phys. Oceanogr., 12 , 989-993.

Garrett, C., 1982b. On the parameterization of diapycnal fluxes due to doublediffusive intrusions. Jour. Phys. Oceanogr., 12, 952-959.

Green, J.S.A., 1970. Transfer properties of the large-scale eddies and the general circulation of the atmosphere. Q. J. R. Met. Soc., 96, 157-185.

Muller, P., 1976. On the diffusion of momentum and mass by internal gravity waves. Jour. Fluid Mech., 77, 789-823. 
Pingree, R.T., 1979. Baroclinic eddies bordering the Celtic sea in late summer. Jour. Mar. Biol. Ass. U.K., 59, 689-698.

Ruddick, B.R., and J.S. Turner, 1979. The vertical scale of double-diffusive intrusions. Deep-Sea Res., 26A, 903-913.

Stone, P.H., 1974. The meridional variation of the eddy heat fluxes and their parameterization. Jour. Atmos. Sci., 31, 444-456.

Toole, J.M., and D.T. Georgi, 1981. On the dynamics and effects of doublediffusively driven intrusions. Prog. Oceanogr., 10, 123-145. 
BAROCLINIC INSTABILITY OF OCEANIC FRONIS

\section{Lee Branscome}

Oceanic frontal regions have strong vertical variations of the geostrophic mean current and stratification. In particular, the baroclinity of major current systems and the subtropical convergence fronts is concentrated in the upper $1000 \mathrm{~m}$. Large variations in $\mathbb{N}^{2}$ are also seen, especially if a permanent thermocline is present at depth. The influence of these strong vertical variations of the mean state on baroclinic instability is examined in a continuously stratified model in which $\partial \overline{\boldsymbol{u}} / \boldsymbol{\partial}$ and $\mathbf{N}^{2}$ are functions of depth.

We start with Boussinesq, f-plane equations and consider only depth dependence in the mean current velocity and stratification. In the context of Killworth's lectures (this volume), we examine the baroclinic stability of a wide symmetric jet. The $\boldsymbol{\beta}$-effect is weak except in the weak baroclinic flow of the open ocean. A single equation in the vertical structure of the vertical velocity is formulated. Before making the quasi-geostrophic approximation, we note that nongeostrophic instabilities are possible for low Richardson number (Ri) (Stone, 1966).

Considering quasi-geostrophic perturbations $(R \mathbb{I}>\mathbf{1}$ ) we make a long-wave expansion ( $\mathrm{L}>>\mathrm{NH} / \mathrm{f}$ ) and find the lowest order phase speed is given by the vertical mean of the current velocity. The growth rate is proportional to mean baroclinity. The waves have maximum amplitude and phase shift where the velocity and shear are maximized. The most unstable mode is given by a vertical averaged radius of deformation weighted to levels of strong shear and away from boundaries.

Numerical solutions show that short waves are destabilized when the potential vorticity gradient is nonzero. These short modes are trapped near the surface. A numerical result confirms that all wavelengths are independent of stratification below the levels of strong baroclinity.

An idealized oceanic profile which includes a strong main thermocline and baroclinic shear maximized in upper layers is examined. The potential vorticity gradient has a sign change at the thermocline and thus we find two types of modes, an external surface-trapped mode and a deep internal mode which becomes trapped at the thermocline for short wavelengths. An equivalent two-layer model only captures the longest internal modes. The surface-trapped mode has a phase speed given by the near-surface mean velocity, whereas the long internal modes have phase speeds given by the vertical mean velocity.

Similar structures and behavior of modes are seen for realistic profiles. Evidence for surface-trapped modes in the Kuroshio Extension (Bernstein and White, 1982) is given. Growth rates and phase speeds for Gulf Stream meanders (Watts and Johns, 1982) are compared with the instability calculations. 


\section{REFERENCES}

Bernstein, R.L., and W.B. White, 1982. Meridional eddy heat flux in the Kuroshio extension current. Jour. Phys. Oceanogr., 12, 154-159.

Stone, P., 1966. On non-geostrophic instability. Jour. Atmos. Sci., 23, $390-400$.

Watts, D.R., and WE Johns, 1982. Gulf Stream meanders: Observations on propagation and growth. Jour. Geophys. Res. 87, 9467-9476.

\section{COMPUIING HOWS WIIH SHARP INTERFACES USING BOUNDARY INTEGRAL MEIHODS}

\section{Hassan Aref}

Some sharply stratified flows in which certain physical properties (such as density or viscosity) have jump discontinuities at all interfaces admit representations in terms of the dynamics of generalized vortex sheet(s). Boundary integral representations of the entire flow field may be formulated for several well-known stratified flow situations including the Rayleigh-Taylor and Taylor-Saffman instabilities and the problem of wave motion at a density interface. A review of the origins of this type of representation was given and results from several numerical implementations were shown. It was stressed that whereas the theoretical formulations go back at least a couple of decades, the implementations are of more recent vintage. Technical issues that arise were mentioned. A detailed discussion of finite amplitude motion in sharply stratified Hele Shaw flow was given following Tryggvason and Aref (1983) and Aref and Tryggvason (1984). A simple demonstration experiment was shown.

\section{REFERENCES}

Aref, H., and G. Tryggvason, 1984. Vortex dynamics of passive and active interfaces. Physica D, to appear.

Baker, G.R., D.I. Meiron, and S.A. Orszag, 1982. Generalized vortex methods for free-surface flow problems. J. Fluid Mech., 123, 477-501.

Tryggvason, G., and H. Aref, 1983. Numerical experiments on Hele Shaw flow with a sharp interface. J. Fluid Mech., to appear. 


\section{AMPLITUDE EQUATIONS FOR FINITE AMPLITUDE BAROCLINIC WAVES}

Joseph Pedlosky

A finite amplitude theory for the dynamics of unstable baroclinic waves is required if we try to answer the following, very fundamental questions:

(1) Does an unstable wave equilibrate? If so, what is the mechanism for equilibration?

(2) What is the long term behavior of the wave subsequent to equilibration?

(3) What is the appropriate selection principle which determines which wave is observed in finite amplitude (e.g., the wave of maximum linear growth rate?)

(4) What alterations of the basic flow, which supports the wave, occur as a consequence of energy and potential vorticity transfers?

In these lectures, a brief review is given of recent work in the weakly nonlinear theory of unstable baroclinic waves. In all cases the model which is used is the finite amplitude extension of the traditional two-layer model for baroclinic instability (Phillips, 1951) with the further addition of Ekman friction. In all cases the $\beta$-effect has been ignored.

The first problem described the finite amplitude evolution of a single wave under conditions where such a wave is slightly supercritical with regard to the linear theory's threshold for instability.

In a channel of width $\mathrm{L}$, rotating at angular velocity $\mathbf{f} / \mathbf{2}$, subject to gravity $g$, the two layers, whose densities differ by Ap and whose undisturbed depths are each $D$, will support an instability if

$$
F \equiv \frac{f^{2} L^{2}}{g \Delta_{p} D}>_{0}=\frac{a^{2}}{2}+\frac{2 r^{2} a^{2} / k^{2}}{\left(U_{1}-U_{2}\right)^{2}}
$$

where

$$
a^{2}=\left(k^{2}+m^{2} \pi^{2}\right) / L^{2}
$$

is the total wave number of the perturbation. Its zonal wave number is $\mathrm{k} / \mathrm{L}$. The parameter $\mathbf{r}$ is a nondimensional measure of the potency of Ekman dissipation, operating equally at rigid upper and lower boundaries while $\left(U_{1}-U_{2}\right)$ is the nondimensional, vertical shear of the basic state.

For small supercriticalities

$$
F=F_{C}+\Delta ; A \ll F_{C}
$$

standard multiple scaling techniques (Pedlosky, 1970) yield the amplitude evolution equations 


$$
\begin{aligned}
& \frac{d^{2} A}{d t^{2}}+\frac{3}{2} \gamma \frac{d A}{d t}-A+A \int_{0}^{1} \sin 2 m \pi y \frac{\partial^{2}}{\partial y^{2}} \psi d y=0 \\
& \frac{\partial}{\partial T}\left\{\frac{\partial^{2} \psi}{\partial y^{2}}-a^{2} \psi\right\}+\gamma \frac{\partial^{2} \psi}{\partial y^{2}}=\left\{\frac{d}{d t}|A|^{2}+2 \gamma|A|^{2}\right\} \sin 2 m \pi y
\end{aligned}
$$

The time variable, $t$, is scaled by the small linear growth rate, $0 . \quad A$ is the amplitude of the wave. $\psi(\mathrm{y}, \mathrm{t})$ is the correction to the vertical shear of the zonal flow. The key parameter is

$$
r=\frac{r}{a}=\text { timear o folding time of instability }
$$

For $Y=0(1)$, the wave monotonically approaches a steady state with $|A|=1$ (the phase depends on initial data). The mechanism for equilibration is entirely due to wave-mean flow interaction. In particular, potential vorticity fluxes by the developing wave alter the relative vorticity of the basic current in such a manner that the instantaneous mean flow profile becomes a stable one.

For small $\boldsymbol{\gamma},\left(\leq .12\right.$ - although this depends weakly on $\mathbf{a}^{2}$ ), the solutions for $\mathbf{A}$ are simple limit cycles which describe a perpetual amplitude vacillation. As $y$ is increased from small values, a sequence of more complex vacillations are found by numerical integrations of (2) and (3) (Pedlosky and Frenzen, 1980) through a sequence of period doubling transitions. Beyond the limit point of these transitions (which for $\mathbf{a}^{2}=2 \pi^{2}$ occurs at $Y=$ .1307) chaotic, aperiodic behavior is found. For large enough y $(z .18)$ the eventual state of the wave amplitude is steady. Within the aperiodic parameter domain, isolated instances of elegant periodic behavior have been found numerically.

When the initial state consists of several wave numbers, modifications of the above theory are required. The waves will each compete for the available potential energy in the shear flow and may also interact. A modification of the theory described above can be most easily carried out for small friction near the minimum of the critical $F$ curve as $r \rightarrow 0$ for long waves $[k=0)$ A) ]. In this limit a set of amplitude equations for $A_{k}$ (the amplitude of the plane wave of zonal wave number $\mathbf{k}$ ) can be derived in the form

$$
\begin{aligned}
& \frac{d^{\sigma} A_{k}}{d t^{2}}+\frac{3}{2} \pi \frac{d A_{k}}{d t}-k^{2} A_{k}\left\{q(k)-2 \int_{0}^{1} \sin 2 x y \frac{\partial^{2} \phi}{\partial y^{2}} d y\right\}=0 \\
& \frac{\partial}{\partial T}\left\{\frac{\partial^{2}}{d y^{2}}-\pi^{2}\right)+n \frac{\partial^{2} \psi}{\partial y^{2}}=\frac{\sin 2 x y}{2}\left\{\frac{d E}{d t}+2 \pi E\right\}
\end{aligned}
$$


where $\mathrm{k}$ is the (scaled $\mathrm{x}$-wave number. The parameters are

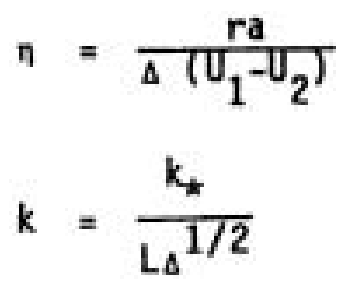

(where $k$, is the dimensional wave number), and

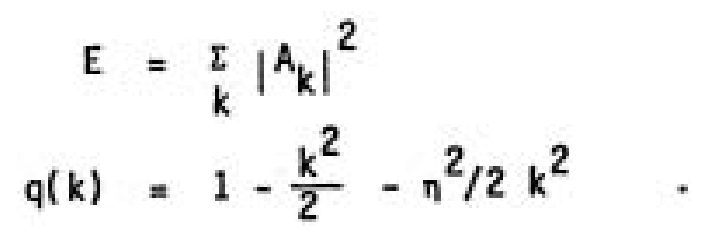

A study of (4) and (5) reveals the following (Pedlosky, 1981). The linear growth rate of disturbances has a peak at $\mathrm{k}=1$. In a competition among waves, the linearly most unstable wave will dominate the field of waves only briefly. The asymptotic wave state is quite dramatically different. The linearly most unstable wave gives way to a wave of smaller $\mathrm{k}$. In particular, the asymptotically dominant wave is that wave whose $\mathrm{k}$ is closest to the $\mathrm{k}$ which maximizes $q(k)$, i.e., $k=q^{1 / 2}$. Since $n$ must be $<1$ for linear instability, this requires that a wavelength shift to longer waves occurs in finite amplitude.' The wave which is realized in finite amplitude (i.e., the wave of maximum q) is the wave which, were it alone, would have the maximum steady state amplitude. The linearly most unstable wave $(\mathbf{k}=1)$ tends to equilibrate at a smaller amplitude. This leaves the mean.field still unstable to longer waves which grow inexorably and reduce the available energy in the mean state below the level required to maintain the $\mathrm{k}=1$ wave which eventually is extinguished.

The longer waves are more sensitive to friction. Hence the theory implies that as the supercriticality is increased the first effect is a transfer of states to longer steady waves. When the minimum zonal wave number consistent with the geometry is then achieved, a further lowering of dissipation (or increase in shear) will initiate the period doubling described earlier. Hart (1981) reports a similar nonlinear transfer to longer waves in a somewhat different two-wave model and also reports qualitative agreement with his experiments (references to these interesting experiments may be found in Hart's paper cited above).

The final problem described in the lectures involved a return to the examination of single wave dynamics. The new feature in this example is the choice of unequal Ekman friction coefficients for the two layers. This will occur, for example, if one layer (say the upper) has a free rather than a rigid surface so that Ekman friction is absent. The resulting problem has several interesting features. 
(a) The inequality of the friction coefficients destabilizes waves which are stable in the symmetric friction case.

(b) In the limiting case of zero upper layer friction, no true neutral curve exists.

(c) Nevertheless, for small friction a qualitative enhancement of the growth rate occurs at the inviscid threshold $\left(\varepsilon=a^{2} / 2\right)$ which arguably remains the physically meaningful wave.

(d) The absence of a true threshold renders the normal asymptotic expansion techniques formally useless.

Heroic methods must be used. At this point we are guided by Virgil (Dante, 1300) and Spiegel (1981). The inviscid neutral curve $\left(\bar{F}=\mathbf{a}^{\mathbf{2} / 2}\right)$ is used as a pivot point for the perturbation analysis. Two long time variables are introduced. On the first time scale the destabilizing role of friction is included. A second, longer time scale is introduced to capture the dissipative role of friction. The resulting sequence of mathematical problems is intractable. If the sequence of asymptotic equations is'recombined, a single amplitude equation is constructed. The method of construction has been called the method of reconstitution (Spiegel, 1981). If this route is followed, the amplitude equation (Pedlosky, 1983) becomes

$$
\begin{aligned}
& \frac{d^{2}}{d t^{2}} A+\frac{d A}{d t}\left\{\frac{3}{2} \frac{\left(r_{1}{ }^{+} r_{2}\right)}{2}+1 q \int_{0}^{1}\left(3 \frac{a^{2} \Phi_{B}}{\partial y^{2}}+4 a^{2} \Psi_{B}\right) \sin 2 \pi y d y\right\} \\
& +A\left\{-1-i\left(r_{1}-r_{2}\right) / 4 q+\int_{0}^{1} \sin 2 \pi y \frac{a^{2} \Psi_{T}}{a y^{2}} d y\right\}=0 \text {. } \\
& \frac{\partial}{\partial t}\left\{\frac{\partial^{2} \psi_{T}}{\partial y^{2}}-a^{2} \psi_{T}\right\}+\frac{\left(\gamma_{1}{ }^{+} \gamma_{2}\right)}{2} \frac{\partial^{2} \psi_{T}}{\partial y^{2}}+\frac{\left(\gamma_{1}-\gamma_{2}\right)}{2} \frac{\partial^{2} \psi_{B}}{\partial y^{2}} \\
& =\sin 2 \pi y\left\{\frac{d|A|^{2}}{d t}+\left(Y_{1}{ }^{+}{ }_{Y_{2}}\right)|A|^{2}\right\} \\
& \frac{\partial}{\partial t} \frac{\partial^{2} \psi_{B}}{\partial y^{2}}+\frac{\left(\gamma_{1}{ }^{+} \gamma_{2}\right)}{2} \frac{\partial^{2} \Psi_{B}}{\partial y^{2}}+\frac{\left(\gamma_{1}-\gamma_{2}\right)}{2} \frac{\partial^{2} \psi_{T}}{\partial y^{2}}=0
\end{aligned}
$$

where $\psi_{\mathbf{r}}$ and $\psi_{\mathbf{s}}$ are the corrections to the baroclinic and barotropic zonal streamfunctions. The parameter $q=\Delta^{1 / 2} / \mathrm{a}$. If $Y_{1}=Y_{2}=y$ then $\psi_{\mathbf{B}}$, initially zero, remains zero and (7) and (8) reduce to (2) and (3).

If $\left(\gamma_{1}-\gamma_{2}\right)$ is $0\left(\Delta^{1 / 2}\right),(7)$ and (8) could be derived by standard multiple scaling expansions. It is worth noting in that case that when $Y_{1} \# Y_{2}$ the 
chaotic behavior found by Pedlosky and Frenzen (1980) disappears (although it will remain if $\left(\gamma_{1}-\gamma_{2}\right) /\left(\gamma_{1}+\gamma_{2}\right)$ is very small, i.e., $\left.\sim 10^{-3}\right)$ in favor of the re-emergence of steady solutions.

If $\gamma_{1} \rightarrow 0$, the wave amplitude, after an initial equilibration at $0(1)$ amplitude levels starts a slow but persistent decline to zero! The reason is quite simple. Summing (7) and (8) shows that as $\boldsymbol{\gamma}_{\mathbf{1}}+\mathbf{0}$, there is no dissipative sink of zonal flow potential vorticity in the upper layer while there remains a wave-induced flux convergence of potential vorticity porportional to $\boldsymbol{\gamma}_{\mathbf{2}}|\mathbf{A}|^{2}$. In order for the mean flow to remain finite, the long term average of the wave flux must remain finite or equivalently

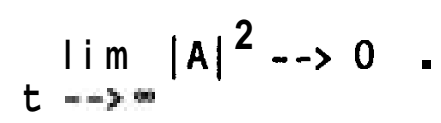

Numerical integrations of (6), (7) and (8) yield just such behavior. The mean field, however, is altered by the ephemeral wave. If a slight dissipative sink for potential vorticity, too'small to affect the above dynamics is added, the basic flow will relax to the initial state. It is hypothesized that this will lead, in the presence of background noise, to a stochastic sequence of episodes of baroclinic wave growth and decay. This model also emphasizes the vital role dissipation plays in the nature of the long term behavior of the wave amplitude.

\section{REFERENCES}

Dante, 1300. La Divina Commedia, J. Desp. Meas., Canto 1.

Hart, J. E., 1981. Wavenumber selection in nonlinear baroclinic instability. J. Atmos. Sci. 38; 400-408.

Pedlosky, J., 1970. Finite-amplitude baroclinic waves. J. Atmos. Sci., 27: 15-30.

Pedlosky, J. and C. Frenzen, 1980. Chaotic and periodic behavior of finiteamplitude baroclinic waves. J. Atmos. Sci. 37: 1177-1196.

Pedlosky, J., 1981. The nonlinear dynamics of baroclinic wave ensembles. J. Fluid Mech. 102: 169-209.

Pedlosky, J., 1983. The growth and decay of finite amplitude baroclinic waves. J. Atmos. Sci., (in press).

Phillips, N. A., 1951. A simple three-dimensional model for the study of large-scale extra tropical flow patterns. J. Meteor. 8: 381-394.

Spiege1, Edward A., 1981. Physics and Convection. 1981 Summer Study Program in Geophysical Fluid Dynamics. WHOI 81-102, 54-57. 


\title{
INERTIAL EFFECTS IN FRONIS
}

(WHY DO FRONIS BECOME SHARP?)

\author{
Francis P. Bretherton
}

This lecture discusses processes giving rise to sharp fronts from the point of view of a meteorologist, assuming an $f$-plane, Boussinesq frictionless fluid and geostrophic balance for the cross front pressure gradient. It will also be assumed that conditions are effectively uniform along the front (an important restriction that is often violated in practice, leading to complications in classifying fronts). Large-scale horizontal gradients of temperature can be intensified by geostrophic confluence or by differential advection of a long front temperature gradient. In a stably stratified fluid vertical motion generally acts to reduce contrasts.

Intensifying cross front contrasts due to large-scale confluence or differential advection are associated with an ageostrophic cross front circulation, which can at each stage be described diagnostically in terms of the potential vorticity distribution. As the local Rossby number approaches unity, the redistribution of potential vorticity by this ageostrophic circulation dominates, leading to discontinuities in a finite time. This process is illustrated by solutions of the Eady problem, reinterpreted to allow for ageostrophic effects.

\section{VORTICITY FRONTOGENESIS IN SHEAR HOW}

\section{Melvin E. Stern}

A quasi-laminar flow with a parabolic velocity profile emerges from a round nozzel at moderate Reynolds number $\left(10^{2}\right)$. The outflow is then increased so that the centerline velocity increased from $U_{\text {to }} \boldsymbol{V}+$. The fast-moving parcels catch up (converge on) the slower ones, producing a vorticity front in the intervening region. A "shock" joining theory predicts that the front propagates with speed $\left(\boldsymbol{U}_{-}+\boldsymbol{U}_{+}\right) / 2$, and the amplitude of the shock in its initial stage of formation is also given. This work is part of a study (Stern and Paldor, 1983) of the strong nonlinear interactions in coherent disturbances of a shear flow.

\section{REFERENCE}

Stern, M.E., and N. Paldor, 1983. Phys. Fluids, April. 


\section{DYNAMICAL GENERATION OF EASTERN BOUNDARY CURRENIS}

\section{George Veronis}

Boundary currents near the eastern sides of the ocean basins tend to be weaker and broader than those on the western sides. They exist along the eastern sides of all the oceans and may be equatorward or poleward depending on latitudinal position and depth. Theories for eastern boundary currents have mostly been based on longshore winds and upwelling dynamics. Yet from their ubiquity and relatively large scale they appear to be an integral part of the large-scale circulation of the oceans.

Roger Hughes and I have developed models showing how eastern boundary currents can be generated dynamically by the contribution of kinetic energy to the Bernoulli function. A sufficiently strong equatorward flow near the eastern boundary will flow in the direction of decreasing pressure gradient. If the latter is related to the depth of the layer through hydrostatic balance, the layer depth will decrease, thus generating anticyclonic vorticity. Models have been developed in which this induced anticyclonic flow requires a large flow near the eastern boundary, i.e. an eastern boundary current.

The basic idea has been applied to a simple thought experiment and to a laboratory model in which fluid is sucked out of the apex of a pie-shaped basin.' A model has been developed in which the flow in the intermediate layer of a three-layer ocean is poleward near the eastern boundary and fans out into the interior to become a westward, equatorward flow. This configuration is intended to model the flow in the region that Luyten, Pedlosky, and Stommel (1983) call the shadow zone near the eastern side of the ocean. The existence of the eastern boundary current allows one to relax the unrealistic condition of zero depth of subducted layers along the eastern boundary itself. Since the model requires nonlinear dynamics only in the boundary layer, it can be appended to the interior of the large-scale circulation model with subduction and the inviscid geostrophic dynamics of the latter can be preserved.

\section{REFERENCE}

Luyten, J., J. Pedlosky, and H. Stommel, 1983. The ventilated thermocline. Jour. Phys. Oceanog., 13, 292-309. 


\section{AMPLITUDE EQUATIONS}

Edward Spiegel

In the 1981 proceedings, Pierre Coullet and I described a way to find amplitude equations for systems with competing instabilities that we later wrote out in extenso (1). There is still much to be said on the topic, and this talk is really an account of various improvements and extensions that we have worked on with Alain Arneodo, who passed through here in the first week of this course. Let me 'remind' you of the original scheme.

We want to describe the derivation and the solution of nonlinear amplitude equations for fluid systems in physical conditions not far from marginal stability. We lay stress on polycritical conditions in which the spectrum of the linear stability theory has multiple roots with zero real parts. Then the general forms of the amplitude equations may be found for given configurations of characteristic values of linear theory. Assume that the state of the fluid may be characterized by $N$ functions $\mathrm{U}=\left(\mathrm{U}_{1}, \mathrm{U}_{2}, \ldots, \mathrm{U}_{\mathrm{N}}\right)$, where the $\mathrm{U}_{\mathrm{I}}(\mathrm{x}, \mathrm{t})$ are such quantities as stream function, temperature, or magnetic flux function. Normally, the fluid equations may be put into the form

$$
\partial_{t} \mathbf{v}=M_{\lambda} \mathbf{v}+X(\mathbf{v})
$$

where $\boldsymbol{M}$ and $\boldsymbol{X}$ are (respectively) linear and nonlinear operators and $\boldsymbol{\lambda}=\left(\boldsymbol{\lambda}_{1}, \boldsymbol{\lambda}_{2}, \ldots, \boldsymbol{\lambda}_{\mathrm{p}}\right)$ is a set of $\mathrm{p}$ parameters such as Rayleigh, Taylor, or Prandtl number. We suppose that $U=0$ is a solution of (1) and that the linear theory for small |ul admits solutions like $\boldsymbol{\phi}_{\mathbf{k}}(\mathbf{x})$ exp (st) where $\mathrm{k}$ is a wave vector.

$\boldsymbol{\lambda}$ Consider finite systems with discrete spectra for s. Suppose that for c there are $\boldsymbol{d}$ critical roots of the characteristic value equation with Res $=0$ and that, for all the other roots, Res 0 . Corresponding sets of basis vectors may be constructed: the (generalized) critical vectors, $\mathbf{c}_{\mathbf{f}}(\mathbf{x})$, and the stable modes $\mathbf{s}_{\mathbf{j}}(\mathbf{x})$. We may expand

$$
\boldsymbol{v}(x, t)=\sum_{i=1}^{\downarrow} a_{i}(t) \mathbf{c}_{i}(\mathbf{x})+\sum_{j=1}^{\infty} \beta_{j}(t) \mathbf{s}_{j}(\mathbf{x}) .
$$

if $\boldsymbol{\alpha}=\left(\boldsymbol{\alpha}_{1}, \boldsymbol{\alpha}_{2}, \ldots, \boldsymbol{\alpha}\right)=0, \boldsymbol{\beta}$ tends to be zero as $t \rightarrow \infty$. Unstable modes are required to excite stable modes if the latter are to maintain nonzero amplitudes for long times. Hence [2], at polycriticality $\boldsymbol{\beta}-\boldsymbol{\beta}(\boldsymbol{\sigma})$. As in the Bogoliubov-Mitropolsky procedure [2] we make the anstaz [1]

$$
\begin{aligned}
& U(x, t)=V(x, A(t)) \\
& \AA=J A+g(A)
\end{aligned}
$$

where $\mathrm{J}$ is an $\mathrm{n} \times \mathrm{n}$ Jordan matrix whose characteristic values have zero real parts and $g$ is a nonlinear function. The new variables $A=\left(A_{1}, \mathbf{A}_{2}, \ldots\right)$ are related to $\boldsymbol{\alpha}$ by a nonlinear transformation that puts the equation for in the simplest possible form (where simplicity is in the eye of the simplifier). Thus, the true amplitude $\mathbf{1 s} \boldsymbol{\sigma}$, while $A$ is some new variable 
that might be called an internal variable. (For diagonal $\mathbf{J}$, this is really related to the Stuart-Watson approach [3].) We obtain

$$
\mathcal{L} \equiv\left\{(\mathbf{J a}) \cdot \frac{\partial}{\partial \mathbf{A}}-M_{\boldsymbol{\lambda}_{\mathrm{c}}}\right\} \mathbf{v}=K(\mathbf{v})-\mathbf{g} \cdot \frac{\partial}{\partial \mathbf{A}} \mathbf{\nabla} .
$$

We expand $V$ and $g$ in Taylor series in $A_{\mathbf{i}} ; V_{\mathbf{r}}$, and $\mathbf{g}_{\mathbf{r}}$ are the sums of terms of degree $r$. Then

$$
L_{\mathbf{r}}=\mathbf{r}_{\mathrm{r}}-\mathbf{g}_{\mathrm{r}} \cdot \frac{\partial}{\partial \mathbf{A}} \mathbf{v}_{1}
$$

where $I_{r}$ is known from results of lower order and $I_{1}=0$. The technical problem 1 ies in finding the adjoint operator $\mathcal{L}+$ and its null vectors, $\mathrm{z}_{\mathbf{r}}$. The solvability conditions,

$$
\left(\mathbf{z}_{\mathbf{r}} \mid \mathbf{r}_{\mathbf{r}}-\mathbf{g}_{\mathbf{r}} \cdot \frac{\partial}{\partial \mathbf{A}} \mathbf{v}_{1}\right)=0,
$$

determine the $g_{r}$ up to a certain gauge. When the gauge is chosen so that $g_{r}$ has as few terms as possible, (4) is in normal form [4].

This talk outlined our work on simplifying the calculations by using analogies to quantum mechanical calculations and profiting from the tricks of that trade and indicated how we have been able to extend the method to include (rather simply all things considered) the treatment of problems in which continuous bands of instability arise. Of course, it was not possible to cover all that in detail as there were a few questions from the audience, but I did get to write down Equation (1).

\section{PERTINENT REFERENCES}

Coullet, P.H., and E.A. Spiegel, 1983. Amplitude equations for systems with competing instabilities. SIAM J. Appl. Math., 43, 775-819.

Haken, H., 1977. Synergetics, Chapter 7, Springer Verlag.

Knobloch, E., and J. Guckenheimer, 1983. Convective transitions induced by a varying aspect ratio. Phys. Rev. A., 27, Appendix.

Nayfeh, A.H., 1973. Perturbation Methods, Wiley, pp. 165 ff. 


\section{"MONIN-OBUKHOV" AND STABILITY THEORY APPLIED TO CIRCULAR COUETIE FLOW}

Willem V. R. Malkus

Smith and Townsend (1983) report results of an excellent experiment on cylindrical Couette turbulence at high Reynolds numbers. M. Claussen (1983), in a review and replotting of the Smith and Townsend data, finds that the "universal" logarithmic boundary velocity law is not achieved even at a Reynolds number of 100,000 . Instead he observes that meteorological-like "Monin-Obukhov" relations describe the mean circulation. In particular, an extensive $z^{-1 / 3}$ region of circulation is found (where $z$ is measured from the boundary), before the flow achieves a constant circulation far from the boundary.

Coincidentally, Leibovich and Stewartson (1983) have published a sufficient condition for the instability of columnar vortices. They conclude that such flow is unstable if

$$
C\left(\gamma^{\prime}\right) \equiv\left(\Gamma^{\prime}-\frac{2}{\gamma} \Gamma\right)\left[\left(\Gamma^{\prime}-\frac{\partial}{r} \Gamma\right) \Gamma^{\prime}+r^{2} W^{\prime 2}\right]<0
$$

where $\Gamma_{\text {is }}$ the circulation, a prime indicates differentiation with respect to the radial coordinate, $r$, and $w$ is the velocity parallel to the cylinder. This stability condition is related to Rayleigh's linear, inviscid mechanism of centrifugal instability, but admits axial flows and three-dimensional disturbances.

Possible relations between linear inviscid stability arguments and observed fully turbulent flow were discussed recently by this author (1979). When a turbulent flow achieves statistical stability, it was proposed that the most stable state was one of marginal inviscid stability in the body of the flow. It was shown that a general positive definite local stability condition, $C(\dot{\varphi})$ may be written

$$
C^{0}(\varphi)=\frac{I_{0}^{2}}{\sin ^{2} \varphi / 2}+\frac{(\Delta I)^{2}}{\sin ^{4} \varphi / 2}+\cdots
$$

where $\mathcal{q}$, like $\mathbf{z}$, is a coordinate normal to the boundary, $\mathrm{I}_{\mathrm{o}}$ and $\Delta \mathrm{I}_{\boldsymbol{0}}$ are constants related to stability conditions on the smallest scales, and where only the leading terms of $C(\mathscr{\varphi})$ are important in the interior of the flow. Application of Equation (2) to (1), for small $)^{+} \mathrm{W} / \Gamma$, leads to a $z^{-1 / 3}$ boundary law for $\Gamma$ and the possibility of a small negative value for $\Gamma \boldsymbol{J}_{i n}$ the interior, both features appearing in the Smith and Townsend data. A numerical integration of (1), (2), and further assessment of the validity limits of the theoretical proposal will be undertaken this fall.

\section{REFERENCES}

Claussen, M., 1983. J. Fluid Mech., submitted.

Leibovich, S., and K. Stewartson, 1983. J. Fluid Mech., 126, 335-356.

Malkus, W.V.R., 1979. $\quad$ J. Fluid Mech., 90, 401-414.

Smith, G.P., and A.A. Townsend, 1983. J. Fluid Mech., 123, 187-217. 


\title{
TURBULENCE MEASUREMENT HROM DOLPHIN
}

\author{
Thomas Osborn
}

The USS Dolphin has been instrumented to make horizontal measurements of turbulence in the ocean. The measurements consist of salinity, temperature, velocity relative to the submarine, and small-scale velocity and temperature turbulence. The results show the distribution of turbulence and doubly diffusive convection (predominately salt fingering) in the waters studied. The experimental apparatus as mounted on the Dolphin is shown in Figure 1. The turbulence probes are located atop the tripod in a manner designed to minimize data contamination due to vibrations.

The submarine is often operated in a slow dive, moving forward a t about $1 \mathrm{~m} / \mathrm{s}$ and sinking at about $10 \mathrm{~cm} / \mathrm{s}$. The data i $\mathrm{n}$ Figure $2 \mathrm{was}$ collected during such a dive with a bit of depth cycling between $35 \mathrm{~m}$ and $40 \mathrm{~m}$ depth. The dissipation rate shows a turbulent patch near $15 \mathrm{~m}$ depth that is well mixed in temperature (also density and salinity see Figure 5). The high dissipation rates about $42 \mathrm{~m}$ depth are in the stratified portion of the water column. The turbulence data is displayed in Figure 3. The velocity turbulence is associated with the temperature microstructure above the well-mixed region. The temperature microstructure above the patch forced by the velocity fluctuations are salt fingers. It is band limited and not associated with measureable velocity fluctuations.

Interestingly enough the fast thermistor on the Neil Brown Instrument System CID was also able to resolve the salt fingers (Figure 4). Here we clearly see the forced temperature fluctuations above the well-mixed layer and the salt finger signature above that in the water column. The NBIS CID resolves the signature because the speed of the submarine is slow enough and the scale of the fingers large enough that the peak of the spectrum is just below the Nyquist frequency of the CID system. A faster moving vehicle or a smaller scale of finger would not have been visible in the CID data.

To study the causes of the fingers we examined the temperature salinity relation for the data set. This curve along with other mean parameters is plotted in Figure 5. The data in Figure 4 forms a small central region of the temperature versus depth plot shown in Figure 5. The salt fingers are at the base of the salinity minimum in a region where the salinity decrease weakens the stability due to the temperature decrease with depth. There is evidence in the temperature microstructure data of salt fingers and irregular temperature fluctuations throughout the region of decreasing salinity from $36 \mathrm{~m}$ depth on down. This result was a little surprising since the stability ratio $R_{p}$ is seldom between 1 and 2 , rather is often as high as 4 in this interval. There is a lot of other evidence for salt fingering in this data set.

The relative importance of the fingers is still to be assessed. They are surpressed by the turbulence quite easily. The reasoning is as follows. The velocity signal from the fingers is below the noise level of our instrumentation. That observational result is consistent with the measured noise level and calculated finger speeds. Linden (1971) has shown that 
turbulence surpresses fingers when turbulent velocity fluctuations are comparable to fingering velocities. Hence turbulent velocity fluctuations that we can measure are sufficient to surpress the fingers. Now we need to examine the data to see if the role of fingers is surpressed much of the time by the turbulence.

Another subject that is interesting is understanding the role of turbulence in the energetics of the ocean. Our past measurements of turbulent velocity fluctuations from free-fall vehicles have shown patches of $1 \mathrm{~m}$ to $45 \mathrm{~m}$ in vertical extent in the ocean. A reasonable argument can be made that forcing by inertial current shears is an important energy source for the thick patches. If this result is true, then much of the variability in the turbulent field and the associated scalar fields will be related to variability of inertial currents rather than internal waves. Osborn (1980a and 1980b) outlines my arguments along these lines, the several papers involving Gargett and others describe much of the present data base. The paper by Simpson (1975) is very interesting for its portrayal of variability in the shear field in the ocean. That paper shows substantial variability for the velocity field.

\section{REFERENCES}

Crawford, W.R., and T.R. Osborn, 1979. Microstructure measurements in the Atlantic Equatorial Undercurrent during GATE. DSR supplement to vol. $26,285-324$.

Crawford, W.R., and T.R. Osborn, 1979. Energetics of the Atlantic Equatorial Undercurrent. DSR supplement to vol. 26, 309-324.

Crawford, W.R., and T.R. Osborn, 1981. Control of equatorial currents by turbulent diffusion. Science, 212, 539-540.

Gargett, A.E., 1978. Data report and calibrations for turbulence measurements in Knight Inlet, B.C. from the Pisces IV submersible. Pacific Marine Sciences Report, $80-86$.

Gargett, A.E., 1978. Microstructure and fine structure in an upper ocean frontal regime. J. Geophys. Res., 83, 5123-5134.

Gargett, A.E., and T.R. Osborn, 1981. Small-scale shear measurements during the fine and microstructure experiment (Fame). J. Geophys. Res., 86, 1929-1944.

Gargett, A.E., P.J. Hendricks, T.B. Sanford, T.R. Osborn, and A.J. Williams III, 1981. A composite spectrum of vertical shear in the upper ocean. J. Phys. Oceanogr., 11, 1258-1271.

Linden, P.F., 1971. Salt fingers in the presence of grid-generated turbulence. J. Fluid Mech., 49 611-624.

Osborn, T.R., 1973. Temperature microstructure in Powell Lake. $\underline{\text { J. Phys. }}$ Oceanogr., 3, 302-307. 
Osborn, T.R., and W.R. Crawford, 1978. Turbulent velocity measurements with and airfoil probe, NATO Advanced Study Institute on Instruments and Methods of Air Sea Interaction.

Osborn, T.R., 1978. Measurement of energy dissipation adjacent to an island. J. Geophys. Res., $\underline{\mathbf{8 3}}, 2939-2957$.

Osborn, T.R., 1980a. Estimates of the local rate of vertical diffusion from dissipation measurements. J. Phys. Oceanogr., 10, 83-89.

Osborn, T.R., 1980b. Dissipation measurements of oceanic turbulence. Marine Turbulence, $143-155$.

Simpson, J.H., and J. Stratford, 1975. Towards the measurement of Richardson Number in the ocean. IERE Conference Proceedings no. 32, 29-46. 


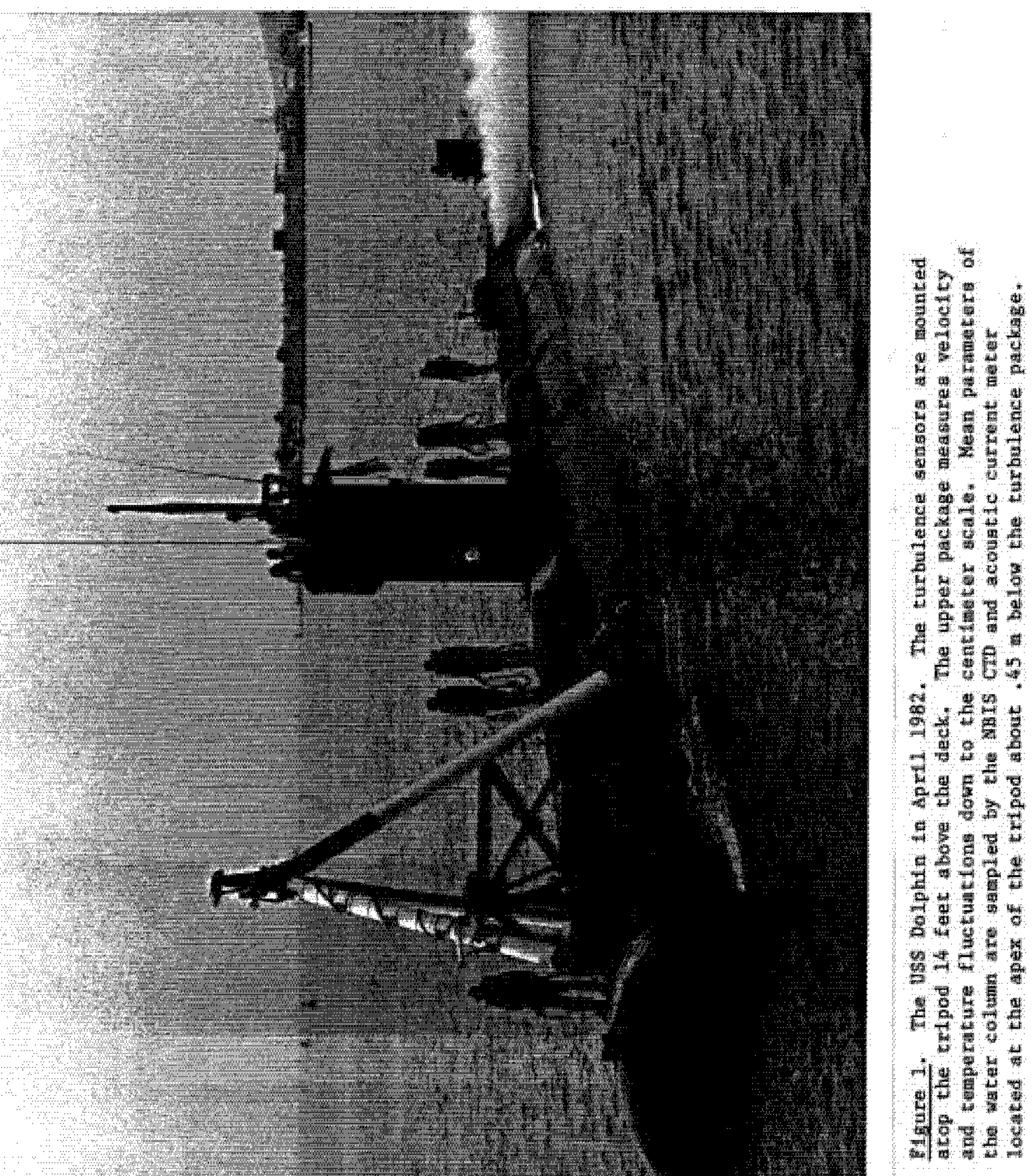



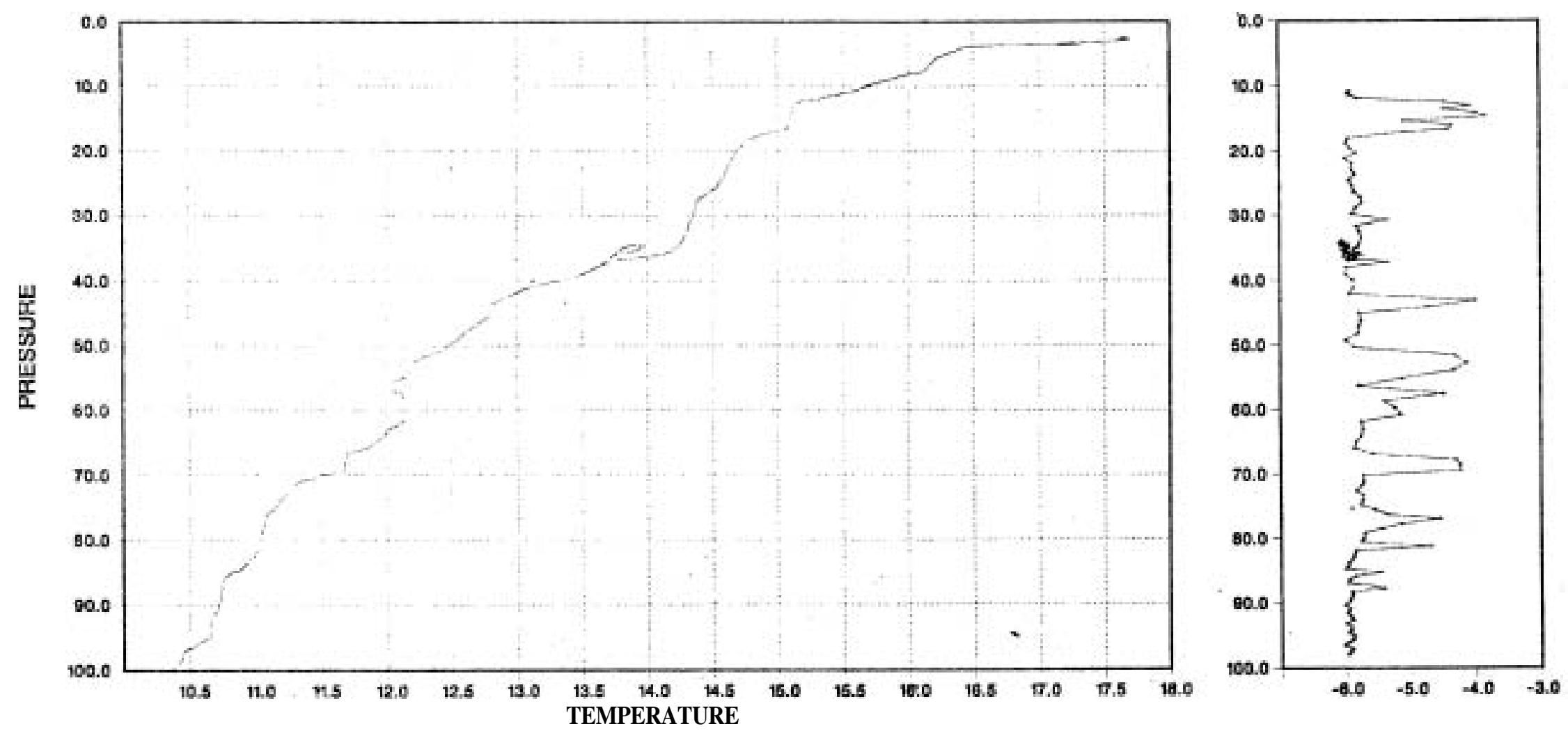

Figure 2. Temperature and turbulent energy dissipation rate from 22 April 1982. The temperature is from the NBIS CID and the dissipation rate is calculated from the turbulent velocity sensors. The dissipation scale is logarithimic and the units are watts/m**3. The dissipation data has been adjusted in depth to account for the vertical displacement between the sensors. 


$$
\text { in }-86-
$$
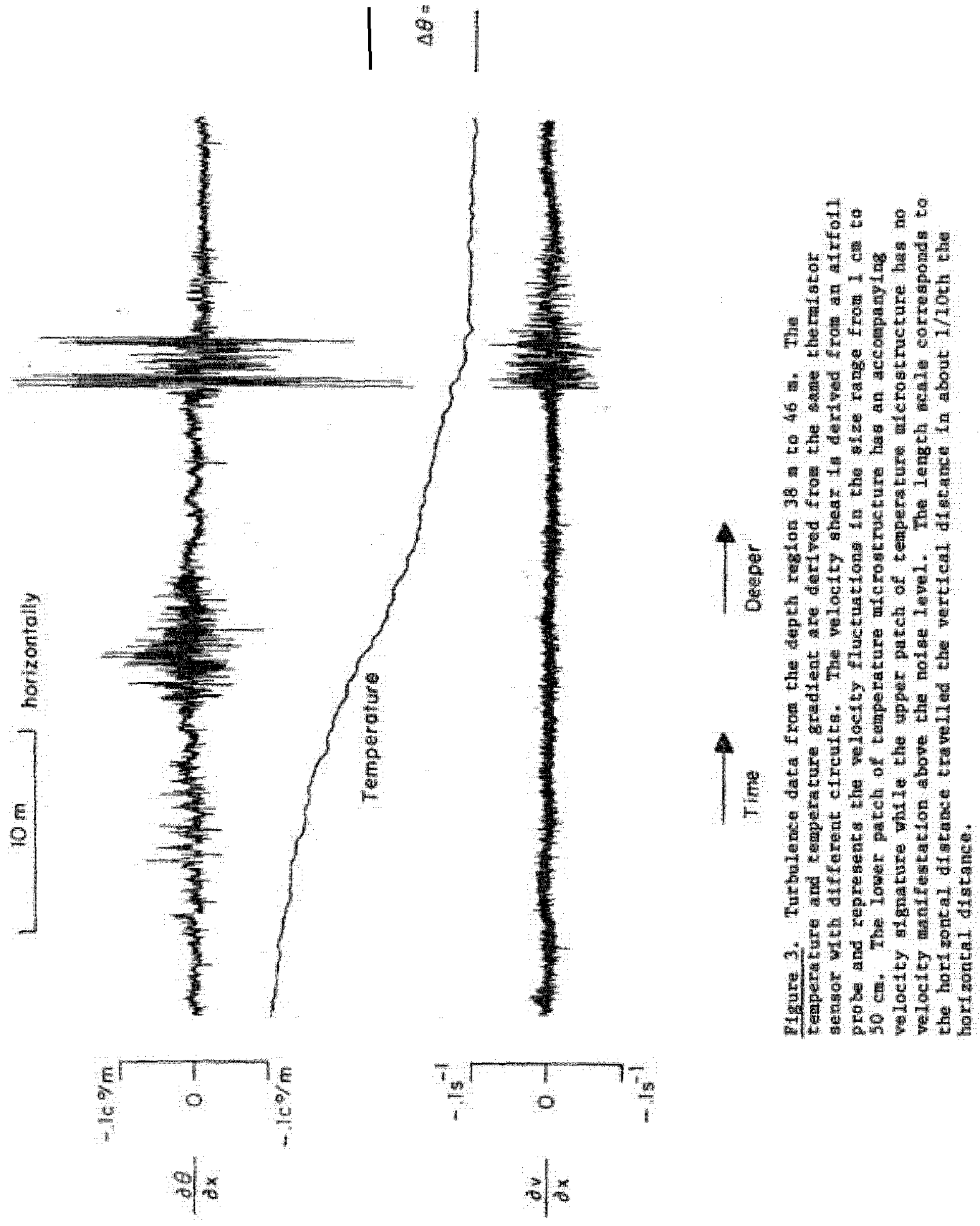


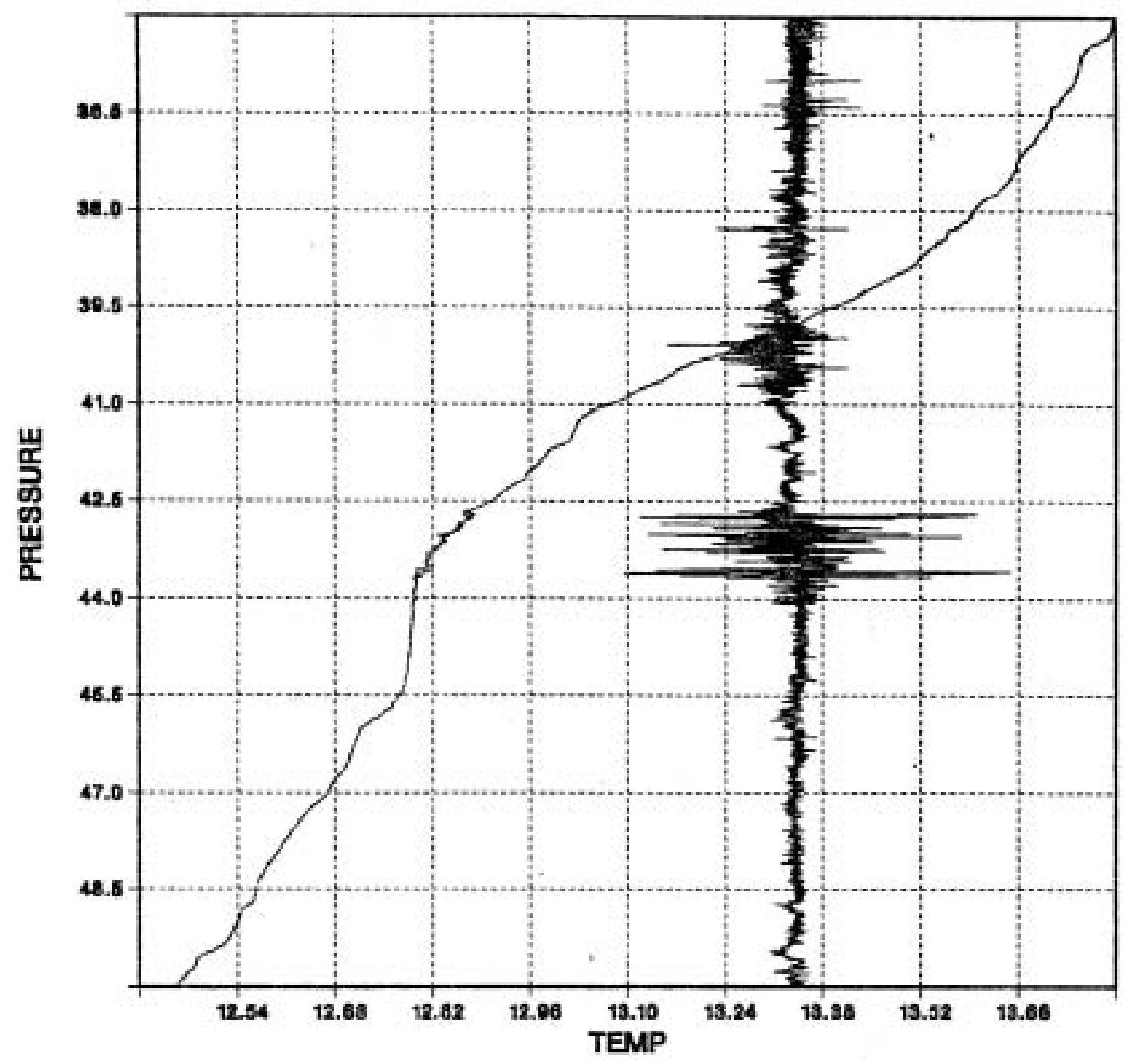

Figure 4. This data is the temperature measure by the NBIS CID. The temperature difference between adjacent time samples is also plotted after multiplication by a factor of 15 . Note the strong similarity between the temperature difference signal from the CTD in Figure 5 and the temperature derivative signal from the turbulence package in Figure 4. If you look carefully you will note that the gradient signal of Figure 3 and difference calculation in Figure 4 differ by a factor of -1 . 
DIVE NO. 822 APRIL 1982

START TIME $15: 06$

TAPE NUMBER D82055

FROM SCAN NUMBER 100884 TO 136752
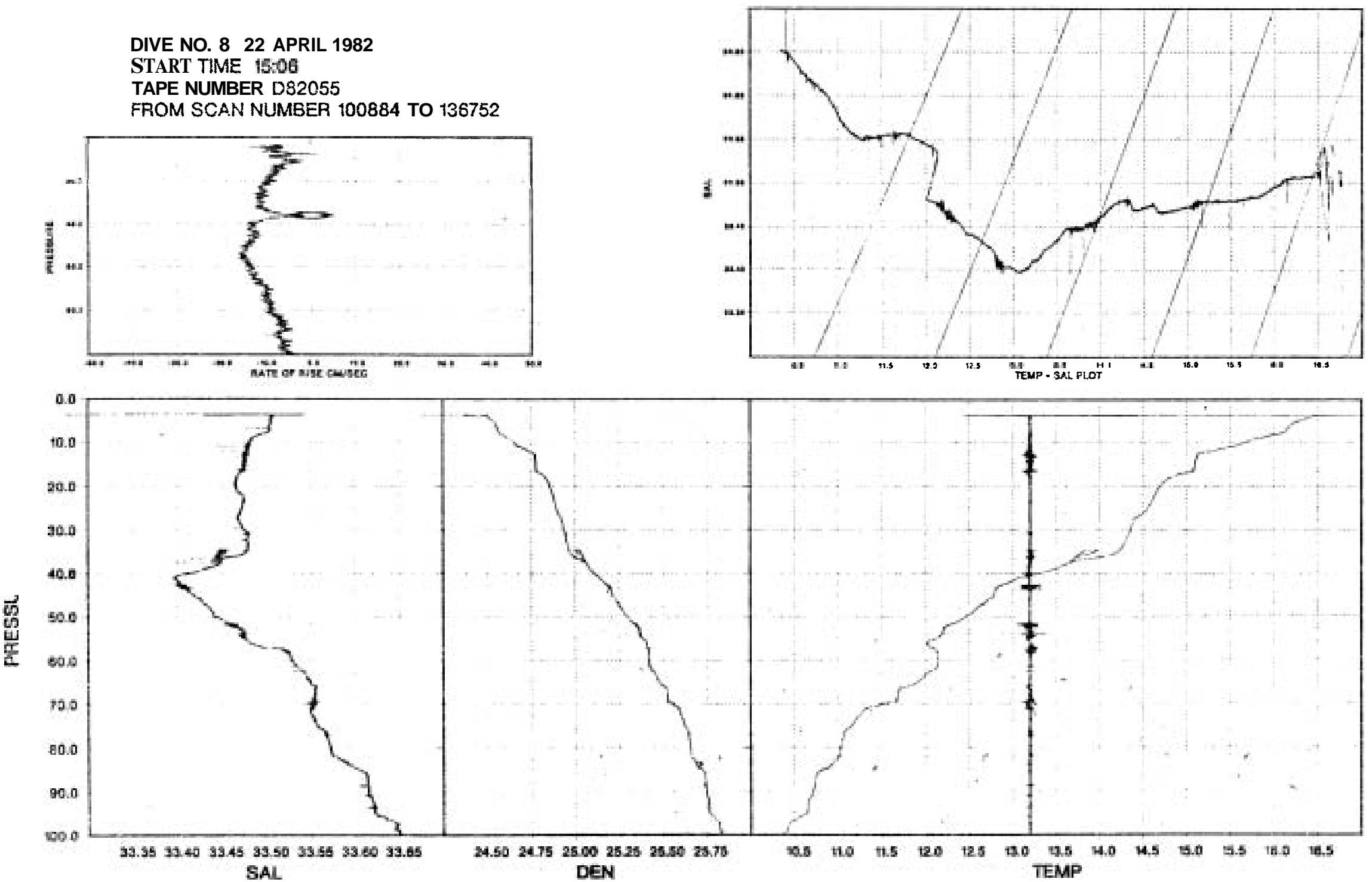

Figure 5. Data from NBIS CTD system. The temperature versus depth plot is the same data as Figure 2. The salinity and density are derived from the temperature and electrical conductivity data. The spikes in salinity (and density) are due to bad data values. The rate of rise is calculated by differencing the pressure signal. The temperature salinity plot is not in the normal format since we wanted to align it above the temperature plot. The sloping lines represent constant value of potential density and represent the slope of $R_{p}=1$. A line with twice ( $n$ times) the slope represents $R_{p}=2$ $\left(R_{p}=n\right)$. 


\section{JFD (JOVIAN FLUID DYNAMICS) \\ 1. NON-HYDROSTATIC, QUASI-GEOSTROPHIC HOW \\ 2. INERTIAL OSCILLATIONS, BAROTROPIC AND BAROCLINIC \\ INSTABILITY I N A ROTATING FLUID PLANET}

Andrew P. Ingersoll

Jupiter and Saturn are rotating, convecting, fluid objects

(radius $\approx 70,000 \mathrm{~km}$, period of rotation $\approx 10$ hours, gravity $\approx 20 \mathrm{~m} / \mathrm{sec}^{2}$, thermal emission $\approx 1.7$ times absorbed sunlight). Winds are mostly zonal $\left.\left(\left\langle\bar{u}^{2}\right\rangle\right\rangle^{1 / 2} \approx 100 \mathrm{~m} / \mathrm{sec}\right)$, with eastward and westward jets alternating in latitude $\left(-3 \beta \leq d^{2} \bar{u} / d y^{2} \leq 2 \beta\right)$. Eddy winds around the large, long-lived oval spots are comparable to the zonal winds. Eddy winds in the turbulent, convective regions are smaller than in the large ovals, but they convert significant amounts of energy into zonal mean kinetic energy ( $\left\langle\bar{u}^{\top} v^{\top} \cdot \mathrm{d} \bar{u} / \mathrm{dy}\right\rangle \approx 1 / 2\left\langle\bar{u}^{2}\right\rangle / 100 \mathrm{days} ;\left\langle\left(\mathrm{u}^{\top}\right)^{2}\right\rangle^{1 / 2} \approx 10 \mathrm{~m} / \mathrm{sec}$ ).

Nevertheless, the large ovals and zonal jets have existed for many years with little change (Ingersoll e t al., 1981; Ingersoll and Cuong, 1981).

Ingersoll and Pollard (1982) have considered the motions that might exist in the interiors of the giant planets. They examine the possibility that the interior is weakly but stably stratified $\left(\mathbf{a}^{2} \gg \mathrm{L}^{2} \approx \mathrm{T} \mathbf{\Delta S} / \mathbf{4} \boldsymbol{\Omega}^{2}\right.$, where a is the planetary radius, $L$ is the scale of motion in a direction perpendicular to the axis of rotation, $T$ is a typical internal temperature, $\Delta S$ is the specific entropy drop from surface to interior, and $\boldsymbol{\Omega}$ is the planetary angular velocity). Surfaces of constant S slope downward toward lower latitudes relative to surfaces of constant pressure, temperature and gravitational potential. Columnar motions, whose length scale parallel to the axis of rotation is comparable to the planetary radius, are quasi-geostrophic and anelastic but not hydrostatic. Accelerations appear in the axial momentum equation, and buoyancy terms appear in the axial vorticity equation.

Several problems (Ingersoll and Miller, in prep.) have been solved for this quasi-geostrophic system: low-frequency inertial oscillations of an adiabatic (homentropic) sphere (periods long compared to the planetary rotation period), low-frequency inertia-gravity oscillations in a weakly stratified sphere, stability of a barotropic zonal flow $\vec{u}(r)$ where $r$ is cylindrical radius, stability of a baroclinic zonal flow $\vec{u}(\mathbf{z})$ where $\mathbf{z}$ is the axial coordinate. For barotropic flow the stability parameter analogous to $\beta$ is $(2 \Omega / M) d M / d r$, where $M(r)$ is the integrated mass per unit area parallel to the axis of rotation. The surface expression of a marginally stable deep barotropic flow has $d^{2} \bar{u} / d^{2} \geqslant-3 \beta$, approximately. For baroclinic flow stability depends on the slope of the constant-entropy surfaces. Unstable disturbances, which appear when the slope exceeds a critical value of order 0.2 , convect heat outward across spherical surfaces and outward across cylindrical surfaces. These results are consistent with observations of the surface zonal flow and heat balance. Further studies may test whether nonlinear baroclinically unstable eddies can transport heat from the interior to the surface and maintain the zonal jets in the interior.

\section{REFERENCES}

Ingersol1, A.P., and P.G. Cuong, 1981. JAS, 38, 2067.

Ingersoll, A.P., et al., 1981. LGR, 86, 8733.

Ingersoll, A.P., and D. Pollard, 1982. Icarus, 52, 62.

Ingersol1, A.P., and R.L. Miller, in preparation. 


\section{DOUBLE-DIFFUSIVELY DRIVEN MIXING ACROSS THERMOHALINE FRONIS IN THE LABORATORY}

\section{Barry Ruddick}

After a brief introduction to the basic physics and jargon of doublediffusive phenomena, the discussion focussed on laboratory experiments on the driving by salt fingers of horizontal layers across a thermohaline front.

MacVean and Woods (1980) modelled the formation of a baroclinic (density) and thermoclinic (property) front in a zone of large-scale convergence. The property front was typically much sharper than the density front ( 2 and $15 \mathrm{~km}$ respectively after a few days) because of the tendency for the density front to adjust geostrophically. A laboratory model of the thermoclinic part of the front was described, in which the sharp property contrast and vertical density stratification are included, but the effects of rotation, tilted isopycnals, and thermal wind shear are not. A movie was shown depicting the development and spreading of intrusive layers at the front. The main balance of advection and vertical mixing due to salt fingers was described. Following Ruddick and Turner (1979) it was argued that the observed layer thickness, h, could be deduced by considering the energetics of the intrusion process, and agrees with the scale of oceanic frontal intrusions. The vertical scales predicted by several linearized instability theories were summarized. It was pointed out that theories for which the basic state consists of lateral property gradients extending infinitely far in either direction are intrinsically unstable to predict the length scales found by Ruddick and Turner (1979). They must instead predict scales based on a Rayleigh number, and so be proportional to $(\nu \mathcal{X})^{1 / 4}$.

The lateral flux of sugar was calculated in several ways from the laboratory observations. All were found to agree with the hypothesis that the sugar contours spread, on the average, with the frontal noses, so the lateral flux of sugar was proportional to the nose velocity times the cross-front property difference. The velocity, c, of each nose was observed to be constant in time, obeying the empirical rule $\mathrm{c}=5 \times \mathbf{1 0}^{-3} \mathrm{Nh}$, where $\mathrm{N}$ is the buoyancy frequency and $h$, the layer thickness. This steady spread is quite different from the $t^{1 / 2}$ spreading expected from a linear diffusion law and suggests an inertial control of the finite-amplitude layer system.

\section{REFERENCES}

MacVean, M.K., and J.D. Woods, 1980. Redistribution of scalars during upper ocean frontogenesis: a numerical model. Quart. Jour. Roy. Met. Soc., 106, 293-311.

Ruddick, B.R., and J.S. Turner, 1979. The verfical length scale of doublediffusive intrusions. Deep-Sea Res., $\mathbf{7} 903-913$. 


\title{
A THREEHAYER BAROCUINIC INSTABILITY ANALYSIS AND ITS APPLICATION TO SUBTROPICAL FRONIS I N THE OCEAN
}

\author{
Donald B. Olson
}

Subtropical fronts are found between 20 and 300 of latitude in most of the world's oceans. They are characterized by eastward near-surface flows extending well into the interior of the subtropical gyres from the west. The vertical stratification in these regions is dominated by two permanent thermoclines. The first of these tilts upward towards the poles and is mixed away seasonally poleward of approximately $30^{\circ}$. This near-surface feature is separated from the main thermocline by a thick layer of weak stratification with subtropical mode water characteristics. The main thermocline deepens poleward in most of the areas within the subtropical frontal zone although there is some evidence for eastward shear across it in narrow bands in the North Atlantic.

The simplest model for the vertical structure in the subtropical frontal zones which maintains the reversals in vertical shear observed in these regions is a three-layer model. In the present study a three-layer, quasigeostrophic analytical model of channel flows on a $\boldsymbol{P}$-plane is used to explore the nature of the eddies and meanders observed along the subtropical front.

Preliminary calculations suggest that the flows do not satisfy the necessary conditions for barotropic instability. Therefore, although the horizontal shears probably affect the details of the meanders which are observed on the fronts, they cannot account for the meanders in terms of a linear theory. The susceptability of constant cross-channel flows of varying directions in the vertical to baroclinic instability is explored. The model is similar to that discussed by Davey (1977) except that the layer thicknesses and the density contrasts across the interfaces are allowed to vary between layers.

The three-layer model allows one additional degree of freedom over the common two-layer approximation. The asymmetry between eastward (stabilized) and westward (destabilized) shear on a $\boldsymbol{\rho}$-plane, as found in the two-layer model is recovered. The cases in which the vertical shear changes signs between successive interfaces leads to decreased stability (higher growth rates) as compared to either the case of entirely eastward or westward shear across the interfaces. There are two basic vertical modes which appear in the analysis. The first long wavelength mode has a maximum amplitude in the surface layer and is equivalent to a first baroclinic mode for the actual stratification. The second unstable mode has its maximum amplitude in the middle layer and is associated with a short-wave branch of the stability curves.

The actual shears observed in the subtropical frontal zone of the North Atlantic consist of two narrow (-200-300 $\mathrm{km}$ wide) bands with deep westward but near surface eastward shear separated by zones with westward shears across both interfaces. Both types of flow are unstable in the linear analysis. The analysis suggests that the regions with eastward shear should have shorter wavelengths and wave growth rates two to four times those in the intervening westward flows. This is in qualitative agreement with the observation of peaks in eddy available potential energy in the regions with eastward nearsurface shear. The wavelengths of meanders observed in the North Atlantic $(300-400 \mathrm{~km})$ are also consistent with those expected in the linear analysis.

\section{REFERENCES}

Davey, M.K., 1977. J.Atmos. Sc1., 34, 1224. 


\section{Arnold L. Gordon}

The most intense and perhaps most unstable fronts within the world ocean are formed at the western margins, where opposing western boundary currents collide. These currents, driven by the Sverdrup interior flow of the subtropical and subpolar gyres respectively, spread seaward after confluence forming a multitude of meanders and eddies. Since these currents carry water of vastly differing characteristics, warm-saline subtropical thermocline water versus cold-low salinity subpolar water a strong thermohaline front is formed.

An example of a front by confluence is that of the Brazil and Falkland Current, which meet near $38^{\circ} \mathrm{S}$, over the continental slope adjacent to Mar del Plata, Argentina. The Falkland Current often considered a branch of the Antarctic Circumpolar Current, carries northern Drake Passage (subantarctic) water into the Argentina Basin, closely flowing along the isobaths of the continental slope. Volume transport is probably between 10 to $20 \times 10^{6} \mathrm{~m}^{3} / \mathrm{sec}$, though without an obvious reference level, transport is not known to a reasonable degree of certainty.

The Brazil Current is relatively weak for a western boundary current. Between $10^{\circ}-20^{\circ} \mathrm{S}$ the highly variable Brazil Current may amount to only $5 \times 10^{6} \mathrm{~m}^{3} / \mathrm{sec}$. Further south near $24^{\circ} \mathrm{S}$ transport of nearly $10 \times 10^{6} \mathrm{~m}^{3} / \mathrm{sec}$ is determined. At the confluence the geostrophic transport is about $25 \times 10^{6} \mathrm{~m}^{3} / \mathrm{sec}$.

After confluence, the Brazil Current momentum dominates, allowing further poleward advection of South Atlantic Central Water (SACW). An abrupt return northward occurs, forming a quasi-stationary poleward extension or loop of SACW near $50^{\circ} \mathrm{W}$, which reaches a times to $46^{\circ}-490^{\circ} \mathrm{S}$. Often warm SACW eddies are shed, which then drift south and southeast, lost to the SACW, though some subsurface return may occur. As warm eddies are shed, filaments of the Falkland Current rapidly spread eastward. Cycles of southward migration of the poleward loops with periods of eddy shedding occur on a time scale of months.

Within the poleward loop relative warm subtropical water forces a regional maximum in oceanic heat loss to the atmosphere. This forms deep winter mixed layers (extending to 350 meters). Cooling without an input of fresh water forces the mixed layer $\theta / S$ point to fall to the saline side of the parent SACW $\theta / S$ curve. The local excess in evaporation enhances this effect. The positive salinity anomaly (relative to the SACW curve) sets up layers of low $\mathrm{R}=\alpha \Delta \mathrm{T} / \boldsymbol{\beta} \boldsymbol{\Delta} \mathrm{S}$ (down to 1.3) making the poleward loop susceptible to active salt fingers with associated boosting of the $\mathrm{K}_{\mathbf{z}}$ value. Welldeveloped thermohaline steps usually taken as signs of double diffusion are indeed observed below the remnant winter mixed layers.

Within the mid-depth $(400-500 \mathrm{~m})$ thermocline stratification north of the loop, intrusions of remnant winter mixed layer water is observed. These intra-thermocline eddies derived from poleward winter surface water represent ventilation of the South Atlantic thermocline. They too are unstable to salt finger. Note salt finger instability is aided by the presence of low salinity Antarctic Intermediate Water at the base of the thermocline. 
Two research questions may be asked: 1) Could a regional enhanced $K_{z}$ value, due to the action of salt fingers, drive or contribute to a regional anticyclonic circulation cell and poleward loop of the Brazil Current? and 2) Do other oceans have similar quasi-stationary poleward loops of thermocline water with thermohaline "ventilation" processes set up by the confluence of oppposing western boundary currents?

\section{SATELLITE DERIVED KINEMATICS OF WARM CORE RINGS - MN OVERVIEW}

\section{O t is B. Brown}

A brief introduction to satellite remote sensing technique and data analysis procedures is given as an introduction. A bulk atmospheric correction scheme based on multiple infrared windows is derived and applied to April, 1982 satellite and ship observations of the U.S. East Coast slope water. A bias of approximately $0.6 \mathrm{C}$ (warm) with a RMS of $\sim 0.4 \mathrm{C}$ is determined from an intercomparison of continuous flow through ship observations and satellite SST derived temperatures. Implications of cloud detection procedures to improvement of satellite SST retrievals are discussed.

An overview of the Wam Core Rings Experiment is presented as background to kinematic studies of WCR 82-B. The location, motion, areal extent, and orientation of 82-B as derived from satellite imagery, and other measurements, are discussed. The role of shelf-slope Gulf Stream streamers in modifying ring surface water properties is explored by viewing a selected subset of $82-B$ satellite imagery. Temperature/Chlorophyll time series derived from satellite data for areal averages on the shelf, slope, Gulf Stream, and ring center show seasonal warming, variations in the efficacy atmospheric correction procedures and streamer influences. A video tape time series (120 images) of 82-B from April to August is then shown. This time series provides graphic illustration of the earlier results on ring kinematics and the influence of streamers on warm core rings. Dramatic changes in ring areal extent and surface temperatures are observed as a direct result of Gulf Stream-ring interaction. 


\section{MEANDERING OF THE GULF STREAM AND LONG WAVE INSTABILITY}

\section{Glenn Flier1}

Two types of models for the wavelike meandering of the Gulf Stream have developed in the literature: the first is based on linearized perturbation analyses while the second attempts to describe the path of the jet axis in terms of average properties such as the net mass and momentum transport of the current. We demonstrate that these two approaches yield the same dispersion relations for small amplitude, longwave, sinus mode motions of the current.

The simplest relevant instability problem considers perturbations upon a zonal barotropic "top-hat" jet profile. The dispersion relation for the sinus mode is

$$
c^{2} \sqrt{1+\frac{\beta}{k^{2} c}}+\left(u_{0}-c\right)^{2} \sqrt{1-\frac{\beta}{k^{2}\left(u_{0}-c\right)}} \tanh \frac{1}{2} k l \sqrt{1-\frac{\beta}{k^{2}\left(u_{0}-c\right)}}=0
$$

where $U_{0}$ is the mean current strength and $l$ is the north-south width of the current. In the long wave 1 imit $k \ell \ll 1$ but $\beta / \mathbb{U}_{0} k^{2}$ still order 1 , this simplifies to

$$
c^{3 / 2} \beta^{1 / 2}=\frac{1}{2} 2\left(\beta u_{0}-k^{2} u_{0}\right)
$$

Showing that the jet is stable for long waves $1 / k<\sqrt{u_{0} / \beta}$. This is a good approximation to the full dispersion relation for very small $\ell$ but for reasonable $\ell$ is only good for $\mathrm{k}$ in the stable long wave regime. Guided by the scaling c $\left(\ell \beta^{2 / 3} u_{s}\right)^{2 / 3} \sim U_{0}(k \ell)^{2 / 3}$ given by the long wave limit (2), we can also solve the perturbation equation for long wave meanders on a baroclinic jet. The perturbation equation is solved in three regions, corresponding to $|\mathrm{U}| \gg|\mathbf{c},| \mathrm{U}|\boldsymbol{k}| \mathrm{k}$ and $\mathrm{U}=0$ (outside of the jet) and the solutions are matched together, giving

$$
c^{3 / 2} \beta^{1 / 2}=\frac{1}{2} k\left[\beta \int u-k^{2} \iint u^{2}\right]
$$

where $\iint U d y d z$ and $\iint U^{2} d y d z$ are the mass and momentum transports of the jet.

The thin jet approach starts by transforming the equations to a downstream and cross-stream coordinate system based upon the (unknown) position of the jet axis, assuming that the cross-stream scale is small (order $b$ ) compared to the scale of the waves along the stream. The velocity field can be split up into the main downstream flow, the motions associated with translations of the axis, and the residual flows. Integrating the vorticity across the current and vertically gives a relationship between the tangential barotropic velocities on either side of the jet and the motions of the axis, with the mass and momentum transports being parameters. In the exterior regions, the vorticity equation must be solved and fitted to the normal velocities at the edge of the jet. The matching of the tangential flow then determines the evolution of the position of the path. The dominant motions in the exterior are barotropic but become nonlinear as the meanders grow to finite amplitude. In the small-amplitude limit, however, the exterior flow is solvable, giving motions decaying on a scale $(\ell)^{1 / 3} \sqrt{u_{s} / \beta}$ to the north and the south. The tangential flows can be solved readily and, with the linearized form of the cross-jet and vertically integrated vorticity equation, yield the same dispersion relation (3) as the instability approach. In 
addition it is possible to find the study finite amplitude meander pattern by solving

$$
\theta_{\tau \tau}+\frac{\beta \delta \int u}{\delta S u^{2}} \sin \theta=0
$$

where $\theta$ is the angle of the jet from east and $\tau$ is the coordinate along the jet axis. The location of the axis in $x-y$ space can then be readily found.

Thus the thin jet model reproduces known linear and nonlinear results, though the accuracy may not be as good as one might wish. In addition, it seems to offer some possibilities for simplifying the finite amplitude timedependent problem; however, this method does rely upon obtaining solutions in the region exterior to the jet so that the equation for the development of the meanders does not appear to be a simple one-dimensional problem. The model suggests that the meandering of the Gulf Stream is not simply the cause of the mesoscale eddy field in the recirculation region and the Slope Water; rather, the two are strongly coupled.

\section{REFERENCES}

Flierl, G.R., and A.R. Robinson, 1983. On the time-dependent meandering of a thin jet. J. Phys. Oceanogr.

Howard, L.N., and P.G. Drazin, 1964. On instability of parallel flow of inviscid fluid in a rotating system with variable Coriolis parameter. $\underline{\text { J. Math. and Phys., }}$ 43, 83.

Robinson, A.R., and P.P. Niiler, 1967. Theory of free inertial jets (I): Path and structure. Tellus, 19, 269. 


\title{
BAROCLINIC AMPLITUDE VACILLATION
}

\author{
Philip Drazin
}

This seminar was a progress report of work with Albert Barcilon of Florida State University.

When a differentially heated rotating annulus contains a liquid, many types of baroclinic oscillations may be observed according to the values of the temperature difference and angular velocity of the annulus. For one type, called amplitude vacillation, the surface of the liquid appears like a standing wave which $\mathrm{drifts}$ around the annulus. Lindzen, Farrell and Jacqmin (1982) suggested that this is due to the superposition of two unstable modes of the basic state with the same zonal wavenumber. Using a linear theory, they calculated two such modes which grow exponentially with time while they propagate around the annulus.

However, amplitude vacillation may be seen to persist for a long time i n the laboratory, so nonlinearity is needed for the modes to equilibrate and thereby for the theory of Lindzen et al. to be completed. On first hearing of their work, we speculated, on the general basis of bifurcation theory, that weakly nonlinear modes with complex amplitudes At and A- should be governed by a coupled pair of equations of the form

$$
d A_{t} / d t=\left(\sigma_{ \pm}-l_{ \pm}\left|A_{ \pm}\right|^{e}-m_{ \pm}\left|A_{\mp}\right|^{2}\right) A_{ \pm}+\text {higher-order terms, }
$$

where $\sigma_{ \pm}$are the complex relative growth rates given by the linear theory, and $1_{ \pm}$and $n+$ are some complex interaction coefficients.

For a substantial model, we consider a quasi-geostrophic flow of a Boussinesq fluid. The flow is in a long rigid channel in the zonal direction and with a rectangular cross-section, there being thin Ekman layers at the top and the bottom. The basic state is a zonal flow of the form $U=\{z+E F(z)\}$ with square of the Brunt-Väisälä frequency $N^{2}=N_{0}^{2} /\{1+\varepsilon G(z)\}$ for $0<\varepsilon \ll 1$, where $N_{0}$ is some given constant and $F$ and $G$ given functions. This becomes the basic state of Eady (1949) when $\varepsilon=0$. Perturbation theory (McIntyre 1970) shows that the two waves of Eady which are neutrally stable when $\varepsilon=0$ become unstable for small values of $\varepsilon$ and many functions $F$ and G. This is an explanation of the results of Lindzen et al. Proceeding to the nonlinear theory, we took a double expansion of the perturbation stream function in powers of $\Delta^{2}$ and $\varepsilon$, where $\Delta^{t_{2}}$ is the order of magnitude of the stream function. The leading term is the sm of the two stable Eady modes with slowly varying amplitudes $A_{+}$and $A_{-}$. The solvability conditions for the system eventually give a coupled pair of amplitude equations of the anticipated form above.

We assume that the basic quasi-geostrophic potential vorticity gradient is antisymmetric about the level $\mathbf{z}=0$ of the middle of the channel. This is a satisfactory representation of laboratory observations of the mean flow, and it ensures that $\sigma_{-}=\sigma_{+}$, so that viscosity renders the two linear modes marginally stable together. It follows that the higher-order terms of the amplitude equations are relatively small in the limit as $\Delta \rightarrow 0$. Then the 
equations may be solved by methods of the phase plane and shown to admit a stable solution, with constant values of $\left|A_{+}\right|^{2}$ and $\left|A_{-}\right|^{2}$. This theory then describes the superficial observations of amplitude vacillation, but needs more thorough corroboration.

\title{
REFERENCES
}

Eady, E. T., 1949. Tellus, 1, 33-52.

Lindzen, R. S., B. Farrell and D. Jacqmin, 1982. J. Atmos. Sci., 39, 14-23, McIntyre, M. E., 1970. J. Fluid Mech., 40, 273-306.

\author{
THE GULF STREAM: BARRIER OR BLENDER \\ OR \\ ISOPYCNAL SIGNATURES OF ADVECTION AND MIXING
}

Thomas Rossby

Using the Gulf Stream '60 hydrographic data set, we have prepared isopycnal analyses (maps) of pressure, acceleration potential, temperature, and oxygen at the potential densities of $\sigma_{\theta}=26.8,27.2$, and 27.6. These have mean Sargasso Sea depths of $-600,850$, and $1050 \mathrm{~m}$ and shoal about $600 \mathrm{~m}$ across the stream. We use the acceleration potential to define the path of the dynamical front and use the property analyses to determine the existence of a property front and its position relative to the dynamical front.

We find strong coincidence of the property fronts (both $\mathrm{T}$ and 02 ) and the dynamical front a 11 along the Gulf Stream (to $50^{\circ} \mathrm{W}$ ) on the $\sigma_{\theta^{-}}=26.8$ suggesting that the Gulf Stream acts substantially as a barrier between the slope and Sargasso Sea waters, particularly west of the New England Seamounts. On the two deeper surfaces and especially on the $\sigma_{\boldsymbol{\theta}}=27.6$ there are no concentrated property gradients along or near the dynamical front. Instead the temperature and oxygen fields are characterized by eddy stirring across the entire region between the cold, high oxygen waters to the north of Labrador origin and the warm (saline) waters to the south of the Mediterranean origin.

The study was motivated in part by a desire to determine whether isopycnal Swallow floats drifting in the Gulf Stream were likely to remain trapped in it. The results suggest that this is so on shallow density surfaces, and that when escape does occur, it is much more likely to the south than across the cyclonic side into the slope waters. 
MIXING, WAVES, AND GRAVITY CURRENIS I N THE LABORATORY AND I N NATURE

Tony Maxworthy

We attempt to put a number of recent experiments and field observations into a more-or-less coherent framework. The interconnections are shown in the diagram below.

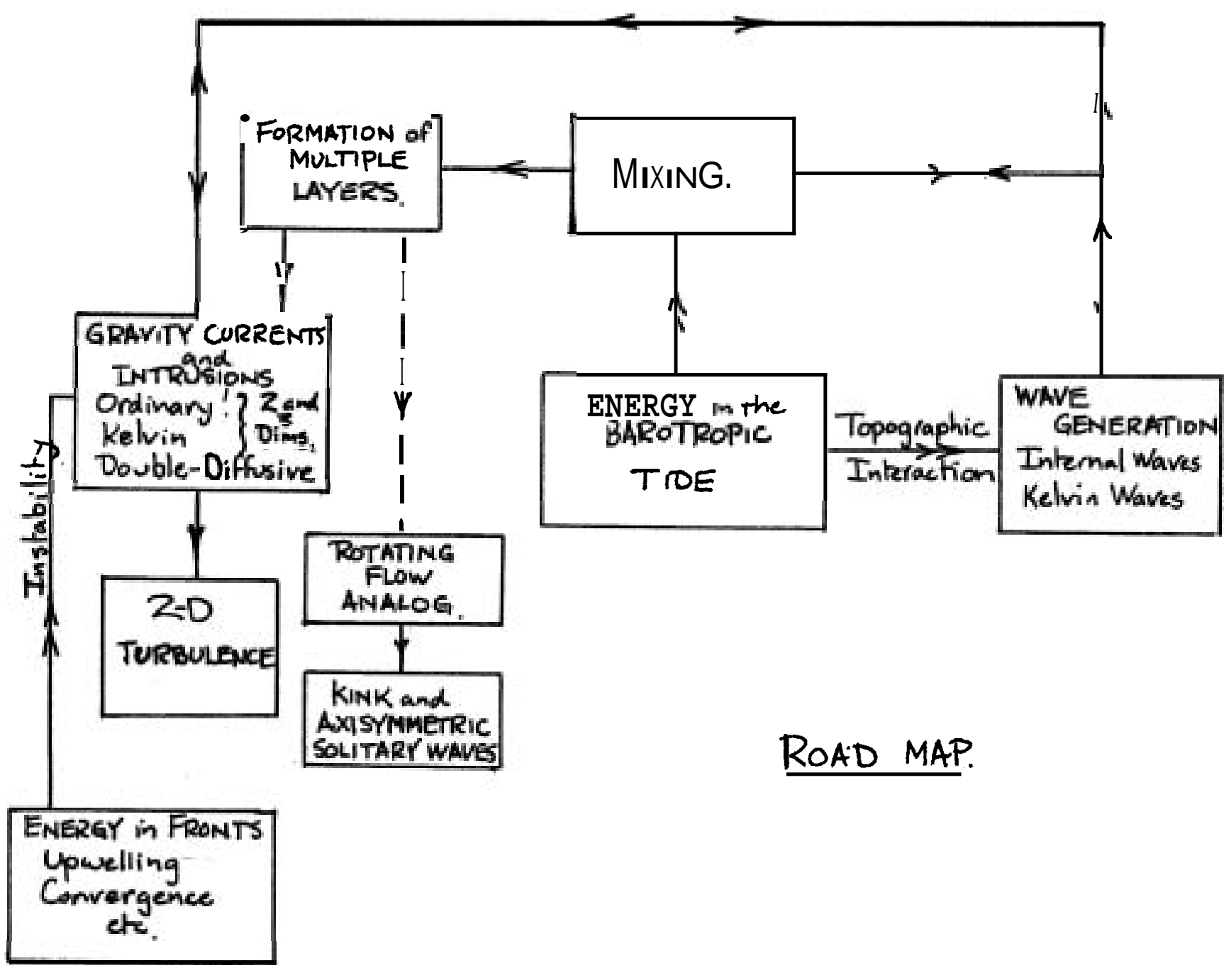

The basic energy source is due to a tidal motion, although the available energy potential of oceanic fronts (upwelling or otherwise) is another possibility.

The Gulf of California in the neighborhood of the Isla de la Guarda appears to contain many of the phenomena of interest including the generation and propagation of internal waves, gravity currents created by mixing processes, and upwelling fronts and plumes. These are especially clear in satellite observations using active (Seasat) or passive (Landsat, GOES) sensors. This area is by no means unique, however, and data from Knight Inlet, the North Atlantic continental margins, among many others can also be considered. 
Firstly we consider the generation of solitary internal waves by tidal flow over bottom topography. The main reference here is Maxworthy (1979). In this paper it was demonstrated experimentally that an ordered sequence of solitary waves could be generated from the lee waves formed behind an obstacle being oscillated in a stratified fluid. It was also shown that mixing in a stratified fluid could generate waves by collapse of the mixed region which contains an excess of potential energy over its surroundings. This mechanism has been explored further by Lansing (1983) and Lansing and Maxworthy (in press) in a numerical and experimental study using a two-layer model. In the numerical model the interface between the two fluids is replaced by a vortex sheet and the obstacle by a distribution of sources. The results compare well with the experiments and show the rapid evolution of a sequence of solitary waves from the initial disturbance created close to the topographic feature.

A new series of experiments on the 13 m rotating table at the University of Grenoble show further details of solitary wave evolution from a more complex lee wave field and indicate the importance of rotation in determining the initial plan-form of the waves.

The second main thrust of our recent work concerns the generation of waves and gravity currents from mixed region collapse. The work has been reported in Maxworthy (1980) and Maxworthy (1983). In particular the latter shows how, in a rotating stratified fluid, solitary Kelvin waves can be produced. They look like ordinary nonrotating waves in side elevation but have an amplitude which decreases away from the wall on which they are supported. Since local wave speed depends on wave amplitude, the wave has a curved plan-form in order for the wave as a whole to propagate at a speed given by its maximum amplitude. In the limit of very large amplitude these waves propagate in gravity currents and should be similar to those studied by Stern, Whitehead, and Hua (1982) and Griffiths (private communication).

The dynamics of viscous gravity currents are considered in two recent papers, Maxworthy (1983). One is concerned with gravity current flows in which the value of the current varies as $t \alpha$. It is shown that the value $\alpha=7 / 4$ separates two distinctly different regimes. For $\alpha<7 / 4$ the flow is initially in a state of buoyancy-inertia balance and undergoes a transition to buoyancy-viscous balance at a later time. For $\alpha>7 / 4$ this sequence is reversed. In the second paper the fluid within the gravity current has a diffusivity which is different from that of the main body of fluid. This gives rise to a double-diffusive interface between the two fluid regions across which both mass and momentum can be exchanged. This results in a large stress being applied to the interface which in most cases dominates the viscous stress and results in a different power-law behavior with time. Experiments show that under suitable circumstances these power laws can be observed and support the basic hypothesis. Secondary effects tend to make these effects in other parameter ranges and the theory should be extended to take them into account.

Up to now we have assumed that mixing creates only one layer of mixed fluid. However, recent experiments by Browand and Hopfinger (1981), and Thorpe (1982) suggest that in a mixed stratified fluid multiple layers can form and each one can then evolve as an intrusion. If a grid is oscillated in a stratified fluid, a front is formed separating mixed from unmixed fluid. When this front has propagated some distance from the grid the local Richardson number, based on the local integral scale and fluctuating of 
velocity exceeds on a critical value and the front collapses into sequence of intrusions the vertical separation of which is given by the value of the Osmidov length $\left(L_{o}=\left(\epsilon / N^{3}\right)^{1 / 2}\right)$ at collapse multiplied by a constant of order one ( $\epsilon$ is the local dissipation and $N$ the Brünt frequency). Experiments in tanks of different widths suggest that three-dimensional effects may also be important in deciding this scale since in a wider tank the collapse of three-dimensional turbulent blobs is not constrained by the presence of side walls and would be expected to be thinner than the intrusions in a narrow tank. Recent experiments on the flow created by towed grids in a stratified fluid show this result very clearly. After collapse the vertical velocities are very small while the horizontal velocities are large and independent of direction. The horizontal scales of motion increases with time suggesting an upscale transfer of energy reminiscent of the predictions of the theory of two-dimensional turbulence, and these possibilities are currently under quantitative study.

\section{REFERENCES}

Browand, F., and E. Hopfinger, 1981. A.P.S.-D.F.D. Mtg. Paper D.E.1.

Lansing, F., 1983. Ph.D. Thesis, Univ. Southern Calif.

Lansing, F., and T. Maxworthy, in press. Jour. Fluid Mech.

Maxworthy, T., 1983. Jour. Fluid Mech., 128, 375-396.

Maxworthy, T., 1983. Jour. Fluid Mech., 129, 365-383.

Maxworthy, T., 1980. Jour. Fluid Mech., 96, 47-64.

Maxworthy, T., 1979. Jour. Geophys. Res., 84, 338-346.

Stern, M., J. Whitehead, and B. Hua, 1982. Jour. Fluid Mech., 123, 237-265.

Thorpe, S., 1982. Jour. Fluid Mech., 124, 391-409. 


\title{
BOUNDARY FORCED NONLINEAR PLANETARY RADIATION
}

\author{
Paola Malanotte Rizzoli
}

In recent years, a renewed interest has been focused upon the Gulf Stream system and its interaction with the mesoscale oceanic eddy field. From the experimental evidence accumulated in experiments like MOE and POLYMODE, important questions emerge not yet adequately treated even in the context of simple theoretical models. One of such questions concerns the possible generation mechanisms of the mesoscale eddy field and, in the limit of highamplitude radiation, the formation of ring-like structures from an eastward meandering jet, like those observed to emerge from the Gulf Stream.

This question is related to the general problem of radiation of mesoscale energy from a meandering current, which was studied by several authors. Flierl et al. (1975) examined the behavior of semi-infinite domains driven by boundary forcing, using the linear barotropic vorticity equation on the -plane. They found that the existence of eddy-like motions depends cruclally on westward phase propagation of the boundary motion. Pedlosky (1972) treated the same problem with a 2-layer quasi-geostrophic model including a mean current. Harrison and Robinson (1979) studied the question of radiation from a northern boundary into a finite domain, with zero-boundary conditions at the meridional boundaries, which makes it rather different from the infinite northern wall problem. In this latter case, the basic result is that in the quiescent ocean the far field can transmit energy radiation by the northern boundary only if this has a westward phase speed.

A 11 these models are however linear. Nonlinear effects may be expected to modify the above results, as indicated by numerical experiments carried out with fully nonlinear models, in which mesoscale radiation is observed to be excited and radiate away from the eastward moving jet.

The above problem has been investigated in the context of a fully nonlinear, although simple, model, in which again the meandering current is idealized as a moving northern boundary.

The model used is the quasi-geostrophic equivalent barotropic potential vorticity equation in a zonal channel over variable topography. For the free case, this model admits as solutions free nonlinear waves, which for weakbut-finite nonlinearity and dispersion are periodic cnoidal waves and, in the limit of infinite axial wavelength, solitary waves. This nonlinear Rossby radiation is asymmetric in the channel. In the limit of high nonlinearity and east-west versus north-south wavelengths of the same order of magnitude, the free nonlinear wave is a high-amplitude monopole, having closed recirculation regions, similar to the ring-like structures observed to emerge from the Gulf Stream.

The problem of boundary forced radiation has been completely examined in the weakly nonlinear parameter range. The forced response - which has the same $x$-structure as the boundary wave - exists for all those linear phase speeds of the northern boundary which do not correspond to the eigenfrequencies of the free modes of the zonal channel. For this specific set of eigenfrequencies, nonlinearity allows the solution of the problem, while in the purely linear case the response of the interior field would be infinite. 
Thus, a weak boundary forcing of $O(E)$, if $\varepsilon$ is a measure of the nonlinearity, excites a response of $O(1)$ in the interior field. The equilibrium boundary forced resonant response obeys a forced Korteweg-deVries equation. For the specific forcing of a $\operatorname{sech}^{4}$, the equilibrium resonant response is again an cnoidal - or solitary - wave, whose amplitude can have multiple equilibrium states, specifically two. The equilibrium states are found in general for arbitrary values of the detuning, nonlinear phase speed $c_{1}-$ of $0(\varepsilon)$ - of the boundary wave.

Two effects of nonlinearity can be demonstrated analytically.

First, let the detuning phase speed $C_{1}$ of the northern boundary be allowed to slowly vary in time, corresponding to a slow modulation of the forcing. Introducing the long time scale $T=\boldsymbol{E} t$, this leads to the time-dependent version of the Korteweg-deVries equation. An initial condition corresponding to the steady equilibrium solution can then be let to evolve over the long time scale $\mathrm{T}$. The observed behavior is soliton production in the region of the zonal channel directly affected by the forcing. When the produced solitons do not feel any longer the northern forcing, they will propagate as nonlinear waves in the direction allowed by the unforced Korteweg-deVries equation. In the opposite direction, they will evolve into dispersive wave packets - this means that the nonlinear resonant response will evolve on the long time scale producing in one direction nonlinear radiation of shorter wavelength and deterministic nature - smaller eddies.

The second effect of nonlinearity can be demonstrated when allowing the northern forcing amplitude to increase to $0(1)$, namely passing to the highly nonlinear case. Then, a boundary forcing of $0(1)$ will excite an interior resonant response of $O\left(\frac{7}{\varepsilon}\right)$, always treating the case of long radiation in zonal direction. In this case, the cross-channel structure of the nonlinear response can be profoundly modified. In the linear case, over a topography which is essentially northward sloping, an eastward moving boundary wave will excite a response which, at the best, has an oscillatory nature in some interior, limited region. Near the northern boundary itself, this response is decaying exponentially to zero. On the other side, in the previous highly nonlinear case, the resonant response excited in the interior can be shown to have an oscillatory behavior near the northern boundary even when this one is eastward moving. These results imply that the limit of high nonlinearity can profoundly modify the results of the linear theory, even in the context of simple analytical treatments like that here performed. Obviously, numerical experiments are necessary to confirm the above analytical theory and to explore more complex effects which cannot be treated analytically.

\section{REFERENCES}

Flie r1, G.R., Kamenkovich, V., and A.R. Robinson, 1975. Gulf Stream meandering and Gulf Stream rings. MOE Dynamics Group, unpublished manuscript.

Harrison, D.E., and A.R. Robinson, 1979. Boundary forced planetary waves: a simple model mid-ocean response to strong current variability. Jour. Phys. Oceanog., 9, 919-929.

Malanotte-Rizzoli, P., 1983. Boundary forced nonlinear planetary radiation. In preparation.

Pedlosky, J., 1977. On the radiation of mesoscale energy in the mid-ocean. Deep Sea Res., 24, 591-600. 
WHAT MAINTAINS THE SUBTROPICAL FRONT AND ITS ASSOCIATED COUNTERCURRENT?

Benoit Cushman-Roisin

The Subtropical Countercurrent is an eastward flow across the subtropical gyre at a latitude where a classical wind-driven circulation theory would predict a westward flow feeding the western boundary current. Japanese investigators documented the Countercurrent as it is found in the upper $150 \mathrm{~m}$ of the North Pacific at about $20^{\circ} \mathrm{N}$ (Uda and Hasunuma 1969 , and references therein). Only later was the current associated with a zonal density front, the Subtropical Front (Hasunuma and Yoshida, 1978; Roden, 1975; and references therein). A rapid check of orders of magnitude reveals that the countercurrent can be easily explained as the zonal thermal current associated with the meridional density gradient across the front. They are thus two components of one phenomenon. But, what is the mechanism responsible for such structure?

Takeuchi (1980) modelled the ocean circulation between $00^{\circ}$ and $50^{\circ} \mathrm{N}$ as forced dynamically (surface wind) and thermodynamically (surface heat flux). This latter drive consisted of warming at low latitudes and cooling at higher latitudes depending on a temperature difference between the ocean and the atmosphere. The numerical results show a shallow eastward flow along a density front at about the central latitude of the subtropical gyre. Moreover, the front tilts, as observed, slightly north toward the east, from $20^{\circ} \mathrm{N}$ to $30^{\circ} \mathrm{N}$. Very similar results were obtained when the zonal winds were replaced by meridional winds with the same curl. As a consequence, convergence of Ekman transports cannot be the main explanation for the existence of the subtropical front, although it can strengthen it. It thus remains to discern among a 11 processes included in Takeuchi's model, besides Ekman convergence, which one is responsible for the formation of the front.

To simplify the problem to a maximum, one assumes that the dynamics yield an upper-level flow field consisting of a nondivergent wind-driven Sverdrup flow and of geostrophic but divergent thermal flow:

$$
\begin{aligned}
& u=\frac{x}{B H} \tau_{y y}^{x}-\frac{a_{1} H_{2}}{2 f H} T_{y} \quad v=-\frac{1}{\beta H} \tau_{y}^{x}+\frac{a_{1} H_{1} H_{2}}{2 f H} T_{x} \\
& w=H_{1}\left(u_{x}+v_{y}\right)=-\beta \frac{a g H_{1}^{2} H_{2}}{2 f^{2} H} T_{x}
\end{aligned}
$$

where $\tau^{\mathbf{x}}$ is the zonal wind stress, $\mathrm{H}_{1}$ the upper-level depth, $\mathrm{H}_{2}$ the lowerlevel depth, $\mathrm{H}=\mathrm{H}_{1}+\mathrm{H}_{2}, \mathrm{~T}$ the upper-level temperature, and $\mathrm{w}$ the vertical velocity between the two levels. The model is closed by an equation for $\mathrm{T}$ :

$$
\mathrm{u} \mathrm{T}_{\mathrm{x}}+\mathrm{v} \mathrm{T}_{\mathrm{y}}+\frac{1}{\mathrm{H}_{1}} \mathrm{wT}=-\mathrm{k}\left(\mathrm{T}-\mathrm{T}_{\mathrm{a}}(\mathrm{y})\right) \text {, }
$$

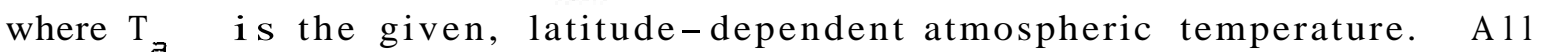
temperatures are measured as an increment above the uniform temperature of the lower level; the wT term thus represents vertical exchange of heat between the two levels. Replacing $u, v$ and w by their known expressions, one obtains 
a single nonlinear equation for $\mathrm{T}$ :

$$
\left(\frac{x}{\beta H} \tau_{y y}^{x}-\beta \frac{\alpha g H_{1} H_{2}}{2 f^{2} H} T\right) T_{x}-\frac{1}{\beta H} \tau_{y}^{x} T_{y}=-k\left(T-T_{a}(y)\right) .
$$

This hyperbolic equation is best solved by the method of characteristics. Close inspection shows that the subtropical gyre $\left(15^{\circ} \mathrm{N}-45^{\circ} \mathrm{N}\right)$ is covered by two families of characteristics: one originates from the western boundary region while the other originates from the eastern wall. Moreover, for the parameters corresponding to the Pacific Ocean, these characteristics intersect, forming an envelope line running across the basin splitting it in two regions of independent properties. This line corresponds to the Subtropical Front. With some diffusion added, the resulting temperature field is as shown on Figure 1. One can clearly see a front starting at $20^{\circ} \mathrm{N}$ to the west and tilting northward toward the east. The associated flow field (not shown here) consists of the subtropical circulation superimposed on which is a thermal current flowing eastward along the front. The Subtropical Front and Countercurrent are thus well reproduced by this very simple model.

The frontogenetical process is now clear, as a direct hopshot of the intersecting characteristics. Without the BTT term, the characteristics would be the Sverdrup streamlines, but with thăt term present, they do intersect. That term is traced back to the vertical exchange of heat between the two levels as forced by the vertical velocity resulting from the convergence of isothermal flow on a beta-plane. It is this term, in combination with the others, which is responsible for the existence of the front. It can thus be stated that the combined effect of Sverdrup flow with the convergence of the isothermal flow on a beta-plane is the mechanism responsible for the maintenance of the Subtropical Front and Countercurrent.

\section{REFERENCES}

Hasunuma, K., and K. Yoshida, 1978. Splitting of the Subtropical Gyre in the Western North Pacific. Jour. Oceanogr. Soc. Japan, 34, 160-171.

Roden, G.I., 1975. On North Pacific temperature, salinity, sound velocity and density fronts and their relation to the wind and energy flux fields. Jour. Phys. Oceanogr., $\underline{5}, 557-571$.

Takeuchi, K., 1980. Numerical study of the Subtropical Front and the Subtropical Countercurrent. Ph.D. dissertation, Tokyo University.

Uda, M., and K. Hasunuma, 1969. The eastward Subtropical Countercurrent in the western North Pacific Ocean. Jour. Oceanogr. Soc. Japan, 25 , 201-210. 
UPPER-LEVEL TEMPERATURE

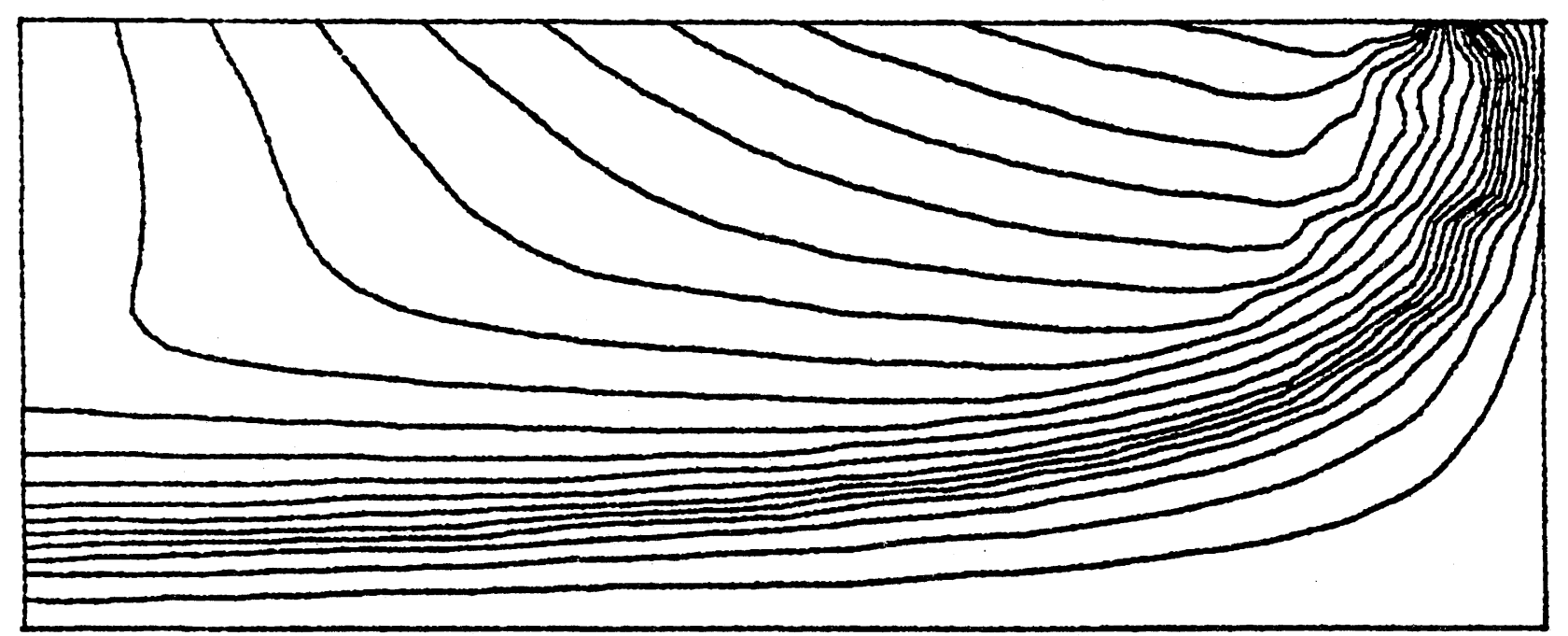

\section{$\angle A M B D A=15.00 \quad A=.65 \quad B=7.70$}

Figure 1. The upper-level temperature field as obtained by the present sample mode1. 


\title{
TURBULENILY GENERATED EASTERN BOUNDARY CURRENIS
}

\author{
Roger L. Hughes
}

The appearance of eastern boundary currents in the finite Froude number models discussed by George Veronis, suggests that it might be possible to obtain such a current in a model with slow mean flow but large amplitude geostrophic turbulence. Such a boundary layer appears to occur in the eddy resolving model of Robinson et al. (1977). A simple analytical model is given in which such a boundary current is driven by the nonisotropic nature of the direct Reynolds stress of the eddy field near the eastern boundaries. The structure predicted agrees with that calculated in the eddy resolving model.

\section{REFERENCE}

Robinson, A.R., D.E. Harrison, Y. Mintz, and A.J. Semtner, 1977. Eddies and the general circulation of an idealized oceanic gyre: a wind and thermally driven primitive equation numerical experiment. Jour. Phys. Oceanog., I, 182-209.

\section{A TWOLAYER MODH OF THE SUBPOLAR GYRE AND THE SUBTROPICAL GYRE}

\section{Rui Xin Huang}

A study is made of the current structure of a subpolar gyre and a double gyre basin. A simple two-layer model is used, and its behavior is explored as the amount of water in the upper layer is gradually reduced. A dynamically consistent picture includes a strong, isolated western boundary current flowing southward and an internal boundary current flowing northward. The isolated western boundary current may represent the Labrador Current and the internal boundary current may represent the North Atlantic Current.

The analysis follows Parsons' (1969) idea, 1.e. we assume that the lower layer has an infinite depth, so that the simplest flow pattern can be found with relatively simple algebra.

We also try to analyze the case when the lower layer thickness is finite so that motion can occur within the lower layer.

\section{REFERENCE}

Parsons, A.T., 1969. A two-layer model of Gulf Stream separation. Fluid Mech., 39, 511-528. 


\title{
VISCO-DOUBLE-DIFFUSIVE DENSITY INTRUSIONS
}

\author{
William R. Young
}

Consider a layer of Boussinesq fluid which is initially stratified by two components, say heat and salt ( $T$ and $S$ ). Suppose that the fluid is confined by solid boundaries through which there is no vertical flux of heat or salt. Thus, unless the system is forced by horizontal fluxes, diffusion and viscosity ensure that the compositional gradients are eventually erased. In this note the initial value problem whose outcome is this motionless state with uniform distributions of heat and salt is discussed. In view of its conceptual simplicity, and also recent laboratory experiments (Ruddick and Turner, 1979), a particularly interesting special case is the spread of a compositional front. (Note that it is possible to contrive the jumps in compositional concentration so that the density is uniform across the front.) This problem was first treated by Erdogan and Chatwin (1967) who limited their discussion to spread of a single buoyant contaminent.

The related problem of a fluid whose compositional gradients are maintained by externally imposed horizontal fluxes is also discussed. The most important issue in this steady state problem is how the flux of a quantity is related to its gradient. Because of buoyancy forces, heat and salt are dynamically active and consequently the flux-gradient relations of the two quantities are nonlinear and coupled.

If one is familiar with Taylor's (1953) theory of dispersion, it is very easy to understand physically how this nonlinear coupling arises. Taylor considered the advection-diffusion equation:

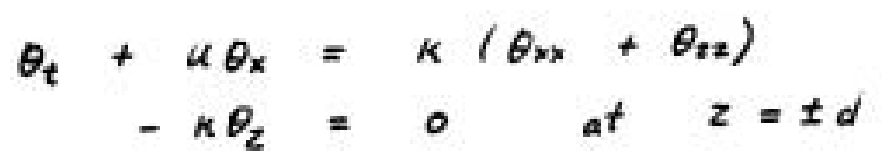

for dye concentration, $\theta$, in a pipe. When the horizontal length scale, L, of the dye is much greater than d, then

$$
\theta \simeq \bar{\theta}(x, t)
$$

where $\bar{\theta}$ is the vertically averaged dye concentration

$$
\bar{\theta} \equiv\left(\frac{1}{2 u}\right)_{i=d}^{d} \theta d z
$$

The approximation (2), which is correct to order $(d / L)$, is a consequence of rapid cross current mixing (i.e. the last term in (la) is bigger than all the others) and no flux boundary conditions. Taylor derived the following approximate evolution equation for $\bar{\theta}$ :

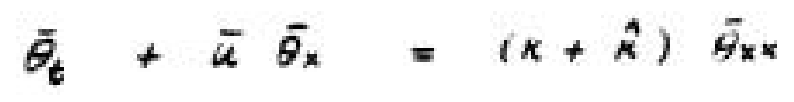

where $(\boldsymbol{k} \boldsymbol{t} \hat{\mathcal{R}})$ is the enhanced diffusivity, and

$$
\hat{k}=c J^{2} d^{\prime} / k
$$

where $\mathrm{C}$ is a dimensionless constant which depends on the shape of the velocity profile, $U$ is a scale estimate of the velocity, d is the width of the channel, 
and $K$ is the diffusivity (either molecular or turbulent eddy) which effects cross channel mixing. Equation (4) is simpler than (1) because one spatial dimension has been "projected" out of the problem.

The condition for validity of Taylor's theory is

$$
(U \alpha / k)(\alpha / i) \leqslant 1
$$

and it is vital to realize that it is possible to satisfy (6) and simultaneously have

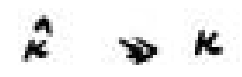

provided the aspect ratio, ( $d / L)$, is sufficiently small. Thus, from a mathematical point of view, Taylor's theory is an aspect ratio expansion and likewise in the dynamical problem treated here it is assumed that the compositional fields have horizontal length scales which vastly exceed the depth of the layer. As in the kinematic shear dispersion theory, the most immediate consequence of this disparity is that variations in $T$ and $S$ across the layer are much smaller than variations along the layer. Thus, if we define vertically averaged fields:

$$
\begin{aligned}
& \bar{T}=\left(\frac{1}{2 d}\right) \int_{-d}^{d} T d z \\
& \left.\bar{\xi}=\left(\frac{1}{2 d}\right)\right)_{-d}^{d} s d z
\end{aligned}
$$

then because $\kappa_{\boldsymbol{Y}} S_{x z}$ and $\kappa_{\tau} T_{x x}$ dominate all the other processes in the conservation equations for heat and salt:

$$
\begin{aligned}
& T \simeq \bar{T}(x, t) \\
& s \simeq \bar{s}(x, t)
\end{aligned}
$$

with errors of order d/L. Given $(9 a, b)$ it is very easy to calculate the pressure from the hydrostatic relation and then the horizontal velocity from the $x$-momentum equation. In this latter relation the small aspect ratio ensures that, as in lubrication theory, the dominant balance is between vertical viscosity and horizontal pressure gradients. One finds that

$$
\mu=\left(g x^{2}, \nu\right)\left(\bar{\rho}_{k}, \rho_{0}\right) P^{\prime}(z / d)
$$

where $P(S)$ is a fourth order polynomial. Given $u$, it is straightforward to use Taylor's theory to calculate the enhanced diffusivities for heat and salt. From (4) and (5)

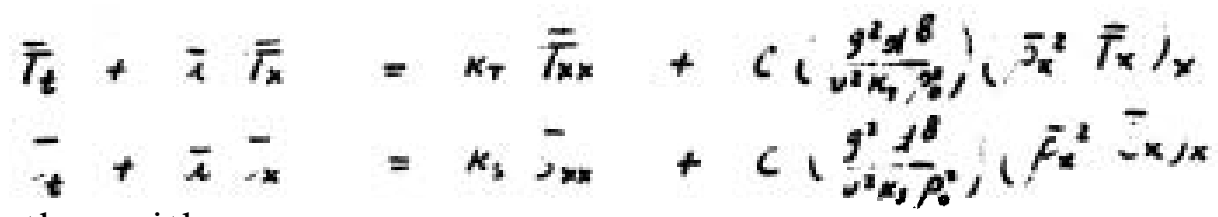

which together with

$$
\bar{k}=-x \bar{r}+\Delta \bar{r}
$$

is a closed set of nonlinear equations for $\overline{\mathbf{T}}(\mathbf{x}, t)$ and $\overline{\mathbf{s}}(\mathbf{x}, \mathbf{t})$. In (11) C is a dimensionless number. It is interesting to note that if the viscosity and 
diffusivities are nonconstant (i.e. depend on $z$ ), then one can still derive (11) and only the value of $C$ is different.

To summarize, compositional gradients produce a density driven shear flow, (10), which in turn shear-disperses the compositional properties. This is the source of the nonlinearity in (11).

The preceding derivation of (11) was informal and heuristic. Using the aspect ratio expansion technique of Childress and Spiegel (1981), the derivation can be formalized: one expands the fields in powers of $\varepsilon=d / L$. At zero order $(0)$ one obtains (9) and consideration of higher order terms eventually yields (11) as a solvability condition.

Now given (11) we turn to the task of obtaining some solutions which illustrate the effects of the nonlinear terms. Smith (1978 and 1982) has discussed solutions of a special case of (II) viz $\boldsymbol{K}_{\boldsymbol{s}}=\boldsymbol{K}_{\boldsymbol{T}}$ so that there is only one component. Suffice it to say that the techniques he used are reasonably effective and straightforward when applied to (11).

\section{REFERENCES}

Childress, S., and E.A. Spiegel, 1981. Variations of the solar constant. S. Sofia, ed., NASA Conference Publication 2191.

Erdogan, M.E., and P.C. Chatwin, 1967. The effects of curvature and buoyancy on the laminar dispersion of solute in a horizontal tube. Mech., 29, 465-484.

Ruddick, B.R., and G.S. Turner, 1979. The vertical length scale of double diffusive intrusions. Deep-Sea Res.. 26A, 903-913.

Smith, R., 1978. Asymptotic solutions of the Erdojan-Chatwin equation. J. Fluid Mech., 88, 323-337.

Smith, R., 1982. Similarity solutions of a nonlinear diffusion equation. IMA J. of Applied Math., 28, 149-160.

Taylor, G.I., 1953. Dispersion of soluble matter in solvent flowing slowly through a tube. Proc. Roy. Soc. A, 219, 186-203.

Taylor, G.I., 1954. The dispersion of matter in turbulent flow through a pipe. Proc. Roy. Soc. A, 223, 446-468. 
ON THE INTERACTION BETWEEN WIND AND THERMOHALINE FORCING IN SUBTROPICAL GYRES

\author{
Claes G. H. Rooth
}

The general problem of synergisms between wind stress, heating, and fresh water fluxes at the sea surface as forcing functions for the large-scale ocean circulation still eludes our understanding. The central difficulty lies in the apparent diversity and inhomogeneity of the turbulent mixing processes. Empirical bounds on the latter, based mainly on global transient tracer studies, suggest the existence of an intermediate time scale range, however, wherein the transient adjustment of the density fields in the main body of the deep oceans is achieved primarily by a mechanical adjustment (spin-up) process. This time scale range depends on the vertical scale of the transient process. For scale depths of a few hundred to a thousand meters it varies from several decades to a few centuries. These time scales are of special interest in the context of possible oceanic feedback on climate transients. There is, in addition, a substantial interest in understanding oceanic signatures of ongoing climatic change. This, in fact, is a major motivation for the study reported here.

There are two central precepts for this study. One is that while the main formation of dense water masses may involve air-sea interaction processes over vast areas of ocean surface, their actual sinking (and effective injection into the interior) occurs within quite limited and geographically well-defined regions. This implies that in most of the ocean volume the response can be considered as a far field effect of a virtual source-sink system in density space. The second precept is that the net changes in water mass volume distribution may be less informative of the process than the dynamic signatures of the adjustment. The archetypal case is the abyssal source problem originally analyzed by stommel and brons (1960) (SA, hereafter). While questionable as a model of the steady-state abyssal circulation because of the demonstrable inhomogeneity of the interior mixing processes, the SA theory should apply very well in the circumstances described above. If one considers a water mass transformation transient where the scale height is between one half and one kilometer, then it is easily seen that, for an Atlantic-size basin, the cross-basin slope induced is about $100 \mathrm{~m}$ for a source-sink pair strength of one Sverdrup (Megaton/second). This implies that the dynamic baroclinic signature dominates or is comparable to the mean height change for several decades. A climatic monitoring strategy aimed at diagnosing large-scale transients in water mass transformation characterstics should therefore concern itself to a substantial degree with the decadal average cross basin thermocline slope.

Now, the primary limitation to the SA theory associated with inertial effects is associated with its dependence on the adequacy of Beta as an estimator for the large-scale potential vorticity gradient in the water masses involved. Recent observations as well as numerical experiments tend to support the theoretically based suggestion that the main wind-driven gyres should include deep domains of substantially weakened gradients, or even of approximate homogeneity in the linearized potential vorticity ( $\mathrm{f} / \mathrm{h}$, where $\mathrm{h}$ is the layer thickness associated with a specific inteval in potential density). Such domains cannot support the SA adjustment process without a first order modification in the induced motion fields. Numerical experiments to explore the character of such deviations were conducted at NCAR in cooperation with W.R. Holland, using an existing and well-documented eddy resolving gyre domain 
circulation model (quasigeostrophic). A reference case has been integrated by Holland (1983) to a state of approximate statistical equilibrium. This case involved an antisymmetric wind stress pattern relative to the zonal (E-W) center line. It produces a well-defined separated jet along the latter, in the mean, with a strongly concentrated eddy energy distribution. The vertical structure is represented by three layers of 300, 700, and $4000 \mathrm{M}$ depth, respectively. We perturbed the forcing of this model by imposing a vertical velocity at the interface at $1000 \mathrm{M}$ mean depth with initial conditions given by the final state of the wind-driven run. A number of forcing configurations and intensities were run. The overall results suggest that the gyre separation process for a strongly eddying free jet is strongly sensitive to the combined forcing of the two upper layers, but insensitive to the divergence imposed in the deep lower layer. Specifically, we found that the separation point could be "pulled" latitudinally by the imposed divergence in the second layer, with retention of the well-defined structure of the mean separated jet, as long as the latitudinal displacement was comparable to the width of the region of high eddy kinetic energy. Increased forcing intensity lead to a destruction of the well-defined mean jet in the interior, and to a strongly vacillating dynamics in the separation region, as indicated by the eddy field intensity. Further experiments and, above all, analytical studies are needed before these phenomena can be considered understood. It is clear already, though, that one has to be suspicious about the relevance of many efforts to refine, by inclusion of higher order mechanical processes, the agreement between purely wind-driven models and observations of the western boundary current separation process.

\section{REFERENCES}

Holland, W.R., 1983. The role of mesoscale eddies in the general circulation of the ocean-numerical experiments using a quasi-geostrophic model. Jour. of Phys. Oceanogr. 8, 363-392.

Stommel, H., and A.B. Arons, 1960. On the abyssal circulation of the world ocean. II. An idealized model of the circulation pattern and amplitude in oceanic basin. Deep-sea Res. 6: 217-233.

Holland, W. R., 1983. Personal communication. 


\title{
RESTRAINING BAROCLINIC INSTABILITY: SOME EFFECTS OF HORZONTAL SHEAR
}

\author{
Stephen P. Meacham
}

In the theory of baroclinic instability, nonlinearity is important as a mechanism for curtailing the unbounded growth of an unstable wave that linear theory alone would predict. It also affords a route for the transfer of energy between the length scales of the unstable linear perturbations and other length scales. By using multiple time-scale techniques, one can investigate the finite-amplitude dynamics of a slowly growing wave analytically.

In this fashion Pedlosky (1970) has examined the weakly non-linear evolution of baroclinic instability in a two-layer model (e.g. Phillips, 1954). In this example, the potential vorticity gradient of the basic state is independent of the horizontal coordinates. A wave/mean flow interaction equilibrates the unstable wave whose amplitude then exhibits a simple oscillation on a long time-scale comparable to the e-folding time of the linear instability. One can show that it is possible for the unstable wave to interact with neutral Rossby waves through a resonant triad interaction on a similar time scale. The amplitude scale that the initially linear unstable wave must reach before either the wave/mean flow or the wave/wave interaction manifests itself is the same for each interaction. One can think of the two mechanisms as being equally powerful in this example.

The work described here differs from the example considered by Pedlosky (1970) in that it looks a the same two-layer, zonal channel model but includes meridionally varying topography so that the potential vorticity gradient in the lower layer is now a function of the cross-channel (meridional) coordinate. The model is configured so that the basic potential vorticity gradient in the upper layer is uniform and positive while the potential vorticity gradient in the lower layer, $\Pi_{2 Y}$, has a minimum in the middle of the channel, $\left(T_{2 Y}\right)_{m} 1_{n}$, say. When the planetary vorticity gradient, $\boldsymbol{\beta}$, is chosen so that $\left(\pi_{2 Y}\right)_{\min }=0$, the basic flow is critical. Decreasing $\beta$ below its critical value, $\beta_{\mathrm{c}}$, to $=\beta_{\mathrm{c}}-\Delta$ results in a basic state in which $\pi_{2 Y}$ is negative in a region around the mid-line of the channel. When $0<\boldsymbol{A}<\mathbf{1}$, this region is narrow, of width $0(\Delta 1 / 2)$. The flow now satisfies the integral conditions necessary for instability, and one can indeed find an unstable mode by numerical procedures. For small values of the supercriticality parameter, ${ }^{*}$, the unstable modes can also be found analytically.

The growth rate of the slightly supercritical instabililty is small, $\mathrm{O}\left(\mathrm{A}^{2}\right)$, when compared to that of the meridionally homogeneous Phillips model (which is $0\left(\Delta^{1 / 2}\right)$, with $\Delta$ similarly defined). The unstable eigenfunction exhibits an internal layer at the mid-line of the channel within which it varies on an $0\left(\Delta^{1 / 2}\right)$ length scale. Over the remainder of the channel width the meridional scale is $0(1)$. The stream function in the lower layer is much weaker than that in upper layer, being $O(\Delta)$ in the internal layer and $0\left(\Delta^{2}\right)$ elsewhere. The range of unstable wavenumbers is only $0\left(\Delta^{3 / 2}\right)$ in width.

The internal layer structure of the linear solution is crucial to the nonlinear dynamics of weakly supercritical wave which differ from those of the meridionally homogeneous case by Pedlosky (1970). The principal effect of 
nonlinearity is to modify this internal layer. Analytically it can be shown that wave/wave interactions of the resonant triad type (unstable wave and two neutral Rossby waves) are potentially a more effective nonlinear mechanism than wave/mean flow interaction in that the unstable wave can be modified by triad interactions at a smaller amplitude, $O\left({ }^{2}\right)$, than that at which wave/mean flow interactions would be felt.

A numerical investigation of the 3-wave problem is in its infancy but has demonstrated that it is possible for a pair of side-bands to halt the growth of the unstable wave.

\section{REFERENCES}

Pedlosky, J., 1970. Finite amplitude baroclinic waves. J. Atmos. Sci., 27, $15-30$.

Phillips, N.A., 1954. Energy transformations and meridional circulation associated with simple baroclinic waves in a two-level quasigeostrophic model. Tellus, 6, 273-286.

THE INTERACTION OF THE WINTER BERING SEA ICE EDGE WITH AN OCEANIC FRONT

\section{Seelye Martin}

A winter experiment called MIZEX WEST conducted during February 1983 at the Bering Sea ice edge studied the interaction of the edge with an Oceanic front. The experiment was carried out from the NOAA ship DISCOVERER and the Coast Guard icebreaker WESTWIND. In the experiment, the WESTWIND went into the ice interior, deployed a series of twelve satellite- and radar-tracked buoys on scales of 1 to $40 \mathrm{~km}$, then drifted under the influence of strong northeast winds out toward the ice edge, where the DISCOVERER carried out a series of CTD and ice drift observations. Analysis of the motion of buoy pairs shows that the ice motion toward the edge divides into two parts: a near solid-body motion, followed by a transition to strong variable motion as the ice floes move over the oceanic front. Analysis of the data suggests that the ice over th front diffuses in a similar non-Fickian manner to dye patch diffusion in the temperate ocean. The data suggests that the ice melting maintains the front, while the instabilities in the geostrophic flow associated with the front cause the ice diffusion, which in turn contributes to the melting and the front maintenance. 


\title{
LECTURES OF FEUOWS
}

\section{THEORY AND EXPERIMENT OF ISOLATED BAROCLINIC VORTICES}

\author{
Mathieu Mory
}

Long life time eddies have been the object of intense research in past years. Numerous examples have been pointed out by oceanographers or astrophysicists, for instance warm core rings in the ocean or the red spot on Jupiter. These eddies show a very coherent and permanent structure. They can persist during months (warm core rings) or centuries (red spot). An explanation of this persistence has been given by analyzing energy transfer between the mean shear flow and the eddy. It is obvious however that the $\boldsymbol{B}$ effect will play a key rule in the dynamics of these eddies. Any vortex will radiate energy through Rossby waves. Nevertheless, under particular circumstances, solutions for isolated eddies are found, which do not radiate (Flierl, 1983; Flierl et al, 1980; Flierl et al., 1983). Such solutions are known for barotropic and baroclinic flows.

The present paper is an attempt to describe theoretically and experimentally isolated solutions for a baroclinic vortex on a half beta plane. This work was suggested by a previous experiment of Griffiths (1983). Griffiths's experiment is conducted in a tank with a parabolic shape at the bottom, on which a gravity current is released. The formation of vortices is observed behind the nose of the current. Griffiths argues that these vortices are due to Kelvin Helmholtz instability at the interface of the boundary current in a similar way as in Griffiths and Hopfinger's (1983) study of gravity currents moving along a lateral boundary. The vortices are very intense and they break up the current into a series of intense baroclinic eddies. However, Griffiths's experiment shows very complicated features. It is highly desirable to simplify it. A straightforward simplification is to replace the parabolic bottom by a linear slope and this paper discusses this case.

In this more simple framework, replacing the parabolic slope by a linear one, Stern (private communication) found an integral relation which suggested some analogy with previous work of Flierlet al. (1983), and Flierl (1983). Detailed theoretical solutions, using different scalings are presented here, which show the existence of isolated baroclinic vortices on a linear slope. The solutions are described and compared with experimental observations.

Another important approach to baroclinic vortices is the study of their stability. Although stability studies are not the purpose of this paper, this approach cannot be neglected if one wishes to carry out a tractable experiment. Experimental studies of the stability of baroclinic vortices have been done by Saunders (1973); Griffiths and Linden (1982). They do not involve the $\beta$ effect, but they provide an interesting framework for the experiment.

This paper will address three different points. First the framework is presented and Stern's integral relation is demonstrated. In the second section solutions for baroclinic isolated eddies are derived. The third part describes the experiment and presents its results. Comparison with theoretical solutions is provided and the discussion of the baroclinic stability of the eddy is addressed. 
1. Framework and Stern's Integral Relation

The basic idea of the experiment consists of a flow over a linear slope bottom (see Figure 1).
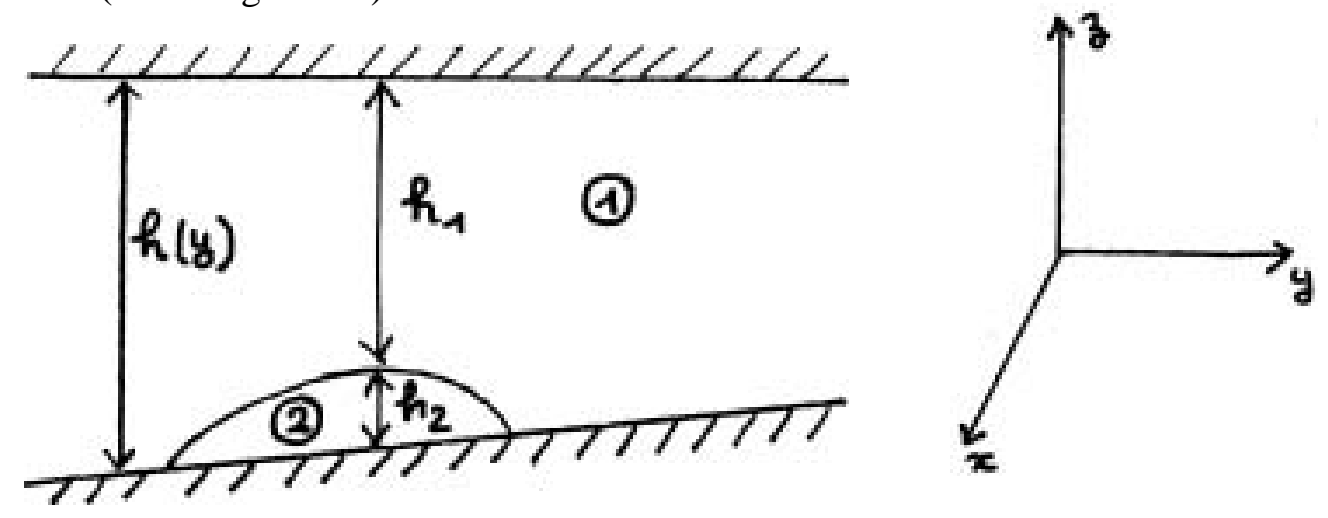

Figure 1

The top surface can be either free or limited by a rigid lid. The model is a two-layer baroclinic one. The lower layer (denoted 2) is slightly denser than the upper layer (denoted 1 ). Only those cases for which the lower layer is finite in space are considered in this paper. It consists therefore in a heavy dome over a slope.

With the system of coordinates drawn on Figure $1, h(y)$ designates the depth of the whole fluid, and $h_{1}(x, y), h_{2}(x, y)$ are respectively the depths of layer 1 and layer 2

$$
\text { then } \quad h(y)=h_{1}(x, y)+h_{2}(x, y)
$$

The shallow water equations are written for both layers, using the Boussinesq approximation

$$
\begin{aligned}
& \frac{\partial}{\partial t} \underline{v}_{1}+\left(\underline{v}_{1} \cdot \nabla\right) \underline{v}_{1}+f \underline{k} \times \underline{v}_{1}=-\nabla \frac{\rho_{1}}{\rho} \\
& \frac{\partial}{\partial t} \underline{v}+\left(\underline{v}_{2} \cdot \nabla\right) \underline{v}_{2}+f \underline{k} k \underline{v}_{2}=-\nabla \frac{\rho_{2}}{\rho}
\end{aligned}
$$

$\boldsymbol{\rho}$ is the mean density and $f$ the Coriolis parameter. The pressures are supposed hydrostatic and are therefore related by

$$
\nabla_{\mathrm{P}_{2}}=\nabla_{\mathrm{P}_{1}}-\mathrm{g}^{\prime} \nabla_{\mathrm{h}_{1}}
$$

where $g^{\prime}$ is the reduced gravity: $g^{\prime}=g \Delta \xi / \xi$

Two final equations come out of the continuity equation for each layer

$$
\begin{aligned}
& \frac{\partial}{\partial t} h_{1}+\nabla \cdot\left(\underline{v}_{1} h_{1}\right)=0 \\
& \frac{\partial}{\partial t} h_{2}+\nabla \cdot\left(\underline{v}_{2} h_{2}\right)=0
\end{aligned}
$$


By adding Equations (5) and (6), and since $\frac{\partial}{\partial t} h=0$, we obtain

$$
\nabla .\left(\underline{v}_{1} h_{1}+\underline{v}_{2} h_{2}\right)=0
$$

The equation ( 7 ) assures the existence of a function $\psi$ given by

$$
\underline{\mathrm{v}}_{1} \mathrm{~h}_{1}+\underline{\mathrm{v}}_{2} \mathrm{~h}_{2}=\underline{\mathrm{K}} \times \nabla \psi
$$

This paper considers isolated solutions of that problem, 1.e. $\psi$ and its first and second derivatives decrease toward zero far away from the dome. This only supposes that velocities, vorticity and circulation are zero at infinity. After integrating (8) over the plane $(x, y)$, we get

$$
\iint_{-\infty}^{+\infty}\left(\underline{v}_{1} h_{1}+\underline{v}_{2} h_{2}\right) d x d y=0
$$

As a convention we write the integration of $\underline{v}_{2} \mathbf{h}_{2}$ over the whole plane instead of integration over the dome. This is valid since $h_{2}=0$ outside the dome. Therefore equation (9) expresses that the total linear momentum is zero.

The evolution equation for the total momentum is obtained after multiplying equations (5) and (6) by $h_{1}$ and $h_{2}$ respectively, integrating over respective domains, and adding both integral equations. The resultant equation has three terms. The integrated Coriolis term

$$
f \underline{k} \times \iint_{-\infty}^{+\infty}\left(\underline{v}_{1} h_{1}+\underline{v}_{2} h_{2}\right) d x d y
$$

vanishes because of equation (9). Next we have the nonlinear term

$$
\iint_{-\infty}^{+\infty}\left\{h_{1} \frac{\partial}{\partial t} \underline{v}_{1}+h_{2} \frac{\partial}{\partial t} \underline{v}_{2}+h_{1}: \underline{v}_{-1} \nabla \mid \underline{v}_{-1}+h_{2}\left(v_{-2} \nabla\right) \underline{v}_{2}\right\} d x d y
$$

After introducing equations (5) and (6) it becomes

$$
\iint_{-\infty}^{+\infty}\left\{\frac{\partial}{\partial t}\left(\underline{v}_{1} h_{1}+\underline{v}_{2} h_{2}\right)+\nabla \cdot\left(h_{1} \underline{v}_{1} \otimes \underline{v}_{1}+h_{2} \underline{v}_{2} \otimes \underline{v}_{2}\right)\right\} d x d y
$$

The time derivative here may be taken outside the integral since $\underline{v}_{1}=0 \mathrm{far}$ away from the dome and $\mathrm{h}_{2}=0$ on dome perimeter. It is zero as aconsequence of (9). The remaining nonlinear terms represent a flux far away from the dome, and therefore vanishes for an isolated flow. Finally we have the integrated pressure term

$$
\iint_{-\infty}^{+\infty}\left(-\frac{\nabla p_{1}}{\rho} h_{1}-\frac{\nabla p_{2}}{\rho} h_{2}\right) d x d y
$$

which also must vanish since we have shown that the Coriolis and nonlinear terms are zerofor an isolated flow. Using hydrostatic relation (4), we obtain

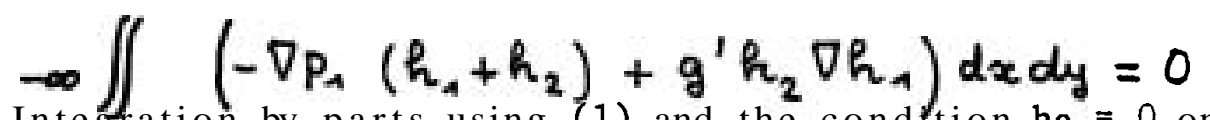

Integration by parts using (1) and the condfion $h_{2}=0$ on the perimeter of the dome leads to the simple form:

$$
\iint_{-\infty}^{+\infty}\left(p_{1}+g^{\prime} h_{2}\right) \nabla h \quad d x d y=0
$$


where $p_{1}$ is the departure from a reference pressure far away from the dome, which is taken to be zero. For a linear slope $\boldsymbol{\nabla} \mathrm{h}=\mathrm{cte}$ and

$$
\iint_{-\infty}^{+\infty}\left(P_{1}+g^{\prime} h_{2}\right) d x d y=0
$$

Two physical remarks may be noted about this integral theorem. First it should be pointed out that the integral theorem is a hydrostatic relation which expresses a "mean" static equilibrium of the gravity dome on the slope. The buoyancy force is balanced by the integrated pressure forces at the interface.

It could also seem surprising that the $\boldsymbol{\beta}$ effect does not appear in the theorem. However, this effect is present. In an analogy with a solid body on the slope, this body will either move uphill or downhill if the balance between buoyancy and pressure forces is not realized. And the guess is that there is no way for the dome to change its latitude without radiating Rossby waves.

\section{Theoretical Models}

We presented in the previous section the basic equations describing the evolution of baroclinic flows. In what follows we will only look for isolated solutions, i.e. solutions going to zero at large distance. Furthermore the radial flux of momentum also has to vanish far away from the center of the eddy, so that the vortex is not radiating.

Solutions in other cases have been found, and the mathematical framework is now well known. It is more difficult to provide a scaling which is relevant to the process. Physical arguments also have to be discussed to infer realistic potential vorticity distribution. However, the model will be an inviscid one.

\section{$\underline{2.1 \quad \text { The scaling }}$}

In the upper and lower layers the horizontal velocities are scaled by $V_{1}$ and $V_{2}$ respectively. The same horizontal length scale $L$ is assumed for both layers which as a convention gives a measure of the radius of the eddy. The scale for the pressure is given to be of the same order of magnitude as the Coriolis force, an assumption which is consistent with the geostrophic approximation.

The depth $h$ is scaled by $H$ and $h_{1}$ and $h_{2}$ by $(1-\delta) H$ and $\delta H$ respectively. Finally the time scale is given to be consistent with the speed of topographic Rossby waves which scales as $F \operatorname{tg} \mathbf{L}^{\mathbf{2}} / \mathbf{H}$. Again $f$ is the Coriolis parameter and $\alpha$ is the angle of the slope with the horizontal plane.

The scaling is summarized in Table 1 . 


\begin{tabular}{lll}
\hline$(\mathrm{x}, \mathrm{y}) \sim \mathrm{L}$ & $\left(\mathrm{u}_{1}, \mathrm{v}_{1}\right) \sim \mathrm{v}_{1}$ & $\mathrm{P}_{1} \sim \rho^{\mathrm{fLV}}$ \\
$\mathrm{h} \sim \mathrm{H}$ & $\left(\mathrm{u}_{2}, \mathrm{v}_{2}\right) \sim \mathrm{v}_{2}$ & $\mathrm{P}_{2} \sim \rho \mathrm{fLV}_{2}$ \\
$\mathrm{~h}_{1} \sim(1-\delta) \mathrm{H}$ & $\mathrm{t} \sim \mathrm{H} / \mathrm{L} \operatorname{tg} \alpha \mathrm{f}$ & \\
$\mathrm{h}_{2} \sim 6 \mathrm{H}$ & & \\
\hline
\end{tabular}

\section{TABLE 1}

The equations are written for each layer in a dimensionless form upper layer

$$
\begin{gathered}
\delta^{\prime} \frac{\partial}{\mathrm{at}} \underline{v}_{1}+\varepsilon_{1}\left(\underline{v}_{1} \cdot \nabla\right) \underline{v}_{1}+\underline{k} \times \underline{v}_{1}=-\nabla p_{1} \\
\delta^{\prime} \frac{\partial}{\partial t} h_{1}+\varepsilon_{1} \nabla \cdot\left(\underline{v}_{1} h_{1}\right)=0
\end{gathered}
$$

lower layer

$$
\begin{gathered}
\delta^{\prime} \frac{\partial}{\partial t} \underline{v}_{2}+\epsilon_{2}\left(\underline{v}_{2} \cdot \nabla\right) \underline{v}_{2}+\underline{k} \times \underline{v}_{2}=-\nabla p_{2} \\
\delta^{\prime} \frac{\partial}{\partial t} h_{2}+\epsilon_{2} \nabla \cdot\left(\underline{v}_{2} h_{2}\right)=0
\end{gathered}
$$

Later on, Equation (1) and the hydrostatic Equation (4) become

$$
\begin{aligned}
& 1-\delta_{\mathrm{y}}=(1-\delta) \mathrm{h}_{1}+\delta \mathrm{h}_{2} \\
& \varepsilon_{2} \nabla_{\mathrm{P}_{2}}=\varepsilon_{1} \nabla_{\mathrm{P}_{1}}+\mathrm{F} \delta \cdot \nabla \mathrm{y}+\mathrm{F} \delta \nabla_{\mathrm{h}_{2}}
\end{aligned}
$$

We introduce four dimensionless parameters:

$\underline{\text { the Rossby number for each layer }} \quad \varepsilon_{1}-\frac{V_{2}}{F L} \quad \varepsilon_{2}=\frac{V_{2}}{F L}$

$$
\begin{array}{ll}
\text { the Froude number } & F=\frac{g^{\prime} H}{F^{2} L^{2}} \\
\text { the slope parameter } & \delta^{\prime}=t^{\prime} \propto L / H
\end{array}
$$

and $\boldsymbol{\delta}$ measures the relative depth of the lower layer. In the following, the Froude number is taken as $0(1)$ and the slope parameter $\delta^{\prime}$ is $\ll 1$. For $\boldsymbol{\sigma}$ and the Rossby numbers $\boldsymbol{E}_{1}$ and $\varepsilon_{2}$, two cases are considered:

\section{$1-E_{1} \sim E_{2} \sim \delta \sim \delta^{\prime} \quad$ and all these parameters are} supposed $\ll 1$. The model is therefore a geostrophic model for each layer.

$$
\text { 2- } E_{1} \sim \delta=0(1) \text { and } E_{2} \sim \delta^{\prime} \ll 1
$$

The model is geostrophic for the lower layer but not for the upper layer. 


\subsection{The geostrophic model $\quad E_{1} \sim \varepsilon_{2} \sim \delta \sim \delta^{\prime} \ll 1$}

The stream functions $\psi_{1}$ and $\psi_{2}$ for layer 1 and 2 are given by the respective pressure in each layer. For simplicity, $\boldsymbol{E}_{1}$ and $\boldsymbol{E}_{2}$ are taken as equal and the labels are omitted.

Then the velocity field is determined from

$$
\begin{array}{lll}
u_{1}=-\psi_{1 y} & v_{1}=\psi_{1 x} & \psi_{1}=P_{1} \\
u_{2}=-\psi_{2 y} & v_{2}=\psi_{2 x} & \psi_{2}=P_{2}
\end{array}
$$

vorticit $\Psi_{1}$ and $\Psi_{2}$ satisfy the quasigeostrophic equations for potential

$$
\begin{aligned}
& \left\{\delta^{\prime} \frac{\partial}{\partial t}-\varepsilon \Psi_{1 y} \frac{\partial}{\partial x}+\varepsilon \Psi_{1 x} \frac{\partial}{\partial y}\right\}\left\{\nabla^{2} \Psi_{1}+\frac{\delta^{\prime}}{\varepsilon} y+\frac{\delta}{\varepsilon} h_{2}\right\}=0 \\
& \left\{\delta^{\prime} \frac{\partial}{\partial t}-\varepsilon \Psi_{2 y} \frac{\partial}{\partial x}+\varepsilon \Psi_{2 x} \frac{\partial}{\partial y}\right\}\left\{\nabla^{2} \Psi_{2}-\frac{\delta}{\varepsilon} R_{2}\right\}=0
\end{aligned}
$$

Equations (18) and (19) are easily integrated for solutions with uniform translation at speed $c$ along the $x$ axis, i.e. translating east-west.

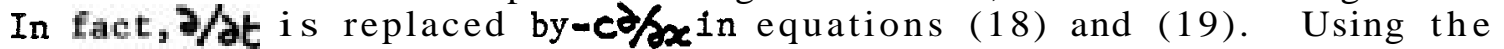
Jacobian $\mathbf{J}$, equations (18) and (19) are now written

$$
\begin{aligned}
& J\left(\varepsilon \Psi_{1}+c \delta^{\prime} y, \nabla^{2} \Psi_{1}+\frac{\delta^{\prime}}{\varepsilon} y+\frac{\delta}{\varepsilon} h_{2}\right)=0 \\
& J\left(\varepsilon \Psi_{2}+c \delta^{\prime} y, \nabla^{2} \Psi_{2}-\frac{\delta}{\varepsilon} h_{2}\right)=0
\end{aligned}
$$

which are easily integrated in

and

$$
\begin{aligned}
& \nabla^{2} \psi_{1}+\frac{\delta^{\prime}}{\varepsilon} y+\frac{\delta}{\varepsilon} h_{2}=G\left(\varepsilon \psi_{1}+c \delta^{\prime} y\right) \\
& \nabla^{2} \psi_{2}-\frac{\delta}{\varepsilon} h_{2}=H\left(\varepsilon \psi_{2}+c \delta^{\prime} y\right)
\end{aligned}
$$

$\mathrm{G}$ and $\mathrm{H}$ are unknown functions, which are determined from physical arguments.

It is beyond the scope of this paper to carry out the whole analysis of this problem. In particular we will only consider solutions translating to the west $(c<0)$. It can be shown that there is no isolated eastward translating solution. A justification of the non-existence of eastward translating eddies will come out of the analysis for westward translating eddies. 
Moreoever, we will also only consider axisymmetric solutions. Asymmetric motions of modon type (i.e. of the form $\varphi(r) \sin \theta$ ) are shown to be necessarily of 0 amplitude. Our solution will be derived in a cylindrical coordinate system. Since all equations are dimensionless, it is valid to impose the further condition:

$$
h_{2}(r)=0 \quad \text { for } \quad r>1
$$

From considering equations (22) and (23) it is obvious that two domains have to be considered in the $(x, y)$ plane whether $h_{2}=0$ or $h_{2} \neq 0$. In other words, two solutions are expressed for the upper layer, one in the domain $r<1$ and the other for $r>1$.

Necessarily, the dome perimeter $\left(h_{2}=0, r=1\right)$ is a streamline in the moving frame for the lower layer. Because of the $\boldsymbol{\beta}$ effect, it comes out of the hydrostatic equation (16) that the dome perimeter cannot be a streamline for the upper layer. Fluid moves from outside the eddy throughout it. Potential vorticity conservation implies therefore that the shape of the function $G$ is the same for $r>1$ and $r<1$. G is determined by considering the motion outside the eddy. Since for $x \rightarrow \pm \infty, \Psi_{1} \rightarrow 0$ and $\nabla^{2} \Psi_{1} \rightarrow 0$, the only possible form for $G$ is $G(Z)=Z / c \varepsilon$ : (22) then becomes

$$
\nabla^{2} \psi_{1}-\frac{\psi_{1}}{c}=-\frac{\delta}{\varepsilon} h_{2}
$$

For $r>1 \quad h_{2} \equiv 0$ and an axisymmetric solution for (25) is

$$
\Psi_{1}(n)=A J_{0}\left(\sqrt{\frac{-T}{C}} n\right)
$$

The nonradiating condition imposes $\mathbf{A}=0$ and then

$$
\psi_{1} \equiv 0 \quad \text { for } r ; 1
$$

The motion for $\mathrm{r}<1$ is the solution of (25) with the boundary conditions

$$
\psi_{1}=0 \quad \psi_{1 n}=0 \quad \text { at } r=1
$$

which ensures the continuity of pressure across the line $\mathbf{r}=\mathbf{1}$ and also that the fluid crossing this line will not support a discontinuity in velocity.

The form for the unknown function H (Eq. (23)) is not uniquely determined as for G. The simplest way is to suppose $\mathrm{H}=\mathrm{P}$ where $\mathrm{P}$ is constant. This could be physically justified by noting that mixing inside the dome would homogenize the potential vorticity.

The hydrostatic equation (16) is integrated as

$$
\varepsilon \Psi_{2}=\varepsilon \Psi_{1}+F S^{\prime} y+F \zeta h_{2}+\text { const }
$$

The line $\mathbf{r}=1$ is a stream line in the moving frame for the lower layer.

$$
\varepsilon \Psi_{2}+c \delta^{\prime} y=\text { const } \quad \text { on } r=1
$$


Since $h_{2}=0$ on that line and because of (27) it comes from equation (28)

$$
\mathbf{c}=-\mathbf{F}
$$

As mentioned above, this justifies the nonexistence of eastward propagating eddies, at least with this simple geometry.

The whole set of equations to be solved may be summarized as follows

$$
\begin{array}{ll}
\nabla^{2} \Psi_{1}+\frac{\Psi_{1}}{F}=-\frac{S}{\varepsilon} h_{2} & \\
\nabla^{2} \Psi_{2}-\frac{\delta}{\varepsilon} h_{2}=P & \text { for } r \leqslant 1 \\
\varepsilon \psi_{2}=\varepsilon \psi_{1}+F^{\prime} y+F S h_{2} &
\end{array}
$$

with the boundary conditions

$$
\begin{aligned}
& \Psi_{1}=0 \quad \Psi_{1 n}=0 \\
& \varepsilon \Psi_{2}-F \delta^{\prime} y=0 \\
& h_{2}=0
\end{aligned}
$$

The system (31), (32), (33) is linear and we get a solution for it which matches the boundary conditions (27), (29), (24). The solution is expressed

$$
\begin{aligned}
& \psi_{1}(x)=\frac{P F^{2}}{\sqrt{5}}\left\{\lambda_{+}\left[1-\frac{J_{0}\left(\sqrt{\lambda_{-}} r\right)}{J_{0}\left(\sqrt{\lambda_{-}}\right)}\right]+\lambda_{-}\left[1-\frac{I_{0}\left(\sqrt{\lambda_{+}} x\right)}{I_{0}\left(\sqrt{\lambda_{+}}\right)}\right]\right\} \\
& \Psi_{2}(r)=\frac{P F}{\sqrt{5}}\left\{\frac{J_{0}\left(\sqrt{\lambda_{-}} x\right)}{J_{0}\left(\sqrt{\lambda_{-}}\right)}-\frac{I_{0}\left(\sqrt{\lambda_{+}} x\right)}{I_{0}\left(\sqrt{\lambda_{+}}\right)}\right\}+\frac{F \delta^{\prime}}{\varepsilon} \sin \theta \\
& h_{2}(r)=-\frac{P \varepsilon}{\sqrt{5} \delta}\left\{\lambda_{+}\left[1-\frac{I_{0}\left(\sqrt{\lambda_{+}} r\right)}{I_{0}\left(\sqrt{\lambda_{+}}\right)}\right]+\lambda_{-}\left[1-\frac{J_{0}(\sqrt{\lambda} r)}{J_{0}\left(\sqrt{\lambda_{-}}\right)}\right]\right\} \\
& \text {with } \\
& \lambda_{+}=\sqrt{5}+1 \quad \lambda_{-}=\frac{\sqrt{5}-1}{2 F} \\
& \text { 2F }
\end{aligned}
$$

The condition (27) leads to the determination of the Froude number by solving numerically the following equation

$$
\begin{gathered}
\sqrt{\lambda_{+}} \frac{J_{1}\left(\sqrt{\lambda_{-}}\right)}{J_{0}\left(\sqrt{\lambda_{-}}\right)}-\sqrt{\lambda_{-}} \frac{I_{1}\left(\sqrt{\lambda_{+}}\right)}{I_{0}\left(\sqrt{\lambda_{+}}\right)}=0 \\
F=0.032
\end{gathered}
$$

Then 
The condition (27) is strictly equivalent to the fact that the integral relation (10) has to be satisfied.

The scaling imposes two other conditions, namely $\mathrm{h}_{2}(0)=\mathbf{1}$ and

which lead numerically to

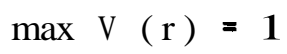

r

and

$$
\begin{aligned}
& P=1.04 \\
& \frac{\varepsilon}{\delta}=0.53
\end{aligned}
$$

We will not discuss here in detail this solution. This will be done in Section 3, where the theoretical solutions will be compared to experimental observations. We note, however, that the scaling gives a consistent solution. In fact, $\mathrm{P}$ is $\mathbf{O}(1)$ and $\boldsymbol{E}$ and $\boldsymbol{\delta}$ are of the same order. The model predicts large scales since the Froude number is small.

The azimuthal velocities in the upper and lower layers as well as the depth $h_{2}$ of the heavy dome are plotted in Figure 2. The motion is cyclonic above the dome and mostly anticyclonic inside.

Figure $3 a$ and $3 b$ show the mapping of the streamlines in upper and lower layers respectively. The frame of reference is the frame translating with the eddy.

\subsection{Non-geostrophic model $\quad \varepsilon_{\boldsymbol{1}} \sim \boldsymbol{\delta}=0(\mathbf{1})$}

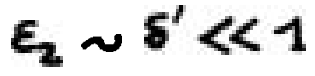

In fact this model is geostrophic for one part. Since $\boldsymbol{E}_{2} \ll \mathbf{1}$ the geostrophic assumption is still valid in the lower layer.

Similarities exist between this model and Flierl's model for warm core rings. However, although the mathematics are similar in both formulations, the physics in our model is quite different than in Flierl's theory. For warm core rings, the vortex (for which $\boldsymbol{E}_{1} \boldsymbol{\sim} 1$ ) is contained in a region of finite volume. In our case, the upper layer, where we look for an ageostrophic solution, extends radially to infinity. As we shall see later, this imposes strong conditions.

The formulation for the lower layer is close to the presentation of the previous subsection. The geostrophic approximation is used and velocities inside the dome are determined by the streamfunction $\boldsymbol{\psi}_{2}$ (equal to the pressure $\mathbf{p}_{2}$ ) as in Equation (17). $\Psi_{2}$ satisfies the quasigeostrophic equation (19) which is simplified to lowest order in

$$
\left\{\delta^{\prime} \frac{\partial}{\partial t}-\varepsilon_{2} \Psi_{2 y} \frac{\partial}{\partial x}+\varepsilon_{2} \Psi_{2 x} \frac{\partial}{\partial y}\right\} h_{2}=0
$$

As done in 2.3, integration of this equation is easily performed for westward translating eddies. We get

$$
\varepsilon_{2} \Psi_{2}+c \delta^{\prime} y=H\left(h_{2}\right)
$$

There are few guidelines for choosing for $\mathrm{H}$. If $\mathrm{H}$ is a constant, the motion of the heavy dome is a uniform westward translation at speed c. If $\mathrm{H}$ is taken 


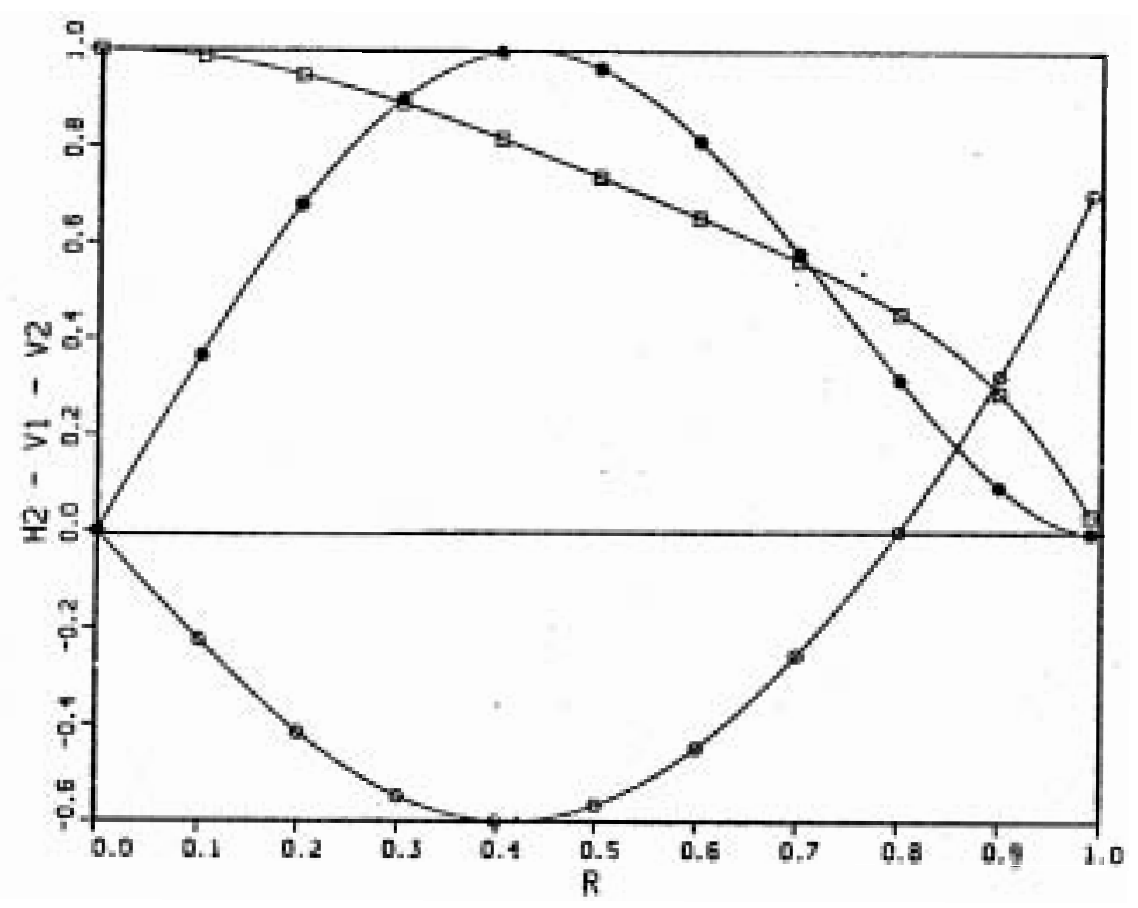

Eigure 2: Plot of the velocities $v_{4}(r) \rightarrow-, v_{2}(r) \bullet-$
and of the shape of the dome $h_{2}(r)$
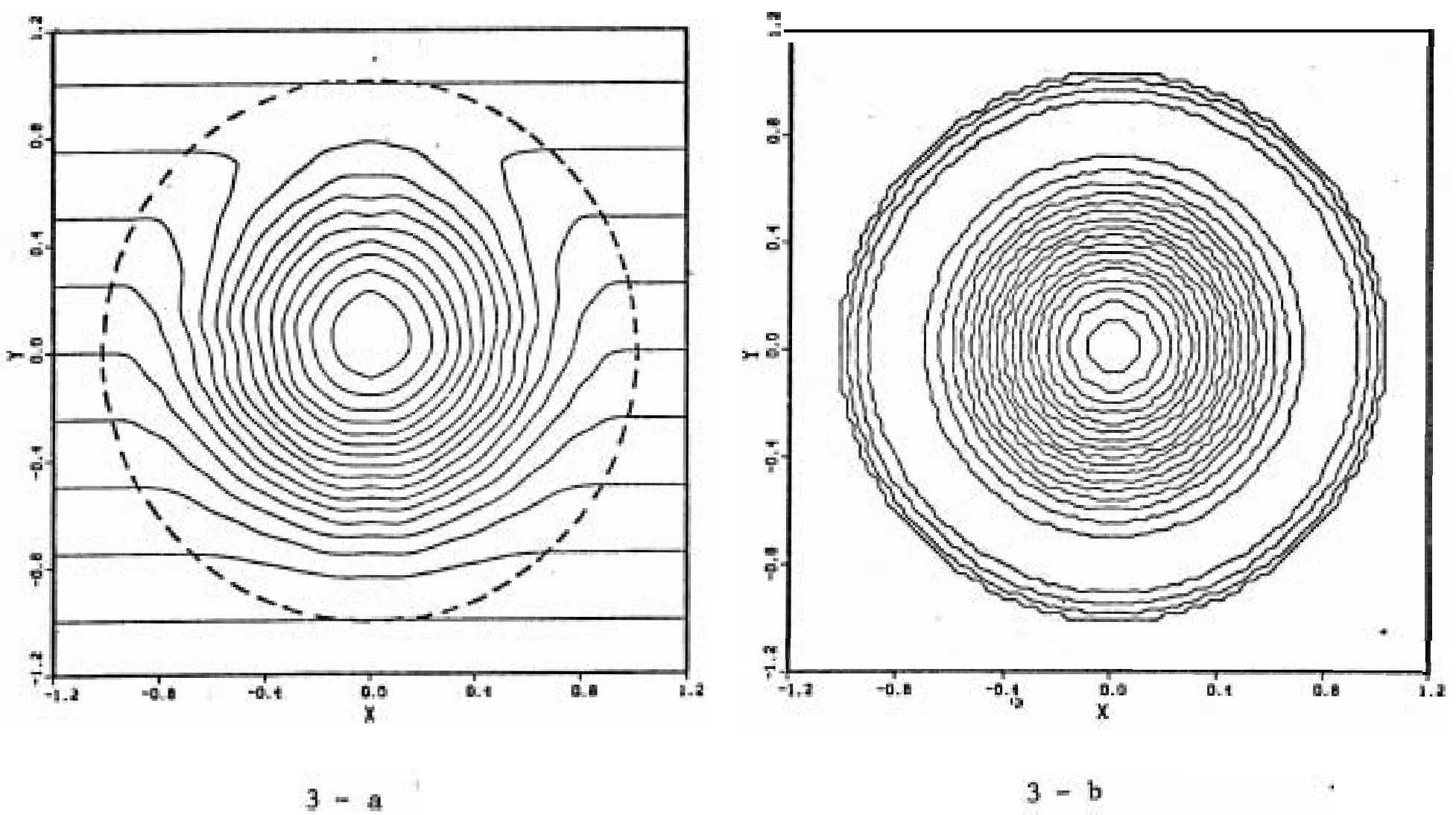

$3-b$

Figure 3 : Mapping of the streamlines in the upper (3a) and the lower (3b) layers. The circle- - indicatesthe line $r=1$. 
as a linear function of $h_{2}$, some coupling between the lower and upper layer is obtained. It comes out from further analysis that the velocities in the lower layer are then proportional to the velocities in the upper layer (but should be smaller)

The geostrophic approximation is no longer valid in the upper layer since $\sim 1$. We have to take into account terms of order of magnitude of the Rossby number $\boldsymbol{E}_{1}$. The analysis is simplified, since time derivative terms vanish $\left(\boldsymbol{\delta}^{\prime} \ll 1\right)$. Equations (11) and (12) become

$$
\begin{gathered}
\varepsilon_{1}\left(\underline{v}_{1} \cdot \nabla\right) \underline{v}_{1}+\underline{k} \times \underline{v}_{1}=-\nabla_{P_{1}} \\
\nabla \cdot\left(\underline{v}_{1} h_{1}\right)=0
\end{gathered}
$$

Equation (42) ensures the existence of a function $\boldsymbol{\psi}_{1}$ as

$$
u_{1}=-\frac{1}{h_{1}} \Psi_{1 y} \quad v_{1}=\frac{1}{h_{1}} \Psi_{1 x}
$$

Incorporating these expressions, a simpler form is obtained for (41):

$$
\nabla\left\{\frac{\varepsilon_{1}}{2}\left[\left(\frac{\Psi_{1 x}}{h_{1}}\right)^{2}+\left(\frac{\Psi_{1 y}}{h_{1}}\right)^{2}\right]+P_{1}\right\}=\left\{\frac{1+\varepsilon_{1} \nabla \cdot\left(\frac{\nabla \psi_{1}}{h_{1}}\right)}{h_{1}}\right\} \nabla \Psi_{1}
$$

An integral form of the later equation is

$$
\begin{aligned}
& \frac{\varepsilon_{1}}{2}\left[\left(\frac{\Psi_{13}}{h_{1}}\right)^{2}+\left(\frac{\Psi_{14}}{h_{1}}\right)^{2}\right]+P_{1}=B\left(\Psi_{1}\right) \\
& 1+\varepsilon_{1} \nabla \cdot\left(\frac{\nabla \Psi_{1}}{h_{1}}\right)=h_{1} B^{\prime}\left(\Psi_{1}\right)
\end{aligned}
$$

The pressure pl is known from the hydrostatic equation (16) whose integral form to lowest order is

$$
\varepsilon_{1} P_{1}+F \delta h_{2}=0
$$

Also, using Stern's integral theorem (10), we get

$$
\mathrm{p}_{1}=0 \quad \text { if } \quad r>1
$$

For an isolated solution $\boldsymbol{\Psi}_{1}$ vanishes far from the eddy. Therefore it comes from (44) that the only solution'is

$$
\Psi_{1} \equiv 0 \quad \text { for } r>1
$$

Inside the eddy $(r<1)$ the simplest form for $B$ is given by uniform potential vorticity in the upper layer i.e.

$$
\mathrm{B}^{\prime}\left(\Psi_{1}\right)=\mathrm{P} \text { and } \mathrm{B}\left(\Psi_{1}\right)=\mathrm{P} \Psi_{1}
$$

In a nongeostrophic vortex, the velocities in the upper layer are much larger than the translation speed, which is neglected. Streamlines in the moving frame do not penetrate into the eddy or, at least, they remain close to the perimeter of the eddy $(r=1)$. This allows some potential vorticity jump between the inside of the eddy and the outside. 
$-125-$

Using (48) and (15), an axisymmetric solution of (44) is obtained by solving

$$
\begin{aligned}
\frac{\epsilon_{1}}{2} v_{1}^{2}-\frac{F \delta}{\varepsilon_{1}} h_{2} & =P \Psi_{1} \\
1+\varepsilon_{1} \frac{1}{r} \frac{\partial}{\partial r}\left(r v_{1}\right) & =P\left\{1-\frac{\delta}{1-\delta} h_{2}\right\}
\end{aligned}
$$

where $v_{1}$ is now defined as the azimuthal velocity

$$
v_{1}=\frac{\psi_{1 n}}{h_{1}}
$$

and the solution verifies the boundary condition

$$
\mathbf{h}_{2}(1)=0
$$

After some algebra, derivation of (49) leads to

$$
\begin{gathered}
v_{1}+\varepsilon_{1} \frac{v_{1}^{2}}{r}=-\frac{F \delta}{\varepsilon_{1}} h_{2 r} \\
\varepsilon_{1} \frac{\partial}{\partial r}\left(\frac{1}{r} \frac{\partial}{\partial r}\left(r v_{1}\right)\right)=-\frac{\mathrm{P} \delta}{1-\delta} h_{2 r}
\end{gathered}
$$
When the centrifugal term $\frac{\varepsilon_{-1}}{\boldsymbol{v}_{\boldsymbol{l}}^{2}}$ is neglected, (52) transforms to a simple
system:

$$
\begin{gathered}
h_{2 r}=-\frac{\varepsilon_{1}}{F \delta} v_{1} \\
r^{2} \frac{\partial^{2} v_{1}}{\partial r^{2}}+r \frac{\partial v_{1}}{\partial r}-\left(1+\frac{P}{(1-\delta) F} r^{2}\right) v_{1}=0
\end{gathered}
$$

Simple solutions are found for (53), which must satisfy the integral form (49). This implies

and then

$$
\begin{gathered}
\text { p }>0 \\
v_{1}(r)=\frac{I_{1}\left(\sqrt{\frac{P}{(1-\delta) F}} r\right)}{I_{1}\left(\sqrt{\frac{P}{(1-\delta) F}}\right)} \\
h_{2}(r)=\frac{\varepsilon}{\delta} \sqrt{\frac{(1-\delta)}{F P}}\left\{\frac{I_{0}\left(\sqrt{\frac{P}{(1-\delta) F}}\right)-I_{0}\left(\sqrt{\frac{P}{(1-\delta) F}}\right)}{I_{1}\left(\sqrt{\frac{P}{(1-\delta) F}}\right)}\right\} \text { for } r<1
\end{gathered}
$$

with the conditions

$$
\begin{aligned}
& 1=P-\varepsilon \sqrt{\frac{P}{(1-\delta) F}} \frac{I_{0}\left(\sqrt{\frac{P}{(1-\delta) F}}\right)}{I_{1}\left(\sqrt{\frac{P}{(1-\delta) F}}\right)} \\
& 1=\frac{\varepsilon}{\delta} \sqrt{\frac{(1-\delta)}{F P}}\left\{\frac{I_{0}\left(\sqrt{\frac{P}{(1-\xi) F}}-1\right.}{I_{1}\left(\sqrt{\frac{P}{(1-\delta) F}}\right)}\right\}
\end{aligned}
$$

Equation (57) expresses $h_{2}(0)=1$. Also the velocity $v_{1}$ is normalized i.e. $\max _{\boldsymbol{r}} \mathrm{v}_{1}(r)=1$. This maximum is reached for $\mathbf{r}=\mathbf{1}$. 
This does not lead to a physically meaningful solution. The nonradiating solution imposes $v_{1}=0$ for $r>1$. Such a strong shear at $r=1$ is consistent in an inviscid model, but not acceptable in a physical sense. Flierl's model for warm core rings also gives a maximum velocity at the perimeter of the eddy. In that case, however, the perimeter is also the interface between the two fluids. In our case it is expected that the eddy will induce a flow outside the vortex $(r>1)$ in the upper layer. There is no way for this induced flow to-be isolated and the vortex will then radiate energy. In a further analysis, solutions with variable potential vorticity distribution have to be examined. In any case, since $\psi_{1} 30 \mathrm{for} r>1$ (eq. 47) and since it is desirable to eliminate shear at the edge of the eddy, potential vorticity distributions have to be studied whose kinematic solutions have a zero velocity at $\mathbf{r}=\mathbf{1}$.

3. Experimental Observations - Stability - Comparison with Theory

3.1 Experimental set-up - observation of baroclinic eddies

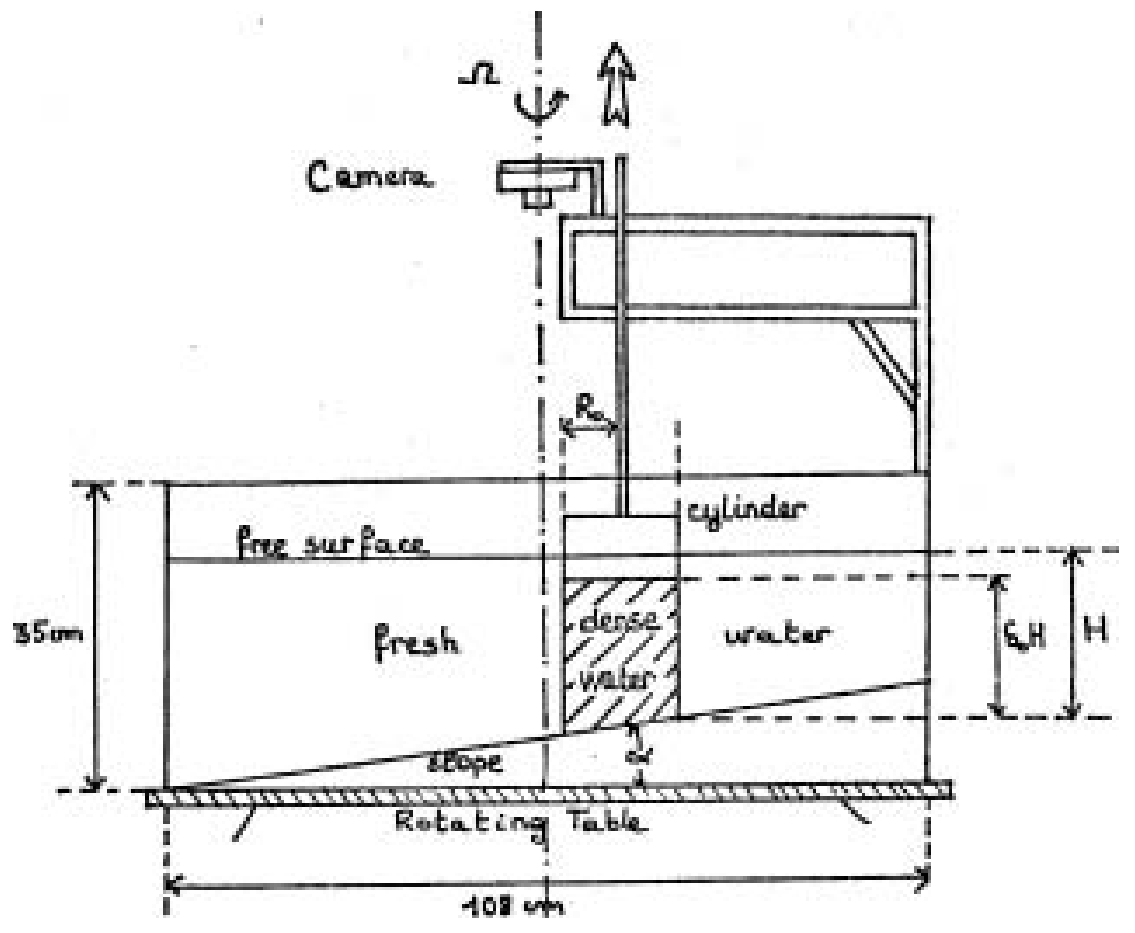

Figure 4. Experimental set-up.

The experiment is conducted in a square tank $(108 \mathrm{~cm}$ wide, $35 \mathrm{~cm}$ depth), and the tank is rotated at a rate between 0.6 and 0.8 rad $\mathrm{s}^{-1}$. A linear slope is put at the bottom with an angle $\alpha$ with the horizontal plane. Two angles have been used, $\alpha \simeq 4^{\circ}$ and $\alpha \simeq 10^{\circ}$. An experiment was also carried out with no slope $(\boldsymbol{\alpha}=0)$.

The way of releasing a heavy dome on the slope is quite similar to Saunders and Griffiths (1973) and Linden (1982) experiments. A cylinder (diameter $R_{o}$ ) on the slope is filled up with denser fluid until the height $\delta_{0} H$ ( $H$ being the total depth of the fresh water). In our experiments the relative initial depth $\boldsymbol{\sigma}_{\boldsymbol{e}}$ of salt water is varied between 0.4 and 0.9 . 
The density difference $\Delta \boldsymbol{\xi}$ between salt water and fresh water is varied between $4 \%$ and $10 \%$. The radius of deformation $L_{D}$ is then $L_{D}=\sqrt{g^{\prime}} E^{\prime} / f$.

The denser fluid is made visible by dark dye and the motion on the free surface is obtained from the displacements of small white paper pellets strewed on it. The flow is recorded by taking photographs of the experiment from above, with the camera rotating at the same rate as the table. The exposure time was in most cases $5 \mathrm{~s}$ so that it was possible to estimate the velocities in upper layer from the length of the streaks due to the displacement of the paper pellets. Although no picture was taken, a side view of the experiment was obtained by looking through the plexiglass side walls of the tank.

As in Saunders's experiment, the heavy fluid is released by pulling the cylinder up. The heavy fluid collapses and spreads out. This first adjustment realizes a balance between momentum and pressure. Depending on the initial conditions, a stable baroclinic vortex is observed or the pattern becomes unstable and breaks down into several baroclinic vortices.

An accurate dimensionless number to describe the stability in terms of the initial input conditions is a Froude number $F_{0}$ as

$$
F_{0}=\frac{g^{\prime} H \delta_{0}}{f^{2} R_{0}^{2}}
$$

where $\delta_{g}$ is the relative depth of dense fluid in the cylinder before starting the experiment. Experiments were carried out with the initial Froude number $\mathbf{F}_{0}$ ranging from 1 to 2.

An experimental estimation for the stability criterion is

$$
F_{0} \gtrsim 1.6-1.9
$$

For initial Froude number exceeding this value, stable baroclinic vortices are observed.

A typical example is shown on Figure 5. The value of $F_{0}$ in this experiment is $\mathrm{F}_{0} \simeq 1.6$. The experimental conditions are summarized in Table 2.

\begin{tabular}{lllll}
$\alpha$ & $\mathrm{g}^{\prime}$ & $\vdots$ & $\mathrm{H}$ & $\boldsymbol{\delta}_{\circ}$ \\
$10^{\circ}$ & $8 \mathrm{~cm} \cdot \mathrm{s}^{-2}$ & $1.3 \mathrm{~s}^{-1}$ & $20 \mathrm{~cm}$ & 0.9 \\
\hline
\end{tabular}

TABLE 2

The sequence of pictures shows the pattern from a top view at successive times, respectively $10 \mathrm{~s}, 30 \mathrm{~s}, 50 \mathrm{~s}, 75 \mathrm{~s}$, and $135 \mathrm{~s}$ after the cylinder has been pulled out.

The conditions for this experiment are weakly unstable ( $F_{0}=1.6$ ). In fact, one strong cyclonic and one weak anticlonic vortices appear. The strong one forms rapidly, within two or three rotation periods. The whole set of pictures shows a westward and northward translation of the cyclonic eddy. 

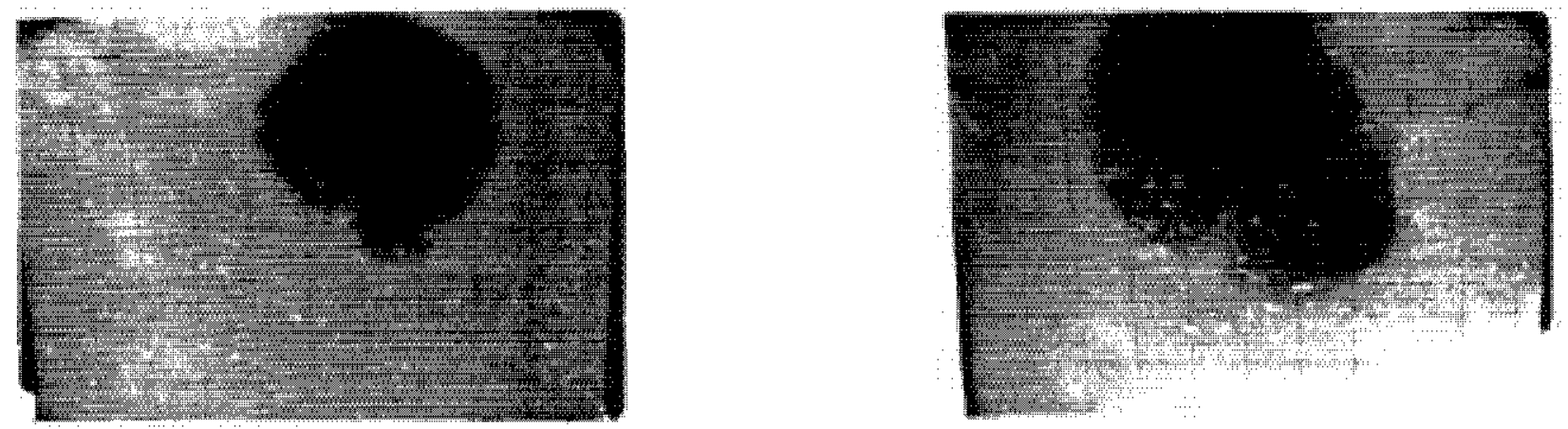

$5=8$

$$
5-6
$$


$$
5-4
$$

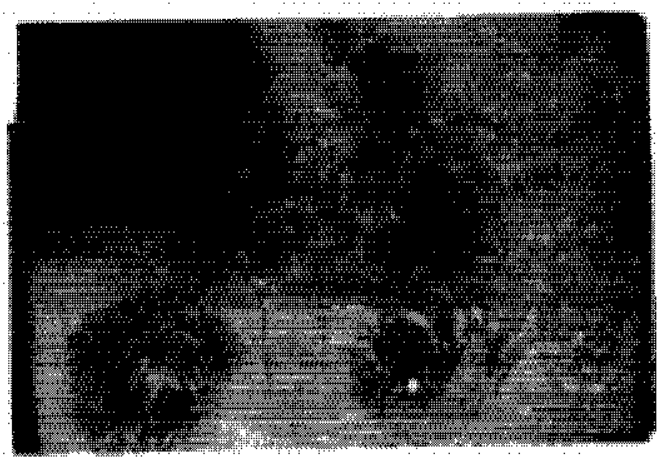

$$
5-1
$$

Figure 5: Baroclinic vortex experiment .

$\mathrm{g}^{\prime}=8 \mathrm{~cm} . \mathrm{s}, \alpha=10^{\circ}, \quad=1.3 \mathrm{~s}^{-4}, \mathrm{H}=20 \mathrm{am}, \delta_{1}=0.9$ Successive exposure times are $10 \mathrm{~s}(5 a), 30 \mathrm{~s}(5 \mathrm{~b}), 50 \mathrm{~s}(5 \mathrm{c})$, $75 \mathrm{~s}$ (5d) and $135 \mathrm{~s}$ (5e) after pulling up the cylinder. 
A more detailed analysis indicates however that the northward motion occurs mostly during the formation time of the eddy and when it approaches close enough to the western wall. In pictures $5 b$ and $5 c$ the eddy has moved slightly downhill from its position in picture 5 a.

The westward translation is very clear. Although a significant westward translation of the eddy is due to the first instability, the translation speed is later much slower, about $0.2-0.4 \mathrm{~cm}^{-1}$. This speed is in fact much smaller than the topographic Rossby wave velocity, as predicted by our theoretical solution.

Typical diameter and velocity scales for the eddy are about

$$
2 \mathrm{~L} \simeq 30 \mathrm{~cm} \quad \nabla_{1} \simeq 2-5{\mathrm{~cm} . \mathrm{s}^{-1}}^{-1}
$$

which indicates a relative vorticity in the eddy between $f / 2$ and $f$.

A significant scatter appears in our experimental results. Further experiments have to be performed, after having improved our set-up. In fact the ratio of the scale of the eddy to the size of the tank is actually too large to describe with accuracy the time evolution of the eddy and to reduce sufficiently the influence of the wall. However, we emphasize from our experimental observation the existence of strong stable baroclinic vortices moving slowly westward. We refrained from calling the vortices isolated vortices since some uncertainty remains about that point. For instance, a small tail is observed on Figure 5 behind the cyclonic vortex. We have presently no argument to decide whether this indicates some interaction with the weak anticyclonic vortex or whether this is due to Rossby wave propagation.

\subsection{Comparison with theoretical models}

Comparison of experimental velocity profiles and of the shape of the dome with theoretical predictions is actually beyond our experimental abilities. Nevertheless values or relationships between the potential vorticity P, the Froude number F, the Rossby number $\boldsymbol{E}$, and the relative depth of the dome have been deduced from the theoretical models. These theoretical results are tested with our experimental results.

The scaling obtained from the experiment is summarized in Table 3.

\begin{tabular}{llllllll}
\hline & $\alpha$ & $g^{\prime}$ & H & $2 \mathrm{~L}$ & S $^{\prime}$ & F & S \\
$1.3 s^{-1}$ & $10^{\circ}$ & $8 \mathrm{~cm} \cdot \mathrm{s}^{-2}$ & $20 \mathrm{~cm}$ & $30 \mathrm{~cm}$ & 0.26 & 0.19 & 0.7 \\
\hline
\end{tabular}

TABLE 3

Comparisons of the experiment with theory have to be done very carefully. It is first noted that the theoretical and experimental shapes for the dome do not compare well. A rough drawing of an experimental shape (dashed line) is compared on Figure 6 with the shape obtained from the geostrophic model (solid line). 


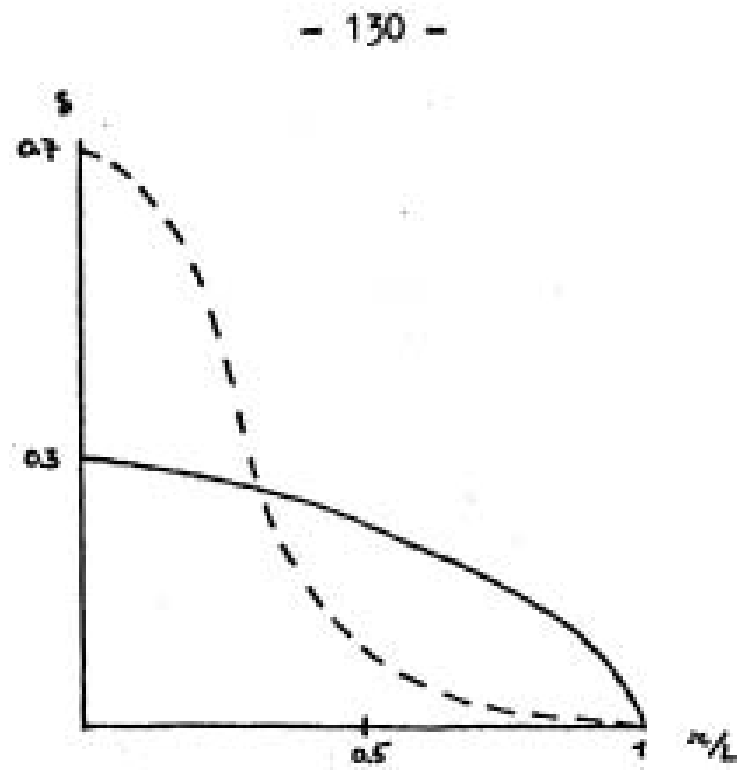

Figure 6. Comparison of the shape of the dome deduced from the geostrophic modet ( $)$ with a qualitative experimental estimation $(-\infty-\infty)$.

In order to estimate the velocity scale, careful estimates of $\delta$ and L are required. The volume of dense fluid in the heavy dome is conserved. Taking and $L$ from Table 3 overestimates the volume of the dome. It is more justified to calculate the length scale $\mathrm{L}_{\mathbf{5}}$ associated with $\mathbf{5}$, for which the volume of the dome is accurately estimated. The results are plotted in Table 4 as well as the corresponding estimates sf the Rossby number $\varepsilon_{1}$ and the velocity scale $\mathbf{V}_{\mathbf{1}}$, deduced from the nongeostrophic model.

\begin{tabular}{|c|c|c|c|}
\hline$\delta$ & $2 L_{6}$ & $\varepsilon_{1}$ & $\mathrm{v}_{1}$ \\
\hline 0,3 & $35 \mathrm{~cm}$ & 0.29 & $6.6 \mathrm{~cm} . \mathrm{s}^{-1}$ \\
\hline 0.4 & $30 \mathrm{~cm}$ & 0.62 & $12 \mathrm{~cm}^{-1} \mathrm{~s}^{-1}$ \\
\hline 0.5 & $26 \mathrm{~cm}$ & 2,1 & $35 \mathrm{~cm}, \mathrm{~s}^{-1}$ \\
\hline 0.7 & $22 \mathrm{~cm}$ & 4.1 & $59 \mathrm{~cm} \cdot \mathrm{s}^{-1}$ \\
\hline
\end{tabular}

TABLE 4

The most plausible result is for $\boldsymbol{S}=0.3$, even if it still overestimates the velocity scale. For higher values of $\delta$ the nongeostrophic model is no longer valid since the Rossby number becomes too large. The centrifugal term is not negligible.

The geostrophic solution does not compare well with observations either. This is not too surprising since $\boldsymbol{\varepsilon} \sim \boldsymbol{\delta}<\boldsymbol{1}$ is assumed for deriving this solution. Furthermore the value of the Froude number (eq. 37) predicts a very large scale. The diameter of the dome is about $1 \mathrm{~m}, \mathbf{1} . e .10-12$ times the radius of deformation. Such range lies far outside the abilities of our experiment. 


\subsection{Baroclinic stability of vortices}

As indicated in subsection (3.1), baroclinic instability conditions impose very strong limitations on our experiment. A complicated pattern results when instability occurs after pulling the cylinder up. The motion of vortices cannot be controlled and strong vortices generally move rapidly uphill toward the wall during the instability events.

A rough instability criterion is proposed in the subsection 3.1:

$$
F_{0}=\frac{g^{\prime} H \delta_{0}}{f^{2} R_{0}^{2}} \quad \gtrsim \quad 1.6-1.9
$$

This estimation of the stability criteria agrees with the experimental work of Sauders (1973) (in which the effect is not present). Saunders used a technique similar to ours except that the heavy fluid filled the whole

cylinder (i.e. =1) before starting the experiment. The following stability criteria was obtained by Saunders

\section{$\frac{8^{\prime} H}{f^{2} R_{0}^{2}}>4.8$}

Saunders also showed that the dense fluid spreads out one deformation radius $L_{D}=\sqrt{g^{\prime} H} / \mathbb{F}$. This result is verified in our experiments and the diameter of the baroclinic vortex is then

$$
2 L \simeq 2\left(R_{O}+L_{D}\right)
$$

Some interesting remarks are deduced from the relationships (59) and $(60)$.

First we should point out that there is no way to observe in our laboratory experiment baroclinic eddies as large as predicted by our geostrophic model. Baroclinic instability will occur in any case. Whenever such a solution could be observed in other situations, there remains some uncertainty about its stability.

In our experiments, we did not explore the whole possible range for stable baroclinic vortices. Only large value of $\boldsymbol{\delta}_{0}$ have been used. It comes out from (59) and (60) that stable baroclinic vortices with small relative depth must have a large radius of deformation. For instance

$$
\text { for } \delta_{0}=0.2 \quad \mathrm{~L}_{\mathrm{D}} \sim 3 \mathrm{R}_{\mathrm{O}}
$$

and this predicts the scale of the vortex to be about $8 R_{0}$. A much larger tank than those we used is required to study such an eddy.

\section{Conclusion}

This paper addresses both theoretical and experimental aspects of isolated baroclinic eddies. A geostrophic solution is derived (equations 33, 34 and 35) and demonstrates the existence of isolated baroclinic eddies characterized by a large length scale (eq. 37). These vortices translate westward with a small velocity compared to topographic Rossby wave speed (eqs. 29 and 37). A nongeostrophic solution with constant potential vorticity is also presented (eq. 55). This solution is valid in an inviscid model but 
imposes a nonphysical shear at the edge of the eddy. The study of nongeostrophic solutions has to be continued with the case of nonconstant distribution of potential vorticity.

Laboratory experiments demonstrate the existence of stable baroclinic eddies (Fig. 5). As predicted, the vortices translate westward at a small speed. However, an important discrepancy appears between observations and theory. Our observations are limited to a limited range of strongly ageostrophic vortices for practical reasons, and therefore the discrepancy is not too surprising. This suggests a more careful scaling of the set up in order to possibly observe weakly ageostrophic stable vortices, determine their translation properties, and examine more carefully their radiation properties.

\section{ACKNOWBGMENIS}

I would like to thank Melvin Stern and Ross Griffiths for suggesting and supporting this project. Discussions with them and Glenn Flierl were very helpful. Experimental facilities were provided by Jack Whitehead, and Bob Frazel's assistance made the experiment possible. G.F.D. program is acknowledged, and $I$ am grateful to the people of WHOI for introducing me to all facilities.

\section{BIBLIOGRAPHY}

Flierl, G.R., 1983. A model of the structure and motion of a warm core ring; Austr. J. Mar. and F. Res., (U.S.-Australian-New Zealand workshop on warm core rings). (In press)

Flierl, G.R., ME. Stern, and J.A. Whitehead, 1983. The physical significance of modons: laboratory experiments and general integral constraints. Dyn. of Atmos. and Oceans. (In press).

Griffiths, R.W., 1983. Inertial wave drag and the production of intense vortices by turbulent gravity currents, with implications for the sinking of bottom waters. Ocean Modelling, March.

Griffiths, R.W., and E.J. Hopfinger, 1983 - Gravity currents moving along a lateral boundary in a rotating fluid. J. Fluid Mech. (In press)

Griffiths, R.W., and P. F. Linden, 1982. The stability of vortices in a rotating stratified fluid. J. Fluid Mech., 폴, 283-316.

Pedlosky, J., 19 - Geophysical Fluid Dynamics, Springer-Verlag.

Saunders, P.M., 1973. The instability of a baroclinic vortex. J. Phys. Oceanography, $3,61-65$. 


\title{
LANGMUIR CIRCULATIONS IN A STRONG SHEAR FLOW
}

\author{
Sanjiva Lele
}

\section{Introduction}

Langmuir circulations are organized convective motions which form in the surface layers of ponds, lakes, and oceans when moderate wind blows over them. These motions have the form of longitudinal vortices whose axis is approximately aligned with the direction of the wind. The vortices produce surface convergence and thus lead to the collection of floatsam, seaweeds, oils, or other organic films into streaks or bands which then provides a visible surface manifestation of the circulation. The downwelling speeds in these motions can at times approach about $1 \%$ of the wind speed.

Langmuir circulations bring about significant vertical transport of heat, momentum, nutrients, and other biological materials - and hence their understanding is fundamental to the physics of the mixing and transport processes in the surface layers of naturally occurring bodies of water.

In a series of papers, Craik and Leibovich (1976) have provided a theoretical model in which Langmuir circulations arise through a nonlinear interaction between the surface gravity waves and a weak current. This model has been used by Leibovich and Paolucci (1980) and more recently by Leibovich and Lele (1982) to systematically explore the dynamical characteristics of Langmuir circulations such as the vertical fluxes of momentum and heat, their effectiveness in overturning stable density gradients and producing mixing, etc. Recently Leibovich (1983) has reviewed the literature on Langmuir circulations, and we refer to this review for further details of the phenomena.

The theoretical model developed by Craik and Leibovich is appropriate when the orbital speeds due to the surface gravity waves greatly exceed any mean currents with which they interact. Though in most circumstances this is a valid assumption yet there are many situations where the mean current is not small compared to the wave induced orbital speeds, but rather equal or large. Windrows or surface streaks are known to occur in the latter case as well, but the Craik-Leibovich model is inapplicable to account for their generation. It is the latter situation which we wish to address in the present work.

Craik (1982) has argued that longitudinal vortex instabilities can arise in general shear flows due to the interaction of small amplitude waves with the shear flow. He adopts the generalized Lagrangian mean (GLM) formulation which unfortunately obscures the instability mechanism for strong shear flows. In the present work we consider the nonlinear interaction of surface gravity waves with a strong shear flow in a density stratified medium. The problem is posed entirely in terms of Eulerian variables which we find easier to interpret. Some of the ideas inherent in Craik's work are directly reflected in the present development.

The principal result is that we provide a mechanism through which the interaction of gravity waves with a strong shear flow drives longitudinal vortices. It is found that these instabilities have an essential anisotropy with the windward anomaly larger than the cross stream anomaly, and it grows 
at a rate much faster than the Langmuir circulation on a weak shear flow. The nondimensional growth rate depends on two nondimensional parameters in addition to its dependence on the wavenumber. One of these parameters which is like a Richardson Number measures the static stability of the basic flow and the growth rate decreases as this parameter is increased. The other nondimensional parameter is like a Froude Number, being a ratio of the velocity difference due to the shear across a scale height to the phase speed of gravity waves. As the basic flow shear is decreased, the growth rate of the instability is seen to decrease. We further find that our linear stability problem is unaffected by a small correction to mean flow which allows for arbitrary vorticity distribution. Hence the present stability problem is applicable to a large class of shear flows which have a small but arbitrary vorticity variation.

In Section 2 we discuss the nature of the basic flow. Section 3 poses the linear stability problem for the basic flow. In Section 4 appropriate boundary conditions are derived for the eigenvalue problem governing the linear stability. Section 5 presents the numerical results and explores the variation of the growth rate with the nondimensional parameters governing the problem. Section 6 presents a brief discussion of the energetics of the instability. Finally we discuss some implications of the present instability mechanism to more general shear flows.

\section{Basic Flow}

The basic flow whose stability we wish to analyze here has three contributions to it.

a) A unidirectional mean flow (whose direction we choose to be the $\mathrm{x}$-axis) having a uniform shear $\left(\overrightarrow{\mathrm{U}}_{\mathrm{b}}^{(0)}\right)$.

\section{distribution $\left(\boldsymbol{u}^{(2)}(\mathbf{z})\right)$. \\ b) A smaller mean flow along $x$ which has an arbitrary vorticity}

c) A small amplitude surface gravity wave on the shear flow described by (a)-(b) above, with all the harmonics and higher order nonlinear

corrections it might imply ( $\vec{U}_{b}(1)$ ).

Hence, the basic flow can be written as

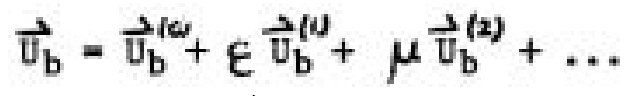

where

$$
\begin{aligned}
& \overrightarrow{\mathrm{U}}_{\mathrm{b}}^{(a)}=\mathrm{U}^{\prime} \mathrm{z} \hat{\imath} \\
& \overrightarrow{\mathrm{U}}_{\mathrm{b}}^{(2)}=\mathrm{U}_{\mathrm{b}}^{(2)}(\mathrm{z}) \hat{\imath}
\end{aligned}
$$

and $\mathrm{z}$ is the coordinate pointing vertically upwards, and $\boldsymbol{E}, \boldsymbol{\mu}$ are small parameters. In what follows, $E$ will be chosen to be the wave slope of the gravity waves. The ... refers to higher order terms which we have not bothered to write. 
The density field for the basic state is written as

$$
P_{b}=P_{r}+\nu P_{b}^{(0)}(z)+\ldots
$$

where $P_{r}$ is the reference density. $\quad P_{b}^{(0)}$ provides a stable stratification whose magnitude is governed by a small parameter, $\boldsymbol{V}$.

The momentum equations are then used to solve for the surface gravity waves on the prescribed mean shear flow. Some important features of these small amplitude gravity waves are summarized below.

i) The waves travelling in $\mathrm{x}$-direction are irrotational to leading order. Oblique waves are, however, rotational, and their velocity field is three dimensional.

i i) The dispersion relation for the waves is given by

$$
\omega^{2}+v^{\prime} \cos \theta \omega-g \hat{L}=0
$$

where $\boldsymbol{\omega}$ is the frequency of the waves, $\theta$ is the angle between the direction of wave propagation and the basic flow, and $\boldsymbol{l}$ is the magnitude of the wave number. The phase speed $\mathrm{C}$ for waves travelling parallel to $\mathrm{x}$-axis is then given by

$$
c= \pm\left(U^{12}+4 \underset{L}{\operatorname{sig} l)^{1 / 2}-U^{\prime}}\right.
$$

with the positive sign corresponding to the wave travelling in tx direction.

i i i) Craik (1968) has shown that certain triads of these waves lead to second order resonant interactions if $U^{\prime}<0$. We further recall that for $\left.U^{\prime}\right\rangle 0$ which is case of interest in the present problem, there are no second order resonances.

\section{Linear Stability Problem}

We consider infinitesimal disturbances ( $u, p, P$ ) on the basic state $\left(\mathrm{U}_{\mathrm{b}}, \mathrm{P}_{\mathrm{b}}, P_{\mathrm{b}}\right)$. The evolution equations for the disturbance fields are:

$$
\begin{aligned}
& \frac{\partial \vec{u}}{\partial t}+(\vec{u} \cdot \nabla) \vec{U}_{b}+\left(\vec{U}_{b} \cdot \nabla\right) \vec{u}+\frac{1}{P_{r}} \nabla p+g \frac{\rho}{P_{r}} \hat{k}=0 \\
& \quad \nabla \cdot \vec{u}=0 \\
& \frac{\partial \rho}{\partial t}+(\vec{u} \cdot \nabla) \rho_{b}+\left(\vec{U}_{b} \cdot \nabla\right) \rho=0
\end{aligned}
$$

Since the basic state whose stability we wish to examine is available as a power series in a small parameter $\mathcal{E}$, we expand the perturbation fields in a similar power series, i.e.

$$
\begin{aligned}
& \vec{u}=\vec{u}^{(0)}+\varepsilon \vec{u}^{(1)}+\cdots \\
& p=p^{(0)}+\varepsilon p^{(1)}+\cdots \\
& p=p^{(0)}+\varepsilon p^{(1)}+\cdots
\end{aligned}
$$


At leading order, the problem governing the perturbation fields is formally identical to the problem governing the leading order surface gravity waves on $\overrightarrow{\mathrm{U}}_{\mathrm{b}}^{(0)}$ shear flow. As a result, the solution corresponds to some arbitary combination of these waves. Since we already know that for $U^{\prime}>0$ the nonlinear interaction of these waves with the primary wave cannot lead to a second-order resonance, we chose to restrict our attention to a special class of disturbances. These disturbances are:

i Independent of $\mathrm{x}$-direction

ii) Periodic in y-direction

i i i) Evolve on a time scale 3 which is slower than the time scale of the surface gravity waves.

An inspection of the leading order problem then reveals that the disturbance velocity field must have a special form for the above conditions to be consistent with the governing equations, which is

$$
\vec{u}^{(0)}=\left(u^{(0)}, \varepsilon v^{(0)} \varepsilon w^{(0)}\right)
$$

With the above form of distubance, the leading order problem implies $\frac{\partial u^{(0)}}{\partial t}=0$.

The next step is then to proceed to higher orders in the perturbation expansions. At each order one takes an $x$-average of the equations to derive the equation which governs the $x$-averaged fields. This equation is then subtracted from the original equation to render an equation governing the $x-$ periodic fields.

At $O(\varepsilon)$ this process identifies the appropriate slow time $\tau$ to be $\boldsymbol{E} \mathbf{t}$ and yields

a) Equation governing the slow time evolution of $\mathrm{u}^{(0)}$.

b) Equations governing the wave distribution produced by the perturbation.

These equations are:

$$
\frac{\partial u^{(0)}}{\partial \tau}+w^{(0)} U^{\prime}=0
$$

where $\tau=\varepsilon t$ is the appropriate slow time for the problem, and

$$
\begin{aligned}
& \mathcal{L} \omega_{1}^{(1)}=\omega_{3}^{(1)} u^{\prime}+\frac{\partial u^{(1)}}{\partial y} u^{\prime}+\omega_{3}^{(0)} \frac{\partial U_{b}^{(1)}}{\partial z} \\
& \mathcal{L} \omega_{2}^{(1)}=-w_{b}^{(1)} \frac{\partial \omega_{2}^{(0)}}{\partial z}+\frac{\partial v^{(1)}}{\partial y} u^{\prime} \\
& \mathcal{L} \omega_{3}^{(1)}=-w_{b}^{(1)} \frac{\partial \omega_{3}^{(0)}}{\partial z}+\frac{\partial u^{(1)}}{\partial y} U^{\prime}+\omega_{3}^{(0)} \frac{\partial w_{b}^{(1)}}{\partial z}
\end{aligned}
$$

where

$$
\begin{aligned}
& \mathcal{L} \equiv \frac{\partial}{\partial t}+v_{b}^{(0)} \frac{\partial}{\partial x} \\
& \vec{\omega}^{(1)}=\left(\omega_{1}^{(1)}, \omega_{2}^{(1)}, \omega_{3}^{(1)}\right)=\text { curl } \vec{u}^{(1)} \\
& \omega_{2}^{(0)}=\frac{\partial u^{(0)}}{\partial z} ; \quad \omega_{3}^{(0)}=-\frac{\partial u^{(0)}}{\partial y}
\end{aligned}
$$


and $\left(U_{b}^{(1)}, 0, W_{b}^{(1)}\right)$ are the primary wave velocities. In Equations (3.7) above we have taken the curl of the momentum equations.

It is possible to provide a simple interpretation for Equation (3.6) if we work through the corresponding vorticity equation. These are

$$
\begin{aligned}
& \frac{\partial \omega_{2}^{(0)}}{\partial \tau}=u^{\prime} \frac{\partial v^{(0)}}{\partial y} \\
& \frac{\partial \omega_{3}^{(0)}}{\partial \tau}=u^{\prime} \frac{\partial w^{(0)}}{\partial y}
\end{aligned}
$$

which state that $\omega_{2}^{(0)}$ is produced by stretching the basic flow vorticity and $\omega_{3}$ is produced by tilting the basic flow vorticity.

Equations (3.7) which govern the wave distortion, however, are complicated, and we have been unable to discover some other set of variables which would simplify its form. Lagrangian displacements following the basic mean flow are of no help in this regard.

At $O\left(E^{2}\right)$ one derives an evolution equation for the streamwise vorticity. This equation can be written as

$$
\frac{\partial \omega^{(0)}}{\partial \tau}=\left\{\operatorname{curl}\left(\left\langle\vec{u}_{b}^{(1)} \times \vec{\omega}^{(1)}\right\rangle\right)\right\} \cdot \hat{\imath}-\frac{g \partial\left\langle p^{(2)}\right\rangle}{P_{r}} \frac{\partial y}{\partial y}
$$

where $\omega_{1}^{(0)}=\partial \omega^{(0)} \quad \partial v^{(0)} \quad$ and

$\langle>$ represents an $\mathbf{x}$-average of the quantity. We have further assumed that the small parameter $\nu$ is equal to $E^{2}$. In dimensional terms it implies that the nondimensional ratio

$$
\frac{N^{2}}{\varepsilon^{2} \omega^{2}} \sim O(1)
$$

where $N$ is the Brunt Vaisalä frequency, $\omega$ is the frequency of the surface gravity waves and $\mathcal{E}$ is the wave slope. If we substitute typical values we expect in the $1 \mathrm{e} f \mathrm{t}$ hand side of Equation (3.12), we indeed find a number of order unity. $\left\langle\rho^{(2)}\right\rangle$ appearing in Equation 3.11 can be found by systematically expanding the incompressibility condition (3.3) at various orders of $E$. The result of such an expansion is that

$$
\frac{\partial\left\langle\rho^{(2)}\right\rangle}{\partial \tau}=-\omega^{(0)} \frac{d f_{b}^{(0)}}{d z}
$$

This formally completes the set of equations we need to solve. 
We now look for disturbances which are periodic in y with a wave number $\mathrm{k}$ and have a growth rate $\sigma$ (to be found). Without any loss of generality, we can write down the following expressions;

$$
\begin{aligned}
& U_{b}^{(1)}=u e^{l z} \cos \{l(x-c t)\} \\
& W_{b}^{(1)}=\eta e^{(z} \sin \{l(x-c t)\} \\
& u^{(0)}=W(z) e^{\sigma \tau} \cos k y \\
& v^{(0)}=-\frac{1}{k} W(z) e^{\sigma \tau} \sin k y \\
& w^{(0)}=W(z) e^{\sigma \tau} \cos k y
\end{aligned}
$$

When these are substituted in Equations (3.7) governing the distortion of the waves, they yield the following relationships.

$$
\begin{aligned}
& u^{(1)}=u \Phi_{u}(z) \cos k y \cos \{l(x-c t)\} e^{\sigma \tau} \\
& v^{(1)}=-u \Phi_{v}(z) \sin k y \sin \{l(x-c t)\} e^{\sigma \tau} \\
& u^{(1)}=u \Phi_{w}(z) \cos k y \sin \{l(x-c t)\} e^{\sigma \tau}
\end{aligned}
$$

Functions $\Phi_{u}, \Phi_{v}$, and $\Phi_{w}$ describe the vertical structure of the wave distortion. There is a continuity condition which can be written as

$$
\Phi_{u}=\frac{k \Phi_{v}-D \Phi_{w}}{\ell} \quad \text { where } D \equiv \frac{\partial}{\partial z}
$$

Equations (3.7) then provide the differential equations which relate the vertical structure of the wave distortion to the vertical structure of the primary wave and the longitudinal vortex perturbations. These are

$$
\begin{aligned}
\left(U_{b}^{(0)}-c\right)\left(D^{2}-k^{2}-l^{2}\right) \Phi_{w} & =e^{(3}\left(D^{2}+k^{2}\right) \mathbb{U} \\
D\left\{\left(U_{b}^{(0)}-c\right) \Phi_{v}\right\} & =k\left[\left(U_{b}^{(0)}-c\right) \Phi_{w}+\mathbb{U} e^{l 3}\right]
\end{aligned}
$$

Given the forms in (3.14) and (3.16) it is now possible to evaluate the vortex force term appearing in (3.11).

It is convenient to make the equations dimensionless by choosing $\boldsymbol{\ell}^{-1}$ as the length scale, $1 / U^{\prime}$ as the time scale and $1 / \boldsymbol{\ell} U^{\prime}$ as the velocity scale.

The dimensionless equations are

$$
\begin{aligned}
& \left(D^{2}-k^{2}-k^{2} \frac{R}{\sigma^{2}}\right) U=\frac{k^{2}}{2 \sigma^{2}} e^{3}\left[\left(D^{2}+k^{2}+1\right) \Phi_{v}+2 D \Phi_{V}-2(D+1) \Phi_{w}\right] \\
& (Z-C)\left(D^{2}-k^{2}-1\right) \Phi_{w}=e^{3}\left(D^{2}+k^{2}\right) \\
& D\left[(Z-C) \Phi_{V}\right]=(Z-C) \Phi_{w}+\mathbb{W} e^{3}
\end{aligned}
$$

where

$$
c=(1+4 F)^{1 / 2}-1
$$


and $\Phi_{\mathrm{v} / \mathrm{k}}$ has been renamed as $\Phi_{\mathrm{v}^{*}}$. The nondimensional parameter $R=N^{2} / \varepsilon^{2} \omega^{2}$ is like a Richardson Number for the problem and is a measure of the static stability of the basic state. The parameter $F=g l / \mathbf{u}^{\prime 2}$ is like an inverse Froude number and is a measure of the destabilizing effect of the mean flow shear.

We further note that if we choose the small parameter $\boldsymbol{\mu}_{\text {to }}$ be equal to $E$, i.e. the basic flow part which allows for arbitrary vorticity distribution is as large as the magnitude of wave orbital speeds the analysis remains unaffected to the present order in the perturbation expansions. This implies that the leading order growth rate of the instability is insensitive to an arbitrary $O(E)$ vorticity distribution in the basic mean flow. The significance of this result is that it assures us that the instability we analyze here is, in fact, applicable to a wide class of basic mean flows.

\section{Boundary Conditions}

Boundary conditions at the free surface are derived by taking into account the fact that the disturbed free surface is a streamline (kinematic boundary condition) and requiring that the pressure at the disturbed interface remains undisturbed. Both of these conditions need to be expanded in a power series involving the small parameter $\mathcal{E}$. We skip all details of these expansions and just quote the final result, viz,

$$
\begin{aligned}
& W(0)=0 \\
& \Phi_{\mathrm{V}}(0)=\frac{c^{2}}{F} \Phi_{\mathrm{v}}(0)
\end{aligned}
$$

where all quantities are dimensionless. We further require that a 11 perturbation fields decay as $Z \rightarrow-\infty$, which implies:

$$
W, \mathbb{W}, \Phi_{u}, \Phi_{v}, \Phi_{w} \rightarrow 0 \quad \text { as } z \rightarrow-\infty
$$

Equations (3-19) to (3.22) and the boundary conditions (4.1) to (4.3) now complete the statement of the eigenvalue problem.

\section{Numerical Results}

The eigenvalue problem which is posed in the previous sections involves a fifth order ordinary differential equation with variable coefficients. Although it may be possible to make some general statements about the nature of the eigenvalues, we choose to proceed to numerical integration to find the eigenvalues as a function of the nondimensional parameters governing the problem.

We used a shooting technique to find the eigenvalues, and the integration was performed starting from a finite bottom at $Z=-D$ using the asymptotic boundary conditions. All computations were performed in double precision arithmetic using a differential equation integrator DVERK from the IMSL library. The convergence of eigenvalues was checked simply by increasing D. As the parameters of the problem were changed, we changed $D$ to assure the accuracy of the eigenvalues found. Typical value needed for D ranged from 3 to 15 . 
Figures 1 and 2 show the eigenfunctions for the first mode of the instability. For higher modes, the function $\mathbb{W}$ has intermediate zeroes. Figure 3 shows the depth variation of the two components of the vortex force. It is seen that the transverse (y) component of the vortex force points towards the planes of maximum in the U(O) anomaly. This force decreases with depth and produces an overturning torque which drives the instability. In this sense. the present instability mechanism is analogous to that for Langmuir circulations on a weak shear flow.

Figure 4 shows the variation of the growth rate of the first mode as function of the wave number $\mathrm{k}$ while $\mathrm{F}$ and $\mathrm{R}$ are kept fixed. It is noted that the growth rate increases as $k$ is increased. The slope of the growth $r a t e$ curve, however, decreases as $\mathrm{k}$ is increased.

Figure 5 shows the variation of the growth rate as a function of $F$ when $\mathrm{k}$ and $\mathrm{R}$ are held fixed. It is seen that the growth rate decreases as the shear of the basic flow is decreased. For very large shear $(F \rightarrow 0)$, the growth rate increases indefinitely.

Figure 6 shows the variation of the growth rate as a function of $R$ when $\mathrm{k}$ and $\mathrm{F}$ are held fixed. It is seen that as the static stability of the basic state is increased, the growth rate of the instability decreases. It is not evident from the present calculations if there is some critical value of $\mathrm{R}$ for which the instability could be suppressed for a fixed F. It is clear, however, that if there is such a critical value of $R$, it must be a function of F. For $F=1$, this critical value must be much larger than unity. Very large values of $\mathrm{R}$ are beyond the applicability of the present model.

Figure 7 shows how the elgenfunction $\mathbb{W}$ changes when the wave number $\mathrm{k}$ of the perturbation is varied. As $k$ increases, the vertical scale of the instability shrinks, and the opposite holds when k decreases. For small k the computational domain required to yield accurate growth rates progressively increases.

\section{Energetles of the Instabil1}

It is instructive to now look at the energetics of the longitudinal vortex instability and try to identify the energy source that the instability feeds on.

The first thing to note is that the strain rate of the basic flow has two contributions to it. A mean strain rate due to the shear of the basic flow and an $O(\varepsilon) x$-periodic strain rate due to the primary wave. The perturbation fields which form the Reynolds stresses are.

$$
\begin{aligned}
& \text { 1) } \vec{u}^{(0)}=\left(u^{(0)}, \varepsilon v^{(0)}, \varepsilon w^{(0)}\right) \\
& \text { 1i) } \varepsilon \vec{u}^{(1)}=\varepsilon\left(u^{(1)}, v^{(1)}, w^{(1)}\right)
\end{aligned}
$$

Now we form the energy equation from the governing equations in the usual manner and then look for the balances that obtain at various orders of the small parameter $\boldsymbol{E}$. This analysis leads to two conclusions: 
i) The $0(\varepsilon)$ Reynolds stress $\varepsilon u^{(0)} \omega^{(0)}$ extracts energy for the mean flow and feeds $u^{(0) 2 / 2}$ kinetic energy leading to its growth on a slow time scale $\tau=E \boldsymbol{t}$.

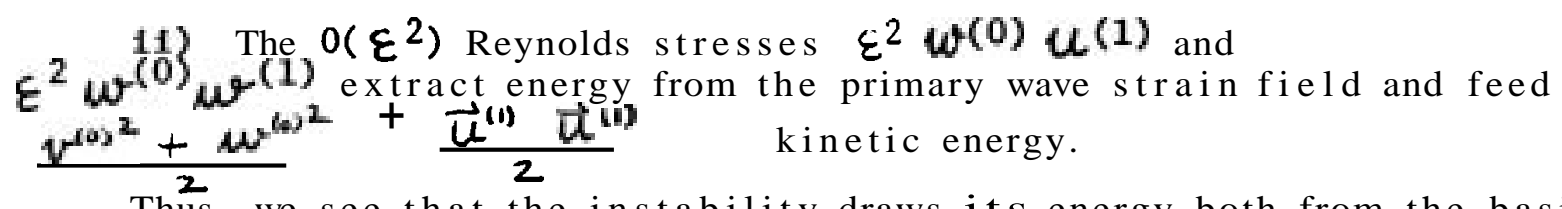

Thus, we see that the instability draws its energy both from the basic mean flow and the primary wave field. It is important to note that in a description of the nonlinear development of the instability, one would have to explicitly account for the energy drained from the wave field.

\section{Discussion}

We have successfully analyzed a mechanism which drives longitudinal vortices through a nonlinear interaction of surface gravity waves with a strong shear flow. The instability mechanism has its origin in a vortex force term which shows its relationship to the instability mechanism of the Langmuir circulations on a weak shear flow. There is, however, an important difference. In the weak shear case, one can proceed to the nonlinear equations governing the instability without having to worry about the drain of energy from the primary wave field. This is no longer possible when the mean flow shear is strong. A systematic computation of the nonlinear development of the present instability would reveal its potential ability to transport momentum and heat downwards.

It seems that the instability mechanism we have explored is likely to be of significance to more general flows than we have analyzed in the present work. The generalization of present analysis to an arbitrary shear flow is straightforward in principle. As a matter of fact, the present work was initiated with such a motivation - but after developing a general set of equations it seemed appropriate to focus on a simple case first. For a general shear flow, one is faced with a more demanding algebraic and numerical task. The primary waves are governed by the Rayleigh equation for the inviscid problem. These waves are rotational with the result that additional terms arise in the equations which govern the evolution of the primary wave distortion and streamwise vorticity. The influence of these additional terms on the instability mechanism explored here is unknown at the present time. Nonetheless it is plausible to suppose that there would be circumstances where these additional terms only lead to a modification of the present instability.

\section{Conclusions}

1. An instability mechanism is established which drives longitudinal vortices through a nonlinear interaction of surface gravity waves with a strong shear flow.

2. The longitudinal vortices which arise have an essential anisotropy in their structure with the windward anomaly much larger than the cross wind anomaly. We note here Langmuir circulations on a weak shear flow do not possess this feature. 
3. The time scale of the present instability is much faster than the time scale for the Langmuir circulation formation on a weak shear flow.

4. The instability is essentially inviscid and its growth rate decreases as the static stability of the basic flow increases or as the shear of the basic flow is decreased.

5. It is conjectured that the present instability mechanism may be of significance in more general shear flows.

\section{ACKNOWLEDGMENTS}

The author wishes to thank the entire staff of the 1983 G.F.D. program at Woods Hole for providing a stimulating environment when the present work was done. He is indebted to Professors Louis Howard, Francis Bretherton, and Willem Malkus for many inspiring suggestions and discussions.

\section{REFERENCES}

Craik, A.D.D., 1968. Resonant gravity wave interactions in a shear flow. J. Fluid Mech., 34, 531-549.

Craik, A.D.D., 1982. Wave-induced longitudinal-vortex instability in shear flows. J. Fluid Mech., 125, 37-52.

Craik, A.D.D., and S. Leibovich, 1976. A rational model for Langmuir circulations. J. Fluid Mech., 73, 401-426.

Leibovich, S., 1983, The form and dynamics of Langmuir circulations. Ann Rev. of Fluid Mech., 15, 391-427.

Leibovich, S., and S.K. Lele, 1982. Thermocline erosion and surface temperature variability due to Langmuir circulations, FDA Rep. 82-07, Sibley School Mech. \& Aerosp. Eng., Cornell Univ., Ithaca, NY.

Leibovich, S., and S. Paolucci, 1980. The Langmuir circulation instability as a mixing mechanism in the upper ocean. J. Phys. Oceanogr., 10, 186-207. 


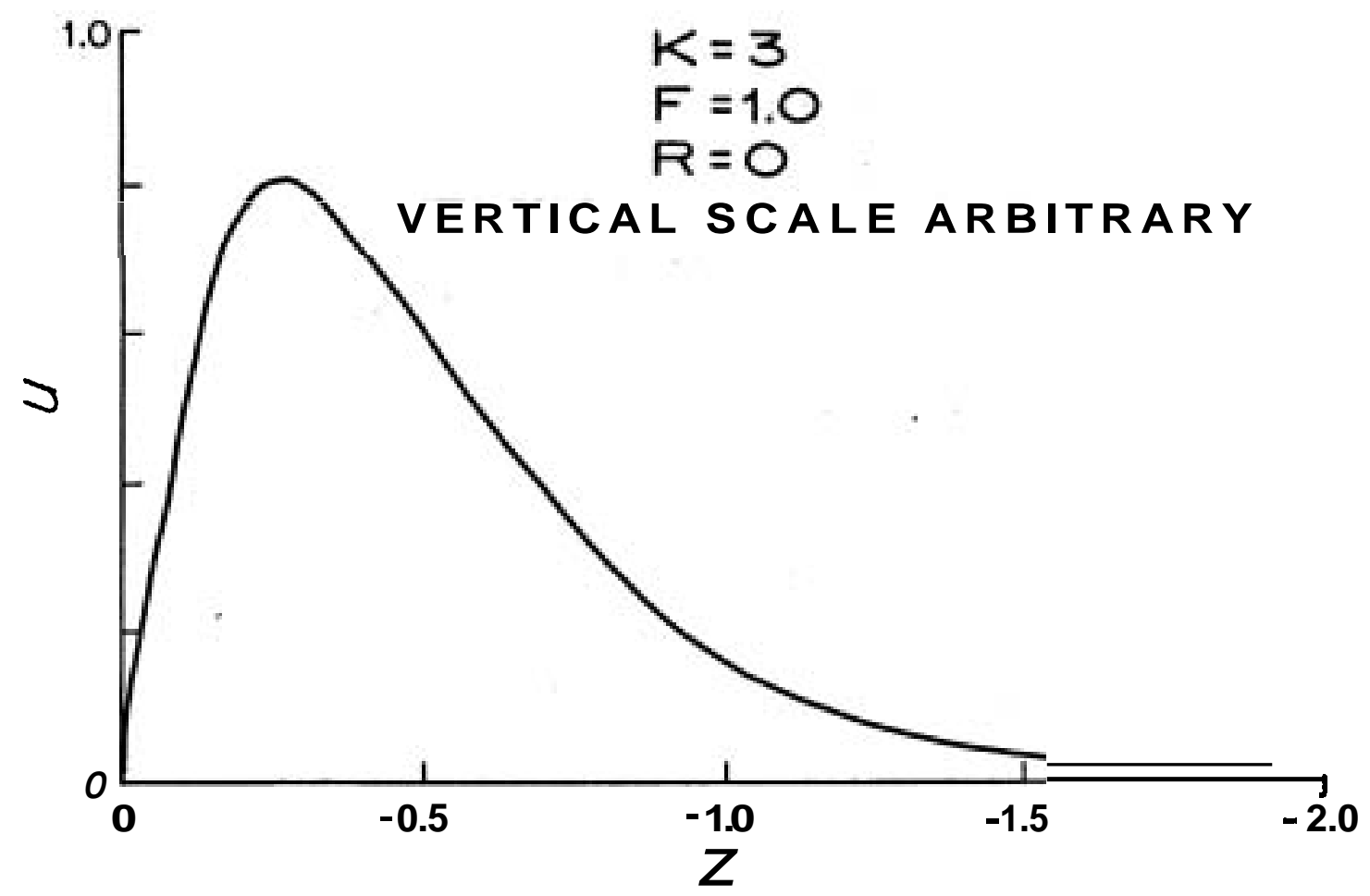

Fig. 1 Vertical structure of windward perturbation.

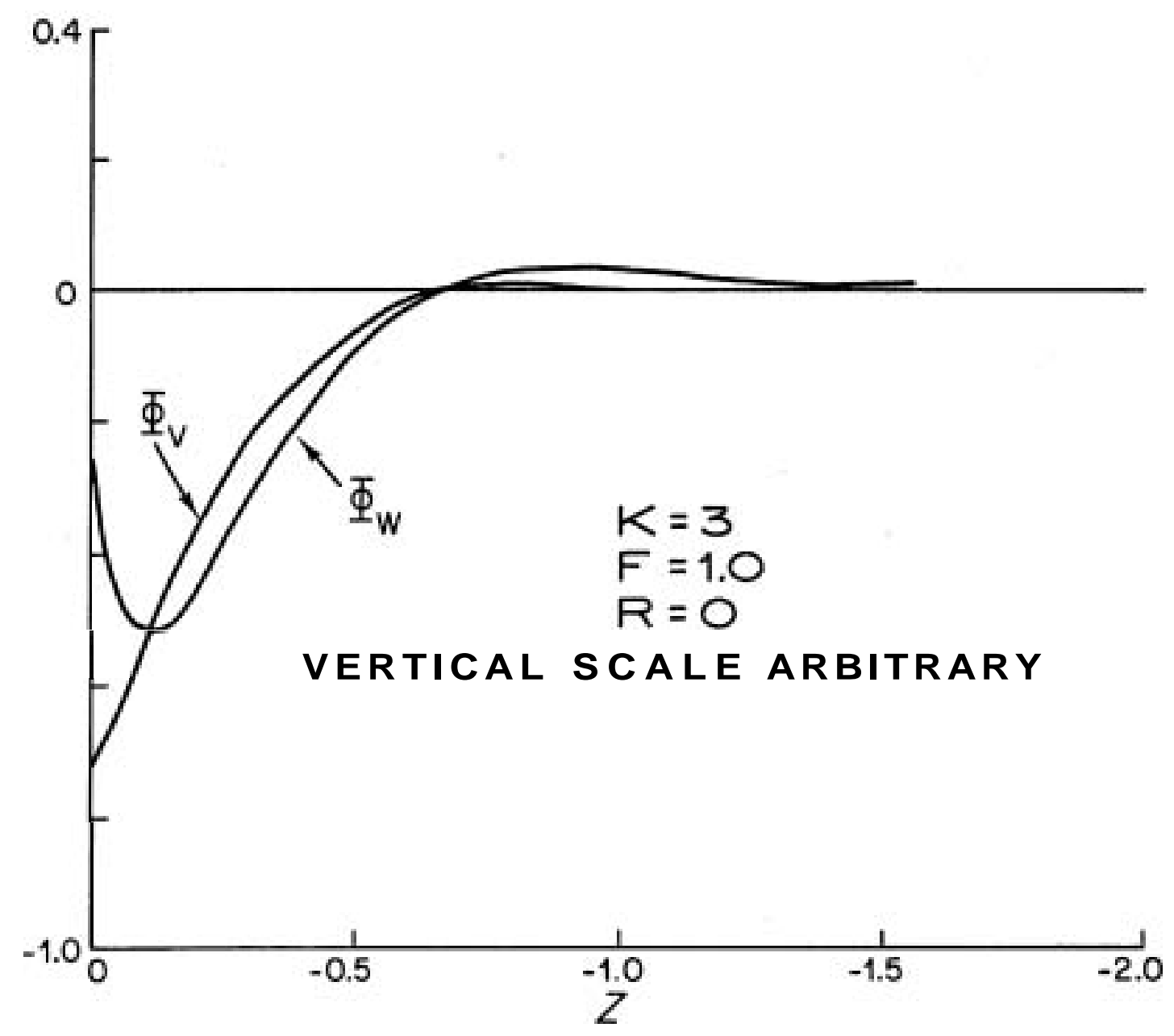

Fig. 2 Vertical structure of the wave distortion. 


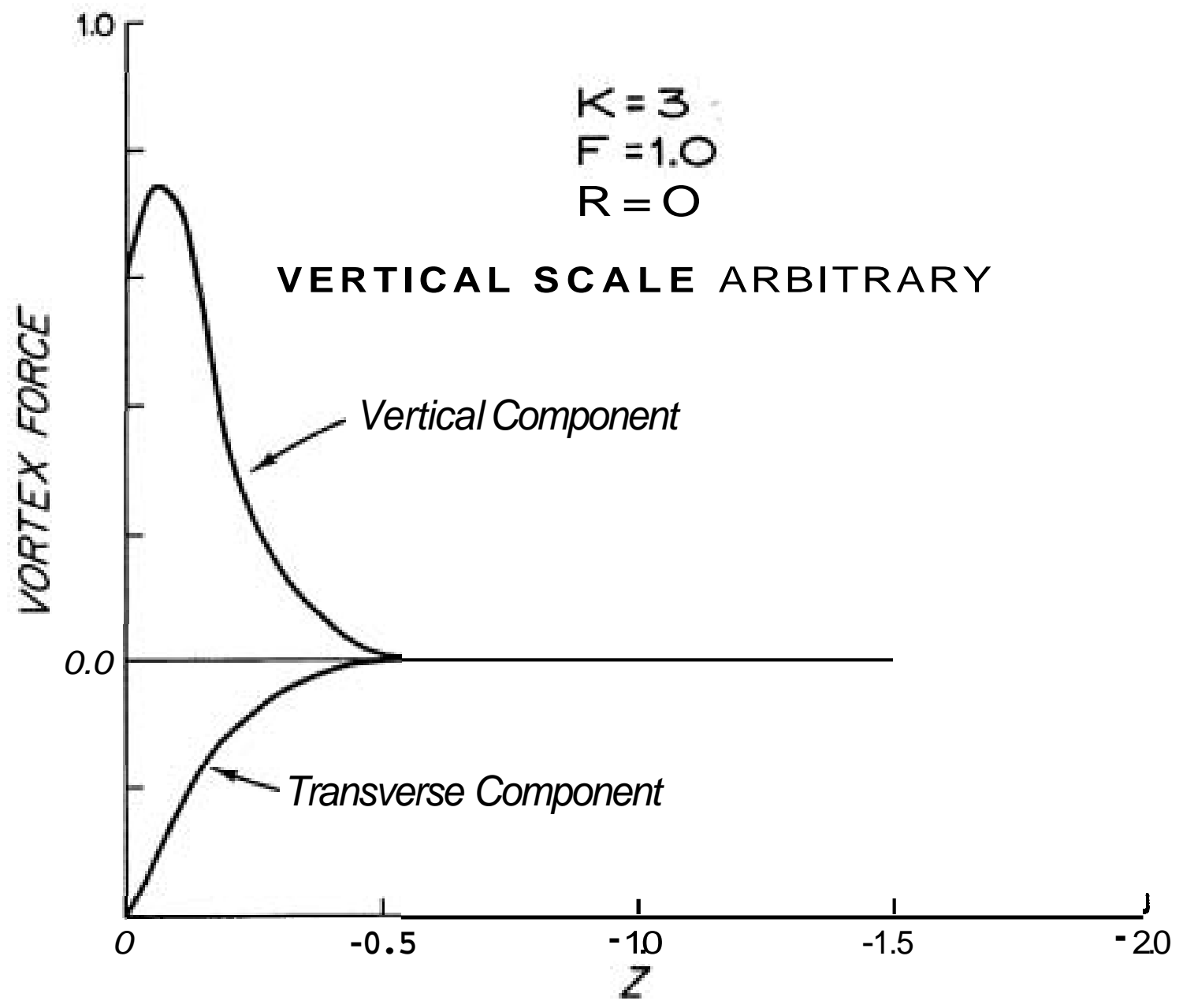

Fig. 3 Vertical structure of the vortex force.

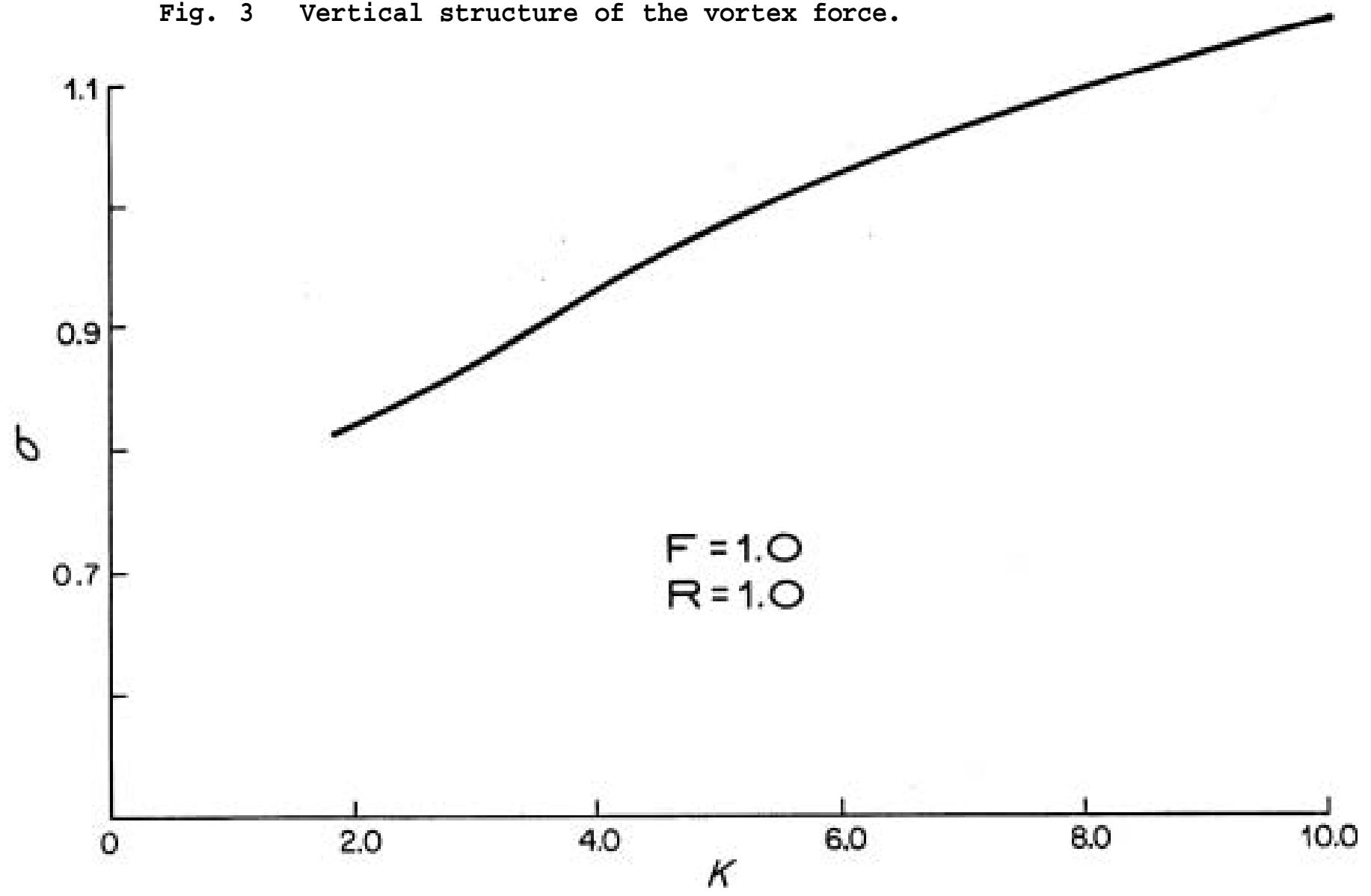

Fig. 4 Dependence of the growth rate of the first mode on the wave number of the perturbation 


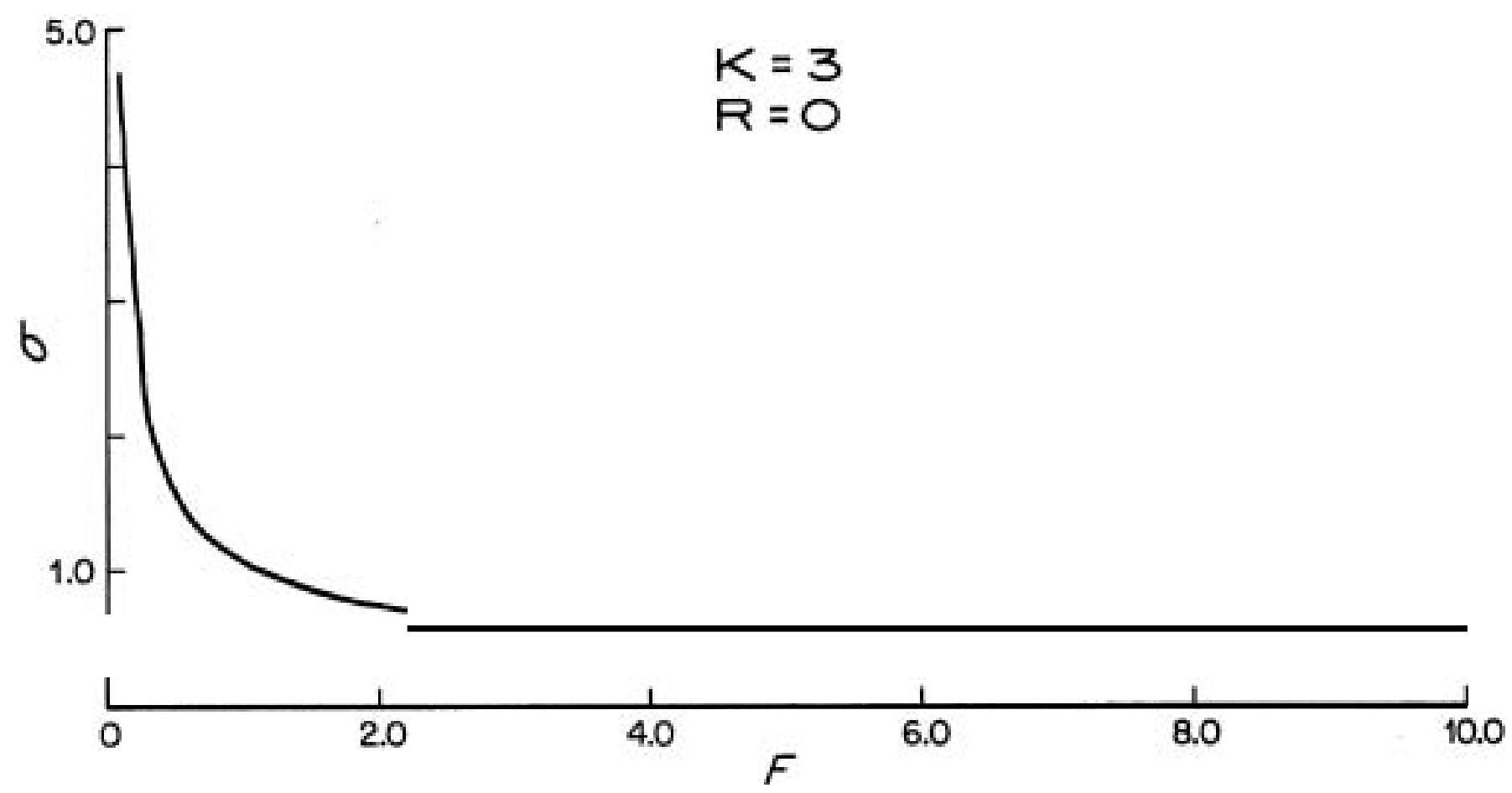

Fig. 5 Dependence of the growth rate on the nondimensional shear of the mean flow.

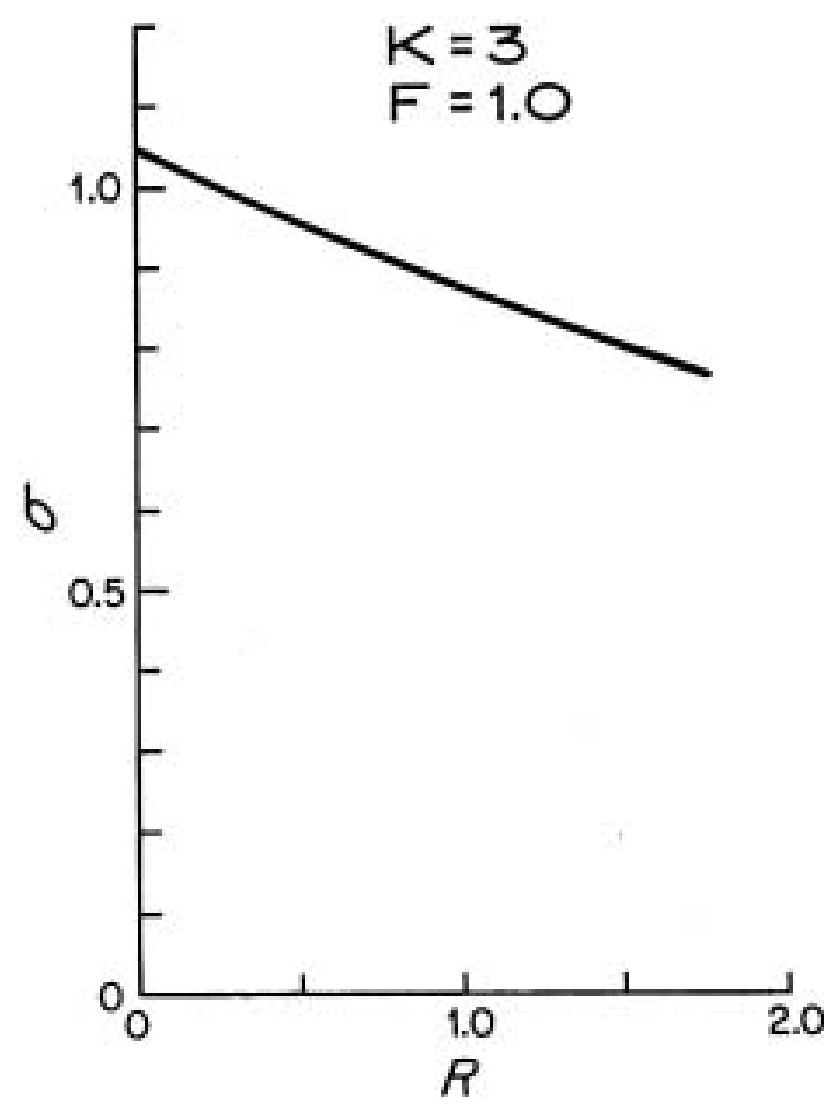

Fig. 6 Dependence of the growth rate on the static stability of the mean flow. 


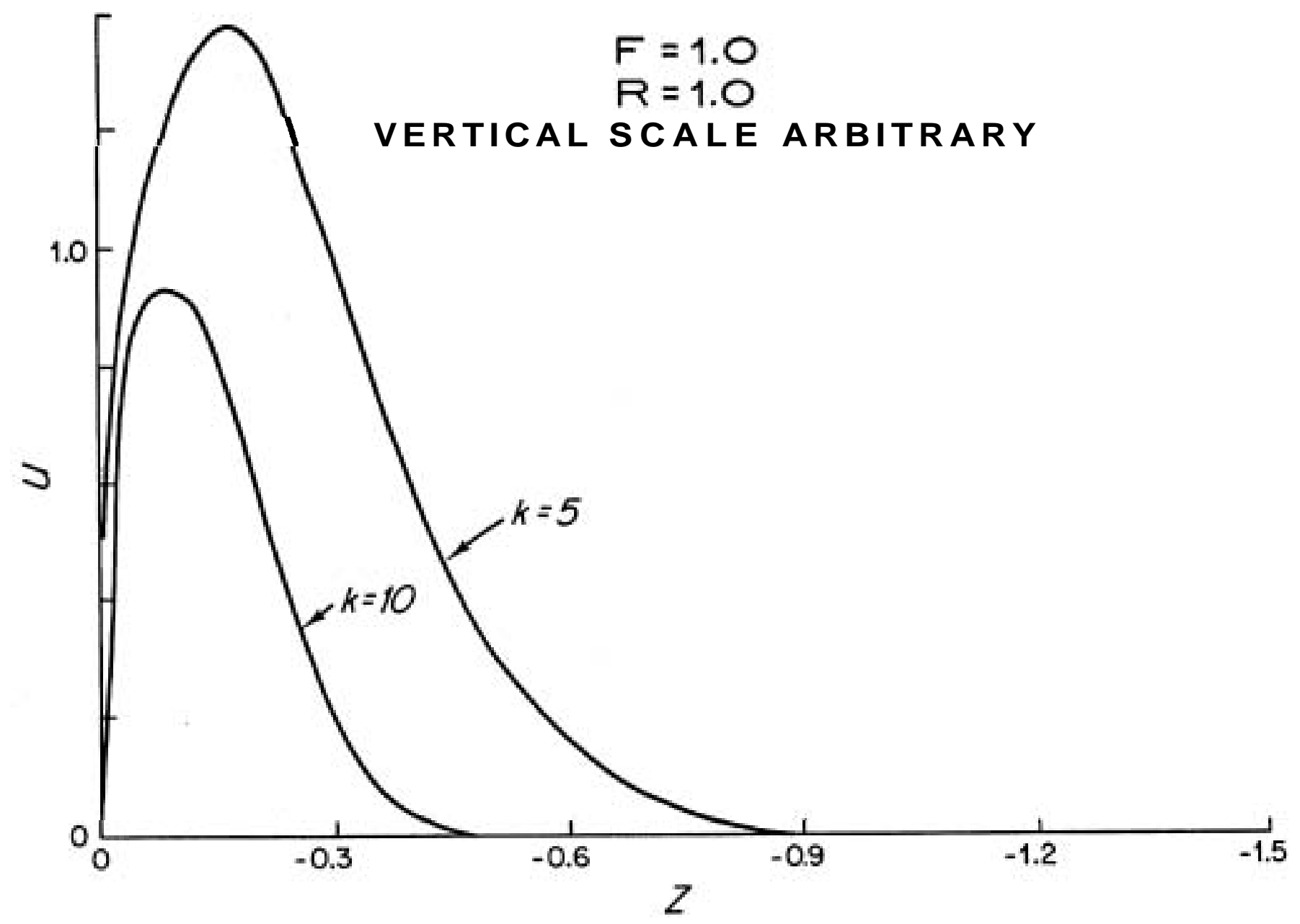

Fig. 7 Dependence of the vertical scale of the eigenfunctions on the wavenumber of the perturbation. 
FREE JET AND FRONMGENESIS EXPERIMENTS IN SHEAR FLOW

Sergey I. Voropaev

\section{Introduction}

The problem of the development of a free round jet intruding into a homogeneous or linearly stratified fluid was investigated experimentally in Voropaev (in press). The Reynolds number in this experiment was equal to $\operatorname{Re}=U D / \rightarrow \quad 10^{2}(U=$ mean velocity at the exit of nozzle, $D=$ diameter of nozzle, $\boldsymbol{\gamma}=$ viscosity of fluid). The main result of the paper is as follows. Just immediately after the jet starts, a spherical eddy forms on its front edge. In a homogeneous fluid the cross size $Y$ of the eddy increases with the distance $\bar{X}$ from nozzle (Figure 1). In a stratified fluid this eddy rapidly collapses and becomes two-dimensional (Figure 2). According to- the experimental data from (1)

$$
\begin{aligned}
& X=\left(I t^{2} / \rho\right) \phi\left(I / \rho \nu^{2}\right) \psi(N t) \\
& Y=\Phi\left(I / \nu^{2} \rho\right) \Phi
\end{aligned}
$$

where $\phi, \psi, \Phi$ are some simple power low dependent functions, $I=\pi D^{2} V^{2} \rho / 4$ is the momentum of the jet, $U=4 Q / \pi D^{2}, Q$ is the volume flux rate through the nozzle, $P$ is the density, $N$ is Brunt-Vasaila frequency of the surrounding fluid. These formulas, relating $I$ to $Q$, assume a uniform velocity (U) emerging from the nozzle.

In this paper we continued the measurements which were begun in Voropaev (in press). Additionally, we simplified the experimental procedure that permits us to test the conclusions of Stern's (1983) theory for propagation of disturbances on the jet: weak disburbances, when the flux rate $\mathbf{Q}=$ const and strong disturbances, when $\mathbf{Q}$ rapidly increases from Q- to Q+. In Stern (1983) it was shown that the propagation velocity of weak disturbances is equal to the velocity on the jet axis, and propagation velocity of strong disturbances is equal to

$$
v_{0}=\left(v_{-}+v_{+}\right) / 2
$$

where $V_{-}, V_{+}$are velocities on the jet axis before and behind the strong disturbance. We tested this conclusion in an indirect way, using the following assumption. The velocity $V$ on the jet axis for a steady jet from a point source of momentum located at $x=0$ is (3)

$$
\bar{V}=3 / 8 \pi \quad\left(I / p_{\rho}\right) \frac{1}{\bar{x}}
$$

From (4) for position of some particle on the axis which at $\mathbf{t}=0$ was $\mathrm{x}=0$ we have

$$
\bar{x}=(3 / 4 \pi)^{1 / 2}\left(I t^{2} / \rho\right)^{1 / 4}\left(I / \rho \nu^{2}\right)^{1 / 4}
$$


It follows from the above assumptions that the measured position $\mathrm{x}$ of weak disturbances should be equal to $\vec{x}$.

Figure 4)

For the case of strong disturbances we have from definitions (see

$$
V_{-}=d x_{-} / d t, V_{0}=d x_{0}\left|d \tau, \quad V_{+}=d x_{+}\right| d t
$$

So from (4) for any arbitrary $\mathbf{x}_{0}$ we have

$$
\frac{d x_{0}}{d t}=\frac{1}{2}\left(d x_{-} / d t+d x_{+} / d t\right)
$$

Integrating (7) we have

$$
\mathrm{X}_{\mathrm{O}}=\left(\mathrm{X}_{-}+\mathrm{x}_{+}\right) / 2+\text { const }
$$

where const $=0$, because $X_{-}, X_{0}, X_{+}=0$ for $t=0$.

2. The Apparatus and Technique

The scheme of the experiment is shown in Figure 3. The working fluid was distilled water with a little amount of Thimol-Blue indicator. The color of this indicator strongly depends on the $\mathrm{Ph}$ of the water. In acid medium the color is light yellow; in alkaline it is dark blue. We added one drop of HC1 to the water in the tank and one drop of $\mathrm{NaOH}$ solution to the water supply from the nozzle. After each experimental run we simply mixed the water in the tank and it became almost transparent again. The method for increasing the flow rate is as follows: At the beginning of each run (see Figure 3) stopcock \#5 was closed and stopcock f 4 was partly opened. After the stopcock 5 was fully opened, the jet with a round eddy at its front edge started from the nozzle. The flux rate at this stage of the experiment was equal to Q-

Then we made a slight strike to the nozzle so that a weak disturbance appeared on the jet (see Figure 5). After that we increased the flux rate to $Q_{+}$by fully opening the stopcock (\$4) and eventually a strong disturbance formed on the jet (Figures 4 and 5). Then we again excited a weak disturbance on the Q+ flow. The experimental data discussed in this papen were obtained as the result of an analysis of the motion pictures. From the movies we obtained the motion of the shock and the positions of weak disturbances immediately before and after the shock wave. The starting time $\mathbf{t}=0$ for every run was determined with error $+1 / f$, where $f=12$, frame $\mathbf{s}^{-1}$ is the filming rate. The error in the measurements of the position of the disturbance was approximately t0.1 cm (correction for parrallax was made). To provide a better comparison of the behavior of the experimental points with theoretical dependences we corrected the measured $x$ values in terms of a virtual origin located at distance $x$ (Figure 4) behind the nozzle. This origin corresponds to the point source in the theory for Eq. (4), and the nozzle correction as given in (4) Is

$$
\Delta x=Q / 8 \pi \rho \nu
$$

3. Experimental Results

The experimental data were obtained in eight series of measurements ( see Table 1). 


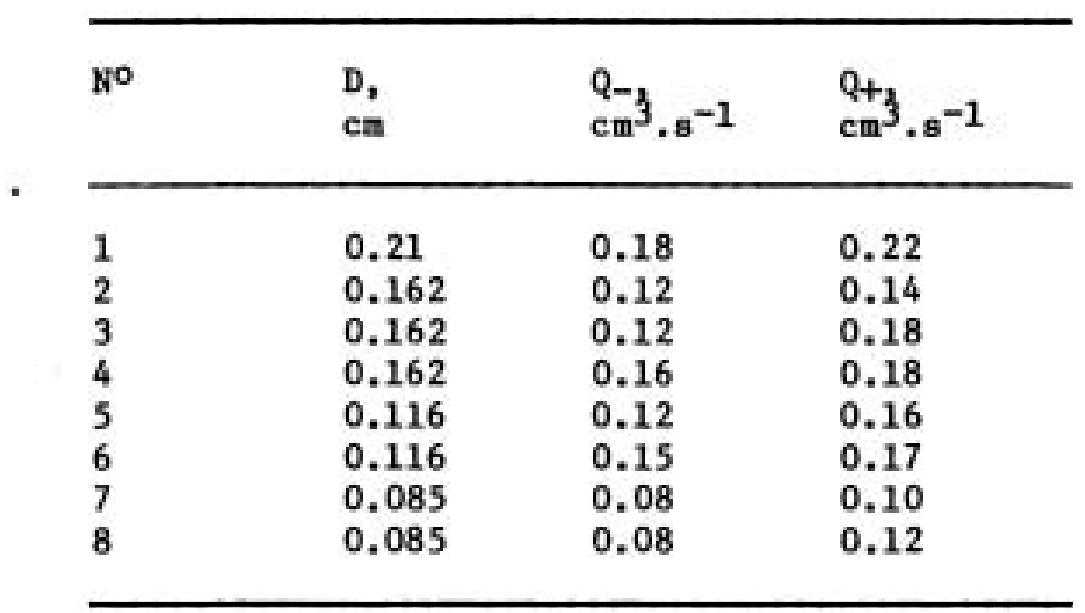

Table 1

Results for Leading Eddy in Figure 4

The experimental data for the cross size $Y$ and length $X$ are shown in Figure 6. It is seen that points 1 ie well along a straight line.

$$
\mathrm{Y}=\mathrm{c} \mathrm{X}
$$

The statistical analysis of the points in Figure 6 gave $C=0.14 \pm 0.02$ with correlation coefficient $\mathbf{r}=0.96$.

\section{Propagation of Disturbances}

As mentioned previously, the propagation velocity on the undisturbed jet mentioned above was measured by introducing a small disturbance and following the position $\mathrm{X}$ 二 of some characteristic signature of the dye pattern (e.g. point b in Figure 4 ) which indicated the group velocity. The experimental $x_{+}$values were obtained in a similar way from small disturbances (symbolized by $b^{\prime}$ in Figure 4) after the flow was increased from Q - to Q+ In Figures 7 and 8 these experimental values $\left(X_{4}\right)$ for the weak disturbances are compared with values $\left(X_{+}\right)$calculated from (5). It is seen that experimental points are in good agreement with calculated values. A statistical analysis of the 232 points in Figure 8 gave

$$
x_{\underline{4}}=A_{1}+C_{1} x_{\underline{H}}
$$

where $\mathbf{A}_{1}=0.3 \mathrm{~cm}, \mathbf{C}_{1}=0.96$ for $\mathbf{r}=0.994$.

5. Propagation of the Strong Disturbance

Figure 5 shows the completely different signature of the strong disturbance which forms in between the $Q_{-}$and $Q_{+}$flow. The position of $x_{0}$ of the nose of the shock was determined from the leading edge of the dye pattern labeled 2 of Figure 5. The three sets of data were reduced according to (8). Figures 9 and 10 show the results for one run and Figure 10 for a 11 runs. A statistical analysis gave

$$
\mathrm{X}_{\mathrm{o}}=\mathrm{C}_{2}+\mathrm{A}_{2}\left(\mathrm{X}_{-}+\mathrm{X}_{+}\right) / 2
$$

where $C_{0}=0.25, \quad A_{2}=.1 .008$ for $r=0.998$. 
So, the experimental results are in agreement with the main conclusions of Stern's (1983) theory.

\section{ACKNOMLDGMENIS}

I wish to thank Melvin Stern, who suggested that I make this experiment, Bob Frazel, who helped me every day, and Louis Howard for helpful discussions.

\section{REFERENCES}

1. Voropaev, S.I., I.A. Fillipov, in press. Horizontal jet in stratified and homogeneous fluid; laboratory experiment. Izvestya Academy of Sciences of the USSR, Atmospheric and Ocean Physics.

2. Stern, M.E., 1983. Vorticity frontogenesis in shear flow (abstract). This volume.

3. Goldstein, S. (ed.), 1950. Moden Development in Fluid Dynamics, Vol. 1, Oxford at the Clarendon Press, 148.

4. Batchelor, G.K., 1920. An Introduction to Fluid Dynamics, Cambridge University Press, 211. 

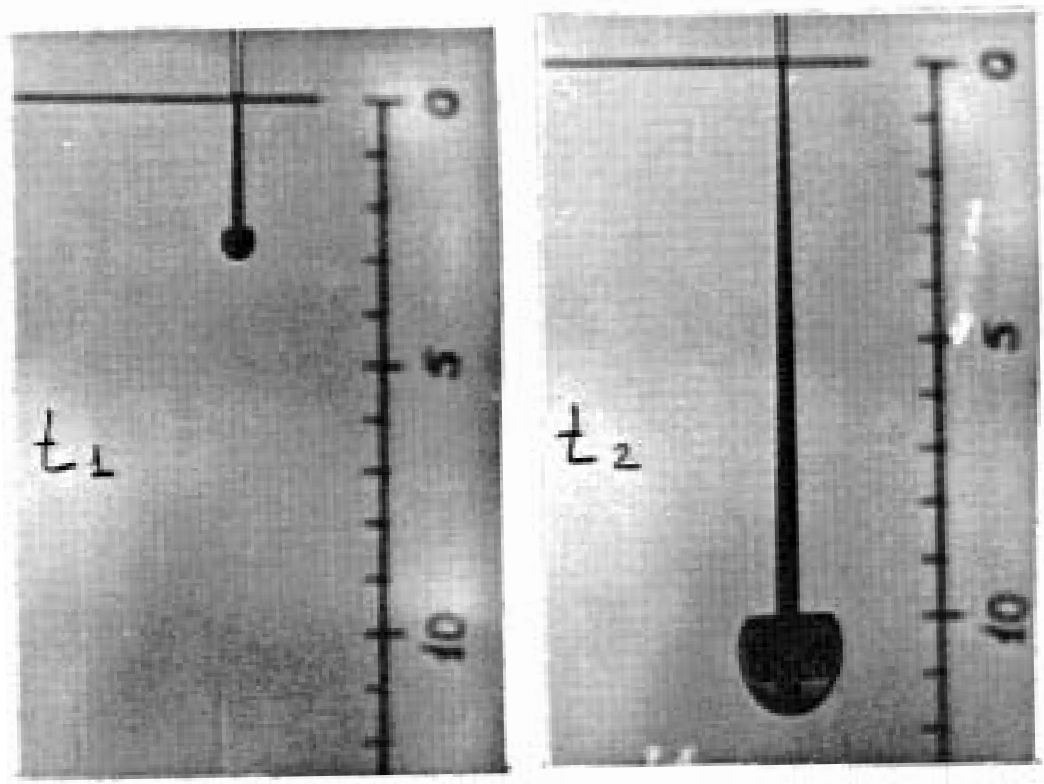
Fig. 1. The jet front in homogeneous fluid: $t_{1}<t_{2}, D=0.162 \mathrm{~cm}$,
$Q=1.6 \cdot 10^{-1} \mathrm{~cm}^{3} \cdot \mathrm{s}^{-1}$
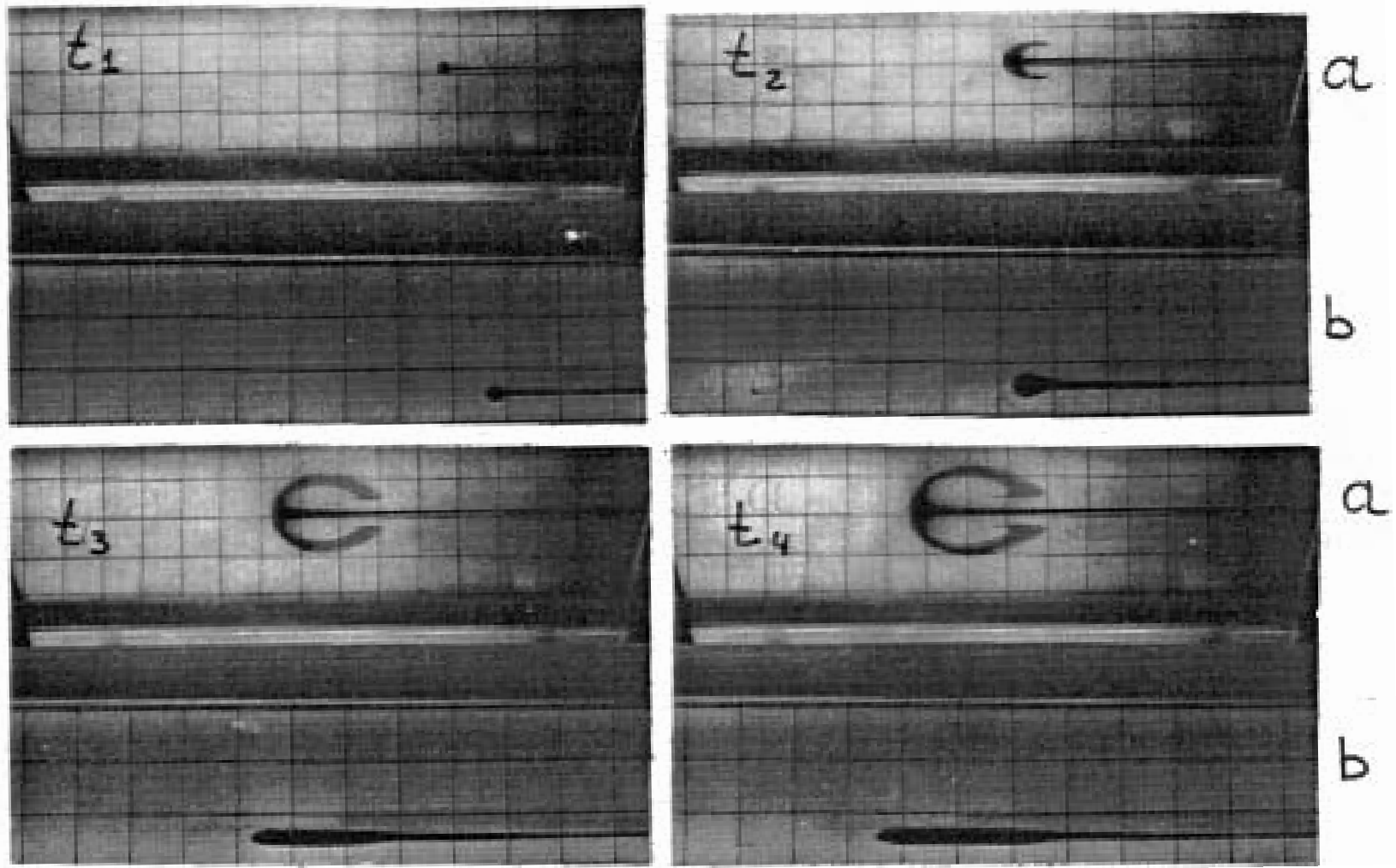

Fig. 2. The jet front in stratifjed fluid: a - top view; b - side view; $t_{1}<t_{2}<\frac{\mathrm{c}}{4}, \mathrm{~N}=0.78 \mathrm{~s}^{-1}, \mathrm{D}=0.058 \mathrm{~cm}, Q=4 \cdot 10^{-2} \mathrm{~cm}^{3} \cdot \mathrm{s}^{-1}$. 


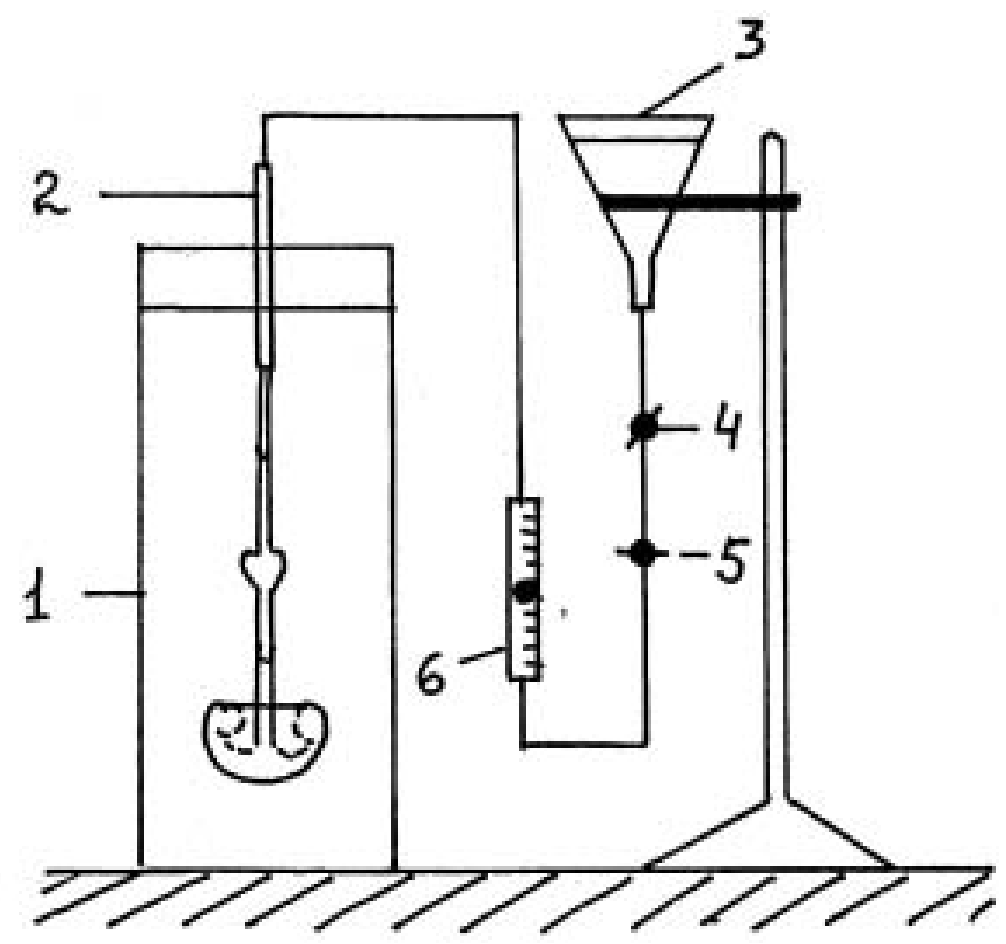

Fig. 3. Scheme of experiment: 1 - tank; 2 - nozzle; 3 - water supply; 4,5 - stopcock, 6 - flow meter.

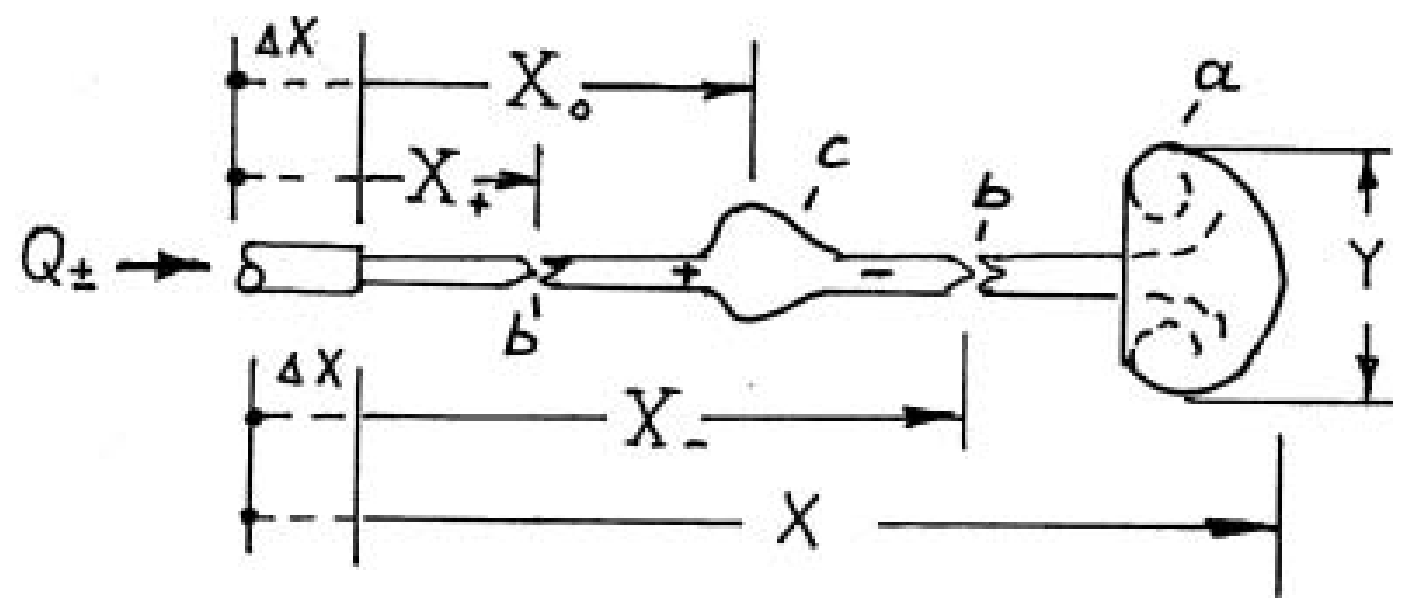

Fig. 4. Scheme of measurements: $a$ - front; b - weak; and $c$ - strong disturbance. 


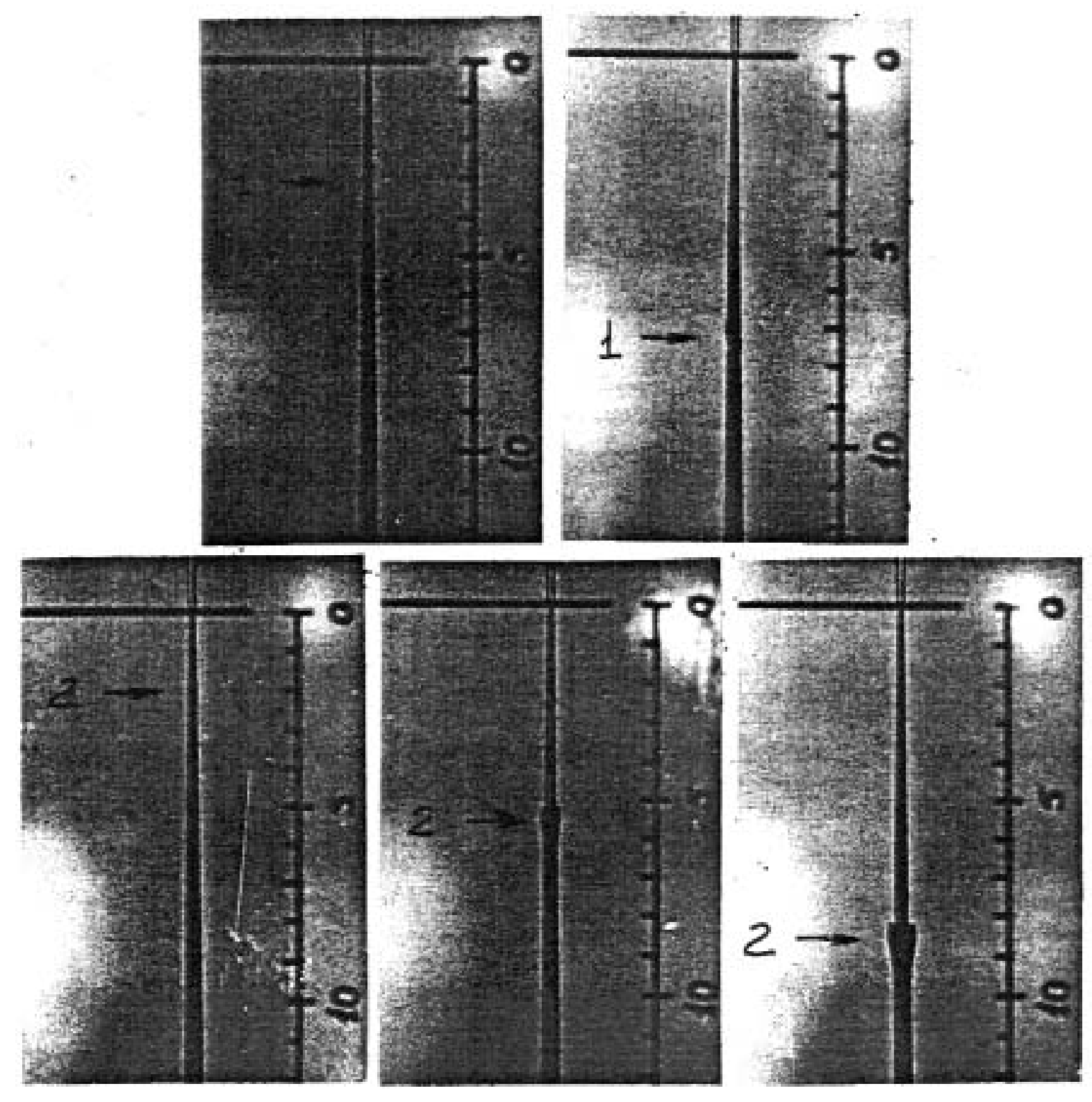

Pig. 5. The weak (1) and strong (2) disturbances on the jet (scale in $\mathrm{cm}$ ). 


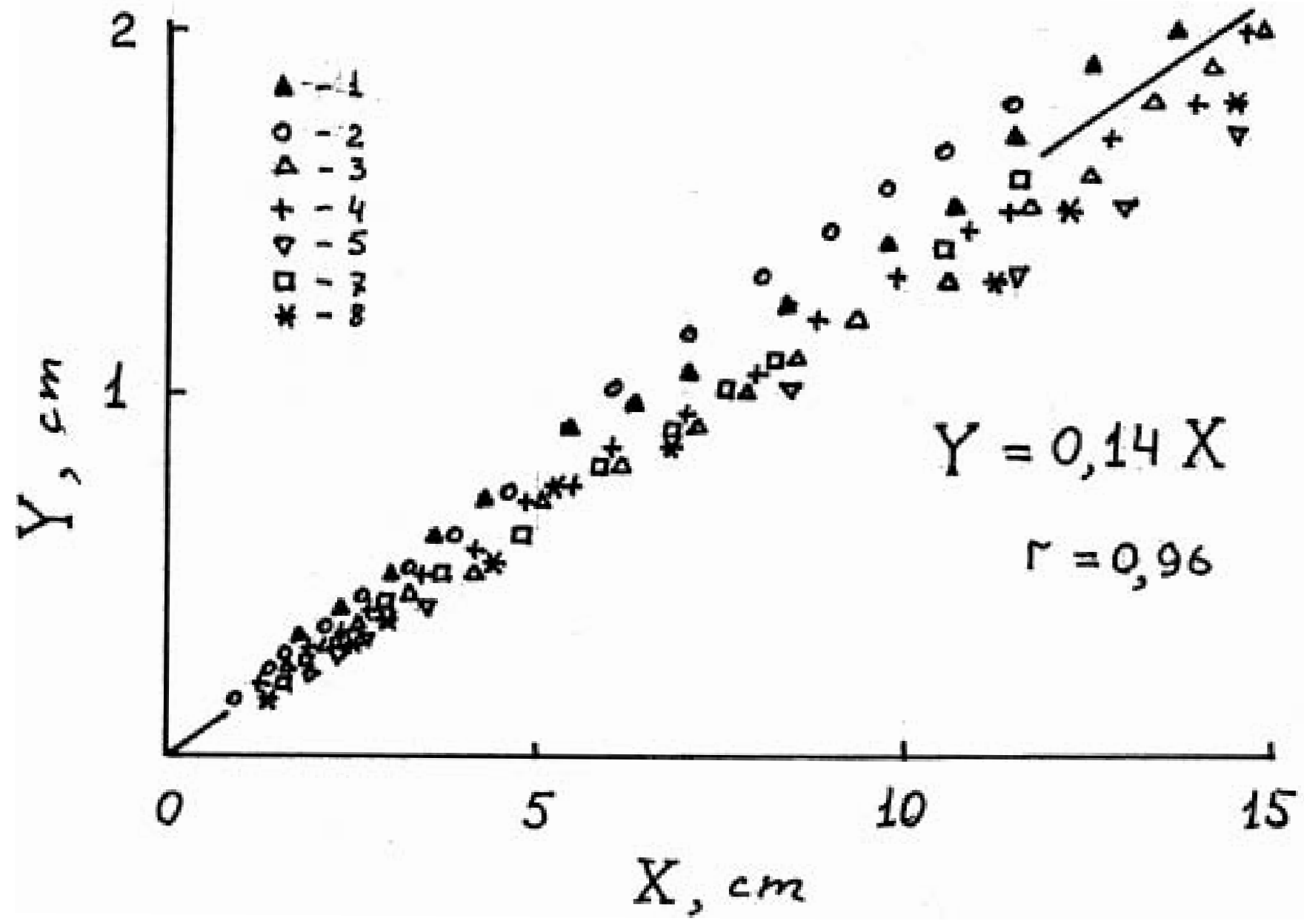

Fig. 6. The dependence $Y$ from $X$ : figures - numbers of runs in table. 



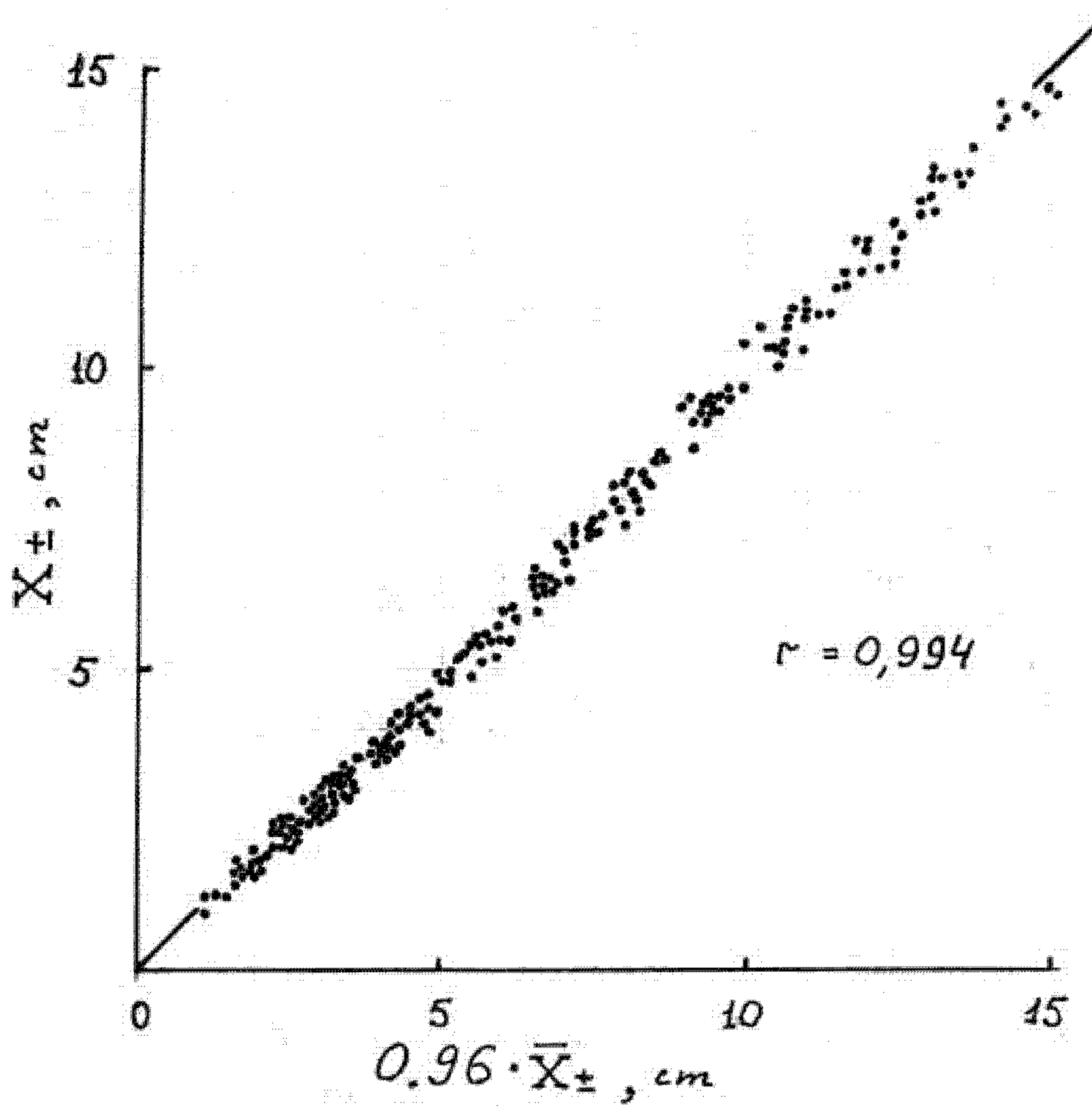

Fig. 8. The measured $\left(x_{1}\right)$ and calculated $\left(\bar{x}_{ \pm}\right)$values of $x_{ \pm}$ for all experiments. 


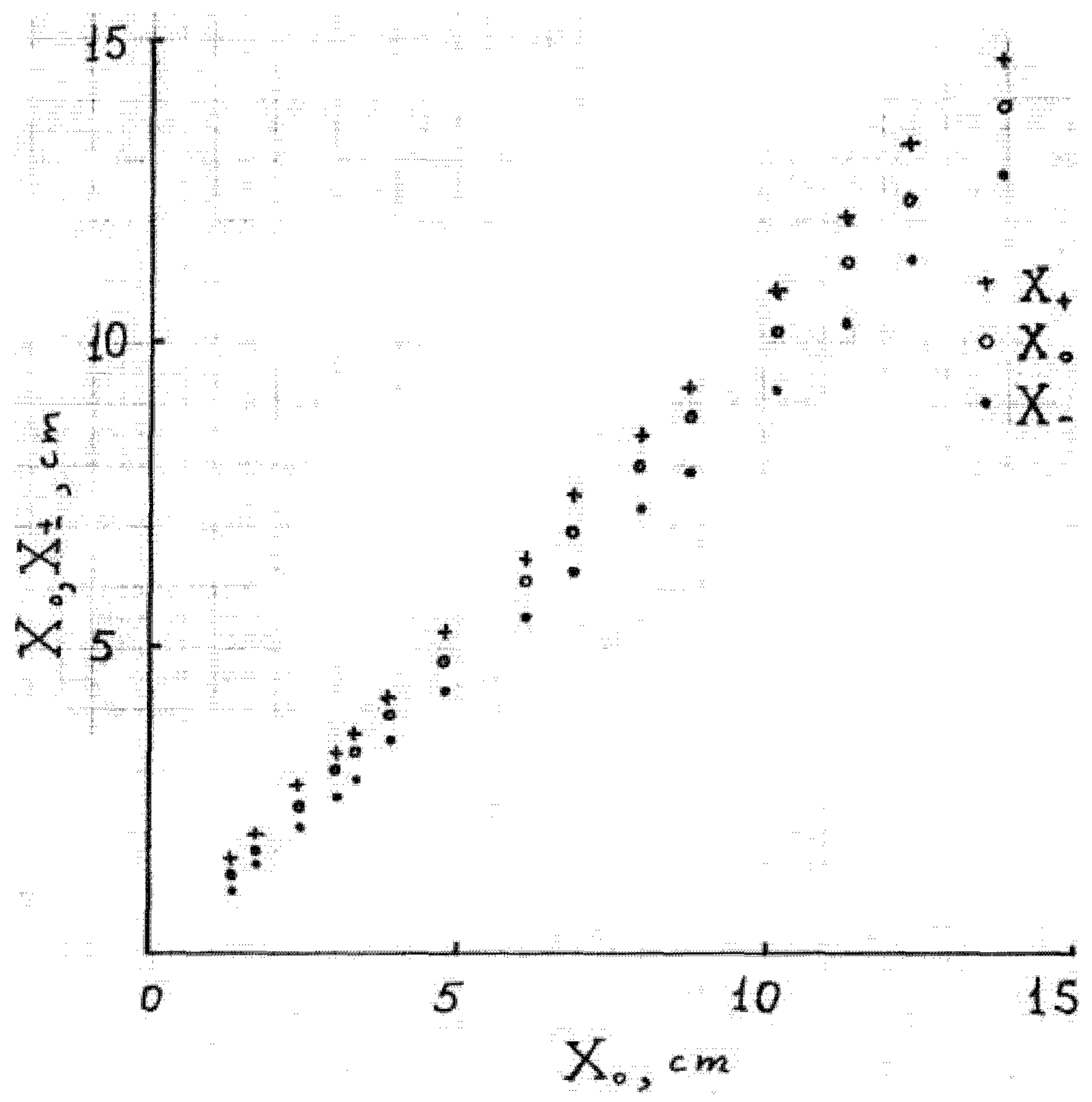

Fig. 9. The values of $x_{ \pm}$and $x_{0}$ for experiment $N 4$. 


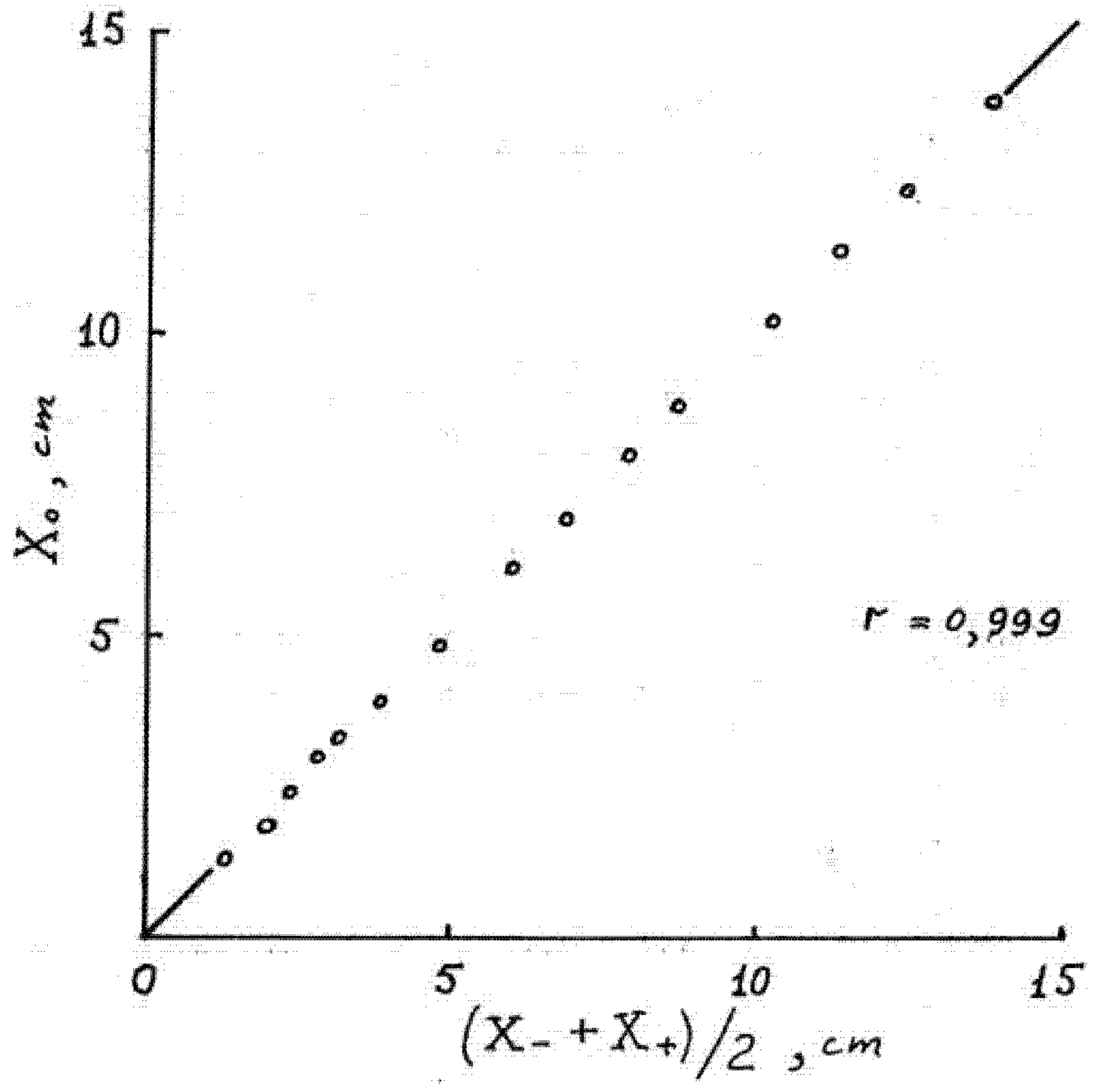

Fig. 10. The measured $\left(\mathrm{X}_{0}\right)$ and calculated positions of strong disturbance for experiment N4. 



\title{
WAVES I N A POLYTROPIC ATMOSPIARE
}

\author{
Charles Corfield
}

\section{Introduction}

The present study has been motivated by a problem in solar physics. Acoustic oscillations have been observed on the solar surface since the early 1960's (for extensive reviews of this see Stein \& Leibacher 1974 and 1981; Deubner, 1981). Numerical models calculating the linear eigenmodes using spherical geometry and current models for the structure of the sun yiald results that are in good agreement with the observations. Less clear is the mechanism by which these oscillations are excited, and how they interact with the convective motions at the top of the convection zone (the oscillations are confined mainly to a thin layer above the coavection zone).

The most prominent oscillations have a period of five minutes, wavelengths of $10,000 \mathrm{~km}$ and penetrate $10,000 \mathrm{~km}$ into the sun. In recent high resolution observations, the waves are seen in packets of half a dozen wavelengths (see Figure 1).

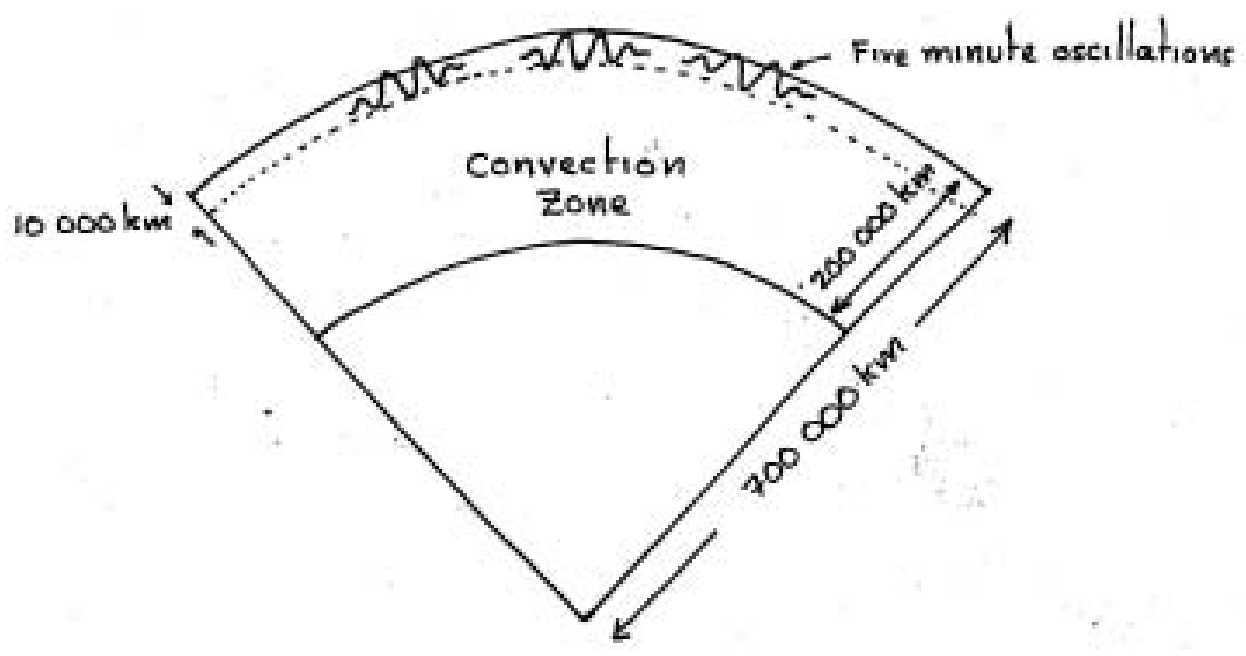

\section{Fig.1. Location of the five minute oscillations in the sun.}

Since the sun has radius $700,000 \mathrm{kr}$, which is much larger than the length scales of the oscillations, spherical effects are small. This suggests that in an analytic treatment of these waves, a plane parallel atmosphere will be a good approximation, at any rate for wavelengths which are not too long. For the sake of tractability I have used an atmosphere, which has a linear (vertical) temperature gradient and constant acceleration due to gravity. Physically this corresponds to having a constant thermal conductivity and constant flux of heat through the layer (Spiegel 1964). However, in the sun the conductivity changes with depth, though not by so much as to vitiate the assumption of a linear temperature grad-tent. Further, it is assumed that ths atmosphere consists of a perfect gas (wizich is a good approximation). In hydrostatic equilibrium we have pressure $(p)$, density $(p)$ and temperature in the following relation:

$$
\begin{aligned}
& \rho \propto T^{m} \text { and } P \propto T^{m+1} \\
& \text { where } m=\frac{g}{k \beta_{0}}-1 \text { and } \beta_{0}=\frac{d T}{d z}
\end{aligned}
$$


where $m$ is the the "polytropic index". Any system which satisfies (1.1) is known as a "polytrope". The polytropic atmosphere was used by Lamb (1908) to discuss long atmospheric (gravity) waves. Spiegel and Unno (1962) used it to estimate convective growth rates for unstable gravity waves of large wave number. Spiegel (1964) went on to investigate the effects of radiative dissipation on the convective growth rates and found an acoustic mode which became overstable (i.e. growing amplitude) when he assumed a cooling rate proportional to the temperature fluctuation (Newtonian cooling). Physically, this case corresponds to disturbances whose length scale is much less than the photon mean free path; such disturbances are "optically thin". In the other limit, where the photon mean free path is much less than the length scale of the disturbances, the cooling rate ( $Q$ ) is given by

$$
Q=\kappa \nabla^{2} \theta
$$

where $\mathrm{K}$ is the thermal conductivity and $\boldsymbol{\theta} \mathbf{1 s}$ the temperature perturbation; these disturbances are "optically thick". There have been several numerical studies of the effects of thermal dissipation in the optically thick and thin limits (Chitre \& Gokhale 1975; Jones 1976; Antia, Chitre \& Gokhale 1977), which a 11 have their problems. Jones (1976) also carried out a semi-analytic treatment by taking the solutions to the nondissipative problem and inserting them into the energy integral for the system to estimate the growth rates of acoustic waves. His results agree with Spiegel's in the optically thin limit and he finds a subadiabatic (1.e. the medium is stably stratified) overstability at the small wave number limit of the acoustic waves. In the optically thick limit his analysis is faulty because he fails to take thermal boundary layers into account. To date there has been no satisfactory analytic derivation of the growth rates in the optically thick limit. In the optically thin case the only analytically derived result is Spiegel's (1964) for the pl-mode (acoustic modes are labelled by an integer $\mathrm{N}$ and the $\mathrm{Nth}$ mode is called the pN-mode, where $N=0,1,2 \ldots$.$) , which is trivially generalizable to$ higher modes, though not to the Lamb wave (the lowest acoustic mode).

In this study I have generalized Spiegel's wave equation (Spiegel 1964) for optically thin disturbances to disturbances of arbitrary optical thickness. The equation is then analyzed in the case of constant cooling coefficient q, so that his analysis may be extended. I have not completed analysis of the optically thick disturbances, which involve thermal boundary layers, so I do not present any results here. I have, however, gone on to obtain amplitude equations for weakly nonlinear disturbances in an isothermal layer and I anticipate that the analysis will go over to the polytropic case.

2. Linear disturbances influenced by radiative dissipation

2.1. Equations of motion

The equilibrium state of the atmosphere is taken to be

$$
\begin{aligned}
& 0=-\frac{d \bar{p}}{d z}+g \bar{\rho} \\
& 0=\frac{d F}{d \bar{z}} \equiv \frac{d}{d z}\left(k \frac{d}{d \bar{z}} \bar{T}\right) \\
& \bar{p}=R \bar{p} \bar{T}
\end{aligned}
$$


Where $F$ is the heat flux through the layer, $K$ is the thermal conductivity, $\mathrm{R}$ is the gas constant, and $\overline{\bar{\rho}}, \bar{\rho}, \overline{7}$ are the equilibrium distributions of the thermodynamic variables. I have taken $\mathrm{K}$ and $\mathrm{F}$ to be constant and following Lamb (1945) I measure $z$ downwards from the top of the layer, so that the temperature gradient is positive in this coordinate. Define

$$
\beta_{0}=\frac{d}{d z} \bar{T}=F / K \quad(>0)
$$

It follows from $(2.1)=(2.4)$ that

$$
\bar{T}=\beta_{0} z, \bar{P} \propto \bar{T}^{m}, \bar{p} \propto \bar{T} m+1
$$

where

$$
m=\frac{q}{k \beta_{0}}-1
$$

Note that $\beta_{0} \rightarrow 0 \Rightarrow m \rightarrow \infty$ which would correspond to an isothermal layer. It is convenient to define

$$
\Gamma=1+\frac{1}{m}
$$

so that $\bar{p} \propto \bar{\rho}^{\Gamma}$. When $\gamma>\Gamma$ the medium is stably stratified and when $\gamma<$ it is unstably stratified.

The linearized equations of motion for disturbances about the equilibrium state are:

$$
\begin{aligned}
& \bar{\rho} \partial t \underline{\mu}=-\nabla p+\rho g \\
& \partial t \rho+w \frac{d \bar{g}}{d \underline{\varepsilon}}+\bar{\rho} \nabla \cdot \underline{u}=0 \\
& \left(\partial t p+w \frac{d \bar{d}}{d}\right)-c^{2}\left(\partial t \rho+w \frac{d \bar{g}}{d}\right)=-R Q(\theta) \\
& \frac{p}{\bar{p}}=\frac{\rho}{\bar{p}}+\frac{\theta}{\bar{p}}
\end{aligned}
$$

where $c^{2}=\frac{\gamma_{\overline{7}}}{\beta}$ is the (adiabatic) sound speed, $\gamma$ is the ratio of specific heats, and $P, \rho, \theta$ are the perturbations of the thermodynamic variables. The third equation is the heat equation which takes into account the leaking of heat due to radiation. The operator $Q$ is in general an integral operator acting on the temperature perturbation - In an homogeneous medium it takes the form:

$$
Q(\theta)=-q \int k\left(1 \underline{x}-\underline{x}^{\prime} 1\right) \theta\left(\underline{x}^{\prime}\right) d^{3} \underline{x}^{\prime}
$$

where

$$
\begin{aligned}
& q=(\gamma-1) 16 \sigma T^{3} \pi \\
& K(|x|)=\pi \frac{\exp (-\psi|x|)}{4 \pi|\underline{x}|^{2}}-\delta(\underline{x})
\end{aligned}
$$

4 is the absorption per unit length and $\mathbf{a}$ is the Stephan Boltzmann constant. (See Spiegel (1960); Spiegel \& Unno (1966) for a discussion of the 
radiative transfer problem.) The integral expresses the balance between the heat radiated away (the $-\delta(\underline{z})$ ) by the fluid parcel and the radiation from other parts of the medium absorbed by the parcel at $x$ (the $\left.x \exp (-*|x|) / 4 \pi|z|^{*}\right)$. The $\left(\left.4 m|x|\right|^{2}\right)^{-1}$ is the geometric attenuation, the $\exp (-\infty|x|)$ is the attenuation due to absorption of the radiation before it reaches $\underline{x}$, and the factor $x$ represents the fraction of radiation arriving at $x$ that is absorbed there. When is small (weak absorption)

$$
Q \simeq q \theta
$$

i.e. when the mean free path of the photons is large we get Newtonian cooling. When is large (strong absorption)

$$
\theta=\frac{-q}{3 x^{2}} \nabla^{2} \theta
$$

i.e when the photon mean free path is small we get diffusive cooling.

The linearized equations can be reduced to one equation in one unknown (see Appendix A), which can be identified as the rate of change of internal energy of the gas multiplied by a normalization factor. The resulting equation is:

$$
\begin{aligned}
\gamma_{m}\left[z D^{2}+(m+2-2 k z) D-2 \alpha k\right] \psi & = \\
& =Q\left[\left[\left[(m+1)\left(\frac{n^{2}}{g}+k+\frac{1}{z}\right)+\left(2 k z-(m+1) D-z D^{2}\right] \psi\right]\right.\right.
\end{aligned}
$$

where $D \equiv \frac{d}{d z}$

$$
\begin{aligned}
& \psi=e^{k z}\left[\nabla \cdot \mu+\frac{1}{c^{2}} R Q(\theta)\right] \propto e^{n t+i k x} \\
& 2 \alpha=\frac{a k}{\hbar}\left[\frac{\beta_{1}}{\beta}-1\right]+\frac{m+1}{\gamma} \frac{n^{2}}{g k}+(m+2) \\
& \beta_{1}=\frac{\gamma-1}{\gamma}, \beta=\frac{r-1}{r}
\end{aligned}
$$

The equation ( 2.17 ) can, in principle, be integrated and the dispersion relation follows from applying suitable boundary conditions. In the case of Newtonian cooling with constant cooling coefficient:

$$
Q(\theta)=q \theta
$$

(where $\mathrm{q}$ is constant) equation ( 2.17 ) reduces to the equation derived by Spiegel (1964) :

$$
\zeta \Phi^{\prime \prime}-\left(m+\frac{\omega}{\sigma+\omega}+2 a \xi\right) \Phi^{\prime}-\left[\frac{2 \mu \sigma+\left(\sigma^{2}-a\right)(m+1) \omega}{\sigma+\omega}\right] \Phi=0 \quad(2.21)
$$


where

$$
\begin{aligned}
\zeta= & z / d, \quad a=k d, \quad \sigma^{2}=\frac{n^{2} d}{g} \\
\omega & =\frac{q}{\gamma(g / d)^{p_{2}}} \\
2 \mu & =\frac{m+\downarrow}{\gamma}\left(\frac{\sigma^{4}-a^{2}}{\sigma^{2}}\right)+m\left(\frac{a-\sigma^{2}}{\sigma^{2}}\right)_{a} \\
\Phi & =\bar{p}\left(\nabla \cdot \underline{\mu}+\frac{g \theta}{\gamma t}\right) e^{k z}
\end{aligned}
$$

and $\mathrm{d}$ is the thickness of the layer. The vertical velocity, w, can be written as

$$
\bar{p} w=\frac{\gamma e^{-a \xi}}{g\left(\sigma^{4}-a^{2}\right)}\left[\sigma^{2} \Phi^{\prime}+a\left(a-\sigma^{2}\right) \Phi\right]
$$

The boundary conditions that I have used are

$$
p w=0 \text { at } 5=0,1
$$

The solution of ( 2.21 ) which satisfies the top boundary condition is

$$
\Phi=(2 a 5)^{1-B} M(A-B+1 ; 2-8 ; 2 a 5)
$$

where

$$
\begin{aligned}
& A=\frac{\mu \sigma+\frac{1}{2}\left(\sigma^{2}-a\right)(m+1) \omega}{a\left(\sigma^{2}+\frac{\omega}{\omega}\right)} \\
& B=-m-\frac{\omega}{\sigma+\omega}
\end{aligned}
$$

and $M(a, b, x)$ is a confluent hypergeometric function which has the following power series representation

$$
M(a, b, x)=1+\frac{a}{b} \frac{x}{1 !}+\frac{a(a+1)}{b(b+1)} \frac{x^{2}}{2 !}+\frac{a(a+1)(a+2)}{b(b+1)(b+2)} \frac{x^{3}}{3 !}+\cdots
$$

which converges for all finite $x$, provided $b$ is not a negative integer or zero. On substituting (2.28) into ( 2.26 ) and applying the boundary condition at $\mathbf{5}=1$ the following dispersion relation is obtained (see Appendix $B$ ).

$$
\frac{1}{\sigma^{4}-a^{2}}\left[\sigma^{2}(b-1) M(a, b-1,2 a)+a\left(a-\sigma^{2}\right) M(a, b, 2 a)\right]=0
$$

where

$$
\begin{aligned}
& a=A-B+1 \\
& b=2-B
\end{aligned}
$$

(In this form it is easier to handle than Spiegel's version). 
In what follows I have assumed that

$$
\omega \ll \sigma
$$

1.e. the cooling time due to dissipation is much longer than the period of the oscillation. I have consigned the mathematical nitty gritty to Appendix C. Further, since $\omega$ is small, it is possible to write $\sigma$ as

$$
\sigma=\sigma_{0}+\omega \sigma_{1}+\cdots
$$

2.2. The large wave number $1 \mathrm{i} \mathrm{m} \mathrm{it}$

The dispersion relation (2.32) is satisfied by

$$
\sigma_{0}^{2} \simeq-a \text { or } a=-N, N=0,1,2, \ldots
$$

The same would be obtained if the rigid bottom boundary was replaced by a radiation condition that the wave should decay sufficiently fast as $z \rightarrow \infty$. $\sigma_{0}^{2} \approx-a$ is the Lamb wave, which in dimensional units has the dispersion relation

$$
\Omega^{2}=g k
$$

where $\Omega$ is the frequency. It is interesting to note that: ( 2.38 ) is independent of the stratification (i.e. no dependence on $\mathrm{m}$ ). I have compared this result to solar observations (see fig 2 ; the Lamb wave is the lowest of the parabolic arcs) and $I$ find from some measurements taken from the figure that

$$
\Omega^{2} / k \simeq 268 \mathrm{~ms}^{-2}
$$

compared with the actual value of the surface gravity which is

$$
g_{\odot}=274 \mathrm{~ms}^{-2}
$$

The Lamb wave is an acoustic wave whose motions are almost entirely horizontal and corresponds to the fundamental mode in a conventional wave guide (which has no transverse component to its velocity field). The Lamb wave is damped :

$$
\sigma_{1}=-\frac{m+1}{2 a}
$$

When $\mathrm{a}=-\mathrm{N}$ the other acoustic modes and the gravity modes are obtained. Let

$$
f=\frac{\gamma(m+2 N+2)}{2(m+1)}
$$




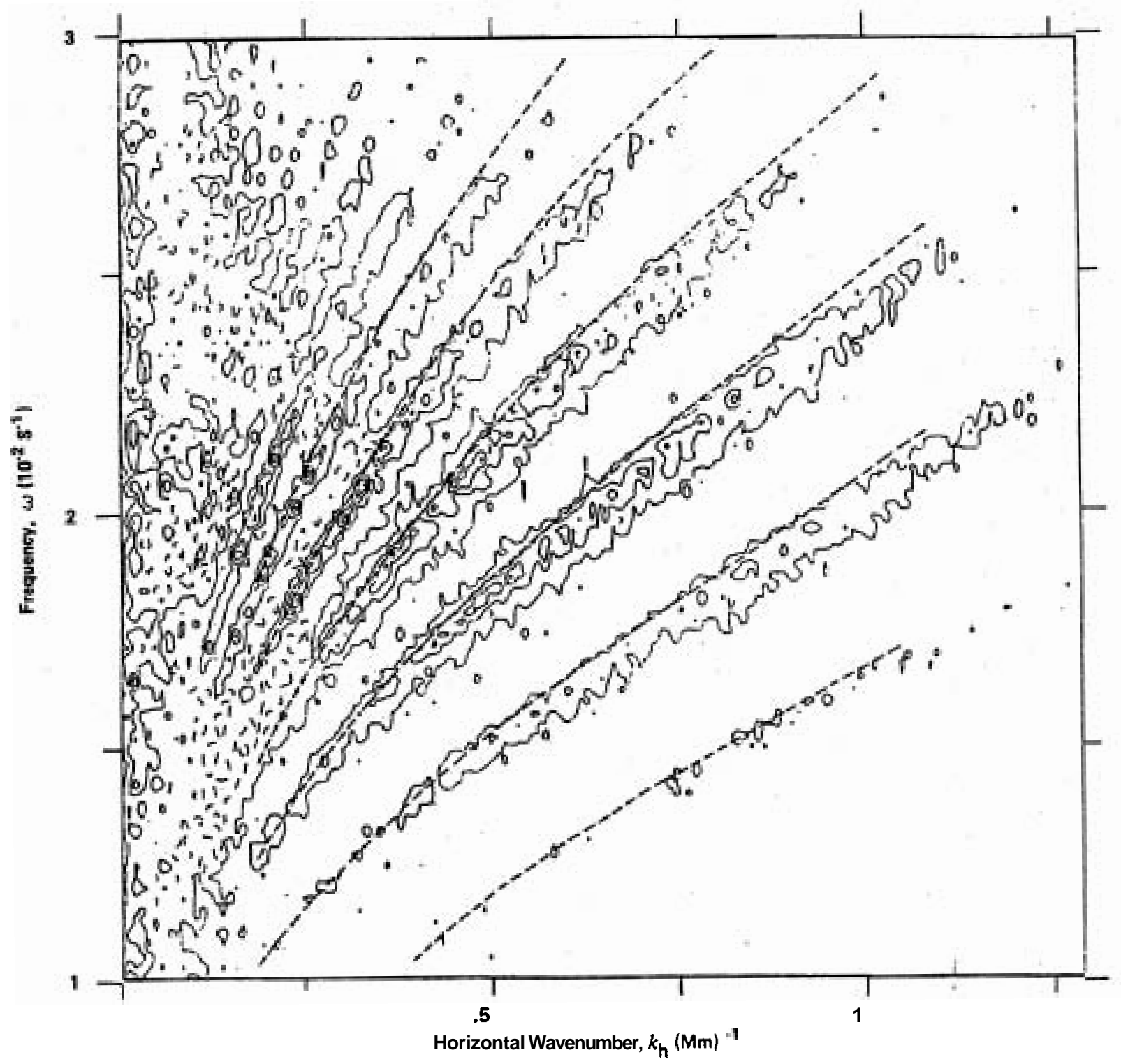

Fig2 Diagnostic diagram of spatially resolved solar Doppler shift fluctuations of the FeI 5576.1 \& line. The dashed lines are the positions of the ridges predicted by a nomerical model of the sun (from Ieubner et ad 1979). 
then the dispersion relation for the acoustic modes is

$$
\sigma_{0}^{2} / a=-f-\sqrt{f^{2}+1-\gamma / \Gamma}
$$

and the dispersion relation for the gravity modes is

$$
\sigma_{0}^{2} / \alpha=-f+\sqrt{f^{2}+1-\gamma / \Gamma}
$$

Note that curves defined by (2.43) and (2.44) are parabolas. This contrasts with results from the isothermal atmosphere where frequencies of the gravity modes are bounded by the buoyancy frequency and the acoustic modes, for fixed vertical wave number, are hyperbolas tending to $\Omega=\mathbf{c k}$ (see Figure 3 ). In the polytrope the gravity waves have no upper bound to their frequencies because the buoyancy frequency

$$
N^{2}(z)=g\left[D \ln \bar{\rho}-\frac{1}{\gamma} D \ln \bar{\phi}\right]=m g(1-\Gamma / \gamma) \frac{1}{z}
$$

becomes infinite at the top of the atmosphere $(z=0)$, so that waves confined to a thin layer at the top feel large values of $N$. Note also that the gravity waves go unstable when $\gamma<\Gamma$.

The correction due to thermal dissipation for the acoustic waves is

$$
\sigma_{1}=-\frac{\gamma}{4} \frac{(m+1) \sigma_{0} \frac{\gamma}{a}+(m+2 N+2)+1}{(m+1) \sigma_{0}^{2} / a+\frac{\gamma}{2}(m+2 N+2)}
$$

which differs slightly from Spiegel's result (Spiegel 1964) due to misprints. Overstability occurs when

$$
\Rightarrow \gamma<1+\frac{1}{2 m+2 N+3}
$$

In other words, the layer must be sufficiently unstably stratified before the acoustic nodes may grow. Only when $\gamma<1$ can a 11 the modes grow, but in that case the overstability would occur without any stratification, as is explained later.

The correction due to thermal dissipation on the gravity modes is

$$
\sigma_{1}=-\frac{\gamma}{4}\left[\frac{\frac{2-\gamma}{\gamma}+\sqrt{f^{2}+1-\gamma / \Gamma}+\frac{1}{m+1}}{\sqrt{f^{2}+1-\gamma / \Gamma}}\right]
$$

which is always damping.

\subsection{The case when $\sigma_{0} \propto \mathbf{a} \gg 1$}

In this limit I have looked at acoustic waves which propogate throughout the layer rather than being confined to the top. The lowest mode is when the turning point coincides with the bottom boundary which gives

$$
\begin{aligned}
& \sigma_{0}^{2}=-\frac{\gamma}{m+1} a^{2} \\
& \sigma_{1}=-\frac{1}{2}(\gamma-1)
\end{aligned}
$$




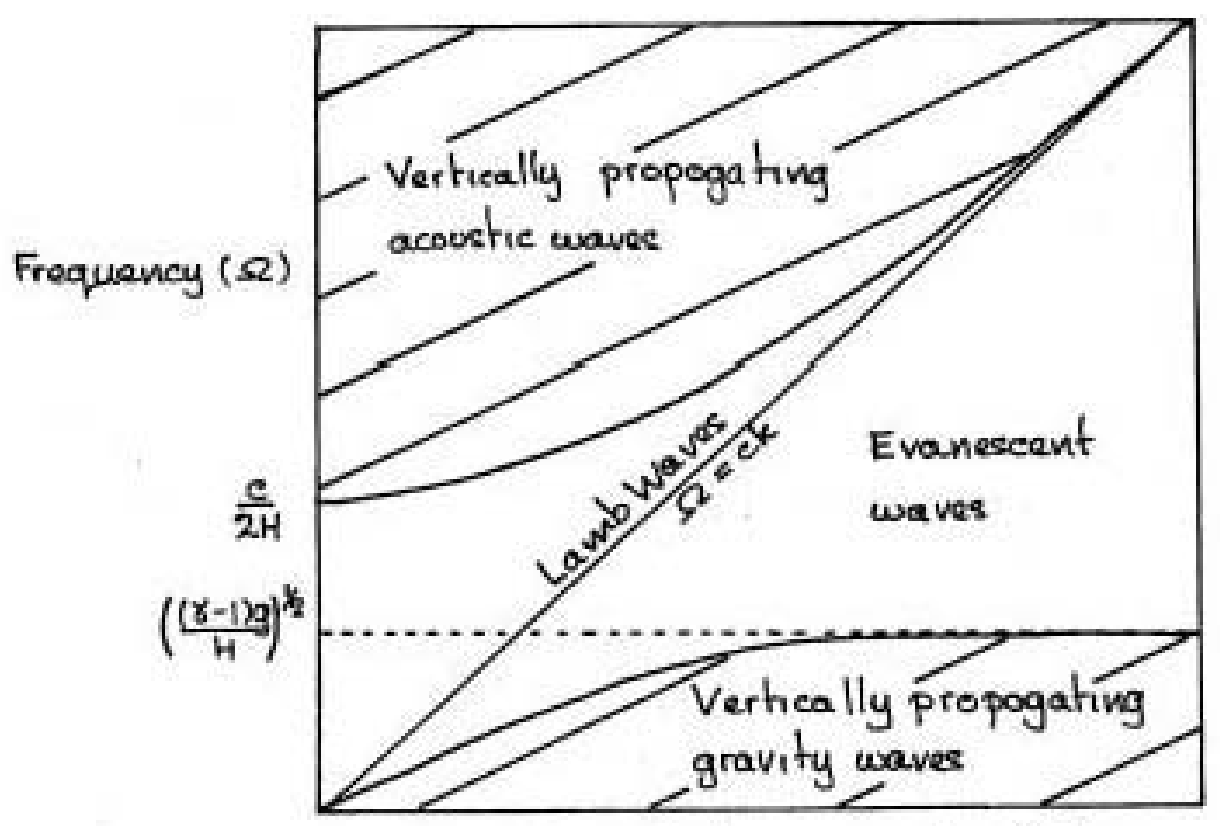

Horizontal wave number ( $k$ )

Fig 3 Diagnoshic diagram for an isothermal atmosphere.

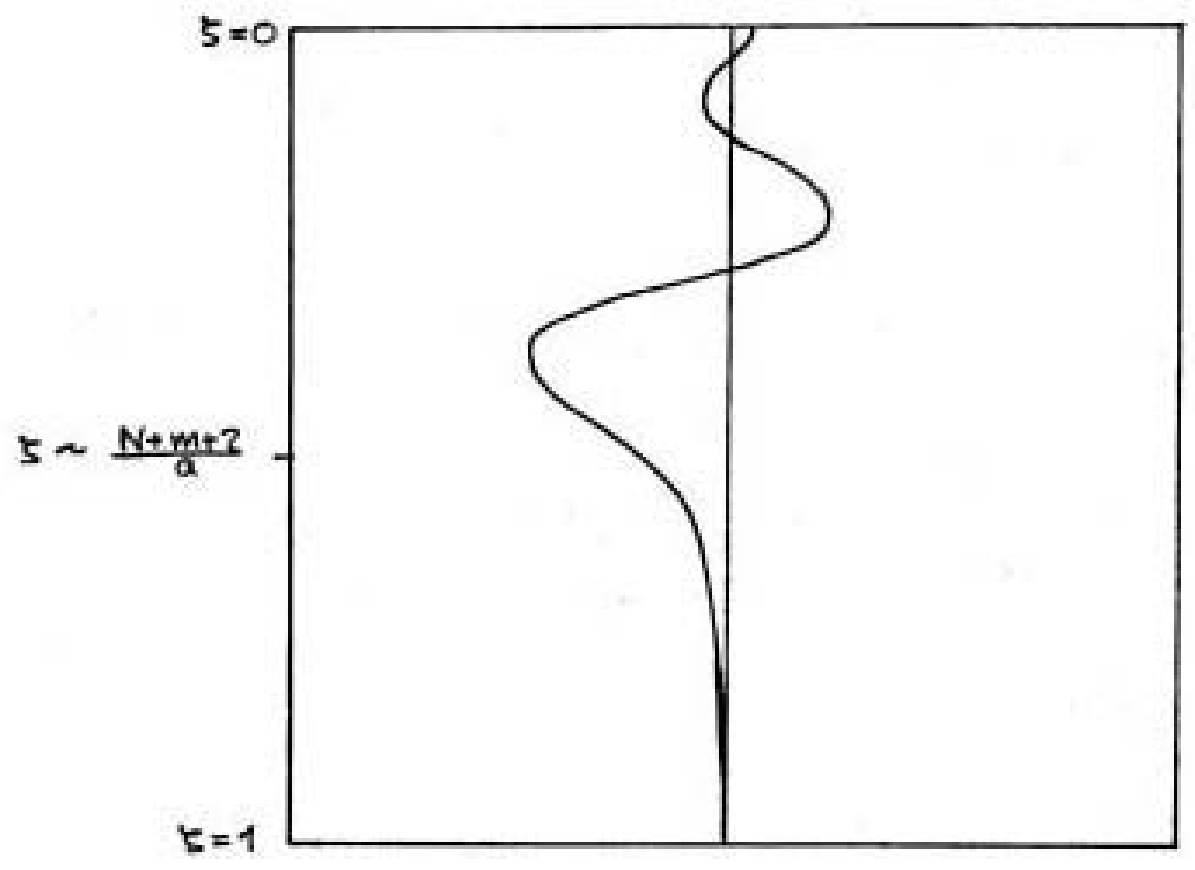

Fig 4 A sketch of $x \propto e^{-0.5} M(-N, m+2,205)$. 
These waves are always damped unless $\gamma<1$, the significance of which is explained later.

\subsection{The acoustic cut-off}

When $a \rightarrow 0$ (the long wave limit) the frequencies of the acoustic modes (with the exception of the Lamb wave) tend to finite values. These cut-off frequencies are given by

$$
\sigma_{0}^{2}=-\frac{1}{4} \lambda_{j m}^{2} \frac{Y}{m+1}
$$

where $\lambda_{\mathrm{j} m}$ is the $\mathrm{jth}$ zero of the Bessel function Jm. The correction due to thermal dissipation is

$$
\sigma_{1}=\frac{r_{j m}}{\lambda_{y m}}-\frac{1}{2}(\gamma-1)
$$

where

$$
r_{j m}=-\left(\frac{\frac{\partial}{\partial m} J_{m}(x)}{\frac{\partial}{\partial x} J_{m}(x)}\right)_{x=\lambda_{y m}}
$$

has been defined so that it is a positive quantity. The waves are overstable when $\sigma_{1}>0$, l.e.

$$
\gamma<1+\frac{2 r_{j m}}{\lambda_{j m}}
$$

I find that for $m \approx 3$ the lowest acoustic mode is overstable even when the medium is stably stratified (1.e. $\gamma>\Gamma$ ). It should be noted that, even though it is unphysical in this model to consider disturbances whose length scale is much greater than the layer thickness as being optically thin, there is nothing unphysical about the mechanism of the instability (as is discussed later).

2.5. The Lamb wave and gravity waves a t low wave number

These waves have $\sigma_{0} \propto a$. In the case of the Lamb wave

$$
\sigma_{0}^{2}=-\frac{a^{2}}{m+1}
$$

while for the gravity waves $\sigma_{0}^{2}=-\frac{4 m(1-\Gamma(\gamma)}{\Lambda_{j m}^{2}} a^{2}$

where $\Lambda_{j m}$ is a factor defined in Appendix $C$. Note that these waves are unstable when $\gamma<\Gamma$. The correction due to thermal dissipation leads to damping in both cases.

\subsection{The structure of the nondissipative motions}

It is instructive to look at the form, which the nondissipative motions take, in order to see why the dispersion curves take the shape they do, and to see how the acoustic overstability arises. The mathematical details are in 
Appendix D. The variables that I have used to describe the motions are the divergence of the velocity field $(\boldsymbol{x})$, the vertical velocity (w), and the temperature perturbation $(\theta)$ :

$$
\begin{aligned}
& w=\frac{2 a \gamma(2 a 5)^{m} e^{-a 5}}{g \bar{p}\left(\sigma^{4}-a^{2}\right)}\left[\sigma^{2}(m+1) M(a, m+1,2 a s)+\left(a-\sigma^{2}\right) a 5 M(a, m+2,2 a 5)\right] \\
& x=\frac{1}{p}(2 a s)^{m+1} e^{-a 5} M(a, m+2,2 a s) \\
& \begin{aligned}
\theta=\frac{(2 a 5)^{m} \beta_{a}}{n g P} e^{-a 5}\left[(1-\gamma)(m+1) 2 a 5 M(a, m+2,2 a 5)-\frac{2 a \gamma}{\sigma^{4}-a^{2}}\left(\sigma^{2}(m+1) M(a, m+1,2 a r)+\right.\right. \\
+(a-\sigma \eta a 5 M(a, m+2,2 a s)]
\end{aligned}
\end{aligned}
$$

where due regard has been taken of relative signs, and $\mathbf{M}(\mathbf{a}, \mathbf{b}, \mathbf{x})$ is a confluent hypergeometric function.

In the large wave number limit the Lamb wave takes the form

$$
\begin{aligned}
& w \sim e^{-05} \\
& x \sim(2 a 5)^{-(m+1)} e^{a 5} \\
& \theta \sim(2 a 5)^{-m} e^{a 5}
\end{aligned}
$$

In other words, the vertical velocity is much weaker than the horizontal velocity, and the wave is concentrated towards the bottom of the layer. For the remaining acoustic modes and the gravity waves

$$
\begin{aligned}
& a=-N \quad N=0,1,2, \ldots . \\
& w \sim e^{-a .5} P_{w(15)} \\
& x \sim e^{-a 5} L_{N}^{(m+1)}(2 a .5) \\
& \theta \sim e^{-a .5} P_{\theta}(0.5)
\end{aligned}
$$

where $P_{w}, L_{N}^{(m+1)}, p_{\theta}$ are polynomials.

The function $\mathbf{M}(-\mathbf{N}, \mathbf{m}+\mathbf{2}, \mathbf{z})$ is a polynomial of degree $\mathrm{N}$ with $\mathrm{N}$ roots in $\boldsymbol{z}>0$. (It is in fact a Laguerre polynomial.) Its extrema vary as

$$
\Gamma(m+2) \pi^{-1 / 2} e^{a s}[(m+2) a b+2 N a s]^{-1-\frac{m}{2}}\left[1-\frac{a 5}{m+2)+2 N}\right]^{-1 / 4}
$$

(see Figure 4). In other words the modes have an oscillatory vertical structure until the turning the turning point

$$
\zeta \sim \frac{1}{a}(N+m+2)
$$

after which there is an exponential decay. So the wave is confined to a layer of thickness

$$
5 \sim \frac{1}{a}(N+m+2)
$$


It is now clear why the acoustic and gravity modes take on a parabolic structure. In the case of an acoustic mode the usual formula

$$
\Omega^{2}=c^{2} k^{2}
$$

is now modified by inserting the value of $c^{7}$ that corresponds to the depth to which the wave penetrates:

$$
c^{2} \propto z^{-1}
$$

Thus

$$
\Omega^{2} \propto k
$$

In the case of gravity waves the usual formula

$$
\Omega^{2} \approx N^{2}
$$

is now modified by inserting the value of $N^{2}$ that corresponds to the depth to which the wave penetrates:

$$
N^{2} \propto 1 / z \propto k
$$

Thus

$$
\Omega^{2} \propto k
$$

At the low wave number limit, the acoustic wave exists throughout the layer, and

$$
\begin{aligned}
& w \sim M(a, m+1,2 a 5) \\
& x \sim M(a, m+2,2 a 5) \\
& \theta \sim(1-\gamma) \zeta M(a, m+2,2 a 5)-\frac{\gamma}{\sigma 2} M(a, m+1,2 a, 5)
\end{aligned}
$$

(see Figure 5).

2.7. The overstability mechanism

The mechanism by which the acoustic waves can become overstable was described by Lord Rayleigh (Rayleigh 1878):

"If heat be periodically communicated to, and abstracted from a mass of air vibrating (for example) in cylinder bounded by a piston, the effect produced will depend upon the phase of the vibration at which the transfer of heat takes place. If the heat be given to the air at the moment of greatest 


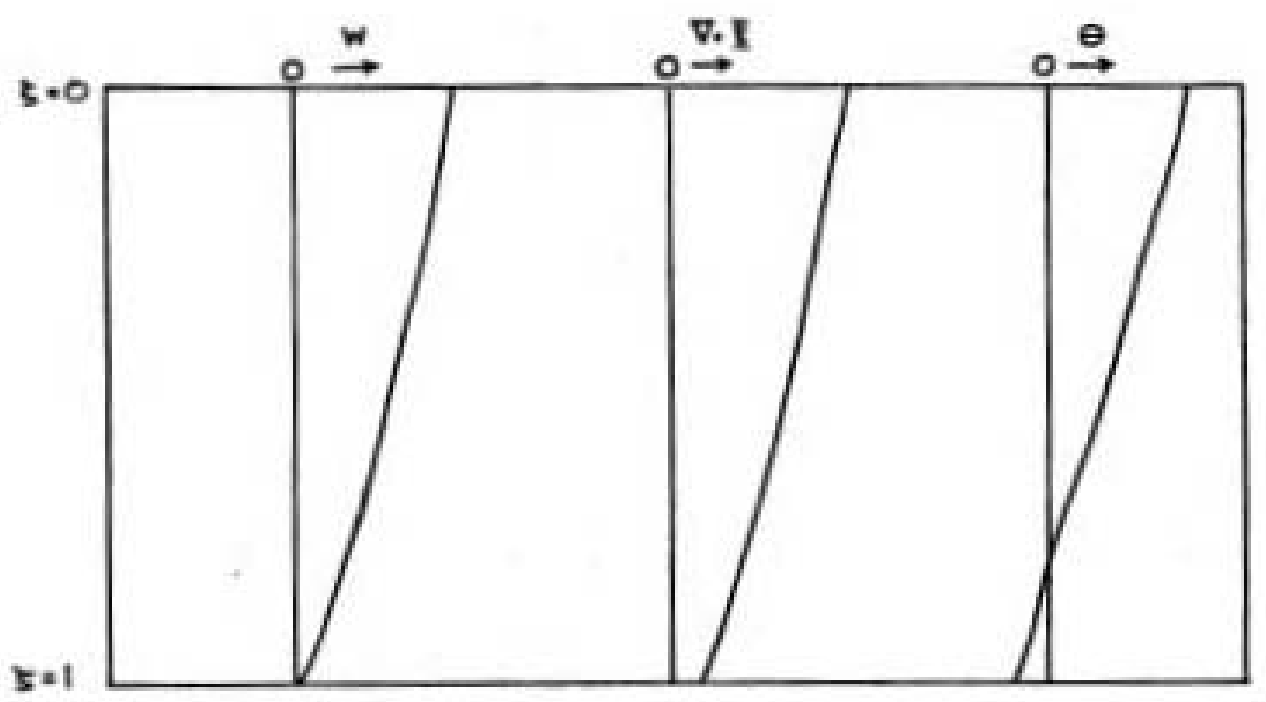

Fig 5 A sketch of the spatial structure of $w, \nabla . \xi, \epsilon$ for the bowest acoustic cot off mode whan $Y$ and $m$ takes values that allow oversbablity in the presonce of weak Newtonian thermal dissipation.

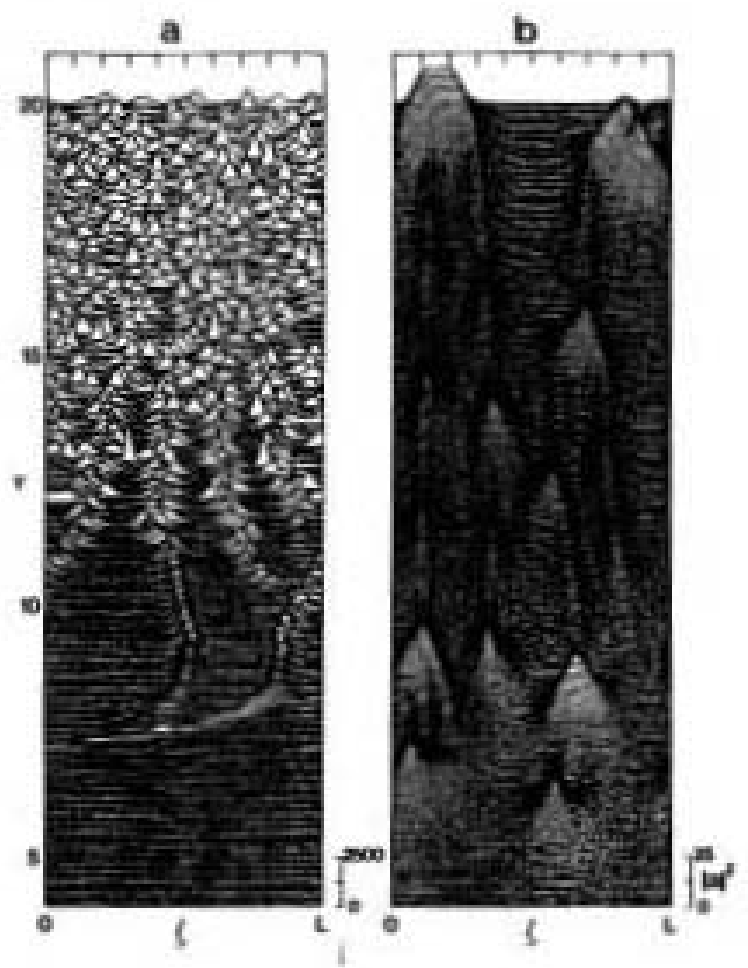

Fig 6 The amplitude $|a|^{2}$ for $L=20$ and (a) $\mu=100$ (b) $\mu=1$ ofter evolving from white noise of mean equare amplitude $\overline{|a|^{2}}=0.01$ at time $\tau=0$. (From Bretherton \& Spiegal 1983) 
condensation, or be taken from it at the moment of greatest rarefaction, the vibration is encouraged. On the other hand, if heat be given a the moment of greatest rarefaction, or abstracted at the moment of greatest condensation, the vibration is discouraged."

This may be expressed mathematically by forming the work integral of the linear equations of motion (Simon 1957). With the form of dissipation that has been used in this study it can be shown (Jones 1976) that the growth rate has the same sign as

$$
\int_{0}^{1}(\nabla, \underline{\xi}) \theta d \zeta
$$

where $\mathbf{E}$ is the displacement and $\theta$ is the temperature perturbation. In the case of an homogeneous fluid the elementary theory of sound gives

$$
\begin{aligned}
\rho+\bar{\rho} \nabla \cdot \underline{\xi} & =0 \text { and } \rho / \bar{\rho}=\frac{1}{\gamma-1}(\theta / \bar{T}) \\
\Rightarrow(\nabla \cdot \underline{\xi}) \theta & =(1-\gamma)(\rho / \bar{p})^{2} \bar{\tau}
\end{aligned}
$$

which is positive for $Y<1$ 1.e. the gas cools as it goes into compression. In which case no stratification is needed to produce overstability, In the polytrope the stratification can make the sound wave go overstable by ensuring that as the wave goes (locally) into compression the fluid is displaced into a region where it is cooler than its surroundings, so that heat leaks into ths compressed fluid; and when the wave goes into rarefaction the fluid is displaced into a region where it is hotter than its surroundings, so that heat leaks out. This is well illustrated in the lowest acoustic cut off mode where the correlation between $\theta$ and $\nabla . \underline{\xi}$ is clear (see Figure 5).

It should be noted in passing that, if the polytrope is truncated (i.e. does not extend all the way to zero temperature), it will be more difficult to get overstability, since the truncation will'remsve'the lowest modes which are the most easily destabilized. This effect has been observed i n numerical studies (Antia, Chitre \& Gokhale 1977, Jones 1976).

\section{Weakly nonlinear waves}

\subsection{The evolution of acoustic waves}

The generic equation for a weakly nonlinear wave train in a nondissipative system is the cubic Schroedinger equation (see Whitham 1974). In a frame moving with the group velocity the disturbance may be written as

$$
\phi=A(x, T) F(5) \sin (\omega t-k x+\theta(x, T))
$$

or equivalently as

$$
\phi=\operatorname{Re} B(\bar{x}, T) F(5) \sin (\omega t-k x)
$$

where $\mathbb{Z}, T$ are the slow space and time variables of the modulation and $F(\zeta)$ is the vertical structure of the mode. In Appendix $E$ I have derived the cubic Schroedinger for a neutrally'stratified isothermal atmosphere with 
rigid upper and lower boundaries. The resulting equation for the complex amplitude may be written as

$$
i B_{T}+B_{X X}+h|B|^{2} B=0
$$

where $h$ is a function of $k$ and $l$. It has solutions of the form

$$
B=a e^{\left[i\left(\beta^{2}-\frac{v^{2}}{4}\right) \tau+i \frac{v}{2} x\right]} \operatorname{sech} \beta(x-v \tau)
$$

where $|\alpha|^{2}=\frac{2}{h} \beta^{2} \quad(h>0)$.

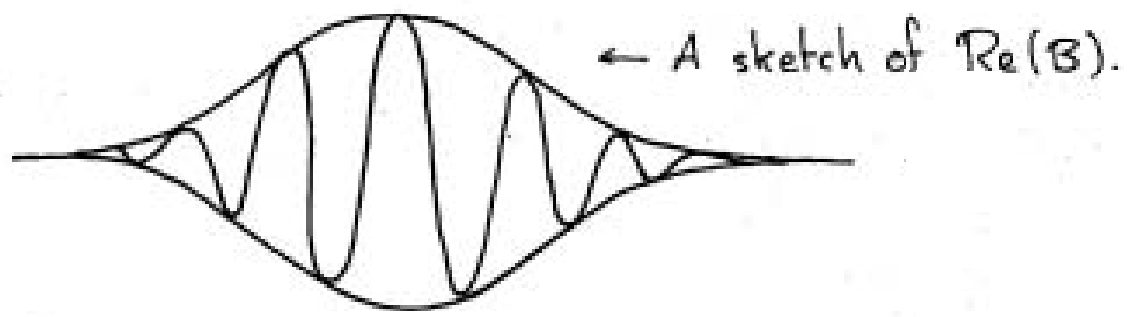

I anticipate that the polytropic atmosphere also admits the cubic Schroedinger equation for its acoustic waves. In order to incorporate the effects of dissipation I have drawn upon numerical results from Antia, Chitre \& Gokhale (1977), pending an analytic treatment. In his study of a polytropic layer between rigid boundaries using thermal diffusion (as against Newtonian cooling) he finds that the growth rate depends on the wave number in the following manner:

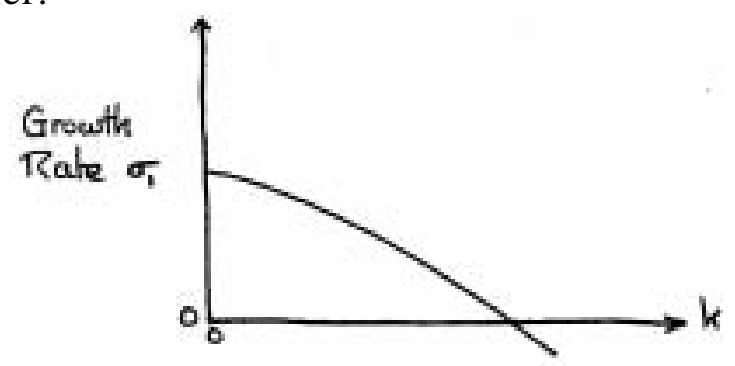

This can be incorporated into the amplitude equation as additional linear terms. I have made the approximation

$$
\sigma_{1}=q\left(1-\nu k^{7}\right)
$$

where $q$ and $\nu$ are constants. This suggests the following equation:

$$
i B_{T}+B_{x x}+h \mid B P^{P} B=i q\left(1+\nu \partial_{x}^{*}\right) B
$$

Bretherton \& Spiegel (1983) have studied the equation

$$
i a_{T}+\mu \partial_{x z}+|a|^{2} a=i\left(a+a_{x x}\right)
$$

in connection with double diffusive convection. See Figure 6 for an example of the output. This is reminiscent of what is seen for the five minute oscillations on the sun. 


\subsection{The evolution of long gravity waves}

As an example of a different amplitude equation for weakly nonlinear wave, I have derived the $\mathrm{KdV}$ equation for long gravity waves i $\mathrm{n}$ an isothermal atmosphere with rigid upper and lower boundaries (see Appendix F). Let the dfsturbance have the form

$$
u=U(x, t, T) F(5) \equiv G_{x}(x, t, T) F(5)
$$

for the horizontal velocity, where $x, t, T$ are the fast space, fast time, and slow time variables. I get two equations

$$
\begin{gathered}
G_{t t}=\frac{E}{L^{2}} G_{x x} \quad\left(L^{2}=1 / 4+d^{2}\right) \\
2 G_{t T}+\frac{8}{3}\left(G_{x}^{2}\right)_{t}+3 / 3 G_{x x} G_{t}+E G_{t t}-\frac{1}{E} G_{t t t t}
\end{gathered}
$$

where E is a stability parameter such that the layer is stably stratified when $E$ is positive and unstably stratified when it is negative. In the case that $E$ is positive it is possible to eliminate $\partial_{t}$ from ( 3.10 ) by setting

$$
G(x, t, T) \equiv G(x+c t, T) \text { and } U(x, t, T) \equiv U(x+c t, T)
$$

where $c^{2}=\frac{E}{L^{2}}$, so that

$$
2 U_{T}+6 U U_{x}+c E U_{x}-\left(\frac{C^{3}}{E}\right) U_{x x x}=0
$$

The $U_{X}$ term can now be eliminated by setting $\partial_{T} \rightarrow \partial_{T}-\frac{c 5}{2} \partial_{x}, U=2 \tilde{U}$

$$
\tilde{U}_{T}+6 \tilde{U} \tilde{U}_{x}-\left(\frac{c^{3}}{2 E}\right) \tilde{U}_{x x x}=0
$$

which is the KdV equation.

If, however, E is negative (3.9) is elliptic and U blows up on the fast time scale t. The nonlinearity is too weak to contain the instability in this scaling. In order to halt the run away, energy considerations suggest that the velocity field should be scaled an order larger than it has been, though this has not been carried out in the present study.

The XdV equation for compressible gravity waves contrasts with the result for an incompressible fluid, where the first nonlinear tern is cubic and the modified KdV equation results. This is because in linear theory $\nabla . \mathrm{y}=0$ i.e $\underline{u} \cdot \underline{\mathrm{k}}=0$ which means that $\underline{u} \cdot \nabla \underline{u}=0$ for the linear components of the velocity field, and so the quadratic tern vanishes. The question arises: where is the crossover between KdV and modified KdV? This awaits future analysis. 


\section{Conclusion}

The polytropic atmosphere is a good testing ground for fully compressible dynamics in a (strongly) stratified medium. Although the mathematics is more involved, it is still tractable. In this study I have only begun to scratch the surface of the nonlinear dynamics; I hope in the not too distant future to be able to get amplitude equations coupling acoustic and convective motions. The connection between acoustic and convective motions is important to the understanding of the dynamics at the top of the solar convection zone.

On a more general problem, it seems that whenever simplifications of the full equations of motion are made, it is to filter out high frequency motions. Implicit in this is an assumption that they average out to nothing on the slow time scale(s). Is it possible to do the opposite process and, in the case of the polytropic atmosphere, arrive at an approximate set of equations which a t leading order include the acoustic waves, but not the gravity waves and at a higher order connect the two?

\section{ACKNOWLEDGENTS}

I would like to thank Ed. Spiegel for many stimulating discussions and his encouragement. I would also like to thank Melvin Stern and the steering committee for providing the beautiful beaches on which to conduct this research.

\section{REFERENCES}

Abramowit z, M. , and Stegun, I ^., 1970. Handbook of Mathematical Functions, Dover.

Antia, H.M., S.M. Chitre, and D.M. Gokhale, 1977. Solar Phys., 56, 275.

Bretherton, C., and E. Spiegel, 1983. Physics Letters, 96A, 152 .s.

Chitre, S.M., and D.M. Gokhale, 1975. Solar Phys., $\underline{43}, 49$.

Deubner, F-L., 1981. The Sun a s a Star, NASA SP 450, Ed. Jordan.

Deubner, F-L., R. K. Ulrich and E. J. Rhodes, 1979. Astron. Astrophys. $\underline{77}$, 177.

Jones, C,A., 1976. Monthly Notices Roy. Astron. Soc., 176, 145.

Lamb, H., 1908, Proc. Roy. Soc. A , 84, 551.

Lamb, H., 1945, Hydrodynamics, Sixth Edition, Dover.

Rayleigh, L., 1878. Proc. Roy. Inst., 8, 536.

Simon, RL., 1957. Bull. Acad. R. Belg. C1. Sci., 5th ser., 43, 610.

Spiegel, Ed., 1960. Astrophys. J., 132, 716.

Spiegel, E.A., 1964. Astrophys. J., 139, 959. 
Spiegel, E.A., and W. Unno, 1962. Publications of the Astronomical Society of Japan, 14,28 .

Spiegel, EA., and W. Unno, 1966. Publications of the Astronomical Society of Japan, $\underline{18}, 85$.

Stein, R.F., and J. Leibacher, 1974. Ann. Rev. Stron. Astrophys., 12, 407.

Stein, R.F., and J. Leibacher, 1981. The Sun as a Star, NASA SP 450, Ed Jordan.

Whi tham, G.B., 1974. Linear and Nonlinear Oscillations, Wiley and Sons. 


\section{APPENDIX A}

The linearized equations of motion (2.8) - (2.11) can be written in the form:

$$
\begin{aligned}
& \partial_{t}^{2} u=\partial_{x}[g w+\not \mathscr{x}] \\
& \partial_{t}^{2} v=\partial_{y}[g w+\not \mathscr{x}] \\
& \partial_{t}^{2} w=\partial_{z}[g w+\not{z}]-\left[\frac{d c^{2}}{d z}-(\gamma-1) g\right] x+\psi / H
\end{aligned}
$$

where

$$
\begin{gathered}
\not=c^{2} \chi+R Q(\theta), \quad H=\bar{p} / \frac{d \bar{p}}{d z}=z / m \\
\psi=R Q(\theta), \quad x=\nabla \cdot \psi
\end{gathered}
$$

It is possible to get an equation for $W$ in terms of $\mathbb{W}$ by taking the divergence of equations (A1) and (A2):

$$
\left(n^{2}-g \partial_{z}\right) w=\partial_{z} \not{x}-\left[\partial_{z} c^{2}-(\gamma-1) g\right] x+\psi / H
$$

where the disturbance is taken to be $\approx e^{i \underline{k} \cdot \underline{x}+n t}$. The daw may be eliminated from (A5) using (A3)

$$
w=\frac{1}{n^{4}-k^{2} g^{2}}\left[n^{2} \partial_{2}+g k^{2}+n^{2} / H\right]
$$

(A3) and A6) can be combined to yield an equation for $\boldsymbol{X}$ in terms of $\boldsymbol{\Psi}$ :

$$
\chi=\frac{1}{n^{4}-k^{2} g^{2}}\left[n^{2} \nabla^{2}+\left(n^{2} \partial z-g k^{2}\right) \frac{1}{H}\right] \not \mathscr{L}
$$

To make further progress, the equations for the thermodynamic variables must now be considered

$$
\begin{aligned}
& \partial_{t} p+g \bar{\rho} w=-\tilde{\rho} \not{X} \\
& \partial_{t} \rho+\bar{p} w / H=-\bar{\rho} x \\
& \theta=\left(\frac{\bar{p}}{\bar{p}}\right)_{p}-\left(\frac{\bar{T} / \bar{\rho}}{\rho}\right)
\end{aligned}
$$


The equations represent the heat equation, continuity equation and equation of state respectively. The pressure and density fluctuations, $p$ and $p$, may be eliminated from (A10) using (A8) and (A9):

$$
n \theta=[\bar{F} / H-g / R] w-\frac{1}{R} \not+\bar{x}
$$

Since $\tilde{\mathbb{Z}}=c^{\mathbb{R}} \boldsymbol{X}+\mathrm{R}(\boldsymbol{\theta} \theta)$, it follows from (All) that

$$
n \not{z}=Q\left[-R \beta_{0} w-\not{x}+R T \chi x\right]+n c^{2} \chi
$$

where $\beta_{0}=d \bar{T}$ (and $m+1=9 / R \beta_{0}$ ). (A7) can be used to eliminate $\chi$ from (A12)

$$
n\left[n^{2} c^{2} \nabla^{2}+n^{2} c^{2} d_{+} 1 / H-g k^{2} c^{2} / H+\left(g^{2} k^{2}-n^{4}\right)\right] \mathscr{X}=
$$

= Q[[ $\left.\left[R \beta_{0}\left(n^{2} \partial_{1}+g k^{2}+n^{2} / H\right)+\left(n^{4}-g^{2} k^{2}\right)-R T\left(n^{2} \nabla^{2}+\left(n^{2} d z-g k^{2}\right)+1\right)\right] \mathbb{Z}\right]$

Now let $\boldsymbol{\Psi}=c^{\boldsymbol{z}} e^{-\mathbf{k}} \boldsymbol{\psi}$, and the following equation for $\boldsymbol{\psi}$ may be obtained:

$8 n \mathbb{L} E D^{2}+(m+2-2 k z) D-2 \alpha k \rrbracket \psi=$

$=Q \llbracket\left[(m+1)\left(n^{2} / g+k+1 / z\right)+(2 k z-(m+1)) D-z D^{2}\right] \psi \rrbracket$

(where $D=\partial \mathbf{z}$ ). This is equation(2.17) .

\section{APPENDIX B}

Spiegel's dispersion relation (Eq. (82) in Spiegel 1964) is

$$
2 a \sigma^{2}\left(\frac{A-B+1}{2-B}\right) M(A-B+2,3-B, 2 a)+\left[\sigma^{2}(1-B)+a\left(a-\sigma^{2}\right)\right] M(A-B+1,2-B, 2 a)=0
$$

which may be rewritten as

$$
\begin{aligned}
\sigma^{2} & {\left[2 a \frac{a}{b} M(a+1, b+1,2 a)+(b-1) M(a, b, 2 a)\right]+} \\
+ & a\left(a-\sigma^{2}\right) M(a, b, 2 a)=0
\end{aligned}
$$

where

$$
a=A-B+1, b=2-B
$$

The relation

$$
M(a+1, b, z)-M(a, b, z)=\frac{z}{b} M(a+1, b+1, z)
$$


may be used to rewrite (B1) as

$$
\sigma^{2}[a M(a+1, b, 2 a)+(b-1-a) M(a, b, 2 a)]+a\left(a-\sigma^{2}\right) M(a, b, 2 a)=0
$$

The relation

$$
a M(a+1, b, z)+(b-1-a) M(a, b, z)=(b-1) M(a, b-1, z)
$$

may be used to rewrite (B4) as

$$
\sigma^{2}(b-1) M(a, b-1,2 a)+a\left(a-\sigma^{2}\right) M(a, b, 2 a)=0
$$

It should be noted that in Spiegel's equation (81)

$$
\bar{p} w \propto \frac{1}{\sigma^{4}-a^{7}}
$$

hence, the complete dispersion relation is

$$
\frac{1}{\sigma^{4}-a^{2}}\left[\sigma^{2}(b-1) M(a, b-1,2 a)+a\left(a-\sigma^{2}\right) M(a, b, 2 a)\right]=0
$$

which is equation (2.32). See Abramowitz and Stegun (1970) for details of confluent hypergeomatic equations.

\section{APPENDIX C}

The asymptotic results used in this section can be found in Abramowitz \& Stegun, unless otherwise stated.

c1. The Large Wave Number Limit

When a is large, the following limit is appropriate

$$
M(a, b, 2 a) \sim \frac{\Gamma(b)}{\Gamma(a)} e^{2 a}(2 a)^{a-b}
$$

in which case (2.32) becomes

$$
\frac{1}{\sigma^{4}-a^{2}} \frac{\Gamma(b)}{\Gamma(a)}(2 a)^{a-b}\left[2 a \sigma^{2}+a\left(a-\sigma^{2}\right)\right] \simeq 0
$$

This is satisfied by putting

$$
\begin{array}{ll}
\text { or } & =-N, N=0,1,2, \ldots \\
& \sigma^{2} \simeq-a
\end{array}
$$

In the latter case, the singular nature of (C2) when $\sigma^{2} \simeq-a$ is more apparent than real, since we are dealing with an asymptotic representation, not an 
exact one, The $\sigma^{2}=-a \quad$ relation corresponds to a $=\mathbf{1}$ and so it could be included in the classification scheme given by (C3). However, to get the corrections due to small $\omega$ on (C4) it is necessary to use an extended form of $(\mathrm{Cl})$ :

$$
M(a, b, 2 a) \sim \frac{\Gamma(b)}{\Gamma(a)} e^{2 a}(2 a)^{a-b}\left[1+\frac{(b-a)(1-a)}{2 a}\right]
$$

i n which case (2.32) becomes

$$
\begin{gathered}
2 a \sigma^{2}+a\left(a-\sigma^{2}\right)+\sigma^{2}(b-1-a)(1-a)+\frac{1}{2}\left(a-\sigma^{2}\right)(b-a)(1-a) \simeq 0 \\
\begin{array}{c}
\text { (C3) can be expanded using }(2.29),(2.30) \text { and }(2.3) \text { to yield } \\
\frac{m+1}{\gamma} \sigma^{4}+(m+1) \omega \sigma^{3}+a(m+2 N+2) \sigma^{2}+(m+3+2 N) a \omega \sigma+a^{2}\left(m-\frac{m+1}{\gamma}\right)= \\
=0
\end{array}
\end{gathered}
$$

Let

$$
\begin{aligned}
& \sigma=\sigma_{0}+\omega \sigma_{1} \\
& f=\frac{\gamma(m+2 N+2)}{2(m+1)}
\end{aligned}
$$

then

$$
\sigma_{0}^{2} / Q=-f \pm \sqrt{f^{2}+1-\gamma / \Gamma}
$$

The upper sign corresponds to gravity waves and the lower sign to acoustic waves. The gravity waves are directly unstable when $\gamma<\Gamma$. The correction to the acoustic frequency is

$$
\sigma_{1}=-\frac{\gamma}{4} \frac{\sigma_{6}^{2} / a+2 f / \gamma+\frac{1}{m+1}}{\sigma_{0}^{2} / a+f}=\frac{\gamma}{4} \frac{\frac{z-\gamma}{\gamma} f+\frac{1}{m+1}-\sqrt{f^{2}+1-\gamma / n}}{\sqrt{f^{2}+1-\gamma / \Gamma}}
$$

Overstability occurs when the numerator is positive, which requires

$$
d<1+\frac{1}{2 m+2 N+3}
$$

The correction to the gravity wave frequency is

$$
\sigma_{1}=-\frac{\gamma}{4} \frac{\sigma_{0}^{2} / a+2 f / \gamma+\frac{1}{m+1}}{\sigma_{0}^{2} / a+f}=-\frac{\gamma}{4} \frac{\frac{z-\gamma}{\gamma} f+\frac{1}{m+1}+\sqrt{f^{2}+1-\gamma / \Gamma}}{\sqrt{f^{2}+1-\gamma / \Gamma}}
$$

This is always negative.

When $\boldsymbol{\gamma}=1$ a 11 the acoustic modes become overstable, but the lowest modes have the highest growth rates. For large $\mathbf{N}$

$$
\sigma_{1} \sim \frac{1}{4(N+1)}
$$


Returning to (C5) the leading order frequency is given by

$$
\sigma_{0}^{2}=-\mathbf{a}
$$

The $O(\omega)$ correction is obtained by substituting (2.33) and (2.34) for a and b. This gives

$$
\sigma_{1}=-\frac{m+1}{2 a}
$$

This wave is weakly damped.

c2 The Limit $\sigma a \mathrm{a} \gg 1$

In this limit I have examined the form of the dispersion relation when the turning point is near the bottom boundary. The appropriate asymptotic representation is

$$
M(a, b, z) \sim e^{z / 2}(b-2 a)^{3 / b} \Gamma(b)[A i(t) \cos (a n)+B i(t) \sin (a \pi)]
$$

where

$$
t=(b-2 a)^{3 / 3}[z-2 b+4 a]
$$

When the turning point is below the bottom boundary the wave is vertically propagating throughout the layer. The lowest such mode is given by

$$
\begin{gathered}
2 a+4 a \simeq 0 \\
a^{2}(\sigma+\omega)+\frac{m+1}{\gamma} \sigma^{-3}+\sigma^{2}(m+1) \omega \simeq 0
\end{gathered}
$$

To leading order in $\omega$ this gives

$$
\sigma_{b}^{2}=-\frac{\gamma}{m+1} a^{2}
$$

with a correction at $O(\omega)$ of

$$
\sigma_{1}=-\frac{1}{2}(\gamma-1)
$$

Hence, overstability only occurs when

$$
\gamma<1
$$

\section{C3 The Acoustic Cut Off}

In this $1 \mathrm{imit}$ the frequency remains finite but the wave number goes to zero, This means that

$$
\text { da } \sim O(1) \text { as } a \rightarrow 0 ; \text { a } a<0
$$


The dispersion relation (2.32) reduces to

$$
\sigma^{2}(b-1) M(a, b-1,2 a) \simeq 0
$$

The appropriate asymptotic representation is

$$
M(a, b, z) \sim \Gamma(b)(-d z)^{\frac{1}{2}(1-b)} J_{b-1}(2 \sqrt{-\partial z})
$$

In which case (C24) becomes

$$
J_{b-2}(2 \sqrt{-2 a a})=0
$$

a,b can be expanded in powers of using (2.29), (2.30) and (2.34):

$$
\begin{aligned}
2 a a \sim \frac{m+1}{\gamma} \sigma_{0}^{2} & +\left[2 \frac{m+1}{\gamma} \sigma_{0} \sigma_{1}+(m+1) \frac{\gamma-1}{\gamma} \sigma_{0}\right] \omega \\
b-2 & \sim m+\frac{\omega}{\sigma_{0}}
\end{aligned}
$$

(C26) now becomes

$$
J_{m}(x)+\frac{\omega}{\sigma_{0}} \frac{\partial}{\partial m} J_{m}(x)+\frac{\omega}{\sigma_{0}}\left(\sigma_{1}+\frac{1}{z}(\gamma-1)\right) x J_{m}^{\prime}(x)=0
$$

where

$$
x=2\left[-\frac{m+1}{\gamma} \sigma_{0}^{2}\right]^{1 / 2}
$$

(C29) can be reconstituted as

$$
J_{m}\left(x+\frac{\omega}{\sigma_{\sigma}}\left(\sigma_{G}+\frac{1}{2}(\gamma-1)\right) x+\frac{\omega}{\sigma_{\sigma}} \frac{\frac{\partial}{\delta_{m}} \operatorname{l} \ln (x)}{\frac{\partial}{\partial x} \operatorname{Jm}(x)}\right)=0
$$

Let $\lambda_{j m}$ be the $j$-th $\operatorname{root}$ of $J_{m}(x)$ then

$$
x+\frac{\omega}{\sigma_{0}}\left[\sigma_{1}+\frac{1}{2}(\gamma-1)-r_{j m}\right]=\lambda_{j m}
$$

where

$$
r_{j m}=-\frac{\frac{\partial}{\partial m} \ln \left(\lambda_{j m}\right)}{\frac{\partial}{\partial x} J_{m}\left(\lambda_{j m}\right)}>0
$$


To leading order (C32) is

$$
\sigma_{i}^{2}=-\frac{1}{4} \lambda_{j m}^{2} \frac{\gamma}{m+1}
$$

with a correction

$$
\sigma_{4}=\frac{r_{j m}}{\lambda_{j m}}-\frac{1}{2}(\gamma-1)
$$

Hence, there is overstability if

$$
\gamma<1+\frac{2 r_{j m}}{\lambda_{j m}}
$$

In particular for $\mathrm{J}=1$ :

\begin{tabular}{|c|c|c|c|}
\hline $\mathrm{m}$ & $r_{\mathrm{m}}$ & $1+\frac{2 r_{\mathrm{m}}}{\lambda_{\mathrm{mm}}}$ & $\Gamma$ \\
\hline 1 & 1.27 & 1.67 & 2 \\
3 & 1.16 & 1.36 & 1.33 \\
5 & 1.08 & 1.25 & 1.20 \\
\hline
\end{tabular}

For $m \geqslant 3$ it is possible to have subadiabatic overstability. For large $\mathrm{J}$ :

$$
\begin{aligned}
& \mathrm{j} m \rightarrow \pi / 2, \quad \lambda_{\mathrm{j} m} \rightarrow\left(\mathrm{j}+\frac{\mathrm{m}}{2}-\frac{1}{4}\right) \\
& 1+\frac{2 r_{\mathrm{jm}}}{\lambda_{\mathrm{j} m}} \rightarrow 1+\frac{1}{\mathrm{j}+\frac{m}{2}-\frac{1}{4}}
\end{aligned}
$$

For large $\mathbf{m}$ and $\quad \jmath=1$ :

$$
\begin{aligned}
& r_{1 m} \rightarrow 1, \lambda_{1 m} \rightarrow m+1.86 m^{1 / 3}+1.03 m^{-1 / s}+O\left(m^{-1}\right) \\
& 1+\frac{2 r_{i m}}{\lambda_{1 m}} \rightarrow 1+\frac{2}{m+1.86 m^{1 / 3}+1.03 m^{-13}+0\left(m^{-1}\right)}
\end{aligned}
$$

(Asymptotic results for $r_{\jmath m}$ are not given in Abramowitz \& Stegun, but are not difficult to derive.)

C4 The Lamb and Gravity Waves at Low Wave Number

In this limit the dispersion relation (2.32) becomes

$$
(b-1) M(a, b-1,2 a)+\frac{a^{2}}{\sigma^{2}} M(a, b, 2 a)=0
$$


$-185$

where

$$
\sigma^{2} \times a^{2}, a a=0(1) \text { and } a a<0 \text { as a } 40
$$

The appropriate asymptotic form is

$$
M(a, b, z) \sim \Gamma(b)(-a z)^{1(1-b)} J_{b-1}(2 \sqrt{-a z})
$$

Hence (C41) can be rewritten

$$
(-2 a a)^{\frac{1}{2}} J_{b-2}(2 \sqrt{-2 a a})+\frac{a^{2}}{\sigma^{-2}} J_{b-1}(2 \sqrt{-2 a a})
$$

The terms - aaa and b can be expended in powers of $\omega$ using (2.29), (2.30), (2.33) and (2.34). To $O(\omega)$ (C44) becomes

$$
\begin{aligned}
\frac{1}{2} \times[1 & \left.-\frac{\omega}{2 \sigma_{0}}\left(1+2 \sigma_{t}\right)\right] J_{m}+\frac{\omega}{\sigma_{0}}\left[x-\frac{\omega x}{2 \sigma_{0}}\left(1+2 \sigma_{t}\right)\right]- \\
& -\frac{1}{m x^{2}} \frac{\omega x}{m(1-\Gamma / x)}\left(1-\frac{2 \omega}{\sigma_{0}} \sigma_{1}\right) J_{m+1}+\frac{\omega}{\sigma_{0}}\left[x-\frac{\omega x}{2 \sigma_{0}}\left(1+2 \sigma_{1}\right)\right]=0
\end{aligned}
$$

To leading order this is

$$
J_{m}(x)=\frac{\frac{1}{2} x}{m(1-\Gamma / x)} J_{m+1}(x)=0
$$

where

$$
x=2\left[-m(1-\Gamma / \gamma) \frac{a^{2}}{\sigma_{0}^{2}}\right]^{1 / 2}
$$

The smallest root of (C46) is the Lamb wave, which for small $x$ is seen to be

$$
\sigma_{0}^{2}=-\frac{a^{2}}{m+1}
$$

(take the first term of the power series for $J_{m}(x)$. The remaining roots of (C46) are sandwiched between pairs of zeros of $J_{m}$ and $J_{m+1}$. Let these be denoted by $\Lambda_{\mathrm{j} m}$, so that

$$
\sigma_{0}^{2}=-\frac{4 m(1-\pi / \gamma)}{\Lambda_{j m}^{2}} a^{2}
$$

These are the gravity waves which are directly unstable for $\gamma<\Gamma$. At $O(\omega) \quad(\mathrm{C} 45)$ is

$$
\begin{aligned}
{\left[-J_{m}(x)-x J_{m}^{\prime}(x)+\frac{x}{m(1-\Gamma / n)} J_{m+1}(x)\right.} & \left.+\frac{\frac{1}{2} x^{2}}{m(1-\Gamma / x)} J_{m+1}^{\prime}(x)\right] \sigma_{q}+ \\
+\left[-\frac{1}{2} J_{m}(x)+\frac{\partial}{\partial m} J_{m}(x)-\frac{1}{2} x J_{m}^{\prime}(x)\right. & -\frac{\frac{1}{2} x}{m(1-\Gamma / r)} \frac{\partial}{\partial m} J_{m+1}(x)+ \\
& \left.+\frac{\frac{1}{4} x^{2}}{m(1-\Gamma / y)} J_{m+1}^{\prime}(x)\right]=0
\end{aligned}
$$


Using (C46) allows (C50) to be written as

$$
\sigma_{1}=-\frac{1}{2}+\frac{\ln (x)-\frac{\partial}{\partial m} J_{m}(x)+\frac{\frac{1}{2} x}{m(1-\Gamma / y)} \frac{\partial}{\partial m} J_{m+1}(x)}{J_{m}(x)-x\left[J_{m}^{\prime}(x)-\frac{1 x}{m\left(1-\Gamma_{y}\right)} J_{m+1}^{\prime}(x)\right]}
$$

When $\mathrm{x}$ is small the Bessel functions may be approximated by the first terms in their power series, in which case (C51) can be reduced to

$$
\sigma_{1}=-\frac{1}{2(m+2)}
$$

i.e., the Lamb wave is damped. For the gravity waves $x$ is finite, but (C51) may be simplified by taking $1-\Gamma / y$ to be small, in which case

$$
\sigma_{1} \simeq-\frac{1}{2}+\frac{\frac{\partial}{m} J_{m+1}(x)}{x J_{m+1}^{\prime}(x)} \simeq-\frac{1}{2}-\frac{1}{x} \Gamma_{j m+1}
$$

since $J_{m+1}(x)=0$ from $(\mathbf{C} 46)$. However, $\boldsymbol{J}_{\mathbf{m}+1}, 0$ and we see that the gravity modes are damped.

APPENDIX D

Starting from Spiegel (1964) a few trivial manipulations on his equations (78) and (81) yield expressions for $\chi$ and $\mathbf{w}$. His equation for can be simplified by using the recurrence relations as in Appendix B.

So

$$
\begin{aligned}
& \bar{\rho} w=\frac{2 a \gamma(2 a \zeta)^{m} e^{-a \xi}}{g\left(\sigma^{4}-a^{2}\right)}\left[\sigma^{-1}(m+1) M(a, m+1,2 a 5)+\xi M(a, m+2,2 a \xi)\left(a-\sigma^{2}\right) a\right] \\
& \bar{p} X=(2 a \zeta)^{m+1} e^{-a 5} M(a, m+2,2 a 5)
\end{aligned}
$$

where

$$
a=\frac{m+1}{2 \gamma}\left(\frac{\sigma^{-4}-a^{2}}{a \sigma^{2}}\right)+\frac{m}{2}\left(\frac{a-\sigma^{2}}{\sigma^{2}}\right)+(m+1)
$$

An equation for $\boldsymbol{\theta}$ may be obtaind as follows:

In the absence of dissipation, the equations for the thermodynamic fluctuations are

$$
\partial_{t} \rho+\rho g w=c^{2}\left(\partial_{t} \rho+w \frac{d \bar{s}}{d z}\right)
$$




$$
\begin{aligned}
& d_{t} \rho+w \frac{d \bar{\rho}}{d \bar{t}}=-\bar{\rho} x \\
& \theta / T=p / \bar{\rho}+\rho / \bar{\rho}
\end{aligned}
$$

(D4), (D5) and (D6) may be combined to give an equation for $\theta$ in terms of $\mathrm{w}$ and $x$

$$
\partial_{t} \theta=\bar{T}[(1-\gamma) x-w / z]
$$

$\chi$ and $\mathbf{w}$ can then be eliminated using (D1) and (D2). After some tidying up and replacing by by $n$ :

$$
\begin{aligned}
\theta= & \frac{\beta_{0}(2 a 5)^{m} e^{-a 5}}{n g P} \llbracket(1-\gamma)(m+1)(2 a 5) M(a, m+2,2 a 5)- \\
& \quad-\frac{2 a \gamma}{\sigma^{4}-a^{2}}\left[\sigma^{2}(m+1) M(a, m+1,2 a 5)+a\left(a-\sigma^{2}\right) 5 M(a, m+2,2 a 5)\right] \rrbracket \\
\propto & e^{-a .5} \llbracket(1-\gamma)(m+1)(2 a 5) M(a, m+2,2 a 5)- \\
& \quad-\frac{2 a \gamma}{\sigma-a^{2}}\left[\sigma^{2}(m+1) M(a, m+1,2 a 5)+a\left(a-\sigma^{2}\right) 5 M(a, m+2,2 a 5)\right] \rrbracket
\end{aligned}
$$

In the case of a Lamb wave

$$
a \simeq 1, \sigma^{2} \simeq-a
$$

and the expression for $\mathbf{w}$ simplifies considerably:

$$
\begin{aligned}
& w \propto e^{-a 5}\left[-a(m+1) M(1, m+1,2 a \zeta)+2 a^{2} 5 M(1, m+2,2 a 5)\right] \\
&=a e^{-a 5}[-(m+1)\left(1+\frac{2 a 5}{m+1}+\frac{(2 a 5)^{2}}{(m+1)(m+2)}+\cdots\right)+ \\
&\left.+2 a \zeta\left(1+\frac{2 a 5}{m+2}+\frac{(2 a 5)^{2}}{(m+2)(m+3)}+\cdots\right)\right] \\
&=-(m+1) a e^{-a 5}
\end{aligned}
$$

This expression is asymptotically valid in the interior of the layer away from a thin bounday layer at the bottom. Expressions for $\boldsymbol{x}$ and $\boldsymbol{\theta}$ may be obtained by using the asymptotic result

$$
M(1, b, z) \sim \Gamma(b) z^{1-b} e^{z}
$$


so that

$$
\begin{aligned}
& x \sim(2 a .5)^{-(m+1)} e^{0.5} \\
& \theta \sim(2 a 5)^{-m} e^{0.5}
\end{aligned}
$$

in the interior of the layer away from both top and bottom boundaries.

For the remaining acoustic and gravity modes

$$
s=-N, N=0,1,2, \ldots
$$

so that

$$
M(-N, b, z)=1+\frac{(-N) z}{b 1 !}+\frac{(-N)(-N+1) z^{2}}{b(b+1) 2 !}+\cdots+\frac{(-N)(-N+1) \ldots(-1) z^{N}}{b(b+1) \ldots(b+N-1) N !}
$$

Therefore, $w, \boldsymbol{x}$ and $\theta$ are proportional to $e^{-a 5}$ as multiplied by polynomials of as

$$
\begin{aligned}
& w \propto e^{-05} p_{w}(0.5) \\
& x \propto e^{-0.5} L_{N}^{(m+1)}(a 5) \\
& \theta \propto e^{-05} P_{\theta}(a 5)
\end{aligned}
$$

where $\quad L\left(\mathrm{~m}_{\mathbf{N}}+\mathbf{1}\right)(\mathbf{a} \mathbf{5})$ is a Laguerre polynomial. In the limit $a \rightarrow 0$ for the acoustic waves, the leading contributions to (D1), (D2) and (D9) yield:

$$
\begin{aligned}
& w \sim M(a, m+1,2 a s) \\
& x \sim M(a, m+2,2 a 5) \\
& \theta \sim(1-\gamma) 5 M(a, m+2,2 a 5)-\frac{\gamma}{\sigma 2} M(a, m+1,2 a 5)
\end{aligned}
$$

The appropriate asymptotic form of the confluent hypergeometric functions is

$$
M(a, b, z) \sim \Gamma(b)(-a z)^{\frac{d}{2}(1-b)} J_{b-1}(2 \sqrt{-a z})
$$

so that

$$
w \sim \Gamma(m+1)(-2 a a .5)^{-\frac{m}{2}} J_{m}(2 \sqrt{-2 a a b})
$$




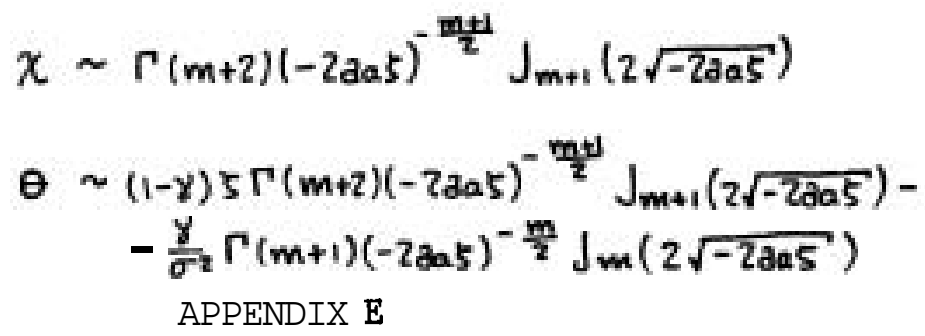

Since acoustic waves are weakly rotational, unless the stratification is very strong, it is reasonable to let $\mathbf{d} / \mathbf{d z}=0$, which means that the buoyance frequency is zero and the acoustic waves can be completely irrotational. In order to ease the analysis I have taken the equilibrium layer to be isothermal and confined between rigid horizontal boundaries. The assumption of neutral buoyancy is equivalent to $\gamma=1$. The equations of motion are

$$
\begin{aligned}
& \bar{P} \frac{D U}{D t}=-\nabla p+g \rho \\
& \frac{D f}{D t}+\rho \nabla \cdot x=0 \\
& \frac{D S}{D E}=0 \\
& P=R_{\rho} T
\end{aligned}
$$

Since

$$
s \propto \ln (P / \rho \gamma)
$$

and $Y=1$, it follows from (E3) that

$$
D(P / \rho)=0
$$

In equilibrium the pressure and density are distributed as follows:

$$
p \propto e^{z / H}, \rho \propto e^{z / H}, p=g H \rho
$$

in which case $P=g H \rho$ in the disturbed fluid. Equation(El) may be rewritten as

$$
\partial t \chi+\frac{1}{2} \nabla x^{2}=-g H \nabla \ln \rho+\nabla(g z)
$$

Let

$$
\underline{\nu}=\nabla \phi
$$

then an equation for $\phi$ can be obtained by substituting (E9) into ( 82 ) and (E8) and eliminating $P$ between (E2) and (E8) :

$$
\frac{D}{D t}\left[\partial t \phi+\frac{1}{2}(\nabla \phi)^{2}-g z\right]=g H \nabla^{2} \phi
$$


Now adimensionlize using $g$ and $\mathrm{H}$ to define time, length and velocity scales:

$$
t \rightarrow\left(\frac{H}{g}\right)^{1 / 2} t, \underline{x} \rightarrow H \underline{x}, \underline{X} \rightarrow(g H)^{n /} \underline{u}
$$

(E10) becomes

$$
\begin{gathered}
\underset{D_{t}}{D}\left[\partial_{t} \phi+\frac{1}{2}(\nabla \phi)^{2}-\zeta\right]=\nabla^{2} \phi \\
\text { or, after rearrangement, } \\
\nabla^{2} \phi+\partial_{z} \phi-\partial_{t}^{2} \phi=\partial_{t}(\nabla \phi)^{2}+\frac{1}{2} \nabla \phi \cdot \nabla(\nabla \phi)^{2}
\end{gathered}
$$

The Lone Wave Limit

Let the layer have its boundaries at

$$
\zeta=0, \pi / l
$$

so that at the boundaries

$$
\partial z \phi=0
$$

In order to get an amplitude equation for the gravest acoustic cut off mode the following scalings are appropriate

$$
\phi \rightarrow \epsilon \phi, \partial_{x} \rightarrow \epsilon \partial_{x}, \partial_{t} \rightarrow \partial_{t}+\epsilon^{2} \partial_{T}, \partial_{z} \rightarrow \partial_{z}
$$

Write D for $\partial z$, then (E13) bcomes

$$
\begin{aligned}
\left(D^{2}+\epsilon^{2} \partial x\right) \phi+D \phi-\left(\partial_{t} \partial^{2}+\epsilon^{2} \partial \tau\right)^{2} \phi= & \epsilon\left(\partial_{t}+\epsilon^{2} \partial \tau\right)\left[(D \phi)^{2}+\epsilon^{7}(\partial x \phi)^{2}\right]+ \\
& +\frac{1}{2} \epsilon^{2}(\nabla \phi) \cdot V(\nabla \phi)^{2}
\end{aligned}
$$

where $\nabla=(\epsilon \partial \mathrm{x}, \mathcal{D})$ and the disturbances are assumed to be independent of $\mathrm{y}$. Let

$$
\phi=\phi_{0}+\epsilon \phi_{1}+\epsilon^{\prime} \phi_{2}+\cdots \cdots
$$

then (E17) may be expanded as follows:

$O(1)$ :

$$
D^{2} \phi_{a}+D \phi_{0}-\partial_{t}^{2} \phi_{0}=0
$$

$O(\epsilon):$

$$
D^{2} \phi_{1}+D \phi_{1}-\partial_{t}^{c} \phi_{1}=\partial_{t}\left(D \phi_{0}\right)^{2}
$$

$O\left(\epsilon^{2}\right): \quad D^{2} \phi_{2}+D \phi_{2}-\partial_{t}^{2} \phi_{2}=-\partial_{x}^{2} \phi_{0}+2 \partial_{t} \partial_{\tau} \phi_{0}+2 \partial_{t}\left[\left(D \phi_{0}\right)\left(D \phi_{1}\right)\right]+$

$$
+\frac{1}{3} D\left[\left(D \phi_{0}\right)^{3}\right]
$$


with boundary conditions

$$
D \phi_{0}=D \phi_{1}=D \phi_{2}=0 \text { at } 5=0, \pi / 2
$$

At $0(1)$ :

$$
\phi_{0}=A(x, T) e^{-5 / 2} f(5) \sin \tau
$$

where

$$
\begin{gathered}
f(5)=\sin 15+2 l \cos l 5, \tau=\omega t+\theta(x, T) \\
\text { and } \omega^{2}=l^{2}+1 / 4
\end{gathered}
$$

At $O(\epsilon)$ :

$$
\left[D(D+1)-\partial_{t}^{2}\right] \phi_{1}=4 \omega^{5} A^{2} e^{-5} \sin ^{2} l 5 \sin 2 \tau
$$

It is only necessary to find a particular integral of this equation, since the complementary function makes no contribuiton to the solvability condition applied at the next order. One particular integral is

$$
\phi_{1}=\frac{1}{2} \omega^{3} A^{2} e^{-5}[1-\cos 2 \ell r+2 l \sin 2 \ell 5] \sin 2 \tau
$$

At $O\left(\epsilon^{2}\right)$; (B21) by

The solvability condition at this order is obtained by multiplying

$$
\phi_{0}^{+}=e^{5 / 2} f(\xi)
$$

and integrating from $\xi=0$ to $\pi / 0$. (The $\partial_{t}^{2}$ on the Lis of (E21) is replaced by $\omega^{*}$. This results in two equations for $A$ and $\theta$ which are the coefficients of $\sin r$ and $\cos \tau$.

$$
\begin{gathered}
A_{x x}-A \theta_{x}^{2}-2 \omega A \theta_{T}+\lambda \omega^{4} A^{3}=0 \\
A \theta_{x x}+2 A_{x} \theta_{x}+2 \omega A_{T}=0
\end{gathered}
$$

where

$$
\lambda=\left(\frac{7+8 l^{2}-144 L^{4}-1024 l^{6}}{16\left(1+16 l^{2}\right)\left(1+4 l^{2}\right)}\right) \cdot\left(\frac{\left(1-e^{-1}\right) l}{\pi}\right)
$$

Let

$$
\partial_{T} \rightarrow \frac{1}{2 \omega} \partial_{T} \text { and } B=A e^{i \theta}
$$


then

$$
i B_{T}+B_{x x}+\lambda \omega^{4}|B|^{2} B=0
$$

Providing $\ell$ is not too large, $\lambda>0$ and soliton solutions exist to (E32). The Case When Horizontal Scales are of Order $\mathrm{H}$

In this case the appropriate scaling is

$$
\partial_{x} \rightarrow \partial_{x}+\epsilon \partial_{\mathbf{X}}, \partial_{t} \rightarrow\left(\partial_{t}+c \partial_{X}\right)+\epsilon c \partial_{\mathbf{X}}+\epsilon^{2} \partial_{T}, \phi \rightarrow \epsilon \phi
$$

and (E13) becomes

$$
\begin{aligned}
& {\left[\left(\partial_{x}+c \partial_{x}\right)^{2}+D^{2}+D-\left(\partial_{t}+c \partial_{x}+\epsilon c \partial_{\mathbf{X}}+\epsilon^{2} \partial_{T}\right)^{2}\right] \phi=} \\
& \left.=\epsilon \overline{\mathbf{D}} \partial_{t}+c \partial_{x}+\epsilon c \partial_{\underline{I}}+\epsilon^{2} \partial_{\mathbf{T}}\right]\left[\left(\partial_{x} \phi+\epsilon \partial_{\mathbf{x}} \phi\right)^{2}+(D \phi)^{2}\right]+\frac{1}{2} \epsilon^{2}(\nabla \phi) \cdot \nabla(\nabla \phi)^{2}
\end{aligned}
$$

where

$$
\nabla=\left(\partial x+\epsilon \partial_{\mathbf{x}}, \boldsymbol{D}\right)
$$

Let

$$
\phi=\phi_{0}+\epsilon \phi_{1}+\epsilon^{2} \phi_{2}+\cdots
$$

then (E34) may be expanded in powers of $\in$ :

$O(1): \quad\left[\partial_{x}^{2}+D(D+1)-(\partial t+C \partial x)^{2}\right] \phi_{0}=0$

$$
\begin{aligned}
O(\epsilon): \quad\left[\partial_{x}^{2}+D(D+1)-\left(\partial_{t}+c \partial_{x}\right)^{2}\right] \phi_{1}= & 2\left[c\left(\partial_{t}+c \partial_{x}\right)-\partial x\right] \partial \mathbf{x} \phi_{0}+ \\
& +\left(\partial t+c \partial_{x}\right)\left[\left(\partial x \phi_{0}\right)^{2}+\left(D \phi_{0}\right)^{2}\right]
\end{aligned}
$$

$O\left(\epsilon^{2}\right):\left[\partial_{x}^{2}+D(D+1)-\left(d_{t}+c \partial_{x}\right)^{2}\right] \phi_{2}=-\partial_{x}^{2} \phi_{0}+c^{2} \partial_{x}^{2} \phi_{0}+2\left(\partial_{t}+c \partial x\right) \partial+\phi_{0}+(E 39)$

$+2\left[c\left(\partial t+c \partial_{x}\right)-\partial_{x}\right] \partial_{x} \phi_{1}+c \partial \mathbf{x}\left[\left(\partial x \phi_{0}\right)^{2}+\left(D \phi_{0}\right)^{2}\right]+$

$$
+\frac{1}{2}\left(\nabla_{0} \phi_{0}\right) \cdot \nabla_{0}\left(\nabla_{0} \phi_{0}\right)^{2}+2\left(\partial t+c \partial_{x}\right)\left[\left(\partial_{x} \phi_{0}\right)\left(\partial \phi_{1}+\partial_{x} \phi_{0}\right)+\left(D \phi_{0}\right)\left(D \phi_{1}\right)\right]
$$

where $\nabla_{0}=\left(\partial_{x}, D\right)$. 
When $\boldsymbol{c}$ is chosen to be the group velocity the first term on the RHS of (E38) vanishes. In (E39) the terms containing resonances are

$$
\begin{aligned}
& \left(c^{2}-1\right) \partial^{2} \phi_{0}+2\left(\partial_{t}+c \partial_{x}\right) \partial_{\tau} \phi_{0}+\frac{1}{2}\left(\nabla \phi_{0}\right) \cdot \nabla\left(\nabla \phi_{0}\right)^{2}+ \\
& +2\left(\partial_{t}+c \partial_{x}\right)\left[\left(\partial_{x} \phi_{0}\right)\left(\partial_{x} \phi_{1}\right)+\left(D \phi_{0}\right)\left(D \phi_{1}\right)\right]
\end{aligned}
$$

It is only necessary to solve for a particular integral of ( $\mathbf{8} 38$ ), since the complementary function makes no contribution to the solvability condition.

At $0(1)$ :

$$
\phi_{0}=A(x, T) e^{-x / 2} f(5) \sin \tau
$$

where

$$
\begin{gathered}
f(5)=\sin 25+22 \cos l 5, \tau=\omega t+k x+\theta(x, T) \\
\text { and } \Omega^{2}=(\omega+c k)^{2}=k^{2}+l^{2}+1 / 4
\end{gathered}
$$

At $O(\in)$ :

$$
\begin{aligned}
\text { Set }[c(\partial t+c \partial x)-\partial x] \partial x \phi_{0}=0 \\
c=k / \Omega=\frac{\partial \Omega}{\partial k}
\end{aligned}
$$

A particular integral of (E38) is

$$
\begin{gathered}
\phi_{1}=\Omega A^{2} \sin 2 \tau e^{-5}\left[\frac{1}{2}\left(L^{2}-k^{2}\right)+\frac{1}{2 L}\left(k^{2}-2 h^{2} L^{2}-L^{2} \Omega^{2}\right) \cos 2 l 5-\right. \\
\left.-\frac{1}{L^{2}}\left(k^{2}-L^{2} \Omega^{2}\right) \sin 2 l 5\right]
\end{gathered}
$$

At $O\left(\epsilon^{2}\right):$

The solvability condition at this order involves multiplying (E39) by

$$
\phi_{0}^{+}=e^{5 / 2} f(5)
$$

and integrating from 0 to $\pi / \mathfrak{l}$. This yields two equations which come from the coefficients of $\sin \tau$ and $\cos \tau$ :

$$
\begin{aligned}
& \left(c^{2}-1\right)\left(A_{Y Y}-A \Theta_{Y}^{2}\right)-2 \Omega A \Theta_{T}+\lambda A^{3}=0 \\
& \left(c^{2}-1\right)\left(A_{\mathbf{I}} \theta_{\mathbf{X}}+A \theta_{\mathbf{x}}\right)+2 \Omega A_{T}=0
\end{aligned}
$$


where

$$
\begin{aligned}
\lambda= & \frac{l}{\pi} \frac{k^{2}}{8 L^{2}} \int_{0}^{\pi / 2} d 5 e^{-5} f(5)^{2}\left[4 L^{4} \sin ^{2} l 5-k^{2} f(5)^{2}\right] \\
& -\frac{l}{4 \pi} \int_{0}^{\pi / 2} d 5 e^{-5} f(5) \sin l 5\left[k ^ { 2 } \left(-2 L^{2}+\left(1 / 2-6 l^{2}\right) \cos 2 l 5+\right.\right. \\
& \left.+\left(4 l^{3}-l\right) \sin 2 l 5+2 L^{4}(-1+\cos 2 l 5+2 l \sin 2 d 5)\right] \\
& -\frac{k^{2} \Omega^{2}}{L^{2}} \frac{L}{\pi} \int_{0}^{\pi / 2} d 5 e^{-5} f(5)^{2}\left[1 / 2\left(L^{2}-k^{2}\right)+\frac{1}{2 L^{2}}\left(k^{2}-2 k^{2} L^{2}-L^{2} \Omega^{2}\right) \cos 2 \Omega 5-\right. \\
& \left.-\frac{l}{L^{2}}\left(k^{2}-L^{2} \Omega^{2}\right) \sin 215\right] \\
& +\frac{\Omega^{2} l}{\pi} \int_{0}^{\pi / Q} d 5 e^{-5} f(5) \sin l 5\left[-1 / 2\left(L^{2}-k^{2}\right)-\left(k^{2}-2 L^{2} \Omega^{2}\right) \cos 215+\right. \\
& \left.+2 k^{2} l \sin 2 l 5\right] \\
\text { and } L^{2}= & L^{2}+1 / 4 .
\end{aligned}
$$

I have not yet had time to evaluate $\lambda$ explicitly, in order to determine where it is positive and negative. If the following rescalings are made

$$
\partial_{\mathbf{X}} \rightarrow \frac{1}{\sqrt{1-c^{2}}} \partial_{\mathbf{X}}, \partial_{\tau} \rightarrow \frac{1}{2 \Omega} \partial_{T}
$$

and $B$ is defined as

$$
B=A e^{-i \theta}
$$

then

$$
i B_{T}+B \mathbf{x x}-\lambda|B|^{2} B=0
$$

When $\lambda<O$, (E52) admits soliton solutions.

\section{APPENDIX F}

In the appendix I derive an amplitude equation for gravity waves of long horizontal length scale in a weakly stratified isothermal layer bounded by two rigid horizontal boundaries. The equations of motion are

$$
\begin{gathered}
(\bar{\rho}+\rho) \stackrel{\frac{D}{D t} u}{=}=-\nabla_{p}+g \rho \\
\frac{D}{D t}(\bar{s}+s)=0
\end{gathered}
$$




$$
\mathcal{D} t(\bar{\rho}+\rho)+(\bar{\rho}+\rho) \nabla \cdot u=0
$$

where $\bar{\psi}$ is the distribution of in hydrostatic equilibrium and $P, \rho, s$ are the fluctuations of pressure, dnsity and entropy. Equation (F2) can be rewritten as

$$
D E(\ln \bar{p}-\gamma \ln \bar{p})+\frac{D}{D} \ln (1+P / \bar{p})-\gamma P_{t} \ln (1+P / \bar{p})=0
$$

since

$$
\bar{s}+s \propto \ln (\bar{p}+p)-\gamma \ln (\bar{\rho}+\rho)
$$

The equilibrium pressure and density are distributed as

$$
\bar{p}=\hat{p} e^{z / H}, \bar{p}=\hat{\rho} e^{z / H} \text { and } \hat{p}=g H \hat{p}
$$

where $\hat{\mathrm{P}}$ and $\hat{p}$ are constants. The equations of motion can be adimensionalized using $\hat{g}, H, \hat{p}, \hat{\rho}$ in the natural way, yielding

$$
\begin{gathered}
\left(e^{5}+\rho\right) D u=-\partial x p \\
\left(e^{5}+\rho\right) D w=-D p+\rho \\
w(1-\gamma)+\frac{D}{D t} \ln \left(1+e^{-5} p\right)-\gamma \frac{D}{D t} \ln \left(1+e^{-5} \rho\right)=0 \\
w e^{5}+D t \rho+\left(e^{5}+\rho\right) \nabla \cdot u=0
\end{gathered}
$$

where

$$
\zeta=z / H \text { and } D=\partial_{\zeta}
$$

Let

$$
\begin{gathered}
\gamma=1+\epsilon^{2} E, \partial_{t} \rightarrow \epsilon^{2} \partial_{t}+\epsilon^{4} \partial_{\tau}, u \rightarrow \epsilon^{3} u, w \rightarrow \epsilon^{4} W \\
p \rightarrow \epsilon^{4} p, \rho \rightarrow \epsilon^{4} \rho, \nabla \rightarrow\left(\partial_{x}, D\right)
\end{gathered}
$$


then (F7) - (F10) become

$$
\begin{aligned}
& \left(e^{5}+\epsilon^{4} \rho\right)\left(\partial_{t}+\epsilon^{2} \partial_{\tau}+\epsilon^{7} U \cdot \nabla\right) u=-\partial x p \\
& \epsilon^{2}\left(e^{5}+\epsilon^{4} \rho\right)\left(\partial_{t}+\epsilon^{2} \partial_{T}+\epsilon^{2} u \cdot \nabla\right) W=-D \rho+\rho \\
& -w E+\left(\partial_{t}+\epsilon^{2} \partial \tau+\epsilon^{2} u, \nabla\right)\left(e^{-5}(p-\gamma \rho)-\frac{1}{2} \epsilon^{q} e^{-25}\left(p^{2}-\gamma \rho^{2}\right)+\cdots\right)=0 \\
& w e^{5}+\epsilon^{2}\left(\partial_{t}+\epsilon^{2} \partial_{T}+\epsilon^{2} \underline{v} \cdot \nabla\right) \rho+\left(e^{5}+\epsilon^{4} \rho\right) \nabla \cdot u=0
\end{aligned}
$$

with the boundary conditions

$$
w=0 \text { at } \Sigma=0, \pi / R
$$

Let

$$
\begin{aligned}
& u=u_{0}+\epsilon^{2} u_{2}+\cdots, \quad w=w_{0}+\epsilon^{x} w_{2}+\cdots \\
& p=p_{0}+\epsilon^{2} p_{2}+\cdots, \quad \rho=p_{0}+\epsilon^{2} p_{2}+\cdots
\end{aligned}
$$

then at $0(1)$

$$
\begin{aligned}
& e^{5} \partial_{t} u_{0}=-\partial \times p_{0} \\
& \boldsymbol{O}=-D p_{0}+p_{0} \\
&-w_{0} E+e^{-5} \partial_{t}\left(p_{0}-p_{0}\right)=0 \\
& w_{0}+\nabla \cdot u_{0}=0
\end{aligned}
$$

and at $O\left(\epsilon^{2}\right)$ :

$$
\begin{gathered}
e^{5}\left[\partial_{t} u_{2}+\left(\partial_{\tau}+u_{0} \cdot \nabla\right) u_{0}\right]=-\partial_{x} p_{2} \\
e^{5} \partial_{t w}=-D p_{2}+\rho_{7} \\
-w_{2} E+e^{-5} \partial_{t}\left(p_{2}-\rho_{2}\right)-E e^{-5} \partial_{t} \rho_{0}+e^{-5} \partial_{T}\left(p_{0}-\rho_{0}\right)+ \\
+\varphi_{0} \cdot \nabla\left[e^{-5}\left(p_{0}-\rho_{0}\right)\right]=0 \\
w_{2} e^{5}+\partial_{t} \rho_{0}+e^{5} \nabla \cdot u_{2}=0
\end{gathered}
$$


The amplitude equation is obtained from the solvability condition at $0\left(\epsilon^{2}\right)$. The $0(1)$ equations are satisfied by

$$
\begin{array}{ll}
u_{0}=G_{x} e^{-5 / 2} f(5) & w_{0}=-2 G_{x \times} e^{-5 / 2} \sin l 5 \\
p_{0}=-G_{t} e^{5 / 2} f(5) & p_{0}=-\frac{1}{2} G_{t} e^{5 / 2}\left[\left(1-4 \Omega^{2}\right) \sin l 5+4 l \cos l 5\right]
\end{array}
$$

where

$$
f(\zeta)=\operatorname{sinl} \zeta+2 l \cos l \zeta \text { and } G=G(x, t, T)
$$

At this order G satisfies

$$
\left(E d_{x}^{2}-L^{2} d_{E}^{2}\right) G=O
$$

where

$$
L^{2}=l^{2}+1 / 4
$$

At $O\left(\epsilon^{2}\right)$, the equations (F23) - (F26) can be tidied up into an equation for $w_{2}$ :

$-\left(E \partial_{x}^{2}+\partial_{t}^{2} D(D+1)\right) w_{2}=\partial_{t}^{3} D\left(e^{-5} \rho_{0}\right)-\partial x \partial t D D+u_{0}+\partial_{t}^{2} \partial_{x}^{2} w_{0}+$

$$
+E e^{-5} \partial t \partial x \rho_{0}^{2}-\partial_{x}^{2} D_{T}\left(e^{-5}\left(p_{0}-\rho_{0}\right)\right)
$$

Now multiply by

$$
w_{0}^{+}=G e^{5 / 2} \sin 15
$$

and integrate over space and time. If $G_{x}, G_{x}, G_{x}$ vanish as $x \rightarrow w$ and $t \rightarrow \infty$ the LHS of the integral of (F31) vanishes. The RHS yields the following amplitude equation:

$$
\begin{aligned}
& \frac{\pi}{2 L} L^{2} G_{t t t t}+\frac{\pi}{l} L^{2} G_{x \times t T}+2 \not L^{2}\left(G_{x}^{2}\right)_{x \times t}-\frac{\pi}{2} G_{x x \times x t t}-\frac{\pi}{4}\left(1-4 Q^{2}\right) E G_{t t \times x}+ \\
& +\frac{\pi}{3} L^{2} G_{x \times t \tau}+\frac{4}{3} A \frac{\pi}{2 l} L^{2}\left(G_{x}^{2}\right)_{x \times t}+\frac{4}{3} A \frac{\pi}{7 l} L^{2}\left(G_{x x} G_{t}\right)_{x x}=0
\end{aligned}
$$

where

$$
A=\frac{2 l}{\pi} \int_{0}^{\pi / 2} e^{-5 / 2} \sin d S d S=\frac{12 L^{4}\left(1-e^{-\pi / 2 L}\right)}{\pi L^{2}\left(L^{2}+8 L^{2}\right)}
$$

(F33) can be tidied up by dividing through by $\pi L^{2} / L$, using (F29) to write

$$
\frac{1}{2} L^{2} G t t t-\frac{1}{4}\left(1-4 l^{2}\right) E G t t x x=E L^{2} G t x x
$$




$$
G_{x x x x t t}=\frac{L^{2}}{E} G_{x x t t t t}
$$

and lifting two $\mathrm{x}$ derivatives:

$$
E G_{t t}+2 G_{t T}+\frac{8}{3} A\left(G_{x}^{2}\right)_{t}+\frac{2}{3} A G_{x x} G_{t}-\frac{1}{E} G_{t t t}=0
$$

In thecase of stable stratification ( $E>0$ ), let

$$
G=G(x+c t, T)
$$

where (from (F29))

$$
c^{2}=E / L^{2}
$$

Rescale $G$ to remove the factor $A$

$$
G \rightarrow G / A
$$

then

$$
2 G_{x T}+3\left(G_{x}^{2}\right)_{x}+E c G_{x x}-\frac{c^{3}}{E} G_{x \times x x}
$$

Let

$$
G_{x}=2 \mu
$$

and adjust the slow time scale by transforming to a moving frame such that

$$
\partial T \rightarrow \partial_{r}-\frac{C E}{2} \partial x
$$

then

$$
u_{T}+6 U U_{x}-\left(\frac{c^{3}}{2 E}\right) U_{x x x}=0
$$

which is the $\mathrm{KdV}$ equation. 


\title{
THE INFLUENCE OF WAVEWAVE INTERACTIONS ON NEAR-INERTIAL WAVES
}

Motivation

\author{
Eric Kunze
}

Wave-mean flow interaction theory predicts critical-layer trapping and amplification on near-inertial waves in regions of negative vorticity (Figure 1) which is consistent with the intense near-inertial wave packets observed on the warm sides of fronts (Figure 2) (Kunze and Sanford, 1984). Near-inertial energy is being continually pumped into these trapping regions at the surface, $F_{f}$. Clearly, this energy cannot accumulate indefinitely; there must be sinks. Three possible sinks are shown schematically in Figure 3:

i) Wave breaking and turbulent dissipation;

i i ) Loss to the mean flow $\vec{N}_{0}(\vec{r})$;

i i i) Loss to higher frequency internal waves (by wave-wave interactions) which are free to propagate out of the trapping region.

Which of these sinks dominates may have important consequences to mixing and the maintenance of the internal wave spectrum.

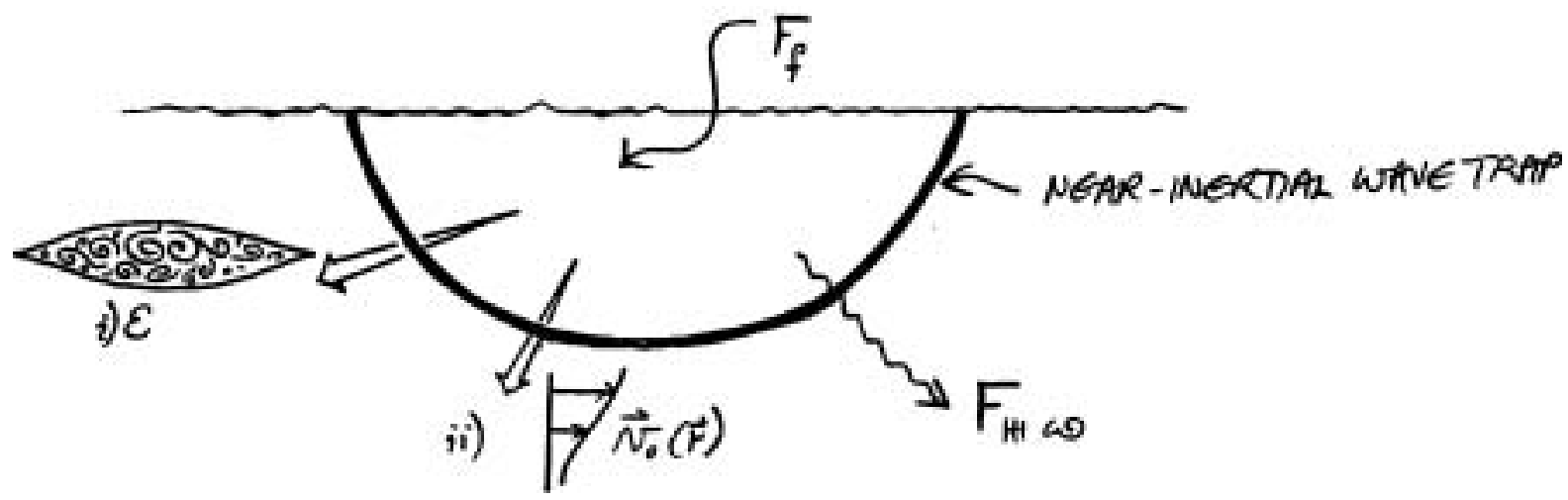

Figure 3

The influence of wave-wave interactions will be focussed on here but a brief discussion of the other sinks is appropriate.

As a near-inertial wave packet approaches its critical-depth, its vertical wavelength shrinks, consistent with the wave becoming more inertial, and the wave amplitude increases, consistent with conservation of action-flux and a diminishing group velocity. Both of these changes enhance the shear and, therefore, the likelihood of internal wave breaking. Turbulent dissipation values of $E \approx 10^{-4} \mathrm{~W} / \mathrm{m}^{3}$ (1000 times larger than typical pycnocline values) have been measured in the core of a warm-core ring in layers $100 \mathrm{~m}$ thick at a depth and $600 \mathrm{~m}$ (Lueck, 1982) in the same depth range that intense near-inertial shear has been observed in other rings. Can dissipation account for all the trapped energy-flux? Taking a typical measured near-inertial flux of $\sim 0.08 \mathrm{~W} / \mathrm{m}^{2}$ (D'Asaro and Perkins, 1984) and assuming it is dissipated in a $100 \mathrm{~m}$ thick layer, one gets dissipation values 

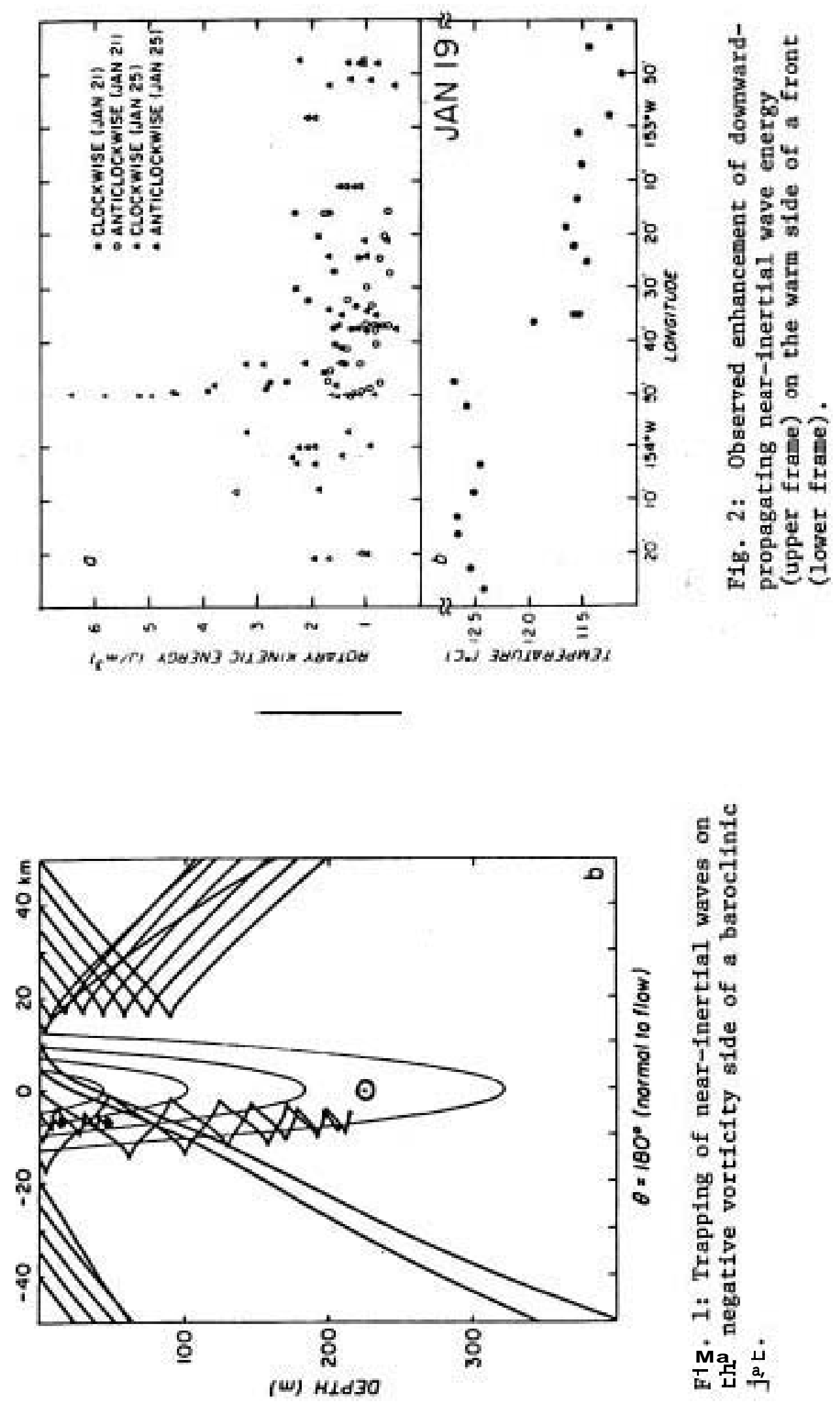
$\mathcal{E} \approx 8 \cdot 10^{-4} \mathrm{~W} / \mathrm{m}^{3}$. Lueck's measurements account for only $=20 \%$ of the near-inertial flux. Though this estimate is crude, it suggests another sink must predominate.

Less can be said about possible energy exchange with the mean flow. Muller (1976) postulated a momentum exchange with internal waves acting as an eddy viscosity. He found a negligible contribution from near-inertial waves. However, he formally excluded critical-layers from his analysis. Measurements have found vertical eddy viscosities much smaller than his estimates (Ruddick and Joyce, 1979), and horizontal viscosities much larger (Brown and Owens, 1982), bringing his formalism into question. The problem of energy exchange between internal waves and the mean flow seems unresolved.

The influence of wave-wave interactions will be presented here. We will be addressing the question: Is there a net loss of energy from a near-inertial wave packet due to wave-wave interaction sufficient to prevent the packet's shear from becoming instable as the packet approaches its critical-depth?

\section{$\underline{\text { Basic Equations }}$}

The loss of energy from a near-inertial internal wave $\left(\vec{k}_{0}, \omega_{0}\right)$ to the rest of the continuum is, to the lowest order, due to a difference reaction with two other waves $\left(\mathrm{k}_{1}, \boldsymbol{\omega}_{1}\right),\left(\overrightarrow{\mathrm{k}}_{2}, \boldsymbol{\omega}_{2}\right)$, and can be most easily expressed in terms of the wave action $A=E / \omega$ (McComas, 1975).

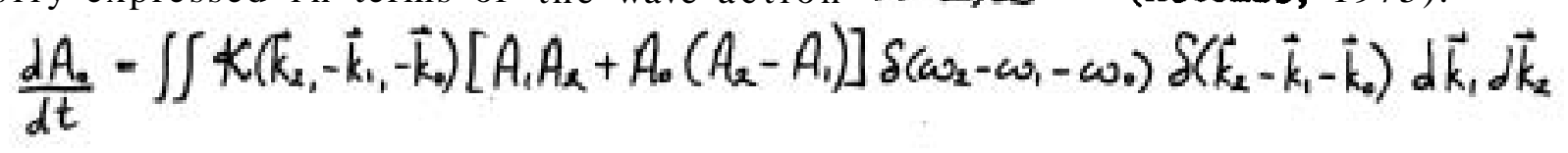

where the $A_{1}\left(\vec{k}_{1}\right)$ are the action-spectra and the interaction coefficient

$$
\begin{aligned}
& K\left(\vec{k}_{2},-\vec{k}_{1},-\vec{k}_{0}\right)=\frac{8 \pi\left(\omega_{0}^{2}-f^{2}\right)\left(\omega_{1}^{2}-f^{2}\right)\left(\omega_{2}^{2}-f^{2}\right)}{N^{2}}\left|\mathbb{D}\left(\vec{k}_{2},-\vec{k}_{1}-\vec{k}_{0}\right)\right|^{2} \\
& \mathbb{D}\left(\vec{k}_{2},-\vec{k}_{1},-\vec{k}_{0}\right)=p_{0}^{*}\left(\vec{k}_{0} \cdot \vec{h}_{1}^{*}\right)\left(\vec{k}_{0} \cdot \vec{h}_{2}\right)+P_{1}^{*}\left(\vec{k}_{1} \cdot \vec{h}_{0}^{*}\right)\left(\vec{k}_{1} \cdot \vec{h}_{2}\right)+P_{2}\left(\vec{k}_{2} \cdot \vec{h}_{0}^{*}\right)\left(\vec{k}_{2} \cdot \vec{h}_{1}^{*}\right) \\
& \text { with } \vec{\lambda}=(x, \eta, 1) \text {; } \\
& \left\{\begin{array}{l}
z=-\frac{k_{z}}{k_{\mu}^{2}}\left(k_{x}+i \frac{f k_{y}}{\omega}\right) \\
\eta=-\frac{k_{z}}{k_{\mu}^{2}}\left(k_{y}-i \frac{f k_{x}}{\omega}\right) \\
p=i \frac{k_{z}}{k_{u}^{2}}\left(\omega^{2}-f^{2}\right)
\end{array}\right.
\end{aligned}
$$

relating the horizontal displacements $\mathcal{Z}, \not{Z}$ and the pressure $p$ to the vertical displacement for internal waves. The integral of (1) will produce a nonzero contribution to $\frac{d \ddot{A}_{s}}{d t}$ only if the resonance conditions are satisfied

$$
\begin{aligned}
& \int \vec{k}_{2}=\vec{k}_{1}+\vec{k}_{1} \\
& \omega_{2}=\omega_{1}+\omega_{0}
\end{aligned}
$$


The Resonance Domain in the $\boldsymbol{\omega}_{\boldsymbol{i}}, \boldsymbol{k}_{\boldsymbol{z}_{1}}-$ Plane

Because the internal wave dispersion relation is horizontallyisotropic, we can assume $k_{H_{*}}=k_{x_{0}}, k_{s_{0}}=0 \quad$ without loss of generality. Then the resonance conditions become

$$
k_{x_{2}}=k_{x_{1}}+k_{x_{0}} ; \quad k_{y_{2}}=k_{y_{1}} ; k_{z_{2}}=k_{z_{1}}+k_{2} ; \omega_{2}=\omega_{1}+\omega .
$$

Each wave must also satisfy the internal wave dispersion relation, implying

$$
\left\{\begin{array}{l}
k_{x_{1}}^{2}+k_{y_{1}}^{2}=\frac{\left(\omega_{1}^{2}-f^{2}\right)}{N^{2}} k_{z_{1}}^{2} \\
k_{x_{0}}^{2}=\frac{\left(\omega_{0}^{2}-f^{2}\right)}{N^{2}} k_{x_{0}}^{2} \\
\left(k_{x_{1}}+k_{x_{0}}\right)^{2}+k_{y_{1}}^{2}=\frac{\left(\left(\omega_{1}+\omega_{0}\right)_{2}-f^{2}\right)\left(k_{z_{1}}+k_{z_{0}}\right)^{2}}{N^{2}}
\end{array}\right.
$$

where equations (4) have been substituted in for wave 2 's variables in the last relation. Equations (5) can be manipulated into an equation for

$$
\frac{k_{x_{1}}}{k_{H_{1}}}=\cos \theta_{1}=\frac{\left(\left(\omega_{1}+\omega_{0}\right)^{2}-f^{2}\right)\left(k_{z_{1}}+k_{e_{0}}\right)^{2}-\left(\omega_{1}^{2}-f^{2}\right) k_{z_{1}}-\left(\omega_{0}^{2}-f^{2}\right) k_{z_{2}}^{2}}{2 \sqrt{\omega_{0}^{2}-f^{2}} \sqrt{\omega_{1}^{2}-f^{2}} k_{z_{1}} k_{z_{0}}}
$$

Resonance occurs provided RHS $\in[-1,1]$ which for fixed $\omega_{1}$ implies for interval(s) in $\mathbf{k z}_{1}$. The bounds for these interval(s) are $\sin \theta_{1}=0$, or $k_{y 1}=0$. Setting $k_{y 1}=0$ in (5) four curves can be found in the $\omega_{1,} k_{z_{1}}-$ plane that bounds the resonance domains:

$$
k_{z_{1}}=-k_{z} \frac{\left(\sqrt{\left(\omega_{1}+\omega_{0}\right)^{2}-f^{2}} \pm \sqrt{\omega_{0}^{2}-f^{2}}\right)}{\left(\sqrt{\left(\omega_{1}+\omega_{0}\right)^{2}-f^{2}} \pm \sqrt{\omega_{1}^{2}-f^{2}}\right)}
$$

These curves are shown in Figure 4a. They clearly bound the numerically determined resonance domain (Figure 4b). There are two resonance branches. One of the branches asymptotes to $-\mathbf{k z}_{\mathbf{z}} / \mathbf{2}_{\mathbf{2}}$ constant for high $\boldsymbol{\omega}_{1}$, so the near-inertial wave does not exchange energy with waves of greater than twice its wavelength. This corresponds to the elastic scattering limit discussed by McComas and Bretherton (1977). The other branch asymptotes to $-\mathbf{k z}_{0} \omega_{1} / \omega_{0}$. which increases linearly with $\omega_{1}$ and corresponds to the induced diffusion limit.

\section{The Action Integral}

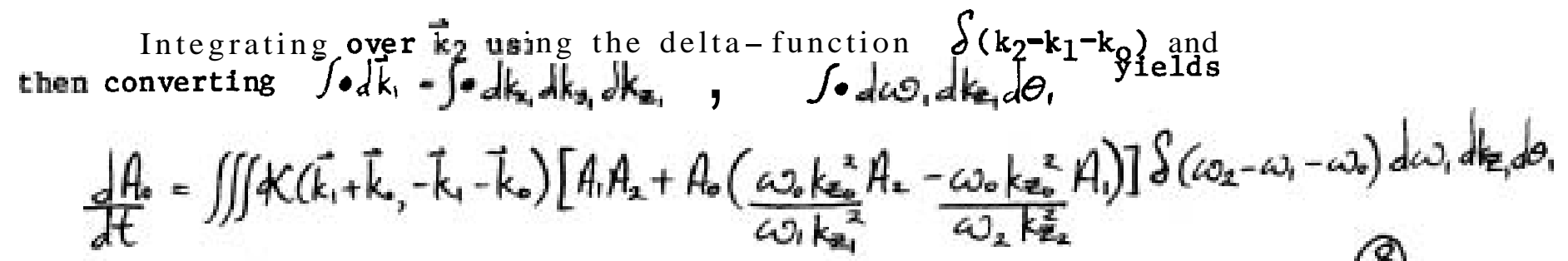



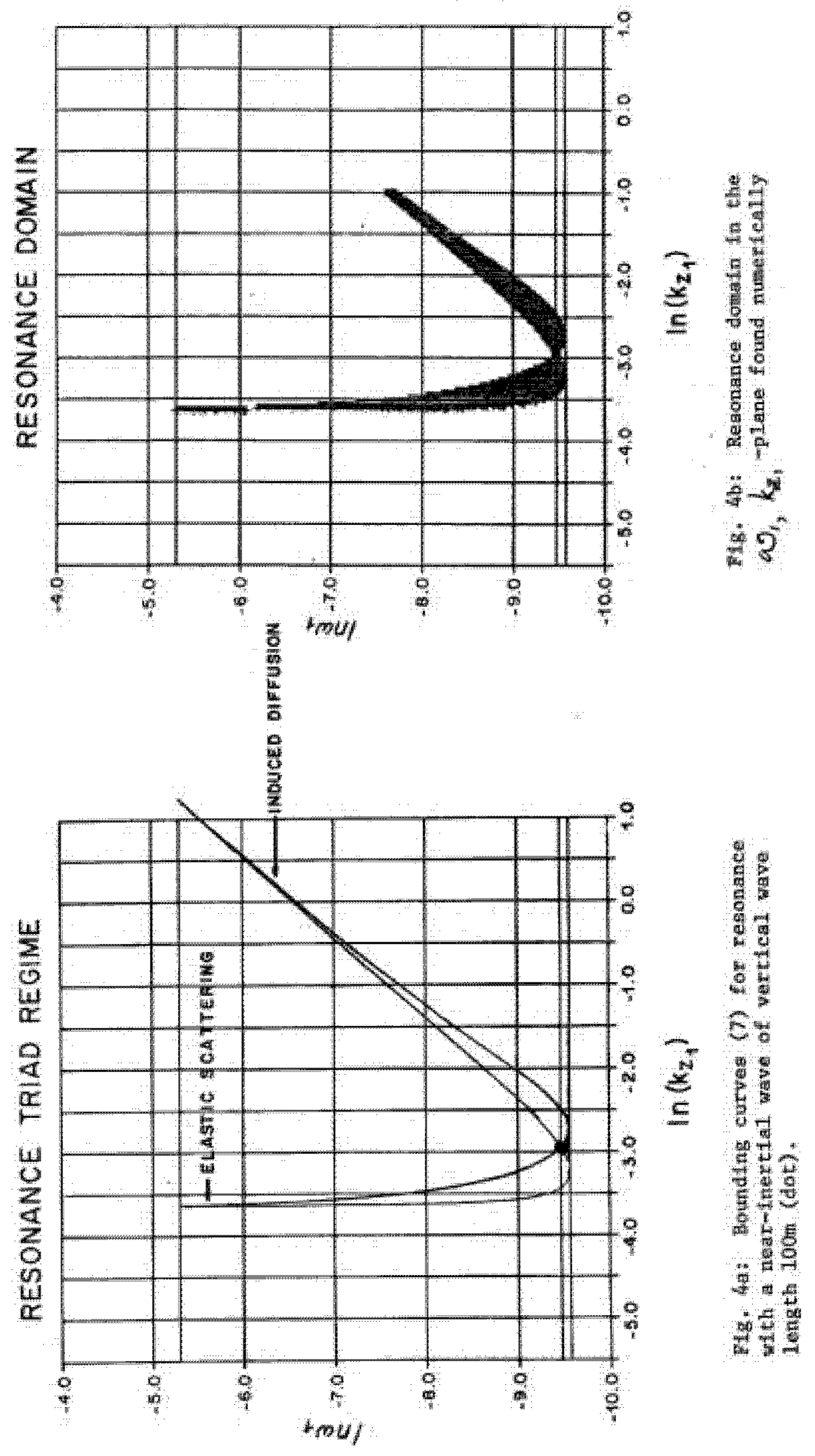
where the action-spectra are now $A(\boldsymbol{\omega}, \mathbf{k z})$. The internal wave spectrum used in the programs is the GM79 model (Munk, 1981) modified to exclude an inertial peak and with a continuous rather than discrete kz-spectrum (i.e., no top or bottom)

$$
A\left(\omega, k_{z}\right)=\frac{3 b N^{2} f E_{0}}{\omega^{3}\left(k_{z}^{2}+k_{z: k}^{2}\right)}
$$

where $b=1300 \mathrm{~m}$, Eo $=63 \cdot 10^{-5}$ and $k z *=3 \pi / b$. Now, to integrate $(8)$ over $\theta_{1}$, taking advantage of the horizontal isotropy, let $\gamma=\left(\omega_{2}-\omega_{1}-\omega_{0}\right)[=0$ for $\cos \theta_{1}$ satisfying (6)]. Then

$$
S(\gamma) d_{\theta_{1}}=\left(\frac{\partial \gamma}{\partial \theta_{1}}\right)^{-1} \delta(\gamma) d \gamma=\frac{-\left(\omega_{0}+\omega_{1}\right)\left(k_{z_{0}}+k_{z_{1}}\right)^{2}}{\sqrt{\omega_{0}^{2}-f^{2} \sqrt{\omega_{1}^{2}-f^{2}} k_{z_{0}} k_{z_{1}}}=\sin \theta_{1}}
$$

Therefore

$$
\frac{\partial A_{0}}{d t}=\iint \frac{K^{*}\left(\vec{k}_{1}+\vec{k}_{0}-k_{1}-k_{0}\right)}{\sin \theta_{1}}\left[A_{1} A_{2}+A_{0}\left(\frac{\omega_{0} k_{2_{0}^{2}}^{2}}{\omega_{1} k_{z_{1}^{2}}^{2}} A_{2}-\frac{\omega_{2} k_{2}^{2}}{\omega_{2} k_{z_{2}}^{2}} A_{1}\right)\right] d \omega_{0} d k_{z_{1}}
$$

where $\sin \theta_{1}=f\left(\omega_{1}, k_{1}\right)$ vanishing on curves (7). Next, integrate over $k z_{1}$ for fixed $\omega_{1}$. The integrand is nonvanishing only within one and the resonance domain branches (Figure 5). Over each of these branches, the integrand of (11) will have integrable cusps at the edges

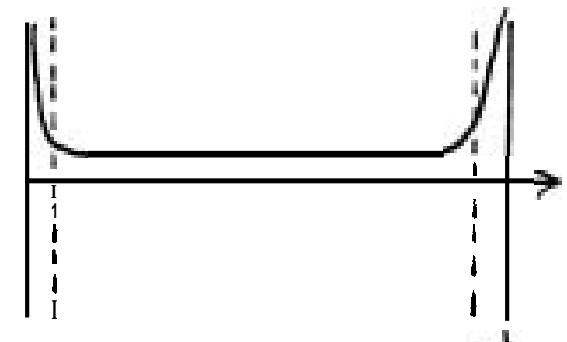

(a)

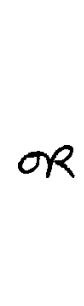

$\Delta k_{z_{1}}$

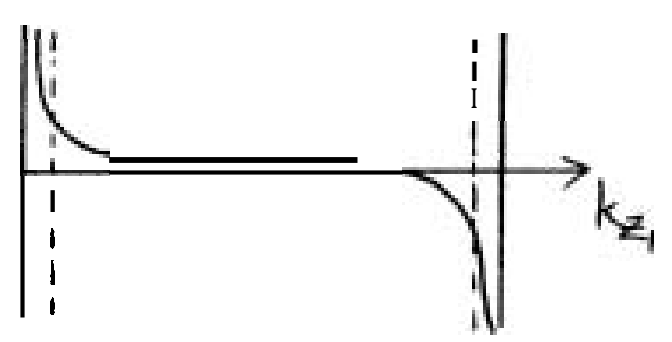

(b)

Figure 5

because the denominator $\sin \theta_{1}$ vanishes in these limits. A coarse, discrete numerical summation is not appropriate near these bounds which will have to be treated differently, i.e integrated analytically over a small interval

$\Delta \mathbf{k z}_{1}$. A suitable choice $\boldsymbol{A} \mathbf{k z}_{\mathbf{l}}$ is

$$
\Delta k_{z_{1}}=\frac{2 \sin ^{2}(\pi / 15)\left(\omega_{1}^{2}-f^{2}\right)\left(\omega_{0}^{2}-f^{2}\right) k_{z_{0}}^{2} k_{z_{1}}}{\left[4\left(\omega_{0}^{2}-f^{2}\right)\left(\omega_{1}^{2}-f^{2}\right) k_{e_{0}}^{2}-\left(2 \omega_{1}+\omega_{0}\right)\left(\left(\left(\omega_{2}+\omega_{1}\right)^{2}-f^{2}\right)\left(k_{z_{0}}+k_{z_{1}}\right)^{2}-\left(\omega_{1}^{2}-f^{2}\right) k_{z_{1}}^{2}-\left(\omega_{0}^{2}-f^{2}\right) k_{z_{0}}^{2}\right]\right.}
$$

The interior region of the domain can be integrated numerically.

The integrand will change sign as in Figure $5(\mathrm{~b})$ if $\left[\mathrm{A}_{1} \mathrm{~A}_{2}+\right.$ $\left.A_{o}\left(A_{2}-A_{1}\right)\right]$ changes sign in the interval, as it does for all $\omega_{1}$ in the low-kz, "elastic-scattering" branch (Figure 6), 1.e., the GM79-spectrum is near equilibrium along this branch and there is little energy gained or lost by the near-inertial wave. 
$\underline{\text { Previous Results }}$

Algebraic and numerical difficulties plagued this problem and were only resolved on the last day of the program. At the time of the oral presentation, I had been unable to reproduce the results of McComas and Bretherton (1977) and Olbers (1976). Corrections were made to the program on the following day and showed close agreement. It will now be possible to go on and examine the problem motivating this work. Some arguments for the importance of wave-wave interactions at critical-layers is presented below.

For near-inertial waves, Mocomas and Bretherton (1977) found the interaction time-scale diminished with increasing vertical wavenumber for the GM75/76 spectra (solid curve, Figure 6). The time-scale became smaller than the wave period for vertical wavelengths less than $120 \mathrm{~m}$. They found flux out of waves for $k_{z} \leqslant k_{z} *$, and an influx for higher wavenumbers. The principal source of influx was parameteric subharmonic instability. They proposed flux from low to high wavenumbers with a sink at high k,

Observations of energetic near-inertial waves in regions of negative vorticity have found enhanced energy only for $\lambda_{2} \simeq 100 \mathrm{~m}$, roughly the same scales where interaction times become smaller than the wave period. However, $\overline{\text { energy }}$-flux is into not out of these waves, i.e. wave-wave interactions are a source not a sink.

However, as energy is piled up in the low wavenumber near-inertial wavepacket approaching its critical-layer, the direction energy-flux may reverse. If we follow this hypothesis, assuming the interaction time scales do not change, a crude estimate of the vertical wavelength a t which the interaction time $t_{1}$ (solid line, Figure 6) will be comparable to the propagation time $t_{p}$ (dashed line, Figure 6) (1.e. the time for a near-inertial wave to propagate one wavelength, $\left.t_{f} \approx\left(\vec{k} \cdot \vec{c}_{j}\right)^{-1}\right)$.

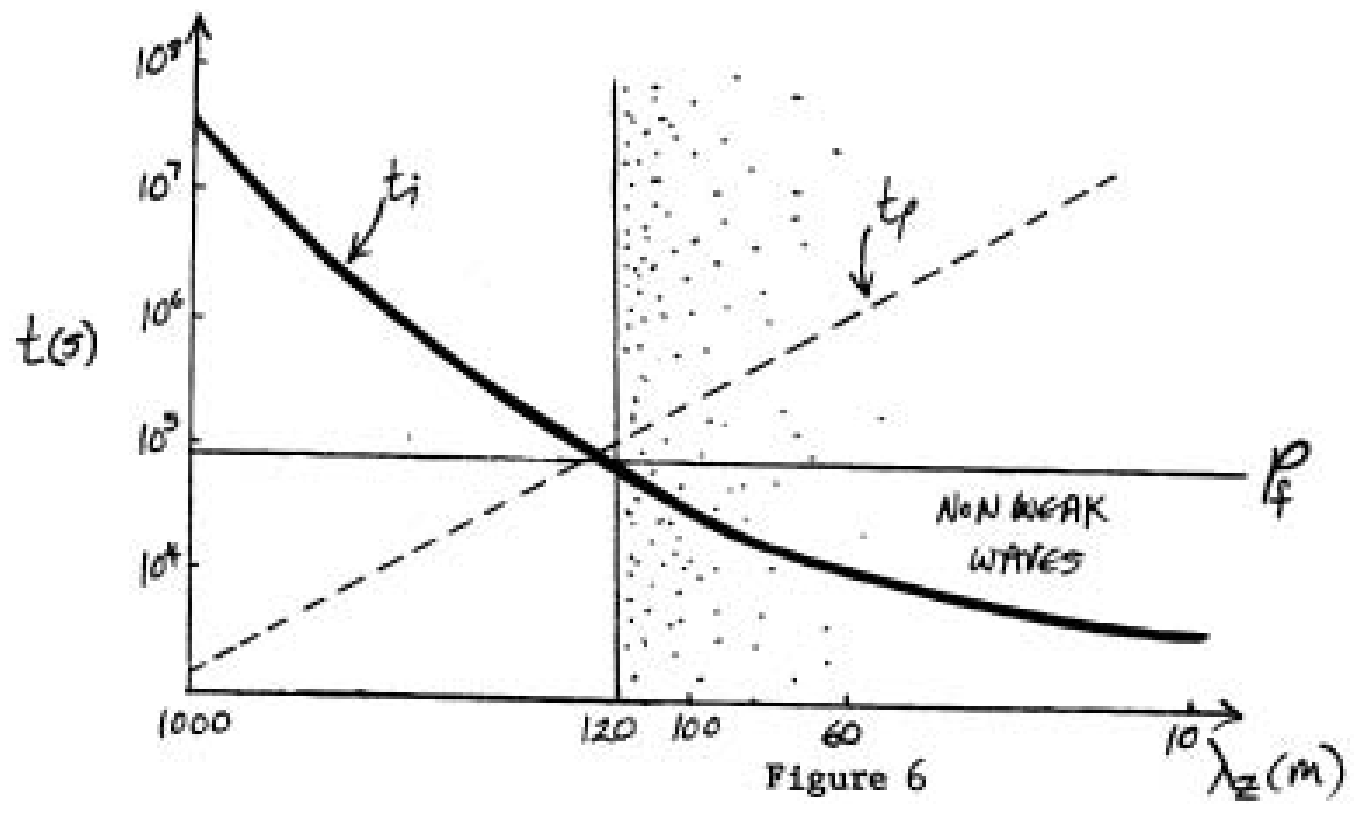


The propagation time increases with $\mathbf{k}_{\mathbf{z}}$ because the group velocity decreases strength with vertical wavenumber. The two curves intersect at

$\lambda_{\text {a }} \quad 120 \mathrm{~m}$. For smaller wavelengths interaction will dominate and if the flux is out of the wave any amplification should be drained away.

This wavelength can be compared to the wavelength at which the Richardson Number will fall below $1 / 4$. The energy-flux

$$
\vec{F} \simeq 1000 \mathrm{~kg} u_{0}{ }^{2} \vec{C}_{g} \simeq 0.08 \mathrm{~W} / \mathrm{m}^{2} \text {. }
$$

From this, the Richardson Number $\mathbb{k}^{2} / \mathrm{k}_{\mathbf{z}}^{2} \mathrm{v}_{0}^{2} \leq 1 / 4$ for $\lambda_{\mathbf{t}} \leq 60 \mathrm{~m}$, smaller than the scales at which wave-wave interactions dominate.

From this crude argument wave-wave interactions may be the dominant sink for trapped near-inertial waves so that strong internal wave-breaking need not occur at the critical depth. It remains to be shown that the direction of energy exchange does change sign when more energy is put into the near-inertial field and that the interaction timescales do not change significantly.

\section{REFERENCES}

Brown, E.D., and W.B. Owens, 1982. Observations of horizontal interactions between the internal wave field and the mesoscale flow. $\underline{\text { J. Phys. }}$ Oceanogr., 11, 1474-1481.

D'Asaro, E.A., and A. Perkins, 1984. The near-inertial spectrum in the late summer Sargasso Sea. Unpublished manuscript.

Kunze, E., and T.B. Sanford, 1984. Observations of near-inertial waves in a front. Submitted to J. Phys. Oceanogr.

Lueck, R.G., 1982. Dissipation in a warm-core ring. BOS Transactions, 63, 999.

McComas, C.H., 1975. Nonlinear interaction of internal gravity waves. Ph.D. Thesis, 111 pp.

McComas, C.H., and F.P. Bretherton, 1977. Nonlinear interaction of oceanic internal waves. J. Geophys. Res., 82, 1397-1411.

Muller, P., 1976. On the diffusion of momentum and mass by internal gravity waves. J. Fluid Mech., 77, 789-823.

Munk, W., 1981. Internal waves and small scale processes. Evolution of Physical Oceanography, B.A. Warren and C. Wunsch, eds., MIT Press, Cambridge, MA, $623 \mathrm{pp}$.

Olbers, D.J., 1976. Nonlinear energy transfer and the energy balance of the internal wave field in the deep ocean. J. Fluid Mech., I, 375-399.

Ruddick, B.R., and T.M. Joyce, 1979. Observations of interaction between the internal wave field and low-frequency flows in the North Atlantic.

J. Phys. Oceanogr., $\underline{9}, 498-517$. 


\section{NUMERICAL EXPERIMENT ON SLUMPING GRAVITY CURRENT}

Grétar Tryggvason

\section{INTRODUCTION}

Gravity currents are fairly common phenomena, and examples range from "avalances and atmospheric pollution at one end of the alphabet to volcanology and zoology at the other" (Simpson, 1982). They occur generally when a fluid of higher (lower) density advances along horizontal boundaries below (above) a fluid of lighter (higher) density. But examples also exist where the current advances along boundaries between two fluids. It is usually referred to as intrusion in such cases. The driving force of a gravity current is in a 11 cases buoyancy, but the balancing force can be due to inertia, viscosity, or surface tension. Here we will only consider gravity currents in the flow regime where inertia and buoyancy comprise the main balance.

Gravity currents where viscosity has small effect are characterized by a "head" at the leading edge. The head is deeper than the following flow, and at the back there are usually unsteady waves and mixing. The foremost point of a gravity current head is raised slightly above the ground as a result of the current running over a thin layer of the ambient fluid (in order to satisfy no slip boundary conditions). This thin layer is gravitationally unstable, being below a heavier fluid, and flows up causing a complex threedimensional motion in the front part of the head. The elevation of the foremost point decreases as viscous effects become less important, and when the overrunning is completely suppressed (by holding the current stationary in a uniform counterflow) it is found that the elevation reduces to zero (Simpson and Britter, 1978). The complex three-dimensional motion also diminishes substantially. In the study presented here, the focus is on the initial motion of a blob of inviscid fluid released into fluid of slightly different density. However, since much of the analytical work has been on steady (or quasisteady) currents, we will review some aspect of those here.

The earliest treatment is due to von Kárman (1940). He considered current advancing under infinitely deep lighter fluid, and by applying the Bernoulli equation along the separating streamline he deduced that the velocity of the front is given by:

$$
u=\sqrt{2 g^{\prime} h}
$$

where $\mathrm{g}^{\prime}=\mathrm{g}\left(\rho_{1}-\rho_{2}\right) / \rho_{2}$ and $\mathrm{h}$ is the depth of the current behind the head. The densities of the current and the surrounding fluid are given by $\rho_{1}$ and $P_{2}$ respectively. Von Kárman (1940) also calculated the slope of the interface, where it meets the ground to be $\pi / 3$. Benjamin (1968), in what is now a classical paper, reconsidered the steady inviscid model of a gravity current. Considering a current of depth h in a channel of total depth $\mathrm{H}$, he showed that mass, momentum, and energy cannot be conserved for all values of $\phi=\mathrm{h} / \mathrm{H}$. Actually only for $\phi=0.5$ are a 11 conservation principles satisfied. He therefore concluded that there must be some head loss along the separating streamline and possibility of hydraulic jump behind the head. Only values of $\phi<0.5$ correspond to head losses, and values greater than 0.5 require input of energy and can therefore not be obtained experimentally. Benjamin (1968) pointed out that the reasoning leading to 
von Kárman's result was inadequate, but obtained the same result using the more refined theory. Benjamin concluded that his treatment more or less exhausted the useful application of inviscid-fluid theory to steady gravity currents. Later treatments have been of semi-empirical type, such as the one of Simpson \& Britter (1979) which tries to incorporate other effects, such as the mixing at the back of the head. An exception is the analysis of Holyer \& Huppert (1980) which uses inviscid steady model, similar to Benjamin's, to study the motion of gravity current (or intrusion) into a two layered fluid. They show that, in contrast to Benjamin's results, the depth of an energyconserving gravity current is not uniquely determined. They also study the effect of head losses.

In experimental work, gravity currents are usually generated by removing a gate in a channel that separated fluids of different densities. When the depth of the fluids is initially equal we have the case of mutual intrusion. This was anlayzed by Yih (1947). He assumed that each fluid formed a tongue of depth $\mathrm{h}=\mathrm{H} / 2$, where $\mathrm{H}$ is the total channel depth. By a simple energy argument he deduced that the velocity of each front would be given by

$$
u=\sqrt{\frac{\rho_{1}-\rho_{2}}{\rho_{1}+\rho_{2}} g h}
$$

Notice that this is identical to von Karman's and Benjamin's results if $\mathbf{2}\left(\rho_{1}-\boldsymbol{\rho}_{2}\right) / \boldsymbol{\rho}_{2}$
is replaced by is replaced by $\left(\rho_{1}-\rho_{2}\right) /\left(\rho_{1}+\rho_{2}\right)$. The case where $\mathrm{h}=\mathrm{H} / 2$ is the only one where a steady, energy conserving, solution can be found according to Benjamin's analysis.

The spread of a fixed quantity of fluid was considered by Fay (1968). He uses an order of magnitude analysis to obtain spreading laws for axisymetric current. Since he is primarily concerned with the spreading of oil on sea he considers the flow regimes where buoyancy is balanced by viscosity or surface tension, in addition to the initial buoyancy-inertia stage. Hoult (1972) obtains the same relationship by solving the depth averaged shallow water equations. As a boundary condition for the advancing nose he specifies the Froude number, Fr $=\left(u / g^{\prime} h\right)^{1 / 2}$, just behind the head to be $\lambda^{1 / 2}$ where $\lambda$ is a constant to be determined from experiments. For a steady state current Benjamin obtained $\mathrm{Fr}=2^{1 / 2}$ when $\phi \rightarrow 0$. After adjusting

$\lambda(=1.4)$ Hoult obtains (an algebraic error was corrected by Huppert and Simpson; this is actually their corrected result)

$$
1=1.6\left(g^{\prime} q\right)^{1 / 3} t^{2 / 3}
$$

where 1 is the distance travelled, and $q$ is the amount of fluid released. Hoult's results are given in terms of a similarity solution and can therefore only be expected to be valid after some time from the release (as Hoult points out). Like Fay, Hoult also considers gravity currents when viscosity or surface tension are important.

Keulegan (1957) performed a series of experiments where he released fixed volumes of salty water into fresh water in channels. Similar experiments were also performed later by Huppert and Simpson (1980). The gravity currents formed in these experiments all start out with essentially constant propagation velocity which in many cases persist until viscous forces become important, thus showing little agreement with the above described power laws. 
This stage, which may or may not be followed by a buoyancy-inertia regime described by Hoult's power law, has been called the slumping stage by Huppert and Simpson (1980). They argue that the correct boundary condition is to let the Froude number at the head depend upon the fractional depth of the current, $\phi=h / H$. From steady state experiment (Simpson and Britter, 1979) they determine $\operatorname{Fr}(\phi)$ as:

$$
\begin{array}{ll}
F_{r}=\frac{1}{2} \phi^{-1 / 3} & 0.075 \leqslant \phi<1 \\
F_{r}=1.19 & \phi<0.075
\end{array}
$$

Notice that for small $\phi$, this is the same as Hoult's boundary condition: $\mathrm{Fr}=1.41 / 2=1.18$. Huppert and Simpson assume further that the slumping takes place through a series of equal-area rectangles, such that

$$
1 \mathrm{~h}=1_{\mathrm{o}} \mathrm{h}_{\mathrm{o}}=\mathrm{q}
$$

where 1 is the length of the rectangle, h the height and the subscript o denotes the initial conditions. The Froud number can then be expressed as

$$
\operatorname{Fr}=1^{1 / 2} i /\left(g^{\prime} q\right)^{1 / 2}
$$

Equating (7) to (4) and integrating, they get (using the initial conditions $1=1_{0}$ at $t=0$ )

$$
\begin{aligned}
I & =\left[I_{0}^{7 / 6}+\frac{7}{12}\left(g^{3} q H^{2}\right)^{1 / 6} t\right]^{6 / 7}, \quad\left(I_{0} \leqslant I \leqslant I_{5}\right) \\
& =\left[I_{0}+\frac{1}{2}\left(g^{13} q H^{2} / I_{0}\right)^{1 / 6} t\right]\left[1+O\left(g^{43} q H^{2} t^{6} / I_{0}^{7}\right)^{1 / 3}\right]
\end{aligned}
$$

where the slumping length $1_{s}$ is the length of the gravity current when $\mathrm{h} / \mathrm{H}=$ 0.075. Equating (7) to (5) they obtain

$$
\begin{aligned}
I & =\left[I_{5}^{3 / 2}+1.78\left(g^{\prime} q\right)^{1 / 2}\left(t-t_{s}\right)\right]^{2 / 3}, \quad\left(I_{5} \leqslant I \leqslant I_{*}\right) \\
& \sim 1.47\left(g^{\prime} q\right)^{1 / 3} t^{2 / 3}, \quad\left(t \gg t_{s}\right)
\end{aligned}
$$

where $t_{\mathbf{s}}$ is evaluated by setting $\mathbf{1}=\boldsymbol{I}_{\mathbf{s}}$ in $(\mathbf{8})$, and the initial conditions $1=I_{s}$ at $t=t_{s}$ are used. $1 *$ is the length when viscous effects become important. The second of those spreading laws (Eqs. (10 and (11)) is essentially Hoult's law. The first describes the slumping phase, and predicts (almost) linear growth initially. Huppert and Simpson compare their theory with Keulegan's (1957) experimental data as well as with data of their own, and find satisfactory agreement when viscous effects are negligible. Although the theory of Hupper and Simpson seems to provide a useful tool for, say, engineering estimation, it does not provide much physical insight into how the head controls the flow. The initial slumping phase and the formation of the head is such an unsteady process that it seems rather surprising that results from steady state experiments can be applied. The assumption of a "box shape" seems also hardly justifiable when compared to experimental observations. It is not clear that steady or quasi-steady models as the one discussed above can 
be expected to describe an inherently unsteady motion as slumping of a gravity current definitely is. The need to incorporate some additional physics, as a Froude number behind the head, is perhaps only a repair, required because of the damage done by neglecting unsteady effects.

In this paper we explore the above made conjecture by simulating numerically the initial motion of an iviscid blob released into a fluid of slightly higher density.

\section{NUMERICAL MEIHOD}

Flows of the type considered here, that is where there is an internal discontinuity of some sort, are generally rather difficult to simulate numerically. Not only is it necessary to track the motion of the interface accurately, but also it is often essential to resolve steep gradients in the vicinity of the interface. For piecewise potential flow the situation is, however, much simpler. Then the motion of the interface is determined by the configuration of the interface itself, and there is no need to accurately calculate the flow field in the interior. The motion of an inviscid fluid with piecewise constant density, started from rest, is exactly of this type. From Bjerknes' theorem it is found that there is a generation of vorticity only at the interface. It can therefore be treated as a vortex sheet with time dependent vortex sheet strength. The velocity of a vortex sheet is given by the integral, due to Birkhoff (1954).

$$
\bar{U}(\bar{x})=\frac{1}{2 \pi} P \int d s \frac{\left(\bar{x}-\bar{x}^{\prime}(s)\right) \times \hat{z}}{\left|\bar{x}-\bar{x}^{\prime}(s)\right|^{2}} \gamma(s)
$$

Here $P$ stands for the principal value. $\quad \gamma=\left(\tilde{u}_{1}-\bar{u}_{2}\right) \cdot \hat{g} \quad$ is the vortex sheet strength and $\tilde{u}_{1}\left(\tilde{u}_{2}\right)$ is the velocity in the lower (upper) fluid at the interface. S is an archlength coordinate and $\hat{\mathbf{s}}$ is a tangent vector. The notation is shown in Figure 1. The Birkhoff equation (2.1) is a solution to Poisson's equation

$$
\nabla^{2} \psi=-\omega
$$

which describes the kinematics of a divergent free velocity field. Here $\psi$ is the usual stream function and $\omega$ is a vorticity field, which in this case is a vortex sheet. Once the velocity of the interface is known its motion is found by integrating

$$
\frac{d \bar{X}(s, t)}{d t}=\bar{U}(s, t)
$$

In general the vortex sheet strength $\gamma(s)$ varies with time and its evolution is governed by the dynamics of the system under consideration.

When simulating the motion of a vortex sheet it is usually replaced by a row of point vortices, each with circulation

$$
\Gamma=\gamma \Delta S
$$

where $\Delta \mathrm{s}$ is the distance between neighboring vortices. The integral (2.1) is then replaced by summation and the velocity of each point vortex is found 
by summing over the contributions from allother vortices. If the number of vortices is $\mathrm{N}$ it is clear that finding the velocity of all vortices requires $\mathrm{O}\left(\mathrm{N}^{2}\right)$ operations. For long interfaces, where large $\mathrm{N}$ is needed, this becomes very time consuming. When the (long) interface occupies relatively small domain, or there are many interfaces, it is often more economical to solve equation (2.2) and find the velocity field by differentiating the stream function. For regular domains fast methods are available to solve Poisson's equation, requiring $O\left(M^{2} \log M\right)$ operations, where $M$ is the number of $g r i d$ spaces in one direction. Since the resolution is determined by $M$ it is sufficient to have of the order one vortex per grid space, therefore $\mathbb{N} \sim \mathbb{M}^{2}$ * This gives $0(\mathrm{~N} \log N)$ operations needed to find the velocities. For 1 arge $N$ this is obviously considerably less than $O\left(\mathbb{N}^{2}\right)$. Finding the velocities by a grid based method and interpolating the velocities of the point vortices is the main feature of the Vortex-In-Cell (VIC) algorithm of Christiansen (1973), which is used here. For general discussion of the VIC algorithm the reader is referred to the orfginal paper by Christiansen (1973). For application to an interface problem see Meng and Thomson (1978), and Tryggvason and Aref (1983). General discussions of vortex methods for flow simulations may be found in Leonard (1980) and Aref (1983). A vortex method based on (2.1) rather than (2.2) is discussed by Baker, Meiron and Orszag (1980) and (1982).

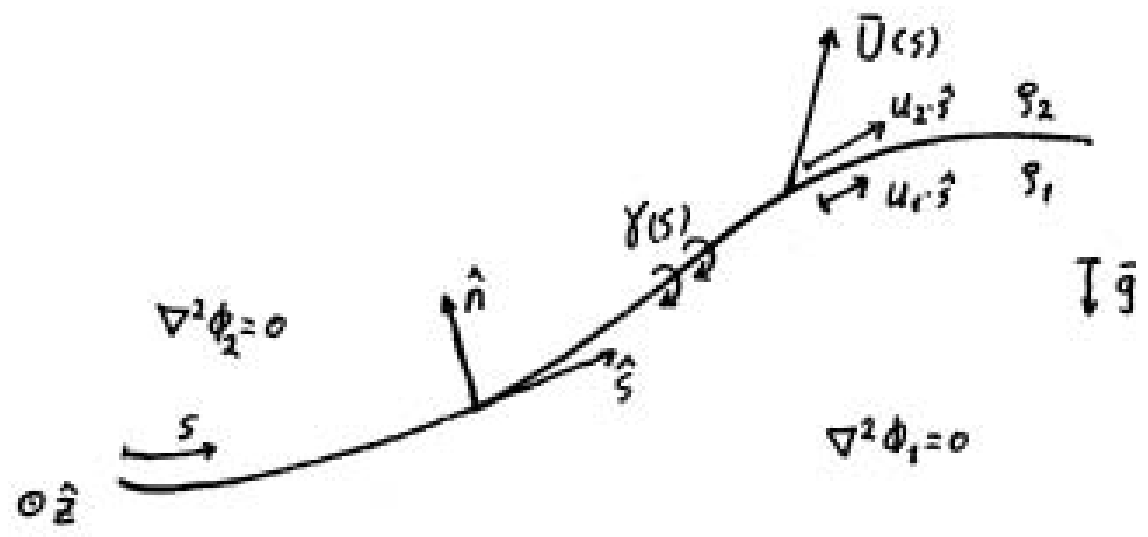

Figure 1. Vortex sheet, notation.

An equation describing the evolution of the vortex sheet strength is obtained from the appropriate momentum equation. Here we consider two layers of inviscid fluid with constant density in each layer. The difference in density is assumed to be very small so it is justified to make the Boussinesq approximation and write the momentum equations as

$$
\rho_{m}\left(\frac{\partial \bar{u}_{i}}{\partial t}+\bar{u}_{i} \nabla \bar{u}_{i}\right)=-\nabla p_{i}+\rho_{i} \bar{g}, \quad 1=1,2
$$

Here $\rho_{m}=\left(\boldsymbol{\rho}_{1}+\rho_{2}\right) / 2$. We also need to remember that the normal velocity is continuous across the sheet, or:

$$
\left(\vec{u}_{1}-\vec{u}_{2}\right) \cdot \hat{\mathrm{n}}=0
$$

The velocity of the vortex sheet is defined as usual by the mean of the velocities of each side of the interface. It can be written as:

$$
\bar{U}=\frac{1}{2}\left(\vec{u}_{1}+\vec{u}_{2}\right)=\vec{u}_{1}-\frac{1}{2} \gamma \hat{s}=\bar{u}_{2}+\frac{1}{2} \gamma \hat{s}
$$


Adding and subtracting $\overline{\mathrm{V}} \cdot \boldsymbol{\nabla} \overline{\mathbf{u}}_{\mathfrak{f}}$ to the left hand side of (2.5) we obtain (after using (2.7))

$$
\rho_{m}\left(\frac{d \bar{u}_{i}}{d t} \pm \frac{1}{2} \gamma \hat{s} \cdot \nabla \bar{u}_{i}\right)=-\nabla p_{i}+\rho_{i} \bar{g}
$$

where the $+(-)$ sign refers to $i=1(2)$, and

$$
\frac{d \bar{u}_{i}}{d t}=\frac{\partial \bar{u}_{i}}{\partial t}+\bar{U} \cdot \nabla \bar{u}_{i}
$$

that is, the acceleration following the interface. We now take the dot product of (2.8) with the tangent vector 6 and subtract the equation for the top fluid from the one for the bottom fluid. Then we obtain

$$
\rho_{m}\left(\frac{d}{d t}\left(\bar{u}_{1}-\bar{u}_{2}\right)+\frac{1}{2} \gamma \hat{s} \cdot \nabla\left(u_{1}+u_{2}\right)\right) \cdot \hat{s}=\left(\rho_{1}-\rho_{2}\right) \bar{g} \cdot \hat{s}-\frac{\partial}{\partial s}\left(P_{1}-\rho_{2}\right)
$$

Rewriting this, and using $\gamma=\left(\bar{u}_{1}-\bar{u}_{2}\right) . \hat{s}$ and $\bar{U}=1 / 2\left(\bar{u}_{1}+\bar{u}_{2}\right)$ we get

$$
\frac{d}{d t} \gamma+\gamma \hat{s} \cdot \frac{\partial \bar{U}}{\partial s}=-2 A g \frac{\partial y}{\partial S}-\frac{2}{\rho_{1}+\rho_{2}} \frac{\partial}{\partial S}\left(P_{1}-P_{2}\right)
$$

where A is the Atwood ratio

$$
A=\frac{\rho_{1}-\rho_{2}}{\rho_{1}+\rho_{2}}
$$

Integrating this equation over a small segment of the interface (between two material points), and assuming that $P_{1}=P_{2}$ on the interface we obtain

$$
\frac{d \Gamma}{d t}=-2 A g \Delta y
$$

Here $\Gamma$ is the total circulation around the segment, and $\Delta \mathrm{y}$ is the difference between the vertical coordinates of the end points. In the numerical simulations the small segment is replaced by a point vortex whose circulation is given by (2.13). The motion of the interface is now described by (2.13), (2.3) and (2.1). In the simulations described here we have not used (2.1) but the VIC algorithm which is based on (2.2).

At this point it is convenient to cast our equations in nondimensional form. Assuming that $L$ is some external length scale we write

$$
x, y=L(\tilde{x}, \tilde{y}), t=\sqrt{\frac{L}{A g}} \tilde{t}, \quad \Gamma=\sqrt{A g L^{3}}, \quad \bar{U}=\sqrt{A g L} \tilde{\tilde{U}}
$$

where the tilda denotes dimensionless variables. Using this we can rewrite (2.13) as (dropping the tilda)

$$
\frac{d \Gamma}{d t}=-2 \Delta y
$$

Equations (2.2) and (2.3) remain unchanged. For the limit of weak stratification there is therefore no variable parameter in the equations, and it is 
sufficient to vary only the initial conditions. In the simulations we have taken $\mathrm{L}=2 \mathrm{H}$, where $\mathrm{H}$ is the depth of the channel. This choice is only for technical conveniences so we can work with computational domain of height 0.5.

For interface with arbitrary density differences the evolution equation has been derived by Baker, Meiron and Orszag (1980). They also showed that it reduces to (2.11) in the limit of weak stratification. In our nondimensional variables their equation is:

$$
\frac{d \gamma}{d t}+\gamma \hat{s} \cdot \frac{\partial \bar{U}}{\partial s}=-2\left(A \frac{d \bar{u}}{d t} \cdot \hat{s}+A \frac{\gamma}{4} \frac{\partial \gamma}{\partial s}+\frac{\partial y}{\partial s}\right)
$$

When $A \rightarrow 0$ this reduces to (2.11).

When following the motion of a vortex sheet with variable vortex sheet strength, $\gamma$, it is necessary to ensure that all parts of the sheet are adequately represented by vortex points. In the simulations presented here this has been achieved by redistributing the vortex points uniformly along the sheet after every time step. This redistributing process can have slight smoothing effects, particularly it tends to cut off sharp corners. In order to reduce this effect we have represented the interface by many more points than formally needed if one simply wants to have the separation of points comparable to a grid space. However, as the interface stretches the separation of points increases, since we use a fixed number throughout the simulations. Consequently, the effect of this smoothing will eventually become important. In this simulation the effect is believed to be small. No difficulties seemed to arise from the fact that the vortex sheet was much better resolved than the velocity field. However, it has been observed in simulations where the vortex sheet is compressed locally that it can develop 'kinks' smaller than a grid space. This can lead to serious errors, particularly if nonzero surface tension is included. We therefore believe that a promising strategy for further development of the numerical method is to keep the separation of point vortices constant and to insert and delete points as needed during the simulations. Since the interface in the simulations presented here is constantly being stretched, the above mentioned difficulties did not arise. For further discussion of the numerical method employed the reader is referred to Tryggvason and Aref (1983).

Finally, we want to discuss shortly a problem arising in most point vortex simulations. For straight vortex sheets with constant $\gamma$ it is found analytically that the growth rate of a small disturbance is proportional to the wave number. When the shear layer is represented by point vortices a maximum wave number is imposed. Therefore, if disturbances of all wave numbers can be expected to be present, there will always be some length scales that are not resolved by the simulations. For vortex sheets with arbitrary shape and vortex sheet strength there is no reason to believe that the situation is in general any better. (Although it should be mentioned that $P$. G. Saffmann has conjectured that sufficiently high stretching may have stabilizing effects (Saffmann and Baker (1980)). A stable stratification across the vortex sheet stabilizes long wave length disturbances, but disturbances with sufficiently high wave numbers will still be unstable. For real physical systems there is of course always some mechanism that provides stabilization for the highest wave numbers. In a fluid system of the sort considered here this is accomplished by viscosity and/or surface tension. Vortex method can only be applied when viscosity can be assumed neglibile, but surface tension is easily incorporated in the formulation presented here. It 
is of course preferable when a physical mechanism can be included in the model to be simulated, that provides a highest unstable wavenumber such that no unresolved length scales are generated (as in Tryggvason and Aref, 1983). However, for gravity current that can be considered essentially inviscid, it seems that surface tension effects must be rather small, if not nonexisting (for immiscible fluids), in most cases. We are therefore left with little option except to live with the stabilization of smallest length scales by the numerics. The standard argument is that the small scale motion has little effect on the large scale evolution of the system and overall accuracy is not seriously affected by misrepresentation of smallest scales. We will confirm this argument for our simulations by comparing runs of different resolution. We also compare results with a simulation employing small surface tension to show that the system is not sensitive to the exact treatment of the small scale motion.

\section{RESULTS AND DISCUSSIONS}

In order to investigate the effect of various initial conditions, a quarter of an ellipse was chosen and the size compared to the channel depth, and the ratio of the major to the minor axis was varied. The initial configuration is shown in Figure 2. For technical reasons four times bigger domain than the one shown in Figure 2 was simulated; the computed domain containing a fullellipse resulting from reflecting the original quarter through the $x$ and $y$ axis. The boundary conditions are that there is no flow through the top and bottom boundaries and that the flow is periodic in the horizontal direction (for the bigger box). In most of the runs the domain shown in Figure 2 was simulated using 256 particles on a 32 by 128 grid. One run employed a 16 by 64 grid. The runs performed are summarized in Table 1 . Figures 3 to 9 show the time evolution for runs 1 to 7 . Run 8, which had identical initial conditions as run 1 except that the total box length was half that of run 1 , showed no different evolution, indicating that end effects are not important. Run 1 to 3 a 11 used a quarter of a circle as initial conditions. Runs 4 and 5 used an ellipse with the same height as the radius in run 2, but different lengths. Runs 6 and 7 had the same initial conditions as run 3. Run 6 employed only half the resolution of run 3, and in run 7 a slight amount of surface tension was added to investigate the effect of treating the smallest scales differently.

The most noticeable feature in all cases is that the blob, soon after release, forms a very distinct head. The head contains a significant fraction of the fluid and has a rather irregular upper surface. Behind the head there is a layer of fluid that flattens out as the head moves outward. In the initial stages of the slumping there appear instabilities on the forward part of the interface. During the subsequent motion these waves grow and new ones are generated at the nose. This generation of new waves is very clearly visible in Figure 3. The mechanism of this instability is generally believed to be of Kelvin Helmholts type. In Figure 4 two waves merge in a way clearly reminiscent of vortex merging. Such merging has also occurred in Figure 5, though it is not as clearly visible from the pictures shown as i n Figure 4. When merging occurs, we note that first the sheet wraps around, but then seems to be stretched out and appear fairly regular again. This seems also to happen without being a consequence of merging. If a sharp spike forms, then it sometimes seems to disappear after a while. It is not clear if this is a real phenomena or some artifact of the numerics. When the points are redistributed, the interface is treated as a polygonline, and when the points 
are put in new positions, sharp corners may be cut off. However, such a thin filament seems usually to be rather inert and probably has rather little net vorticity, so even if the numerics contribute to its annihilation, it is not expected to have serious effects on the overall dynamics of the system. In Figures 3 to 5 (and 8 and 9) the head has approximately the same shape in the sense that the ratio of the height of the head to its length is similar. In Figure 6 where the initial length of the blob was smaller, the head is also smaller, but its height is approximately the same as in Figure 4 . It is difficult to define any head length for run 5 (Figure 7), but it is definitely longer than in the other runs. The head height can be seen to be similar as in runs 2 and 4 . Another distinct feature seen in the simulations is that the backmost part of the blob falls most rapidly. It is completely free of all disturbances and stays very horizontal during the slumping. By estimating the area below the levelled surface from the figures, it is found to be approximately constant once it is formed. An immediate consequence is, of course, that once the head is formed, it does not draw more fluid into it. Notice in particular that for runs 2, 4, and 5 the region under the horizontal smooth surface is similarly long, indicating that the difference is primarily in the length of the head. The merging of two (or three?) waves on the front part of the head in run 5 gives, at first glance, the impression of a shorter head, but closer observation indicates that this is not so.

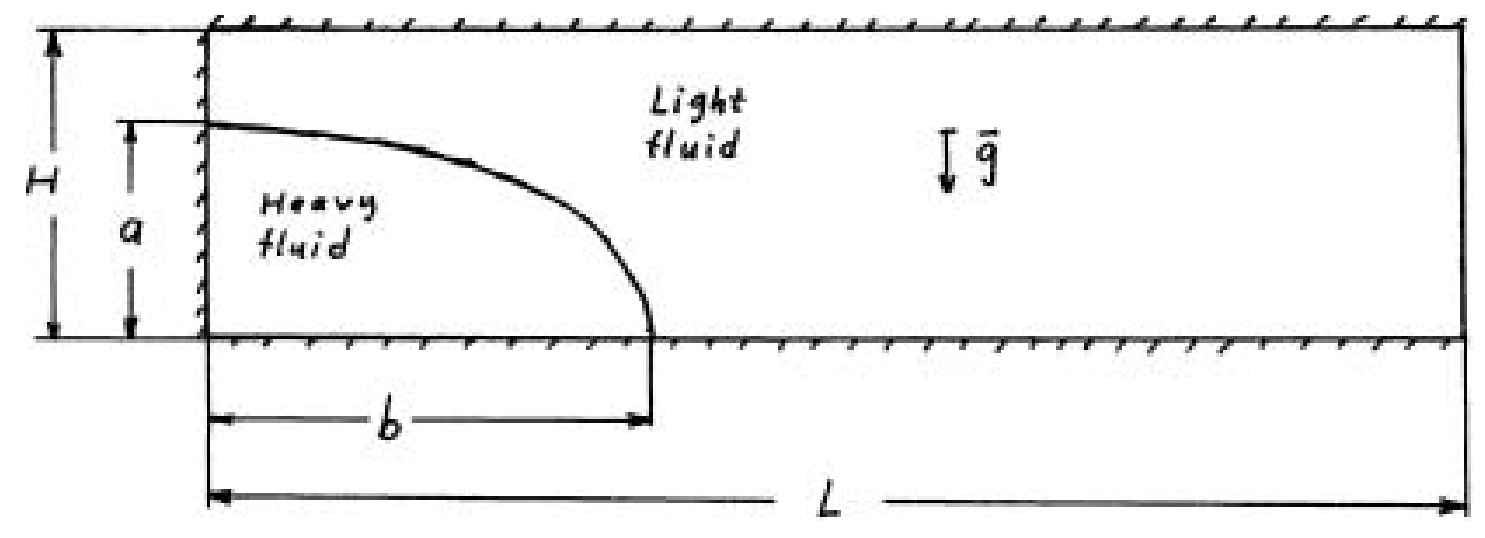

Figure 2. Initial conditions.

\begin{tabular}{l|cccccccc} 
Run no. & $\mathbf{1}$ & $\mathbf{2}$ & $\mathbf{3}$ & 4 & 5 & 6 & 7 & 8 \\
\hline a/H & .5 & .7 & .9 & .7 & .7 & .9 & .9 & .5 \\
b/a & 1 & 1 & 1 & .5 & 2.0 & 1 & 1 & 1
\end{tabular}

Remarks: No surface tension, except in run 6.

Resolution $\mathbf{3 2}$ by 128 gridpoints, except in run 7 where the resolution is 16 by 64 .

$\mathrm{L} / \mathrm{H}=\mathbf{4}$, except in run 8 where $\mathrm{L} / \mathrm{H}=\mathbf{2}$.

Table 1 
It is not clear from the simulations presented here, what the eventual fate of large amplitude disturbances is. In Figure 3 (run 1) the first wave to hit the back of the head seems to be stretched out, whereas the next one shows no sign of similar process. In run 2 one wave seems to be undergoing similar destruction by stretching, but others seem to form a filament or blob that is swept back. That process is also seen in other runs. The shedding of fluid blobs surrounded by parts of the vortex shedd would not be inconsistent with experiments where there is observed to be a turbulent wake behind the head.

The property of gravity currents, perhaps of most interest to investigators, is the propagation speed of the nose. In Figures 10 to 12 we have plotted the position of the nose for the runs performed versus time.

Figure 10 shows the result for runs 1 and 8 . There is no difference, indicating that the length of the computational box has no effect on the result. Figure 11 shows the propagation for runs 2, 4, and 5. There is essentially no difference between them, indicating that the height of the initial blob is the important parameter for determining the propagation speed. (We will discuss the relevant height scale shortly.) Figure 12 shows results for runs 3, 6, and 7. It indicates that the exact treatment of the small scales is not essential, at least not as far as the velocity of the nose is concerned. It is obvious from the graphs that the velocity is constant during the simulated period. The velocity is seen to increase slightly with the height of the initial blob.

If we accept that the motion of the fluid after release is independent of the exact initial shape of the blob, the proper description seems to be specification of the quantity of fluid, q, and the elevation of its centroide, $\mathbf{h}_{\mathrm{c}}$. The motivation is, of course, that these quantities multiplied by the gravity and the density difference, give the initial potential energy. Assuming that the density difference of the fluids is small, using the Boussinesq approximation reduces the number of dependent variables by one, since gravity appears combined with the densities. We can therefore define a reduced gravity $\mathrm{g}^{*}$ as

$$
g^{*}=2 \frac{\rho_{1}-\rho_{2}}{\rho_{1}+\rho_{2}} g=2 A g
$$

In the literature a different definition is often used, namely

$$
g^{\prime}=\frac{\rho_{1}-\rho_{2}}{\rho_{2}} g
$$

In the limit of vanishing density difference these expressions are obviously the same, and knowing one, the other can be found. We prefer to use g* since it occurs more naturally in our formulation of the problem. If the velocity of propagation of the nose, $u$, depends on the above-described parameters and $\mathrm{H}$, the total channel depth, then there exists a relationship such that

$$
f\left(g^{\star}, q, h c, H, u, t\right)=0
$$

Notice that there are three length scales in this relation, $h_{c}, H$, and q. If the depth of the channel is very large, we assume that dependence on $\mathrm{H}$ will be negligible. From the simulations it appeared that volume was not an 
important parameter so far as the propagation velocity was concerned (runs 2, 4, and 5). Therefore, we select the dimensionless group such that the following expression holds:

$$
\frac{u}{\sqrt{g^{x} h_{c}}}=\tilde{f}\left(\frac{\sqrt{q}}{h_{c}}, \frac{H}{h_{c}}, \sqrt{\frac{g^{7}}{h_{c}}} t\right)
$$

From the simulations we have already noticed that the dependence on the first and last group is very weak, if not nonexisting. Therefore, the appropriate form to use is:

$$
\frac{U}{\sqrt{g^{2} h_{c}}}=\hat{f}\left(\frac{H}{h_{c}}\right)
$$

The linearized form of Huppert's and Simpson's equation for the initial propagation speed gives the velocity

$$
u=\frac{1}{2}\left(g^{13} q \frac{H^{2}}{l_{0}}\right)^{1 / 6}=2^{-1 / 9} \sqrt{g^{1} A h_{c}}\left(\frac{H}{h_{c}}\right)^{1 / 3}
$$

The second form is obtained by using that $q=h_{0} 1_{o}$ and $2 h_{c}=h_{o}$ for a rectangle. Replacing g' by $2 \mathrm{Ag}$ (since they are similar for small A), we can write this as

$$
\frac{U}{\sqrt{9 A h_{c}}}=\left(\frac{H}{2 h_{c}}\right)^{I / 3}
$$

which has the same form as obtained earlier, (3.5), except here we have $\mathbf{A}$ explicitly. The linearized form of Huppert's and Simpson's equations is obviously the relevant one to compare our results with, since our velocity is constant. (It is also easily checked that the difference from their exact formula for our simulations is small.) Huppert's and Simpson's formula has been shown to fit experimental observation well, so comparison with it is also a comparison with experiments. In Table II we have listed our calculated velocity and the velocity calculated by (3.7) for the three different values of $h_{c} / H$ that were simulated. It is seen to be in good agreement, except perhaps for the lowest value of $h_{c} / H$, where the fractional depth of the current behind the head is smaller than 0.075, which is the lowest value where (3.7) is valid. It should be pointed out that even in that case, do we observe constant velocity, contrary to the prediction of Huppert and Simpson. It has to be admitted that the three values of $h_{c} / H$ simulated, and the narrow range considered does not allow very general conclusions to be drawn, but it is not contrary to evidence to expect $h_{c}$ to be the most important scaling length in the problem. The effect of finite depth is to reduce the velocity scaled by $h_{c}$. This is as one expects; sidewalls of finite distance usually have a decreasing effect on propagation velocity. The increase in velocity as scaled by the external length $L$ is therefore due to larger amount of initial potential energy and should increase faster with initial height, if it were not for the effects of the finite channel depth.

In order to obtain some understanding of the energy balance of the system, we made the following rough estimation: an ellipse was fitted to the head in runs 1, 2, and 3. The height made it possible to estimate the loss in 
potential energy compared with the initial state, and by knowing its area the kinetic energy was estimated. The kinetic energy in the surrounding fluid was found by assuming infinitely deep channel. From the energy balance a propagation velocity was calculated. The results are shown in Table III. Considering the rough nature of the estimation the agreement is fair, indicating that little energy can be associated with the billows on the back of the head. The reason that the energy balance gives higher velocity than observed when $h_{c} / H$ is large is probably an indication that larger fraction (than assumed in the estimation) of the kinetic energy is contained in the surrounding fluid because of the top surface.

\begin{tabular}{llll}
$\mathrm{h}_{\mathrm{c}} / \mathrm{H}$ & $\mathrm{u} / \sqrt{\mathrm{gAh}}$ & difference \\
\hline .212 & 1.167 & $\mathrm{H} \& \mathrm{~S}$ theory & $12.3 \%$ \\
.298 & 1.142 & 1.188 & $3.7 \%$ \\
.382 & 1.052 & 1.094 & $3.8 \%$
\end{tabular}

Table II. Velocity of the nose.

\begin{tabular}{l|ccccc} 
Run & $\mathrm{R} / \mathrm{L}$ & $\mathrm{a} / \mathrm{L}$ & $\mathrm{a} / \mathrm{b}$ & \multicolumn{4}{c}{$\mathrm{e} / \sqrt{\mathrm{AgL}}$} \\
entimated by & $\begin{array}{l}\text { from } \\
\text { enimulations }\end{array}$ \\
\hline 1 & .039 & .234 & 2.5 & .375 & .380 \\
2 & .055 & .328 & 2.3 & .440 & .440 \\
3 & .070 & .469 & 2.3 & .525 & .460
\end{tabular}

Table III. Estimated energy balance.

\section{CONCLUSIONS}

The results presented here show a fair support for the conjecture made i $n$ the Introduction that the essential missing element in the modelling of slumping inviscid gravity currents is unsteadiness rather than some arbitrary Frode number conditions at the head. However, if the picture of the energetics sketched in the last paragraph of the last section (that is, the kinetic energy of the billows is small compared with the kinetic energy of the mean flow) is correct, then it is plausible that a steady-state solution is relevant after some time. It would probably consist of a blob of fixed material propagating with uniform velocity, thus being rather different from the picture presented in Huppert's and Simpson's theory. The evolution towards this blob is likely to be essentially unsteady. Note also that such a blob would be highly unstable to shear instabilities.

The good agreement of our simulations with experimental results, where the initial blob is essentially a rectangle, suggest that $h_{c}$ is indeed the relevant lengthscale in the problem. However, a study of wider variety of initial conditions is clearly useful and may be performed in future extensions of this work. The same comment applies to the range of $h_{c} / H$ studied. What 
happens when $h_{c} / H \rightarrow O$ is mostly unresolved: Huppert's and Simpson's theory predicts a time-dependent velocity, but in our simulations no trend towards nonconstant velocity was observerd in the run with smallest $h_{c} / H$. Runs that follow the evolution of the blob for longer time are probably difficult, unless some small-scale smoothing is introduced. But even with such artifact they might be illuminating. There is also more information that could be extracted from the runs presented, but time did not allow us to do this. The most obvious ones are more exact energetics and plots of the velocity field inside and outside the head. This information is difficult to obtain experimentally but should be helpful for further understanding of this problem.

\section{ACKNOWLHDMENIS}

I would like to thank H. Aref, L.N. Howard, and T. Maxworthy for helpful discussions. H. Aref kindly provided me with computer time on the Cray-1 computer at NCAR B. Owen allowed me to use his terminal to access NCAR's computers. Last, but not least, I wish to thank M. Stern and other participants in the G.F.D. program for providing a very enjoyable summer.

\section{REFERENCES}

Aref, H., 1983. Integrable, chaotic and turbulent vortex motion in twodimensional flows. Ann. Rev. Fluid Mech., 15, 345-389.

Baker, G.R., Meiron, D.I., and Orszag, S.A., 1980. .Vortex simulation of the Rayleigh-Taylor instability. Phys. Fluid, 23" 1485-1490.

Baker, G.R., Meiron, D.I., and Orszag, S.A., 1982. Generalized vortex methods for free-surface flow problems. J. Fluid Mech., 123. 477-501.

Benjamin, T.B., 1968. Gravity currents and related phenomena. J. Fluid Mech., 31, 209-248.

Birkhoff, G., 1954. Taylor instability and laminar mixing. Los Alamos Science Lab. Rept. LA-1862, 75 pp., appendices i n Rept. LA-1927, 91 pp.

Christiansen, J.P., 1973. Numerical simulation of hydrodynamics by the method of point vortices. J. Comp. Phys., 13, 363-379.

Fay, J.A., 1969. The spread of oil slicks on calm sea. In Oil on the Sea, D.P. Hoult (ed.), Plenum Press, 53-63.

Holyer, J.Y., and H.E. Huppert, 1980. Gravity currents existing in a two layer fluid. $\quad$ J. Fluid Mech., $\underline{100}, 739-769$.

Hoult, D.P., 1972. O 11 spreading on the sea. Ann. Rev. Fluid Mech.. 4 , 341368.

Huppert, H.E., and J.E. Simpson, 1980. The slumping of gravity currents. I. Fluid Mech., 99, 785-799.

von Kärman, T., 1940. The engineer grapples with nonlinear problems. Bull. Am. Math. Soc., 46, 615-683. 
Keulegan, G.H., 1958. The motion of saline fronts in still water. U.S. Nat. Bureau of Standards, Rept. No. 5831.

Leonard, A., 1980. Vortex methods for flow simulations. ‥ Comp. Phys., 37, 289-335.

Meng, J.C.S., and J.A.L. Thompson, 1978. Numerical studies of some nonlinear hydrodynamic problems by discrete vortex element methods. Mech., 84, 433-453.

Saf Emann, P.G., and G.R. Baker, 1979. Vortex interactions. Ann. Rev. Fluid Mech., 11, 95-112.

Simpson, J.E., 1982. Gravity currents in the laboratory, atmosphere, and ocean. Ann. Rev. Fluid Mech., 14, 213-234.

Simpson, J.E., and R.E. Britter, 1978. Experiments on the dynamics of a gravity current head. J. Fluid Mech., 88, 223-240.

Simpson, J.E., and R.E. Britter, 1979. Dynamics of the head of gravity current advancing over a horizontal surface. J. Fluid Mech. 94, 447-495.

Tryggvason, G., and H. Aref, 1983. Numerical experiments on Hele Shaw flow with a sharp interface. J. Fluid Mech., (to appear).

Yih, C-S., 1947, referred to in: Yih, C-S., 1980. Stratified flows. Academic Press, New York, 204-207. 


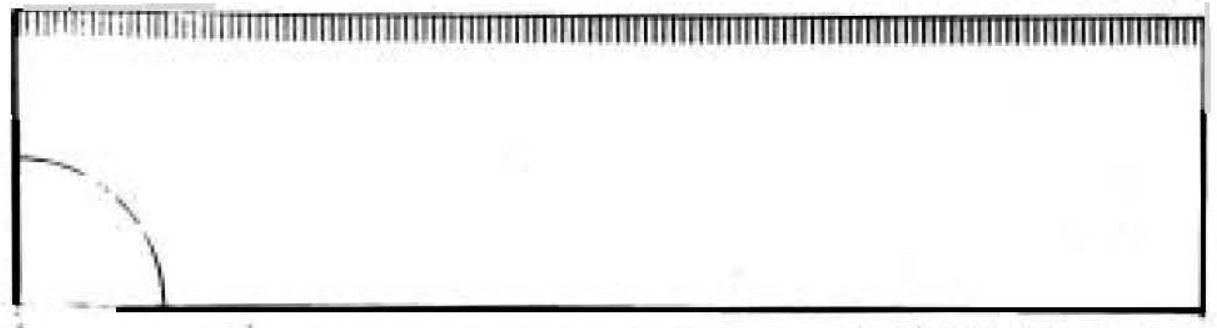

[

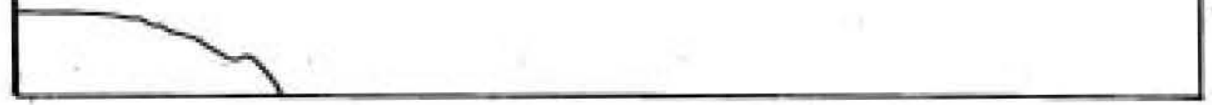

[
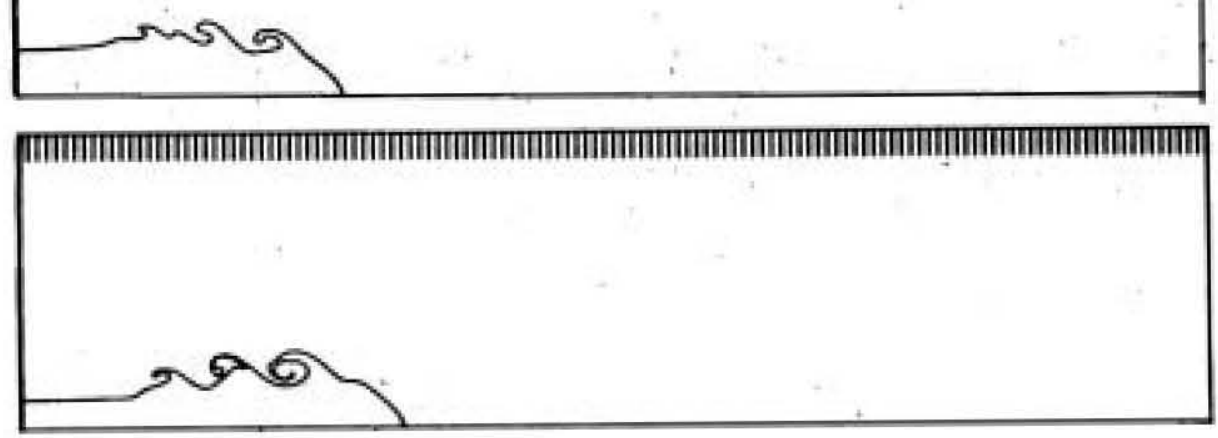

|

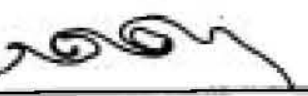

[
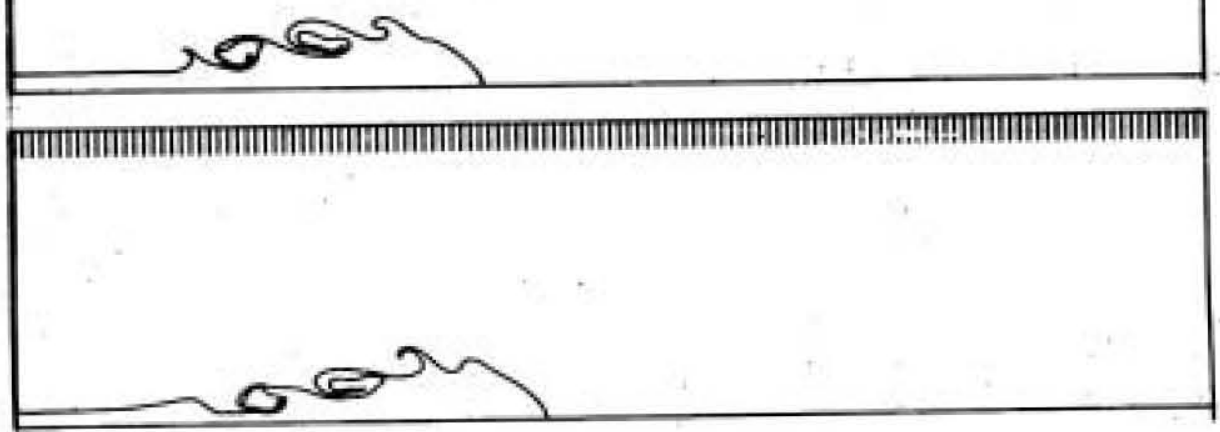

FIGURE 3 
$-222=$
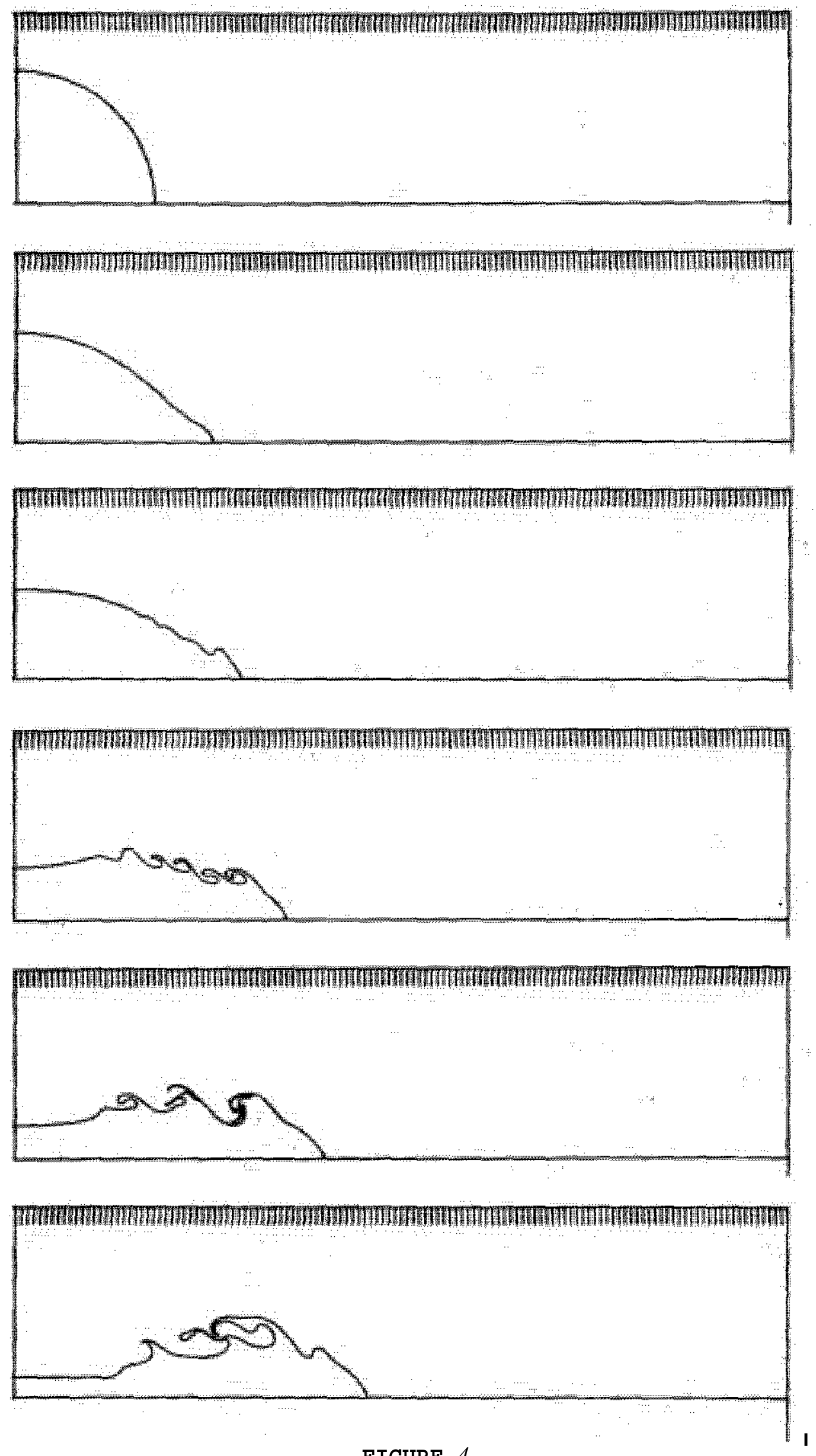

FIGURE 4 


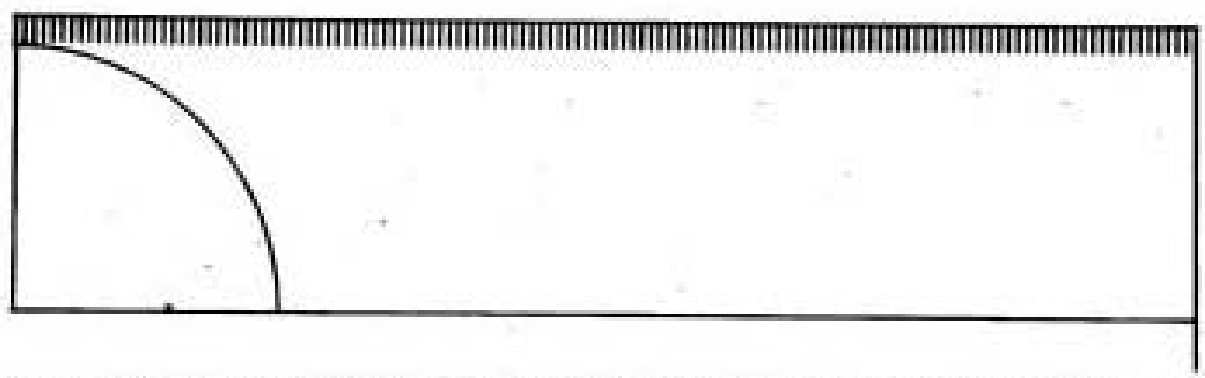

[

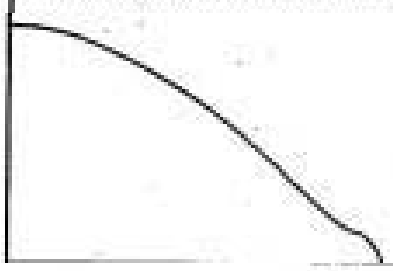

|
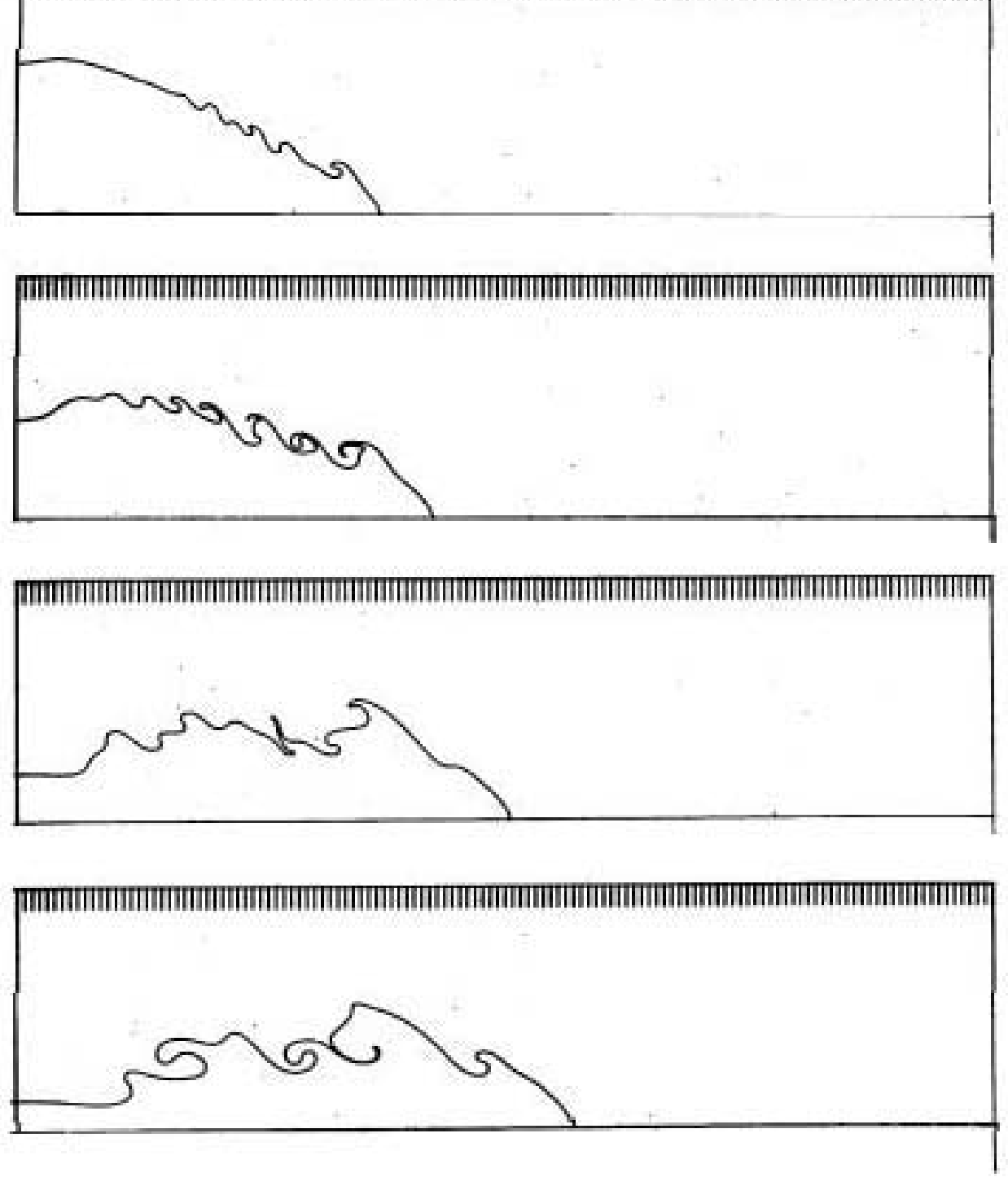

FIGURE 5 

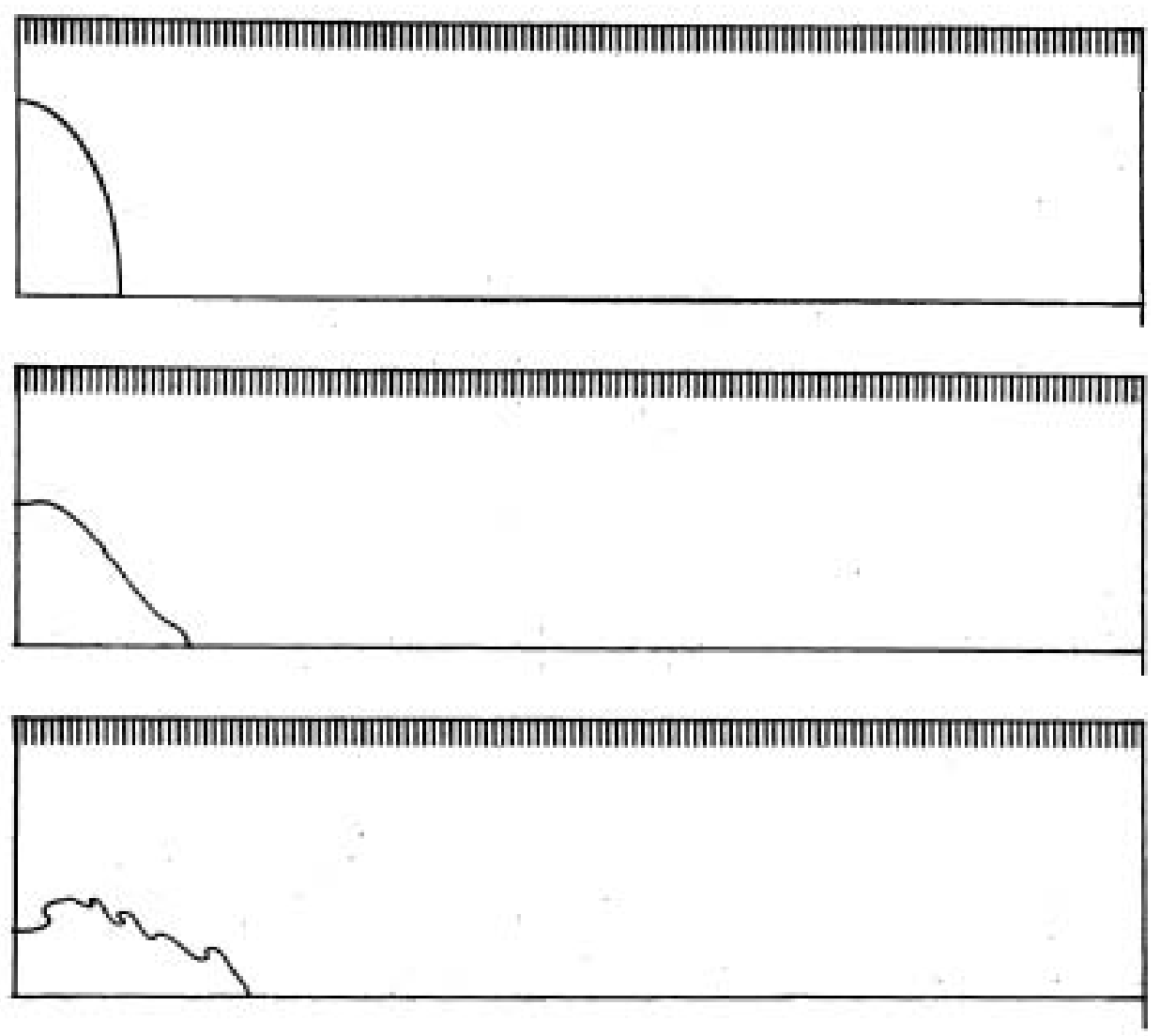

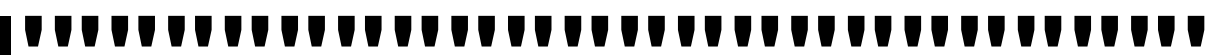

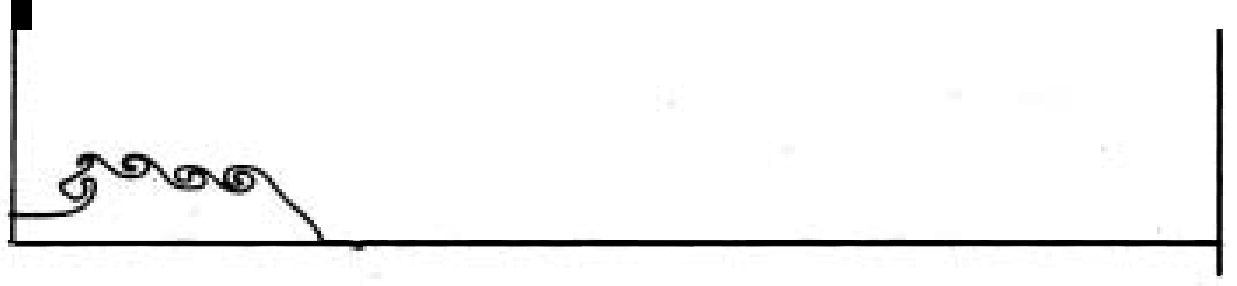

|
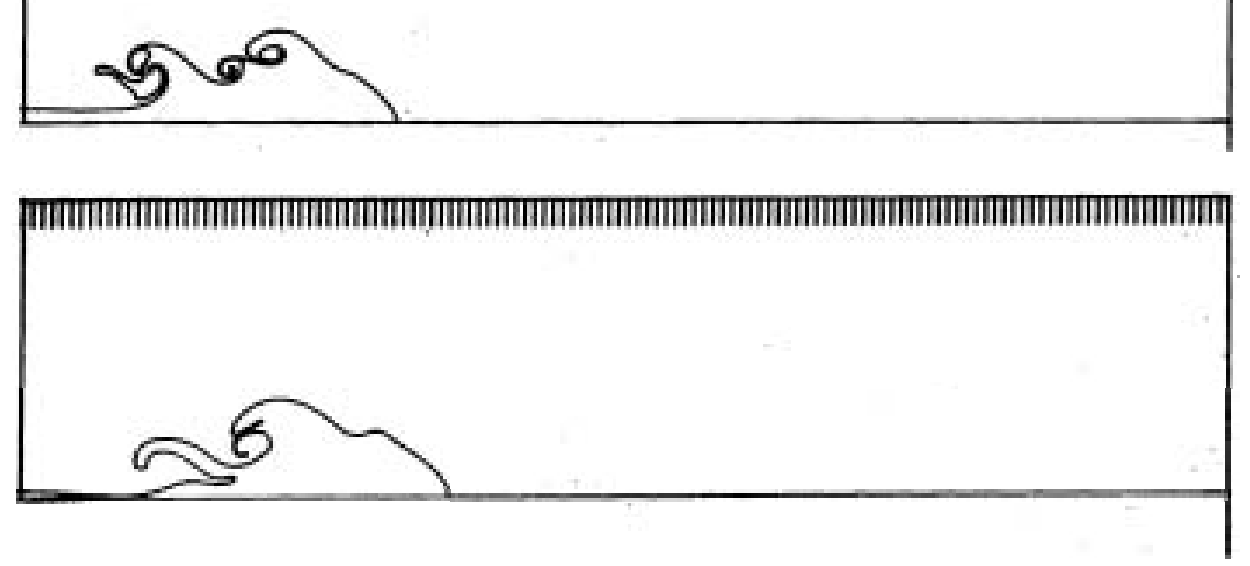

FIGURE 6 

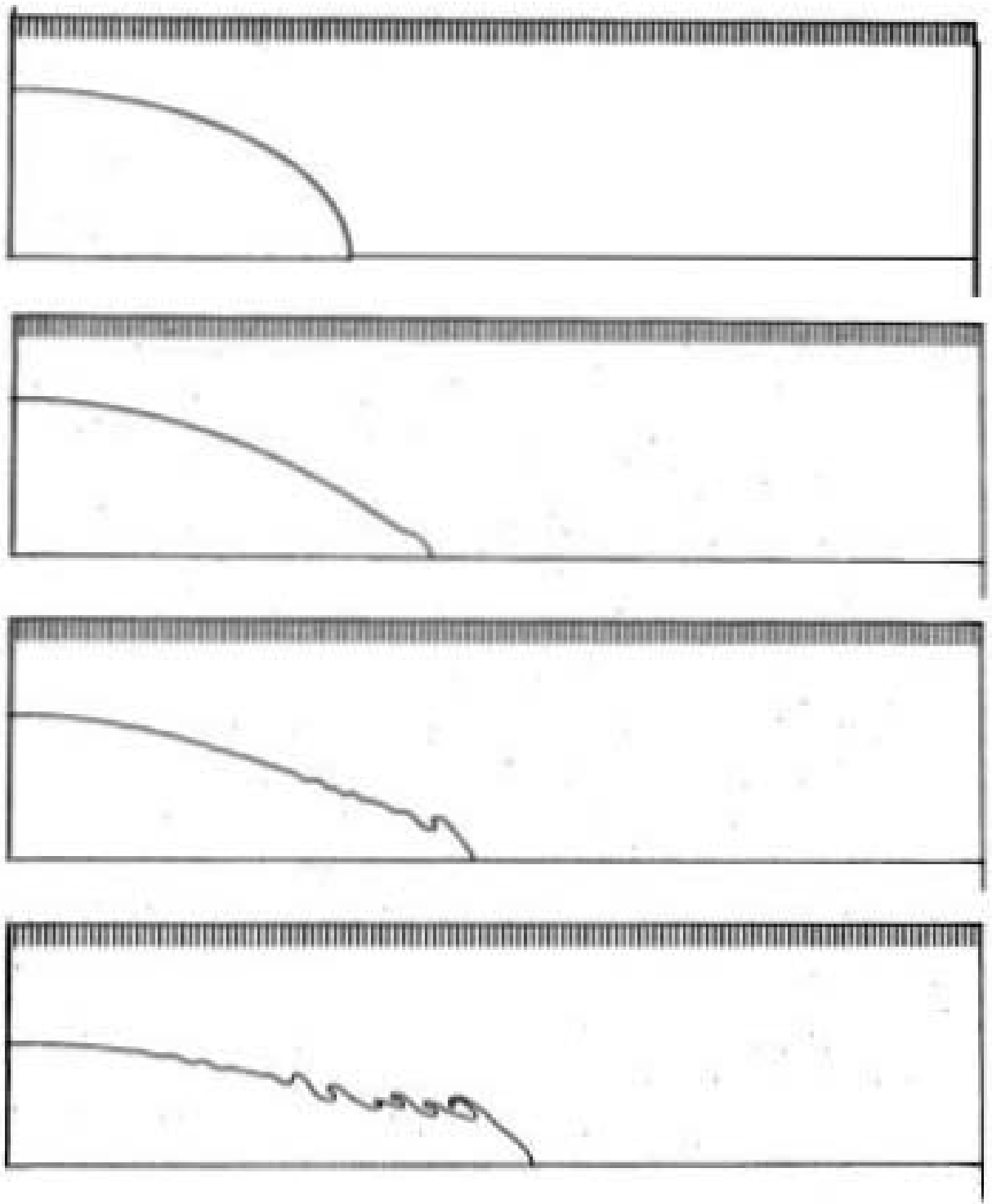

|
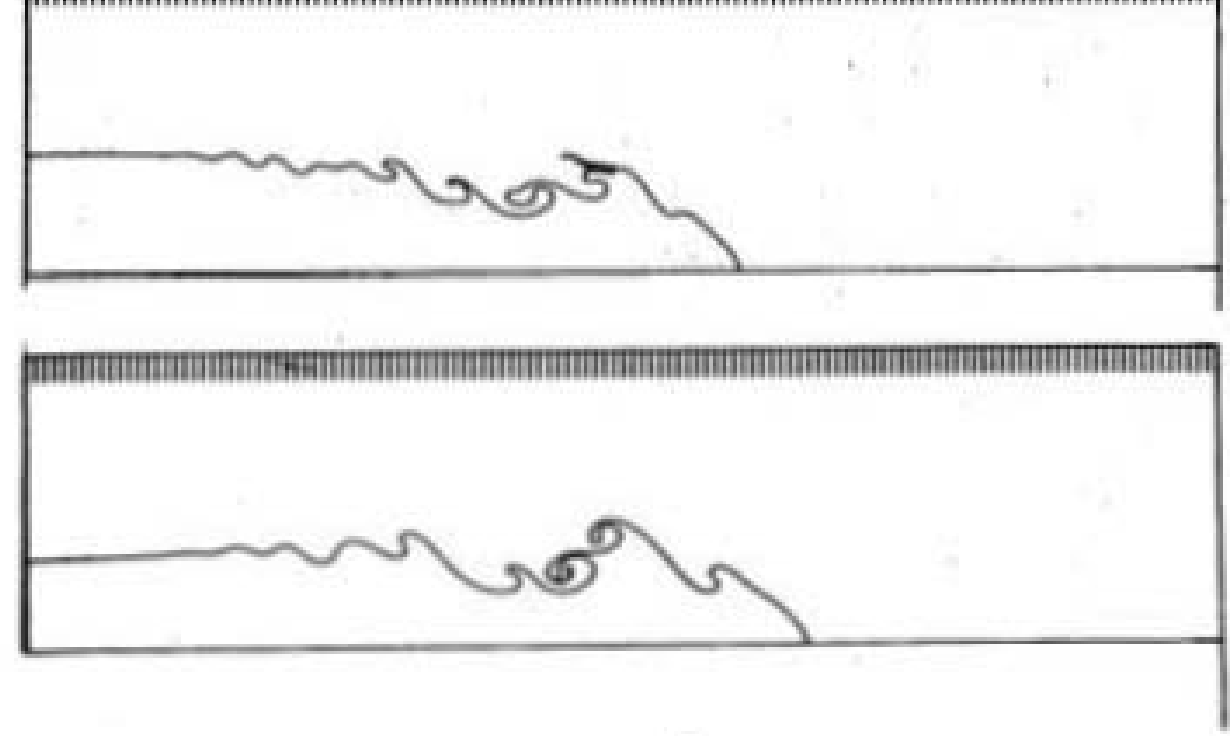

FIGURE ? 

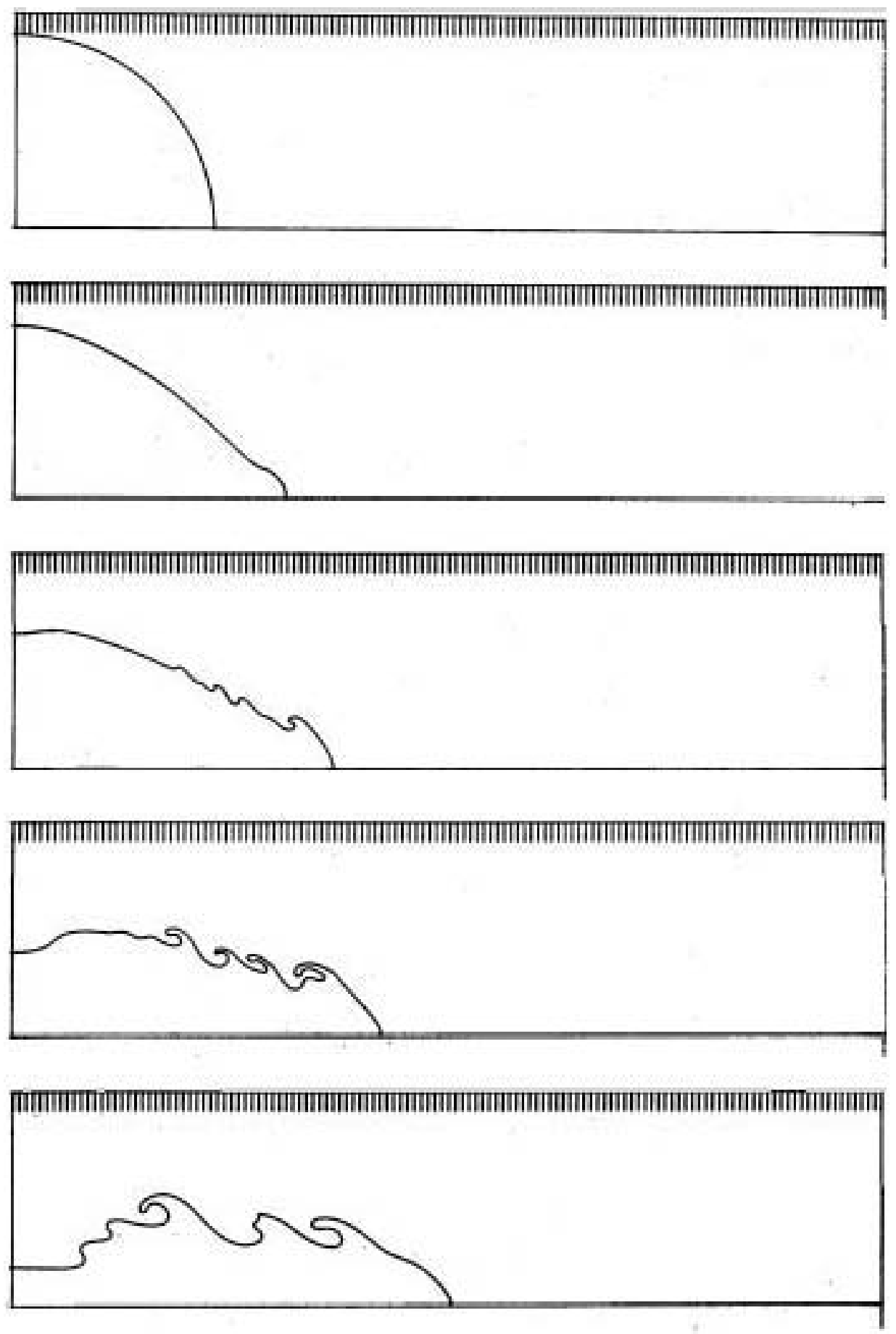

[

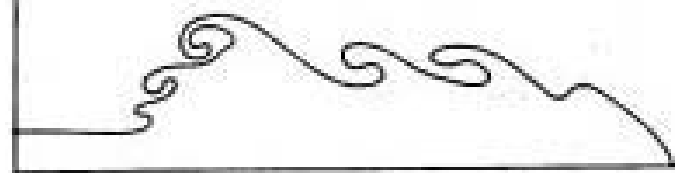



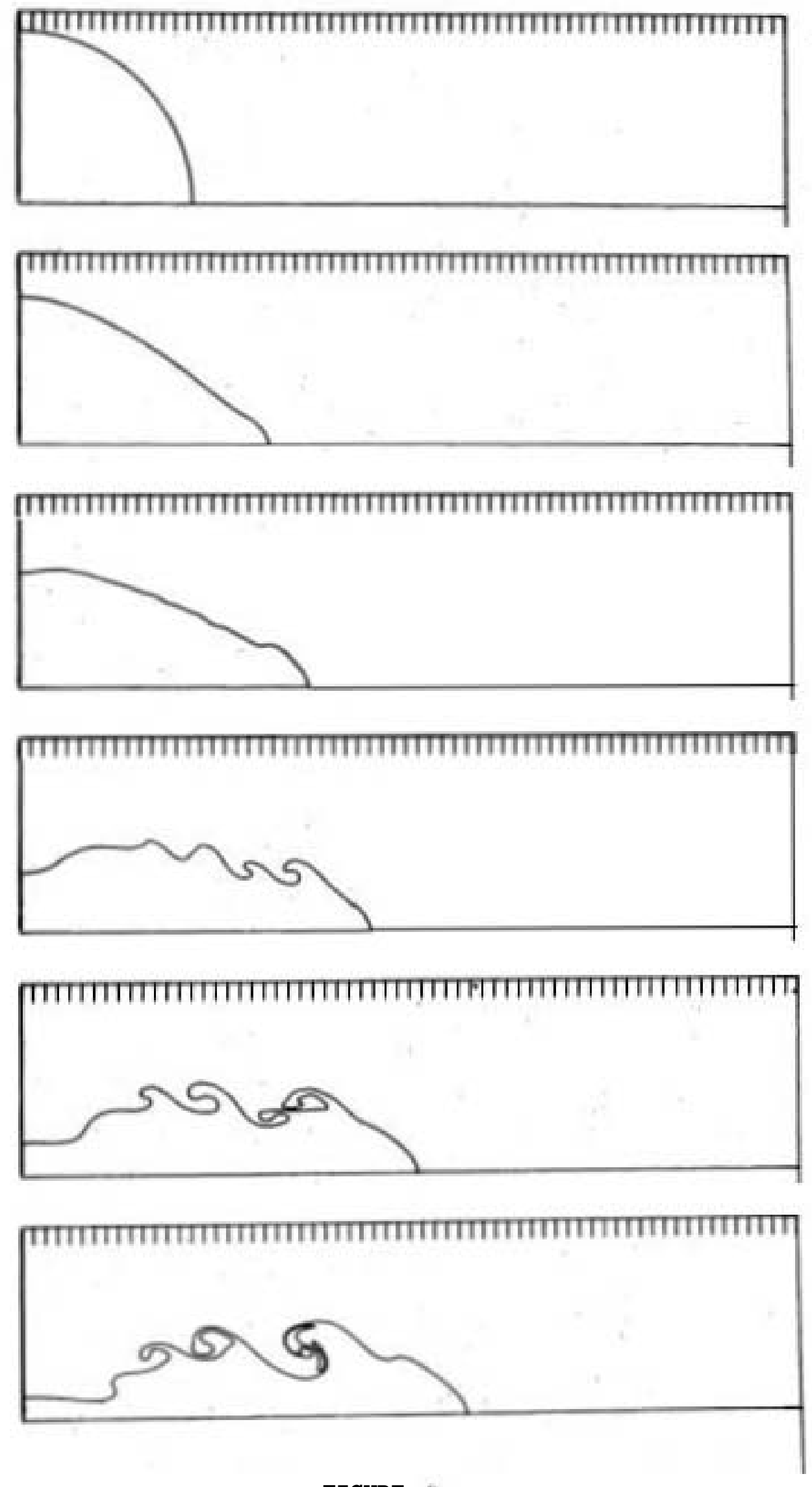


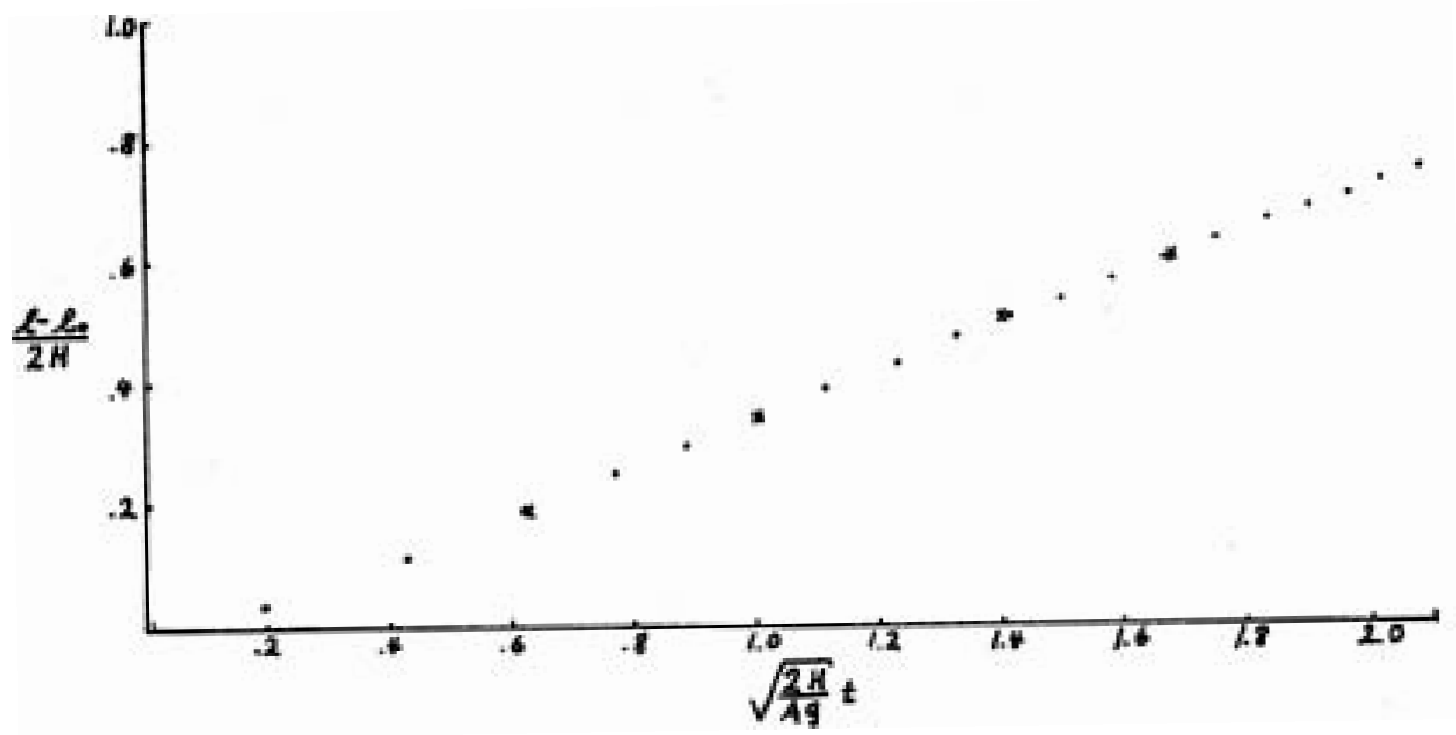

Figure 10. Propagation of the nose. - Run $1, \mathbf{x}$ Run 8.

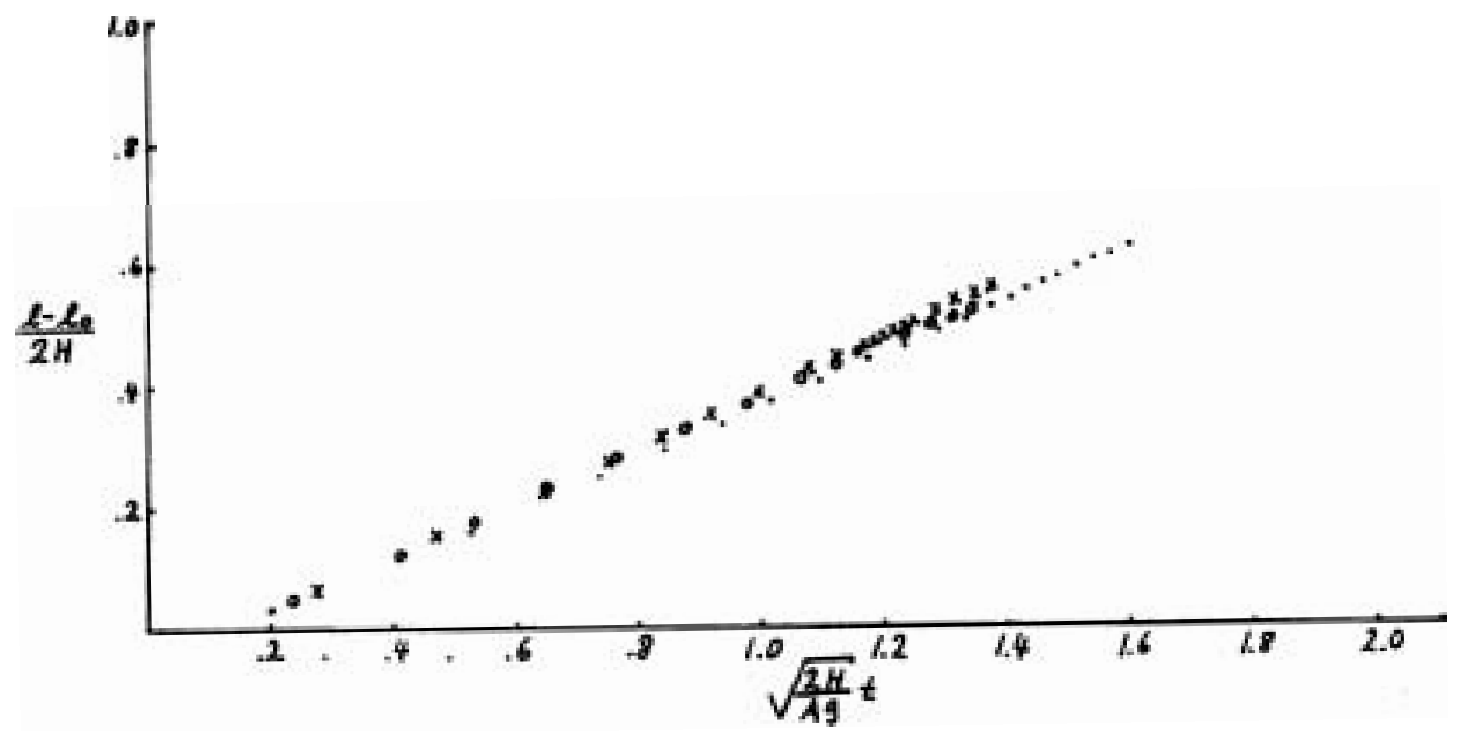

Figure 11. Propagation of the nose. $\quad \times$ Run 2, 0 Run 4, Run 5 .

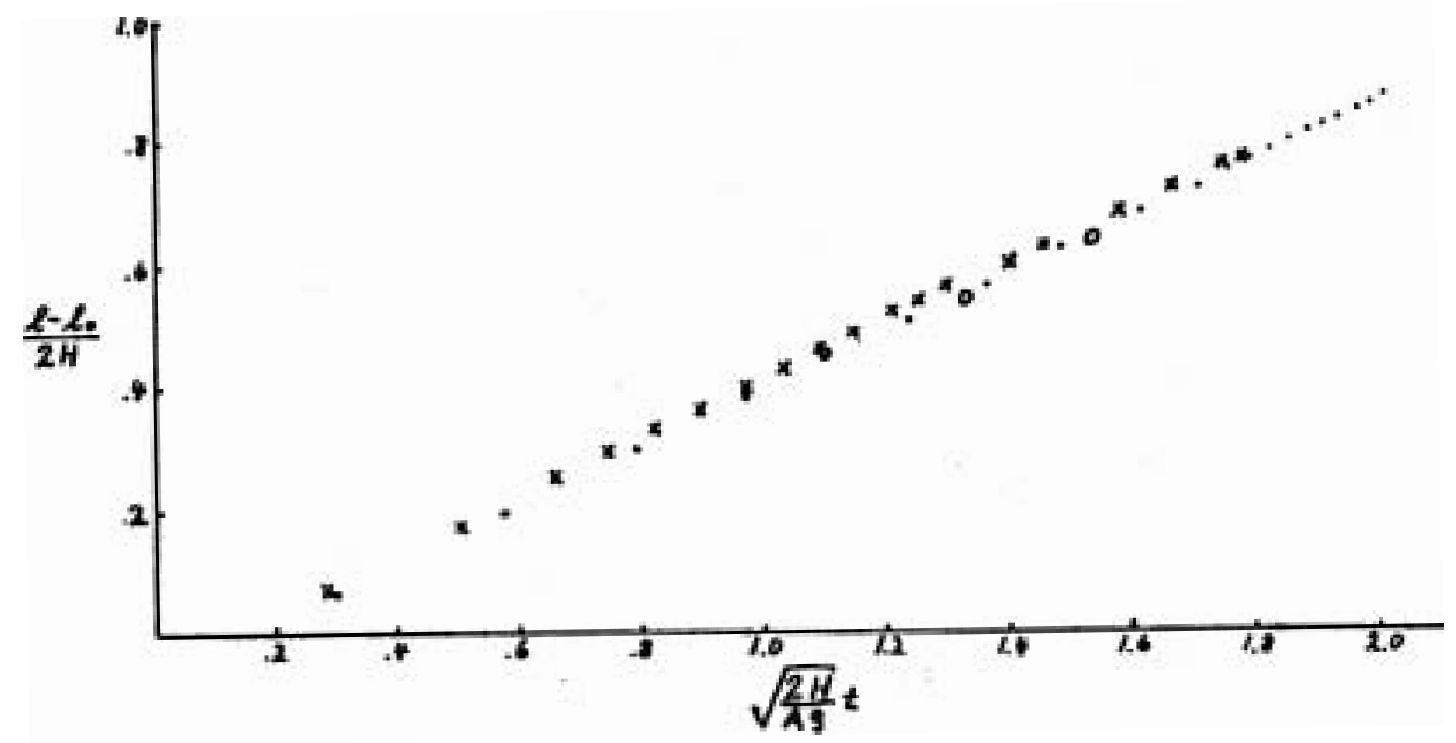




\title{
A LINEAR STABILITY THEORY OF DOUBLE DIFFUSIVE HORZONTAL INTRUSIONS I N A TEMPERATURE-SALINITY FRONT
}

\author{
Hiroshi Niino
}

\section{Introduction}

It is one of the common features in the ocean that interleaving layers are found where there is a strong contrast in temperature and salinity in the horizontal direction. The possibility of a cooperative instability in which salt-fingers drive medium-scale intrusions which, in turn, provide a necessary amount of salt flux to maintain the salt fingers has been theoretically suggested by Stern (1967). Recent observations in oceanic fronts have shown that cold/fresh intrusions are sinking while warm/salt intrusions are rising across isopycnals (Horne, 1978; Joyce, Zenk, and Toole, 1978; Gregg, 1979), which is consistent with Stern's (1967) prediction.

The intrusions have been also studied in the laboratory. Thorpe, Hutt, and Soulsby (1969) and Chen, Briggs, and Wirtz (1971) have made a laboratory experiment on stably stratified salt water heated and cooled through side boundaries. They have found alternating layers of cellular motions which extend from one boundary to the other, and Chen et al. (1971) have suggested that the vertical scale of these layers is scaled by $g \alpha \Delta \mathrm{T}^{2} \mathrm{~N}^{2}$, where $g \alpha \Delta \mathrm{T}$ is the effective buoyancy difference between the heated and cooled boundaries, and $N^{2}$ is the Brunt-Väisälä frequency for the salt stratification. Recently a laboratory experiment which seems to be more relevant to the case of the oceanic front has been suggested by Turner (1978) and performed by Ruddick and Turner (1979); (this paper will be referred to as RT). Here we will briefly summarize the important features found in their experiment. They prepared two fluids on the two sides of a barrier which is located at the middle of a rectangular tank. One of the fluids is stably stratified with salt and the other with sugar but with the same stratification. At a certain instant the barrier was carefully withdrawn. Although the withdrawal of the barrier produced a considerable amount of internal gravity waves because of an initial unbalance of the densities at both sides these waves decayed away within two minutes after the barrier was withdrawn. Then, intrusions started to develop with a typical time scale of a few minutes and penetrate into the other fluid. The penetration velocity was nearly proportional to the depth (i.e., density anomaly). The vertical scale of the intrusion was also proportional to the depth. It is important to mention that these features of the intrusions did not depend on the initial disturbances which were rather arbitrarily produced by the withdrawal of the barrier. RT used an energy argument to derive the possible vertical scale $\mathrm{H}$ and found that $\mathrm{H}$ should be between $(1-\gamma) g \beta \Delta S / N^{2}$ and $2(1-\gamma) g \beta \Delta S / N^{2}$, with $g$ the gravity acceleration, $\beta$ the salinity contraction coefficient, $\Delta \mathbb{S}$ the local salt concentration difference, $\mathbb{N}^{2}$ the Brunt-Väsälä frequency for the density, and $\gamma$ the density flux ratio of salt to sugar $(\approx 0.88$, Griffiths and Ruddick, 1979). Since most of the experimental results for H fell into the above interval, they concluded that the vertical scale of the intrusion is scaled by $g \beta \Delta S / N^{2}$.

Although the energy argument seems to work well in determining the vertical scale of the intrusions, it assumes the presence of layered intrusions from the beginning and does not require any knowledge of the structure of the intrusion nor does it give any information on the structure. 
Thus, it is desirable to develop a theory which explains the vertical scale aswell as the behavior of the intrusions such as the growth rate, the flow pattern, and the salinity and temperature fields, etc. The fact that the development of the intrusions is not influenced by the initial disturbance suggests that those intrusions may be generated as a result of an instability.

Recently, Toole and Georgi (1981) developed a linear stability theory similar to Stern's (1967). They considered a stably stratified fluid which has uniform compensating horizontal temperature and salinity gradients extending to infinity. Although they tried to apply their results to RT's experiment, the wavelength of the fastest growing mode did not agree with RT's vertical scale. This result might be expected, since there is no typical scale based on the salt concentration difference when the salinity gradient extends to infinity.

In this report, an effort is made to interpret the generation of the intrusions in RT's experiment as a result of an instability of a salinitytemperature front*. Although the initial disturbances caused by the withdrawal of the barrier decays off within a few minutes (see Figure 2(b) in RT), it is observed that the mixing caused by the initial disturbances has already spread the initial salinity-temperature front to a finite width. At the same time, small-scale motions which seem to be due to salt fingers can be observed. In the following section the stability problem of this fluid is formulated. In Section 3 the results are presented, and they are discussed in Section 4. The concluding remarks are made in the last section.

\section{Formulation of the Problem}

\subsection{Basic field}

We consider a salinity-temperature front of a finite width which could be regarded as the field immediately after the initial medium-scale disturbances have died away. Although small-scale disturbances such as salt fingers might be present, it is assumed that there is no medium-scale motion in this basic field. Taking the $x$ - and $z^{-}$axes to the cross-frontal and vertical directions respectively, we choose the following distributions of the temperature $\overline{\mathbf{T}}$ and the salt $\overline{\mathrm{S}}$ as a crude model of the front (see Figure 1 ):

$$
\begin{aligned}
& \bar{T}=\frac{N^{2}}{g \alpha} Z+\frac{\Delta \rho}{\alpha \rho_{0}} h(x), \\
& \bar{S}=\frac{\Delta \rho}{\beta \rho_{0}} h(x),
\end{aligned}
$$

where

$h(x)= \begin{cases}1 & \text { for } x>a \\ x / a & \text { for }|x|<a \\ -1 & \text { for } x<-a\end{cases}$

\footnotetext{
*Although sugar and salt were used in RT's experiment instead of salt and temperature, the description will be hereafter made in terms of salt and temperature to imply that the result of the present report can also apply to the oceanic front, provided that the effect of the earth's rotation were not important.
} 
$\alpha$ and $\beta$ are the thermal expansion and salinity contraction coefficients respectively, $\mathbb{N}^{2}$ the Brunt-Vaisala frequency, g the gravity acceleration, $2 \Delta \rho$ the equivalent density change of temperature and/or salinity across the front, $\mathcal{P}_{0}$ a reference density and a the half width of the front. Since the density is given by $\bar{\rho}=\rho_{0}\left[1-\alpha\left(T-T_{0}\right)+\beta\left(S-S_{0}\right)\right]$ with reference temperature and salinity, $\mathrm{T}_{\mathrm{O}}$ and $\mathrm{S}_{\mathrm{O}}$, there is no horizontal gradient of the density. The vertical stratification of the density is characterized by the Brunt-Väisälä frequency $N^{2} \equiv\left(g / \rho_{0}\right) \partial \bar{\rho} / \partial Z(=g \alpha \partial T / \partial Z)$. There is no stratification in the salinity field. RT performed three experiments which.

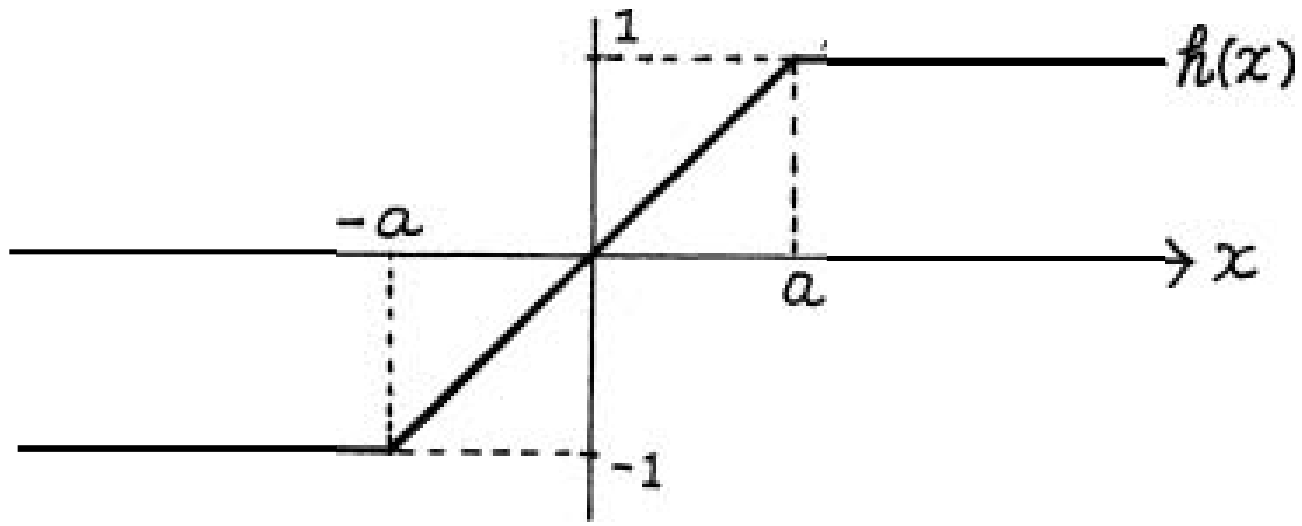

Figure 1

correspond to this configuration and found that the vertical scales of the intrusions did not have a systematic dependence on the depth. Although it would be desirable to consider the configuration for which most of RT's experiments were made, we avoid the complexity which will be introduced by considering the linear dependence of $\Delta \boldsymbol{\rho}$ on $\boldsymbol{Z}$. Once the behaviors of the intrusions are found for the former configuration, however, it might be possible to infer those for the latter configuration by substituting $\Delta \rho$ by its local value.

\subsection{Governing equations}

Consider a medium-scale disturbance of infinitesimal amplitude superimposed on the basic field. The evolution of the disturbance may be described by the following equations:

$$
\begin{aligned}
& \frac{\partial u}{\partial t}=-\frac{\partial p}{\partial x}+\varepsilon \kappa \frac{\partial^{2} u}{\partial z^{2}} \\
& 0=-\frac{\partial p}{\partial z}+g(\alpha T-\beta S) \\
& \frac{\partial u}{\partial x}+\frac{\partial w}{\partial z}=0 \\
& \frac{\partial T}{\partial t}+u \frac{\partial \bar{T}}{\partial x}+w \frac{\partial \bar{T}}{\partial Z}=\frac{\beta}{\alpha} \gamma r \frac{\partial^{2} S}{\partial Z^{2}} \\
& \frac{\partial S}{\partial t}+u \frac{\partial \bar{S}}{\partial x} \quad=n \frac{\partial^{2} S}{\partial \nabla^{2}}
\end{aligned}
$$


The behavior of $\sigma_{\mathrm{n}}$ can be graphically examined. In Figure 2 are plotted the left-hand side (LHS) of (32) (dashed line) and the right-hand side (RHS) of (32) (solid lines) against 6 .

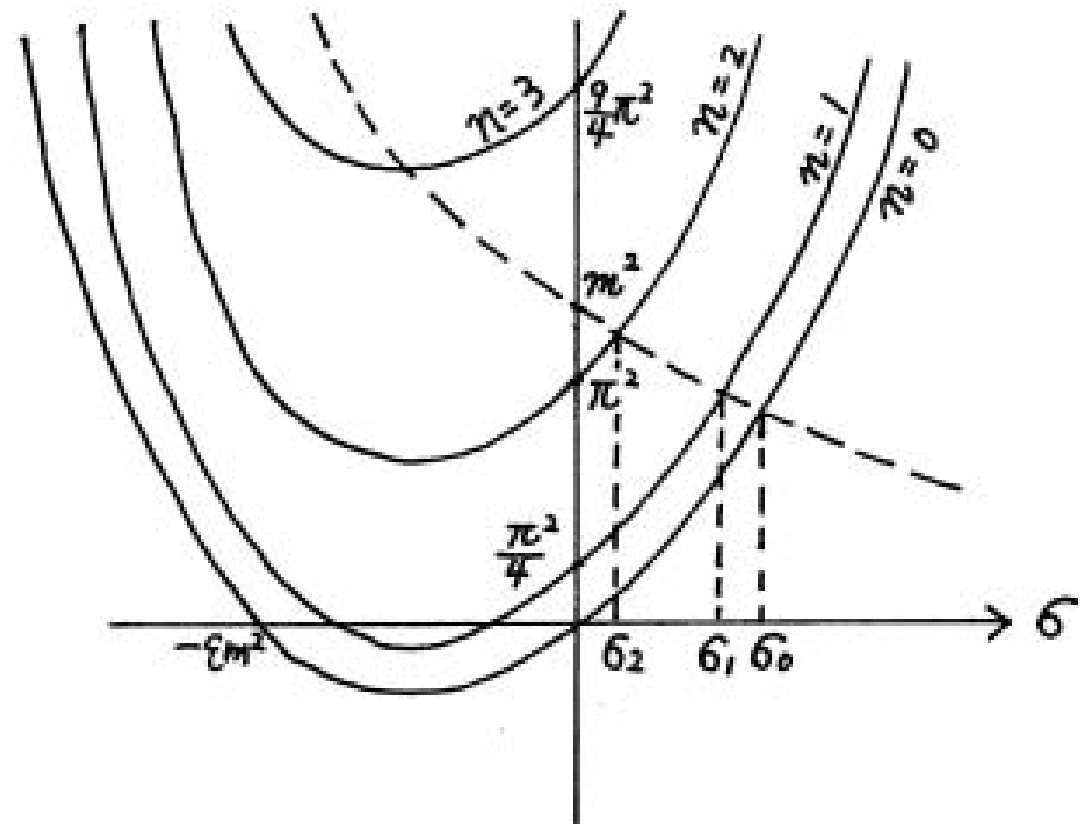

Figure 2

It is seen that $\sigma_{0}$ exists for all values of $m$. The existence of $6 \mathrm{n}$ depends on only $\mathrm{m}$, and the condition for the existence of $6 \mathbf{n}$ is given by $\mathrm{m}^{2}>n^{2} \pi^{2} / 4$. Now, LHS and RHS of (30) are plotted by the solid lines and the dashed line in Figure 3, respectively.

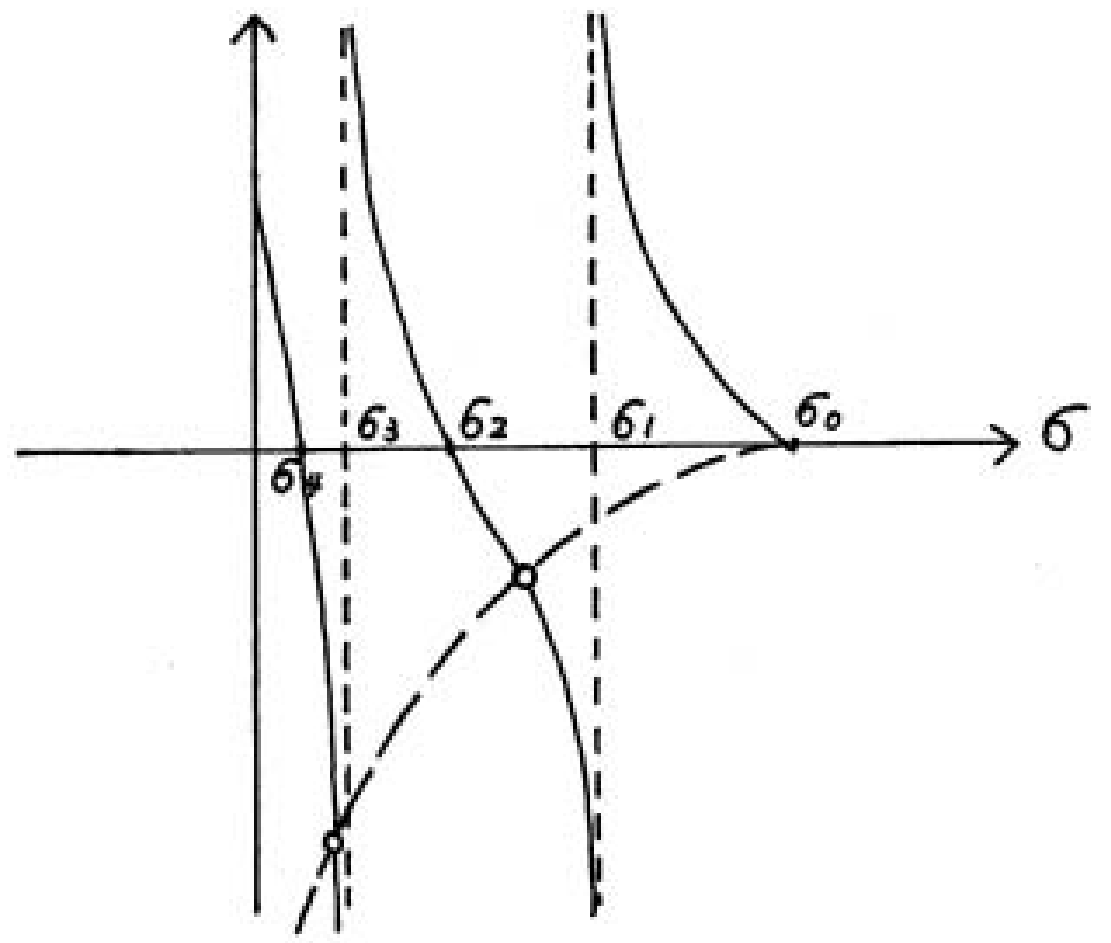

Figure 3 
where $\mathcal{U}$ and $\mathcal{U}$ are the velocities in the $\mathcal{X}$ - and $\boldsymbol{Z}$-directions respectively, $\boldsymbol{p}$ the kinematic pressure, $\mathrm{T}$ the temperature, and $\mathrm{S}$ the salinity.

The following assumptions and approximations are made to derive the above equations:

1) Two dimensionality is assumed, since it can be expected that a disturbance which has a y-dependence has a smaller growth rate.

2) The Bousinessq approximation.

3) The hydrostatic approximation is used, since the aspect ratio of the intrusions found. in RT's experiment is fairly small.

4) In order to express the effect of enhanced vertical mixing of the temperature and the salinity due to salt fingers, Stern's (1967) parameterization are used: i.e. the salt flux is assumed to be given by

$-K \partial S / \partial Z$, where $K$ is a constant eddy diffusivity, while the temperature flux is assumed to be proportional to the salt flux with the constant of proportionality $(\beta / \alpha) \gamma$. The value of $\gamma$ has been determined experimentally and is known to be $\approx 0.56$ for the temperature-salt problem (Turner, 1967) and $\approx 0.88$ for the salt-sugar problem (Griffiths and Ruddick, 1979).

5) In order to retain a possibility that the salt fingers transfer some horizontal momentum, it is also assumed that there is an eddy momentum flux given by $-\varepsilon \kappa \partial u / \partial Z$. However, we have not excluded a possibility that the momentum is transferred by molecular process. $\mathcal{E}$ is assumed to be a constant and may be called a Schmidt number. The parameterizations (4) and (5) may be applicable to the salt finger interfaces but not to the diffusive interfaces. Since the medium-scale motion is driven by the salt-finger process but not by the diffusive process, however, it is assumed that the absence of the diffusive interfaces is not essential to the dynamics of the intrusions. The effect of the diffusive interfaces will be discussed in Section 4.

\subsection{Method of the solution}

Consider a wave disturbance whose vertical wavenumber is $\mathrm{m}$. Then, any variable, say $\boldsymbol{\eta}$, may be expressed as

$$
\eta=\operatorname{Re}\left[\hat{\eta}(x) e^{i m z+\sigma t}\right]
$$

where $\operatorname{Re}[]$ denotes the real part of the quantity in the bracket. $\sigma$ is the eigenvalue to be determined and can be a complex number in general. In this report, however, we will look for a real positive eigenvalue which seems relevant to the intrusion problem. Thus, $\sigma$ may be called the growth rate.

Substituting (10) into (4), (6), (7), and (8), we can express all the variables in terms of $p$ :

$$
u=-i m \psi=-\frac{1}{6+\varepsilon r m^{2}} p^{\prime}
$$




$$
\begin{aligned}
& w=\psi^{\prime}=\frac{1}{i m\left(\sigma+\varepsilon k m^{2}\right)} p^{\prime \prime} \\
& S=\frac{1}{\left(\sigma+r m^{2}\right)\left(\sigma+\varepsilon r m^{2}\right)} \cdot \frac{\Delta \rho}{\beta \rho_{0}} h^{\prime} p^{\prime} \\
& T=-\frac{1}{\sigma}\left[-\frac{\sigma+(1-\gamma) k m^{2}}{\left(\sigma+\pi m^{2}\right)\left(6+\varepsilon r m^{2}\right)} \cdot \frac{\Delta \rho}{\alpha \rho_{0}} h^{\prime} p^{\prime}+\frac{\frac{N^{2}}{g \alpha} p^{\prime \prime}}{i m\left(\sigma+\varepsilon m^{2}\right)}\right]
\end{aligned}
$$

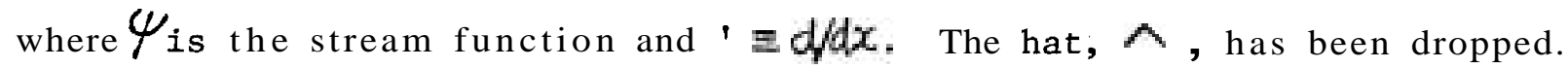

Substitutions of (11)-(14) into (5) give the second-order differential equation for $p$ :

$$
p^{\prime \prime}-\frac{i m^{3} g r h^{\prime}}{N^{2}\left(\sigma+r m^{2}\right)} \not p^{\prime}-m^{2} \sigma\left(\sigma+2 \pi m^{2}\right) \not p=0,
$$

where $\tilde{g}=g \frac{\Delta \rho}{\rho_{0}}(1-\gamma)$.

Now, we nondimensionalize (15) in the following way:

$$
x=a x_{*}, m=d^{-1} m_{*} \text { and } \sigma=k d^{-2} \sigma_{*} \text {, }
$$

where the asterisks denote non-dimensional quantities and

$$
d=\frac{\widetilde{g}}{N^{2}} \text {. }
$$

Dropping the asterisks, we obtain

$$
p^{\prime \prime}-\frac{i m^{3} h^{\prime}}{\sigma+m^{2}} p^{\prime}-\frac{m^{2} \sigma\left(\sigma+\varepsilon m^{2}\right)}{R a} p=0
$$

where $R a=N^{2} d^{6} / r^{2} a^{2}$ is a Rayleigh number and is the only external parameter which describes the stability of the front when $\varepsilon=0$. It is noticed that Eq. (19) does not depend on how u, w, p, T, and S are scaled. Also note that the nondimensionalization introduced above is the only way to have only one external parameter in Eq. (19).

The solutions of (19) for $x>1$ and $x<-1$ are given by

$$
p=A e^{-k x} \quad \text { for } x>1
$$

and

$$
p=B e^{k x} \text { for } x<-1 \text {, }
$$

where $\mathrm{k} 3 R m \sqrt{\sigma\left(\sigma+\varepsilon m^{2}\right)}, R=I / \sqrt{R \bar{a}}$, and $\mathrm{A}$ and $\mathrm{B}$ are complex constants. On the other hand, the solution for $|x|<1$ is given by

$$
p=c e^{i n_{1} x}+D e^{i n_{2} x},
$$


provided that $n_{1}$ and $n_{2}$ are not equal*, where $n_{l}=\left(L+\sqrt{L^{2}-4 k^{2}}\right) / Z$, $n_{2}=\left(L-\sqrt{L^{2}-4 k^{2}}\right) / 2$, and $L \equiv m^{3} /\left(6+m^{2}\right)$.

These solutions (20)-(22) must be matched at $\mathrm{x}=\mathbf{+ 1}$. The matching conditions are that the pressure and the horizontal velocity be continuous, i.e.

and

$$
\boldsymbol{p}=\text { continuous }
$$

$$
\boldsymbol{p}^{\prime}=\text { continuous } * *
$$

Applying these conditions at $\mathrm{x}=\mathbf{= 1}$, we obtain the following equation for the eigenvalue 6 :

$$
2 k^{2}\left(e^{i \sqrt{L^{2}-4 k^{2}}}-e^{-i \sqrt{L^{2}-4 k^{2}}}\right)+i k\left(e^{i \sqrt{L^{2}-4 k^{2}}}+e^{-i \sqrt{L^{2}-4 k^{2}}}\right)=0
$$

When $\mathrm{L}^{2}-4 \mathrm{k}^{2}>0$, (29) can be written as

$$
\tan \sqrt{\frac{m^{6}}{\left(6+m^{2}\right)^{2}}-4 R^{2} m^{2} \sigma\left(\sigma+\varepsilon m^{2}\right)}=-\sqrt{\frac{m^{4}}{4 R^{2} \sigma\left(6+\varepsilon m^{2}\right)\left(6+m^{2}\right)^{2}}-1}
$$

while when $\mathrm{L}^{2}-4 \mathrm{k}^{2}<0$,

$$
\tan \sqrt{4 k^{2}-L^{2}}=-\frac{\sqrt{4 k^{2}-L^{2}}}{2 k} \text {. }
$$

Since $k$ and $\sqrt{4 k^{2}-L^{2}}$ are positive, there is no solution for

(31). It can also be shown that there is no eigenvalue when $\mathrm{L}^{2}=4 \mathrm{k}^{2}$ (see Appendix I). Thus, only the solutions for (30) need to be considered. In the following section, the behaviors of the eigenvalues are examined.

\section{Results}

3.1. Analytical considerations on the behavior of the eigenvalue

Before presenting the results of the numerical calculation of the eigenvalue, we will analytically consider some behavior of the eigenvalue based on Eq. (30).

If we denote the value of $\mathbf{6}$, for which the argument of the tangent in (30) becomes $n \pi / 2(n=0,1, \ldots)$, by $\sigma_{n}$, the equation for $\sigma_{n}$ becomes

$$
\frac{m^{6}}{\left(\sigma_{n}+m^{2}\right)^{2}}=4 R^{2} m^{2} \sigma_{n}\left(\sigma_{n}+8 m^{2}\right)+\frac{n^{2} \pi^{2}}{4} \text {. }
$$

*The case when $\mathrm{L}^{2}=4 \mathrm{k}^{2}$ (when $\mathrm{n}_{1}$ and $\mathrm{n}_{2}$ degenerate) is treated in the Appendix I.

**This condition can be also obtained by integrating (19) between $X-\underline{I}-\varepsilon$ and $\pm 1+\varepsilon(\varepsilon \ll 1)$. 
The points where the two curves cross each other give the eigenvalue. Note that $\sigma=\sigma_{0}$ is not the eigenvalue, since $L^{2}-4 \mathrm{k}^{2}$ vanishes when $\sigma=\sigma_{\mathrm{o}}$. From Figure 3 we can deduce the following important information.

1) There is no eigenvalue for $m<\pi / 2$. This result coincides with the result of the energy argument by RT that the height of the intrusion cannot exceed $4 d$ exactly*. where 2$)$ The number of eigenvalues depends on only m and is equal to $\left\langle\frac{m}{\pi}+\frac{1}{2}\right\rangle$, integers which are smaller than $m / \pi+1 / 2$. $\sigma_{2}$ and $\sigma_{1}$ (or 0 and $\sigma_{1}$ if $\pi / 2<m<\pi$ ).

4) The front is always unstable even if the eddy diffusivity is present; i.e. there is no marginal stability curve.

Figure (3) makes it possible to derive the asymptotic behavior of the eigenvalue for high wavenumbers. Let us assume $\delta \mathrm{n} \sim \mathrm{m} \alpha$ as $\mathrm{m} \rightarrow \infty$ with $\alpha$ a constant to be determined. If $\alpha$ is larger than 2, it can be shown that Eq. (32) has no solution. If $\mathcal{X}$ is less than 2 on the other hand, Eq. (32) becomes

$$
\mathrm{m}^{2}= \begin{cases}4 R^{2} m^{2} \sigma_{n}^{2} & \text { for } \varepsilon=0 \\ 4 R^{2} \varepsilon m^{4} \sigma_{n} & \text { for } \varepsilon \neq 0\end{cases}
$$

which in turn gives

$$
\sigma_{n} \rightarrow \begin{cases}\frac{1}{2 R}-\frac{1}{4 R^{2} m^{2}}+O\left(m^{-4}\right) & \text { for } \varepsilon=0 \\ \frac{1}{4 R^{2} \varepsilon m^{2}}+O\left(m^{-4}\right) & \text { for } \varepsilon \neq 0\end{cases}
$$

Thus, it is expected that when the eddy viscosity is absent, the growth rate increases and approaches the asymptotic value $\frac{1}{2 R}$ as the wavenumber becomes large. If the eddy viscosity is included, however, the growth rate eventually becomes small for high wavenumbers.

\subsection{Results of the numerical calculations}

In this subsection, the results of the numerical calculations are presented. The eigenvalue (growth rate) was calculated from Eq. (30) by using Newton's iterative method for various combinations of $R a$ and $\mathcal{E}$. As the initial guess of the iteration, the average of $\sigma_{1}$ and $\sigma_{2}$ which have been also calculated from Eq. (32) by Newton's method, are used for obtaining the fastest growing mode.

*RT's d is equal to $2(1-\gamma)$ times our d. 
$-237-$

Figure 4 shows the growth rate as a function of the vertical wavenumber $m$ for various values of Ra when the eddy viscosity is absent. It is seen that the growth rate increases nearly in proportion to $\sqrt{\mathrm{Ra}}$. For a fixed value of $\mathrm{Ra}$, the growth rate increases with the wavenumber and approaches the asymptotic value predicted in the previous subsection. The most important feature of this figure is, however, that about $80 \%$ of the asymptotic value is attained for $m<10$. Since motions with high wavenumbers are expected to be damped when there is some viscosity, this feature suggests that our choice of the vertical scale was correct.

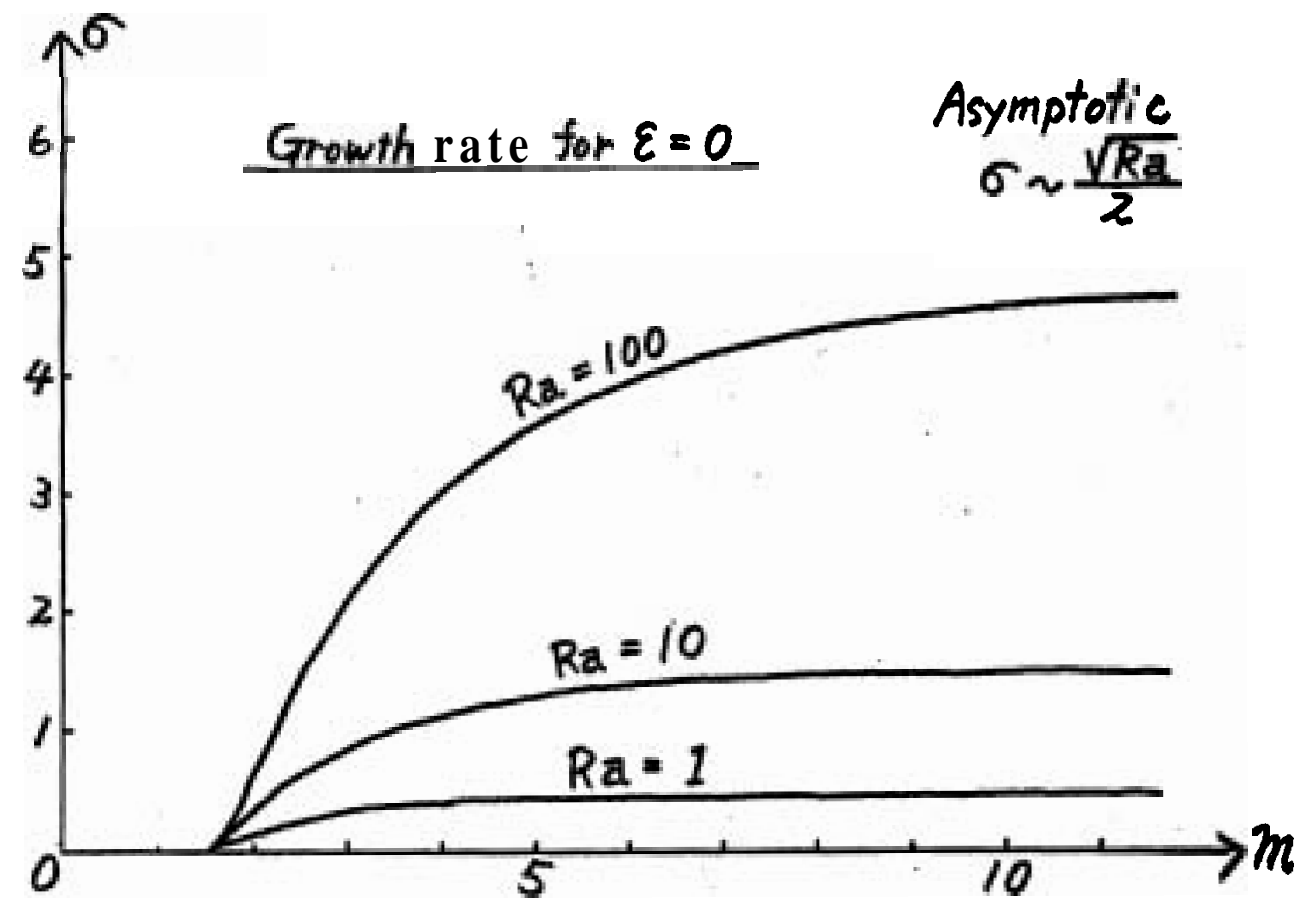

Figure 4

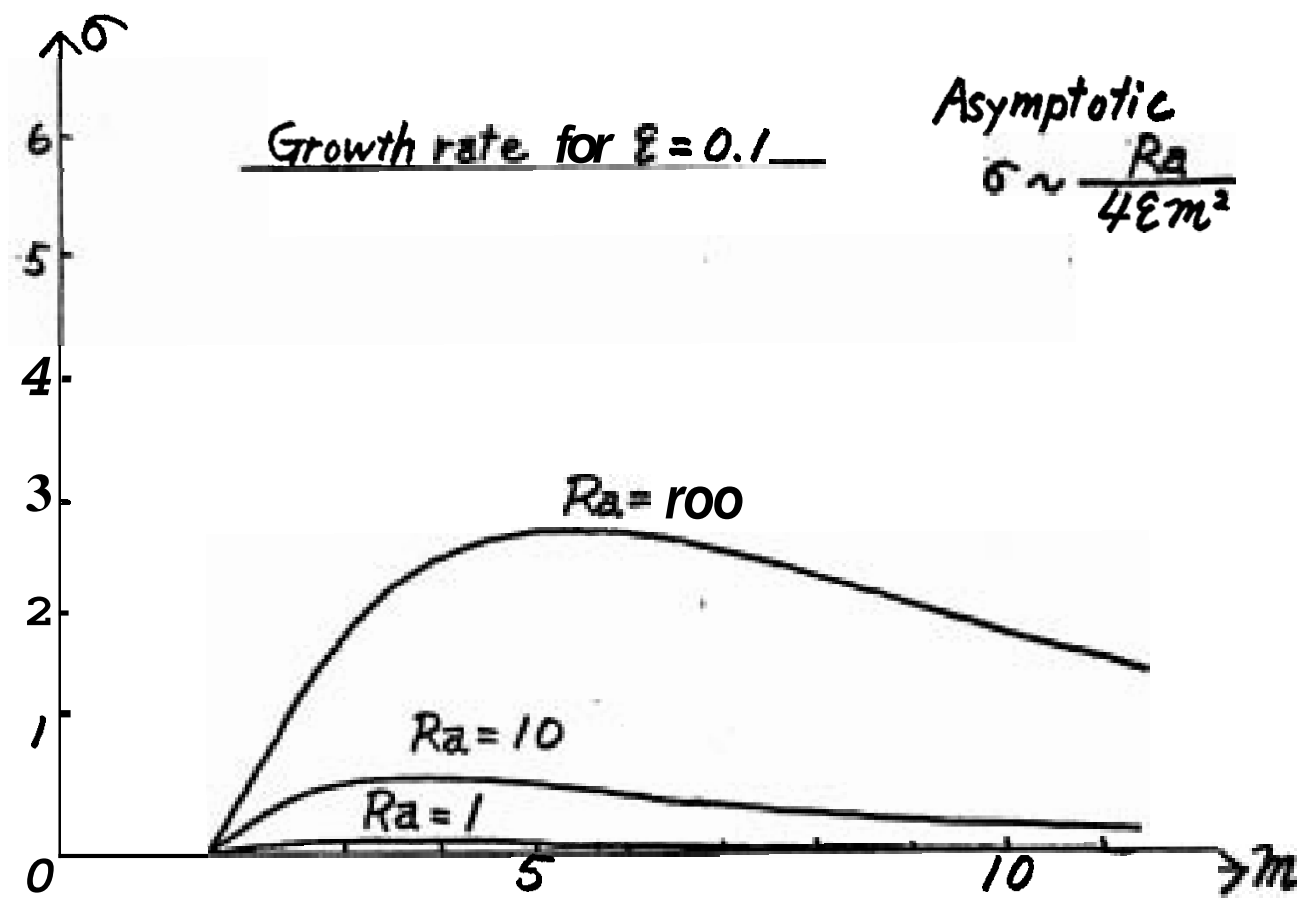

Figure 5 
In fact, if the effect of the eddy viscosity is considered, a maximum in the growth rate is produced at a moderate value of $\mathrm{m}$. Such an example is shown in Figure 5 for $\varepsilon=0.1$ and for $\mathrm{Ra}=1,10$, and $10^{2}$. It is seen that the growth rate again increases with Ra. The wavenumber of the fastest growing mode also increases with Ra.

The streamlines, temperature, and salinity fields of the fastest growing mode for $\mathcal{E}=2.0, \mathrm{Ra}=10^{4}$, and $\mathrm{m}=6.4$ are shown in Figure 6 (a), (b), and (c), respectively.
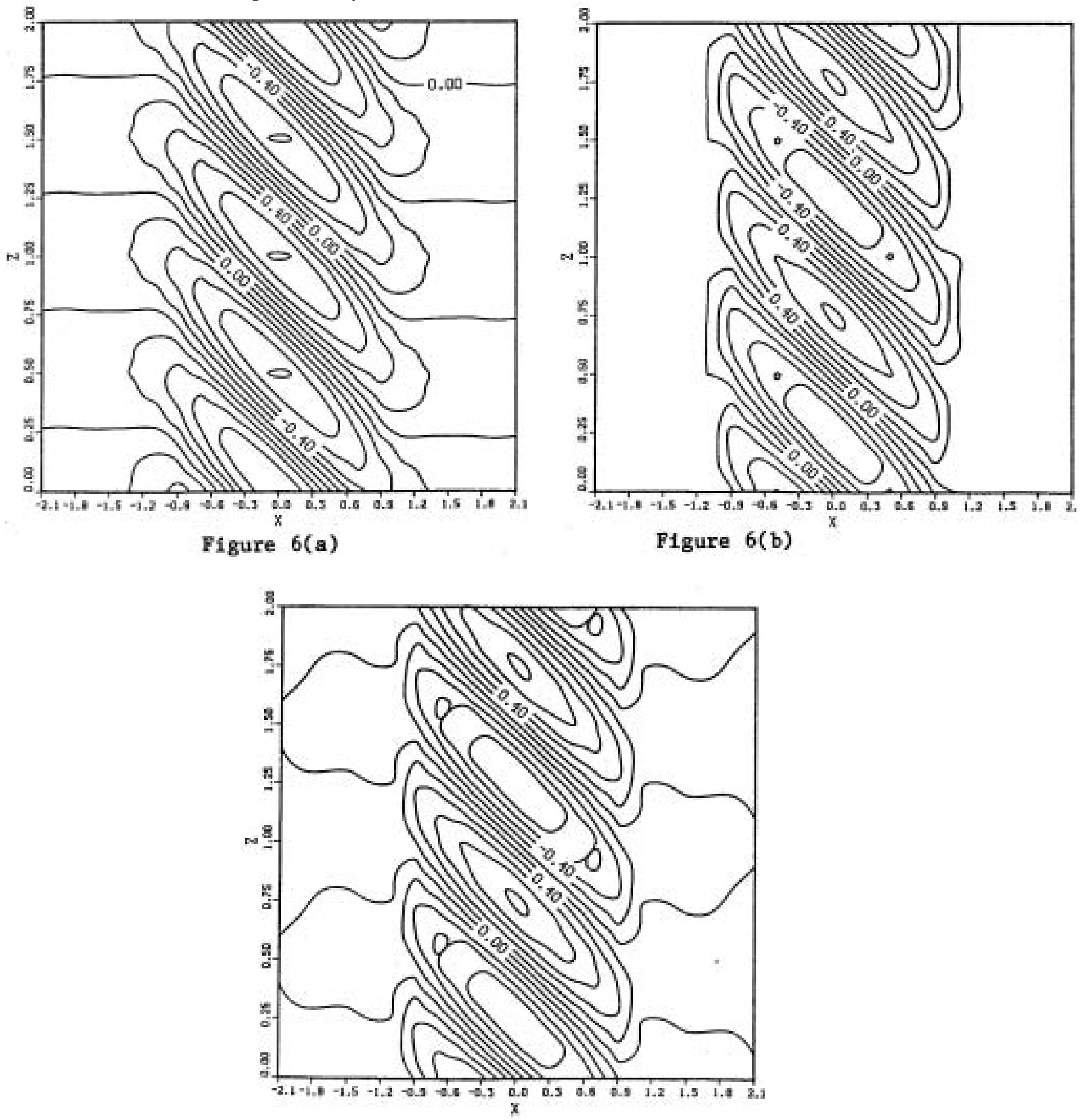

Figure 6(c) 
The direction of the tilt of the intrusion is to cross the isotherms, as RT observed in their experiment. The cold/fresh water is sinking and the warm/salty water is rising, which is characteristic of the intrusion driven by salt fingers. Furthermore, the amount of tilt is about one wavelength across the front. This is also similar to what is observed in the above experiment (see RT's Figure 3(a)). However, a qualitative difference is found for the circulation pattern. The theory predicts an alternation of the clockwise and counterclockwise circulations in the vertical direction, while only clockwise circulations were found in the experiment. The difference is probably due to the fact that the theory does not include diffusive interfaces.

The wavenumber of the fastest growing mode and the corresponding growth rate are shown for various values of $R a$ and $\mathcal{E}$ in Figures 7 and 8 , respectively. Generally speaking, the wavenumber of the fastest growing mode decreases as $\mathrm{Ra}$ is decreased and/or $\varepsilon$ is increased. However, there is an asymptotic wavenumber which gives the smallest wavenumber of the fastest growing mode for a 11 combinations of $R a$ and $\varepsilon$. The asymptotic wavenumber is given by 2.88 and the corresponding growth rate is given by

$$
\frac{0.47 / 9 R a}{4 \varepsilon}
$$

(see Appendix II)

The growth rate of the fastest growing mode also decreases as $R$ a is decreased and/or $\mathcal{E}$ is increased. For small values of $\mathbf{6}$, the growth rates calculated from the asymptotic formula (34) are shown by dotted lines. It is seen that the formula gives a good approximation to the eigenvalue for $\sigma<0.1$.

\section{Discussion of the Results}

Although the results obtained above could also be applied to the oceanic case as long as the earth's rotation is not important, we will confine our discussion here to compare the results with those of RT's experiments.

In Table 1 are presented the parameters used in the three RT experiments in which the salt concentration anomaly ( $\beta \Delta S$ across the front) was constant with depth. ( $(\rho) \partial \rho / \partial Z$ is the density stratification due to temperature and d the vertical scale defined by $d=g \beta \Delta S(1-\gamma) / 2 N^{2}$.

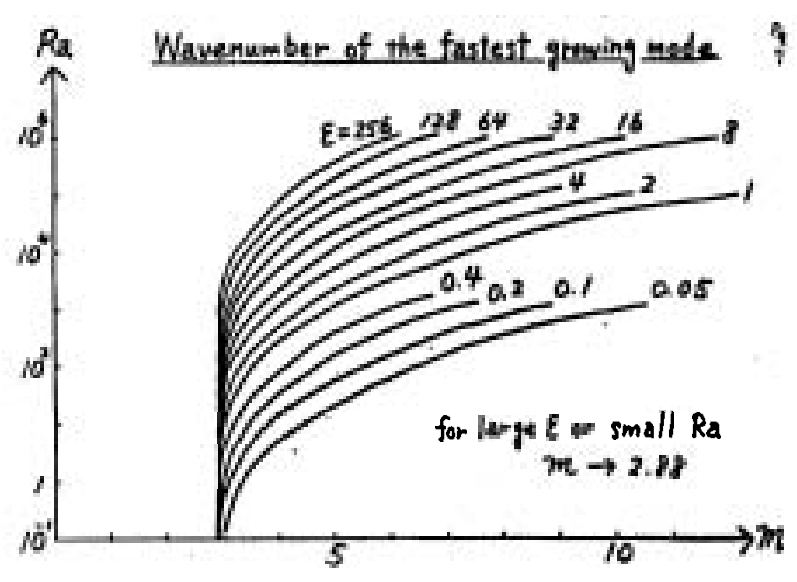

Figure 7

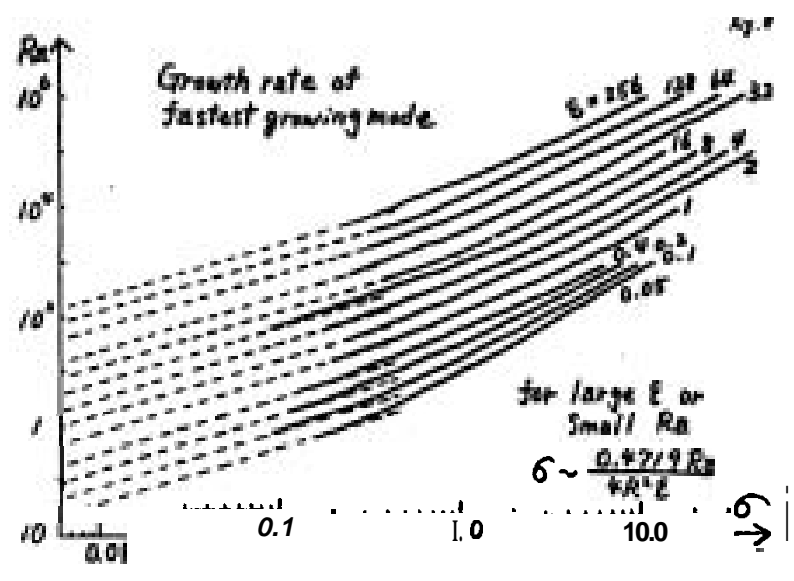

Figure 8 

Exp. 1
Exp. 2
Exp. 3

\begin{tabular}{clll}
\hline \hline$\beta \Delta S$ & 0.025 & 0.003 & 0.011 \\
$\frac{1}{\rho} \frac{\partial \rho}{\partial Z}$ & $1.3 \times 10^{-3}$ & $1.2 \times 10^{-3}$ & $0.67 \times 10^{-3}$ \\
$\mathrm{~d}$ & $1.2 \mathrm{~cm}$ & $0.15 \mathrm{~cm}$ & $1.0 \mathrm{~cm}$ \\
$\mathrm{H}$ & $3.0 \mathrm{~cm}$ & $0.30 \mathrm{~cm}$ & $2.3 \mathrm{~cm}$ \\
$\chi$ & 2.5 & 2.0 & 2.3 \\
$\mathrm{~m}$ & 2.5 & 3.1 & 2.7 \\
$\mathrm{r}$ & $8 \times 10^{-3} \mathrm{~cm}^{2} / \mathrm{s}$ & $4 \times 10^{-4} \mathrm{~cm}^{2} / \mathrm{s}$ & $5 \times 10^{-3} \mathrm{~cm}^{2} / \mathrm{s}$ \\
$\mathrm{Ra}$ & $3 \times 10^{4}$ & $3 \times 10^{3}$ & $3 \times 10^{4}$ \\
$\mathrm{~d}^{2} / \mathrm{r}$ & $2 \times 10^{2} \mathrm{sec}$ & $4 \times 10 \mathrm{sec}$ & $2 \times 10^{2} \mathrm{sec}$ \\
$\varepsilon$ & 1 & $3 \times 10$ & 2 \\
\hline
\end{tabular}

Table 1

$H$ is the height of the intrusion found in the experiments, $\pi$ the nondimensional height defined by $\boldsymbol{X} \equiv \mathrm{H} / \mathrm{d}$, and $\mathrm{m}$ the corresponding nondimensional wavenumber. In order to compare the theoretical results with the experimental ones, it is necessary to estimate the magnitudes of $\mathrm{Ra}$ and $\mathcal{E}$ in the experiment. The Rayleigh number $\mathrm{Ra}$ has been defined by

$$
R_{a}=\frac{N^{2} d^{6}}{r^{2} a^{2}},
$$

where we have two unknown quantities $\mathcal{K}$ and a. The value of a may be determined by the mixing due to initial disturbances. Figure 2(b) of RT shows that the horizontal scale of the mixing region is about the same as the height of the intrusions. Thus, we may assume that $a \sim d$.

To estimate the eddy diffusivity of salt due to salt fingers is a more difficult task. However, we start from the definition of the eddy diffusivity:

$$
r=\frac{\beta F_{S}}{\beta S_{z}}
$$

where $F_{s}$ is the salt flux due to salt fingers and $\mathrm{Sz}$ is the vertical gradient of salt concentration.

If we use Stern and Turner's (1969) experimental result to estimate $\beta$ Fs and an order of magnitude argument to estimate a typical vertical salinity difference in the intrusions, $\boldsymbol{\beta F}_{\mathbf{s}}$ and $\boldsymbol{\beta S}_{\mathbf{z}}$ may be given by

$$
\beta F_{S} \approx A(\beta \Delta S)^{\frac{4}{3}}
$$


and

$$
\beta S z=\frac{1}{H} \beta \Delta S
$$

where A was estimated for salt/sugar fingers to be around $10^{-2} \mathrm{~g} \mathrm{~cm}^{-2}$

$\mathbf{s}^{-1}$ by Stern and Turner (1969). Recent accurate measurement by Griffiths and Ruddick (1979) also suggest that $A$ is around $10^{-2}$ for small values of density anomaly ratio. Substituting (37) and (38) into $\therefore$, we obtain

$$
\kappa \approx H(\beta \Delta S)^{1 / 3} \times 10^{-2} \mathrm{~cm}^{2} / \mathrm{s}
$$

The values of $K$ calculated from (39) are shown i n Table 1 together with the Rayleigh number $\mathrm{Ra}$ and the diffusion time scale $\mathrm{d}^{2} / \mathrm{r}$, which is used to scale the time in the present theory. The values of could be smaller than those presented in Table 1 , since the vertical salinity difference should be less than $\Delta \mathbf{S}$ and also the coefficient $\mathrm{A}$ in (37) could be smaller than $10^{-2} \mathbf{g} \mathbf{c m}^{2} / \mathbf{s}$. These estimates of the eddy diffusivity suggest that the eddy diffusivity is smaller than the molecular viscosity. The vertical momentum transfer process due to salt fingers is one of the subjects which is still not well understood. Here let us consider the ratio of the vertical momentum flux by salt fingers to that by molecular process $\left(\overline{u^{\prime} \omega^{\prime}} / \mathcal{\nu}(\partial u / \partial z)\right.$. If the correlation coefficient between $u^{\prime}$ and $w^{\prime}$ is denoted by $\mathrm{C}_{1}\left(\mathrm{C}_{1}<1\right)$, the ratio may be written as $C_{1} \mathbf{w}^{\top} H / \mathcal{\nu}$. If we multiply this expression by $\Delta S$, we have

$$
c^{\prime} \frac{w^{\prime} \Delta S}{\nu\left(\frac{\Delta S}{H}\right)} \approx \frac{C_{1}}{C_{2}} \frac{\overline{w^{\prime} S^{\prime}}}{\nu \frac{\partial S}{\partial P}}=\frac{C_{1}}{C_{2}} \cdot \frac{\kappa}{\nu}
$$

where $\mathrm{C}_{2}$ is the correlation coefficient between $\mathrm{w}^{\prime}$ and $\mathrm{S}^{\prime}$. Since salt fingers are driven by a buoyancy excess due to salt, it is probable that the correlation coefficient $\mathbf{C}_{2}$ for $w^{\prime}$ and $\mathbf{S}^{\prime}$ is larger than $\mathbf{C}_{1}$ for $w^{\prime}$ and $\mathbf{u}^{\prime}$. Thus, (40) suggests that the momentum flux due to salt fingers could be smaller than that due to molecular processes. If the molecular viscosity is used for the viscosity term, the values of $\mathbb{R} 1 \mathrm{n}$ Table 1 suggest that the Schmidt number $\mathcal{E}$ may be between $1-3 \times 10^{1}$.

Now we will compare the theoretical results with the experimental ones. Given the estimated values of $R a$ and $\mathcal{E}$, we can obtain the wavenumber of the fastest growing mode, which is compared with the observed one in Table 2.

\begin{tabular}{llll}
\hline & Exp. 1 & Exp. 2 & Exp. 3 \\
m (observed) & 2.5 & 3.1 & 2.7 \\
$\mathrm{~m}$ (theory) & 9.0 & 3.4 & 7.9 \\
e-folding time & $1 \times 10 \mathrm{sec}$ & $5 \times 10 \mathrm{sec}$ & $1 \times 10 \mathrm{sec}$ \\
\hline
\end{tabular}


The predicted scales are found to be about three times smaller than those observed except for Exp. 2 where a good agreement is seen. The interval of the wavenumber which is indicated by the arrows near the bottom of Figure 7 shows the observed wavenumbers in a 11 of RT's experiments in which the salt concentration anomaly increased with depth. If we assume that $R a$ is between $10^{3}$ and $10^{5}$ and $\varepsilon$ is between 1 and $10^{2}$, the wavenumber of the fastest growing mode ranges between 3.0 and 12.0. Considering the rough assumptions made in the theory, the agreement seems to be satisfactory. Especially it is noteworthy that the theory predicts the right scaling d for the height of the intrusions. Two possible reasons for the discrepancy between the observed wavenumber and the predicted wavenumber of the fastest growing mode may be considered. First, since the intrusion in the experiment is a nonlinear phenomenon, the wavelength could be different from what is obtained from the linear stability theory. In fact, recent investigations on various instabilities show that the wavenumber of the finite-amplitude waves are different from that of the fastest growing mode by a factor of two or three (e.g. see Yoshizaki (1982) or Niino (1983)). Secondly, the assumption of the constant eddy diffusivity may not be appropriate. Because of this assumption, the theory is unable to produce diffusive interfaces which were present in the experiment. Since the flux through the diffusive interface is quite small compared to that through the salt finger interface, this could result in a factor of two difference in the vertical scale. Furthermore, as Eq. (39) might suggest, the eddy diffusivity could depend on the scale. Although it may be difficult to consider the effect of the scale-dependence of the eddy diffusivity in the linear stability theory, the effect could change the wavenumber of the fastest growing mode.

The theoretical predictions of the growth rates corresponding to the three RT experiments can be also obtained from Figure 8 and are shown in Table 2. The e-folding time for Ex. 1 and 3 is about $10 \mathrm{sec}$ which seems to be somewhat shorter than what was observed in RT's experiment. The e-folding time for Ex. 2 is about $50 \mathrm{sec}$ and seems to be in good agreement with the experiment. Again in spite of the crude model assumed in theory, the agreement seems to be satisfactory.

\section{Summary and Conclusions}

A linear stability theory of double diffusive horizontal intrusions in a temperature-salinity front is formulated and the results are compared with Ruddick and Turner's (1979) experiment. In the theory, constant diffusivities are assumed to express the heat and salt transports due to salt fingers, but it is suggested that the momentum transport due to molecular viscosity may dominate that due to the salt fingers.

The front is found to be always unstable even if the viscosity is included. However, it is found that the disturbances which have a vertical scale larger than $4 d$ cannot grow. The vertical scale of the most unstable wave is found to be of the order of $d$, which is the same scale as found in the RT experiments (1979). The precise value of the wavelength of the fastest growing mode is somewhat smaller than the observed one. This is attributed to the effect of nonlinearity and the crudeness of the assumption of the constant eddy diffusivities. The predictions of the growth rate and the flow characteristics of the intrusions are quite reasonable except that the model is unable to produce the diffusive interfaces. Overall the agreement is satisfactory regarding the crude model assumed in theory. 
The theoretical model employed here is essentially unchanged even if the effects of the rotation and three-dimensionality, which seem to be important in the oceanic front, are included. In fact, some interesting results have been found when these effects are included. The results for these cases will be reported elsewhere.

Appendix I. The Eigenvalue Problem for $\mathrm{L}^{2}=4 \mathrm{k}^{2}$

to

When $\mathrm{L}^{2}=4 \mathrm{k}^{2}$, the solution for the gradient region must be changed

$$
\begin{gathered}
p=C e^{i k x}+D x e^{i k x}, \\
\text { where } k=R m \sqrt{\sigma_{0}\left(\sigma_{0}+2 m^{2}\right)} .
\end{gathered}
$$

Applying the matching conditions (23) and (24) to the solutions (20), (21), and (I.1), we obtain the following algebraic equation

$$
2 k^{2}+k=0
$$

Since $k$ is positive, there is no solution to satisfy this equation.

Appendix II. Asymptotic Wavenumber of the Fastest Growing Mode for Large $\mathcal{E}$ and/or Small Ra

If $\sigma$ is assumed to be proportional to $\quad \varepsilon^{-\alpha}$ or $R^{-\alpha}$ as $\varepsilon$ becomes large or Ra becomes small (R becomes large), where $\alpha$ is a positive number, Eq. (32) gives

$$
\sigma_{n}=\frac{m^{2}-\frac{n^{2} \pi^{2}}{4}}{4 R^{2} \varepsilon m^{4}}
$$

Thus, the growth rate $\sigma$ of the fastest growing mode is located between $\left(m^{2}-\pi^{2}\right) / 4 R^{2} \varepsilon m^{4}$ and $\left(m^{2}-\pi^{2} / 4\right) / 4 R^{2} \varepsilon m^{4}$ when $a>\pi$ or between 0 and $\left(m^{2}-\pi^{2} / 4\right) /$ $4 R^{2} \varepsilon m^{4} \quad$ when $\pi / 2<a<\pi$. If we introduce $x$ by $x=4 R^{2} q \sigma$, Eq. (30) can be written as

$$
\tan m \sqrt{1-m^{2} x}=-\sqrt{\frac{1-m^{2} x}{m^{2} x}}
$$

with $0<x<\left(m^{2}-\pi^{2} / 4\right) / m^{4}$ for $\pi / 2<x<\pi$ and $\left(m^{2}-\pi^{2}\right) / m^{4}<x<\left(m^{2}-\pi^{2} / 4\right) / m^{4}$ for $m>\pi$. (11.2) can be solved numerically and the wavenumber of fastest growing mode is found to be 2.88 while the corresponding value of $x$ is equal to 0.4719 . This gives the asymptotic formula for the maximum growth rate $\sigma$ :

$$
\sigma=\frac{0.4719 R_{a}}{4 \varepsilon}
$$

\section{ACKNOMHDGMENIS}

I am grateful to Professor 0.M. Phillips who made me interested in the present problem through his lecture at the Ocean Engineering Department, Woods Hole Oceanographic Institution. The discussions and suggestions due to Dr. Barry Ruddick and Professors George Veronis and Melvin Stern were very helpful to me. Finally, I would like to thank a 11 the members of the G.F.D. program and the staff of WHOI who kindly provided me with such a comfortable and stimulating environment for research. 


\section{REFERENCES}

Chen, C.F., D.G. Briggs, and R 1. Wirtz, 1971. Stability of thermal convection in a salinity gradient due to lateral heating. Int. J. Heat Mass Transfer, 14, 57-65.

Gregg, M.C., 1979. Thermohaline intrusions lie across isopycnals. Nature, $280,310-311$.

Griffiths, R., and B.R. Ruddick, 1979. Accurate fluxes across a salt-sugar finger interface deduced from direct density measurements. J. Fluid Mech., 99, 85095 .

Horne, E.P.W., 1978. Interleaving at the subsurface front in the slope water off Nova Scotia. J. Geophys. Res., 83, 3659-3671.

Joyce, T.M., W. Zenk, and J.M. Toole, 1978. The anatomy of the Antarctic Polar Front in the Drake Passage. J. Geophys. Res., 83, 6093-6113.

Niino, H., 1983. An experimental and theoretical study of barotropic instability. Submitted to $\underline{\text { J. Atmos. Sci. }}$

Ruddick, B.R., and J.S. Turner, 1979. The vertical length scale of doublediffusive intrusions. Deep Sea Res.,

Stern, M.E., 1967. Lateral mixing of water masses. Deep Sea Res., 14, $747-753$.

Stern, M.E., and J.S. Turner, 1969. Salt fingers and convecting layers. Deep Sea Res., 16, 497-511.

Thorpe, S.A., P.K. Hutt, and R. Soulsby, 1969. The effects of horizontal gradients on thermohaline convection. J. Fluid Mech. 38, 375-400.

Toole, J.M., and D.T. Georgi, 1981. On the dynamics and effects of doublediffusively driven intrusions. Prog. Oceanog., 10, 121-145.

Turner, J.S., 1978. Double-diffusive intrusions into a density gradient. J. Geophys. Res., 83, 2887-2901.

Yoshizaki, M., 1982. Stability of finite amplitude baroclinic waves in a twolayer channel flow. Part II. Moderately non-linear regime. I. Meteor. Soc. Japan, 60, 620-637. 
WEAKLY NONLINEAR FRONTAL WAYES

Nathan Paldor

\section{Introduction}

The linear stability of the two single layer geostrophic fronts shown in Figure 1 has been recently studied by Griffiths, Killworth, and Stern (1982) (hereafter GKS), Killworth and Stern (1983) (hereafter KS) and Paldor (1983). The focus of the present study is the effect of weak nonlinearities in the simple case when the potential vorticity is zero. We limit our discussion to long waves since the short wave, nonlinear problem is too complicated.

Throughout this work the lower layer is considered infinitely deep so that the velocities there are neglected. The assumption of zero potential vorticity greatly simplifies the basic state as it results in a linear shear, and this along with the assumed geostrophy implies that the interface depth is quadratic. In the coupled front the quadratic interface depth intersects the surface twice, while in the wall bounded front, a vertical coastline is introduced in order to eliminate the second free streamline.

The question which we address here is: what is the effect of the nonlinearities in these two fronts? In particular, do they stabilize the linearly unstable coupled front? Do they destabilize the stable wall bounded front? The answers to these questions rest in the fully nonlinear equations of motion and we explore here the longwave, weakly nonlinear asymptote of these equations.

We first review, in Section II, the results of the linear theory, then, in Section III we develop the weakly nonlinear equations, and in Section IV we briefly discuss the solutions and describe how to relate them to laboratory experiments. Since the two fronts are qualitatively dissimilar, each section is divided into two parts, each dealing with another front.

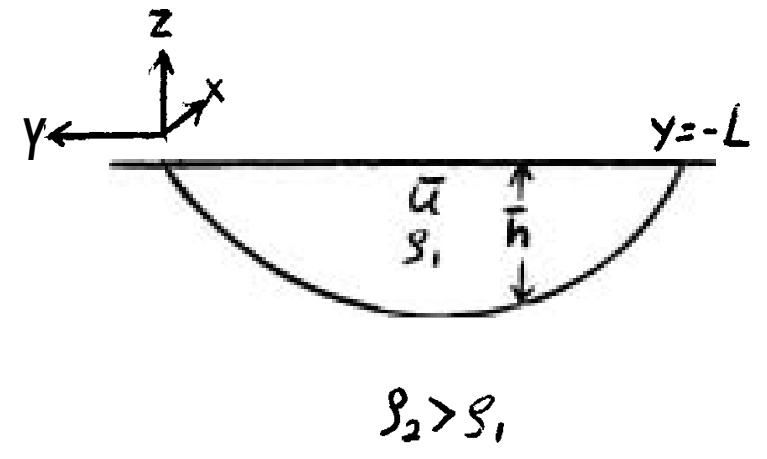

(a)

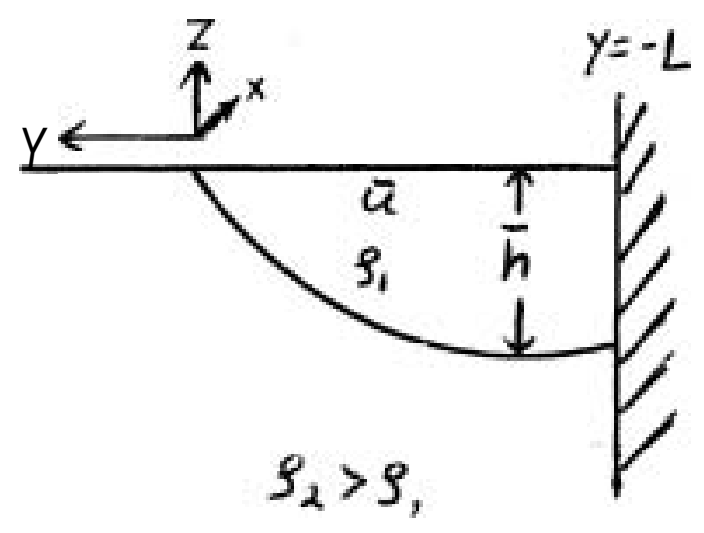

(b)

Fig. 1. The two geostrophic fronts of zero potential vorticity; (a) the coupled front, (b) the wall bounded front. 
II. Review of Linear Theory

A. Wall bounded front

The front has been shown by Paldor (1983) to be stable for a 11 wavenumbers provided the velocity of the basic state is large enough. For slow basic flow Paldor (1983) and KS have shown that the front is stable with regard to longwave perturbations. The temporal evolution of the front is given by $\exp (\mathbf{i} \boldsymbol{w})$ where the dispersion relation $w(k)$ is given for long waves by

$$
w_{ \pm}=k\left[(2 v-1) \pm(2 v-1)^{1 / 2}\right] / 2
$$

where $\boldsymbol{V}$ is the velocity of free streamline velocity.

Equation (2A.1) suggests that in order to compute the slow, nonlinear temporal evolution, one has to use a coordinate system which moves at a speed

\section{B. Coupled front}

$$
C_{ \pm}=\frac{\omega_{ \pm}}{k}=\left[(2 v-1) \pm(2 v-1)^{1 / 2}\right] / 2
$$

The coupled front can be viewed as a special case of the wall bounded front, when the free streamline's velocity $U$ is equal to $1 / 2$ (although the boundary condition is different this is not the profound difference). GKS have shown that the linear theory predicts an exponential temporal growth $\exp (w t)$ where

$$
\mathrm{w} \sim \mathrm{k}^{2}+\mathrm{o}\left(\mathrm{k}^{4}\right), \mathrm{k} \rightarrow 0
$$

The different nature of the temporal evolution of the coupled front was suggested by Paldor (1983) to be the result of the resonant condition $\mathrm{C}_{+}=\mathrm{C}_{-}=0$ which is satisfied when $V=1 / 2$ in (2A.2).

The difference in the power law of the dispersion relation w(k) suggests that the nonlinear effects of the coupled front cannot be derived as a limiting case of the wall bounded front.

III. Weakly Nonlinear Waves

The nondimensionalization used for the two fronts is

$$
\mathrm{x}, \mathrm{y}-\mathrm{L}
$$$$
u, v-f L
$$$$
\text { h }-\mathrm{f}^{2} \mathrm{~L}^{2} / \mathrm{g}^{\prime}
$$$$
\text { t } \quad-1 / \mathrm{f}
$$

(the distance between the free streamline and the wall or the distance between the two free streamlines)

(f is the Coriolis parameter)

$\left(g^{\prime}\right.$ is the reduced gravity $g^{\prime}=\frac{g}{\rho} \rho_{\lambda}$ ) 
The $x, y$ momentum equations and the continuity equation for the shallow, upper layer, are

$$
\begin{aligned}
& u_{t}+u u_{x}+v u_{y}-v+h_{x}=0 \\
& v_{t}+u v_{x}+v v_{y}+u+h y=0 \\
& h_{t}+(h u)_{x}+(h v)_{y}=0
\end{aligned}
$$

The simplifying assumption of zero potential vorticity is written as:

$$
1-u_{y}+v_{x}=0
$$

The basic state is assumed to consist of $\bar{u}, \bar{h}$ which are in steady state and in geostrophic balance. Setting $\mathrm{v}=0$ in (3.2), (3.4) we get

$$
\begin{aligned}
& \bar{u}=y+U \\
& \bar{h}=-y\left(U+\frac{y}{2}\right)
\end{aligned}
$$

where $\boldsymbol{U}$ is the free streamline $(\mathrm{y}=0)$ velocity.

The depth of the interface at the boundary $y=-1$ is $\overline{\mathrm{h}}(-1)=\boldsymbol{U}-1 / 2$ and $i n$ the special case $U=1 / 2$ it becomes $\bar{h}(-1)=0$ so that the coupled front is encountered.

of The phase speeds of the linear theory are derived from the relation $\left[\bar{h}-\left(\bar{u}-C_{o}\right)^{2}\right] d y=0$ so that for $U>1 / 2(2 \mathrm{~A} .2)$ is the answer while for $\forall=1 / 2$ the answer is $\mathrm{C}_{0}=0$. We focus our attention to longwave perturbations with amplitudes not too large so that only the weakly nonlinear effects can be realized.

A. Wall bounded front

Following the results of the linear theory we transform the $\mathrm{x}, \mathrm{t}$ coordinates in (3.1) to (3.4) into new coordinates $\xi, \tau$ moving at the linear phase speed (2A.2) which are given by

$$
\begin{aligned}
& \zeta=\varepsilon\left(x-c_{0} t\right) \\
& \tau=\varepsilon^{3} t
\end{aligned}
$$

where $C_{o}$ is either of the phase speeds $C_{ \pm}$in (2A.2) and $\boldsymbol{E}$ is the small parameter which measures the relative smallness of $2 / \nu_{\mathbf{x}}$ and $\partial / \partial_{\mathbf{t}}(\mathrm{k}, \mathrm{w}$ in the linear theory). In terms of $\xi$ and $\tau$, Eqs. (3.1), (3.3), and (3.4) become

$$
\begin{aligned}
& \varepsilon^{2} u_{\tau}+\left(u-c_{o}\right) u_{\xi}+v v_{\xi}+h_{\gamma}=0 \\
& \varepsilon^{3} h_{\tau}-\varepsilon c_{o} h+\varepsilon(h u)_{\xi}+(h v)_{y}=0 \\
& 1-u_{y}+\varepsilon v_{z}=0
\end{aligned}
$$

where (3A.3) has been used in (3A.I) to replace $v_{y}-v$ by $v_{z}$ and the resulting equation was divided through by $\boldsymbol{\varepsilon}$. 
The boundary conditions associated with the system (3A.1) to (3A.3) are that there is no normal flow at the wall, i.e.

$$
v(-1)=0
$$

and an additional frontal $(y=0)$ boundary condition is derived now.

Let $y=y_{f}(\boldsymbol{\gamma}, \boldsymbol{\tau})$ denote the position of the free streamline, i.e.

h( $\bar{z}, y_{f}$,

)$=0$ with $y_{f}(z, 0)=0$, then $\frac{D}{D t}\left(y-y_{t}\right)=0$

implies

we get

$v\left(y_{t}\right)=\left(\frac{2}{t}+u\left(y_{t}\right) \delta_{x}\right) y_{f}$ and applying the transformation to $\tau$ and $\xi$

$$
v\left(y_{f}\right)=\varepsilon^{3 y_{f} \tau}+\varepsilon\left(u-C_{o}\right) y_{f} \xi
$$

This, along with

$$
h\left(\xi, y_{f}(\xi, \tau), \tau\right)=0
$$

will determine the boundary condition at $y=y_{f}$ once the amplitudes of the perturbations are chosen. The right choice of amplitudes can save the despair of tedious and frustrating algebraic manipulations. Therefore, in anticipation of the resulting KdV equation

$$
\mathrm{u}_{\tau}+\mathrm{uu}_{3}+\mathrm{u}_{333}=0
$$

we let u $\sim$ A, $\quad \partial / \partial \sim \mathrm{B}, \quad \nabla_{\boldsymbol{\xi}} \sim \mathrm{C}$, so that in order for all three terms to exist we require

\section{$\mathrm{AB} \sim \mathrm{A}^{2} \mathrm{C} \sim \mathrm{AC}^{3}$}

The balance $A B \sim A^{3}$ implies $B \sim \mathrm{C}^{3}$ in accordance with our choice of scaling $\partial / \partial t \sim \varepsilon^{3}$ and $\partial / \mathscr{x}_{\mathrm{x}} \sim \mathcal{E}$. The balance $\mathrm{A}^{2} \mathrm{C} \sim \mathrm{AC}^{3}$ implies $\mathrm{A} \sim \mathrm{C}^{2}$ so that we choose the amplitudes to be of order $\varepsilon^{2}$.

We therefore let

$$
\begin{array}{ll}
u=\bar{u}+\varepsilon^{2}\left(u_{o}+\varepsilon u_{1}+\varepsilon^{2} u_{2}+\ldots .\right. & ) \\
h=\bar{h}+\varepsilon^{2}\left(h_{o}+\varepsilon h_{1}+\varepsilon^{2} h_{2}+\ldots .\right. & ) \\
v=\varepsilon^{2}\left(v_{o}+\varepsilon v_{1}+\varepsilon^{2} v_{2}+\ldots .\right. & ) \\
y_{f}=\bar{y}_{f}+\varepsilon^{2}\left(y_{f}^{(4)}+\varepsilon y_{f}^{\prime \prime \prime}+\ldots . . .\right.
\end{array}
$$

where $\overline{\mathrm{u}}, \overline{\mathrm{h}}$ are given by (3.5), (3.6) respectively and $\overline{\mathrm{y}}_{\mathbf{f}}=0$. The set of expansions (3A.6) to (3A.9) is now substituted into (3A.1) to (3A.5) and the coefficients of like power in $\boldsymbol{E}$ are set equal to zero. The result is a set of equations which are manipulated to yield a single equation for $u_{o}$. The y derivatives which arise from the continuity equation are eliminated by integrating the equation with respect to y. Thus, the resulting equation for $u_{o}$ involves only $\boldsymbol{\tau}$ and $\tau$ derivatives and has the form of a classical Korteweg-de Vries equation. 
Appendix A contains the details of the algebraic manipulatioins leading to the equation:

$$
u_{0 z}+\left(\frac{3}{2}+\frac{\gamma^{3}}{v\left(1-2 \gamma^{\prime}\right)}\right) u_{0} u_{0 \gamma}+\frac{g(\gamma)}{1-2 \gamma} u_{0333}=0
$$

where $\gamma=v-c_{0}$

and

$$
\begin{aligned}
g(\gamma)= & \frac{v\left(1+2 \gamma^{2}\right)}{4}-\frac{\gamma^{2}+2 \gamma}{6}-\frac{1}{20}+c_{0}\left(r^{2}-\frac{4 \gamma}{3}+\frac{1}{2}\right) \\
& -2 c_{0}^{2}\left[v^{2}-\frac{3 v}{4}-2 \gamma v+\frac{1}{6}-\frac{\gamma}{2}\right]-4 v c_{0}^{4} \ln \left(1-\frac{1}{2 v}\right)
\end{aligned}
$$

We note that when $V \rightarrow 1 / 2, \mathrm{C}_{\mathrm{o}} \rightarrow 0$, and therefore $\gamma \rightarrow 1 / 2$ so that (3A.10) becomes singular with the trivial solution (when the boundary conditions are imposed) $u_{o}=$ const. Therefore, the coupled front cannot be recovered as a limiting case of the wall bounded front and a different derivation is called for.

We defer a discussion of the solution of (3A.10) to Section IV and derive next the nonlinear equation for the coupled front.

B. Coupled front

The linear dispersion relation (2B.1) suggests the scaling

$$
\begin{aligned}
\xi & =\varepsilon x \\
\tau & =\varepsilon^{2} t
\end{aligned}
$$

and when this is substituted into (3.1) to (3.3) and (3.4) the result is

$$
\begin{aligned}
& \varepsilon u_{\varepsilon}+u u_{3}+v v_{3}+h_{3}=0 \\
& \varepsilon^{2} h_{\tau}+\varepsilon(h u)_{3}+(h v)_{y}=0 \\
& 1-u_{y}+\varepsilon v_{\xi}=0
\end{aligned}
$$

The boundary conditions are the two frontal conditions

$$
h\left(z, y_{f}(z, \tau), \tau\right)=0
$$

and

$$
\frac{D}{D t}\left(y \cdot y_{\theta}\right)=0
$$

where $y_{f}$ is the displacement of the two free streamlines, initially at $y=0,-1$. The latter condition can be written as

$$
v\left(y_{t}\right)=\varepsilon^{2} y_{t}+\varepsilon u\left(y_{t}\right) \cdot y_{t z}
$$

The choice of the amplitudes of the perturbations in this case is less obvious, but if one formally sets the amplitudes of the perturbations proportional to $\varepsilon^{2}$, as for the wall bounded front, the result is precisely the linear theory. Hence the amplitudes of the perturbations have to be bigger 
than $O\left(\varepsilon^{2}\right)$ and a natural choice is $O(S)$. We thus let

$$
\begin{aligned}
& u=\bar{u}+\varepsilon\left(u_{0}+\varepsilon u_{1}+\varepsilon^{2} u_{2}+\ldots, \quad\right) \\
& h=\bar{h}+\varepsilon\left(h_{0}+\varepsilon h_{1}+\varepsilon^{2} h_{2}+\ldots .\right) \\
& \mathrm{v}=\varepsilon\left(\mathrm{v}_{0}+\varepsilon \mathrm{v}_{1}+\varepsilon^{2} \mathrm{v}_{2}+\ldots\right) \\
& y_{f}=\bar{y}_{f}+\varepsilon\left(y_{f}^{(c)}+\varepsilon y_{f}^{(t)}+\ldots\right)
\end{aligned}
$$

where $\overline{\mathrm{h}}, \overline{\mathrm{u}}$ are given by $(3.5),(3.6)$, and $\bar{y}_{\mathbf{f}}=0,-1$. Equations (3B.5) to (3B.8) are substituted in the set (3B.1) to (3B.4) and the coefficients of like power in $\varepsilon$ are set equal to zero. The resulting set of equations is reduced (see Appendix B) to the following single nonlinear equation for $\mathbf{u}_{\mathbf{o}}$

$$
u_{0 \tau \tau}=\frac{1}{240} u_{03333}+\frac{1}{2}\left(u_{0}^{3}\right)_{33}
$$

The linear part of (3В.9) gives the linear instability

$$
\omega=\frac{i}{4 \sqrt{15}} k^{2}
$$

when $e^{i(k z-w)}$ is substituted for $u, \quad$ This is in agreement with the longwave instability found by GKS (note, however, that the width of the front in GKS is $2 \sqrt{2}$, while in the present nondimenslonal scheme the width is 1 so that our lengths (wavenumbers) should be multiplied (divided) by $2 \sqrt{2}$ in order to precisely match their result $\left.w= \pm i(2 / \sqrt{15}) k^{2}\right)$.

We turn now to a discussion of the properties of the solution of $(3 \mathrm{~A} .10),(3 \mathrm{~B} .9)$.

\section{Characteristics of Nonlinear Waves}

The equations which describe the temporal evolution of the nonlinear waves in both fronts are discussed in great detail in text books such as Whitham (1974). The description of the characteristics of the waves closely follows Whitham's exposition of nonlinear waves.

A. Wall bounded front

The KdV equation (3A.10) which describes the temporal evolution of the nonlinear waves admits both solitons and cnoidal waves as solutions. The solitons are compact perturbations which travel at a constant speed without changing its form. In order to compute it we use the similarity transformation $u_{0}(\xi, \tau)=A(\zeta-v \tau)$. The special case when $\phi$ and its derivative tend to zero at infinity yields solitons. The propagation velocity of the soliton, V, and its amplitude, A, are related by

$$
V=\frac{B}{3} A
$$


where $B$ is the coefficient of the nonlinear term $u_{0} u_{0} \xi$ in (3A.10), i.e.

$$
v=\left(\frac{1}{2}+\frac{\gamma^{3}}{3 U(1-2 r)}\right) A
$$

This equation relates the velocity of the soliton in the $\boldsymbol{T}, \boldsymbol{\tau}$ coordinates, to its amplitude. In $x, t$ coordinates the soliton has the additional linear phase speed $\mathrm{C}_{\mathrm{o}}$ given by (2A.2) i.e. the velocity, $\mathrm{C}$, in $\mathrm{x}, \mathrm{t}$ space is given by

$$
c=c_{o}+v=c_{0}+\left(\frac{1}{2}+\frac{\gamma^{3}}{3 v(1-2 \gamma)}\right) A
$$

The cnoidal waves are also similarity solutions but $\phi$ and $\phi^{\prime}$ are not assumed to tend to zero at infinity. The mathematics is more cumbersome but it can still be shown that the solutions can be expressed in standard form in Jacobian elliptic functions and that the wavelength and phase speed are well defined. The dispersion relation of these waves, involves, however, the amplitude of the perturbations. For steady state free wave solutions we set $\nabla u_{c} / \nu \tau=0$ in (3A.10) and integrate once to get

where $F=\frac{20 g(r)}{30(1-2 \gamma)+2 r^{3}}>0$ and $A_{1}$ is a constant
$(4 A .3)$ by $u_{03}$ and integrating again we get
\[ \frac{F}{2}\left(u_{0}\right)^{2}=A_{1} u_{0}+A_{2}-u_{0}^{3} / 3 \]

steady solutions therefore exist for $\boldsymbol{A}_{1}=0, A_{2}>0$, if $u_{0}^{2}<3 A_{2}$.

B. Coupled front

Equation (3B.9) is similar to the cubic diffusion equation. Thus we can try a similarity transformation similar to that used for the KdV equation in the preceding paragraph. Thus, we let

$$
u_{0}(\xi, \tau)=\phi(z-v \tau) \equiv \phi(X)
$$

and substitute it in (3B.9). The result is

$$
v^{2} \phi^{\prime \prime}-\frac{1}{240} \phi^{\prime \prime \prime}-\frac{1}{2}\left(\phi^{3}\right)^{\prime \prime}=0
$$

Integrating this equation twice we get

$$
v^{2} \phi-\frac{1}{240} \phi^{\prime \prime}-\frac{1}{2} \phi^{3}=k_{1} X+k_{2}
$$

where $\mathrm{K}_{1}, \mathrm{~K}_{2}$ are the two constants of integration. Requiring $\boldsymbol{\phi}$ to remain finite at infinity we get $\mathrm{K}_{1}=0$; and for solitary waves we will also require $\mathrm{K}_{2}=0$. Multiplying (4B.1) by @'(assuming $\phi^{\prime} \neq 0$ ) and integrating the resulting equation we get

$$
v^{2} \phi^{2}-\frac{1}{240} \phi^{2}-\frac{j}{4} \phi^{4}=2 k_{2} \phi+k_{3}
$$

where $\mathrm{K}_{3}$ is a constant of integration which will be set to zero for solitary waves. 
Equation (4B.2) can be written in the form

$\left(\frac{\partial \phi}{\partial(4 \sqrt{15} x)}\right)^{2}=F(\phi)=\left(v^{2} \phi^{2}+A \phi+B-\phi^{4} / 4\right)$

where $\mathrm{A}=-2 \mathrm{~K}_{2}, \mathrm{~B}=-\mathrm{K}_{3}$.

For the solitary waves we set $A=B=0$, and $F(\phi)$ will be positive in this case provided $\phi^{2} \leqslant 2 V^{2}$. We therefore get a bound on the amplitude of the solitary waves in terms of their velocity.

We next explore the possibility of having unbounded solutions. We note that a legitimate solution of (3B.9) is

$$
u_{0}=\alpha \tau+\beta
$$

for some constants $\alpha$ and $\beta$. This trivial solution (4B.4) corresponds to a transverse rigid displacement of the whole front (upper layer) in which case the basic downstream velocity $\overline{\mathrm{u}}=1 / 2+\mathrm{y}$ has to be modified at any point, $y$, according to $y \rightarrow y+\varepsilon \alpha \tau$ where $\alpha$ is the velocity of translation. This physically simple solution demonstrates the fact that (3B.9) does indeed have unbounded solution. (The existence of this translatory solution might also be the reason why (3B.9) is second order in time. First order systems do not have this simple solution.) Note however that (4B.4) is not a solution to (3B.1).

Another class of interesting solutions is the steady solutions. These are obtained by letting $V=0$ in (4B.3). If we choose $A=0$ and $B>0$ then we get bounded oscillations in $X$ where the amplitude of oscillation is bounded by $\phi \leqslant(4 B) 1 / 4$.

The cnoidal waves, for which $\mathrm{V} \neq 0$, can be shown to exist by setting $\mathbf{A}=\mathbf{0}$ on the righthand side of (4B.3) and rewriting $\mathbf{F}(\phi)$ as

$$
F(\phi)=B+V^{4}-(1 / 4)\left(\phi^{2}-2 V^{2}\right)^{2}
$$

and since the lefthand side of (4B.3) is positive for real $\phi$ we get that $F(\Phi)$ in (4B.5) has to be positive 1 .e.

$$
\phi^{2} \leqslant 2 v^{2}+2\left(B+v^{4}\right)^{1 / 2}
$$

The steady state solutions can be obtained from (3B.6) by letting $\mathrm{V}=0$. Equation (4B.6) gives the amplitude, velocity relation of the cnoidal waves for which $A=0, B>-V^{4}$.

Summary

The effects of nonlinearities on the wall bounded front and on the coupled front were shown to fall into different categories. For the wall bounded front the nonlinear equation (3A.10) is of the typical KdV form, its solutions are solitons, or cnoidal waves or steady waves, but it can be shown that there are no solutions which grow indefinitely with time (Bona and Smith, 1975) if the initial perturbation is bounded.

The more interesting case of the coupled front, admits the solitons, cnoidal and steady waves like the wall bounded front but in addition there exist solutions which grow with time. 
The amplitudes of all bounded solutions such as solitons, cnoidal and steady waves are bounded by undetermined constants of integration. However, since in addition there exist solutions to the problem which grow indefinitely with time, we conclude that the linearly unstable coupled front is unstable even for weakly nonlinear waves. In contrast all nonlinear solutions of the (linearly stable) wall bounded front are stable.

The interesting problem which will be pursued in the future is how does any given initial perturbation evolve into these solutions and i $n$ particular how is the steady wave realized? When these questions are answered, the solution can be related to waves observed in GKS.

Appendix A - Derivation of (3A.10)

Substitution of (3A.6) to (3A.8) into (3A.1) to (3A.3) yields the following set

$$
\begin{gathered}
-\left(\bar{u}-c_{0}\right) u_{0 z}=h_{03} \\
u_{0 x}+\left(\bar{u}-c_{0}\right) u_{2 \xi}+u_{6} u_{6 \xi}+h_{2 \xi}=0 \\
u_{0 y}=0 \\
-u_{2 y}=v_{13} \\
\left(\bar{h} v_{0}\right)_{y}=0 \\
\bar{h} u_{0 z}+\left(\bar{u}-c_{0}\right) h_{0 y}+\left(\bar{h} v_{1}\right)_{y}=0 \\
h_{0 \tau}+\left(\bar{u}-c_{0}\right) h_{2 \xi}+\bar{h} u_{2 z}+\left(h_{0} u_{4}\right)_{\xi}+\left(\bar{h} v_{3}\right)_{y}+\left(h_{0} v_{1}\right)_{y}+\left(h, v_{0}\right)_{y}=0
\end{gathered}
$$

From the y momentum equation we also get

$$
\mathrm{u}_{\mathrm{o}}=-\mathrm{h}_{\mathrm{oy}}
$$

When the displacement of the free streamline (3A.9) is substituted in (3A.5) we also get

$$
\begin{aligned}
& h_{o}\left(y_{f}\right)=\bar{u}\left(\bar{y}_{f}\right) \cdot y_{f}^{(a)} \\
& v_{1}\left(\bar{y}_{f}\right)=\left(\bar{u}\left(\bar{y}_{f}\right)-c_{o}\right) y_{f}^{(f)}
\end{aligned}
$$

Equation (A.1) implies that

$$
h_{0}=-\left(\bar{u}-c_{0}\right) u_{0}+A^{*}(y, \tau)
$$

where $A^{*}$ is arbitrary. Equations (A.8) and (A.3) along with $\overline{\mathbf{u}}_{\mathbf{y}}=\mathbf{1}$ imply that $A^{*}(y, \tau)=A(\tau)$ so that

$$
h_{0}=-\left(\bar{u}-C_{0}\right) u_{0}+A(\tau)
$$


$A(\tau)$ can be shown to be zero by requiring that the total downstream averaged transport is constant with respect to $\tau$, in the moving coordinate, i.e.

$$
\frac{\partial}{\partial \tau}<\int_{-1}^{D} \mathrm{~h}\left(\mathrm{u}-\mathrm{c}_{\mathrm{o}}\right) \mathrm{dy}>=0
$$

by integrating equation (A.5) we get $\mathrm{v}_{0}=0$. When Equation (A.1) is substituted in equation (A.6) we get

$$
\left(h v_{1}\right)_{y}=-\left(\bar{h}-\left(\bar{u}-c_{0}\right)^{2}\right) u_{o z}
$$

so that with the boundary condition $v(-1)=0$ we get

$$
v_{1}(y)=-\frac{\sqrt[y]{\left[\bar{h}-\left(\bar{u}-c_{0}\right)^{2}\right] d y^{\prime}}}{\bar{h}(y)} u_{0 \xi} \equiv F(y) u_{0 \xi}
$$

where $F(y)$ satisfies $F(-1)=0$ and $F(0)$ is finite on sccount of the definition of $\mathrm{C}_{\mathrm{o}}$ which guarantees the numerator in $\mathrm{F}$ is zero at $\mathrm{y}=0$.

Equation (A.4) yields

$$
u_{2}=\int^{q} \mathrm{~F}\left(\mathrm{y}^{\prime}\right) \mathrm{dy^{ \prime }} \cdot \mathrm{u}_{0} y^{+}+\mathrm{B}(\tau, \zeta) \equiv G(y) \cdot u_{0} z_{3}+B(\tau, \zeta)
$$

where $B(\tau, \zeta)$ is the constant of integration. From Equation (A.2) we then get

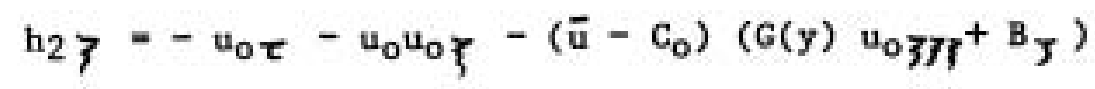

When Equations (A.12) (with $\mathrm{A}=0$ ), (A.15), (A.14), and (A.13) are substituted into Equation (A.7) and the latter is integrated between $\mathrm{y}=-\mathbf{1}$ and $-\mathrm{y}=\mathbf{0}$, we get Equation (3A.10) where $\bar{h}(0)=0=v_{1}(-1)=$ $v_{3}(-1)=f\left(\bar{h}-\left(\bar{u}-C_{0}\right)^{2}\right) d y$ have been used (so that the term with B and $G$ drops out).

Appendix B - Derivation of (3B.9)

When Equations (3B.5) to (3B.8) are substituted into Equations (3B.1) to (3B.4) we get the following set of equations.

$$
\begin{aligned}
& h_{0 \zeta}=-\bar{u} u_{0 z} \\
& u_{o z}+\bar{u} u_{1 \zeta}+u_{0} u_{o z}+h_{1 \xi}=0 \\
& u_{o y}=0 \\
& u_{1 y}=v_{0 z} \\
& u_{2 y}=v_{1 \zeta} \\
& \left(h v_{0}\right)_{y}=0 \\
& \bar{h} u_{0 \zeta}+\bar{u} h_{0 \zeta}+\left(h v_{1}\right)_{y}+\left(h_{o} v_{0}\right)_{y}=0
\end{aligned}
$$




$$
\begin{aligned}
& h_{0 \tau}+\bar{h} u_{1 z}+\left(h_{0} u_{c}\right)_{y}+\bar{u} h_{13}+\left(\bar{h} v_{2}\right)_{y}+\left(h_{0} v_{1}\right)_{y}+\left(h_{1} v_{0}\right)_{y}=0 \\
& h_{1} x+\bar{h} u_{2 z}+\left(h_{0} u_{2}\right)_{3}+\left(h_{1} u_{0}\right)_{z}+\bar{u} h_{2 z}+\left(\bar{h} v_{3}\right)_{y}+\left(h_{0} v_{2}\right)_{y}+\left(h_{1} v_{1}\right)+\left(h_{2} v_{c}\right)_{y}=0( \\
& \vec{u}\left(\bar{y}_{b}\right) \cdot y_{b \xi}^{(0)}=v_{1}\left(\bar{y}_{b}\right) \\
& h_{1}\left(\dot{y}_{f}\right)=\bar{u}\left(\bar{y}_{f}\right) \cdot y_{f}^{(\prime \prime)}+\frac{1}{2}\left(y_{f}^{(i)}\right)^{2}+u_{0}\left(\bar{y}_{f}\right) \cdot y_{f}^{(0)}
\end{aligned}
$$

The y momentum equation also yields

$$
\begin{aligned}
& u_{0}=-h_{0 y} \\
& h_{2 y}=-\left(\bar{u} \cdot u_{2}\right)_{y} \Rightarrow h_{2}=-\bar{u} u_{2}+c(3, \tau)
\end{aligned}
$$

where $C(\zeta, \tau)$ is a constant of integration.

Equation (B.6) when integtrated once implies $v_{0}=0$ and when this is used in (B.7) along with (B.1) we get

$$
V_{1}=-\frac{y_{\varepsilon}\left(\bar{h}-\bar{u}^{2}\right) d y^{\prime}}{\bar{h}(y)} u_{0 \zeta}=-\bar{u} u_{0 \zeta}
$$

When (B.14) is used $\ln ($ B.5) we get

$$
u_{2}=\bar{h} \cdot u_{0} \zeta \bar{\zeta}+B(\zeta, \tau)
$$

where $B$ is the constant of integration. This equation is used in (B.13) to get

$$
h_{2}=-\bar{u} \bar{h} u_{033}-\bar{u} \cdot B(\zeta, \tau)+C(\bar{\gamma}, \tau)
$$

We now integrate equation (B.1) once with respect to 3 to get

$$
h_{0}=-\bar{u} u_{0}+A(\tau)
$$

where (B.12) has been used to eliminate the y dependence of the constant of integration $\mathrm{A}$.

When (B.17) (B.14) are used in the y integral of (B.8) along with $\mathrm{v}_{0}=0$, we get

$$
v_{2}=-2 u_{c r}-\bar{u} u_{1}-\frac{1}{2}\left(u_{0}^{3}\right)_{Y}-\frac{y}{\bar{h}(y)} A_{\tau}
$$

and requiring $v_{2}$ to be finite at both streamlines (where $\bar{h}(y)=0$ ) we get A, $=0$, i.e.

$$
v_{2}=-2 u_{0} \tau-\bar{u} u_{1} \zeta-\frac{1}{2}\left(u_{0}^{2}\right)_{\zeta}
$$

We now differentiate (B.9) once with respect to $\zeta$ and use the derivative of (B.2) to replace $h_{1 \tau}$ and substitute (B.18), (B.17) (with $A=0$ ), (B.16), (B.15), (B.14). When the resulting equation is integrated between the two 
free streamlines and $\int_{1}^{0} \bar{u} d y=0=\int_{0}^{0}\left(\bar{h}-\bar{u}^{2}\right) d y \quad$ are used we get

$$
-u_{0 \tau e}+\frac{1}{240} u_{03333}-\frac{1}{2}\left(u_{c}^{2}\right)_{Y \tau}+\left[\left(h_{0} v_{2}\right)_{Y}+\left(h_{1} v_{1}\right)_{3}\right]_{-1}^{0}=0
$$

Equations (B.14) and (B.17) (with $A=0$ ) can be used to show that

$$
\left(\left.h_{0} v_{2}\right|_{-1} ^{0}\right)_{3}=\left[u_{0}\left(u_{0} \cdot c+\frac{1}{2}\left(u_{0}^{2}\right)_{3}\right]_{3}\right.
$$

whereas the boundary condition (B.1O) and (B.11) can be invoked to show that

$$
\left(h,\left.v_{1}\right|_{-1} ^{0}\right)_{3}=\frac{1}{2}\left(u_{0} u_{0}^{2}\right)_{3}
$$

Substituting (B.21) and (B.20) in (B.19) we get equation (3B.9).

\section{ACKNOMLEDEMENIS}

I would like to thank the entire staff of the 1983 G.F.D. summer program and particularly the director, Melvin E. Stern, for providing a stimulating environment. I am indebted to Edward Spiegel, Peter Killworth,, Glenn Flierl, and Melvin Stern for helpful suggestions and enlightening discussions.

\section{REFERENCES}

Bona, J.L., and R. Smith, 1975. The initial value problem for the Kortevegde Vries equation. Phil. Trans. Roy. Soc. Lond., 278, 555-601.

Griffiths, R.W., P.D. Killworth, and M.E. Stern, 1982. Ageostrophic instability of ocean currents. J. Fluid Mech., 117, 343-377.

Killworth, P.D., and ME. Stern, 1982. Instabilities on density driven boundary currents and fronts. Geophys. Astrophys. Fluid Dyn., 22, $1-28$.

Paldor, N., 1983. Linear stability and stable modes of coastal fronts. Submitted to Geophys. Astrophys. Fluid Dyn.

Whi tham, G.B., 1974. Linear and Nonlinear Waves. John Wiley and Sons, Inc., N.Y. 


\section{A MULTI-TIMING ANALYSIS OF THE HEAT-UP PROBLEM}

Roger Samelson

I. Introduction

The mathematical analysis of heat-up, the process by which a contained stratified fluid adjusts to a small change in thermal boundary conditions of the side wall is very similar to that of spin-up, the process by which a rotating fluid adjusts to a small change in rotation rate of the top and bottom boundaries (see for example Veronis, 1967). For Prandtl number equal to one, the heat-up of a rotating stratified fluid is mathematically analogous to the spin-up. Three distinct time scales appear in spin-up, and multi-timing has been used (St. Maurice and Veronis, 1975) to analyze the process. An analogous treatment of the heat-up process for general Prandtl number is explored in this report. Finally, a few remarks on the relation of the steady solution to boundary layer mixing in the ocean are made.

II. The Problem

Consider the flow of a rotating stratified fluid contained between two infinite planes. The domain is $-L \leq x \leq L,-\infty<z<\infty$, where the $z-a x i s$ is the rotation axis and antiparallel to gravity. The field equations are the Boussinesq equations

$$
\begin{aligned}
& \begin{aligned}
u_{t}+\underline{u} \cdot \nabla \underline{u}+2 \Omega k \times u & =-\frac{1}{d} \nabla p+g \alpha \theta \hat{k}+\nu \Delta \underline{u} \\
\theta_{t} & +\underline{u} \cdot \nabla \theta+\omega \frac{\hat{T}}{L}=k \Delta \theta
\end{aligned} \\
& d v \underset{\sim}{u}=0
\end{aligned}
$$

and the initial and boundary conditions are

$$
\begin{aligned}
& u=v=w=\theta=0 \\
& u=v=w=0, \quad \theta=\delta \theta \cos m z, \quad x= \pm L, t>0
\end{aligned}
$$

The density has been eliminated using a linear equation of state,

$$
\rho=\rho_{0}\left[1-\alpha\left(T-T_{c}\right)\right]
$$

and the temperature has been decomposed into a basic state with linear dependence on $z$ only, and a perturbation,

$$
T=T_{0}+\frac{\delta T}{L} z+\theta(x, y, z, t)
$$

and the basic state has been subtracted from the initial and boundary conditions. The rest of the notation is standard: $\mathbf{u}=(\mathbf{u}, \mathrm{v}, \mathrm{w})$ is velocity, $\mathrm{p}$ is the modified pressure including the centrifugal and hydrostatic gravitational potentials, $\rho_{c}$ is a constant reference density, $\Omega$ is the rotation rate, $g$ is the acceleration of gravity, $\hat{k}$ is a unit vector along the $z$-axis, $\alpha$ is the coefficient of thermal expansion, $\nu$ is the kinematic viscosity, $k$ is the thermometric conductivity, $\nabla$ and $\Delta=\nabla^{2}$ are the gradient and Lapacian operators, and subscripts denote partial derivatives. 
The appropriate nondimensionalization is

$$
\begin{aligned}
& \left(\mathrm{x}, \mathrm{y}, \mathrm{z} ; \frac{1}{\mathrm{~m}}\right)=\mathrm{L}\left(\mathrm{x}^{\prime}, \mathrm{y}^{\prime}, \mathrm{z}^{\prime} ; \frac{1}{\mathrm{~m}^{\prime}}\right) \\
& \mathrm{t}=\mathrm{N}^{-1} \mathrm{t}^{\prime} \\
& \theta=\delta \theta \cdot \theta^{\prime} \\
& (\mathrm{u}, \mathrm{v}, \mathrm{w})=\frac{\delta \theta}{\delta T} \text { NL }\left(\mathrm{u}^{\prime}, \mathrm{v}^{\prime}, w^{\prime}\right) \\
& \mathrm{P}=\mu \mathrm{s} \alpha \mathrm{L} \delta \mathrm{p}^{\prime}
\end{aligned}
$$

where $N^{2}=g \propto \delta T / 2$ is the buoyancy frequency of the basic state. The velocity scale is obtained from the linear balance (ignoring diffusion) in the heat equation, and the pressure scale from the hydrostatic balance. Dropping primes, the nondimensional equations and auxiliary conditions are

$$
\begin{aligned}
& \underline{u} t+\epsilon \underline{u} \cdot \nabla \underline{u}+2 F \hat{k} \times \underline{u}=-\nabla p+\theta \hat{k}+\sigma^{1 / 2} R \Delta \underline{u} \\
& \theta_{t}+\epsilon u_{1} \nabla \theta+\omega=\sigma^{-\frac{1}{2}} R \Delta \theta \\
& \operatorname{div} \underset{\mu}{u}=0 \\
& \mathrm{u}=\mathrm{v}=\mathrm{w}=\theta=0, \quad \mathrm{t} \leq 0 \\
& u=v=w=0, \quad \theta=\cos m z, x= \pm 1, t>0
\end{aligned}
$$

where

$$
\epsilon=\frac{\delta \theta}{\delta T}, F=\frac{\Omega}{N}, \sigma=\frac{\nu}{K}, R=\frac{\sqrt{K \nu}}{N L^{2}}
$$

$F$ is an internal Froude number, $\mathbf{V}$ the Prandtl number, $R$ the universe square root of the Rayleigh number, and $\boldsymbol{E}$ the measure of nonlinearity. The latter pair corresponds respectively to the Ekman and Rossby numbers in spin-up.

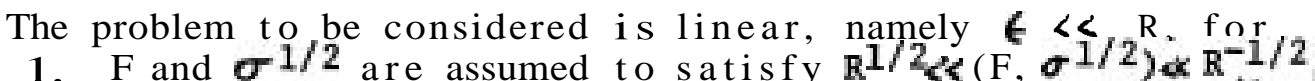
$0<\mathrm{R} \ll 1$. F and $\sigma^{1 / 2}$ are assumed to satisfy $\mathrm{R}^{1 / 2}<\left(\mathrm{F}, \sigma^{1 / 2}\right) \propto \mathrm{R}$
so that the only relevant ordering parameter for the system is $\mathrm{R}^{1 / 2}$.

The $z$-dependence can be removed from the problem by the substitution

$$
\begin{aligned}
& u(x, z, t)=(U(x, t)+\tilde{U}(x, t)) \sin m z \\
& v(x, z, t)=(v(x, t)+\tilde{v}(x, t)) \sin m z \\
& p(x, z, t)=(P(x, t)+\tilde{P}(x, t)) \sin m z \\
& w(x, z, t)=(W(x, t)+\tilde{W}(x, t)) \cos m z \\
& \theta(x, z, t)=(\mathbb{Q}(x, t)+\tilde{Q}(x, t)) \cos m z
\end{aligned}
$$

where the variables with tildes are boundary layer parts which are required to vanish in the interior. Symmetry allows treatment of the problem only in the 
region $0 \leq x \leq 1$, with boundary conditions

$$
\mathrm{U}=\mathrm{V}=0, \mathrm{~W}_{\mathrm{x}}=\mathbb{O}_{\mathrm{x}}=\mathrm{P}_{\mathrm{x}}=0 \quad \text { on } \mathrm{x}=0
$$

since $U$ and V will be antisymetric in $x$, and $W,(\mathbb{H})$, and $P$ symmetric.

In the spin-up problem, the system responds on three separate time scales, given nondimensionally by $t, \mathrm{E}^{1 / 2} \mathrm{t}$, and $\mathrm{Et}$ ( $\mathrm{E}$ is the Ekman number) and corresponding respectively to internal wave motion, the interior spin-up response, and diffusion (Greenspan and Howard, 1963; St. Maurice and Veronis, 1975). The analogy suggests multiscaling in time by $t, R^{1 / 2} t$, and $R t$. Each function is rewritten as a function of three times, and each time derivative as the sum of three time derivatives:

where

$$
\begin{array}{ll}
\phi(x, t) & \mapsto \phi\left(x, t^{\prime}, \tau, T\right) \\
\frac{\partial}{\partial z} & \mapsto \frac{\partial}{\partial z^{\prime}}+R^{1 / 2} \frac{\partial}{\partial t}+R \frac{\partial}{\partial T}
\end{array}
$$

$$
t^{\prime}=t, \tau=R^{1 / 2} t, T=R t
$$

and we will drop the prime immediately. All dependent variables are then expanded in powers of $\mathrm{R}^{1 / 2}$

$$
\phi(x, t, \tau, T)=\phi_{0}(x, t, \tau, T)+R^{1 / 2} \phi_{1}(x, t, \tau, T)+R \phi_{2}(x, t, \tau, T)+\ldots
$$

Equating terms of equal order in $\mathrm{R}^{1 / 2}$ gives the following, where the boundary layer variable $\xi=\mathrm{R}^{-1 / 2}(\mathrm{x}-1)$ has been introduced to make the highest order derivative an order one quantity in the boundary layer equations:

$$
\begin{aligned}
& \text { Interior } u_{n t}+u_{n-1 \tau}+u_{n-2 T}-2 F V_{n}=-P_{n x}+\sigma^{1 / 2}\left(\partial_{x}^{2}-m^{2}\right) u_{n-2} \\
& V_{4 t}+V_{n-12}+V_{H-2 T}+2 F U_{4}=\quad \sigma^{1 / 2}\left(\partial_{x}^{2}-m^{2}\right) V_{H-2} \\
& W_{n t}+W_{n+\tau}+W_{n-2 T}-\mathbb{Q}_{n}=-m P_{n}+\sigma^{1 / 2}\left(\partial_{x}^{2}-m^{2}\right) W_{n-2} \\
& \mathbb{Q}_{n t}+B_{n-1 \tau}+\mathbb{Q}_{n-2 T}+W_{n}=\sigma^{-1 / 2}\left(\partial_{x}^{2}-m^{2}\right) B_{n-2} \\
& U_{n x}-m W_{n}=0
\end{aligned}
$$

$\underline{\text { Boundary layer }}$

$$
\begin{aligned}
& \tilde{U}_{n-1 t}+\tilde{U}_{m-2 z}+\tilde{U}_{n-3 T}-2 F \tilde{V}_{n-1}=-\tilde{P}_{n \xi}+\sigma^{1 / 2}\left(\tilde{U}_{n-1-\zeta 3}-m^{2} \check{U}_{n-3}\right) \\
& \tilde{V}_{n t}+\tilde{V}_{n-1 \tau}+\hat{V}_{n-2 T}+2 F \tilde{V}_{n}=\quad \sigma^{1 / 2}\left(\hat{V}_{n} \xi \xi-m^{2} \hat{V}_{1-2}\right) \\
& \tilde{W}_{n t}+\tilde{W}_{n-1 \tau}+\tilde{W}_{n-2 T}-\tilde{\mathbb{Q}}_{n}=-m \tilde{P}_{n}+\sigma^{1 / 2}\left(\tilde{W}_{n \xi \xi}-m^{2} \tilde{W}_{n-2}\right)
\end{aligned}
$$

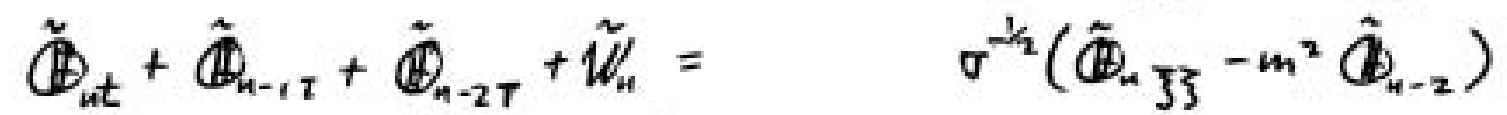

$$
\begin{aligned}
& \tilde{U}_{n \xi}-m \tilde{w}_{-1-1}=0
\end{aligned}
$$


$-260-$

The auxiliary conditions are

$$
\begin{array}{ll}
\phi_{n}=\ddot{\phi}_{n}=0 & , t \leq 0 \\
\phi_{n}^{0}=0 & , t>0 \\
\phi_{n}^{\prime}+\tilde{\phi}_{n}^{\prime}=0 & \text { except } \mathbb{\phi}_{0}^{\prime}+\tilde{\mathbb{Q}}_{0}^{\prime}=1, t>0
\end{array}
$$

$(15 a-d)$

where

$$
\phi_{n}^{0}=\phi_{n}(\mathrm{x}=0), \quad \phi_{n}^{\prime}=\phi_{n}(\mathrm{x}=1) .
$$

III. Analysis

Order zero interior system

$$
\begin{array}{ll}
U_{0 t}-2 F V_{0}=-P_{0 x} & Q_{0 t}+W_{0}=0 \\
V_{c t}+2 F U_{0}=0 & U_{0 x}-m W_{0}=0 \\
W_{0 t}-\mathscr{Q}_{0}=-m P_{0} &
\end{array}
$$

One equation for $U_{0}$ can be derived,

$$
\mathrm{L}_{1} \mathrm{U}_{\mathrm{oxx}}-\mathrm{m}^{2} \mathrm{~L}_{2} \mathrm{U}_{\mathrm{o}}=0
$$

where

$$
\mathrm{L}_{1}=\partial_{t}^{2}+1, \mathrm{~L}_{2}=\partial_{t}^{2}+4 F^{2}
$$

The zero order boundary layer equations give $\tilde{U}_{0}=0$, so homogeneous initial and boundary conditions apply to (17), and since all free oscillations decay in a dissipative system, the natural solution is the trivial solution. Then

$$
\begin{aligned}
& \mathrm{U}_{0}=\mathrm{w}_{0}=0 \\
& \mathbb{B}_{0}=\mathrm{mP}_{0}, \mathrm{v}_{0}=\frac{1}{2 F} \mathrm{P}_{\mathrm{ox}} \\
& \mathbb{H}_{0}=\mathscr{P}_{0}(\mathrm{x}, \tau, \mathrm{T}), \mathrm{v}_{\mathrm{o}}=\mathrm{v}_{\mathrm{o}}(\mathrm{x}, \tau, \mathrm{T})
\end{aligned}
$$

so the system is hydrostatic and geostrophic and the thermal wind relation holds.

Order zero boundary layer system

$$
\begin{aligned}
& =\tilde{P}_{c \zeta}=0 \quad \tilde{w}_{0 t}-\tilde{\theta}_{2}-\sigma^{1 / 2} \tilde{W}_{i \xi \zeta}=0
\end{aligned}
$$

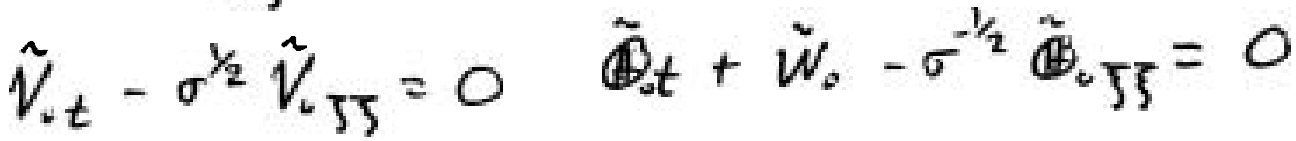

$(19 a-e)$. 
$-261-$

Clearly (19a) implies $\hat{U}_{0}=\check{P}_{\alpha}=0 . \quad$ (19b) is just an ordinary heat equation and has a simple solution by Laplace transform.

$$
\tilde{V}_{0}=-\frac{\mathbb{B}_{0 x}^{1}}{\lambda} \operatorname{erf} c\left(-\frac{1}{2} \xi \sigma^{-1 / 4} t^{-1 / 2}\right)
$$

where

$$
\lambda=2 m F
$$

has been defined for convenience. (19c,d) are difficult to solve for general $\sigma$. For $\sigma=1$ they are analogous to the transient Ekman layer equations, and a solution by Laplace transform gives

$$
\tilde{W}_{0}+\iota \tilde{\Phi}_{0}=-\iota\left(1-Q_{0}^{\prime}\right) \delta_{\zeta}^{t} e^{-\xi^{2} / 4 t^{\prime}} e^{-c t^{\prime} \frac{1 t^{\prime}}{2 t^{\prime \prime}\left(\pi t^{\prime}\right)^{1 / 2}}}
$$

An integral of the continuity equation gives

$$
\tilde{u}_{1}=m\left(1-\Phi_{0}^{\prime}\right) \int_{3}^{t} e^{-\xi^{2} / 4 t^{\prime}} \sin t^{\prime} \frac{d t^{\prime}}{\left(\pi t^{\prime}\right)^{\frac{1}{2}}}
$$

and

$$
U_{1}^{\prime}=-\tilde{U}_{1}^{\prime}=-m\left(1-\mathbb{B}_{0}^{\prime}\right) \sqrt{2} S\left(t^{1 / 2}\right)
$$

where $\mathbf{S}(\mathbf{x})$ is the Fresnel integral defined by

$$
S(x)=\frac{2}{\sqrt{2 \pi}} \int_{0}^{x} \sin t^{2} d t
$$

Further discussion of the equations for general $\sigma$ is relegated to the Appendix. Since the boundary conditions are independent of $\mathbf{t}$ and the behavior of the $\sigma=1$ solution is known, the steady solution is assumed to be the limit of the transient solution as $\mathbf{t} \rightarrow \infty$, yielding

$$
\begin{aligned}
& \tilde{w}_{0} \rightarrow-\sigma^{-1 / 2}\left(1-\omega^{\prime}\right) e^{5 / \sqrt{2}} \sin \frac{3}{\sqrt{2}} \\
& \tilde{\mathbb{B}}_{0} \rightarrow\left(1-\mathbb{1}^{i}\right) e^{\frac{3 / \sqrt{2}}{\sqrt{2}} \cos \frac{3}{\sqrt{2}}}, t \rightarrow \infty
\end{aligned}
$$

and the boundary condition

$$
U_{1}^{\prime}=-\tilde{U}_{1}^{\prime} \rightarrow-m(2 \sigma)^{-\frac{1}{2}}\left(1-B_{0}^{\prime}\right), t \rightarrow \infty
$$

a 11 of which agree with the solution for $\mathbf{\sigma}=1$.

Order one interior equations

$$
\begin{gathered}
U_{1 t}-2 F V_{1}=-P_{1 x} \quad W_{1 t}-\mathbb{Q}_{1}=-m P_{1} \\
V_{1 t}+2 F U_{1}=-V_{0 I} \quad \mathbb{Q}_{1 t}+W_{1}=-\mathbb{Q}_{u x} \\
U_{1 x}-m W_{1}=0
\end{gathered}
$$

$(28 \mathrm{a}-\mathrm{e})$ 
Forming one equation for $\mathbf{P}_{1}$ gives

$$
\mathrm{L}_{1} \mathrm{P}_{1 \times x t}-m^{2} \mathrm{~L}_{2} \mathrm{P}_{1 \mathrm{t}}=\mathrm{m}^{-1}\left(\lambda^{2} \mathscr{O}_{\mathrm{o \tau}}-\mathbb{B}_{\mathrm{oxx} \tau}\right)
$$

Since $\mathbb{P}_{\mathrm{ot}}=0$, integrating once over $\mathbf{t}$ would give secular terms if the righthand side did not vanish. In order that the solution remain uniformly valid as $t \rightarrow \infty$, a nonsecularity condition that the right side vanish must be imposed, yielding

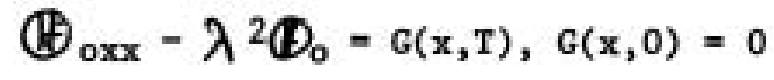

Applying the boundary conditions,

$$
\text { (10) }=\mathrm{A}(\tau, \mathrm{T}) \frac{\cosh \lambda x}{\cosh \lambda}+g(x, T), A(0,0)-g(x, 0)=0
$$

An equation for A can be obtained from the 1 imit as $t \rightarrow \infty$ of (28a) evaluated at $x=1\left(v_{1 t} \rightarrow 0\right.$ as $t \rightarrow \infty$ by a nonsecularity condition) and (27), which gives

so

$$
\oplus_{0}^{\prime} \times \tau+\frac{\lambda^{2}}{\sqrt{2}} \mathbb{B}_{0}^{\prime}=\frac{\lambda^{2}}{\sqrt{2}}
$$

and

$$
A_{\tau}+\frac{\lambda}{\sqrt{2}} \operatorname{coth} \lambda A=[1-g(1, T)] \frac{\lambda}{\sqrt{2}} \operatorname{coth} \lambda
$$

$$
\mathbb{B}_{0}=f(T)\left(1-e^{-\mu \tau}\right) \frac{\cosh \lambda x}{\cosh \lambda}+g(x, T)
$$

where

$$
\mu=\frac{\lambda}{\sqrt{2}} \text { coth } \lambda, g(x, 0)=0, f(T)=1-g(1, \pi), f(0)=1 \text {, }
$$

A Laplace transform of the system (28a-e), some algebra and solution of the boundary value problem yields

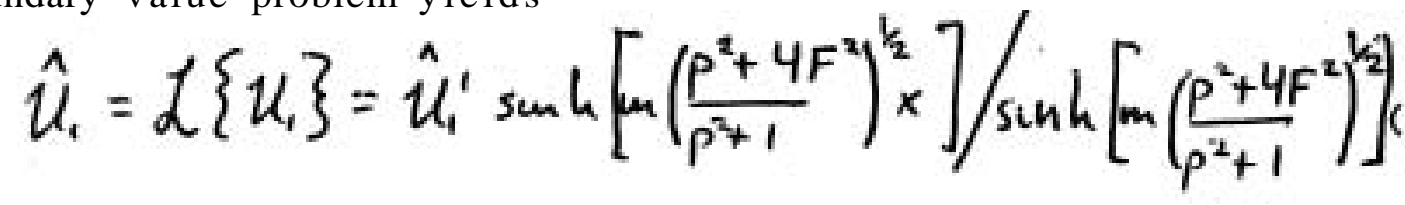

For the case $F=1 / 2$ the Laplace inversion reduces to inversion of the boundary value; for general $F$ the 1 imit of the tranform as $p \rightarrow 0$ suggests

$$
U_{1} \rightarrow-m(2 \sigma)^{-1 / 2}\left(1-\mathbb{D}_{0}^{1}\right) \frac{\sinh \lambda x}{\sinh \lambda}, t \rightarrow \infty
$$

which agrees with the 1 i mit for $F=1 / 2$.

Order one boundary layer system

$$
\begin{array}{ll}
\tilde{P}_{1 \xi}=2 F \tilde{V}_{0} & \tilde{W}_{1 t}-\tilde{B}_{1}-\sigma^{1 / 2} \tilde{W}_{1 \xi \zeta}=-m \tilde{P}_{1}-\tilde{W}_{i \tau} \\
\tilde{U}_{2 \xi}=m \tilde{W}_{1} & \tilde{\mathbb{B}}_{1 t}+\tilde{W}_{1}-\sigma^{-1 / 2} \tilde{\mathbb{B}}_{1 \xi \xi}=-\mathbb{B}_{0 \tau}
\end{array}
$$


$-263-$

The steady system $\left(\phi_{t}=0\right)$ can be solved quickly; this will be used as the limit $t \rightarrow \infty$, The solution is

$$
\begin{aligned}
& \tilde{w}_{1}=f \mu e^{-\mu z} e^{\frac{\pi}{\sqrt{2}} \cos \frac{5}{\sqrt{2}}}+\sigma^{-1 / 2} \mathbb{\theta}_{1} e^{\frac{3}{\sqrt{2}} \sin \frac{3}{\sqrt{2}}} \\
& -\frac{1}{4}\left(\frac{2}{\sigma}\right)^{1 / 2} s \mathbb{Q}_{0 \tau}^{1} \xi e^{\frac{3}{\sqrt{2}}\left(\cos \frac{3}{\sqrt{2}}-\sin \frac{\xi}{\sqrt{2}}\right)}
\end{aligned}
$$

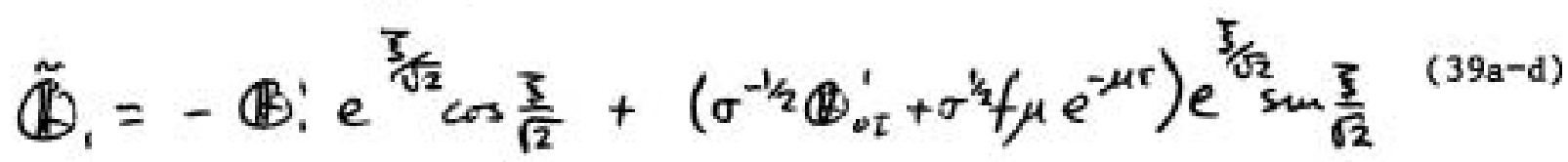

$$
\begin{aligned}
& -\frac{1}{4} \sqrt{2}=\otimes_{0 \tau}^{1} \zeta e^{\frac{3}{\sqrt{2}}\left(\cos \frac{3}{\sqrt{2}}+\sin \frac{3}{\sqrt{2}}\right)}
\end{aligned}
$$

and a boundary condition for $\mathrm{U}_{2}$ is

$$
u_{2}^{\prime}=-\frac{m}{2}\left[\sqrt{2}+\frac{1}{2}\left(\frac{2}{\sigma}\right)^{1 / 2} s\right] f \mu e^{-\mu \tau}+\frac{m}{2}\left(\frac{2}{\sigma}\right)^{1 / 2} \mathbb{G}_{1}^{\prime}
$$

where

$$
s=\frac{1}{2}\left(\sigma^{1 / 2}+\sigma^{-1 / 2}\right)
$$

The case $\sigma=\mathbf{1}$ is analogous to the forced Ekman layer equations in St. Maurice and Veronis (1975), giving

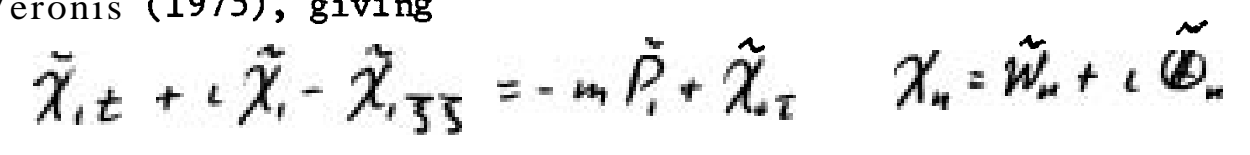

A Laplace transform leads to

$$
(p+i) \stackrel{\hat{X}}{t}^{\wedge}=-m \hat{P}_{1}+\dot{\tilde{X}}_{v \tau}
$$

which can be solved using (22), (23), and $\left(2^{\left.e_{n}\right)}{ }^{1 / 2} \frac{1}{3}\right.$ yields

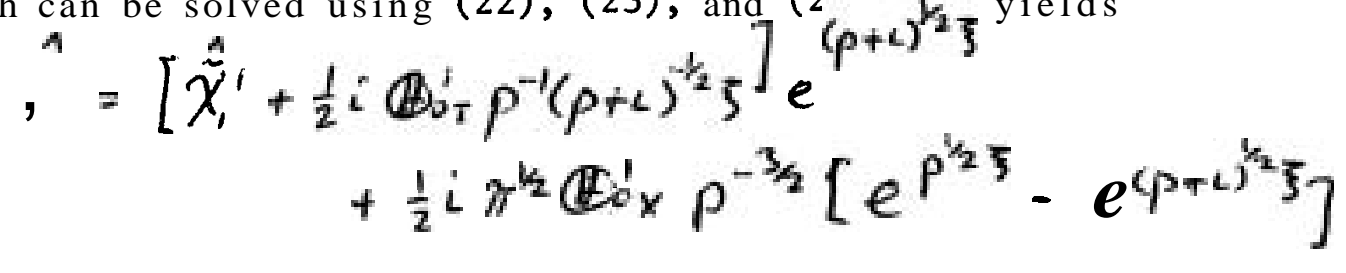

To obtain a boundary condition for U2, which is all we need, we integrate the transformed continuity equation

$$
\tilde{\hat{U}}_{\tau_{\zeta}}=m \text { Re } \hat{X}_{1}
$$

$$
\begin{aligned}
& \text { from - } \infty \text { to zero to get } \\
& \hat{\tilde{U}}_{2}^{\prime}=\operatorname{Re}\left\{m\left[\hat{x}_{1}^{\prime}(\rho+c)^{-1 / 2}-\frac{1}{2}+\mathbb{U}_{2 \tau}^{\prime} \rho^{-1}(\rho+L)^{-3 / 2}\right]\right. \\
& \left.+\frac{1}{2}<x^{1 / 2} \mathbb{\leftrightarrow}_{0 x}^{\prime} p^{-3 / 2}\left[p^{-1 / 2}-(p+c)^{-1 / 2}\right]\right\}
\end{aligned}
$$


$-264-$ The boundary value $\hat{\hat{\chi}_{i}^{\prime}}$, can be determined from $(28 \mathrm{~d}, e),(34)$, aus $(36)$. From
$(\mathbf{2 8 d}, \mathrm{e})$,

$$
m \hat{\tilde{X}}_{1}^{\prime}=-m \hat{X}_{1}^{\prime}=-\frac{\rho-\iota}{\rho} \hat{U}_{1 \times}^{\prime}+c \frac{m}{P^{2}} \mathbb{Q}_{\tau}^{\prime}
$$

so for the case $F=1 / 2$

$$
\begin{aligned}
& \hat{U}_{2}^{\prime}=-\bigcap_{e}\left\{\left[\frac{p-L}{p}\left(1-B_{0}^{\prime}\right) m^{2} \operatorname{cothm} \cdot \sqrt{2} S\left(t^{1 / 2}\right)\langle p+c)^{-1 / 2}\right.\right. \\
& \left.+\left(c \frac{m}{\rho}(\rho+c)^{-k / 2}-c \frac{1}{2} p^{-1}(\rho+c)^{-3 / 2}\right) \boldsymbol{D}_{0}^{1} \tau\right] \\
& \left.+<\frac{1}{2} \pi^{1 / 2} Q_{x}^{\prime} p^{-3 / 2}\left[\rho^{-k_{2}}+(\rho+c)^{-1 / 2}\right]\right\}
\end{aligned}
$$

This can be inverted as

$$
U_{2}^{\prime}=\text { (now growing tens) }+Q_{e_{X}}^{\prime} t^{1 / 2}(\text { oscillatory terms })
$$

Thus the solution for $U_{\boldsymbol{\varepsilon}}^{\prime}$ will not remain uniformly valid in time as $\mathbf{t} \rightarrow \infty$ and will not approach the steady solution (40) unless $\boldsymbol{B}_{\mathbf{x}}$ x vanishes at large times. (The steady solution leads to $v_{0}=\widetilde{P}_{1}=0$, so the forcing term $\widetilde{\mathrm{P}}_{1}$ in (42) and the part of (49) proportional to $\mathbf{B}_{\mathbf{X}}$ do not appear.) Applying the condition $\mathscr{D}_{x}=0$ at small times, however, leads to an overdetermined problem for $0^{-}$he require

$$
B_{0}^{\prime}=0, T>0
$$

where $\mathrm{T}>0$ indicates the condition cannot be applied to the short time problem.

Order two interior system

$$
\begin{aligned}
& U_{2 t}-2 F V_{2}=-P_{2 x}-U_{1 \tau} \\
& V_{2 t}+2 F U_{2}=\sigma^{1 / 2}\left(V_{0 x x}-m^{2} V_{0}\right)-V_{1 \tau} \\
& W_{2 t}-\mathscr{W}_{2}=-m P_{2}-W_{1 \tau} \\
& \mathbb{Q}_{2 t}+W_{2}=\sigma^{-\frac{1}{2}}\left(\mathbb{B}_{0 x x}-m^{2} \otimes_{0}\right)-\mathbb{U}_{1 \tau} \\
& U_{2 x}-m W_{2}=0
\end{aligned}
$$

(51a-e) 
$-265-$

This leads to

$$
\begin{aligned}
& L_{1} P_{2 x x t}-m^{2} L_{2} P_{2 t}=-L_{1} P_{1 x x x}+m^{2} L_{2} P_{1 x}+\left(\frac{\lambda^{2}}{m^{2}}-1\right) U_{1 x t r} \\
& +m^{-1}\left\{\sigma^{1 / 2}\left[\Phi_{0 x x x x}-\left(m^{2}+\frac{\lambda^{2}}{\sigma}\right) \Phi_{0 x x}+\frac{\lambda^{2} m^{2}}{\sigma} \mathbb{\Phi}_{0}\right]\right. \\
& \left.-\left(\Phi_{0 \times x T}-\lambda^{2} \Phi_{0 T}\right)\right\}
\end{aligned}
$$

Imposing a nonsecularity condition as $t \rightarrow \infty$, with $U_{1}$ given by (37) and 1.6. by (34),

$$
\begin{aligned}
& m\left(P_{1 \times x \tau}-\lambda^{2} P_{1 \tau}\right)=\beta f\left(1-e^{-\mu \tau}\right) \frac{\cosh \lambda x}{\cosh \lambda}-\left(g_{x x T}-\lambda^{2} g_{\tau}\right) \\
& +\sigma^{1 / 2}\left[g_{x x x x}-\left(m^{2}+\frac{\lambda^{2}}{\sigma}\right) g_{x x}+\frac{\lambda^{2} m^{2}}{\sigma} g\right]
\end{aligned}
$$

where

$$
B=\sigma^{1 / 2}\left[\lambda^{4}-\left(m^{2}+\frac{\lambda^{2}}{\sigma}\right) \lambda^{x}+\frac{\lambda^{2} m^{2}}{\sigma}\right]
$$

Imposing a nonsecularity condition as $\tau \rightarrow \infty$ gives the two equations

$$
\begin{gathered}
g_{x x T}-\lambda^{2} g_{\tau}-\sigma^{1 / 2}\left[g_{x x x x}-\left(m^{2}+\frac{\lambda^{2}}{\sigma}\right) g_{x x}+\frac{\lambda^{2} m^{2}}{\sigma} g\right]=B f \frac{\cosh \lambda x}{\cosh \lambda} \\
m\left(P_{1 x x \tau}-\lambda^{2} P_{1 \tau}\right)=-\beta f \frac{\cosh \lambda x}{\cosh \lambda} e^{-\mu \tau}
\end{gathered}
$$

The second of these has solution

$$
\text { in } P_{1 \tau}=C(\tau, T) \frac{\cosh \lambda x}{\cosh \lambda}-\frac{B f}{2 \lambda} \frac{x \sinh \lambda x}{\cosh \lambda}
$$

Nonsecularity conditions require

$$
V_{2 t} \rightarrow 0, V_{1} \rightarrow \frac{1}{\lambda} \mathbb{E}_{1 x} \text { as } t \rightarrow \infty
$$

so evaluating $(51 \mathrm{~b})$ at $\mathrm{x}=1$ gives

$$
U_{2}^{\prime}=\frac{m}{\lambda^{2}}\left[\sigma^{1 / 2}\left(\mathbb{E}_{0 \times x x}^{\prime}-m^{2} \mathbb{W}_{0 x}^{\prime}\right)-\mathbb{A}_{b x T}^{\prime}-\mathbb{B}_{i x x}^{\prime}\right]
$$


and equating this with the previous result from the analysis oi the order one boundary layer,

$$
\begin{aligned}
& \mathbb{B}_{1 \times \tau}^{i}+\lambda^{2}(2 \sigma)^{-\frac{1}{2}} \mathbb{Q}_{1}^{\prime}=\sigma^{1 / 2} \lambda \tanh \lambda\left(\lambda^{2}-m^{2}\right) f\left(1-e^{-\mu \tau}\right) \\
& +\sigma^{1 / 2}\left(g_{x \times x}^{\prime}-m^{2} g_{x}^{\prime}\right)-f_{T}\left(1-e^{-\mu \tau}\right) \lambda \tanh \lambda \\
& -g_{x T}^{\prime}+\frac{1}{2} \lambda^{2}\left(\sqrt{2}+\frac{1}{2}\left(\frac{2}{\sigma}\right)^{1 / 2}\right) f_{\mu} e^{-\mu \tau}
\end{aligned}
$$

One differentiation with respect to $\boldsymbol{\tau}$ and the substitution of (57) for Q as $t \rightarrow \infty$ results in

$$
\lambda \tanh \lambda\left(C_{\tau}+\mu c\right)=-\left(f_{T}+\beta f\right) e^{-\mu \tau}
$$

where

$$
\begin{aligned}
\beta=\frac{1}{4}\left(\frac{2}{\sigma} k_{2}+\right. & \left.\frac{s}{\sigma}\right) \lambda^{2} \operatorname{coth} \lambda-\sigma^{1 / 2}\left(\lambda^{2}-m^{2}\right) \\
& \pm \frac{\beta}{2 \lambda^{2}}(1+\lambda \operatorname{coth} \lambda-\lambda \tan 4 \lambda)
\end{aligned}
$$

Since the forcing term is a solution of the differential operator, it will generate secular terms if it does not vanish. These terms will behave like $\tau e^{-\mu x}$, and thus in fact vanish as $\boldsymbol{x} \rightarrow \infty$, but the formal procedure of applying nonsecularity conditions to avoid a power series behavior with increasing order is still legitimate. This leads to an equation for $\mathrm{f}$,

$$
f_{T}+\beta f=0
$$

with the initial condition from (35), this has solution

$$
f(T)=e^{-\beta T}
$$

The solution for $\mathbf{g}(\mathrm{x}, \mathrm{T})$ can be written as the sum of a particular solution to (55) and the solution to the homogeneous equation, that is, as

$$
g(x, T)=g_{p}(x, T)+h(x, T)
$$

where

$$
g_{p}=-\frac{\cosh \lambda x}{\cosh \lambda} e^{-\beta T}
$$

is easily shown to be a particular solution. Thus hust satisfy

$$
\begin{aligned}
& h(x, 0)=\frac{\cosh \lambda x}{\cosh \lambda} \\
& h(1, T)=1
\end{aligned}
$$

A further boundary condition on $h$ is needed and comes from condition (50):

$$
\mathrm{h}_{\mathbf{x}}(1, \mathrm{~T})=0, \mathrm{~T}>0
$$


- 267 -

Then $h$ can be written as a steady part satisfying the time independent boundary conditions and a transient part.

$$
h(x, T)=h_{g}(x)+h_{u}(x, T)
$$

where

$$
\begin{aligned}
& h_{s}(x)=\frac{\operatorname{sinhm} \cosh \sigma^{-1 / 2} \lambda x-\frac{\lambda}{\sigma^{1 / 2}} \sinh \sigma^{-1 / 2} \lambda \cosh m x}{\operatorname{sinhm} \cosh \sigma^{-1 / 2} \lambda-\frac{\lambda}{m \sigma^{1 / 2}} \sinh \sigma^{-1 / 2} \lambda \cosh m} \\
& h_{m \times x T}-\lambda^{2} h_{m T}=\sigma^{1 / 2}\left[h_{m \times x x x}-\left(m^{2}+\frac{\lambda^{2}}{\sigma}\right) h_{m x x}-\frac{\lambda^{2} m^{2}}{\sigma} h_{u}\right] \\
& h_{n}(1, T)=0, \quad h_{u_{x}}(1, T)=0
\end{aligned}
$$

The equation for $h_{u}$ can be solved by separation of variables. Substitution of the form

$$
h_{n}=\sum_{n=1}^{\infty} A_{n}(T) \cos \ln x, l_{n}=\left(n+\frac{1}{2}\right) \pi
$$

and subsequent evaluation of initial conditions leads to the full solution for the lowest order temperature field.

$$
\begin{aligned}
& \theta=\mathbb{B}_{0} \cos m z \\
& \Phi_{0}=-\frac{\cosh \lambda x}{\cosh \lambda} e^{-\mu \tau-\beta T}+h(x, T) \\
& h=h_{s}(x)+\sum_{n=1}^{\infty} A_{n}^{0} e^{-\gamma_{n} T} \cos \ln x \quad, l_{n}=\left(n+\frac{1}{2}\right) \pi \\
& A_{\mu}^{0}=2(-1)^{n}\left[\left(l_{n}^{2}+\lambda^{2}\right)^{-1}\right.
\end{aligned}
$$

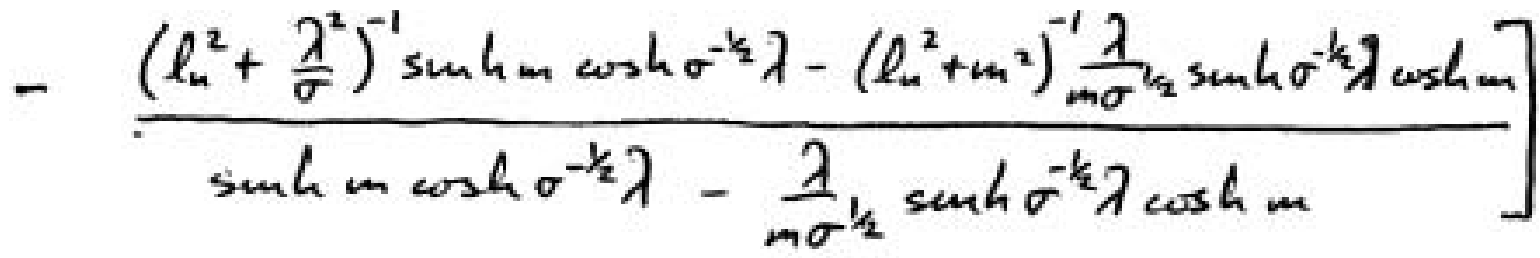

$$
\begin{aligned}
& r_{n}=\sigma^{1 / 2}\left[\left(\ln ^{2}+m^{2}\right)\left(\ln ^{2}+\frac{\lambda^{2}}{\sigma}\right)\left(\ln ^{2}+\lambda^{2}\right)^{-1}\right]
\end{aligned}
$$




\section{Discussion}

Three distinct time scales occur in the heat-up process. The buoyancy layers set up on the time scale given by the inverse of the buoyancy frequency, nondimensionally order 1 . The horizontal velocity arising from the divergence of the boundary layer transport is order $\mathrm{R}^{1 / 2}$; since it must traverse an order 1 distance to affect the interior, the interior responds on a time scale of order $R^{-1 / 2}$. Finally, diffusion occurs on a time scale of order $R^{-1}$. (These correspond to the spin-up time scales $1, E^{-1 / 2}$, $E^{-1 .)}$ Examination of the steady state solution $h_{s}$ in (73) indicates that penetration into the interior of effects due to the boundary forcing will be limited by vertical diffusion roughly to a scale of the order of the layer of $\mathrm{m}^{-1}$ and $1 / 21 / 2 \mathrm{~F}^{-1} \mathrm{~m}^{-1}$. For small $\mathrm{m}$, penetration will occur throughout the interior.

We can put some numbers into the steady solution to get a crude estimate of the penetration of boundary mixed layers in the ocean. Assume a ratio of horizontal to vertical eddy diffusivities $\eta$, where

$$
\eta=10^{7}
$$

then $-m^{2}$ in the diffusion operator-is replaced by- $\frac{m^{2}}{y}$ and the decay scales become $y^{1 / z_{m}},(1 / 2) \sigma^{-1 / 2 \mathrm{~F}^{-1} \mathrm{~m}^{-1}}$. For ordering to be consistent, we require $\mathrm{R}^{1 / 2} \ll \frac{\mathrm{m}^{2}}{7} \ll \mathrm{R}^{-1 / 2}$

With

$$
\begin{aligned}
& Y_{H}=K_{H_{3}}=10^{7} \mathrm{~cm}^{2} \mathrm{~s}^{-1} \\
& N=10^{-3} \mathrm{~s}^{-1} \\
& L=10^{3} \mathrm{~km}=10^{8} \mathrm{~cm} \\
& \Omega=10^{-4} \mathrm{~s}^{-1}
\end{aligned}
$$

we obtain

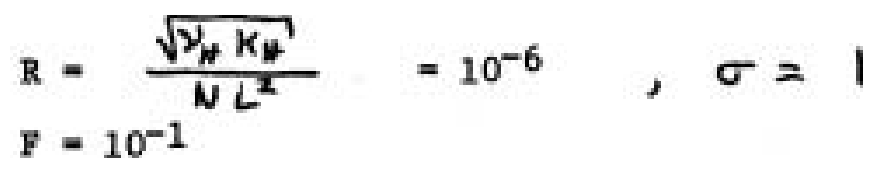

and a boundary layer scale $L^{1 / 2}=1 \mathrm{~km}$. The boundary mixing scale $\ell$ determines $\epsilon$ :

$$
\begin{array}{lcl}
\ell:- & 50 \mathrm{~m} & 1 \mathrm{~km} \\
\epsilon: & 5 \times 10^{-5} & 10^{-3} \\
\mathrm{~m}=2 \pi \epsilon^{-1}: & 10^{5} & 5 \times 10^{3} \\
\ell_{\mathrm{p}}=\eta^{1 / 2}=\mathrm{m}^{-1} & 3 \times 10^{-2} & .6 \\
\left(\epsilon=\frac{\ell}{\delta \rho}=\frac{\ell \cdot N^{2}}{L \cdot N^{2}}=\frac{\ell}{L}\right)
\end{array}
$$

The suggestion is that vertical diffusion may inhibit penetration of the effects of boundary mixing into the oceanic interior. Note that the assumption of $50 \mathrm{~m}$ for $\boldsymbol{\ell}$ (as suggested by Armi, 1979) violates the requirement for consistent ordering. 
$\underline{\text { Appendix - The Transient Buoyancy Layer Equations }}$

Writing ${ }_{\mathrm{w}}=\tilde{\mathrm{W}}_{\mathrm{O}}, \theta=\widetilde{W}_{\mathrm{O}}, \delta \theta=1-\mathscr{Q}_{0}^{\prime}$, the order zero boundary layer problem for the vertical velocity $w$ and temperature 8 is

$$
\begin{aligned}
& \begin{array}{l}
w_{E}-\theta-\sigma^{1 / 2} w_{\text {IJ }}=0 \\
{ }^{E}+\sigma^{-1 / 2}{ }_{\theta}=0
\end{array} \\
& w=\theta=0, \quad t \leq 0 \\
& \text { w, } \theta \rightarrow 0 \text { as } \xi \rightarrow \infty \\
& w=0, \theta=\delta 0, \xi=0, t>0
\end{aligned}
$$

Solution of the boundary value problem for the Laplace transform functions is straightforward, giving

$$
\begin{aligned}
& \hat{\omega}=-\frac{1}{2} \delta \theta \sigma^{-1 / 2} p^{-1}\left(\xi^{2} \rho^{2}-\delta^{-k_{2}}\left(e^{a_{1} \xi}-e^{a_{2} \xi}\right)\right. \\
& \hat{\theta}=\frac{1}{2} \delta \theta\left[\bar{s}\left(\xi^{2} \rho^{2}-1\right)^{k_{2}}\left(e^{a_{1} \xi}-e^{a_{2} \xi}\right)+p^{-1}\left(e^{a_{1} \xi}+e^{a_{2} \xi}\right)\right]
\end{aligned}
$$

where

$$
\begin{array}{ll}
a_{1}=s p+\left(\bar{g}^{2} p^{2}-1\right)^{1 / 2}, & \operatorname{Re} a_{1}>0 \\
a_{2}=s p-\left(\tilde{\sigma}^{2} p^{2}-1\right) 1 / 2, & \operatorname{Re} a_{2}>0 \\
s=(1 / 2)\left(\sigma^{1 / 2}+\sigma^{-1 / 2}\right) & \\
\bar{s}=(1 / 2)\left|\sigma^{1 / 2}-\sigma^{-1 / 2}\right| &
\end{array}
$$

The complex square roots must be dealt with carefully in the inversion of these transforms.

Define $r(p)=\left|s^{2} p^{2}-1\right|^{1 / 2} e^{i \frac{\theta_{1}+\theta_{2}}{2}}$ as analytic except on

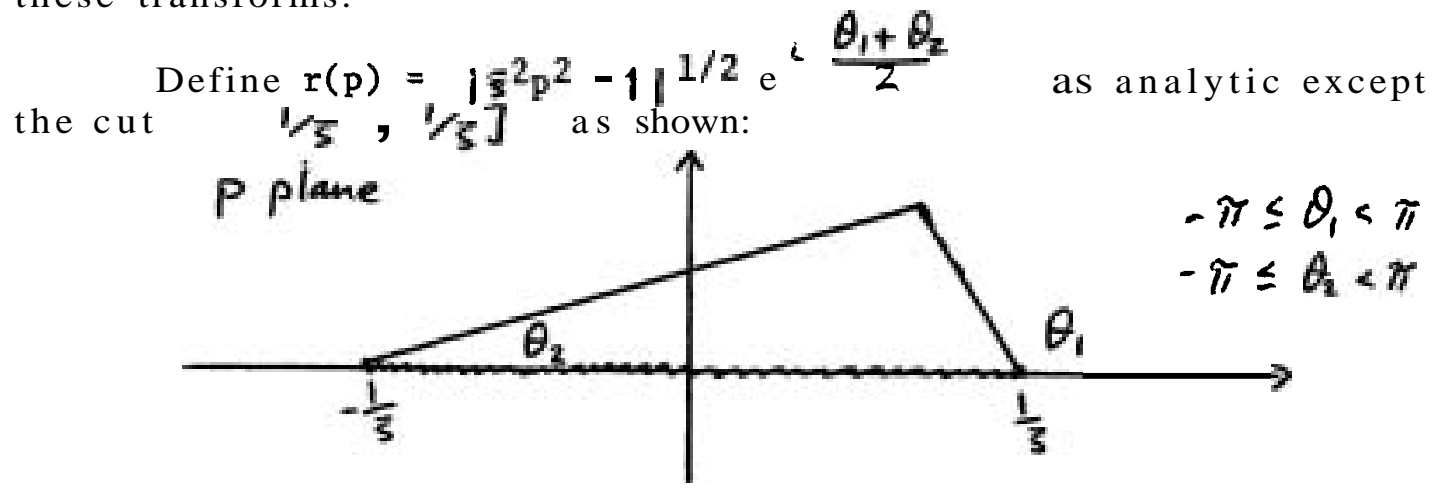

Define

$$
\begin{aligned}
& a_{1}(p)=|s p+r(p)|^{1 / 2} e^{(\theta / 2},-\pi \leq \theta=\operatorname{ar} 4(s p+r)<\pi \\
& a_{2}(p)=|s p-r(p)|^{1 / 2} e^{-\theta / 2},-\pi \leq \theta=\arg (s p-r)<a
\end{aligned}
$$

with branch cuts along the negative real axis in the $s p+r$ and $s p-r$ planes respectively. The corresponding cuts in the p plane, including the cut for $r(p)$, are
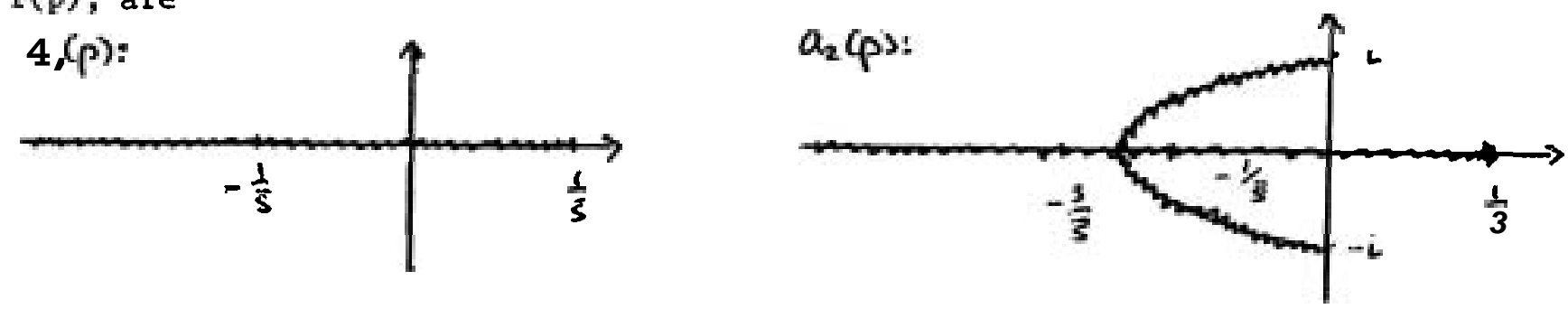
The cut for $\mathbf{a}_{2}$ (p) includes the half-ellipse

$$
p=-\frac{5}{3} \cos \alpha+i \sin \alpha,-\frac{\pi}{2} \leq \alpha \leq \frac{\pi}{2}
$$

The contour $\mathrm{C}$ of the Bromwich inversion integral may be deformed $\mathrm{to} \mathrm{C}^{\prime}$ as shown:

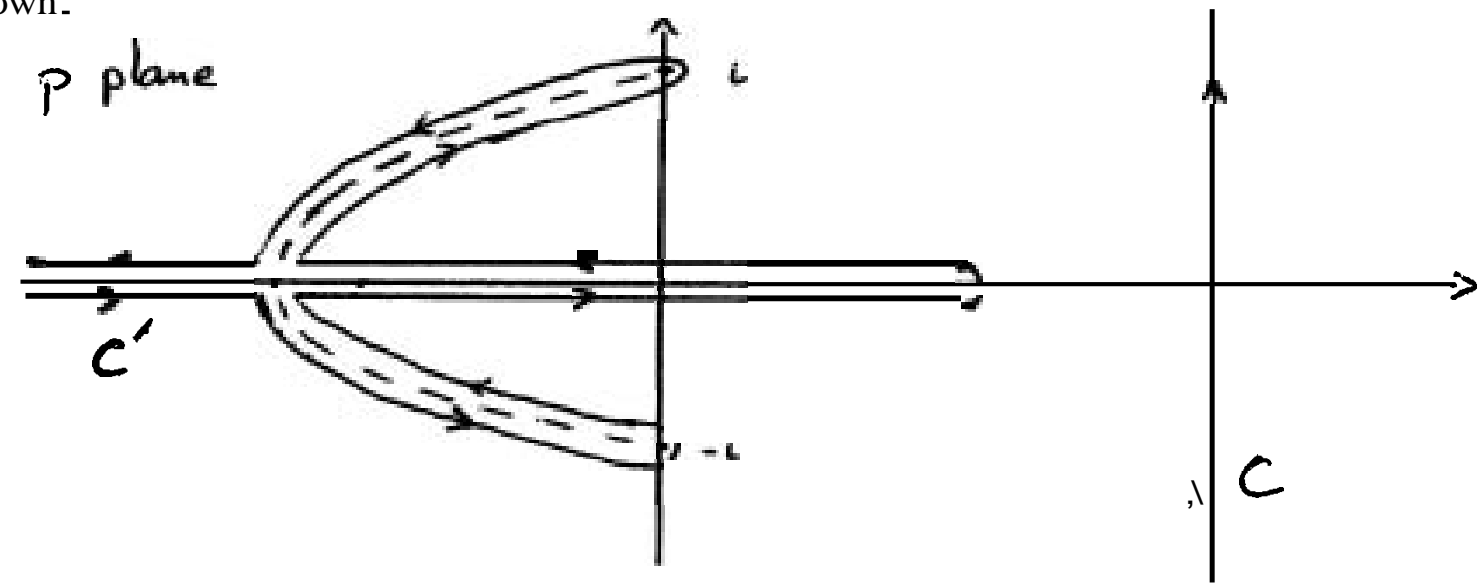

Then

$$
\begin{aligned}
& \omega=\int_{c} \hat{\omega} e^{p t} d \rho
\end{aligned}
$$

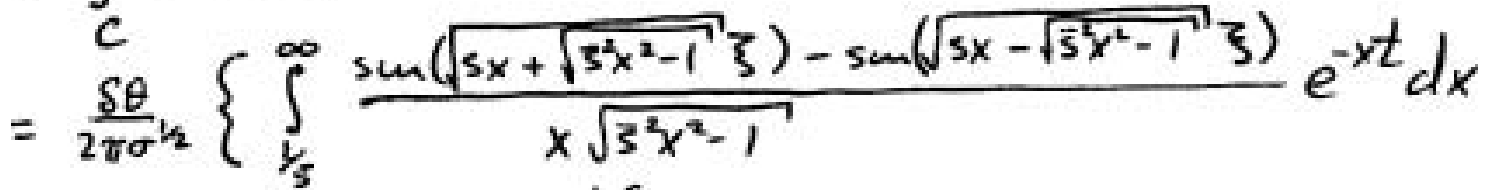

$$
\begin{aligned}
& -\int_{0}^{\pi / 2} \sin \left(\xi|s \rho-r|^{\frac{1}{2}}\right)\left[\frac{2 s}{s} \frac{e^{-t \frac{s}{s} \cos x}}{p\left|s^{2} p^{2}-1\right|^{1 / 2}} \sin \alpha \cos (t \sin \alpha-\phi)\right. \\
& \left.\left.-2 \frac{e^{-t \frac{s}{s} \sin \alpha}}{p\left|s^{2} p^{2}-1\right|^{1 / 2}} \cos \alpha \sin (t \sin \alpha-\phi)\right] d \alpha\right\} \text {, } \\
& \phi=\arcsin \left(\frac{\sin \alpha}{\left|\bar{s}^{2} \rho^{2}-1\right|^{1 / 2}}\right), r(\rho)=\left|\bar{s}^{2} \rho^{2}-1\right|^{1 / 2} e^{1 \phi} \\
& |s p-r|^{1 / 2}=\sqrt{\left(s \rho-\left|s^{2} \beta^{2}-1\right|^{1 / 2} \cos \alpha\right)^{2}+\left|s^{2} \rho^{2}-1\right| \sin ^{2} \alpha}
\end{aligned}
$$

I have not carried out this integration.

\section{ACKNOMHDGMENIS}

I would like to thank George Veronis for suggesting this problem to me and for his patient assistance, and Lou Howard, Me1 Stern, and Peter Killworth for helpful discussions. Thanks also to all the staff and fellows for a stimulating summer. 


\section{REFERENOES}

Armi, L., 1979. Effects of variations in eddy diffusivity in property distributions in the oceans. J. Mar. Res., 37, 515-530.

St. Maurice, J.P., and G. Veronis, 1975. A multi-scaling analysis of the spin-up problem. J. Fluid Mech., 68, 417-445.

Veronis, G., 1967. Analogous behavior of homogeneous, rotating fluids and stratified, non-rotating fluids. Tellus, 19, 326-335. 


\title{
SOME EXPERIMENTS ON ROTATING BAROCLINIC VORTICES
}

\author{
Andrey Zatsepin
}

\section{Introduction}

Recently the work of Griffiths and Linden (1981) was published with the results of laboratory experiments on the stability of vortices in a rotating stratified fluid. Initially they produced axisymmetric flows with a two-layer density stratification by releasing either a constant flux of fluid from a point source or a constant volume of fluid into a rotating environment with a different density. In all experimental runs the axisymmetric baroclinic vortex appeared to be unstable, and there was a transition to nonaxisymmetric flow. Griffiths and Linden described this transition by two parameters: $\theta$, the square of the ratio of the internal fogssy radius of deformation to the horizontal length scale of the flow, and $\mathcal{J}$, the fraction of the total fluid depth occupied by the surrounding density front.

Griffiths and Linden showed that their experimental results on the stability of baroclinic vortices are in good qualitative agreement with the results of inviscid stability analysis done by Phillips (1954). They discovered that according $F 9$ Phillips' model, the instability occurs when the parameter $\delta=\theta(i-\delta / \delta) / 2$ reaches the critical value, which is approximately $0.01-0.04$ in the case of constant flux experiments when $\delta>0.2$. On the other hand they pointed out that the viscous friction plays an important role in the instability processes. Although they did not change the viscosity in the experiment, they were able to show that the value of at which instability occurs is decreasing with decreasing of the nondimensional term which is the ratio of the time scale required for Ekman pumping to spin up the vortex and the time scale required for inviscid radial advection of the fluid in the vortex during the constant flux regime. So, one can assume that the increasing of viscous effects should lead to the occurrence of instability at larger values of $\gamma$. As the parameter $\gamma$ is decreasing with time during the experiment, it seems that the instability will occur as soon as viscous effects become relatively important.

However, it is not yet clear how the frictional effects influence the onset and the development of instability. Especially, there is no information as to what role the frictional effects play on the solid boundaries of the tank and particularly at the bottom.

In the experiments which $I$ am going to describe here $I$ am trying to find the answer to the following questions:

1) How does the bottom friction influence the instability process of the anticyclonical vortex which is created in the case of constant flow-rate of less dense fluid from a small source on the top of a rotating, more dense fluid?

2) What happens to this vortex when the supply is suddenly finished before the vortex reaches the unstable state? How does the bottom friction influence the spinning up process of this vortex and the onset and development of instability? 
II. Description of the Experiment

The scheme of the experiment is shown in Figure 1. The plexiglass tank (1) $\left(50 \times 48 \times 30 \mathrm{~cm}^{3}\right)$ was situated in the central part of the rotative platform (2). Before each experimental run, the tank was filled with salt water solution and put on solid body rotation. Then, fresh water, colored with dye, from a container (4) was added continuously from the small source (3) - a $1.0 \mathrm{~cm}$ diameter vertically mounted tube at the top of the fluid layer. The tube was situated just in the center of the tank and rotative platform, so the incoming fluid had approximately zero angular momentum. The flow rate $Q$ was monitored by a flow meter (7) and held nearly constant by a constant lever device $(5,6)$.

The process of the formation of anticyclonic vortex (dome), its growth and instability, was photographed from the side by a Nicon motor camera (8). In order to have both sides and plane views of the dome, a mirror (9) was mounted on the top of the tank at a $45^{\circ}$ angle to the horizontal. The measurements of the height of the dome were obtained from the side view and the diameter (radius) from the plane view.

In order to investigate the role of bottom friction we provided eight experimental runs with a smooth bottom and the same amount of experimental runs with a rough bottom. All-over experimental parameters such as flow rate $Q$, reduced gravity $g^{\prime}=(\Delta \rho / \rho) g$, where $\Delta f$ is the difference in density between layers, Coriolis parameter $=2 \Omega$, where $\Omega$ is the rotation frequency, viscosity $\boldsymbol{\gamma}$ of the fluid, the initial height of the fluid layer in the tank $\mathrm{H}_{\mathrm{O}}$, was nearly constant through the whole series of experimental runs. The values of these parameters are given in Table 1.

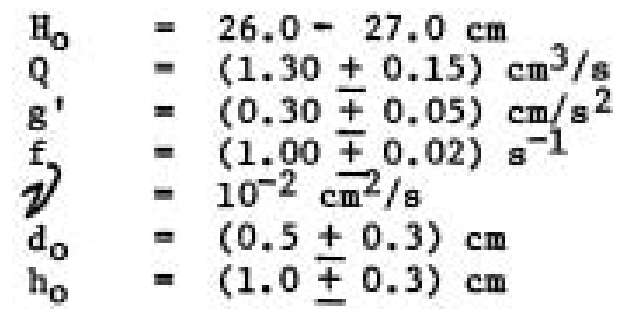

Table 1. The values of experimental parameters.

Half of the experimental runs with smooth and rough bottom were made in the condition of continuous flow supply. In other runs the flow supply was stopped when the vortices were still in stable state.

The roughness on the bottom was produced by using a layer of small stones. The height of the "rocky" layer $h_{0}$ was about $1.0 \mathrm{~cm}$ and the average size of the stones $d_{0} \simeq 0.5$ am (see Table 1 ).

It is clear that the rocky layer will increase the frictional effects near the bottom and will change the time of spin up. Assuming that the rocky bottom is in some way similar to the porous bed, one can estimate the new time of spin up using the results obtained for this case in works of Howard (1969) and Kroll and Veronis (1970). For this case of rocky bottom the new time of spin up can be approximately five times shorter than in the case of smooth bottom. This increasing of frictional effects near the bottom will effectively reduce the anticyclonic motion in the lower layer which is created during the horizontal and vertical expansion of the upper layer vortex because of stretching of vortex lines. Also, because of the increasing of the bottom 
Ekman layer divergency, the downward velocity in the lower layer must increase. The changes in the dynamics of the lower layer may significantly influence the dynamics and stability of the upper layer baroclinic vortex. The experimental results which are presented in the next section seems to support this suggestion.

\section{Experimental Results}

1. The continuous supply experiment

In both cases with rough and smooth bottom, the dome was observed to be unstable to nonaxisymmetrical disturbances with the wavenumber $n=2$. The series of photographs on which the features of instability are clearly seen are illustrated in Figures 2 and 3, correspondingly. In the cases shown in these figures, the instability did not lead to the destruction of the whole dome and to the splitting of the vortex into the pair of vortices as was shown in the experiment of Griffiths and Linden (1981). After the period of developing of instability, the restabilization of the dome occurs. After the process of restabilization of the dome, the second instability can occur. However, in more than one experiment with the smooth bottom, the dome was broken into a pair of vortices during the instability, and no restabilization process was observed. In this particular case the dome had the highest amount of potential energy before the onset of instability. It seems that the intensity of the instability process strongly depends on the amount of potential energy which the dome obtained before. It is obvious that this amount of potential energy will be greater if the dome will be stable for a long time during the constant supply. However, from Figures 2 and 3, one can see that in the case of rough bottom, instability occurs much sooner than in the case of smooth bottom, and the dome at the onset of instability has a much smaller size in the case of rough bottom. In the series of experiments it was obtained that the average time $\frac{\tau}{\tau}$ of the onset of instability counted from the beginning of the experiment is: a) In the case of smooth bottom

b) Inthe case of rough bottom

The ratio between these two times is

$$
\bar{\tau}_{s} / \bar{\tau}_{z} \simeq 2.4
$$

so it seems that there is more chance to achieve good splitting of the dome in the case of smooth bottom during constant sunnly regime. It is also important to menfion that the stability parameter $\gamma=\theta(1-\delta / \delta) 1 / 2$ (where $\delta=$. H/ $\mathrm{H}_{0}^{*}$ and $\mathrm{H}^{*}$ is the depth of the fluid in the tank at the moment of the beginning of lnstability) o has different values in these two cases:

a) The average. $\chi_{S} \quad \gamma_{S}$ at the beginning of instability in the ease of smooth bottom is: $\gamma_{s}=0.017 \pm 0.05$

b) In the case of rough bottom: $\quad \gamma_{z}=0.44 \pm 0.15$

The value $\boldsymbol{\gamma}_{\boldsymbol{S}}$ for the case of smooth bottom obtained in our experiment is approximately the same as in the Griffiths and Linden (1981) experiment, but 
for the rough bottom it is somewhat larger. Another important thing is that there were no significant changes in the value of $\boldsymbol{\gamma}$ just before the onset of instabilit (see Figures $6 a-8$ ). At the beginning of the experiment the value of $\{$ is rapidly decreasing, but then it changes only slightly. It seems that the vortex becomes unstable, not only because the instability parameter $\gamma$ reaches the critical value, but also for some other reasons. One can suppose that the condition of small value of $\gamma$ is an important one, but not sufficient. The whole series of data was used to achieve the laws of dependences of $\mathrm{H}$ and $\mathrm{R}$ on $t$ in the cases of rough and smooth bottom. The results are shown in Figures 8 and 9 correspondingly. It is interesting that in the case of rough bottom, the height $\mathrm{H}$ is growing with time faster than in the case of the smooth bottom. So the vortex in the case of rough bottom has more sharp density fronts and its bottom is closer to the bottom of the tank in the same time than in the case of smooth bottom.

\section{The experiments with finishing supply before the onset of instability}

In both cases of rough and smooth bottom these vortices become unstable to the disturbances with wavenumber $n=2$. The instability leads to the destruction of the dome and to the splitting into two vortex pairs (except, perhaps, the case where the dome is small and does not have enough potential energy to split). No restabilization of the dome was observed. The sequences of photographs illustrating the process of instability are shown in Figures 4 and 5 for the case of rough and smooth bottom correspondingly. The supply was finished at the time $t=650 \mathrm{~s}$ in the case of rough bottom (Figure $4 \mathrm{c}$ ) and at the time $\mathbf{t}=800 \mathrm{~s}$ in the case of smooth bottom (Figure $5 \mathrm{c}$ ). The volumes of fluid in the dome at these moments of time were approximately equal to each other. After this the vortices were stable for some. times. During this time they were slowly decreasing because of frictional effects. After tr. Some . time the instability began. The time 1nterval $\Delta \mathbf{t}$ between the moments of the end of supply and the beginning of instability was different in the cases of rough and smooth bottom.

\section{1) In the case of smooth bottom: $\overline{\Delta t}_{\mathbf{z}}=150 \pm 60 \mathrm{~s}$ \\ 2) In the case of rough bottom: $\overline{\Delta t_{\mathbf{g}}}=370 \pm 40 \mathrm{~s}$}

The ratio between these two intervals of time is

$$
\overline{\Delta t}_{s} / \overline{\Delta t}_{t}=2.5
$$

So, in the case of rough bottom, instability occurs much sooner than in the case of smooth bottom after the end of supply.

It is important to mention that the processes of the developing of instability and splitting sf domes goes very fast. It takes only a few periods of rotation while the period before the beginning of instability after supply is stopped, was more than ten periods of rotation in every case.

We also calculated the values of the stability parameter. In this situation the stability parameter at the beginning of instability does not differ significantly in both cases:
a) In the case of smooth bottom $\gamma=0.031 \pm 0.012$
b) In the case of rough bottom $\gamma=0.038 \pm 0.007$ 
As in the situation with constant flux regime, the value of $Y$ was not changing significantly in the interval between the moments of finishing flux and the beginning of instability (see Figure $7 a$ and $b$ ).

IV. Conclusions

1. During the constant supply regime, in the case of rough bottom, instability begins sooner than in the case of the smooth bottom. The stability parameter $\gamma$ at the beginning of instability is remarkably larger in the case of rough bottom.

2. During the constant supply regime, after a period of development of instability, the dome structure can be restabilized after a period of development of instability. This suggests that the incoming nonrotating fluid plays the stabilizing role on the dome structure. After the process of restabilization, another instability can occur.

3. The growth rate of the height $\mathrm{H}$ of the dome during the supply regime before the instability is greater in the case of rough bottom.

4. After the supply is finished, the instability occurs which leads to the rapid decay of the dome structure and splitting into two vortex pairs.

5. The interval of time between the moments of finishing supply and beginning of instability is compared to the time of viscous spin up of the vortex. This time interval is shorter in the case of rough bottom.

6. The intensity of instability relates on the amount of potential energy which the dome obtained before the onset of instability.

7. It seems that the stability parameter which was used in the work of Griffiths and Linden (1981) and in this paper is not the best one for prediction of instability. One can suppose that the stability parameter must somehow include the frictional effects at the bottom of the tank and in the interior of the fluid.

\section{ACKNOWLEDGMNIS}

I am very thankful to Professor M.E. Stern, Professor J. Whitehead, and Mr. R. Frazel for strong support of all stages of this work. The author is also very thankful to Professor G. Veronis for useful discussions of the results.

\section{REFERENCES}

Griffiths, R.W., and P.F. Linden, 1981. The stability of vortices in a rotating stratified fluid. J. Fluid Mech., 퐁, 283-316.

Howard, L.N., 1969. The effect of side walls on the porous-medium spin-up problem. Deep-Sea Res., 16 (suppl. ), 97-107.

Kroll, J., and G. Veronis, 1970. The spin up of a homogeneous fluid bounded below by a permeable medium. J. Fluid Mech., 40, 225-239.

Phillips, N., 1954. Energy transformations and meridional circulation associated with simple baroclinic waves in a two-level quasigeostrophic model. Tellus, 6, 273-286. 


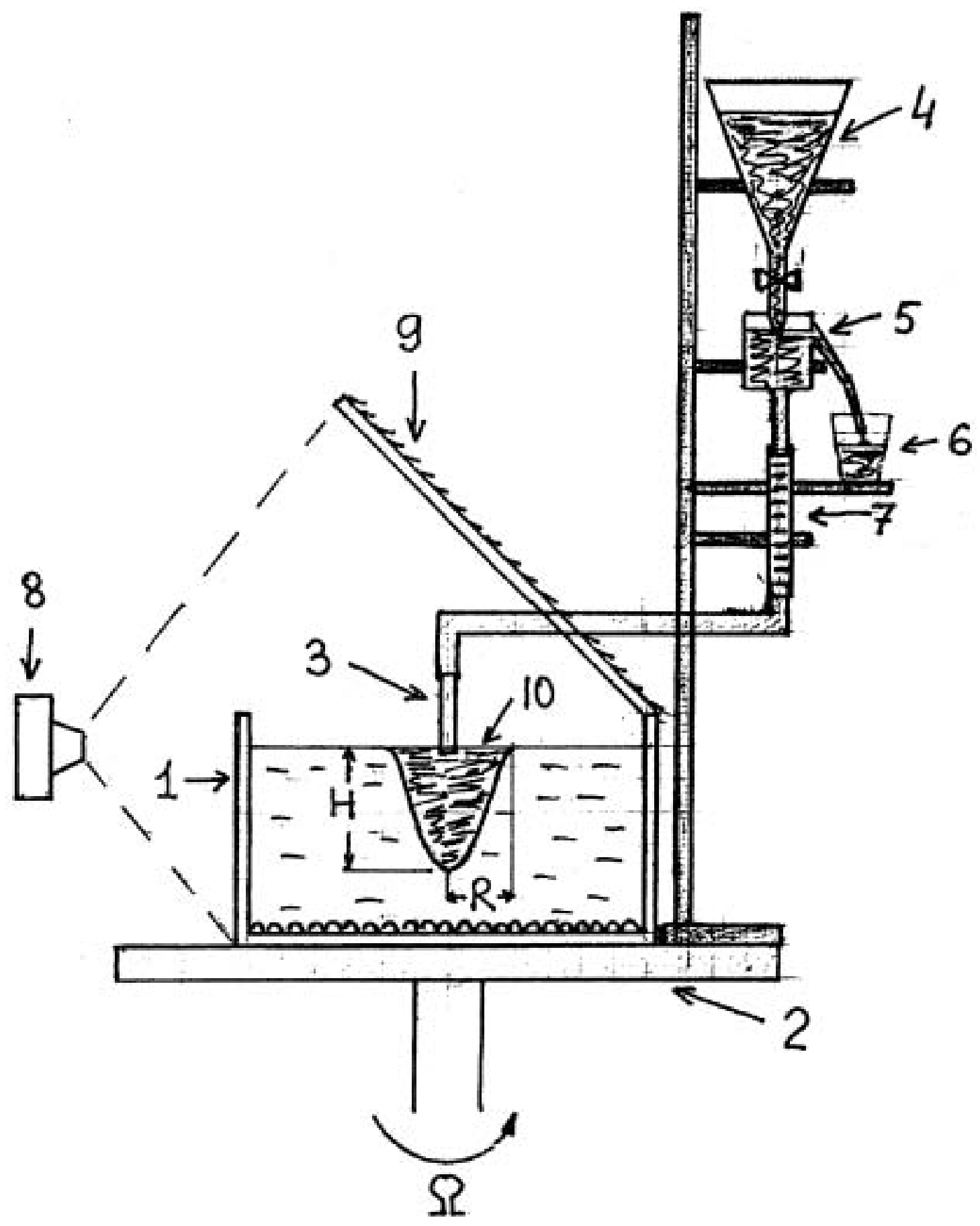

Fig. 1. The scheme of the experiment. 

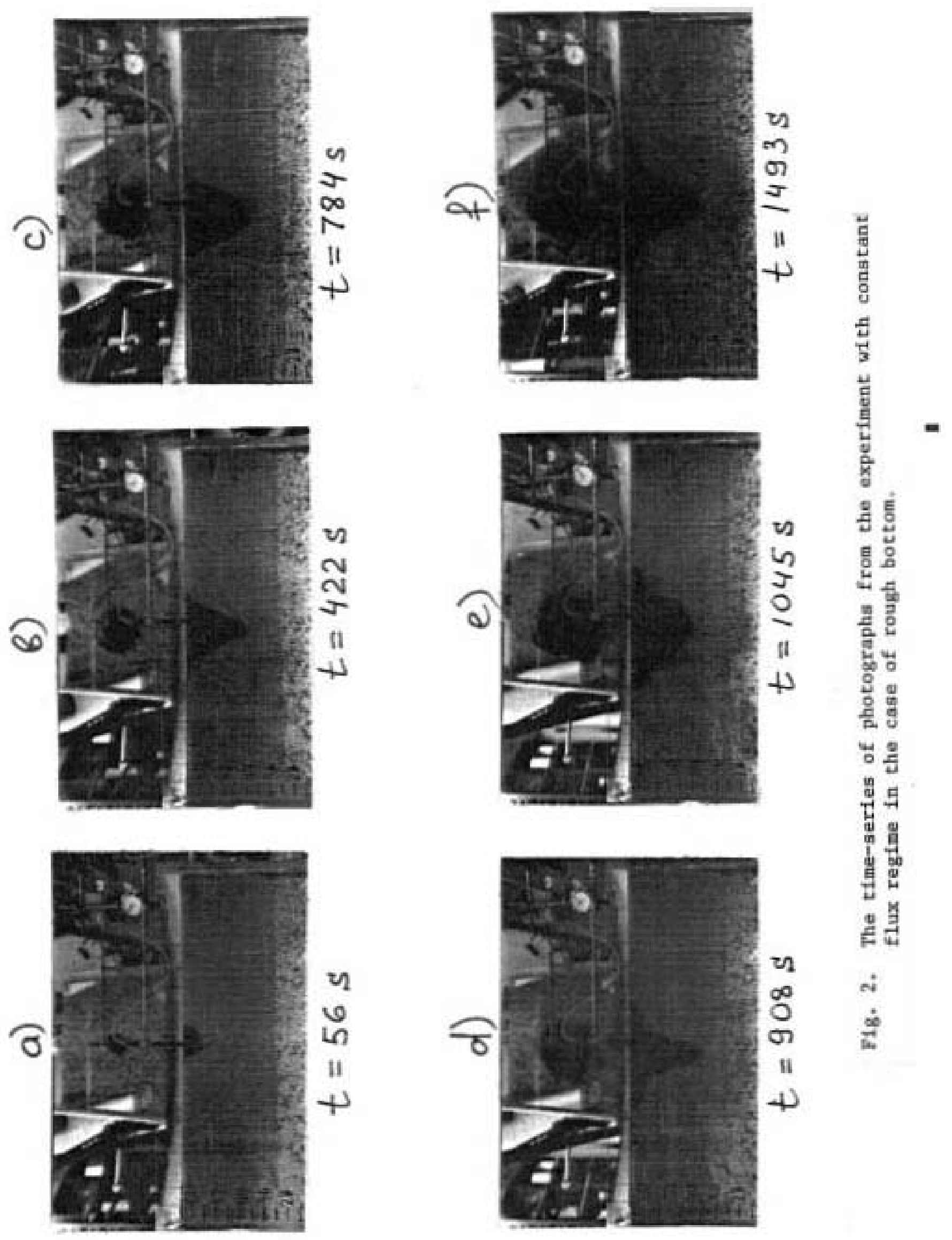

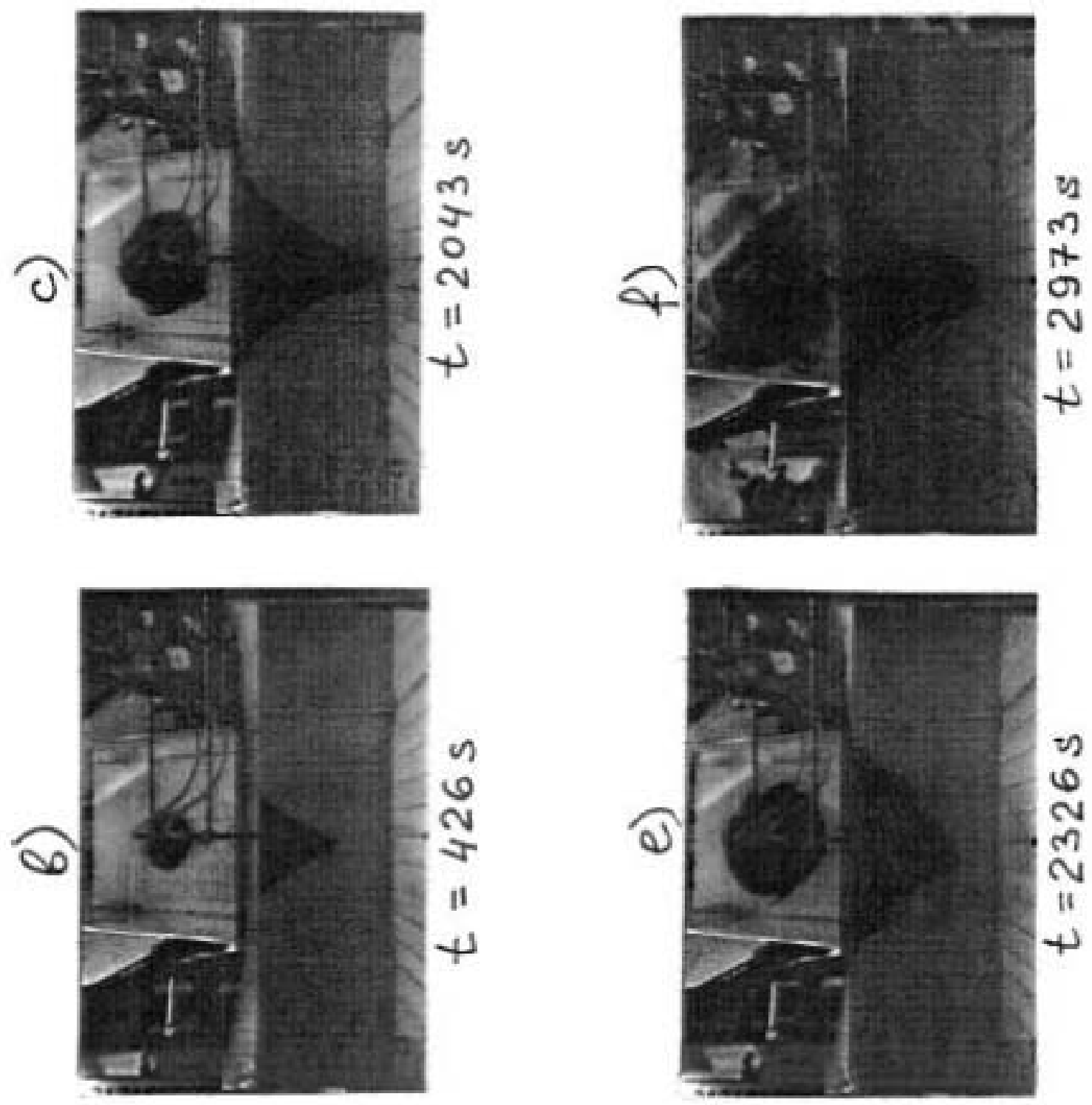

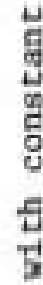
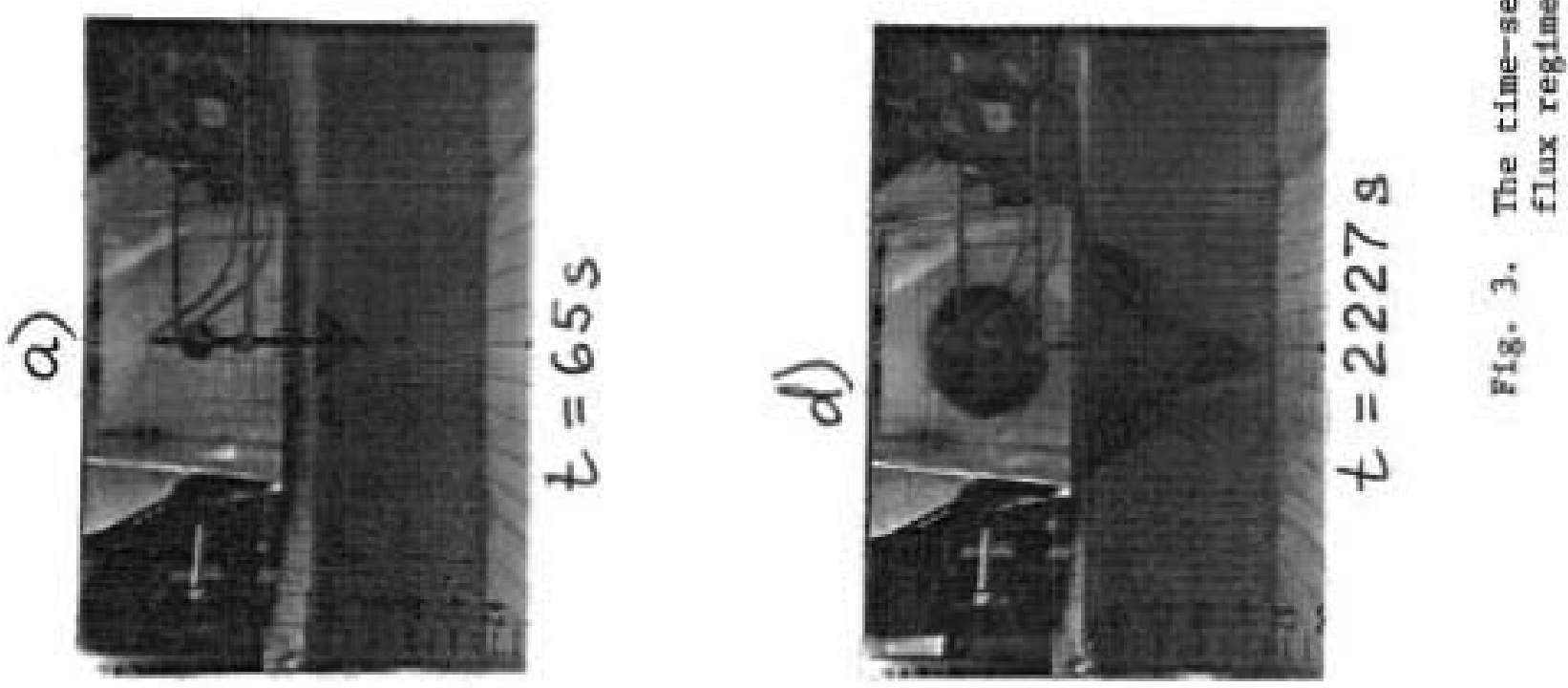

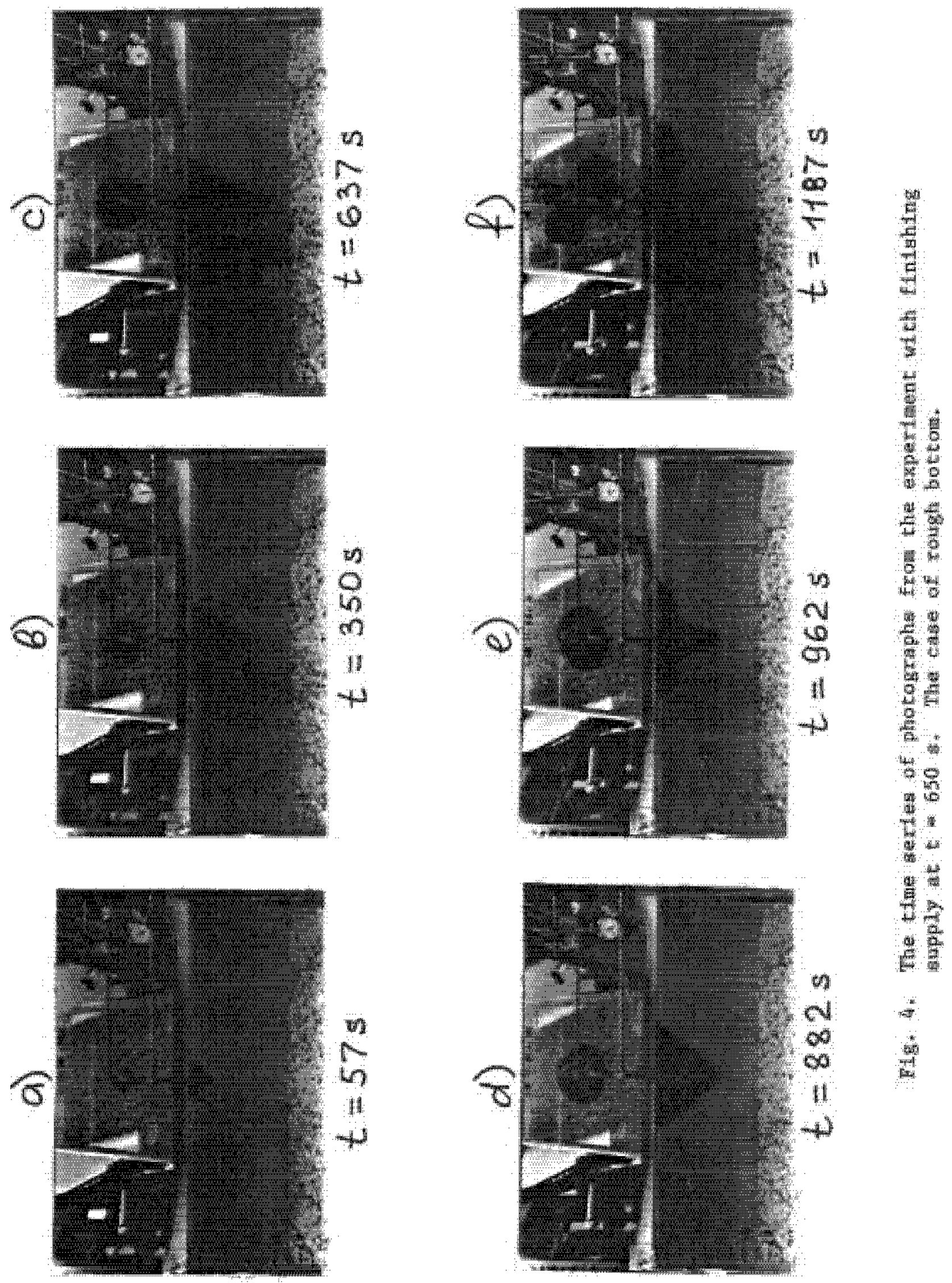

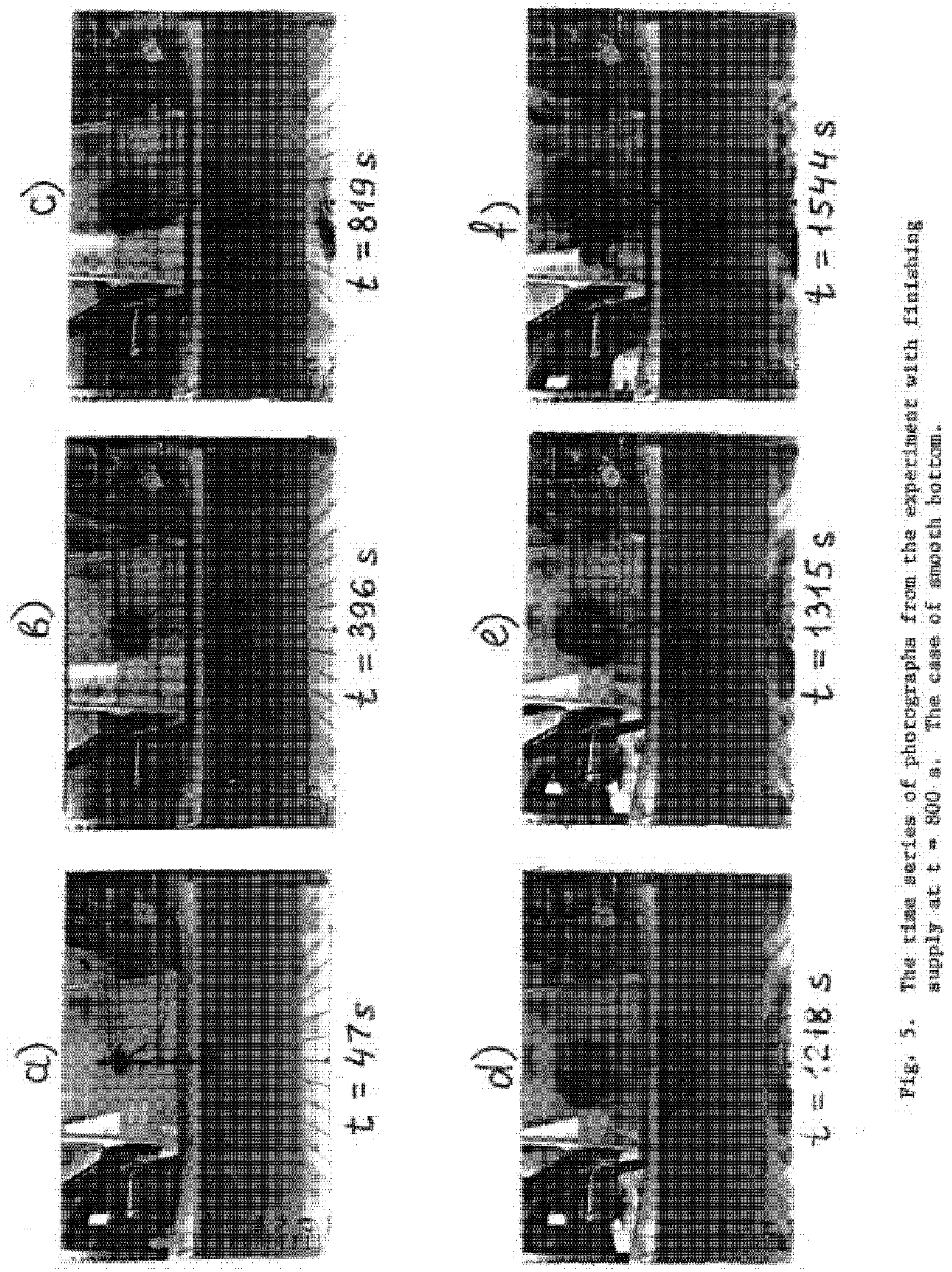


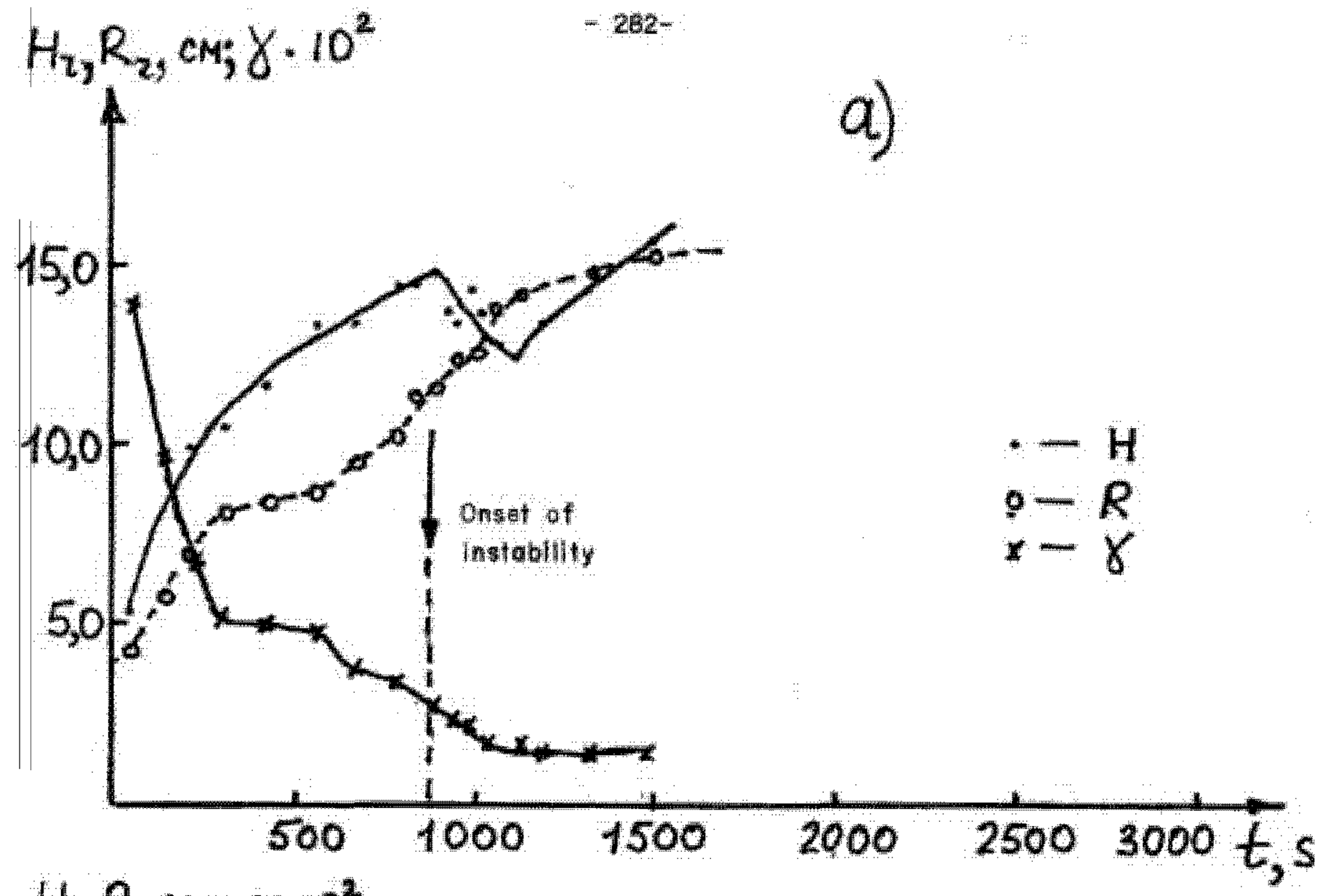

$\mathrm{H}_{s}, \mathrm{R}_{s}, \mathrm{CM} ; \gamma \cdot 10^{2}$

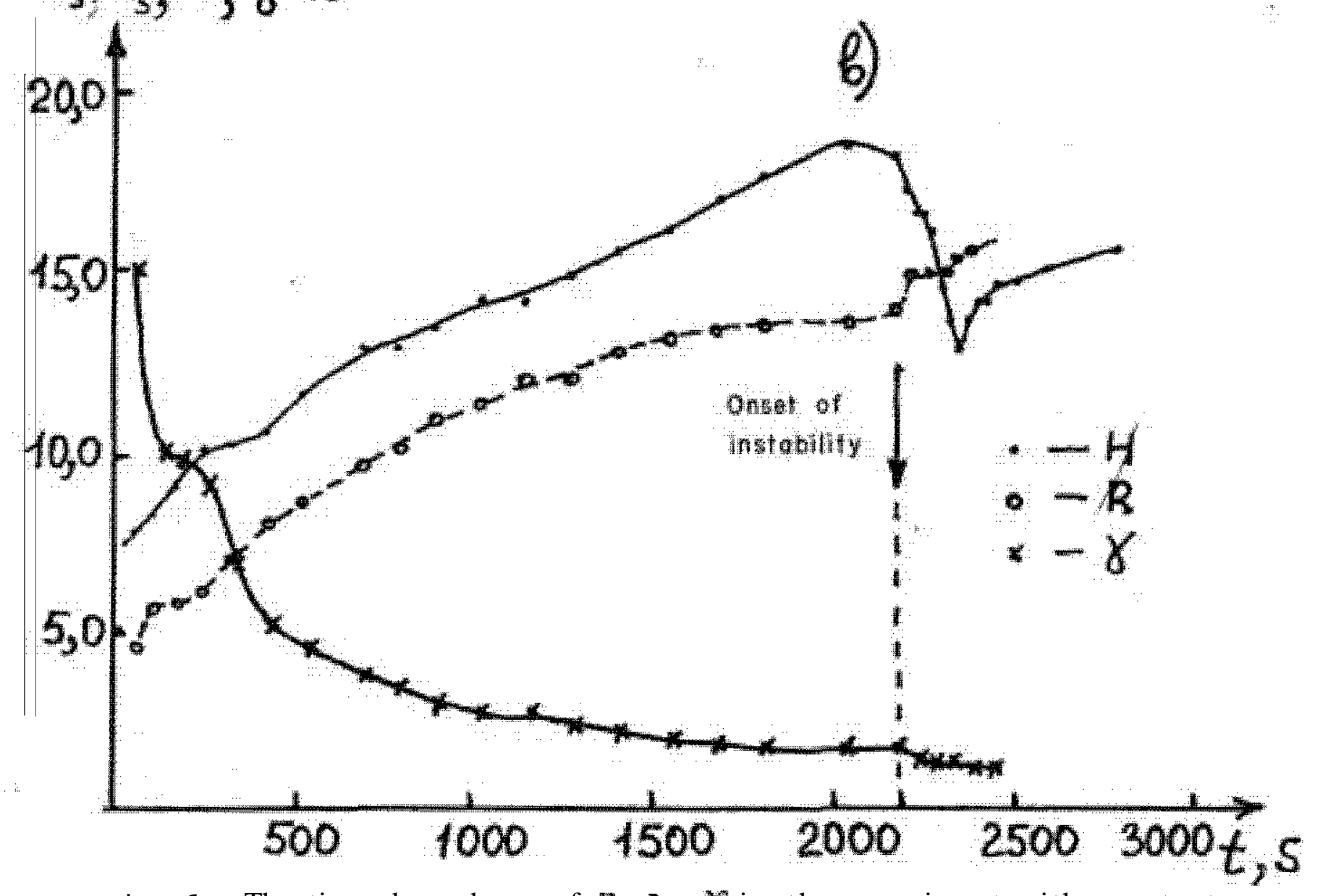

Fig. 6. The time dependence of $H, R, \gamma^{m}$ in the experiment with constant flux regime. a) in the case of rough bottom; b) in the case of smooth bottom. 

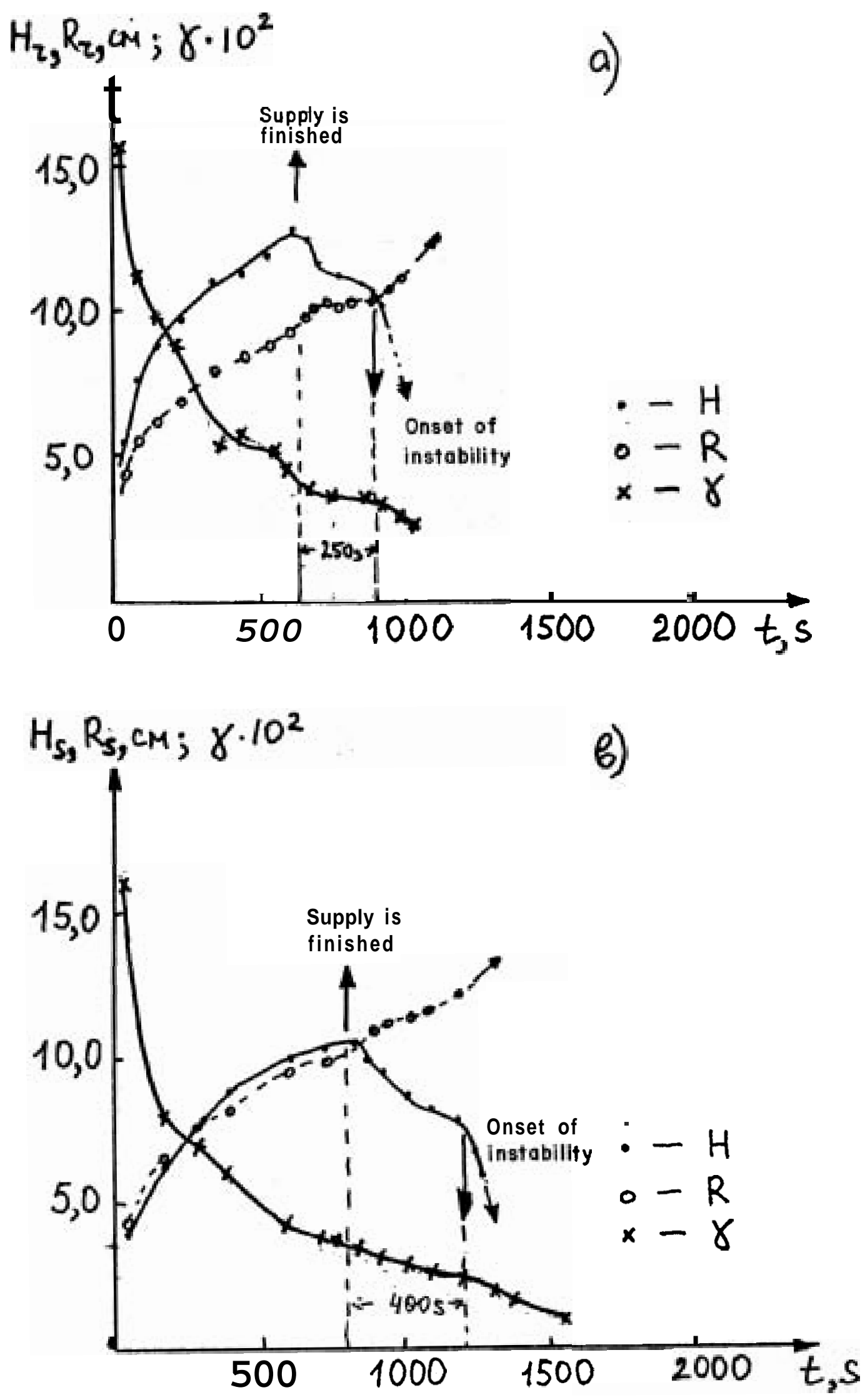

Fig. 7. The time dependence of $\mathrm{H} R, \gamma$ in the experiment with the finishing flux before the beginning of instability. a) in the case of rough bottom; b) in the case of smooth bottom. 

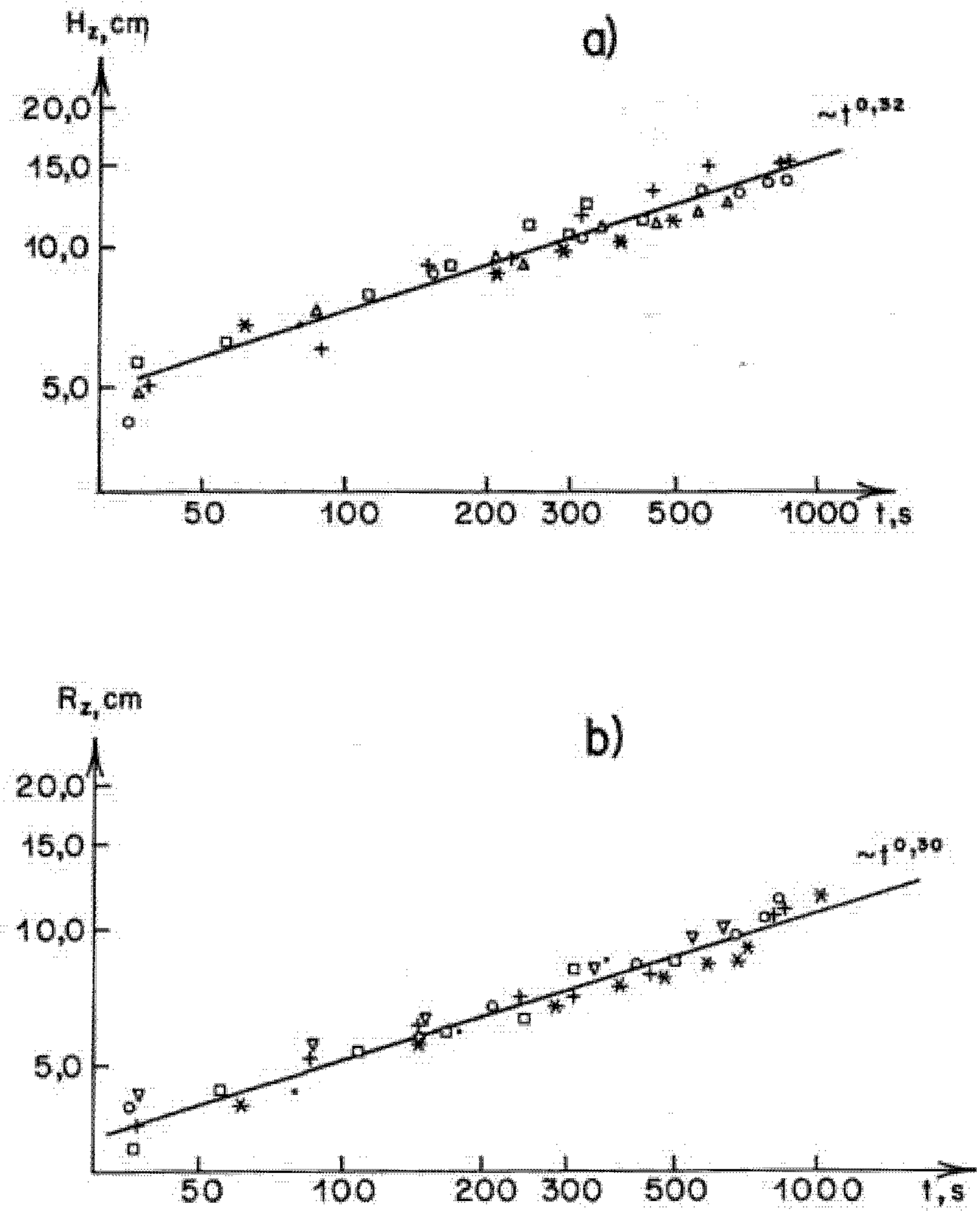

Fig. 8. Statistically determined growth rates $H, R$ with time before the onset of instability in the case of rough bottom. 

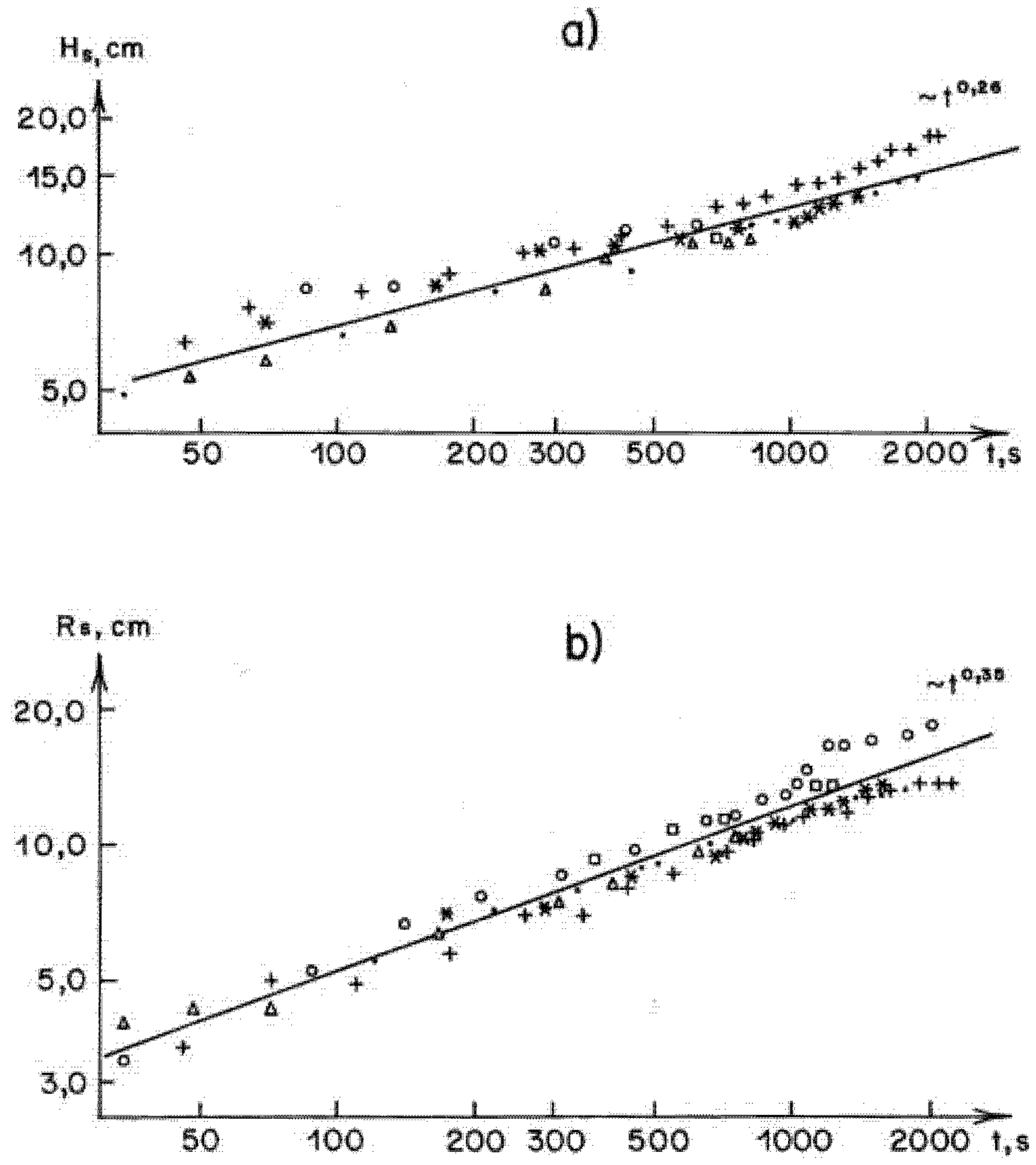

Fig. 9. Statistically determined growth rates $H, R$ with time before the onset of instability in the case of smooth bottom. 


\title{
SOME THREE-DIMENSIONAL ASPECTS OF COASTAL UPWELUNG
}

\author{
Pierre J. Flament
}

\section{Introduction.}

Thermal infrared satellite images have shown that coastal upwelling is often a strongly three-dimensional process, and is therefore not adequately described by two-dimensional models. For reviews, see Allen (1980) and Brink (1983) ; for a recent two-dimensional model, see Foo (1980).

In this paper, I present new observations on three-dimensional aspects of upwelling, and discuss a simple mean flow / mixed layer interaction model consistent with some of the frontal features observed.

\section{Observations.}

Figure 1 is a typical surface temperature pattern off California during upwelling favorable winds (the thermal radiation is emitted by a thin surface layer a few um thick, but the radiometric temperature is correlated with the bulk temperature when there is a well mixed surface layer : in particular, when the wind is strong).

The cold near-shore region is visible on the right (A). The main upwelling front (B) is highly convoluted, and meanders into two cold filaments (C,D) extending more than $100 \mathrm{~km}$ offshore.

Figure 2 is a sequence showing the evolution of a similar but stronger filament.

Surface drifter tracks (Davis et al., 1982) and hydrographic surveys (Huyer, 1982, personal communication) have shown that these narrow filaments are strong dynamical jets (they are not cold water streaks entrained between meso-scale eddies). Their geostrophic signature is confined near the surface, with an e-decaying depth of about $100 \mathrm{~m}$; their typical width is $10 \mathrm{~km}$, and their maximum surface velocity is of the order of $0.5 \mathrm{~m} / \mathrm{s}$, corresponding to an estimated transport of up to $10^{6} \mathrm{~m}^{3} / \mathrm{s} \quad(1 \mathrm{~Sv})$.

The vertical structure of the jet shown on figure 2 has been studied using a yo-yoed conductivity-temperature-depth probe towed along the line A-B, about $200 \mathrm{~km}$ offshore. The results are sketched on figure 3 .

A striking characteristic is the thermal asymetry of the jets: the southern front is generally sharp (often $1 \mathrm{C}$ in less than $100 \mathrm{~m}$ ), while the northern boundary has smoother gradients ( $1 \mathrm{C}$ i $\mathrm{n}$ a few $\mathrm{km}$ ). At this distance from the coast, the sharp front tends to be salinitycompensated : there is little or no density gradient (unlike the main near-shore upwelling front which is usually also a strong density front).

The temperature signature of the jet is confined to the turbulent surface layer : there is no temperature front in the thermocline. The depth of the bottom (E) of the surface layer, which lies approximately on an isopycnal, varies on short distances : in less than $10 \mathrm{~km}$, it increases from $30 \mathrm{~m}$ under the sharp front (C), to $60 \mathrm{~m}$ at (D). Between (C) and (D), the surface layer is not well-mixed and isothermal : there is an intrusion (F) of warm, saline water underneath the cold, fresh near-surface water. 
Many regions with vertical gradients favorable to differential diffusive processes are observed, and a complicated pattern of small thermohaline intrusions is found; only the largest is sketched on figure 3 : it detaches from the mixed layer at (D) where it crosses isopycnals, and lies on a density surface thereafter.

We also note that, because the sharp thermohaline front is in a fully turbulent mixed layer (the wind was blowing at $20 \mathrm{~m} / \mathrm{s}$ ), its dynamics should be different from the model discussed by Ruddick and Turner (1979), which concerns interleaving at a vertical thermohaline front between initially quiescent fluids.

The jets are unstable, and eddies grow on both sides, but the instabilities are more visible on the south front due to the thermal asymetry. Using Killworth's (1980) nomenclature, the non-dimensional parameters of the flow are $\lambda=O(1)$ and $\delta \ll 1$, which classifies the instability as barotropic or mixed : the growing perturbations are fed mainly from the kinetic energy of the mean flow.

The typical wavelength of the instabilities is $30-50 \mathrm{~km}$, and the angular velocity of the eddies is of order $f$ : the Rossby number is of order 1 .

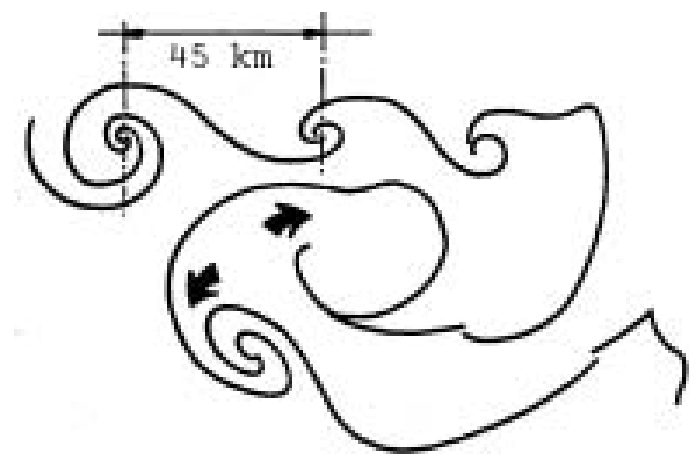

Similar jets are found in the Eastern Pacific from Baja California to Washington. Their spacing is variable, from $50 \mathrm{~km}$ to $300 \mathrm{~km}$, and seems to increase with time during the upwelling season : late summer patterns often display fewer and stronger jets than early spring ones; however, the satellite image set is not yet statistically significant to make this a final statement.

The data set is also insufficient to decide whether the jets are related to upwelling centers (regions of intensified upwelling due to enhanced wind-stress, large coastline curvature or bottom topographic features), and hence are repeatedly found at the same location, or result from the finite amplitude growth of a fundamental instability of the upwelling front (somewhat similar to the instabilities discussed by Griffith in his lecture): the mechanism of the jets, and the origin of their huge kinetic energy is an open question requiring further experimental and theoretical work.

It is interesting to compare the offshore transport due to the jets with a typical wind-driven Ekman transport, both per meter of coastline. For the jets, assuming a rate of flow of $0.5 \mathrm{~Sv}$ and a spacing of $200 \mathrm{~km}$, we find $: 2.5 \mathrm{~m}^{2} / \mathrm{s}$.

For the Ekman transport, assuming a wind speed of $10 \mathrm{~m} / \mathrm{s}: \tau / \rho f=4 \mathrm{~m}^{2} / \mathrm{s}$. They have the same order of magnitude, and it is clear that the jets cannot be ignored in a complete dynamical model of upwelling.

In the next section, I present a simple model of frontogenesis/lysis consistent with the thermal asymetry of the jets. 
3. A linear wind-driven frontogenesis model.

We are interested in the lowest order effects of the interaction between a narrow jet and the Ekman transport of a wind mixed layer.

We consider an incompressible, Boussinesq, hydrostatic fluid with an upper turbulent layer of density $\rho_{0}$ and an infinitely deep lower layer of density $\rho_{0}+\Delta \rho$. The frame of reference is taken as usual : $x$ eastwards, $\mathrm{y}$ northwards and $\mathrm{z}$ upwards.

The interface $z=h(x, y, t)-H$ is defined slightly deeper than the depth of the turbulent layer, so that the turbulent stress vanish on this surface ; at the free surface, the turbulent stress is the applied wind stress $\tau$. We assume that the mixed layer has reached an equilibrium depth, and that further deepening (or shallowing) can only be caused by Ekman downwelling (or upwelling) without entrainment (detrainment) of new fluid. We neglect horizontal gradients of turbulent stresses.

The jet is represented by a steady westward flow

$$
\underline{U}_{0}=\left(\mathrm{U}_{0}(\mathrm{y}), 0\right) \quad ; \quad \mathrm{U}_{0}<0
$$

present at all depths ; in the upper layer, there is an additional velocity that we split in two components:

-

- u' $^{\prime}$ is the residual (Ekman) flow balancing the stresses.

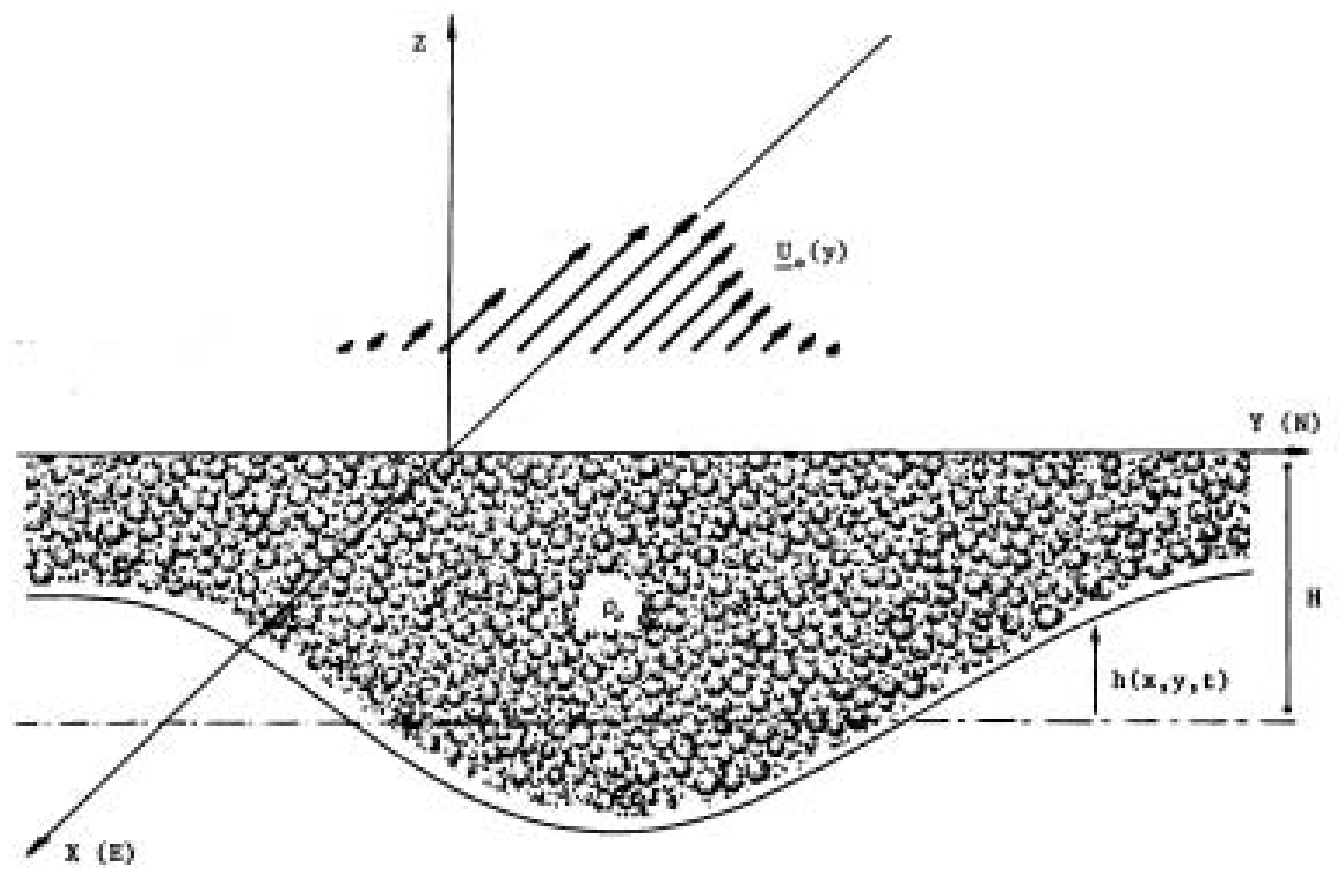


Write mean momentum and mass conservation equations for $\underline{u}$ and $\underline{u}$ ' ; integrate vertically between the interface and the free surface using the boundary conditions on the stress, and get, after some algebra :

$$
\begin{gathered}
\partial_{\mathrm{t} \underline{\mathrm{u}}}+\left(\underline{\mathrm{u}}+\underline{\mathrm{u}}_{0}\right) \cdot \underline{\nabla} \underline{\mathrm{u}}+\underline{\mathrm{u}} \cdot \underline{\nabla} \underline{\mathrm{u}}_{0}+\underline{\mathrm{f}} \times \underline{\mathrm{u}}=\mathrm{g}^{\prime} \underline{\nabla} \mathrm{h} \\
\partial_{\mathrm{t}} \mathrm{h}+\left(\underline{\mathrm{u}}+\underline{\mathrm{u}}_{0}\right) \cdot \underline{\nabla} \mathrm{h}+(\mathrm{h}-\mathrm{H}) \underline{\nabla} \cdot \underline{\mathrm{u}}=\underline{\nabla} \cdot \underline{\tilde{u}} \cdot \underline{\underline{\mathrm{u}}} \cdot \underline{\nabla}\left(\underline{\mathrm{u}}+\underline{\mathrm{u}}_{0}\right)+\underline{\mathrm{u}} \underline{\nabla} \cdot \underline{\mathrm{u}} \\
\left.\partial_{\mathrm{t} \underline{\mathrm{u}}}+\underline{\underline{\mathrm{u}}}+\underline{\mathrm{u}}_{0}\right) \cdot \underline{\nabla} \underline{\mathrm{u}}+\underline{\underline{\mathrm{u}}} \times \underline{\mathrm{u}}=\frac{\underline{\tau}}{\rho_{0}}
\end{gathered}
$$

where

$$
\underline{\mathrm{u}}=\int_{\mathrm{h}-\mathrm{H}}^{0} \underline{\mathrm{u}}^{\prime} \mathrm{d} z \text { is the wind-driven Ekman transport, }
$$

and

$$
\mathrm{g}^{\prime}=\mathrm{g} \Delta \rho / \rho_{\circ} \quad \text { is the reduaed gravity. }
$$

Similar equations are derived in more details by Stern (1975) and Weller (1982) ; they are independent of the detail of the velocity field within the mixed layer.

We want to study the effects of the divergence $\underline{\nabla} \cdot \underline{\underline{u}}$ of the Ekman transport, using an approach formally analogous to the $\overline{1}$ inear model of the onset of coastal upwelling proposed by Yoshida (1955).

This leads us to the following additional assumptions :

- the along-jet component $u$ of $\underline{u}$ is in geostrophic balance with the across-jet pressure gradient $a_{Y} h$

- at $\mathbf{t}=0$, the interface is undisturbed $: h(x, y, 0)=0$ and hence $\underline{\Perp l}(\mathrm{x}, \mathrm{y}, \mathrm{0})=0$

- the equations are linearized : this is justified for short times, III $\& U_{0}$ and $\mathrm{h} \& \mathrm{H}$, and for small wind stresses, $\mid$ ü $\mid \& U_{0}$

- the wind is steady or varies slower than the inertial period, so that the adjustment to Ekman balance can be regarded as instantaneous : $a_{t}$ 능 0 in $(\mathbf{3})$. This removes wind-excited inertial oscillations and their interaction with the mean flow, discussed by Weller (1982)

- the system remains independent of $x$ at all times. This assumption is the most restrictive and even questionable : it removes the effects of the coast and of the finite length of the jets, and excludes a priori horizontal "confluent" motions, leaving downwelling as the only possible consequence of an Ekman convergence. 
With these assumptions, (1)-(3) reduce to :

$$
\begin{aligned}
& \partial_{t} u+v \partial_{y} U_{0}-f v=0 \\
& \text { f u }=g^{\prime} \partial_{y} h \\
& \partial_{t} \text { h }- \text { H } \partial_{y} v=\partial_{y} \tilde{v} \\
& \tilde{u}=\frac{T_{y}}{f \rho_{0}} \\
& \tilde{v}=\frac{-\tau_{x}}{\left(f-\partial_{y} U_{0}\right) \rho_{0}}
\end{aligned}
$$
is

To linearize ( 3 ), we made the small wind stress assumption. The wind stress

$$
\underline{\tau}=c_{\mathrm{d}} \rho_{\text {air }}\left|\underline{\mathrm{U}}_{\mathrm{w}}-\underline{\mathrm{U}}_{0}\right|\left(\underline{\mathrm{U}}_{\mathrm{w}}-\underline{\mathrm{U}}_{0}\right)
$$

where $\mathrm{U}_{\mathrm{w}}$ is the wind velocity; because the scale of the oceanic flows considered here is much smaller than the scale of typical meteorological systems, $\underset{\rightarrow}{U}$ is taken as constant.

For very small winds ( $\underline{\mathrm{u}}_{w}$ of the order of $\underline{\Perp}_{0}$ ), the wind velocity relative to the water varies significantly across the jet, and induces a divergence in the Ekman flow. However, this effect can be ignored here, because winds small enough to be of the order of $U_{0}$, are in general too weak to maintain a well mixed surface layer in the presence of diurnal heating.

"Small wind stresses" therefore means small enough for the quadratic terms in (3) to be neglegible, yet large enough to be uninfluenced by the water velocity:

$$
\underline{\tau}=\mathrm{C}_{\mathrm{d}} \rho_{\text {air }}\left|\underline{u}_{w}\right| \underline{u}_{w}
$$

Equation (8) shows that the across-jet Ekman flow $\vec{v}$ depends on the total vorticity of the flow : north of the axis, the negative local vorticity reduces the total vorticity, and the Ekman flow is larger, while south of the axis, the situation is opposite : these variations of v generate a divergence in (6), which is important for strong, narrow jets.

A positive divergence will decrease the gradient of a passive tracer advected by the flow, and is frontolytic; a convergence increases the gradient and is frontogenetic.

Because we required invariance for $x$-translations, only the component of the wind parallel to the jet generates divergences in the Ekman flow. 
eastwards.

Figure 4 shows a numerical solution of the equations, for a wind blowing

For short times, the southwards Ekman velocity $v^{\prime}$ is slower south of the jet, and faster north of it (figure 4b). There is a strong frontogenetic downwelling near the axis of the jet, and a strong frontolytic upwelling north of it (and a weaker one south, figure 4c).

Thus a westerly wind generates an asymetry similar to the one observed on figures 1 and 2, where de winds were from the northwest : strong gradients south, weak gradients north.

The asymetry should reverse when the wind is blowing from the northeast : correlating frontal gradients with the synoptic wind direction on the available satellite archives would be an excellent test of the mechanism proposed.

The height of the interface after 6 hours (figure 4d) is similar to the observed slope (A-C-D-B) of the isopycnal (figure 3).

Another encouraging result is the variation of the Ekman velocity v', which is similar to an accoustic doppler velocimeter record made during the tow (communicated by Mike Kosro).

The region of strongest gradient does not coincide with the point of maximum downwelling (D) : curiously, it is found several $\mathrm{km}$ south of it, at (C).

Assume that the temperature is a passive scalar advected southwards by the cross-jet Ekman flow :

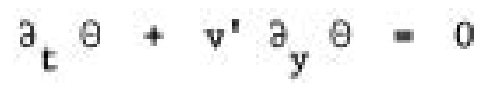

This equation is integrated numerically, with an initial constant gradient of $50 \mu \mathrm{C} / \mathrm{m}$.

For short times, the gradient is enhanced on the axis as expected, and reduced north of it (figure 4e).

For long times, the Ekman flow continuously advects the whole structure southwards, and the region of strongest gradient is found several $\mathrm{km}$ south of the axis, in qualitative agreement with the observations (figure 4f).

This argument is plausible, but we are certainly pushing our simple linear model too far, and the conclusion should be validated by more sophisticated models discussed below. Other mechanisms cannot be ruled out at this stage, for example shear-enhanced gradients on a jet with asymetric velocity profiles.

\section{Conclusions.}

We have developed a simple frontogenetic model consistent with the thermal structure of upwelling filaments.

Of course this model is crude and has many limitations : some of the simplifying assumptions should be relaxed and further work is required to confirm the mechanism proposed.

One way to improve the model is to keep a similar parameterization of the mixed layer turbulence, but to integrate numerically the full non-linear equations (1)-(3) with a two-dimensional imposed mean flow ( $x, y)$. The effects of the meanders and of the instabilities of the jet on the mixed layer could be explored with such a model. 
The other direction is to construct a two-dimensional $(y, z)$ model with a more realistic treatment of the turbulence including entrainment, similar to the parameterization of de Szoeke and Richman (1981) or Cushman-Roisin (1981), but allowing for a more detailed vertical structure of the turbulent layer. One may hope to reproduce, for example, the vertical temperature profile observed between (C) and (D).

Garrett (1982) mentions straining by a field of meso-scale eddies as a mechanism steepening thermohaline gradients to the point where differential diffusive instabilities can develop. In the presence of an eddy field with high local vorticities, the horizontal gradients in the surface Ekman layer are further sharpened by the frontogenesis process proposed here : the implications on the parameterization of near-surface horizontal mixing should be investigated. Similar effects on bottom Ekman layers should also be studied.

Finally, this approach to wind-driven frontal dynamics could be applied to the main upwelling front and the coastal jet, and throw some new light on the problem of cross-shelf circulation cells.

Acknowledgements.

I thank the staff of the 1983 GFD program for a stimulating atmosphere, and particularily Melvin Stern, Peter Killworth, Francis Bretherton, Georges Veronis and Ken Brink with whom I had helpfull discussions.

The experiment on which this work is based has been conducted in collaboration with Larry Armi, principal investigator, and Libe Washburn and Sharon Yamasaki, under an ONR contract with SIO.

The help of the crew of $R / V$ Wecoma during CODE-82, and of the staff of Scripps SSOF and PACODF has been instrumental in the data collection. 
REFERENCES

Allen, J.S., 1980. Models of wind-driven currents on the continental shelf. Ann.Rev.F1uid Mech. 12, pp. 389-433.

Brink, K.H., 1983. The near-surface dynamics of coastal upwelling. Prog. Oceanog., in press.

Cushman-Roisin, B., 1981. Effects of Horizontal Advection on Upper Ocean Mixing. J.Phys.Oceanogr. 11, pp. 1345-1356.

Davis, R.E., 1982. Preliminary drifter results from CODE-1. Unpublished manuscript.

de Szoeke, R.A., and Richman, J.G., 1981. The role of wind-generated mixing in coastal upwelling. J.Phys.Oceanogr. 11, pp. 1534-1547.

Foo, E.C., 1981. A 2-D diabatic isopycnal model simulating the upwelling front. J.Phys.Oceanogr. 11, pp. 604-626.

Garrett, C., 1982. On the parameterization of diapycnal fluxes due to doublediffusive intrusions. J.Phys.Oceanogr. 12, pp. 952-959.

Killworth, P.D., 1980. Barotropic and baroclinic instability in rotating stratified fluids. Dyn.Atm.Oceans 4 , pp. 143-184.

Ruddick, R.R., and Turner, J.S., 1979. The vertical length scale of doublediffusive intrusions. Deep-sea Res. 26A, pp. 903-913.

Stern, M.E., 1975. Ocean circulation physics. Academic press, New York. 246 p.

Weller, R.A., 1982. The relation of near-inertial motions (...) to the local wind stress (...), J.Phys.Oceanogr. 12, pp. 1122-1136.

Yoshida, K., 1955. Coastal upwelling off the California coast. Rec.Oceanogr.Works Japan 2, pp. 8-20. 


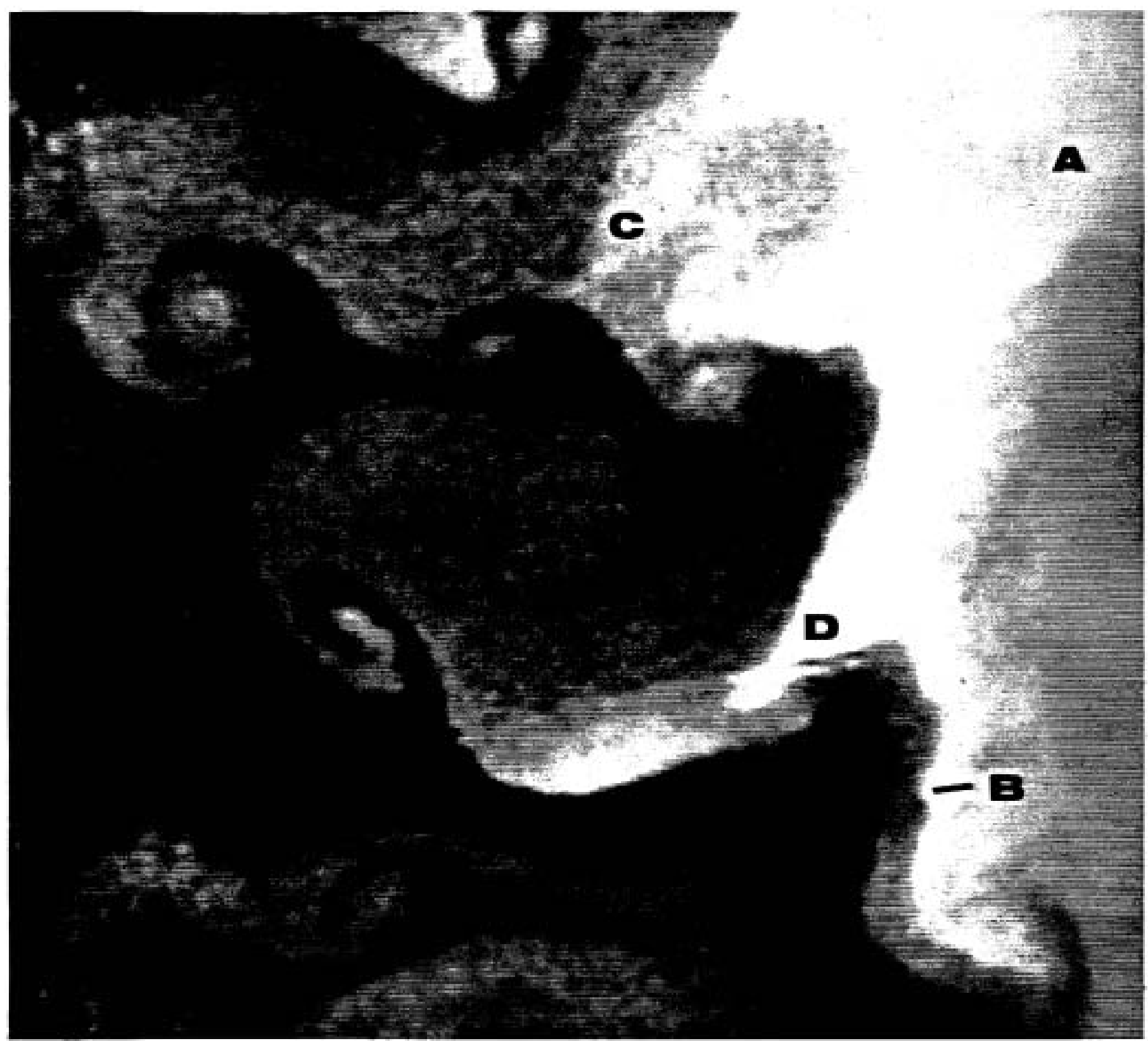

$\underline{\text { Figure } 1}$

Sea surface temperature field off Point Sur, California, May 7th, 1981. The coast is at the right edge of the image, and the continental shelf is about $25 \mathrm{~km}$ wide there. The image width is $125 \mathrm{~km}$. Cold water is white, warm water black ; the temperature range is 3 C. A steady wind from the $\mathrm{NW}$ had been blowing at $15 \mathrm{~m} / \mathrm{s}$ for one week. 

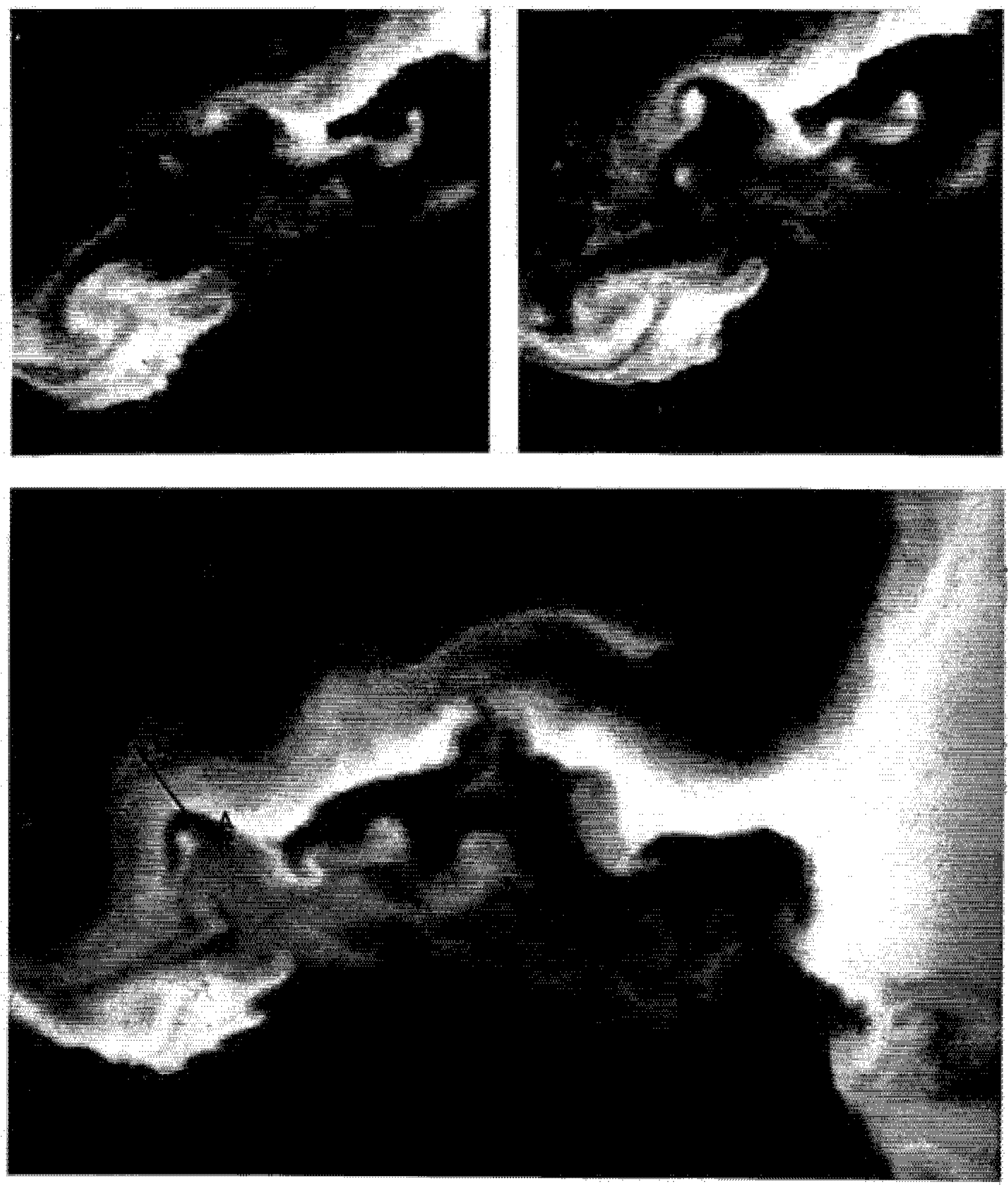

\section{Figure 2}

Sea surface temperature field off Point Arena, California, July $21 \mathrm{st}$ at 23 UT, July 22nd at 11 UT and July 22nd at 22 UT, 1982.

The coast is at the right edge of the lower image; the lower image width is $250 \mathrm{~km}$. A steady wind from the NNW had been blowing at $20 \mathrm{~m} / \mathrm{s}$ for 2 days. 

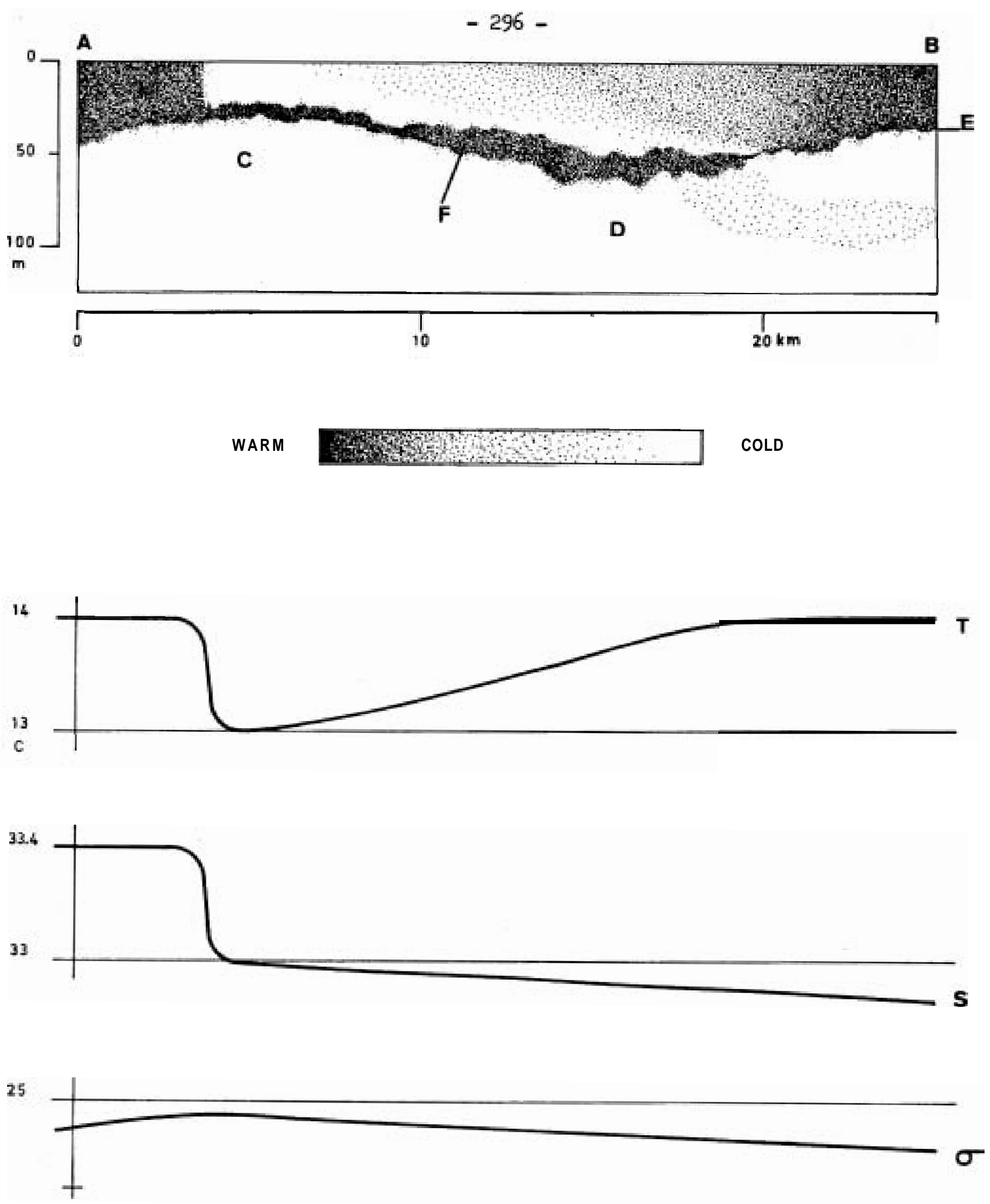

Figure 3.

top : vertical temperature structure above the thermocline along A-B on figure 2. bottom : horizontal profiles of surface temperature, salinity and density. 

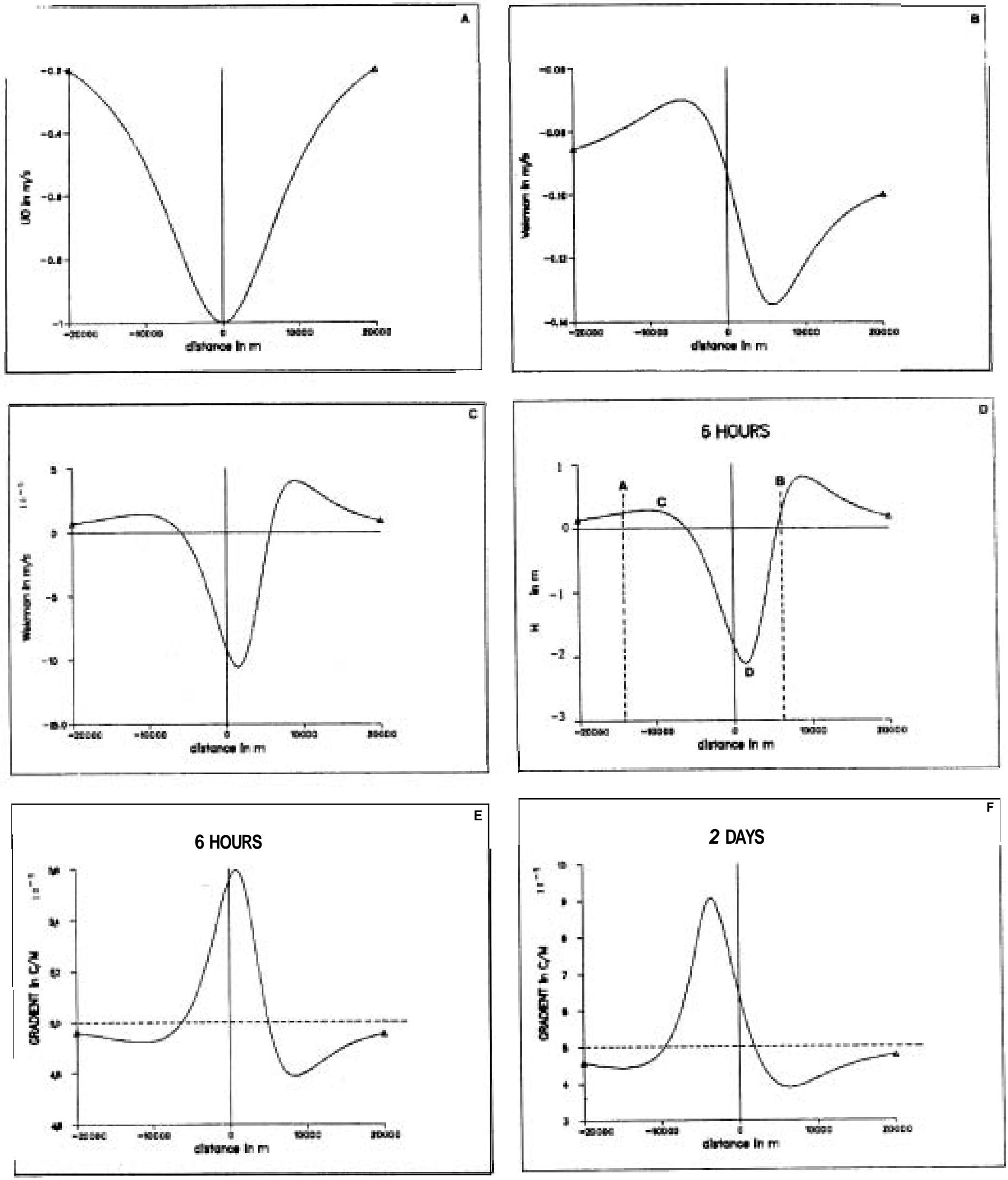

Figure 4.

Numerical solution of equations (4)-(8) and (10), with :

$$
\begin{array}{ll}
\mathrm{U}_{\circ}(\mathrm{y})=-\left(10^{+}(\mathrm{y} / 10 \mathrm{~km})^{2}\right)^{-1} \mathrm{~m} / \mathrm{s} & \mathrm{U}_{\mathrm{w}}=10 \mathrm{~m} / \mathrm{s} \text { eastwar } \\
\text { latitude }=45^{\circ} & \mathrm{H}=20 \mathrm{~m} \\
\Delta \rho / \rho_{\circ}=10^{-3} & \partial_{\mathrm{y}} \theta_{\mathrm{a}}=510^{-5} \mathrm{C} / \mathrm{m}
\end{array}
$$




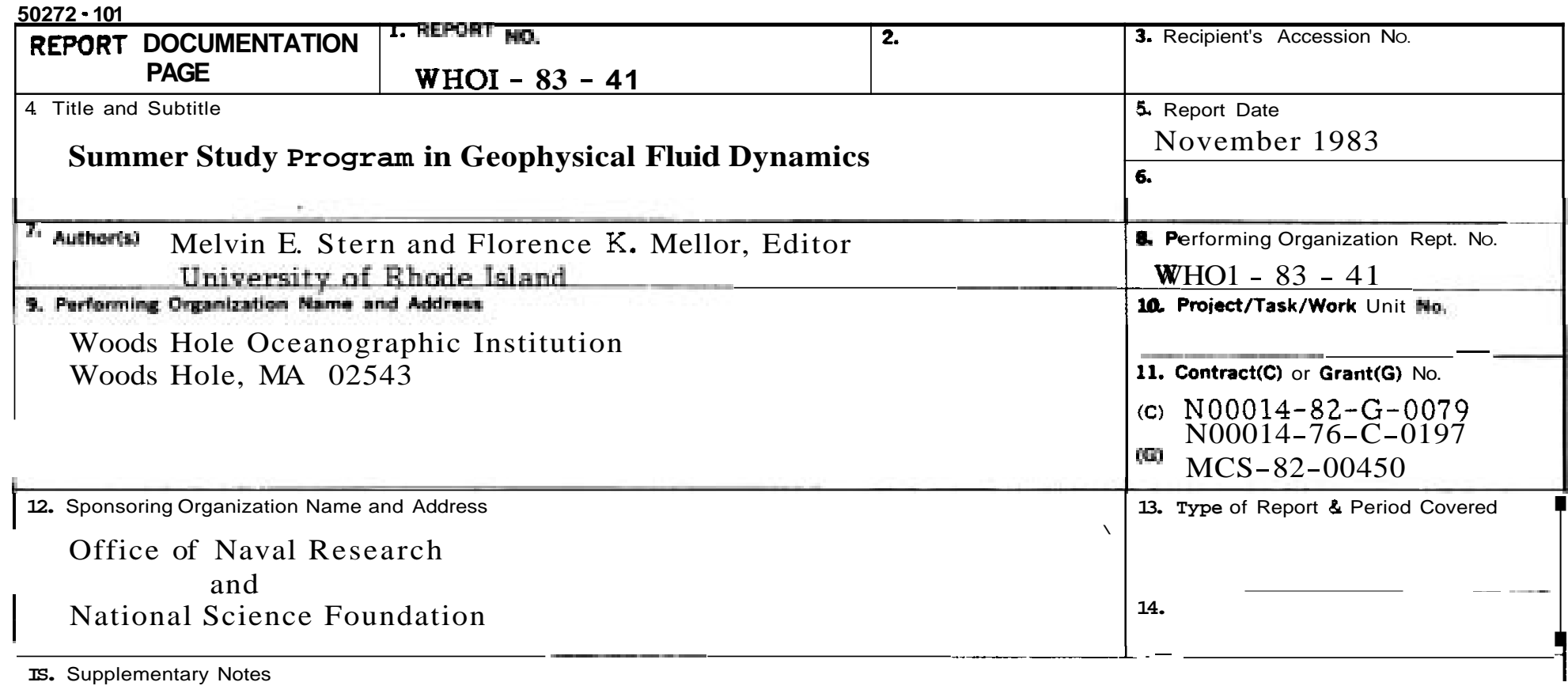

IS. Supplementary Notes

This report should be cited as Woods Hole Oceanog. Inst. Tech. Rept. WHOI - 83 - 41.

16. Abstract (Limit: 200 words)

Quasi-geostrophic baroclinic instability theory was reviewed, leading up to recent ageostrophic theories which consider the distinctive structure of an ocean front. Laboratory experiments on baroclinic instability theory were also reviewed. Surveys of different kinds of ocean fronts were given and observations on widely different scales were presented. The main theme was supplemented by a diversity of geophysical fluid dynamical subjects as exhibited by the research papers of each of the ten G.F.D. fellows.

17. Document Analysis a Descriptors

1. Geophysical Fluid Dynamics

2. Baroclinic instability

3. Ocean fronts

b. Identifiers/Open-Ended Terms

c. CosATI Fieid/Group

18. Availability Stateman:

Unlimited distribution.

\begin{tabular}{|l|l|}
\hline $\begin{array}{c}\text { 19. Security Class (This Report) } \\
\text { Unclassified }\end{array}$ & $\begin{array}{c}\text { 21. No. of Pages } \\
306\end{array}$ \\
\hline 20. Security Class (This Page) & 22 Price \\
\hline
\end{tabular}


DOQUMNT LIBRARY

\author{
June 16, 1982 \\ DISTRIBUTION LIST \\ BIBLIOGRAPHY AND TECHNICAL REPORT EXCHANGE
}

Library

R.S.M.A.S.

University of Miami

4600 Rickenbacker Causeway

Miami, FL 33149

Fisheries-Oceanography Library

151 Oceanography Teaching Bldg.

University of Washington

Seattle, WA 98195

Institute of Marine Sciences Library

University of Alaska

O'Neil1 Building

905 Koyukuk Ave. North

Fairbanks, AR 99701

PellMarine Science Library

University of Rhode Island

Narragansett Bay Campus

Narragansett, R.I. 02882

Institute of Geophysics

University of Hawaii

Library Room 252

2525 Correa Rd.

Honolulu, H I 96822

NOAA/EDIS Miami Library Center 4301 Rickenbacker Causeway

Miami, FL 33149

Library

Chesapeake Bay Institute

4800 Atwel1 Rd.

Shady Side, MD 20867

Working Collection

Texas A \& M University

Dept. of Oceanography

College Station, TX 77843
Hancock Library of Biology \& Oceanography

Alan Hancock Laboratory

University of Southern California

Los Angeles, CA 90007

Library

Lamont-Doherty Geological Observatory

Columbia University

Palisades, NY 10964

Library

Physical Oceanographic Laboratory

Nova University

8000 N. Ocean Drive

Dania, FL 33304

Library

Skidaway Institute of Oceanography

P.O. Box 13687

Savamah, GA 31406

U.S. Coast Guard Oceanographic Unit Library

Bldg. 159 E. Navy Yard Annex

Washington, DC 20590

Library

Virginia Institute of Marine Science

Gloucester Point, VA 23062

MT Libraries

Serial Journal Room 14E-210

Cambridge, MA 02139

Director, Ralph M. Parsons Laboratory

Room 48-311

MIT

Cambridge, MA 02139

Marine Resources Information Center

Bldg E38-320

MIT

Cambridge, MA 02139 